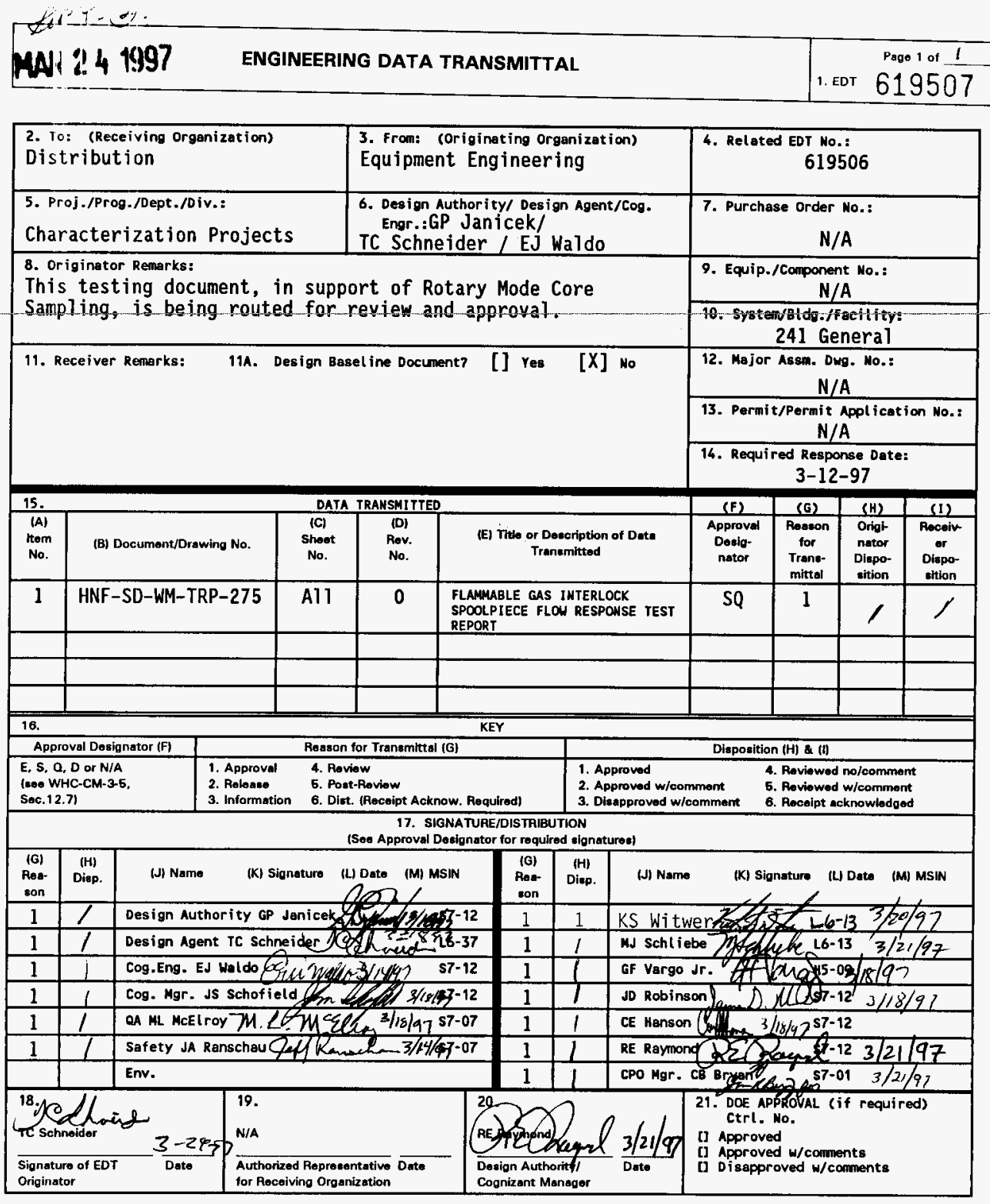

BO-7400-172-2(05/96) GEF097 


\title{
FLAMMABLE GAS INTERLOCK SPOOLPIECE FLOW RESPONSE TEST REPORT
}

\author{
TC Schneider
}

Numatec Hanford Corporation, Richland, WA 99352

U.S. Department of Energy Contract DE-AC06-96RL13200

EDT/ECN: EDT-619507

Org Code: 8C460

B\&R Code: EW3120072

UC: 2030

Charge Code: N4H2B

Total Pages: 300

Key Words: Flammable Gas Interlock, Hydrogen, Whittaker, SMC, Interlock, Electrochemical, Flow

Abstract: The purpose of this test report is to document the testing of the Whittaker electrochemical cell and the Sierra Monitor Corp. flammable gas monitors in a simulated field flow configuration. The sensors are used on the Rotary Mode Core Sampling (RMCS) Flammable Gas Interlock (FGI), to detect flammable gases, including hydrogen and teminate the core sampling activity at a predetermined concentration level.

TRADEMARK DISCLAIMER. Reference herein to any specific comercial product, process, or service by trade name, trademark, manufacturer, or otherwise, does not necesserily constitute or imply its endorsement, recommendation, or favoring by the united states Government or any agency thereof or its contractors or subcontractors.

Printed in the United States of Anerica. To obtain copies of this document, contact: WHC/BCS Document Control Services, P.O. Box 1970, Mailstop H6-08, Richland WA 99352, Phone (509) 372-2420; Fax (509) 376-4989.
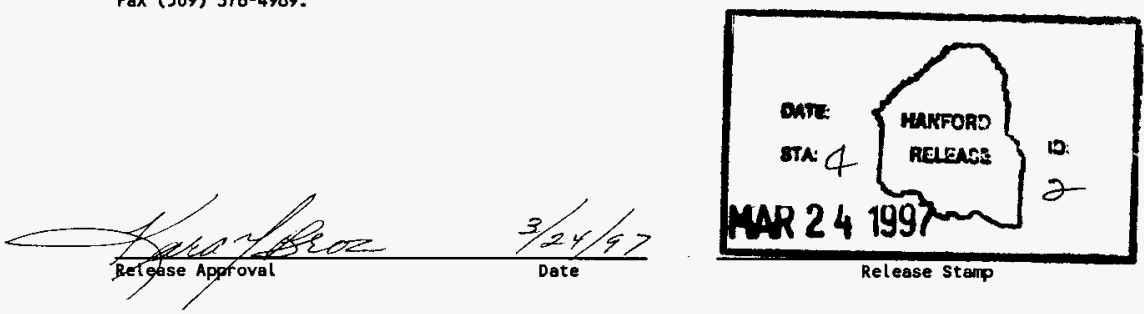

Approved for Public Release 


\title{
FLAMMABLE GAS INTERLOCK SPOOLPIECE FLOW RESPONSE
}

\author{
TEST REPORT
}

REV. 0

APPROVAL DESIGNATOR SQ

\section{T.C. Schneider}

Characterization Equipment Engineering Projects Numatec Hanford Corporation, Richland, Wa 99352

U.S. Department of Energy Contract DE-AC06-96RL13200 March, 1997 


\section{TABLE OF CONTENTS}

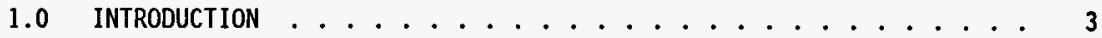

2.0 DESCRIPTION OF TEST ................. 3

3.0 TEST RESULTS ....................... T

3.1 SUMMARY ............................. 7

3.2 TEST EXCEPTIONS $\ldots \ldots \ldots$

4.0 CONCLUSIONS AND RECOMMENDATIONS ............. 13

4.1 CONCLUSIONS ......................... 13

4.2 RECOMMENDATIONS .................. 14

5.0 REFERENCES ......................... 14

APPENDIX A - MEASUREMENT AND TEST EQUIPMENT DATA SHEET . . . . . . 15

APPENDIX B - GAS BOTTLE RECORDS .............. 38

APPENDIX C - DATA RECORDS . . . . . . . . . . . . 40

APPENDIX D - EXCEPTION RECORDS ............... 246

APPENDIX E - TEST LOG RECORDS . . . . . . . . . . . 258

APPENDIX F - SUPPORTING DOCUMENTATION . . . . . . . . . 293 


\subsection{INTRODUCTION}

The purpose of this test report is to document the testing performed under the guidance of HNF-SD-WM-TC-073, Flammable Gas Interlock Spoolpiece Flow Response Test Plan and Procedure. This testing was performed for Lockheed Mart in Hanford Characterization Projects Operations (CPO) in support of Rotary Mode Core Sampling jointly by SGN Eurisys Services Corporation and Numatec Hanford Company. The testing was conducted in the 305 building Engineering Testing Laboratory (ETL). NHC provides the engineering and technical support for the 305 ETL. The key personnel identified for the performance of this task are as follows:

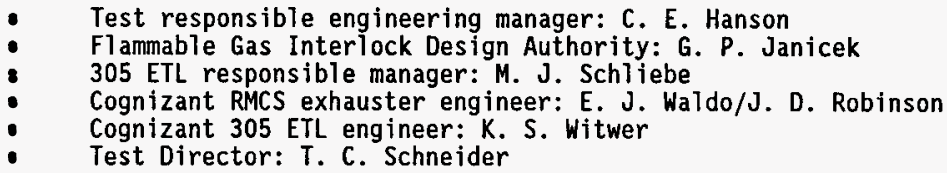

Other support personnel were supplied, as necessary, from 305/306 ETL.

The testing, on the flammable Gas Interlock (FGI) system spoolpiece required to support Rotary Mode Core Sampling (RMCS) of single shell flammable gas watch 1 ist tanks, took place between 2-13-97 and 2-25-97.

\subsection{DESCRIPTION OF TEST}

The primary purpose of the test was to evaluate the sensor gas delivery characteristics of the mechanical spoolpiece system by determining typical sensor response times as installed in their deployed configuration. The main components of the test setup included metallic and flexible conductive ducting, a blower and flow control valve capable of providing the required duct flow, an accurate flow measuring system, an accurate hydrogen injection gas system, a mechanically equivalent FGI spoolpiece with sensors, a gas chromatograph (GC) system to validate the duct gas concentrations, a data logger and associated process temperature and pressure instruments. The piping and instrumentation diagrams (P\&ID) of figures 1 and 2 depict the initial and alternate test hardware configurations. A listing of the measurement and test equipment used and pertinent calibration records are included in Appendix $A$. 
The test required a simulated exhauster flow between 180 and 220 SCFM be provided through the spoolpiece. The initial test set utilized the blower pushing the air through the spoolpiece. This allowed for the hydrogen gas to be mixed very conservatively since there were no spark sources downstream of the injection point. Once confidence was gained in the safe mixing of the hydrogen gas, a blower, qualified to transport flammable gas concentrations, was installed downstream of the sensors. This location provided a more prototypic field configuration by pulling the duct gas through the spoolpiece. The sensor outputs were required to reach $90 \%$ of their final output value in less than 120 seconds when subjected to an instantaneous mixed gas concentration. The final output value is defined as the sensor output after 15 minutes. Hydrogen gas was accurately metered and injected into the main duct 14 feet upstream of the sensors to provide a well mixed range of supplied gas concentrations. The equipment was operated in a laboratory environment with the nominal ambient temperatures between 65 and $75^{\circ} \mathrm{F}$. The FGI spoolpiece tested used two diverse detection sensors, a Whittaker electrochemical cell (WEC) and a Sierra Monitor Corporation (SMC) catalytic bead combustible gas cell. 


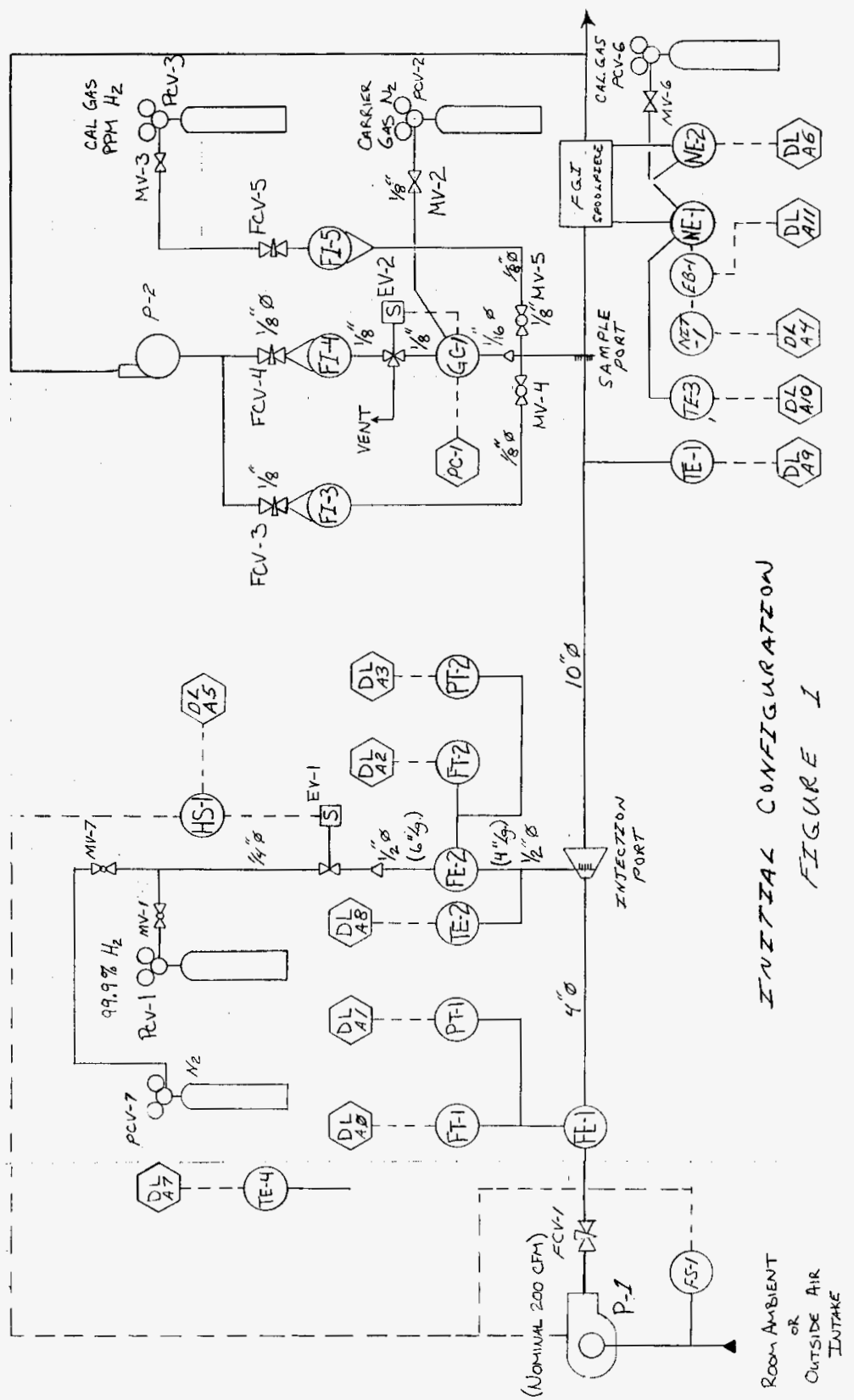




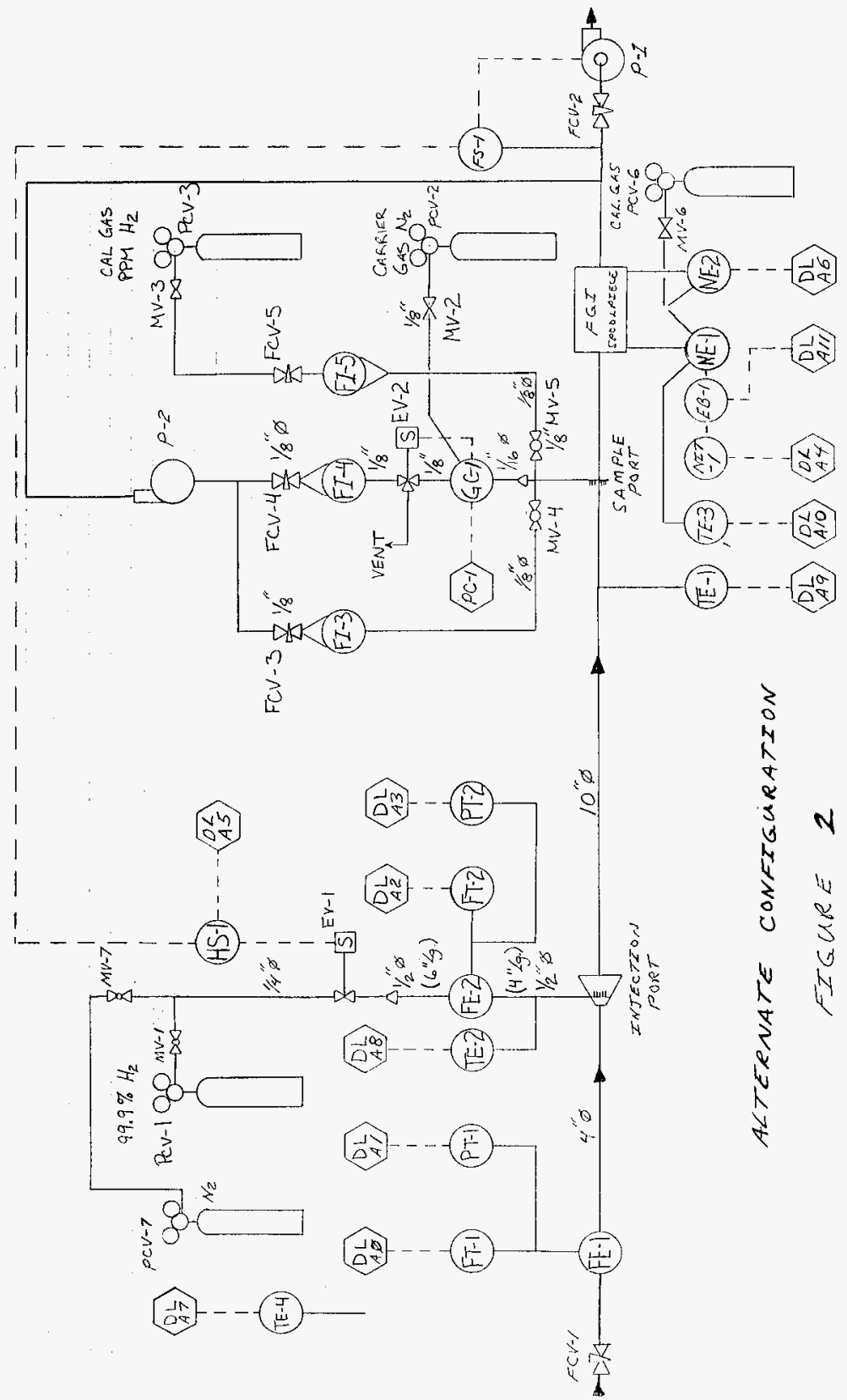


The testing was documented using both manual means and automatic data logging utilizing electronic media. The sequences of the test were recorded in a laboratory notebook, HNF-N-3-1, Testing and System Development Notes for 305 Engineering Testing Laboratory, Hanford Nuclear Facility and included in Appendix $E$. The data for the tests were recorded manually on individual data sheets, included in Appendix $\mathrm{C}$ and automatically by the data logger and GC. The data logger and GC data file printouts are also included in Appendix C.

\subsection{TEST RESULTS}

\subsection{SUMMARY}

The test plan and procedure, HNF-SD-WM-TC-073, allowed for flexibility in the test configuration and performance. The test report, TABLE 1 CONSOLIDATED TEST RESULTS, is used to present results from a combination of manually and automatically recorded data. The data was processed using Microsoft Excel Version 5.0. (Microsoft is a registered trademark of the Microsoft Corporation in the United States and other countries.) The consolidated test data supports the test plan FGI FLOW TEST MATRIX and test exception (TE) resolution testing. The GC and process data recorded on the data sheets were single points, nominally six minutes into the sample run, not averaged from the data files. Post testing evaluation of the data files indicates that only a slightly more precise instrument accuracy analysis could be obtained from averaging the measured concentrations. Since the data logger sample rate was selected to be 5 seconds, the instrument response times will be presented as the next 5 second interval following the achievement of $90 \%$ of the sensor final value. The mixed gas transport time from the injection point to the sensors was calculated to be between 2 and 3 seconds for all tests. That response time is within the data collection interval, will not be included on TABLE 1 and will be disregarded for system response time evaluation.

The test plan and procedure Table 1, FGI FLOW TEST MATRIX, required that a gas concentration mixture of $200-300 \mathrm{ppm}$ hydrogen be used for the first set of tests (ID. 1-1 through 1-3). The SMC sensor is not sensitive enough to accurately detect the low hydrogen concentrations and did not achieve a final value near the measured duct concentration. Although the WEC was able to accurately measure the low hydrogen concentration, the cell output indicated a very poor response time of nominally 460 seconds to $90 \%$ of the final value. A single test (ID. 2-4) was performed at a concentration of nominally $4,000 \mathrm{ppm}$ to confirm the response times measured with the nominal 200ppm duct gas. The WEC cell 4,000ppm response time of 440 seconds was consistent with the lower concentration response times. TE 1 was prepared to document the poor sensor response times. 


\begin{tabular}{|c|c|c|c|c|c|c|c|c|c|c|c|}
\hline \multicolumn{12}{|c|}{$\begin{array}{c}\text { TABLE } 1 \\
\text { CONSOLIDATED TEST RESULTS }\end{array}$} \\
\hline $\begin{array}{l}\text { TEST / DATA } \\
\text { FILE ID }\end{array}$ & $\begin{array}{l}\text { FLOW } \\
\text { SCFM }\end{array}$ & \multicolumn{2}{|c|}{$\begin{array}{l}\mathrm{H}_{2} \text { PPM CONCENTRATION } \\
\text { MEAS. CALC. }\end{array}$} & \multicolumn{2}{|c|}{$\begin{array}{l}\text { 90\% RESPONSE SEC. } \\
\text { SMC WEC }\end{array}$} & \multicolumn{2}{|c|}{$2 \underset{\text { SMC }}{\text { MIN. PPM VALUE }}$} & \multicolumn{2}{|c|}{$\begin{array}{l}14.5 \text { MIN. PPH VALUE } \\
\text { SMC }\end{array}$} & \multicolumn{2}{|c|}{$\begin{array}{l}15 \text { MIN. PPN VALUE } \\
\text { SMC } \\
\text { ACCCURACY TO MEC } \\
\text { VALUES. }\end{array}$} \\
\hline $1-1 / F G I 21311$ & 197.8 & 237.8 & 232.4 & 25 & 400 & 80.2 & 100.3 & 79.3 & 240.4 & $\begin{array}{l}79.7 \\
(-66 x)\end{array}$ & $\begin{array}{l}240.4 \\
(+1 x)\end{array}$ \\
\hline $1-2 /$ FGI21312 & 198.5 & 236 & 232 & 25 & 460 & 88.3 & 90.2 & 84.1 & 240.4 & $\begin{array}{l}84.4 \\
(-64 x) \\
\end{array}$ & $\begin{array}{l}240.4 \\
(+2 x)\end{array}$ \\
\hline $1-3 /$ FGI21313 & 199.0 & 235 & 229 & 30 & 470 & 86.1 & 80.2 & 86.9 & 230.3 & $\begin{array}{l}87.8 \\
(-63 \%)\end{array}$ & $\begin{array}{l}230.4 \\
(-2 x)\end{array}$ \\
\hline $2-4 / F G I 21324$ & 198.7 & 4054 & 3707 & 30 & 440 & 3228 & 1733 & 3958 & 4226 & $\begin{array}{l}3963 \\
(-2 x) \\
\end{array}$ & $\begin{array}{l}4226 \\
(+4 x) \\
\end{array}$ \\
\hline EX1-1/EX102137 & 199.2 & 4040 & 3656 & $N / A$ & 240 & $N / A$ & 2834 & $N / A$ & 4425 & $N / A$ & $\begin{array}{l}4426 \\
(+9 x)\end{array}$ \\
\hline EX1-2/EX202137 & 198.7 & 4100 & 3656 & 25 & 75 & 4007 & 4255 & 3968 & 4406 & $\begin{array}{l}3961 \\
(-3 \%)\end{array}$ & $\begin{array}{l}4386 \\
(+7 x)\end{array}$ \\
\hline EX1-3/EX102147 & 197.8 & 4150 & 3722 & 35 & 65 & 4026 & 4095 & 4078 & 4306 & $\begin{array}{l}4082 \\
(-2 x)\end{array}$ & $\begin{array}{l}4306 \\
(+4 x)\end{array}$ \\
\hline $1-1 /$ FGI22211 & 200.4 & 219 & 207.6 & 25 & 85 & 59.2 & 211.4 & 57.9 & 221 & $\begin{array}{l}57.3 \\
(-74 x)\end{array}$ & ${ }_{(+1 x)}^{221.4}$ \\
\hline $1-2 /$ FGI22212 & 200.9 & 218 & 206.4 & 25 & 110 & 54 & 211.3 & 53 & 231.4 & $\begin{array}{l}52.5 \\
(-76 x)\end{array}$ & $\begin{array}{l}230 \\
(+5 x)\end{array}$ \\
\hline
\end{tabular}




\begin{tabular}{|c|c|c|c|c|c|c|c|c|c|c|c|}
\hline \multicolumn{12}{|c|}{$\begin{array}{c}\text { TABLE } 1 \\
\text { CONSOLIDATED TEST RESULTS }\end{array}$} \\
\hline $\begin{array}{l}\text { TEST ' DATA } \\
\text { FILE ID }\end{array}$ & $\begin{array}{l}\text { FLOW } \\
\text { SCFM }\end{array}$ & \multicolumn{2}{|c|}{$\begin{array}{l}\mathrm{H}_{2} \text { PPM CONCENTRATION } \\
\text { MEAS. CALC. }\end{array}$} & \multicolumn{2}{|c|}{$\begin{array}{l}\text { 90\% RESPONSE SEC. } \\
\text { SMC }\end{array}$} & \multicolumn{2}{|c|}{2 MIN. PPM VALUE } & \multicolumn{2}{|c|}{$\begin{array}{l}14.5 \text { HIN. PPH VALUE } \\
\text { SMC }\end{array}$} & \multicolumn{2}{|c|}{$\begin{array}{l}15 \text { MIN. PPW VALUE } \\
\text { SHC } \\
\text { CACCURACY TO MEC } \\
\text { VALUE) }\end{array}$} \\
\hline $1-3 / F G I 22213$ & 201.1 & 217 & 204.3 & 25 & 70 & 48 & 211.3 & 47.4 & 218 & $\begin{array}{l}48 \\
(-78 \%) \\
\end{array}$ & $\begin{array}{l}210.6 \\
(-3 x)\end{array}$ \\
\hline $2-1 / F G I 22221$ & 204.0 & 2029 & 1918 & 25 & 80 & 1893 & 2012 & 1878 & 2093 & $\begin{array}{l}1885 \\
(-7 x)\end{array}$ & $\begin{array}{l}2093 \\
(+3 x)\end{array}$ \\
\hline $2-2 / F G I 22222$ & 199.6 & 2170 & 2055 & 30 & 80 & 2006 & 2173 & 2023 & 2274 & $\begin{array}{l}2030 \\
(-6 \%)\end{array}$ & $\begin{array}{l}2274 \\
(+5 x)\end{array}$ \\
\hline 2-3/FGI 22223 & 200.1 & 2159 & 2031 & 30 & 90 & 1973 & 2142 & 1988 & 2263 & $\begin{array}{l}1999 \\
(-7 x)\end{array}$ & $\begin{array}{l}2264 \\
(+5 \%)\end{array}$ \\
\hline $2-4 / F G I 22224$ & 199.6 & 4355 & 4182 & 30 & 90 & 4240 & 4335 & 4324 & 4606 & $\begin{array}{l}4335 \\
(-.5 \%)\end{array}$ & $\begin{array}{l}4605 \\
(+6 \%)\end{array}$ \\
\hline $2-5 / F G I 22225$ & 200.1 & 4373 & 4169 & 30 & 90 & 4252 & 4345 & 4324 & 4636 & $\begin{array}{l}4348 \\
(-.6 \times)\end{array}$ & $\begin{array}{l}4636 \\
(+6 \%)\end{array}$ \\
\hline $2-6 / F G I 22226$ & 199.6 & 4377 & 4138 & 25 & 90 & 4267 & 4365 & 4287 & 4655 & $\begin{array}{l}4304 \\
(-2 x)\end{array}$ & $\begin{array}{l}4656 \\
(+6 x)\end{array}$ \\
\hline 2-7/FGI 22227 & 199.6 & 6432 & 6235 & 30 & 95 & 6373 & 6466 & 6477 & 6918 & $\begin{array}{l}6485 \\
(-.8 \times)\end{array}$ & $\begin{array}{l}6928 \\
(+8 x)\end{array}$ \\
\hline $2-8 / F G I 22228$ & 199.2 & 6665 & 6269 & $N / A$ & $N / A$ & $N / A$ & $N / A$ & N/A & $N / A$ & $N / A$ & N/A \\
\hline
\end{tabular}




\begin{tabular}{|c|c|c|c|c|c|c|c|c|c|c|c|}
\hline \multicolumn{12}{|c|}{$\begin{array}{c}\text { TABLE } 1 \\
\text { CONSOLIDATED TEST RESULTS }\end{array}$} \\
\hline $\begin{array}{l}\text { TEST / DATA } \\
\text { FILE ID }\end{array}$ & $\begin{array}{l}\text { FLON } \\
\text { SCFM }\end{array}$ & \multicolumn{2}{|c|}{$\begin{array}{l}\mathrm{H}_{2} \text { PPM CONCENTRATLON } \\
\text { MEAS. CALC. }\end{array}$} & \multicolumn{2}{|c|}{$\begin{array}{l}\text { 90X RESPONSE SEC. } \\
\text { SMC } \quad \text { WEC }\end{array}$} & \multicolumn{2}{|c|}{$2 \underset{\text { SMC }}{\text { MIN. PPM VALUE }}$ MEC } & \multicolumn{2}{|c|}{$\begin{array}{l}14.5 \mathrm{MIN} \text {. PPM VALUE } \\
\text { SHC }\end{array}$} & \multicolumn{2}{|c|}{$\begin{array}{l}15 \text { MIN. PPM VALUE } \\
\text { SMC } \\
\text { (ACCURACY TO MEC } \\
\text { VALUE) }\end{array}$} \\
\hline 2-8A/FG22228A & 200.2 & 6523 & 6279 & 30 & 95 & 6437 & 6566 & 6534 & 7068 & $\begin{array}{l}6547 \\
(+.4 x)\end{array}$ & $\begin{array}{l}7069 \\
(+8 \%)\end{array}$ \\
\hline 2-9/FGI22229 & 199.3 & 6480 & 6289 & 30 & 90 & 6388 & 6586 & 6438 & 7008 & $\begin{array}{l}6475 \\
(0 *)\end{array}$ & $\begin{array}{l}7008 \\
(+8 \%)\end{array}$ \\
\hline EX3-1/EX102227 & 180.8 & 4348 & 4149 & 35 & 95 & 4012 & 4475 & 4041 & 4776 & $\begin{array}{l}4072 \\
(-6 x)\end{array}$ & $\begin{array}{l}4776 \\
(+10 x)\end{array}$ \\
\hline EX3-2/EX202227 & 180.9 & 4340 & 4135 & 30 & 90 & 4013 & 4485 & 4026 & 4766 & $\begin{array}{l}4030 \\
(-7 x)\end{array}$ & $\begin{array}{l}4766 \\
(+10 \%)\end{array}$ \\
\hline EX3-3/EX302227 & 180.7 & 4330 & 4122 & 30 & 90 & 3973 & 4465 & 4049 & 4756 & $\begin{array}{l}4026 \\
(-7 x)\end{array}$ & $\begin{array}{l}4766 \\
(+10 \%)\end{array}$ \\
\hline EX4-1/EX102257 & 198.97 & 3800 & 3696 & 25 & 85 & 3667 & 3745 & 3706 & 3976 & $\begin{array}{l}3707 \\
(-2 x)\end{array}$ & $\begin{array}{l}3976 \\
(+5 \%)\end{array}$ \\
\hline EX4-2/EX202257 & 199.98 & 4217 & 4094 & 25 & 85 & 4067 & 4195 & 4120 & 4436 & $\begin{array}{l}4137 \\
(-2 x)\end{array}$ & $\begin{array}{l}4436 \\
(+5 \%)\end{array}$ \\
\hline EX4-3/EX302257 & 199.0 & 4210 & 4120 & 25 & 85 & 4054 & 4236 & 4135 & 4466 & $\begin{array}{l}4128 \\
(-2 x)\end{array}$ & $\begin{array}{l}4466 \\
(+6 x)\end{array}$ \\
\hline EX4-4/EX402257 & 200.3 & 4210 & 4105 & 25 & 85 & 4046 & 4225 & 4127 & 4486 & $\begin{array}{l}4136 \\
(-2 x)\end{array}$ & $\begin{array}{l}4486 \\
(+6 \%)\end{array}$ \\
\hline
\end{tabular}


Following the spoolpiece modification, required by the resolution to TE 1 , the original test system configuration of Figure 1 was changed to the al ternate system configuration of Figure 2. This allowed a more prototypic field condition to be met by drawing the duct gases through the spoolpiece and having the system under negative pressures. The test duct pressures $(-3.5$ to $\left.-4.5 \mathrm{H}_{2} \mathrm{O}\right)$ are slightly more negative than the field operating pressures $(-1$ to $-2{ }^{2} \mathrm{H}_{2} \mathrm{O}$ ), but will not affect the test outcome.

While performing the initial calibration of the instruments with the alternate configuration, it was noted that the SMC flowmeter was at full scale with 200 SCFM of duct flow. The flow was reduced to 190 SCFM to allow the sensor flow to be duplicated during the SMC calibration procedure. The actual duct flow was then set to 200 SCFM for the next series of tests (ID. 1-1 through 2-9). As can be seen from the consolidated test data Table 1, the flow difference between 190 and 200 SCFM did not make a significant difference in the SMC sensor final output signal. However, when the flow was reduced to 180 SCFM to support TE-3 testing, the SMC read between 3 and 5\% lower than the readings obtained at 200 SCFM. This error is still within the acceptable range for the instrument.

It should be noted that the WEC and signal conditioning calibration span was established only at the beginning of the testing, due to the limited amount of selected calibration gas. Subsequent adjustments to the WEC signal conditioning only involved zero offset, which proved to be adequate.

The testing was successfully completed with the resolutions of TE 2 through TE 4, which are discussed in the following Section 3.2.

\subsection{TEST EXCEPTIONS}

3.2.1 TE 1 was prepared to document the poor WEC response times. The proposed TE resolution involved providing continual duct gas flow through the WEC measurement chamber to enhance the response time.

The initial test misinterpreted the location of the WEC cell relative to the spoolpiece baffle. The SMC high pressure port was routed to the WEC calibration port as a high pressure point to drive the gas through the chamber. Since there was only a slightly higher static pressure at that point, relative to the WEC cell measurement chamber, only a $50 \%$ improvement in the response time was achieved. 
Acceptable WEC cell measurement chamber flow was accomplished using the pressure drop of the spoolpiece baffle to draw duct gas through the $0.10^{\prime \prime}$ diameter diffusion port in the WEC cell housing and out the housing calibration port. Since the WEC housing was located upstream of the baffle, a downstream static pressure pitot tube was installed to provide the low pressure drive source for the chamber flow. The chamber flow improved the WEC response time to well below the required value, as noted by the TABLE 1 consolidated test results data. Engineering change notice (ECN) 640420 , salient portions of which are included in the test $\log$ APPENDIX E, was prepared to provide a metered flow through the WEC cell and a three way isolation valve to select calibration or duct gas flow through the WEC. The test spoolpiece was modified in the $306 \mathrm{E}$ weld shop to incorporate the changes of ECN 640420 .

3.2.2 TE 2 was prepared to document a hydrogen injection gas problem. The repeat of the complete FGI FLOW TEST MATRIX was performed successfully until Test ID No. 2-8. The hydrogen injection gas pressure regulator could not maintain a stable flow due to very low bottle pressure. The test was terminated at approximately 14 minutes into the test run. A new test gas bottle of hydrogen was installed and the test was repeated as ID No. 2-8A. The last test, ID 2-9, was performed at 200 SCFM with the WEC and the SMC successfully meeting the required signal response times for all the tests.

3.2.3 TE 3 was prepared to allow a series of response tests to be performed at 180 SCFM, since the current field spoolpiece flow is nominally 180 SCFM due to the flow lost to the record sampler. The flow was adjusted without adjusting the WEC and SMC instrument calibrations, and three tests were run at nominally $4,000 \mathrm{ppm}$ hydrogen concentration. The sensor response times were consistent with the previous testing and well below the requirement. The SMC output indication was also consistent with the values displayed at 200 SCFM.

3.2.4 TE 4 was prepared to document that the SMC flowmeter was full scale high at 200 SCFM. The TE resolution installed $0-1,000 \mathrm{ccm}$ rotameters to monitor the WEC and SMC duct gas flow. The WEC and SMC sensor signal conditioners were adjusted in preparation for a series of three tests to resolve TE 4 .

The initial hydrogen injection gas parameters were repeated from the last, 180 SCFM test. Due to the higher duct flow, the duct concentration values were below $4,000 \mathrm{ppm}$. The hydrogen injection gas flow was adjusted upward between the first and second test runs to provide the hydrogen concentration closer to $4,000 \mathrm{ppm}$. At approximately 660 seconds into the third test to resolve TE 4, a negative going spike was observed in the WEC signal. Even though there was valve isolation between the purge gas and the WEC cell, it appeared that the monitored duct gas had been momentarily interrupted to the WEC. A fourth test was run to verify proper cell operation. 
In a subsequent conversation with Whittaker Safety Systems Jerry Fuller, it was indicated that the probable cause of the spike was due to a gas bubble in the cell electrolyte solution. Resident gas in the electrolyte solution may move and come in contact with one of the electrodes causing an interference with the cell ion transport process. This interruption is identified by a positive or negative output signal spike. These signal spikes are short lived as the gas bubble passes or comes in contact with one of the electrodes. The vendor recommended that the cell operational orientation be vertical with the internal electrodes pointing down, which allows the gas bubbles to accumulate near the top of the cell away from the active portions of the sensing electrodes. The FGI spoolpiece WEC cell is mounted in a horizontal configuration.

\subsection{CONCLUSIONS AND RECOMMENDATIONS}

The primary purpose of the testing was to verify the response time of the flammable gas sensors in simulated field configuration. The response time requirement from WHC-SD-WM-SAD-035, A Safety Assessment of Rotary Mode Core Sampling in Flammable Gas Single Shell Tanks, was that the sensor output would achieve $90 \%$ of the final value in 120 seconds or less. Although there is no stated accuracy requirement in WHC-SD-WM-SAD-035, the test plan suggested that the sensor outputs be compared to the GC measured gas concentrations to determine their ability to accurately represent the duct gas concentrations.

\subsection{CONCLUSIONS}

4.1.1 The SMC and WEC flammable gas monitoring cells both easily met the required response times in the simulated exhaust duct and spoolpiece flow and pressure conditions, following the modifications to the WEC cell sample delivery system.

4.1.2 Both sensors stayed within $10 \%$ of the actual gas chromatograph measured concentrations between 2,000 and $6,500 \mathrm{ppm}$. Adequate testing was not performed to completely characterize the accuracy of the two sensors over their entire operating range or over an extended time. The SMC was not sensitive enough to accurately measure the very 10w, 200ppm concentrations of hydrogen; however, the limited testing at the additional nominal concentrations of $2,000,4,000$ and $6,000 \mathrm{ppm}$ indicated that the sensors are reasonably accurate. The WEC cell output typically read higher than the SMC and tended to slowly increase with extended exposure to hydrogen. Although this will decrease the absolute accuracy, it provides conservative hydrogen concentration values. 


\subsection{RECOMMENDATIONS}

4.2.1 The vertical mounting orientation recommended by the vendor should be implemented in the future for improved cell output signal stability, since the horizontal orientation of the WEC cell may continue to cause some intermittent instability in the cell output due to the mobile gas bubbles.

\subsection{REFERENCES}

* WHC-SD-WM-SAD-035, A Safety Assessment of Rotary Mode Core Sampling in Flammable Gas Single Shell Tanks, Rev. OA, Los Alamos National Laboratory, Los Alamos New Mexico, August 1996.

* HNF-SD-WM-TC-073, Flammable Gas Interlock Spoolpiece Flow Response Test Plan and Procedure, Rev. 0, Numatec Hanford Corporation, Richland Washington, Feb. 13, 1997

* HNF-N-3-1, Testing and System Development Notes for 305 Engineering Testing Laboratory, Hanford Nuclear Facility. Issued to KS Witwer, $1 / 8 / 97$. 
FE-1 Main Duct Flow Element Laminar Flow Element, Meriam Inst. Model 50MC24, Serial No. 757200-Kl

Range: 0-424.6 SCFM air at std. temp./press.

Accuracy: $\pm 1.0 \%$ of reading

Cal. Std.: Vendor Cal. Record Curve

FT-1 Main Duct Flow Xmitter Diff. Press., Rosemount, Model 3051CD, Serial No. 32686

Range: $0-25$ " $\mathrm{H}_{2} \mathrm{O}$

Accuracy: $\pm 0.1 \%$ FS

Cal. Std.: Std. Lab. No. 750-80-02-026

Ca1. Due Date: 2-3-98

PT-1 Main Duct Press Xmitter Abs. Press., Rosemount, Model 3051CD, Serial No. 65843

Range: $0-30$ psia

Accuracy: $\pm 0.1 \%$ FS

Cal. Std.: Std. Lab. No. 750-80-02-023

Cal. Due Date: 2-3-98

FE-2 Injection Flow Element Laminar Flow Element, Meriam Inst. Model 50MJ1010, Serial No. 753510-J3

Range: 0-1.6087 SCFM air at std. temp./press.

Accuracy: $\pm 1.0 \%$ of reading

Cal. Std.: Vendor Cal. Record Curve

FT-2 Injection Flow Xmitter Diff. Press., Rosemount, Model 3051CD, Serial

No. 33807

Range: $0-25 \mathrm{H}_{2} \mathrm{O}$

Accuracy: $\pm 0.1 \%$ FS

Cal. Std.: Std. Lab. No. 750-80-02-025

Cal. Due Date: 2-3-98

PT-2 Injection Press Xmitter Abs. Press., Rosemount, Model 3051CD, Serial No. 0266492

Range: $0-30$ psia

Accuracy: $\pm 0.1 \%$ FS

Cal. Std.: Std. Lab. No. 355-80-02-055

Cal. Due Date: 5-6-97

FI/FCV-3 GC Bypass Flow

Rotameter with outlet control valve, Wallace \& Tiernan, Mode1 32E083S1XX3

Range: $0-2.5 \mathrm{CFH}$ air

Accuracy: $\pm 10 \%$ FS

Ca1.: Test Log Comparison (Indication Only) 
FI-4 GC Sample Flow

FI/FCV-5 GC Cal. Flow

TE-1

$\mathrm{TE}-2$

TE-3

TE-4

GC-1 $\mathrm{H}_{2}$ Concentration Std.

PC-1 GC Control Computer

DL-1 System Data Logger
Rotameter, Matheson, Model FM-1050 E1-4Y101-E910

Range: $0-100 \mathrm{ccm}$ air

Accuracy: $\pm 5 \%$ FS

Cal.: Test Log Comparison (Indication Only)

Rotameter with inlet control valve, Wallace \& Tiernan, Mode1 32E083S1XX2

Range: $0-2.5 \mathrm{CFH}$ air

Accuracy: $\pm 10 \%$ FS

Cal.: Test Log Comparison (Indication Only)

Type $K$ stainless steel jacketed thermocouple Cal.: Std. Lab. No. 750-78-02-013 S/N 002

Cal. Due Date: 2-5-98

Type $K$ stainless steel jacketed thermocouple Cal.: Std. Lab. No. 750-78-02-013 S/N 236

Cal. Due Date: 2-5-98

Type $\mathrm{K}$ stainless steel jacketed thermocouple Cal.: Std. Lab. No. 750-78-02-013 S/N 001

Cal. Due Date: 2-5-98

Type $K$ stainless steel jacketed thermocouple Cal.: Std. Lab. No. 750-78-02-013 S/N 400

Cal. Due Date: 2-5-98

Dual Column GC, Micro-sensor Tech. Inc., Model M200D, Serial No. 150313

Range: $100-30,000 \mathrm{ppm}$

Accuracy: $10 \%$ Reading

Cal.: Standard Gas per Appendix F-3

Lap Top PC, Toshiba Satellite Pro Model 405CS, Serial No. 02628992-1

Range: N/A

Accuracy: N/A

H-P Data Logger, Model 3497A, Serial No. 2222A09660

Range and Accuracy: $0-0.1 \mathrm{Vdc}( \pm 18 u V d c), 0-100$

$\operatorname{Vdc}( \pm 0.3 \mathrm{mVdc}), \mathrm{T} / \mathrm{C}\left( \pm 1^{\circ} \mathrm{C}\right)$

Ca1.: Std. Lab. No. 752-67-11-002

Ca1. Due Date: 2-5-98 
NIT-1 Whittaker Sig. Cond. Newport Infinity Voltmeter, Model INFU-0010-DC1

Range: Input (Nom. 0-1mV DC), Output 4-20mA DC Accuracy: Input $\pm 0.005 \%$ of readings, Output: $\pm 0.1 \%$ Full Scale Calibration performed by Appendix F-2 using standard gases and input from the WEC under test

NE-1 Whittaker Sensor

NE-2 SMC Sensor

Handheld Digital Multimeter

Reference Rotameter
Electro-Chemical Cell, Whittaker, Model 10900202, Serial No. HS-1226

Range: $0-10 \% \mathrm{H}_{2}$ by Volume

Accuracy: $\pm 0.2 \%$ Absolute

Cal.: Standard Gas per Appendix F-2

Combustible Gas Module, Sierra Monitor Corp., Model 4101-02, Serial No. 96-B-4455

Range: $0-100 \%$ LFL $\mathrm{H}_{2}$

Accuracy: $\pm 3 \%$ FS

Cal.: Standard Gas per Appendix F-1

Fluke Model 702, Serial No. 6240608

Cal.: Std. Lab. No. 750-13-71-001

Cal. Due Date: 12-06-97

Wallace and Tiernan Model NPXQ-143

Range: $0-6 \mathrm{SCFH}$

Accuracy: $\pm 10 \%$ FS

Cal.: Std. Lab. No. 750-28-13-005

Cal. Due Date: 01-23-98 


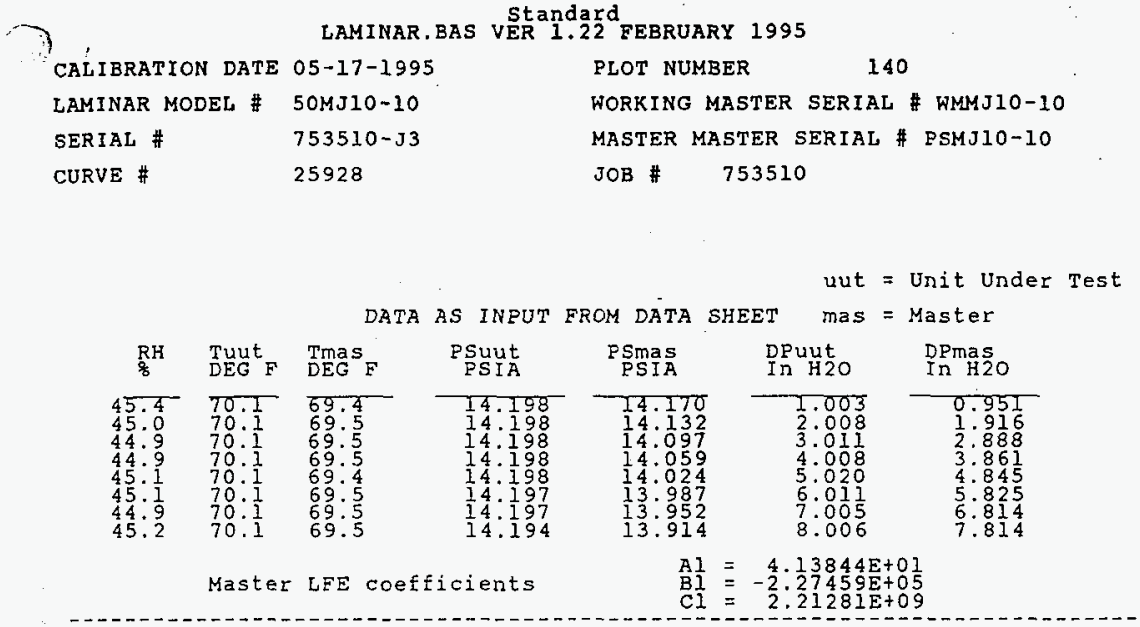

REDUCED DATA, BASED ON MASTER LFE COEFFICIENTS:

\begin{tabular}{|c|c|c|c|c|c|}
\hline $\begin{array}{l}\text { DATA } \\
\text { POINT }\end{array}$ & $\begin{array}{l}\mathrm{DP} \\
\text { InH } 20 \mathrm{OU} \\
4 \mathrm{C}\end{array}$ & $\begin{array}{l}\text { FLOW IN ACFM } \\
\text { BASED ON MASTER }\end{array}$ & $\begin{array}{l}\text { CFM* (DATA) } \\
\text { BASED ON MASTER }\end{array}$ & $\begin{array}{l}C F M * \\
B^{\star} D P+C^{\star} D P^{*}\end{array}$ & $\begin{array}{l}\text { PERCENT } \\
\text { ERROR } \star *\end{array}$ \\
\hline $\begin{array}{l}1 \\
2 \\
3 \\
4 \\
5 \\
6 \\
7 \\
8\end{array}$ & $\begin{array}{l}1.003 \\
2: 008 \\
3.011 \\
4.008 \\
5.020 \\
6.011 \\
7.005 \\
8.006\end{array}$ & $\begin{array}{l}0.214139 \\
0.245349 \\
0.632727 \\
0.835089 \\
1.034980 \\
1.229194 \\
1.411411 \\
1.611889\end{array}$ & $\begin{array}{l}0.214070 \\
0.425211 \\
0.632544 \\
0.834847 \\
1.034681 \\
1.228399 \\
1.421000 \\
1.611424\end{array}$ & $\begin{array}{l}0.214050 \\
0.425200 \\
0.632177 \\
0.834445 \\
1.036035 \\
1.229967 \\
1.420994 \\
1.609749\end{array}$ & $\begin{array}{r}-0.01 \\
-0.00 \\
-0.06 \\
-0.05 \\
0.13 \\
0.09 \\
-0.00 \\
-0.10\end{array}$ \\
\hline
\end{tabular}

A Least Squares Fit of the CEM(DATA) yields the foll lowing formula and LFE uut coefficients used to generate the CFM(CURVE) values:

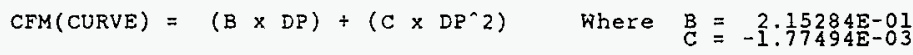

A Flow of $\quad 1.608675$ CFM produces a UUT DP of 8.00 In $H 2 O$ TERMINAL NON-LINEARITY $=3.3158$ FOR DETAILS SEE INDEPENDENT NON-LINEARITY $=1.0698$ FILENO. 501:440

PRINTOUT DATE : 05-18-1995

Filename : 75351003.300 
$\because$

LAMINAR.BAS VER 1.26 NOVEMBER 2995

CALIBRATION DATE 02-07-2996

LAMINAR MODEL \# 5OMC2-4 SERIAL †

CURVE * $757200-\pi 2$

26862
PLOT NOMBER

126

KORKING MASTER SERIAL 68-9606

MASTER MASTER SERIAL $66-8606-2$

JOB $\quad 757200$
DATA AS INPUT FROH DATA SHEET

uut = Unit Under Test

mas = Master

RH
8
16.6
$15: 6$
$15: 6$
$16: 6$
$16: 6$
$16: 6$
$16: 6$
16.6

Tuut
DEG F
68.6
68.7
68.8
69.0
69.2
69.5
69.7
69.9

$\operatorname{Timas}$

PSUUt

69.6

69.6

69.6

69.7

69.7

69.7

69.8

PSIA
14.345
$14: 342$
14.334
$14: 327$
$14: 319$
$14: 305$
14.292
14.278

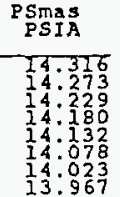

Deunt

In $\mathrm{H} 20$

DPmas

Master LFE coefficients

Al $=9.46020 E+03$

$B I=-2.73395 E+07$

CI $=-2.727140 E+11$

$\therefore$ DATA DP UUt

REDUCED DATA, BASED ON MASTER LFE COEFFICIENTS:

1
2
3
4
5
6
7
8 BASED ON WASTER

BASED ON HASTER

$1.009 \quad 54.652706$

$\frac{2}{3} .0142$

4.028

5.036

5.024

$7: 994$

108.816788

162.897992

216.485642

269.480673

321.781332

374.669687
424.279641

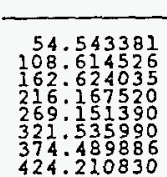

$\underset{B * D E}{C F * C D P}$ (CURVE)

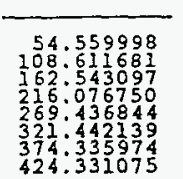

PERCENT
ERROR $\star$

0.03

$-0.00$

$-0.05$

$-0.04$

$-0.03$

$-0.03$

*CFM $=$ ACFM $\times$ (Flowing viscosity in Micropoise / 181.87)

* FERCENT ERROR = ( (CURVE-DATA) / DATA) * 100

A Least squares fit of the CFM(DATA) Yields the following formula and LFE uut coefficients used to generate the CFH(CURVE) values:
$\operatorname{CFH}(C U R V E)=(B \times D P)+\left(C \times D P^{\wedge} 2\right)$
Where
$B=5.42091 E+0 I$
$C=-1.41293 E-01$

A Flow of 424.629974 CEM produces a OJT DP of 8.00 In H2O TERMINALNON-LINEARITY $=1.080 \%$ FOR DETAILS SEE

PRINTOUT DATE :02-08-1996

Filename : 75720001.100

U. 
HANFORD STANDARDS LABORATORY PHYSICAL AND ELECTRICAL REPORT

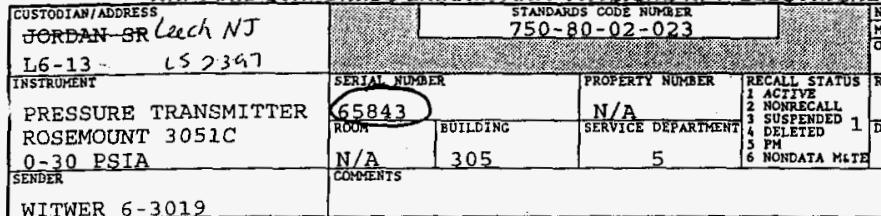

WITWER $6-3019$

meG.spees $\% .1 \%$. $1 \%$

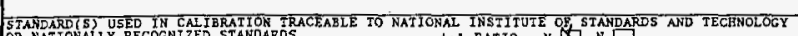

OR NATIONAILY RECOGNIZED STANDARDS

$002.77-01-0056-6-59$

$00245.080472-37-97$

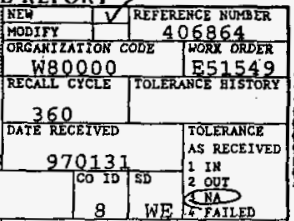

TRAINING HOURS

CALIBFATIOA hOURS 2.0

REPAIR HOURS

OTEER BOURS

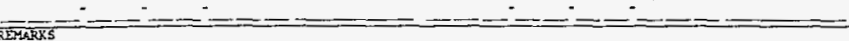

(WP-21.6)

ENG/ADMIN HOURS

MATERIALS

TOTAL CBARGE -

(\$1SO $\times S$ SIM OF HOTRS) + MATERIAL

\section{Used on the Cominer Flow Element}

\section{neasuring Lange Volume $M_{\text {uxed }} \mathrm{G}_{25}$}

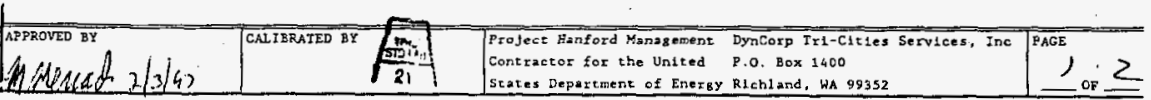


HNF-SD-WM-TRP- 275

Rev 0
Page 21

\begin{tabular}{|l|l|l|}
\hline $\begin{array}{l}\text { PROCEDURE HIME - 304 } \\
\text { WHC-5-RMT-3051CA REY.3 GENERIC }\end{array}$ & $750-80-02-023$ & $406864 \%$ \\
\hline
\end{tabular}

DATA SHEET

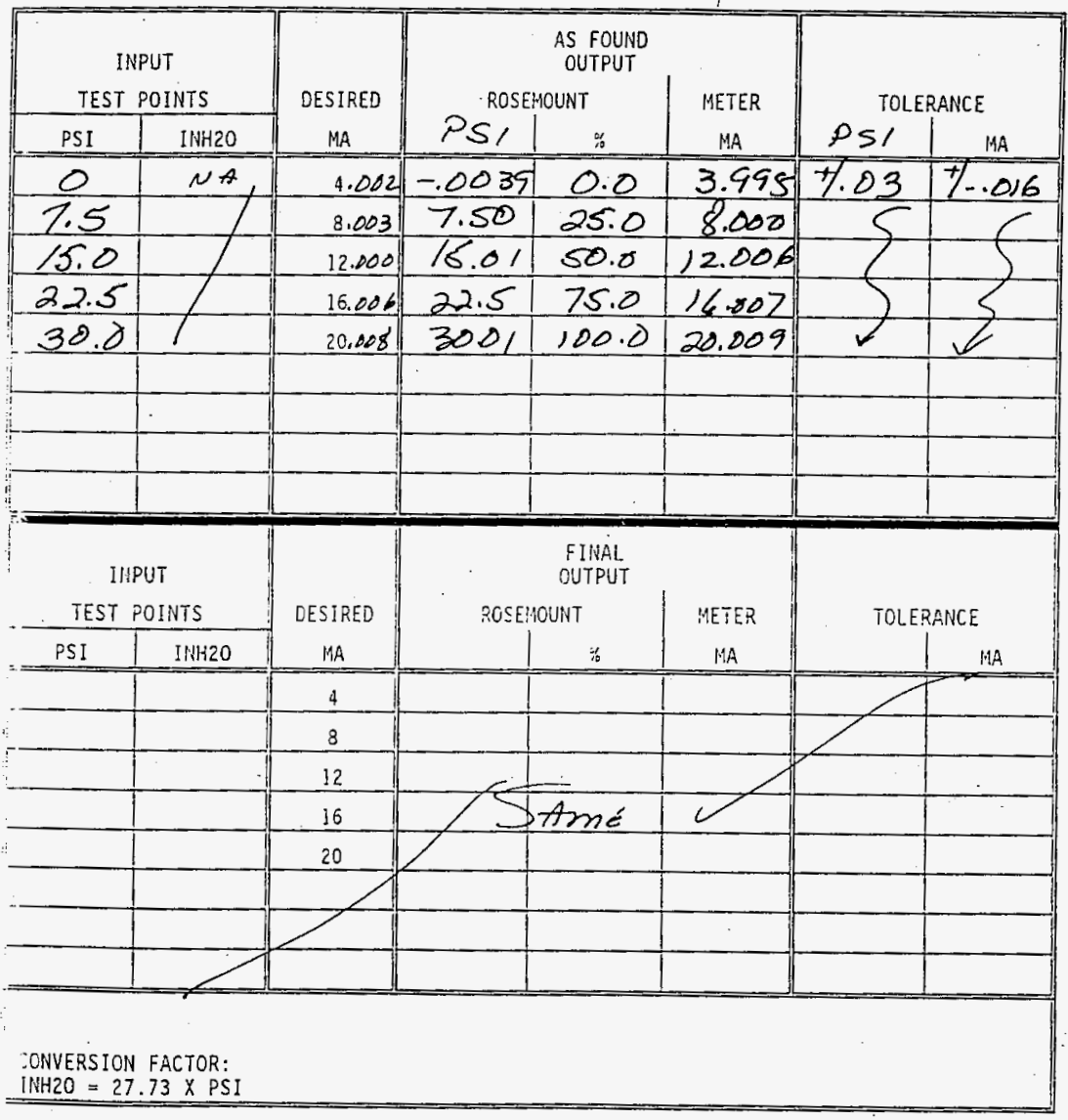

$[\mathrm{HH} 2 \mathrm{O}=27.73 \times$ PS

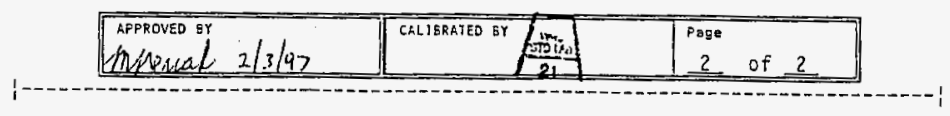


$\therefore \quad \therefore$

HANFORD STANDARDS LABORATORY PHYSICAL AND ELECTRICAL REPORT

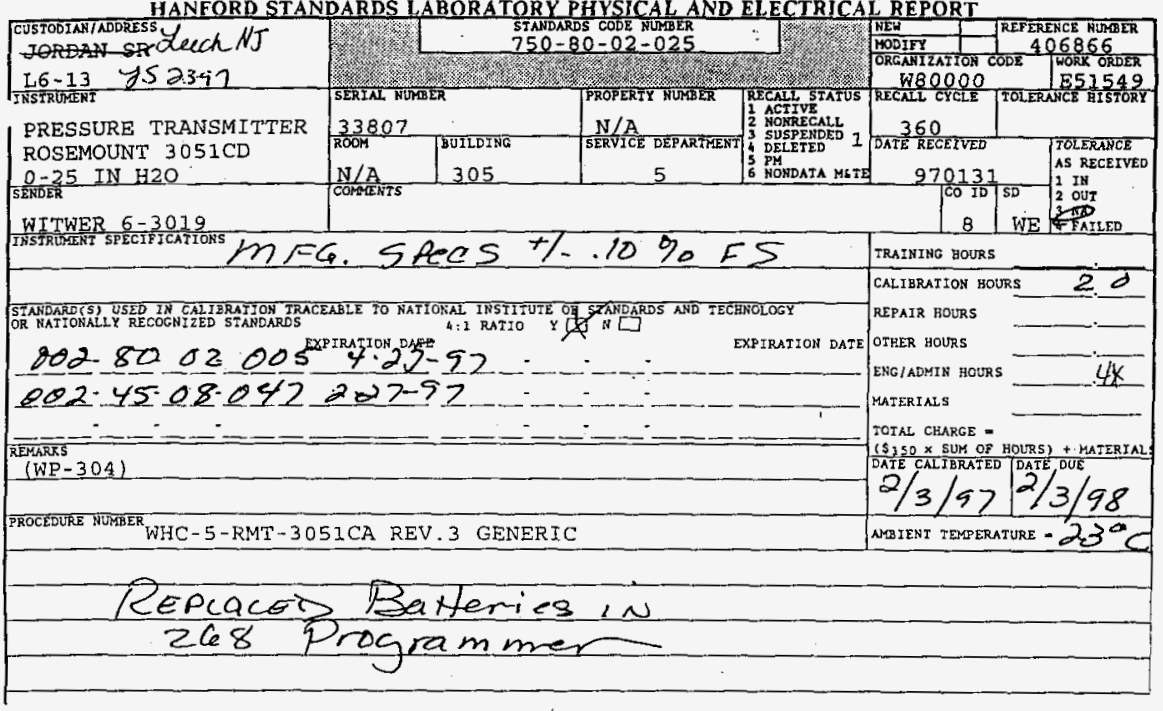

Nissiog Screwsid Coven Te ferminal Strip.

Used with Laninor Flow Elennent

measuring Hoummale gos inlet

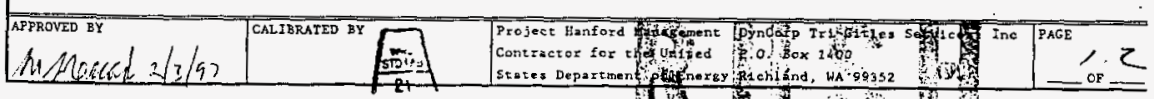

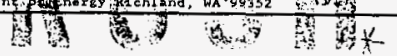




\begin{tabular}{|l|l|l|}
\hline PROCEDURE HAME -304 & STANDARDS COOE NUMBer & REFEREMLE MUMBER \\
WHC-5-RMT-3051CA REV.3 GENERIC & $750 \cdot 80-02-025$ & 406 866 \\
\hline
\end{tabular}

DATA SHEET

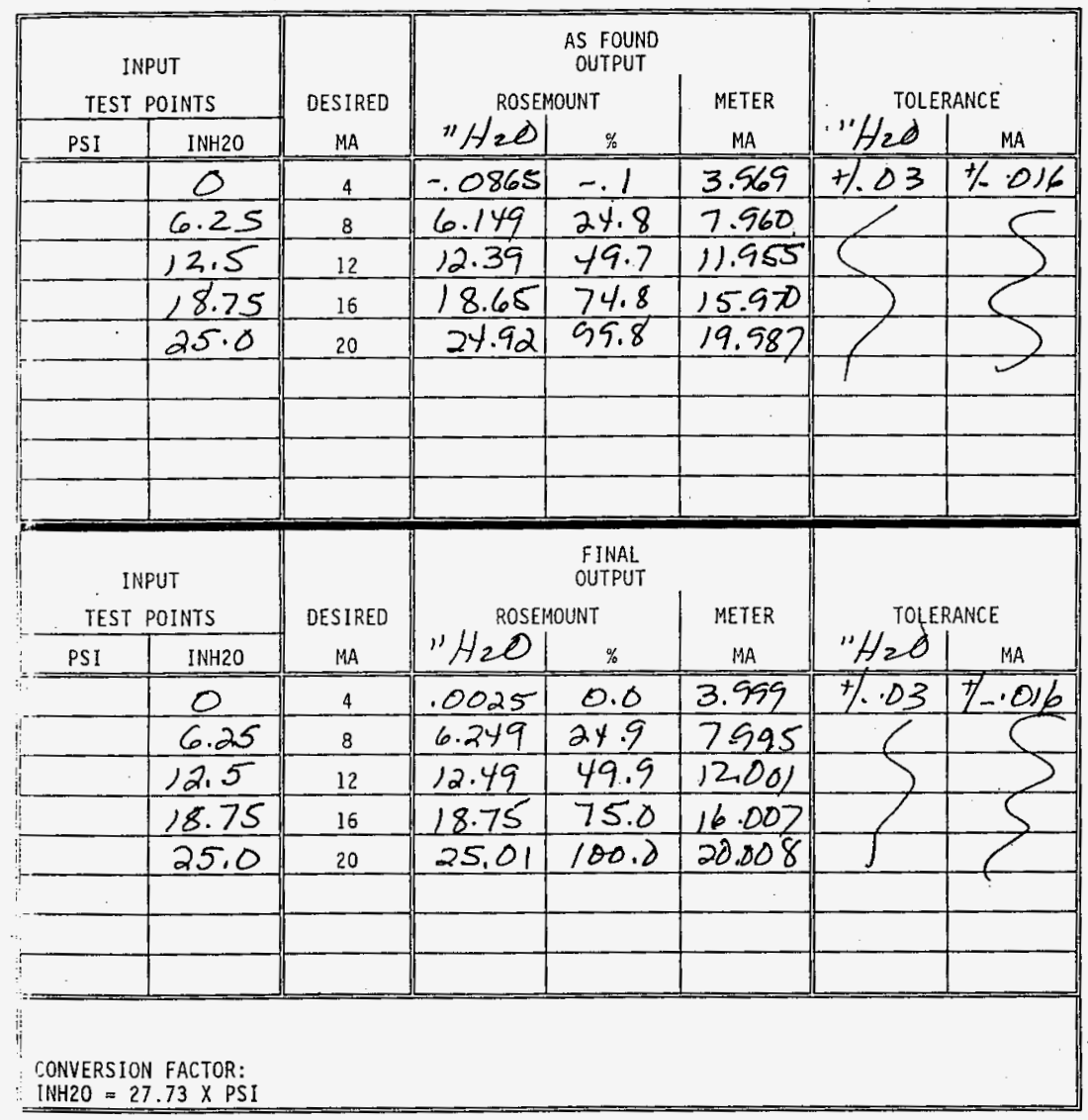

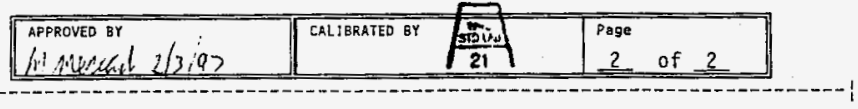


"...

HANFORD STANDARDS LABORATORY PHYSICAL AND ELECTRICAL REPORT

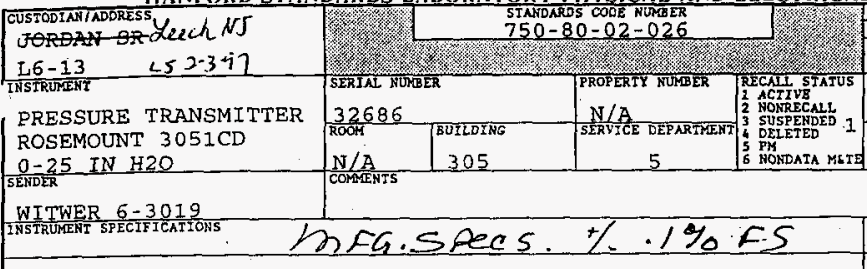

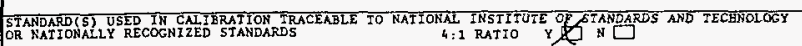

$002-80-02005^{\text {ExpIRATION DATE }}$

$002.45 .08 .047 .2-27-92$

EXPIRATION DATE

REMARS 304$)$
(WP-304)

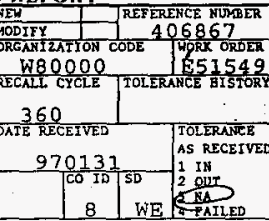

TRAINING BOURS CALIBRATION HOURS $2 d$ REPAIR GOURS

OTBER HOURS

ENG/ADMIN HOURS

MATERIALS

TOTAL CGARGE *

(S150 $\times$ SUM OF BOURS) + MATERIAL

DATE GSLIBRATED DATE PUE

PROCEDURE NUMBER WHC-5-RMT-3051CA REV.3 GENERIC

AMBIENT TEMPERATURE - $23 \%$

\section{Used on the Laminer Flow Element}

measuring Large volume / Mixed Gas

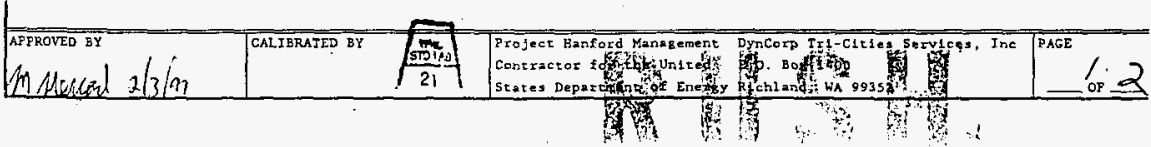




\begin{tabular}{|c|c|c|}
\hline $\begin{array}{l}\text { PROCEDURE NAME - } 304 \\
\text { WHC-5-RMT-305ICA REV.3 GENERIC }\end{array}$ & $\begin{array}{l}\text { STAMOAROS COOE HUMBER } \\
750 \cdot 80-022\end{array}$ & $\begin{array}{l}\text { REFERENCE NUKBER } \\
Y 0<86\end{array}$ \\
\hline
\end{tabular}

DATA SHEET

\begin{tabular}{|c|c|c|c|c|c|c|c|}
\hline \multirow{2}{*}{\multicolumn{2}{|c|}{$\begin{array}{c}\text { INPUT } \\
\text { TEST POINTS } \\
\end{array}$}} & \multirow{3}{*}{$\begin{array}{c}\text { DESIRED } \\
\text { MA } \\
\end{array}$} & \multicolumn{3}{|c|}{$\begin{array}{l}\text { AS FOUND } \\
\text { OUTPUT }\end{array}$} & \multirow{2}{*}{\multicolumn{2}{|c|}{ TOLERANCE }} \\
\hline & & & \multicolumn{2}{|c|}{ ROSEMOUNT } & \multirow{2}{*}{$\begin{array}{c}\text { METER } \\
\text { MA }\end{array}$} & & \\
\hline PSI & INH2O & & $" \mathrm{H}, \mathrm{O}$ & $\%$ & & "H20 & MA \\
\hline & 0 & 4 & -.1956 & -.4 & 3.928 & 4.03 & +.016 \\
\hline & 6.25 & 8 & 6058 & 24.5 & 7.921 & & \\
\hline & 12.5 & 12 & 12.38 & 49.6 & 11.923 & & \\
\hline & 18.75 & 16 & 18.53 & 74.5 & 15.923 & & \\
\hline & 25.0 & 20 & 24.81 & 95.6 & 19.534 & & \\
\hline & & & & & & & \\
\hline & & & & & & & \\
\hline & & & & & & & \\
\hline & & & & & & & \\
\hline & & & & $\begin{array}{l}\text { FINAL } \\
\text { OUTPUT }\end{array}$ & & & \\
\hline & DINTS & DESIRED & ROSE & UNT & METER & TOLE & ANCE \\
\hline PSI & INH2O & MA & $" \mathrm{H}_{2} \mathrm{O}$ & $\%$ & MA & & MA \\
\hline & 0 & 4 & .0024 & 0.0 & 4.001 & 1.03 & $\pi .016$ \\
\hline & 6.25 & 8 & 6.253 & 24.9 & 8.008 & & \\
\hline & 12.5 & 12 & 12.50 & 50.0 & 12.001 & & \\
\hline & 18.75 & 16 & 18.75 & 75.0 & 16.003 & & \\
\hline & 25.0 & 20 & 25.01 & $100 . \delta$ & 20008 & $\sum$ & 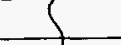 \\
\hline & & & & & & & 1 \\
\hline & & & & & & & \\
\hline & & & & & & & \\
\hline & $\begin{array}{l}\text { CTOR: } \\
\times \text { X PSI }\end{array}$ & & & & & & \\
\hline
\end{tabular}

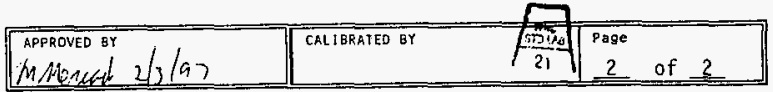


HNF-SD-WM-TRP-275

Rev. 0
Page 26

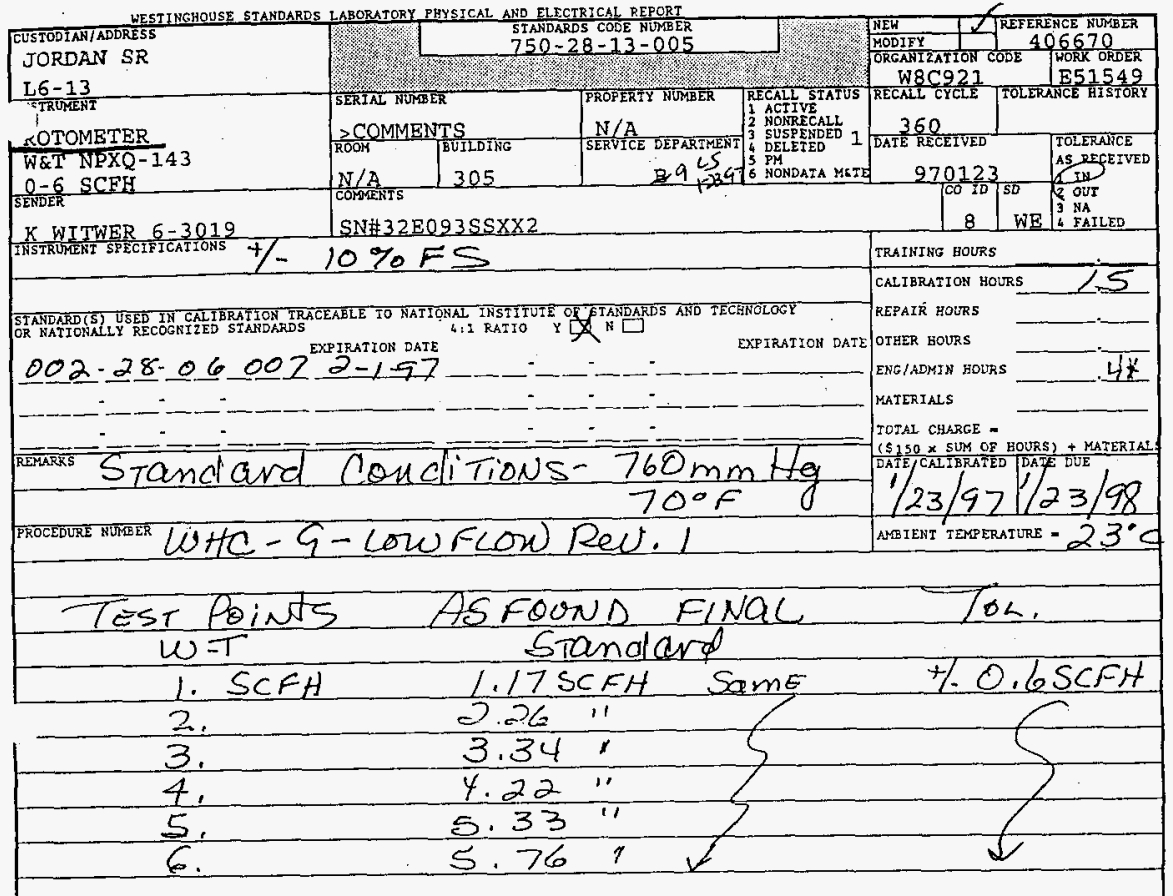

Uned sis secondary calibiation souvce for Zez Dto 2.5 fotometers:

These are used on the bas chromat aqued qus feed lines

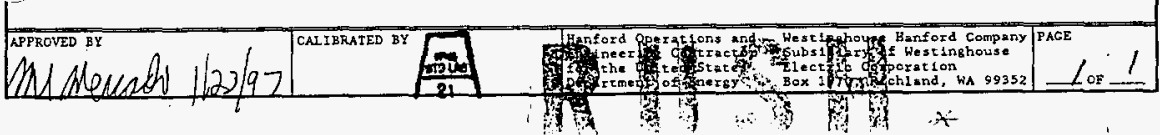




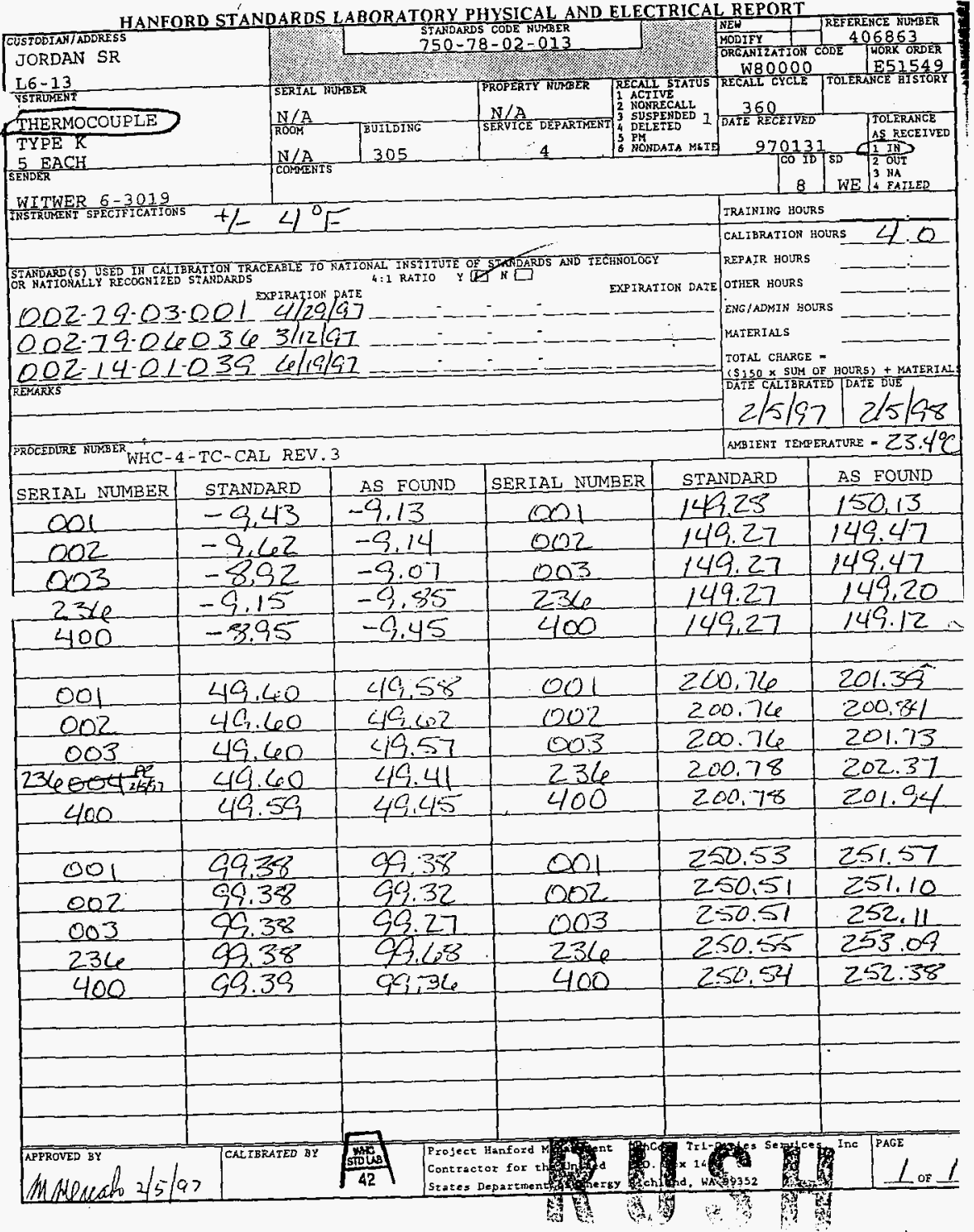




\section{WESTINGHOUSE STANDARDS LABORATORY PHYSICAL AND ELECTRICAL REPCRT}

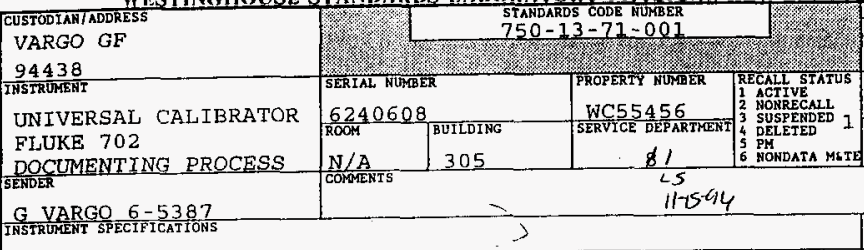

DEFEREICE NUMBER YODIFY 405287 \begin{tabular}{l|l} 
RGANI2ATIOH CODE & WORK OBDER \\
W75250 & $E 58333$ \\
\hline
\end{tabular} TOLERAICE HISTORY

360 1115

AS RECEIVED 1 In Caltbration hovrs 4.0

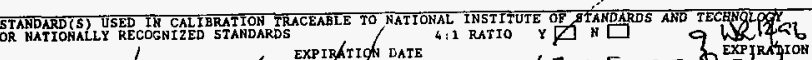
REPAIR HOURS

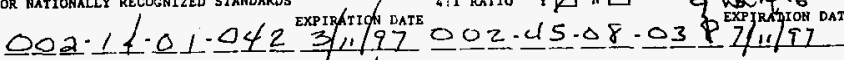
$002.23-01-0107 / 23 / 97$ OTHER HOURS ENG/ADMIN HOURS MATERTALS $002-23 \cdot 01-00665 / 97=\cdots$ NEW - NO WP DATA SHEET IN SYSTEM TOTRL CHARGE (5125 $\times$ SUM OF HOURS) + MATERTAL

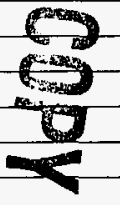


PROCEDURE HAME - 515

WHC-4-TEMPIND REV. 1 FLUKE 701

\begin{tabular}{l|l}
\hline STANDARDS CODE MUMBER & RE fFREHCE NUMBER \\
$750-13-71-001$ & 40 5287 \\
\hline
\end{tabular}

DATA SHEET

\begin{tabular}{|c|c|c|c|c|c|}
\hline \multirow[b]{2}{*}{ FUNCTION } & \multirow{2}{*}{$\begin{array}{l}\text { TC } \\
\text { TYPE } \\
\end{array}$} & \multirow{2}{*}{$\begin{array}{l}\text { STANDARD } \\
{ }^{\circ} \mathrm{C} /{ }^{\circ} \mathrm{F} \\
\end{array}$} & \multicolumn{2}{|c|}{ UUT INDICATION } & \multirow{2}{*}{$\begin{array}{l}\text { MANUFACTURER } \\
\text { SPECIFICATION }\end{array}$} \\
\hline & & & AS FOUND ${ }^{\circ} \mathrm{C}$ & FINAL $c$ & \\
\hline MEASURE & $K$ & $=200^{\circ} \mathrm{C}$ & $=199.9$ & Same & $\pm 1.0^{\circ} \mathrm{C}$ \\
\hline \multirow[t]{17}{*}{${ }^{\circ} \mathrm{C} /{ }^{\circ} \mathrm{F}$} & & $-100^{\circ} \mathrm{C}$ & -99.9 & & 1 \\
\hline & & $0^{\circ} \mathrm{C}$ & .1 & & $\pm 0.5^{\circ} \mathrm{C}$ \\
\hline & & $32^{\circ} \mathrm{F}$ & $\therefore 1$ & & $\pm 0.9^{\circ} \mathrm{C}$ \\
\hline & & $100^{\circ} \mathrm{C}$ & 100.1 & & $\pm 0.5^{\circ} \mathrm{C}$ \\
\hline & & $200^{\circ} \mathrm{C}$ & 200.0 & & H \\
\hline & & $300^{\circ} \mathrm{C}$ & 300.1 & & " \\
\hline & & $400^{\circ} \mathrm{C}$ & 400.0 & & $\pm 0.7^{\circ} \mathrm{C}$ \\
\hline & & $500^{\circ} \mathrm{C}$ & 499.9 & & " \\
\hline & & $600^{\circ} \mathrm{C}$ & 400.0 & & $"$ \\
\hline & & $700^{\circ} \mathrm{C}$ & 700.1 & & " \\
\hline & & $800^{\circ} \mathrm{C}$ & 800.0 & & " \\
\hline & & $900^{\circ} \mathrm{C}$ & 900.1 & & " \\
\hline & & $1000^{\circ} \mathrm{C}$ & 1000.1 & & $1 "$ \\
\hline & & $1100^{\circ} \mathrm{C}$ & 1100.0 & & $"$ \\
\hline & & $1200^{\circ} \mathrm{C}$ & 1199.9 & & $\pm 0.9^{\circ} \mathrm{C}$ \\
\hline & & $1300^{\circ} \mathrm{C}$ & 1300.1 & & " \\
\hline & & $1370^{\circ} \mathrm{C}$ & 1369,9 & & " \\
\hline \multirow[t]{3}{*}{ SOURCE } & $K$ & $-199^{\circ} \mathrm{C}$ & -199.2 & & $\pm 1.0^{\circ} \mathrm{C}$ \\
\hline & & $400^{\circ} \mathrm{C}$ & 399.9 & & $\pm 0.7^{\circ} \mathrm{C}$ \\
\hline & & $1370^{\circ} \mathrm{C}$ & 1369.9 & & $\pm 0.9^{\circ} \mathrm{C}$ \\
\hline MEASURE & $\mathrm{J}$ & $0^{\circ} \mathrm{C}$ & -1 & & $\pm 0.5^{\circ} \mathrm{C}$ \\
\hline \multirow[t]{3}{*}{${ }^{\circ} \mathrm{C}$} & $T$ & $0^{\circ} \mathrm{C}$ & -.1 & & $\pm 0.8^{\circ} \mathrm{C}$ \\
\hline & $E$ & $\mathrm{O}^{\circ} \mathrm{C}$ & -1 & 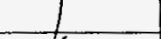 & $\pm 0.5^{\circ} \mathrm{C}$ \\
\hline & $\mathrm{R}$ & $200^{\circ} \mathrm{C}$ & 200.4 & 1 & $\pm 1.2^{\circ} \mathrm{C}$ \\
\hline
\end{tabular}

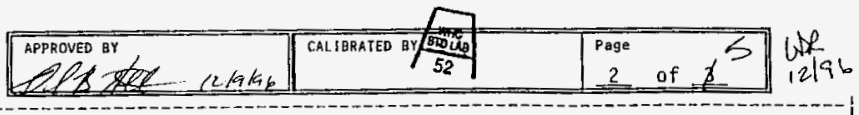




\begin{tabular}{|c|c|c|}
\hline $\begin{array}{l}\text { PROCEDURE NAME - } 515 \\
\text { WHC-4-TEMPIND REV. } 1 \text { FLUKE } 701\end{array}$ & $\begin{array}{l}\text { STAMDARDS COOE MUMBER } \\
750-13.71-401\end{array}$ & $\begin{array}{l}\text { REFEREMCE HUMBER } \\
405287\end{array}$ \\
\hline
\end{tabular}

\section{DATA SHEET}

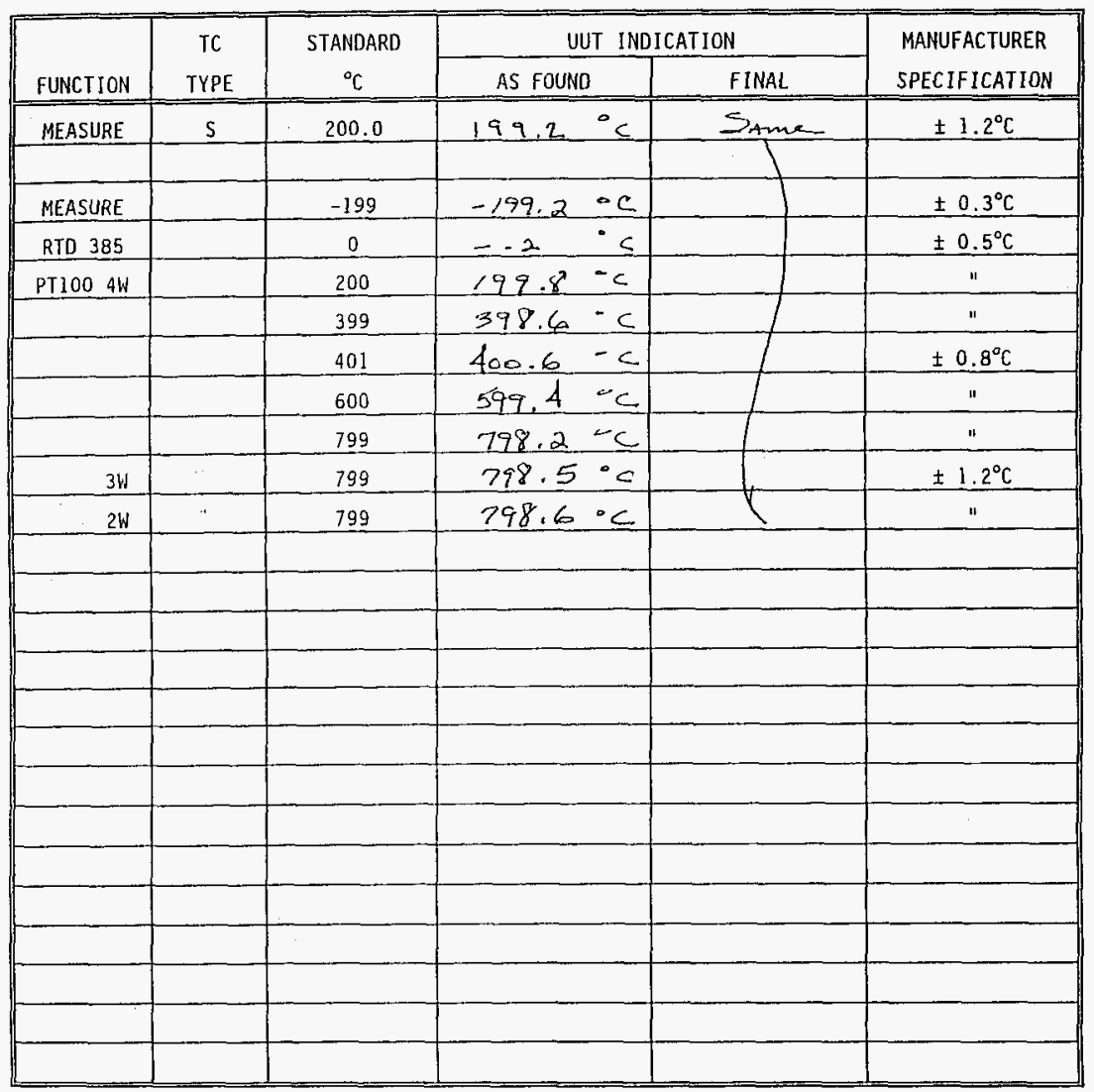

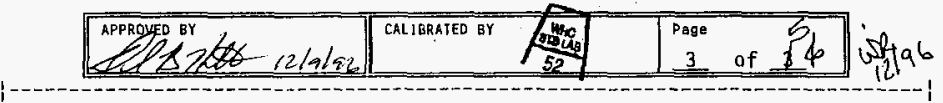




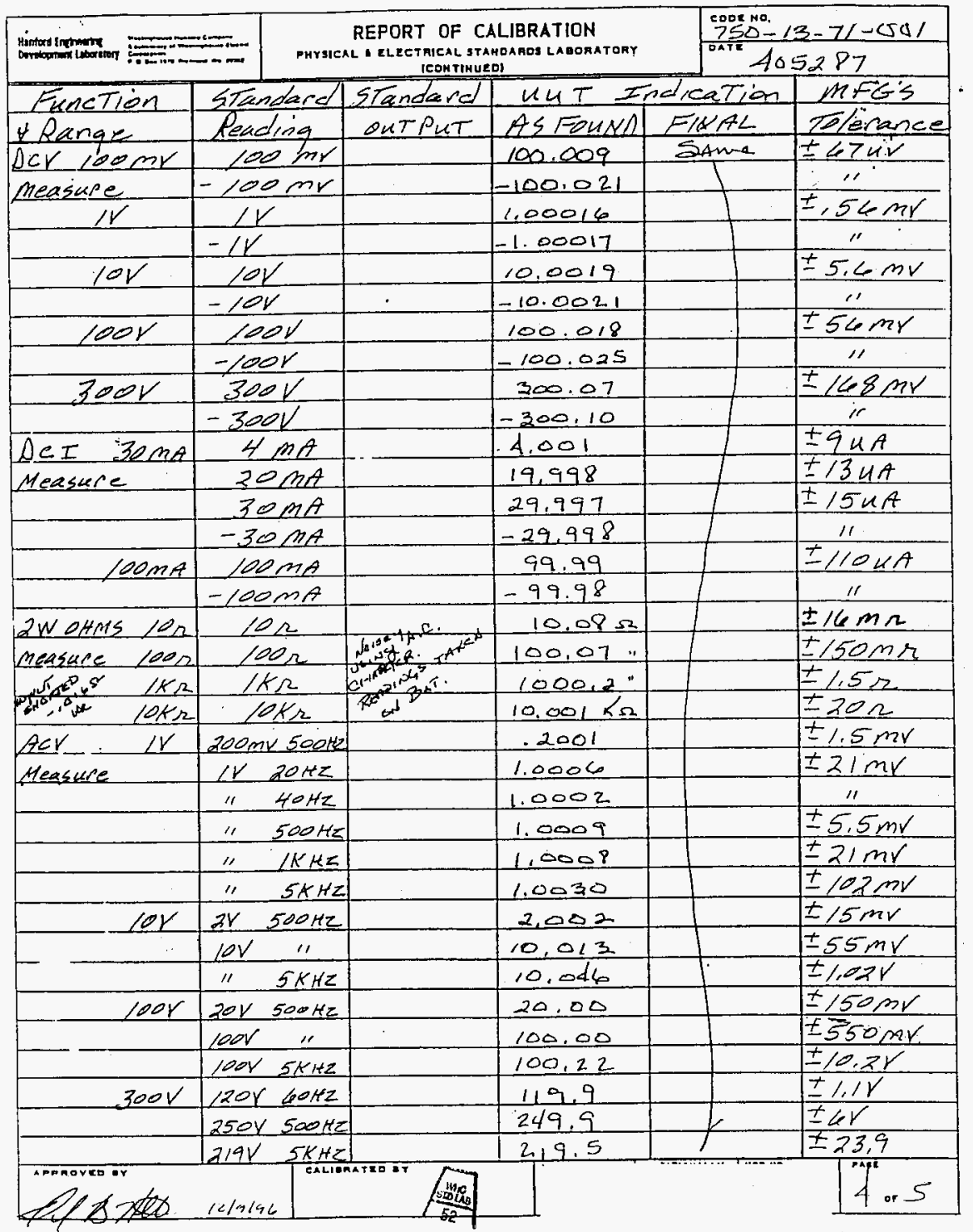




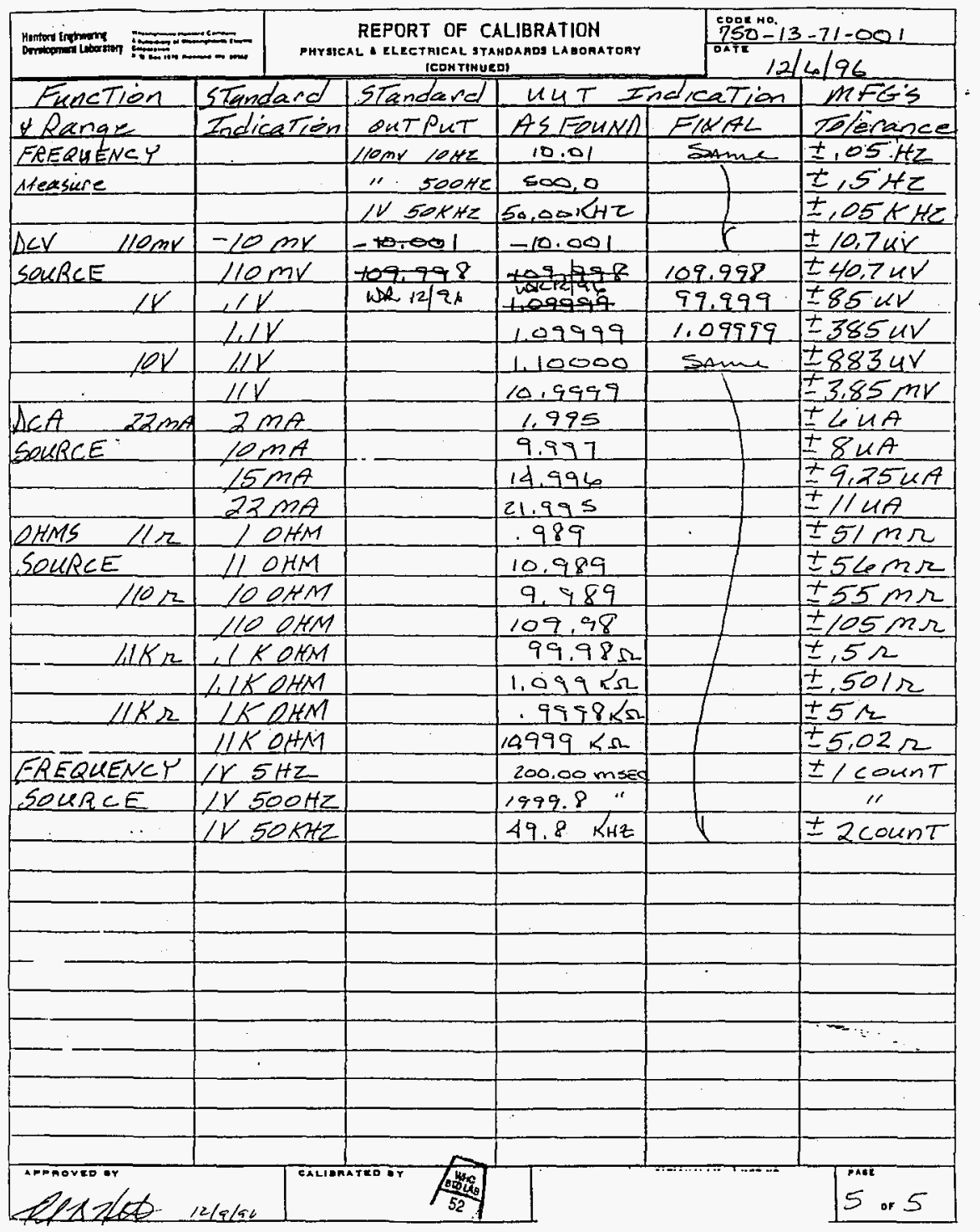


HANFORD STANDARDS LABORATORY PHYSICAL AND ELECTRICAL REPORT

CDISTODIAN/ADORESS JORDAN SR

L6 - 13

INS TRDMEN

¿ATA ACOUSN SYSTEM

H-P $3497 \mathrm{~A}$

CONTROI UNIT

ENDER

K WITWER 6-3019

STANDARES CODE NUMBER

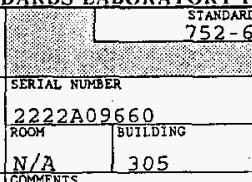

$67-11-002$
FROPERTY NUTBRR
WB96061
SERVICE DEPARTMENT
4

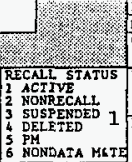

$\mathrm{NEL}$

WOIFY D REF FREHCE NISMBER ORANI2ATION CODE 406861

W8000 TRE ORER RECAIT CYCL

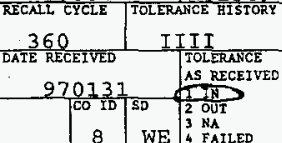

SEE TOLERANCE COLUMN

5TANDARD (S) USED TN CALIBRATION TRACEABLE TO NAT TONAL INSTITUTE OF STAATARES AND TECHNOLOGY

OR NATIOMALIY RECOGNIZED STARDAROS

$002-14.01-0366^{\text {EXPIRATION DATE }}$

AL TASTITUTE OF STAADAR

$002.79 .060360 .3 / 12 / 9$

- $\quad$ - $\quad$ - EXPIRATION DATE

$20 \leq 71060360.3 / 2197$

$002.4508 .052 .9 / 25 / 97$

(WP -16$)$

PROCEDVTRE NWMER WHĆ-67-1.1-3497A (12-82)

CAL IBRATION HOURS

REPAIR HOURS

OTHER HOURS

EMG/ADMIN HOURS

MATERTALS

TOTAL CHARGE -

(S150 $\times$ SUM OF GOURS) + MSTERIAL DATE CALTERATED DATE DUE

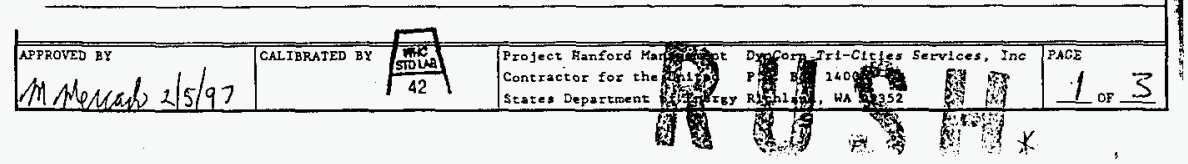

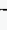

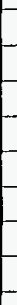




\begin{tabular}{|l|l|l|}
\hline PROCEDURE KAKE - 16 & STAMDARDS CDOE HUKBER & REFERENCE MUMBER \\
WHC-67-11-3497A(12-82) & $752-607-11-002$ & 4068601 \\
\hline
\end{tabular}

DATA SHEET

\begin{tabular}{|c|c|c|c|c|}
\hline \multirow{2}{*}{$\begin{array}{l}\text { DMM RANGE } \\
\text { AND DISPLAY }\end{array}$} & \multirow{2}{*}{$\begin{array}{l}\text { STANDARO } \\
\text { READING } \\
\end{array}$} & \multicolumn{2}{|c|}{ DMM READING } & \multirow{2}{*}{$\begin{array}{l}\text { ONE YEAR } \\
\text { TOLERANCE }\end{array}$} \\
\hline & & AS FOUND & FINAL & \\
\hline .IV 5$\}$ DIGIT & .100000 & .077989 & SAME & $\pm 18 \mu \mathrm{V}$ \\
\hline i.ov & 1.00000 & 1.00000 & & $\pm 160 \mu v$ \\
\hline \multirow[t]{3}{*}{$10.0 \mathrm{~V}$} & 1.1111 & 1.1111 & & $\pm .3 \mathrm{mV}$ \\
\hline & 2.2222 & 2.2222 & & $\pm .4 \mathrm{mV}$ \\
\hline & 3.3333 & 3.3333 & & $\pm .6 \mathrm{mV}$ \\
\hline \multirow[t]{7}{*}{$\therefore$} & 4.4444 & 4.4444 & & $\pm .8 \mathrm{mV}$ \\
\hline & 5.5555 & 5.5555 & & $\pm .9 \mathrm{mV}$ \\
\hline & 6.6666 & 6066060 & & $\pm 1.1 \mathrm{mV}$ \\
\hline & 7.7777 & 77777 & & $\pm 1.3 \mathrm{mV}$ \\
\hline & 8.8888 & 8.8888 & & $\pm 1.4 \mathrm{mV}$ \\
\hline & 9.9999 & 9.9999 & & $\pm 1.6 \mathrm{mV}$ \\
\hline & 10.0000 & 10.0000 & & $\pm 1.6 \mathrm{mV}$ \\
\hline \multirow[t]{4}{*}{$100.0 \mathrm{~V}$} & 50.000 & 49998 & & $\pm 8.0 \mathrm{mV}$ \\
\hline & 100.000 & 99.996 & & $\pm 16.0 \mathrm{mV}$ \\
\hline & -100.000 & -93.907 & & $\pm 16.0 \mathrm{mV}$ \\
\hline & -50.000 & $-49.99 \%$ & & $\pm 8.0 \mathrm{mV}$ \\
\hline \multirow[t]{4}{*}{ AUTO RANGE } & 50.000 & 49998 & & $\pm 8.0 \mathrm{mV}$ \\
\hline & 5.0000 & 5,0000 & & $\pm .8 \mathrm{mV}$ \\
\hline & .50000 & .50002 & & $\pm 80 \mu \mathrm{V}$ \\
\hline & .050000 & .049993 & & $\pm 10 \mu v$ \\
\hline 10V 43 DIGIT & 9.000 & 9.000 & & $\pm 3.0 \mathrm{mV}$ \\
\hline IOV $3 \frac{1}{2}$ DIGIT & 9.00 & 9,00 & $\downarrow$ & $\pm 20 \mathrm{mV}$ \\
\hline & & & & \\
\hline & & & & \\
\hline & & & & \\
\hline
\end{tabular}

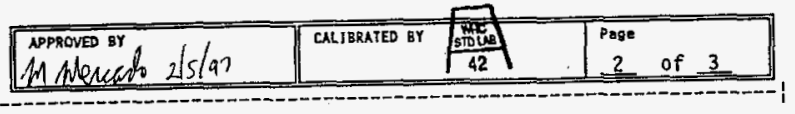


DATA SHEET

\begin{tabular}{|c|c|c|c|c|}
\hline \multirow{2}{*}{$\begin{array}{l}\text { DMM RANGE } \\
\text { AND DISPLAY }\end{array}$} & \multirow{2}{*}{$\begin{array}{l}\text { STANDARD } \\
\text { READING }\end{array}$} & \multicolumn{2}{|c|}{ STANDARD INDICATION } & \multirow{2}{*}{$\begin{array}{c}\text { ONE YEAR } \\
\text { TOLERANCE } \\
\end{array}$} \\
\hline & & AS FOUND & FINAL & \\
\hline \multicolumn{5}{|l|}{ CURRENT OUT } \\
\hline $1 \mathrm{ma}$ & & 1.000021 & SAME & \pm 320 ua \\
\hline 100 ua & & 100.013 & & \pm .032 ua \\
\hline loua & & 9.4997 & $\downarrow$ & \pm .0032 ua \\
\hline & & & & \\
\hline CJ COMPENEATION & $2.5 \mathrm{~V}$ aT $25^{\circ} \mathrm{C} 4$ & $2.2 \sqrt{45}$ & & $+/ 1^{\circ} \mathrm{C}$ or $.1 \mathrm{~V}$ \\
\hline $\mathrm{CH} * 19$ & $100 \mathrm{mv} /{ }^{\circ} \mathrm{C}$ & & & \\
\hline & & & & \\
\hline & & & & \\
\hline & & & & \\
\hline & & & & \\
\hline & & & & \\
\hline & & & & \\
\hline & & & & \\
\hline & & & & \\
\hline & & & & \\
\hline & & & & \\
\hline & & & & \\
\hline & & & & \\
\hline & & & & \\
\hline & & & & \\
\hline & & & & \\
\hline & & & & \\
\hline & & & & \\
\hline & & & & \\
\hline
\end{tabular}

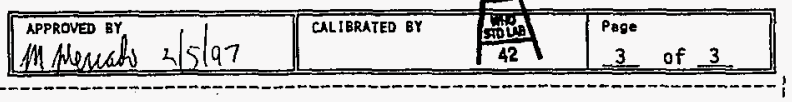


1.

WESTINGHOUSE STANDARDS LABORATORY PHYSICAL AND ELECTRICAL REP

CUSTODIARIADDRESS

SCHNEIDER TC

L6-37

TRLMET

_. $E$ ESSURE TRANSMITTER

ROSEMOUNT $3051 \mathrm{C}$

$0-30$ PSIA

SEMDER
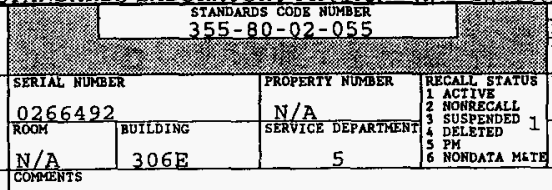

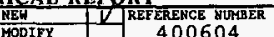

MODIFY 100604

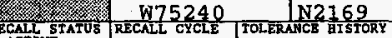

BCALL STATUS

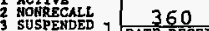

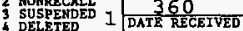

\begin{tabular}{l|l|l}
4 DELETED & DATE RECETVED \\
5 PM POHDATA METE & 960501 \\
\hline
\end{tabular}

960501

SHIPFIWG DAY

w) WTLLINGHAM 36256

MFG.SPECS $+1 \ldots .2 \%$ FS

STARDARD (S) USE IR CATIBRATION TRACEABLE TO NAT TOHAL INSTITUTE OF STANDARDS AND TECEKOLOGY

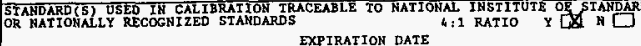

EXPTRATION DATE EXPIRATION DATE

202. $35.40-008 \frac{6-14-56}{2}$.

$002-45-08-042$ 2-27-5?

REMARKS

$(W P-216)$

ROCEOURE NWGER WHC-5-RMT-3051CA REV . 3 3OPSIA

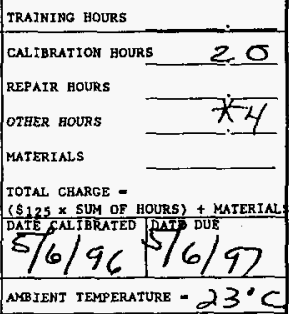

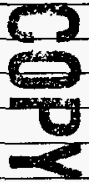

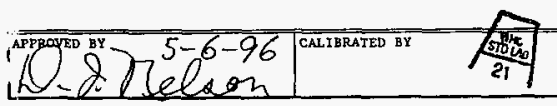

of

Hanford Operations

Eng1neering contraci
for the United Stat

Department of Enerzy 14 Box 19

Department of Energ

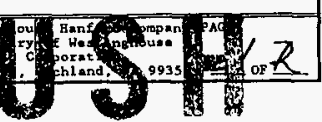


DATA SHEEI
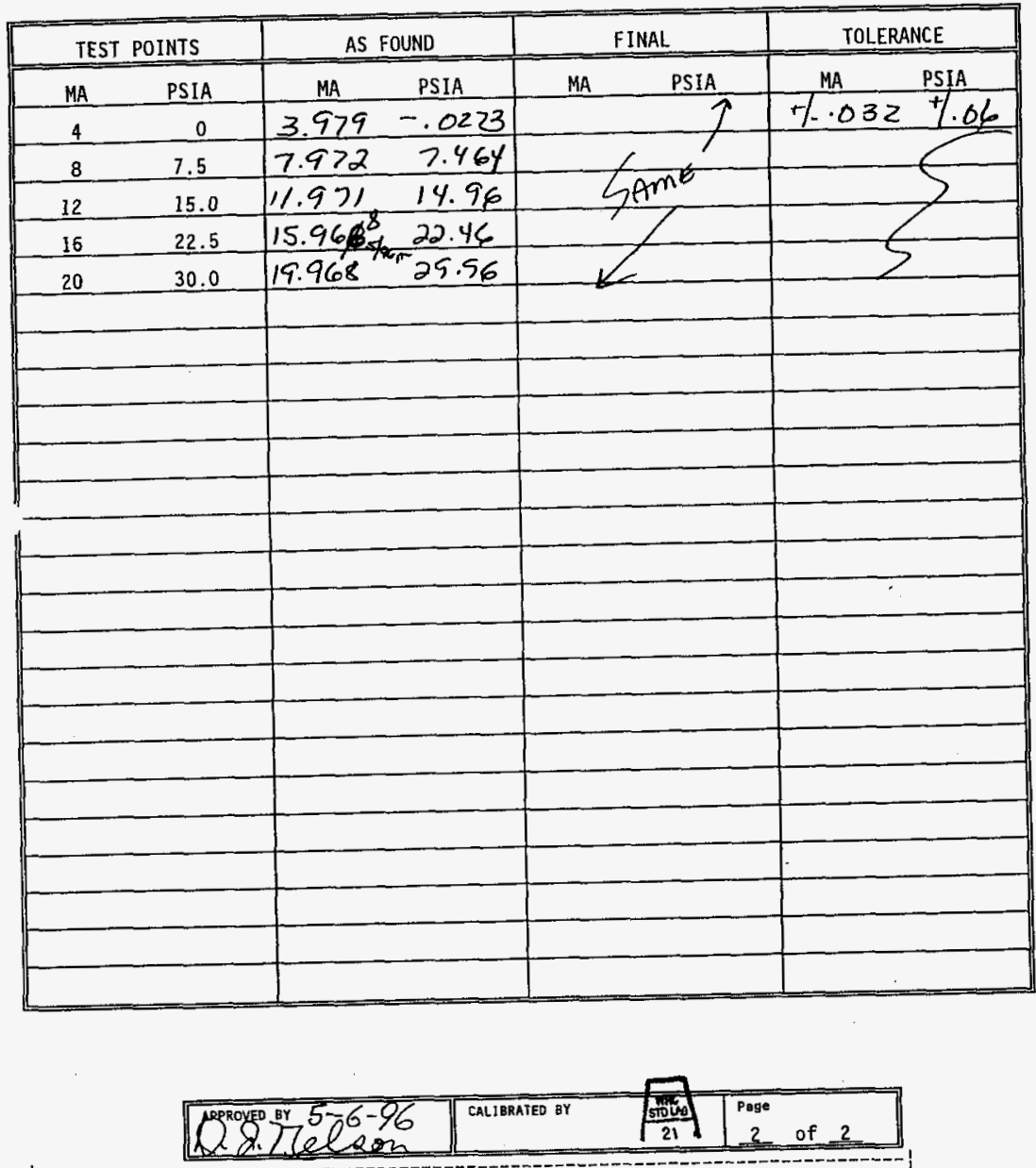


\section{APPENDIX B - GAS BOTTLE RECORDS}

The following information was obtained from cylinder labels attached to the certified standard calibration and injection gases used.

1. The gas used to calibrate the WEC and SMC sensors:

* Vendor: Scott Specialty Gases

* PO No. 023372

* Project No. 08-27000

* Cylinder Item No.

* Certified Concentration $8070 \mathrm{ppm} \mathrm{H}_{2}$, balance air

NOTE: This gas was checked by the test GC following its initial

calibration using the $1010 \mathrm{ppm}$ standard gas. The data file is included in APPENDIX $C$ as 8087 tst.dif, with the other GC data files.

2. The gas used to calibrate the gas chromatograph:

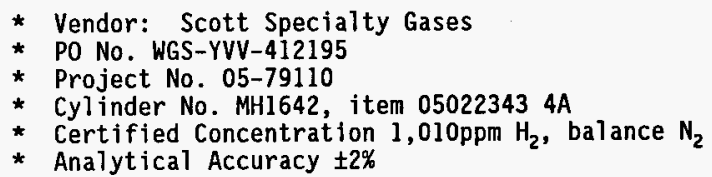

3. The gas initially used for low concentration injection gas:

* Vendor: Scott Specialty Gases

* PO No. WGS-YVV-412195

* Project No. 05-79110

* Cyl inder No. A016648, item 05022342 4A

* Certified Concentration $30,000 \mathrm{ppm} \mathrm{H}_{2}$, balance $\mathrm{N}_{2}$

* Analytical Accuracy $\pm 2 \%$

4. The gas used for subsequent low concentration injection gas:

* Vendor: Scott Specialty Gases

* PO No. WGS-YVV-455801

* Project No. 07-37904

* Cylinder No. ALM046117, item 07022342 4AL

* Certified Concentration $5.00 \% \mathrm{H}_{2}$, balance $\mathrm{N}_{2}$

* Analytical Accuracy $\pm 2 \%$ 
5. The gas used for the high concentration injection gas was the standard high purity $\mathrm{H}_{2}$ supplied from the site stores (Cat. No. 21-2870-100).

6. The gas used for the gas chromatograph carrier and purging of the systems was the standard pre-purified $\mathrm{N}_{2}$ supplied from the site stores (Cat. No. 21$3440-220\rangle$. 
HNF-SD-WM-TRP-275

Rey 0

Page 40

APPENDIX C - DATA RECORDS 


\section{FGI FLOW TEST DATA SHEET}

TEST ID. No. OATA FILE No. GC $02137 \%$

DLFGI213/1

\section{SMC ID. No. $96-\beta-Y 455$} WHITTAKER ID No. $4 / 5-1226$

MAIN DUCT FLOW PARAMETERS:

REQUIRED FLOW 200 CFM CALCULATED FLOW $/ 97.8 \mathrm{CFM}$

DIFF. PRESS. $3.75 \quad \mathrm{H}_{2} \mathrm{O}$ ABS. PRESS. 14.80 PSIA TEMP. Z7 ${ }^{\circ} \mathrm{F}$ DUCT LENGTH $\angle 4 . / 7$ FT. FLOW VELOCITY G.OY FT/SEC TRANSPORT TIME $2.3 Y$ SEC.

INJECTION FLOW PARAMETERS:

$$
\text { Calculated t/z }-232.4 \mathrm{prm}
$$

DESIRED $\mathrm{H}_{2}$ CONCENTRATION $2 \mathrm{CQ}$ PPM MEASURED $\mathrm{H}_{2}$ CONCENTRATION $237.8 \ldots \mathrm{pPm}$

DIFF. PRESS. $6.937 " \mathrm{H}_{2} \mathrm{O}$ ABS. PRESS. 15.89 PSIA TEMP. $\quad 7 /{ }^{\circ} \mathrm{F}$

SENSOR CONCENTRATION OUTPUT:

2 MINUTE CONCENTRATION VALUES:

SMC $80.2 \mathrm{ppm} \quad$ WHITTAKER $100.3 \mathrm{ppm}$

14 MINUTE 30 SECOND CONCENTRATION VALUES:

SMC $79.3 \mathrm{ppm} \quad$ WHITTAKER $240.4 \mathrm{ppm}$

15 MINUTE CONCENTRATION VALUES:

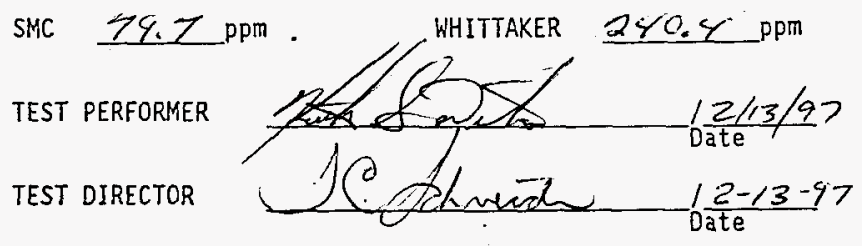




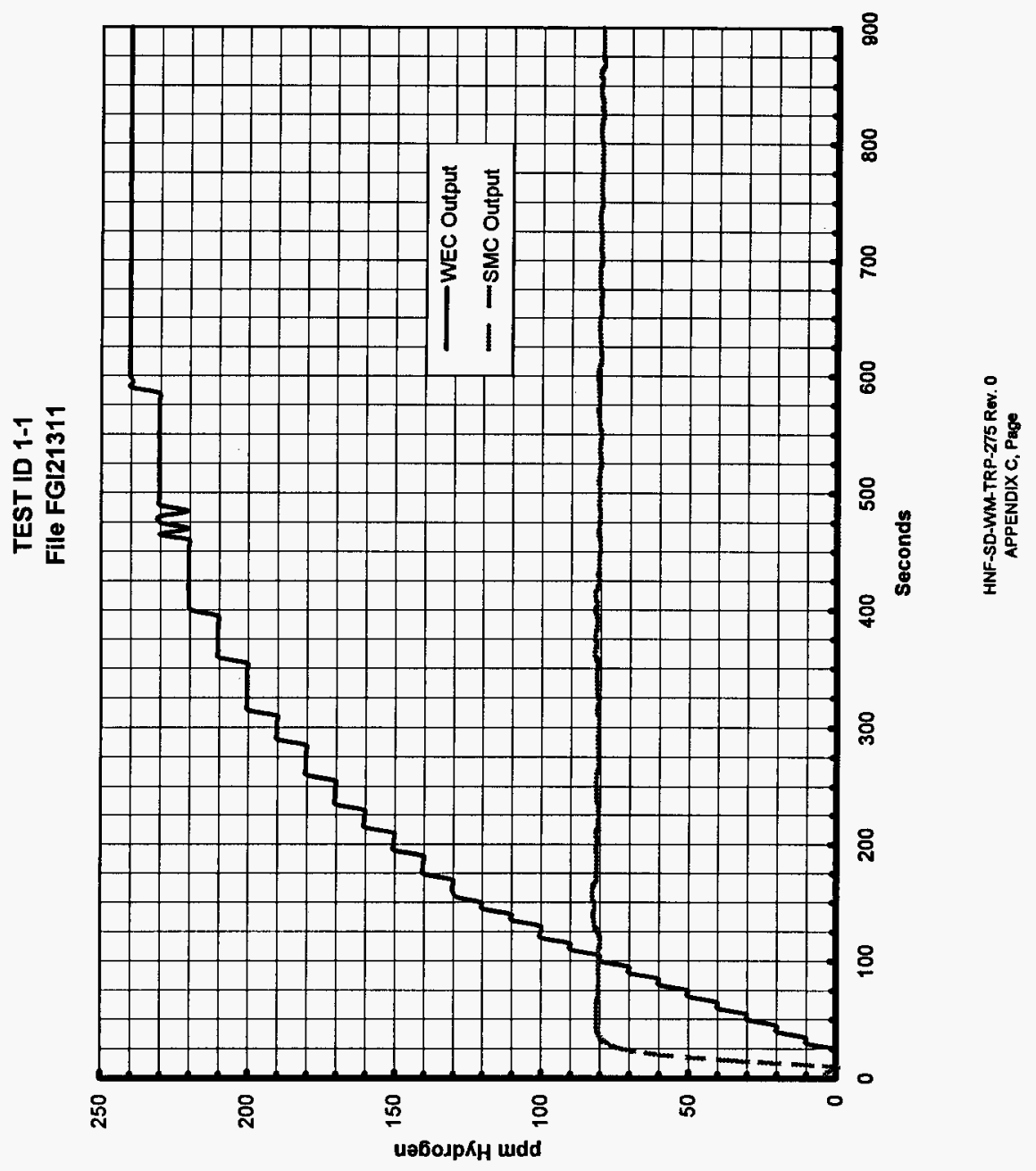


FGI FLOW TEST DATA SHEET

TEST ID. No. $/-Z$

SMC ID. No. $96-\beta-4455$
DATA FILE NO. GC $02 / 37 /-2$

DL FGI213!2 WHITTAKER ID NO. $\$ 15-1226$

MAIN OUCT FLOW PARAMETERS:

REQUIRED FLOW 200 CFM

DIFF. PRESS. 3. $\mathrm{ZZ}$ " $\mathrm{H}_{2} \mathrm{O}$

DUCT LENGTH $/ 4 . / 7$ FT.

TRANSPORT TIME 233 SEC.
CALCULATED FLOW 198.5 CFM ABS. PRESS. 14.80 PSIA TEMP. $788^{\circ} \mathrm{F}$ FLOW VELOCITY $6.07 \mathrm{FT/SEC}$

INJECTION FLOW PARAMETERS:

Calculated H/2 - $232 \mathrm{ffm}$

DESIRED $\mathrm{H}_{2}$ CONCENTRATION $200 \mathrm{pPM}$ MEASURED $\mathrm{H}_{2}$ CONCENTRATION $236 \mathrm{ppm}$ DIFF. PRESS. 6.95 " $\mathrm{H}_{2} \mathrm{O}$ ABS. PRESS. $\$ 5.89$ PSIA TEMP. $7 /{ }^{\circ} \mathrm{F}$

SENSOR CONCENTRATION OUTPUT:

2 MINUTE CONCENTRATION VALUES:

SMC $88.3 \mathrm{ppm} \quad$ WHITTAKER $90.2 \mathrm{ppm}$

14 MINUTE 30 SECOND CONCENTRATION VALUES:

SMC 84.1 pPm WHITTAKER 240.4 ppm

15 MINUTE CONCENTRATION VALUES:

SMC $84.4 \mathrm{pPm}$. WHITTAKER $240.4 \mathrm{ppm}$

TEST PERFORMER

TEST DIRECTOR

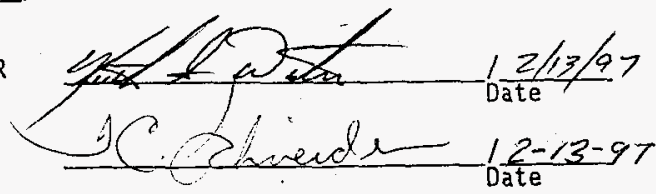




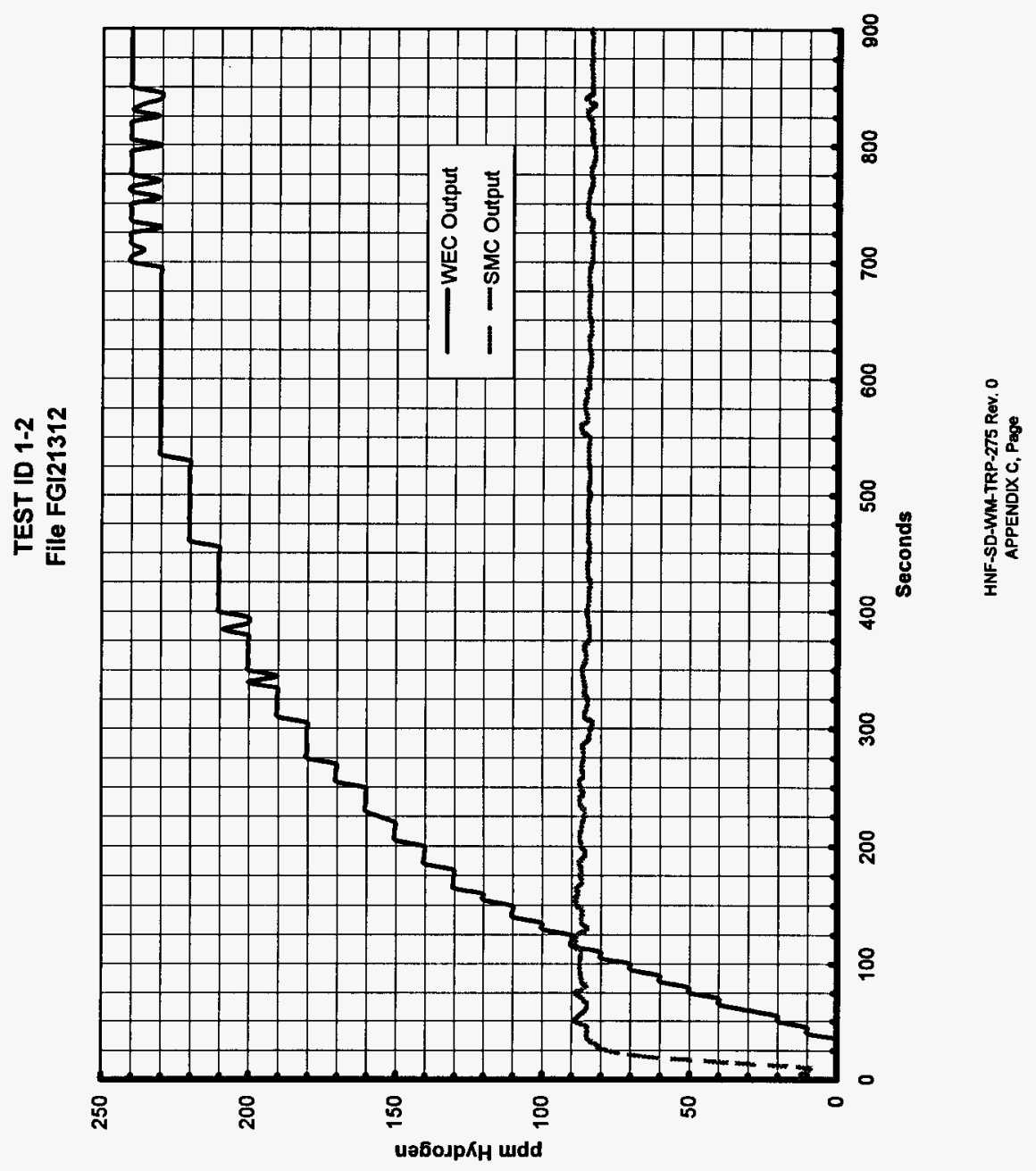




\section{FGI FLOH TEST DATA SHEET}

TEST ID. No. $/-3$ DATA FILE NO. GC $02137 /-3$ DL FGI2/3/3 SMC ID. NO. $96-B-4455$ WHITTAKER ID No. $\not 15-1226$

MAIN DUCT FLOW PARAMETERS:

REQUIRED FLOW 200 CFM CALCULATED FLOW 199.0 CFM

DIFF. PRESS. 3. 78 " $\mathrm{H}_{2} \mathrm{O}$ ABS. PRESS. $\angle Y .80$ PSIA TEMP. $78^{\circ} \mathrm{F}$ DUCT LENGTH $\angle Y, / Z F T$. FLOH VELOCITY 6.08 FT/SEC

TRANSPORT TIME 2.33 SEC.

INUECTION FLOW PARAMETERS:

Calcalated thz - $2298 \mathrm{sm}$

DESIRED $\mathrm{H}_{2}$ CONCENTRATION 200 MEASURED $\mathrm{H}_{2}$ CONCENTRATION $235 \mathrm{pPm}$ DIFF. PRESS. $6.90 \quad " \mathrm{H}_{2} \mathrm{O}$ ABS. PRESS. 15.87 PSIA TEMP. $\mathrm{ZC}{ }^{\circ} \mathrm{F}$

SENSOR CONCENTRATION OUTPUT:

2 MINUTE CONCENTRATION VALUES:

SMC 86./ PPM WHITTAKER \$0.2 pPm

14 MINUTE 30 SECOND CONCENTRATION VALUES:

SMC $86.9 \mathrm{ppm} \quad$ WHITTAKER $230.3 \mathrm{ppm}$

15 MINUTE CONCENTRATION VALUES:

SMC $87.8 \mathrm{ppm}$. WHITTAKER $230.4 \mathrm{ppm}$

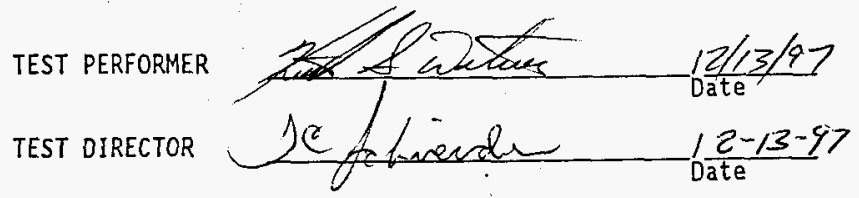


TEST ID 1-3

File FG121313

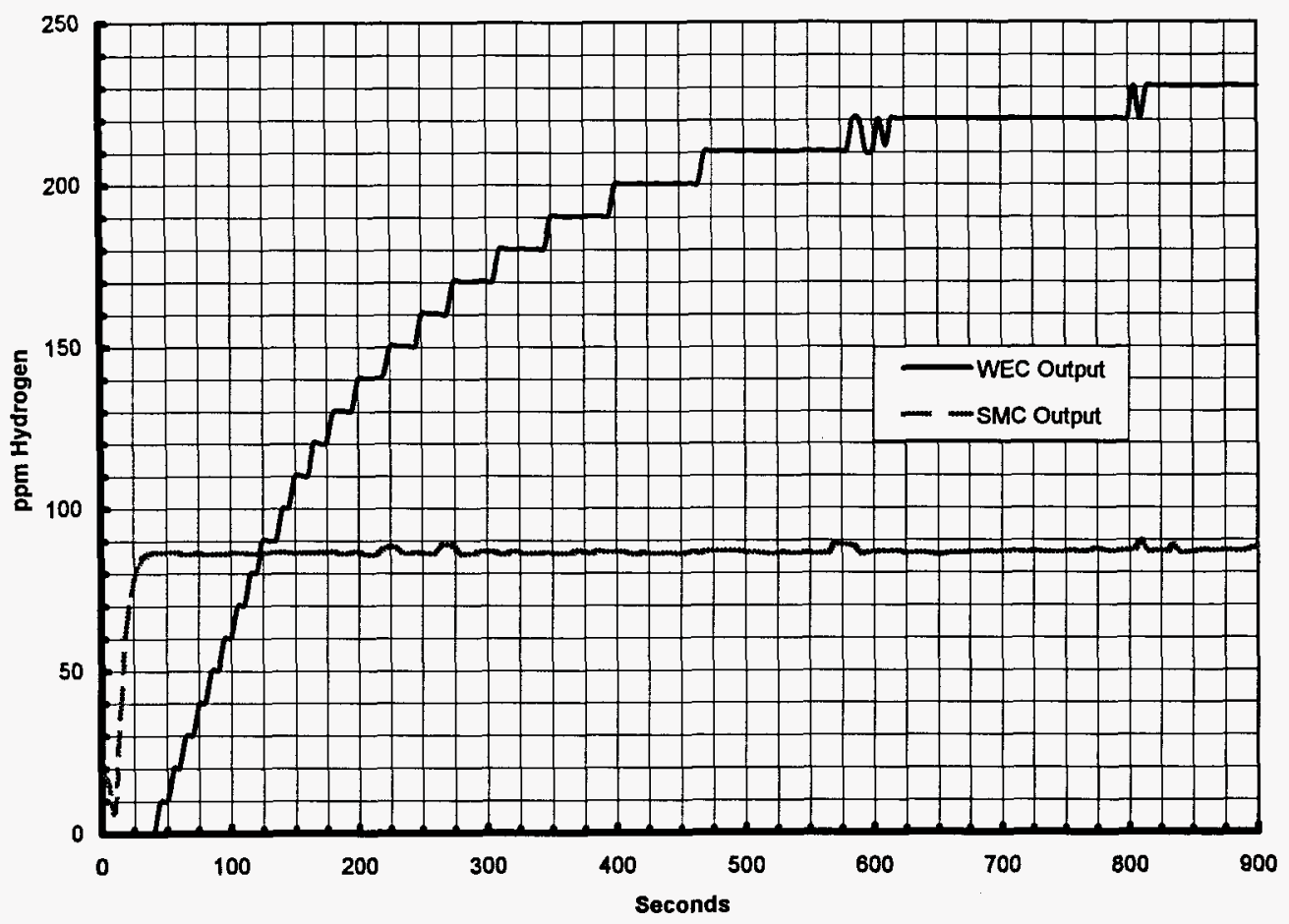

HNF-SD-WM-TRP-275 REV 0 


\section{FGI FLOH TEST DATA SHEET}

TEST ID. No, 2-4

SMC ID. No. 96- $3-4455$
DATA FILE No.

$6 C 021372-4$

WHITTAKER ID NO. $15-1226$

MAIN DUCT FLOW PARAMETERS:

REQUIRED FLOW $200 \quad$ CFM

DIFF. PRESS. $3.77^{~} \mathrm{H}_{2} \mathrm{O}$

CALCULATED FLOW $198.7 \mathrm{CFM}$

DUCT LENGTH $/ Y, / Y$ FT.

ABS. PRESS. $14,8 /$ PSIA

TEMP. $78{ }^{\circ} \mathrm{F}$

FLOH VELOCITY 6.07 FT/SEC

TRANSPORT TIME 2.33 SEC.

INJECTION FLOW PARAMETERS:

Calculated He -3707 pom

DESIRED $\mathrm{H}_{2}$ CONCENTRATION $Y 000 \mathrm{PPM}$ MEASURED $\mathrm{H}_{2}$ CONCENTRATION $\%, 05 Y \mathrm{PPM}$

DIFF. PRESS. $1.76 \quad{ }^{2} \mathrm{H}_{2} \mathrm{O}$ ABS. PRESS. 1.73 PSIA TEMP. $7 /{ }^{\circ} \mathrm{F}$

SENSOR CONCENTRATION OUTPUT:

2 MINUTE CONCENTRATION VALUES:

SMC $\quad 3927.2 \mathrm{ppm}$

WHITTAKER $1732.8 \mathrm{ppm}$

14 MINUTE 30 SECOND CONCENTRATION VALUES:

SMC 3957.8ppm WHITTAKER 4226 ppm

15 MINUTE CONCENTRATION VALUES:

SMC $3962.7 \mathrm{ppm}$. WHITTAKER $4225.8 \mathrm{ppm}$

TEST PERFORMER

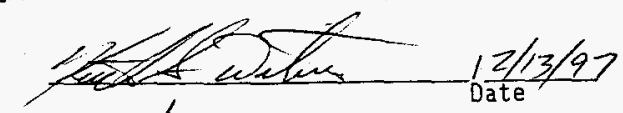

TEST DIRECTOR

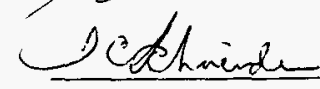
Date $\frac{2-13-97}{10}$ 


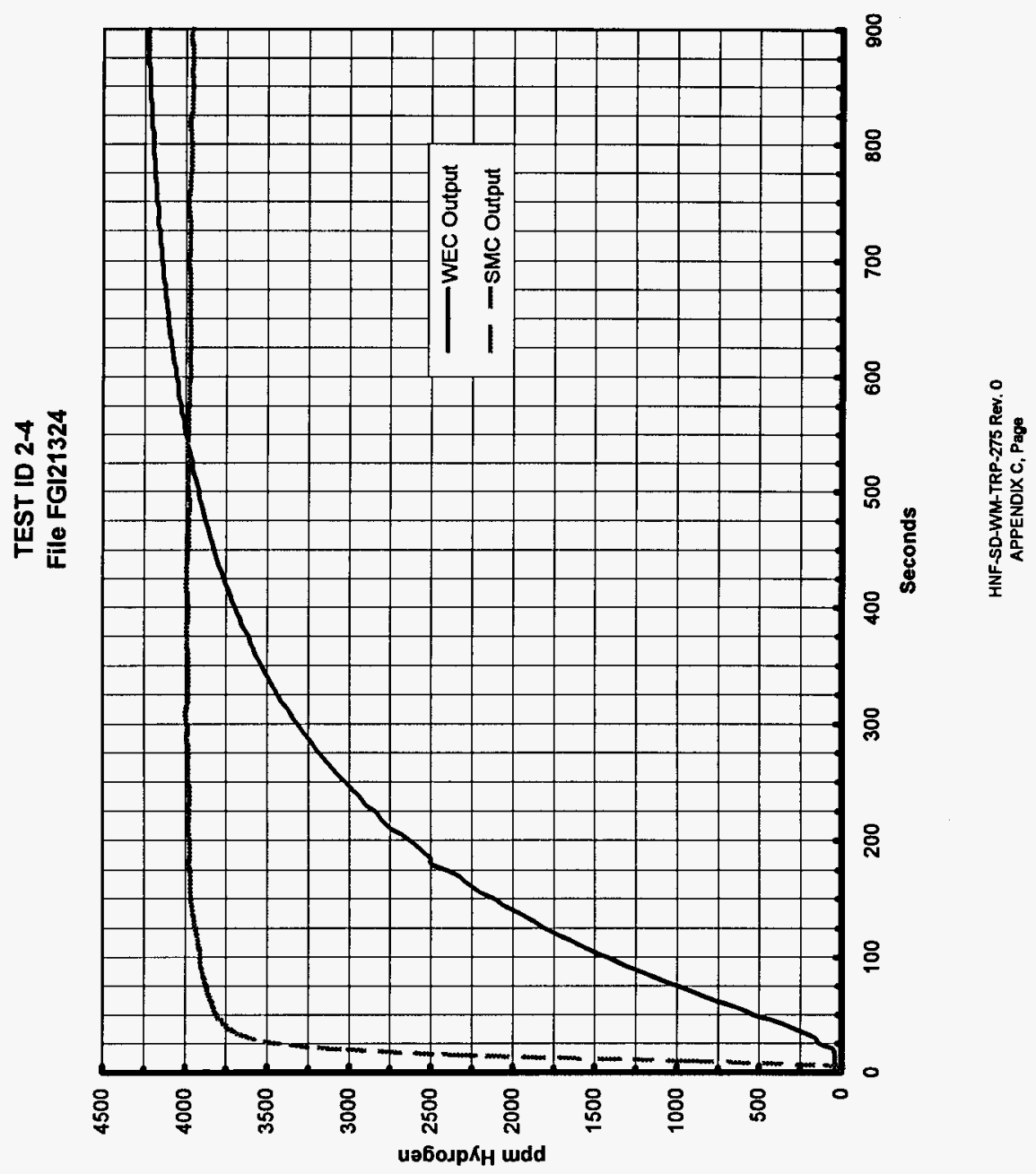




\section{FGI FLOW TEST DATA SHEET.}

TEST ID. No. EX/ $/$ SMC ID. No. $96-3-4455$
DATA FILE NO. GC O2/37EX, DLEX/02/37 WHITTAKER ID NO. $H 5-1226$

MAIN DUCT FLOW PARAMETERS:

REQUIRED FLOW $200 \quad$ CFM CALCULATED FLOW

$199.2 \mathrm{CFM}$

DIFF. PRESS. $3.78 \quad " \mathrm{H}_{2} \mathrm{O}$ ABS. PRESS. $/ 4$, \& $~ P S I A$ TEMP. $78{ }^{\circ} \mathrm{F}$

DUCT LENGTH 14.17 FT. FLOW VELOCITY G.OQFT/SEC

TRANSPORT TIME 2.33 SEC.

INJECTION FLOW PARAMETERS:

$$
\text { Cakalated } H_{2}-3656 \text { ppin }
$$

DESIRED $\mathrm{H}_{2}$ CONCENTRATION

$\lcm{Y 000} \mathrm{ppm}$

MEASURED $\mathrm{H}_{2}$ CONCENTRATION $\$, 040 \mathrm{PPM}$

DIFF. PRESS. $\angle Y 4 \quad \mathrm{H}_{2} \mathrm{O}$

ABS. PRESS. 14.73 PSIA

TEMP. $\quad 700^{\circ} \mathrm{F}$

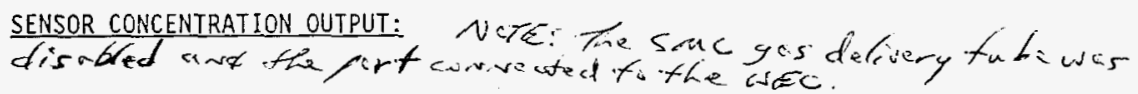
2 MINUTE CONCENTRATION VALUES:

SMC

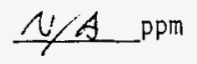

WHITTAKER 2834

14 MINUTE 30 SECOND CONCENTRATION VALUES:

SMC

$$
\text { YAA }
$$

WHITTAKER YYQS PPM

15 MINUTE CONCENTRATION VALUES:

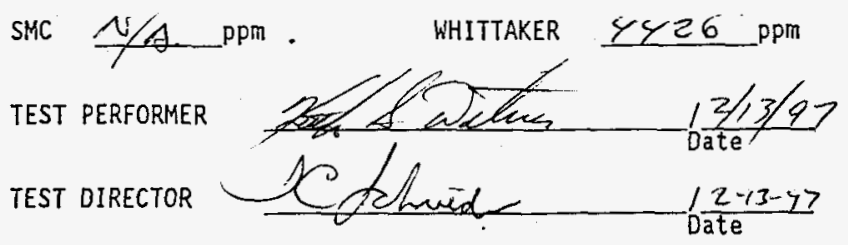




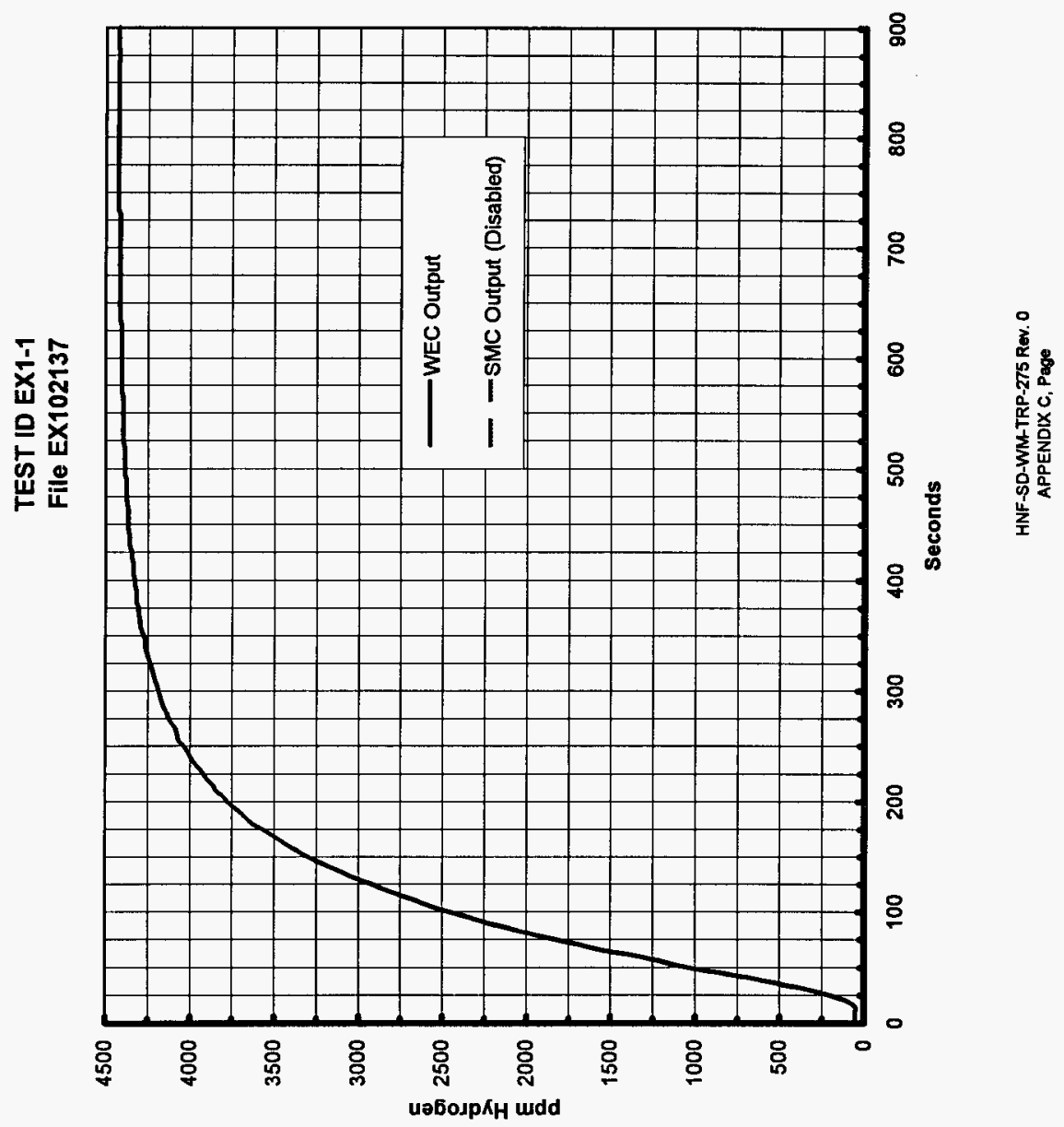


FGI FLOW TEST DATA SHEET.

TEST ID. No. EXX-2 SMC ID. No. 96-16-4455
DATA FILE No. GC O2/37EXZ

DL Ex202137

\section{MAIN DUCT FLOW PARAMETERS:}

REQUIRED FLOW 200 CFM

DIFF. PRESS. $3,77=\mathrm{H}_{2} \mathrm{O}$

DUCT LENGTH $/ 4.17 \mathrm{FT}$.

TRANSPORT TIME 2.33 SEC.
WHITTAKER ID NO. $\not 15 y 226$
INJECTION FLOW PARAMETERS:

DESIRED $\mathrm{H}_{2}$ CONCENTRATION $\underline{Y}_{1}$, O O PPM

DIFF. PRESS. $\angle 7 \% 36$ " $\mathrm{H}_{z} \mathrm{O}$
Calculated Hz -3656 frm MEASURED $\mathrm{H}_{2}$ CONCENTRATION $Y$ COO PPM ABS. PRESS. $\angle 4.73$ PSIA TEMP. $69^{\circ} \mathrm{F}$

SENSOR CONCENTRATION OUTPUT:

2 MINUTE CONCENTRATION VALUES:

SMC YOOZ WPM WHITTAKER Y255

14 MINUTE 30 SECOND CONCENTRATION VALUES:

SMC $3968 \mathrm{pPm} \quad$ WHITTAKER $4406 \mathrm{pPm}$

15 MINUTE CONCENTRATION VALUES:

SMC $396 \angle \mathrm{pPm}$. WHITTAKER \&386

TEST PERFORMER

TEST DIRECTOR

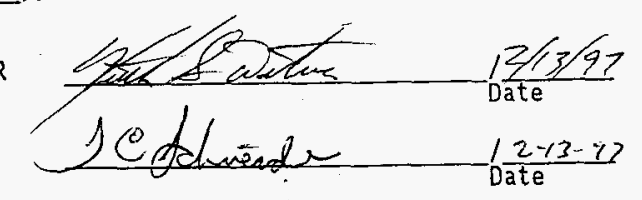




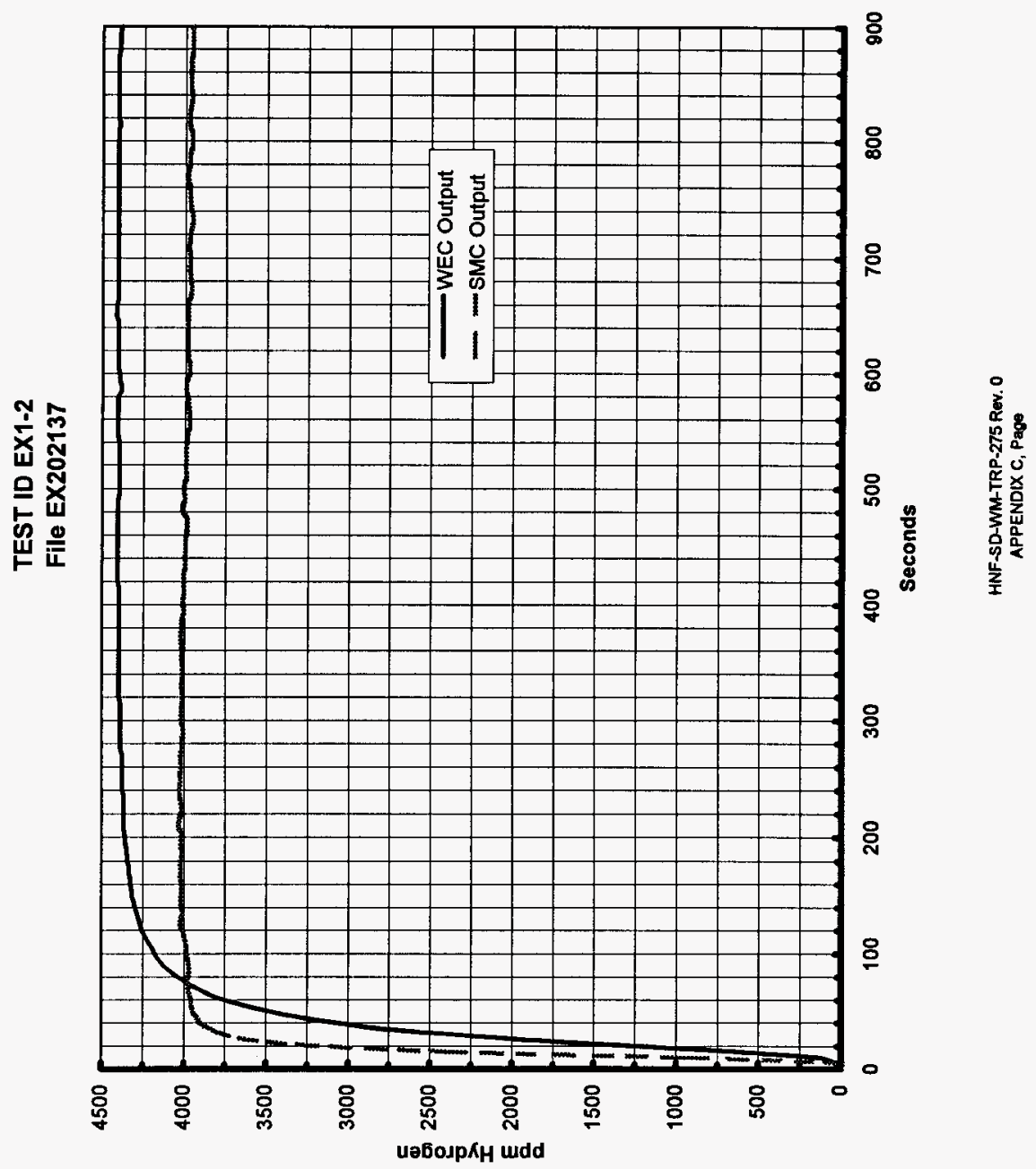



TEST ID. No. $E X /-3$
DATA FILE No.
GC OZ/4TEXI
DL EX102147
SMC ID. No. $96-B-Y 455$ WHITTAKER ID No. $15-1 Z Z 6$

MAIN DUCT FLOW PARAMETERS:

REQUIRED FLOW $20 \mathrm{C}$ CFM

CALCULATED FLOW $197.8 C F M$

DIFF. PRESS. 3.77 " $\mathrm{H}_{2} \mathrm{O}$

ABS. PRESS. 14.75 PSIA

TEMP. $788^{\circ} \mathrm{F}$

DUCT LENGTH $\triangle Y, 1]$ FT.

FLOW VELOCITY 6 .O FT/SEC

TRANSPORT TIME 2.34 SEC.

INUECTION FLOW PARAMETERS:

DESIRED $\mathrm{H}_{2}$ CONCENTRATION YCOCPPM MEASURED $\mathrm{H}_{2}$ CONCENTRATION $4 / 50 \mathrm{pPM}$

DIFF. PRESS. $1,77 " \mathrm{H}_{2} \mathrm{O}$ ABS. PRESS. $/ 4,67$ PSIA TEMP. $\cong 1{ }^{\circ} \mathrm{F}$

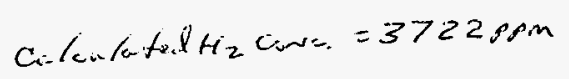

SENSOR CONCENTRATION OUTPUT:

2 MINUTE CONCENTRATION VALUES:

SMC YC26 WPM WHITTAKER 4C95 pPm

14 MINUTE 30 SECOND CONCENTRATION VALUES:

SMC YYC78 $\mathrm{pPm}$ WHITTAKER $\$ 306 \mathrm{PPm}$

15 MINUTE CONCENTRATION VALUES:

SMC $4082 \mathrm{ppm}$. WHITTAKER $4306 \mathrm{ppm}$

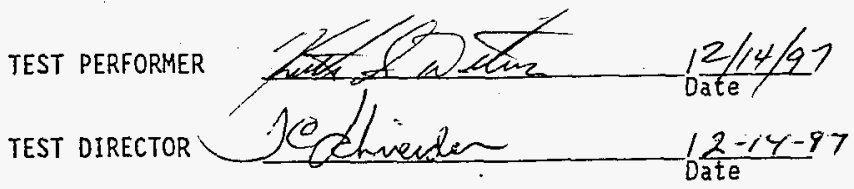




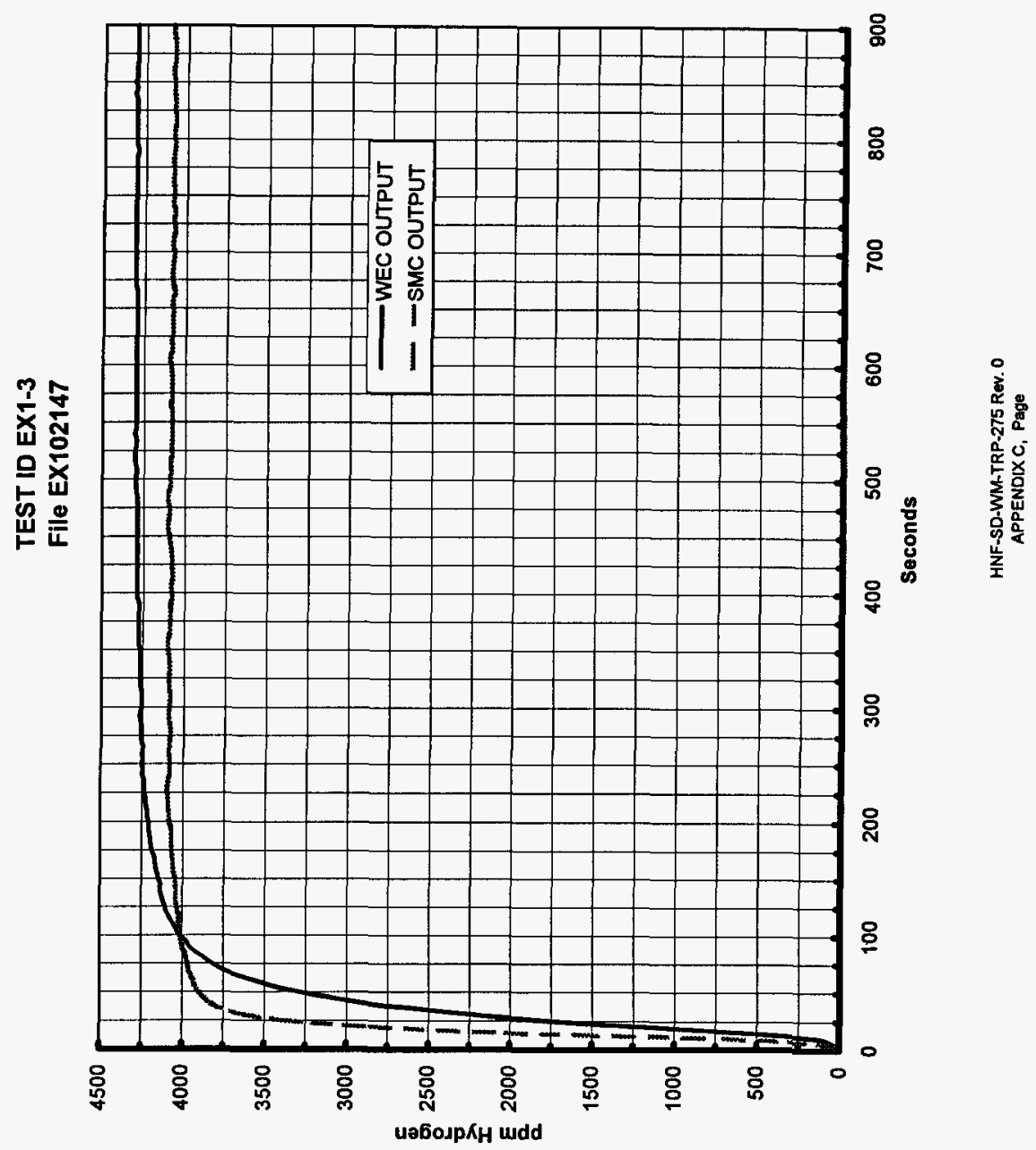


TEST ID. No. $1-1$

SMC ID. No. $46-\beta-Y 455$

MAIN DUCT FLOW PARAMETERS:

REQUIRED FLOW 200 CFM

DIFF. PRESS. $375-\mathrm{H}_{2} \mathrm{O}$

DUCT LENGTH 14.17 FT.

TRANSPORT TIME $2.3 /$ SEC.
DATA FILE No. GCOZZ2Z71-1 DLFGIZZ2Z11 WHITTAKER' ID No. $115-1226$

INJECTION FLOW PARAMETERS:

CALCULATED FLOW $200.4 \mathrm{CFM}$

ABS. PRESS. 4.74 PSIA TEMP. $68{ }^{\circ} \mathrm{F}$

FLOW VELOCITY G./ZFT/SEC

DESIRED $\mathrm{H}_{2}$ CONCENTRATION $20 \mathrm{O}$

ppm

$$
\text { Colenlo-fed Cou }
$$

Cirs. $=207.6 \mathrm{rpm}$

DIFF. PRESS. $4.03 \quad " \mathrm{H}_{2} \mathrm{O}$
SENSOR CONCENTRATION OUTPUT:

2 MINUTE CONCENTRATION VALUES:

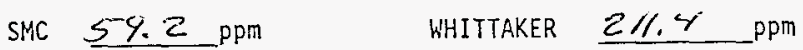

14 MINUTE 30 SECOND CONCENTRATION VALUES:

SMC $57.9^{\circ} \mathrm{ppm} \quad$ WHITTAKER 221 Ppm

15 MINUTE CONCENTRATION VALUES:

SMC $57.3 \mathrm{ppm}$. WHITTAKER $22 / .4 \mathrm{ppm}$

TEST PERFORMER

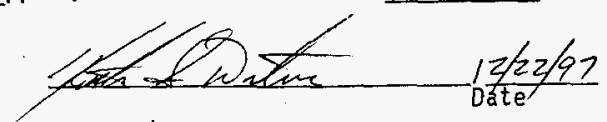

TEST DIRECTOR

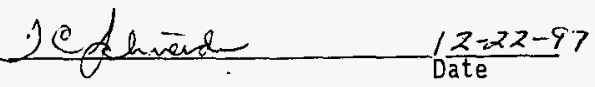


TEST ID 1-1

File FG|22211

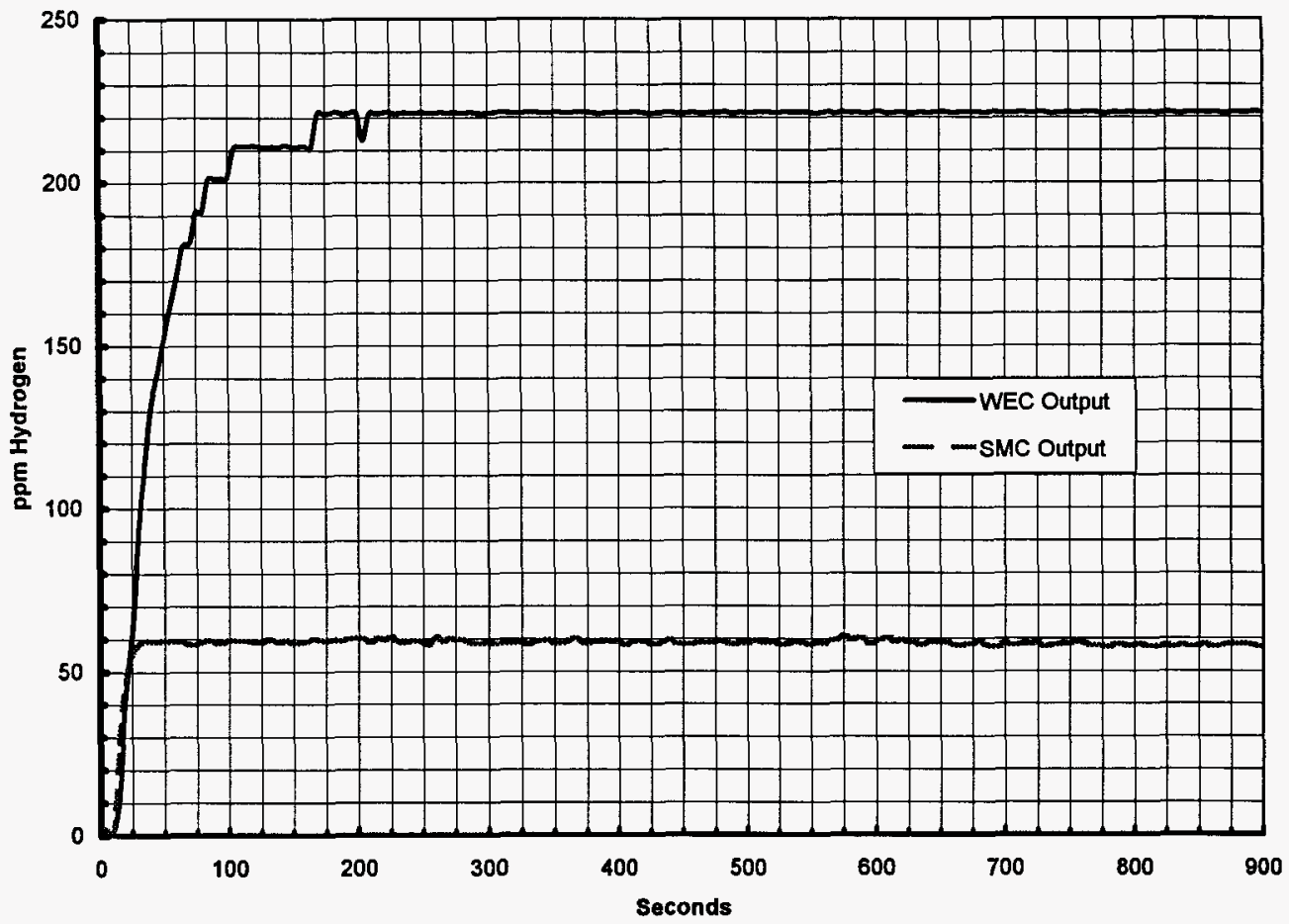

HNF-SD-WM-TRP-275 Rev. 0 


\section{FGI FLOW TEST DATA SHEET}

TEST 10. No. $1-2$

SMC ID. No. 96-6-445-5

MAIN DUCT FLOW PARAMETERS:

REQUIRED FLOW ZOO CFM

DIFF. PRESS. $3.76{ }^{-H_{2} \mathrm{O}}$

DUCT LENGTH $\perp 4,17$ FT.

TRANSPORT TIME $2.3 /$ SEC.

INJECTION FLOW PARAMETERS:

DESIRED $\mathrm{H}_{2}$ CONCENTRATION ZUO PPM

Cakukated cinr. H. $=206.4$

DIFF. PRESS. $\mathrm{Y}, \mathrm{O} / 7 \mathrm{C}^{\mathrm{H}} \mathrm{H}$

ABS. PRESS.
CALCULATED FLOW $200.9 \mathrm{CFM}$

ABS. PRESS. 4 ITY PSIA TEMP. $68{ }^{\circ} \mathrm{F}$

FLOH VELOCITY $6.14 \mathrm{FT} / \mathrm{SEC}$

SENSOR CONCENTRATION OUTPUT:

2 MINUTE CONCENTRATION VALUES:

SMC 54 PPM WHITTAKER $211.3 \mathrm{ppm}$

14 MINUTE 30 SECOND CONCENTRATION VALUES:

SMC $53^{i} \mathrm{pPm} \quad$ WHITTAKER $23 / .4 \mathrm{pPm}$

15 MINUTE CONCENTRATION VALUES:

SMC $52.5 \mathrm{ppm}$. WHITTAKER 230 ppm

TEST PERFORMER

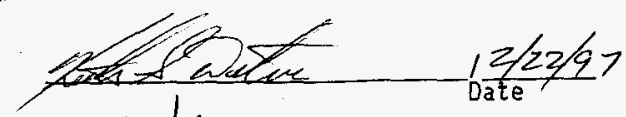

TEST DIRECTOR

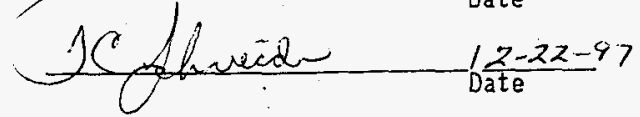




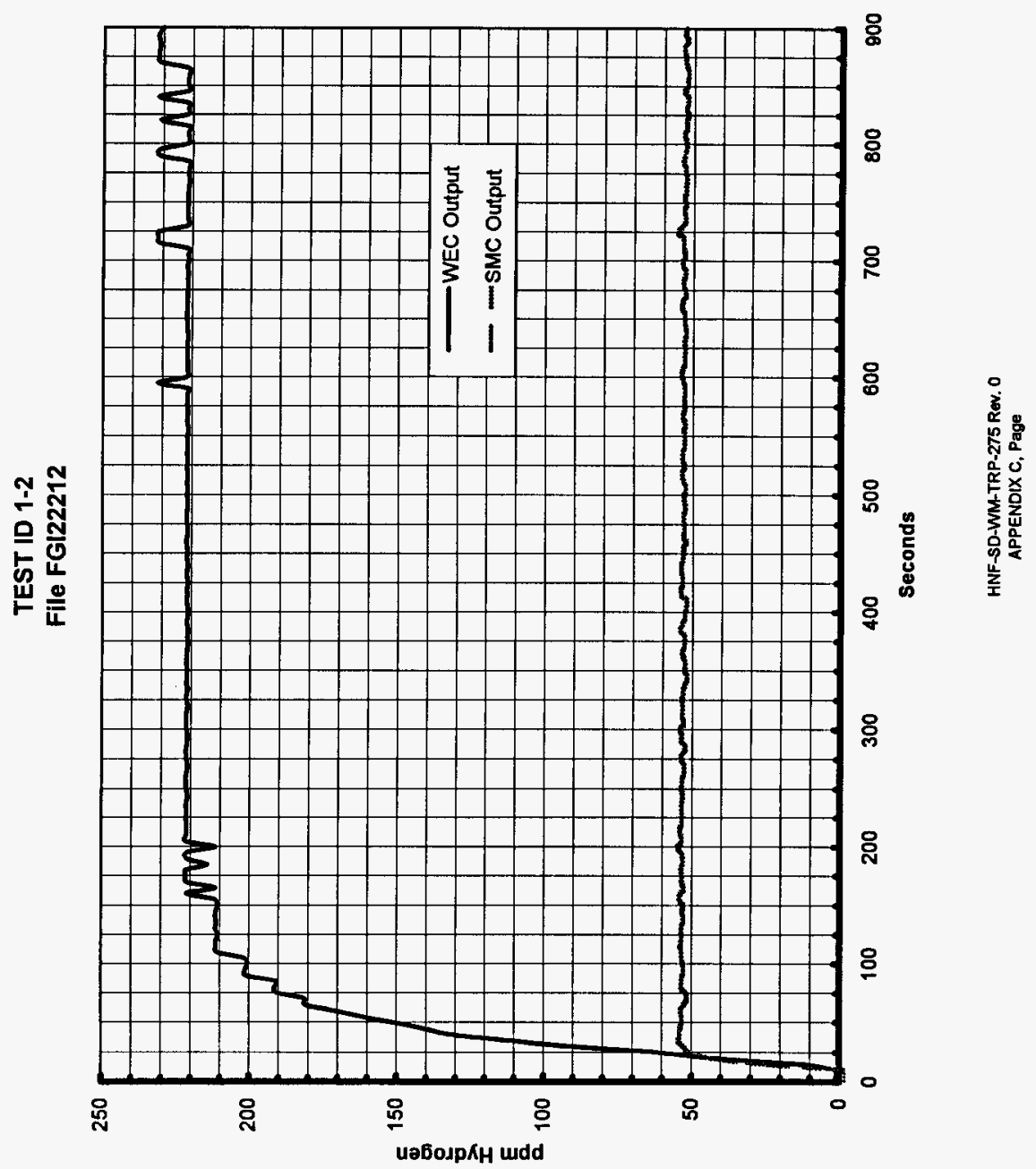


TEST ID. No. $1-3$

DATA FILE No.

GC $02227 /-3$

$\mathrm{DLFGIZ22/3}$

SIMC ID. No. $96-B-4455$ WHITTAKER ID No. H5-1226

MAIN DUCT FLOH PARAMETERS:

REQUIRED FLOW $200 \quad C F M$

DIFF. PRESS. $3.76 \quad \mathrm{H}_{2} \mathrm{O}$

DUCT LENGTH $\ \% 17$ FT.

TRANSPORT TIME $2.3 /$ SEC.
CALCULATED FLOW $20 \% / \mathrm{CFM}$

ABS. PRESS. 14.75 PSIA TEMP. $68^{\circ} \mathrm{F}$

FLOW VELOCITY C. M 4 FT/SEC

\section{INJECTION FLOW PARAMETERS:}

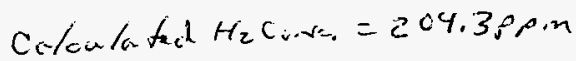

DESIRED $\mathrm{H}_{2}$ CONCENTRATION $\mathrm{ZOO} p P M$ MEASURED $\mathrm{H}_{2}$ CONCENTRATION $2 / 7 \mathrm{ppm}$

DIFF. PRESS. 3.98 " $\mathrm{H}_{2} \mathrm{O}$ ABS. PRESS. 15.06 PSIA

TEMP. $69^{\circ} \mathrm{F}$

\section{SENSOR CONCENTRATION OUTPUT:}

2 MINUTE CONCENTRATION VALUES:

SMC Y Y.0 ppm

WHITTAKER $2 \%, 3 \mathrm{pPm}$

14 MINUTE 30 SECOND CONCENTRATION VALUES:

SMC Y7.4 HPM HITTAKER 2/8 ppm

15 MINUTE CONCENTRATION VALUES:

SMC 48 PPM. WHITTAKER $210.6 \mathrm{ppm}$

TEST PERFORMER

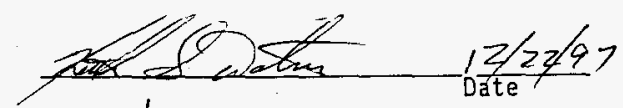

TEST DIRECTOR

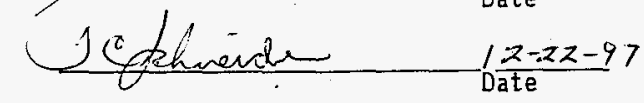




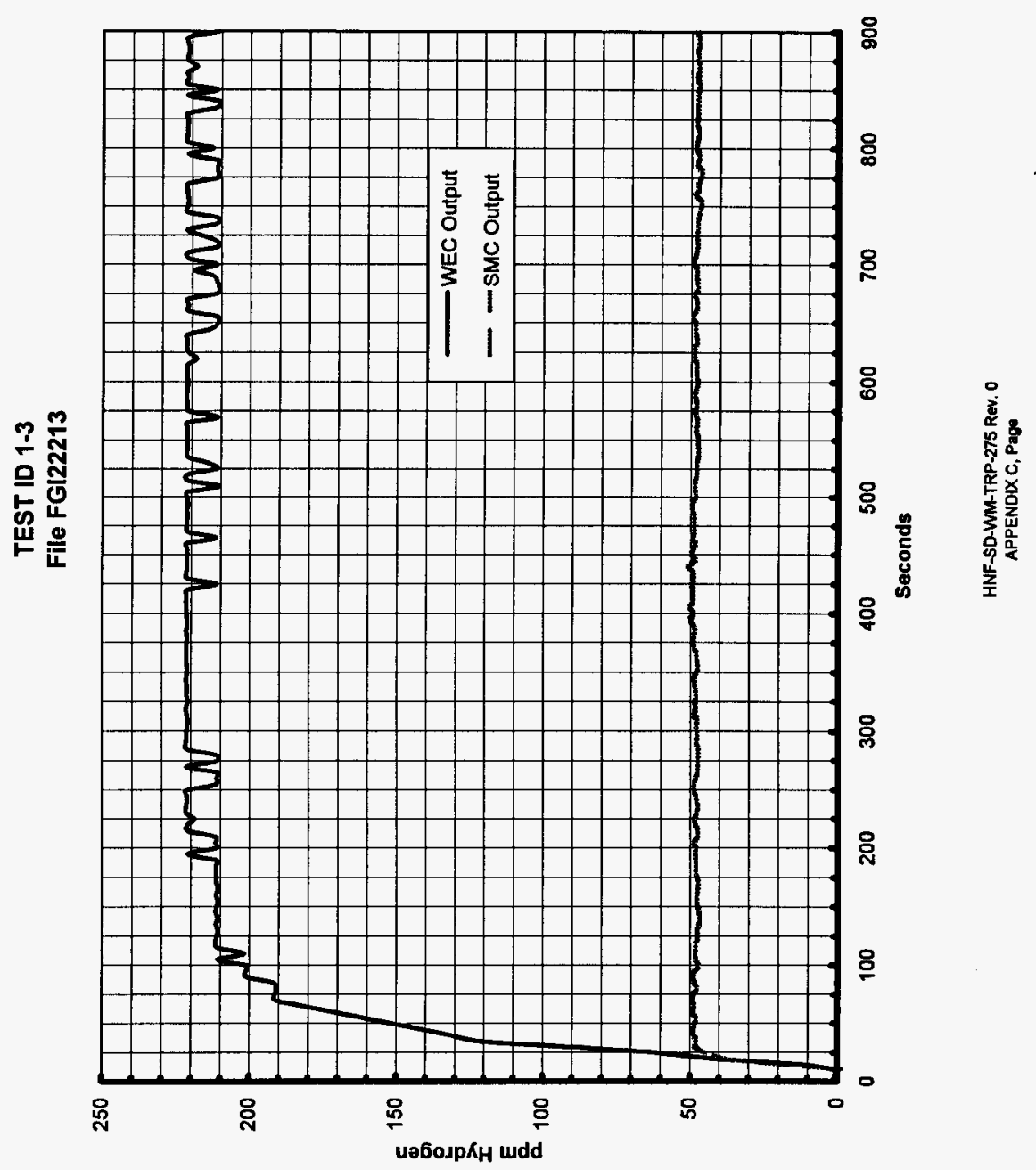




\section{FGI FLOW TEST DATA SHEET}

TEST ID. No. 27 DATA FILE No. GC 022272-1 DLFGI22Z2/

SMC ID. No. 96-B-4455 WHITTAKER ID No. $A 15-1226$

MAIN DUCT FLOW PARAMETERS:

REQUIRED FLOW $200 \mathrm{CFM}$

DIFF. PRESS. 3.815 " $\mathrm{H}_{2} \mathrm{O}$

DUCT LENGTH 14,17 FT.

TRANSPORT TIME 2.27 SEC.
CALCULATED FLOW $20 \% .0 \mathrm{CFM}$

ABS. PRESS. 4.75 PSIA TEMP. $68^{\circ} \mathrm{F}$

FLOW VELOCITY $6.23 \mathrm{FT} / \mathrm{SEC}$

INJECTION FLOW PARAMETERS:

Colculated $H_{2}$ cus. $=/ 9 / 7.5$

DESIRED $\mathrm{H}_{2}$ CONCENTRATION $2,000 \mathrm{pPM}$ MEASURED $\mathrm{H}_{2}$ CONCENTRATION $2029 \mathrm{ppm}$ DIFF. PRESS. $0.940{ }^{\prime \prime} H_{2} \mathrm{O}$ ABS. PRESS. 14.62 PSIA TEMP. $69{ }^{\circ} \mathrm{F}$

SENSOR CONCENTRATION OUTPUT:

2 MINUTE CONCENTRATION VALUES:

SMC $1893 \mathrm{pPm} \quad$ WHITTAKER $2012 \quad \mathrm{pPm}$

14 MINUTE 30 SECOND CONCENTRATION VALUES:

SMC $187 \& \mathrm{ppm} \quad$ WHITTAKER $2093 \mathrm{ppm}$

15 MINUTE CONCENTRATION VALUES:

SMC $\angle 885 \mathrm{pPm}$. WHITTAKER $2093 \mathrm{ppm}$

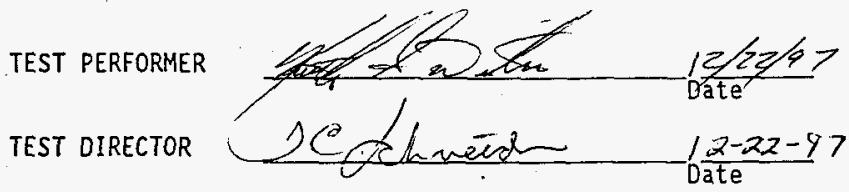


TEST ID 2-1

File FGI22221

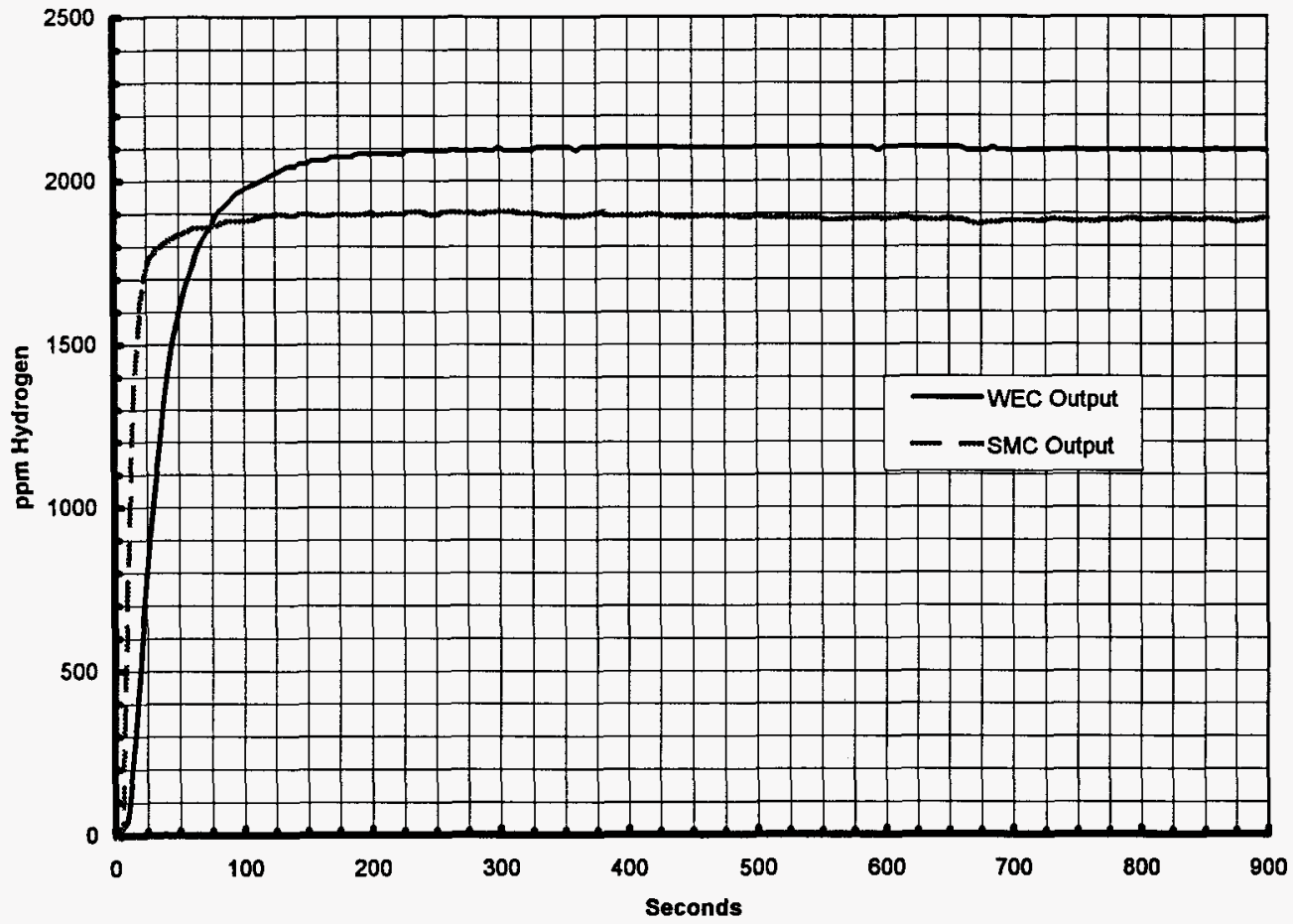

HNF-SD-WM-TRP-275 Rev, 0

APPENDIX C, Page 
TEST ID. No. $2-2$

SIC ID. No. $96-B-4455$
DATA FILE No.

GC $022272-2$

DL F fG<Zz2zz

MAIN DUCT FLOW PARAMETERS:

REQUIRED FLOW Z0O CFM

CALCULATED FLOW $19 \%$ C CFM

DIFF. PRESS: $3.74 \quad " \mathrm{H}_{2} \mathrm{O}$

ABS. PRESS. 14.75 PSIA

TEMP. $69{ }^{\circ} \mathrm{F}$

DUCT LENGTH $1 Y, 17 \mathrm{FT}$.

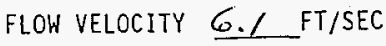

TRANSPORT TIME 2.32 SEC.

INJECTION FLOW PARAMETERS:

Colculatad $\mathrm{H}_{2} \mathrm{C}_{\mathrm{u}} \mathrm{s}=2055$

DESIRED $\mathrm{H}_{2}$ CONCENTRATION

Zicoppm

MEASURED $\mathrm{H}_{2}$ CONCENTRATION $2 / 70 \mathrm{PPM}$

DIFF. PRESS. $\mathrm{C} .787 \mathrm{H}_{2} \mathrm{O}$

ABS. PRESS. 4.63 PSIA

TEMP. $69{ }^{\circ} \mathrm{F}$

SENSOR CONCENTRATION OUTPUT:

2 MINUTE CONCENTRATION VALUES:

SMC $2006 \mathrm{ppm} \quad$ WHITTAKER $2173 \mathrm{ppm}$

14 MINUTE 30 SECOND CONCENTRATION VALUES:

SMC $2923 \mathrm{pPm} \quad$ WHITTAKER $2273.8 \mathrm{ppm}$

15 MINUTE CONCENTRATION VALUES:

SMC $2030 \mathrm{ppm}$. WHITTAKER 2273.8 $\mathrm{ppm}$

TEST PERFORMER

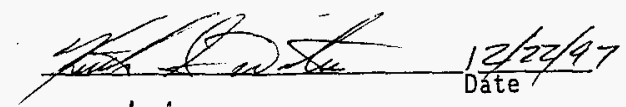

TEST DIRECTOR

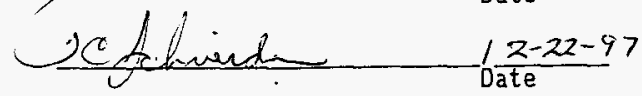




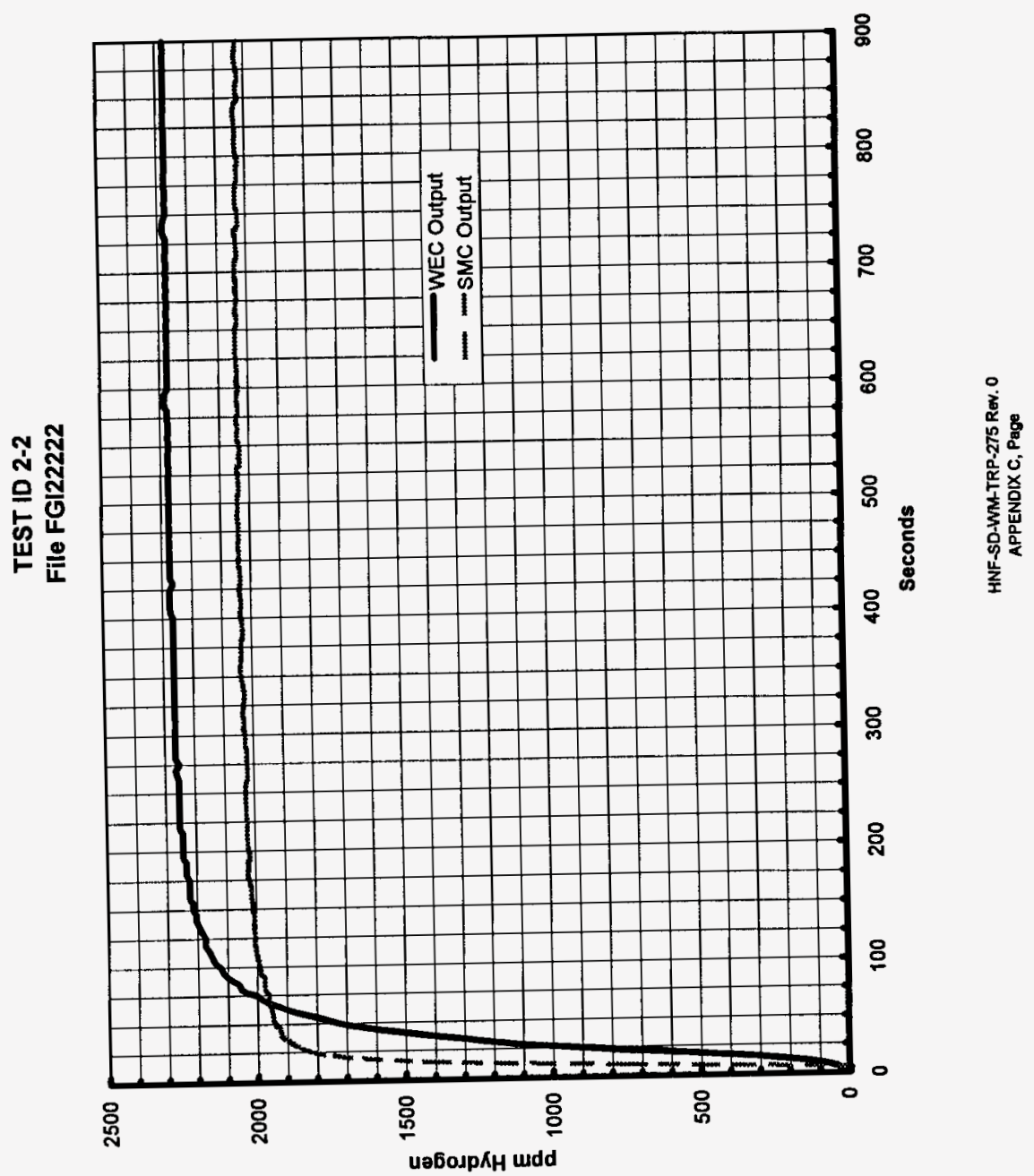




\section{FGI FLOW TEST DATA SHEET}

TEST ID. No. $2-3$ DATA FILE NO.

GC $022272-3$

DL FGE22223

SMC IO. NO. $96-\beta-4455$ WHITTAKER ID NO. HS 1226

MAIN DUCT FLOW PARAMETERS:

REQUIRED FLOW 200 CFM

DIFF. PRESS. $3.75 " \mathrm{H}_{2} \mathrm{O}$

DUCT LENGTH 14.17 FT.

TRANSPORT TIME 2.32 SEC.
CALCULATED FLOW $200.1 \mathrm{CFM}$

ABS. PRESS. 14.75 PSIA

TEMP. $69^{\circ} \mathrm{F}$

\section{INJECTION FLOW PARAMETERS:}

DESIRED $\mathrm{H}_{2}$ CONCENTRATION ZUNOLPPM MEASURED $\mathrm{H}_{2}$ CONCENTRATION $2 / 59 \mathrm{ppm}$ DIFF. PRESS. $0.978 " \mathrm{H}_{2} \mathrm{O}$ ABS. PRESS. 4.63 PSIA TEMP. $70^{\circ}{ }^{\circ} \mathrm{F}$

\section{SENSOR CONCENTRATION OUTPUT:}

2 MINUTE CONCENTRATION VALUES:

SMC 1973 ppm WHITTAKER $2 / 42$ ppm

14 MINUTE 30 SECOND CONCENTRATION VALUES:

SMC $1988 \mathrm{pPm} \quad$ WHITTAKER ZZZ63 $\mathrm{ppm}$

15 MINUTE CONCENTRATION VALUES:

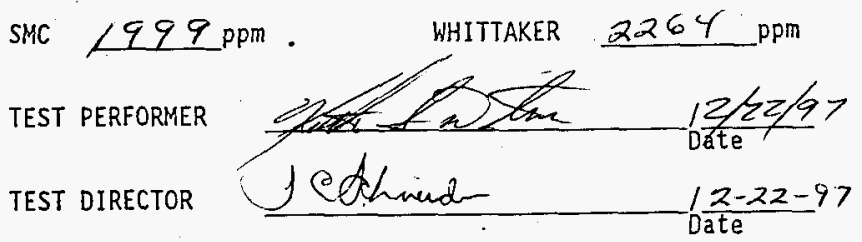


TEST ID 2-3

File FGI22223

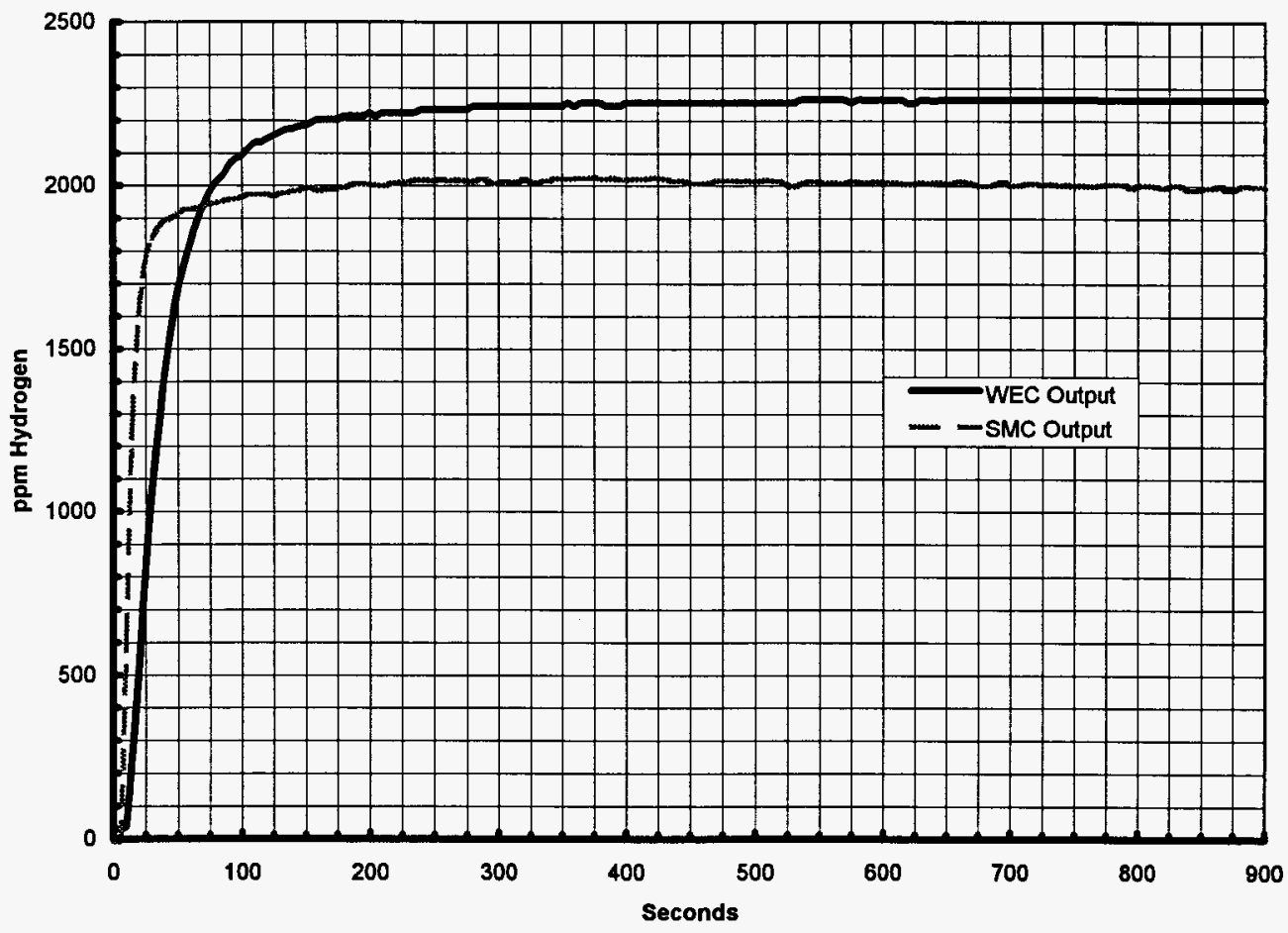

HNF-SD-WM-TRP-275 Rev. 0

APPENDIX C, Page 


\section{FGI FLOW TEST DATA SHEET}

TEST ID. No. 2-4

SMC 10. No. $96-15-4455$

MAIN DUCT FLOW PARAMETERS:

REQUIRED FLOW 200 CFM

DIFF. PRESS. $3.74 " \mathrm{H}_{2} \mathrm{O}$

DUCT LENGTH 14.17 FT.

TRANSPORT TIME 2.32 SEC.
DATA FILE No. GC $022272-4$ DLFGZ2Z224

WHITTAKER ID No. HYS-12z6

INJECTION FLOW PARAMETERS:

CALCULATED FLOW 199.6 CFM

ABS. PRESS. 4.75 PSIA

TEMP. $69{ }^{\circ} \mathrm{F}$

FLOW VELOCITY G. LFT/SEC

INJECTION FLOW PARAKETERS:

c.leulated to Cive $=4182$

DESIRED $\mathrm{H}_{2}$ CONCENTRATION YOCO PPM

MEASUREO $\mathrm{H}_{2}$ CONCENTRATION $4355 \mathrm{pPm}$

DIFF. PRESS. 2. Q CCC " $\mathrm{H}_{2} \mathrm{O}$

ABS. PRESS. 4.69 PSIA

TEMP. $70{ }^{\circ} \mathrm{F}$

\section{SENSOR CONCENTRATION OUTPUT:}

2 MINUTE CONCENTRATION VALUES:

SMC YZYO WPM WHITTAKER 4335

14 MINUTE 30 SECOND CONCENTRATION VALUES:

SMC $\$ 324 \mathrm{ppm}$ WHITTAKER $\$ 606 \mathrm{ppm}$

15 MINUTE CONCENTRATION VALUES:

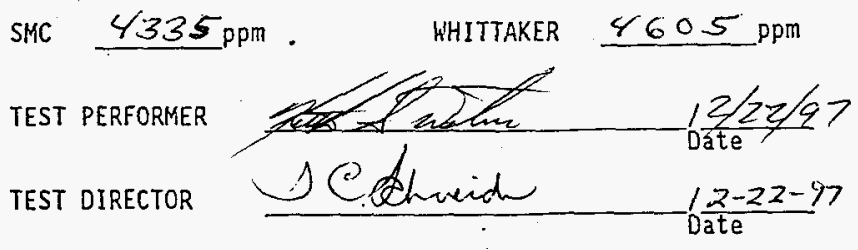


TEST ID 2-4

File FGI22224

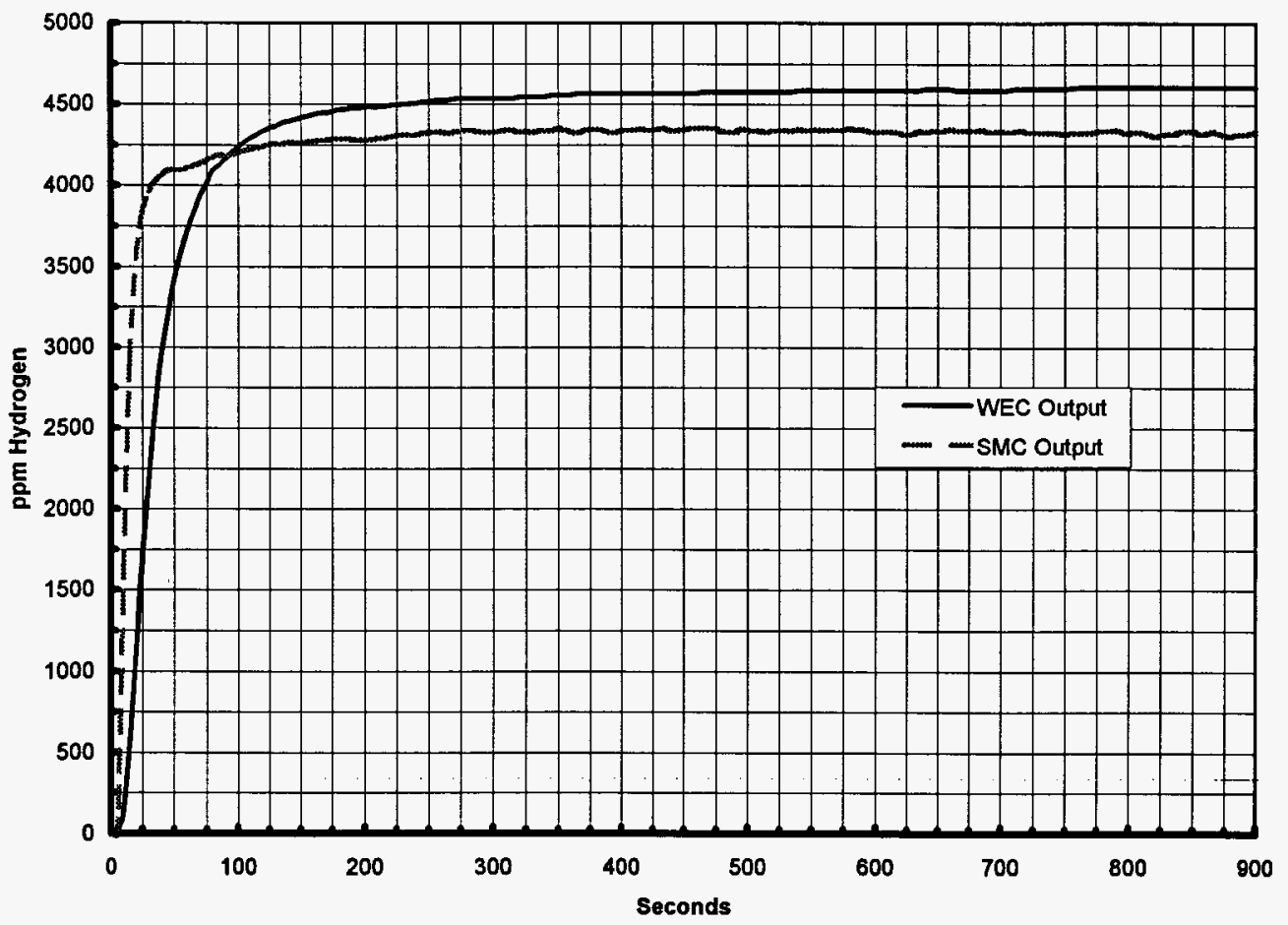

HNF-SD-WM-TRP-275 Rev. 0

APPENDIX C, Page 


\section{FGI FLOW TEST DATA SHEET}

TEST ID. No. $2-5$

SMC ID. No. $96-B-4 Y 55$

MAIN DUCT FLOW PARAMETERS:

REQUIRED FLOH $200 \quad C F M$

DIFF. PRESS. 3,75 " $_{2} \mathrm{O}$

DUCT LENGTH 14.17 FT.

TRANSPORT TIME 2.32 SEC.
DATA FILE No. GC 0 222Z72-5 DL FGZ2Z2z5 WHITTAKER ID NO. HYS-1226

CALCULATED FLOW $200.1^{\mathrm{CFM}}$

ABS. PRESS. 44,75 PSIA TEMP. $\mathrm{Z}^{\circ} \mathrm{F}$

FLOH VELOCITY $6 . / 2$ FT/SEC

INUECTION FLOW PARAMETERS:

$$
\text { Cislculated } \mathrm{H}_{2} \text { Cins. }=4169
$$

DESIRED $\mathrm{H}_{2}$ CONCENTRATION 4 CCCO PPT MEASURED $\mathrm{H}_{2}$ CONCENTRATION $4373 \mathrm{pPm}$ DIFF. PRESS. $1.999 " \mathrm{H}_{2} \mathrm{O}$ ABS. PRESS. $\$ .69$ PSIA TEMP. $70^{\circ}{ }^{\circ} \mathrm{F}$

SENSOR CONCENTRATION OUTPUT:

2 HINUTE CONCENTRATION VALUES:

SMC Y252 WPM WHITTAKER $4345 \mathrm{ppm}$

14 MINUTE 30 SECOND CONCENTRATION VALUES:

SMC $4324 \mathrm{ppm}$ WHITTAKER $46.36 \mathrm{pPm}$ :

15 MINUTE CONCENTRATION VALUES:

SMC $4348 \mathrm{ppm} \quad$ WHITTAKER $4636 \mathrm{ppm}$

TEST PERFORMER

TEST DIRECTOR

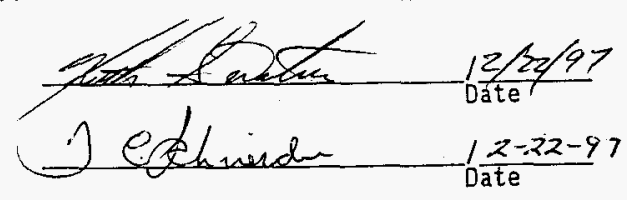




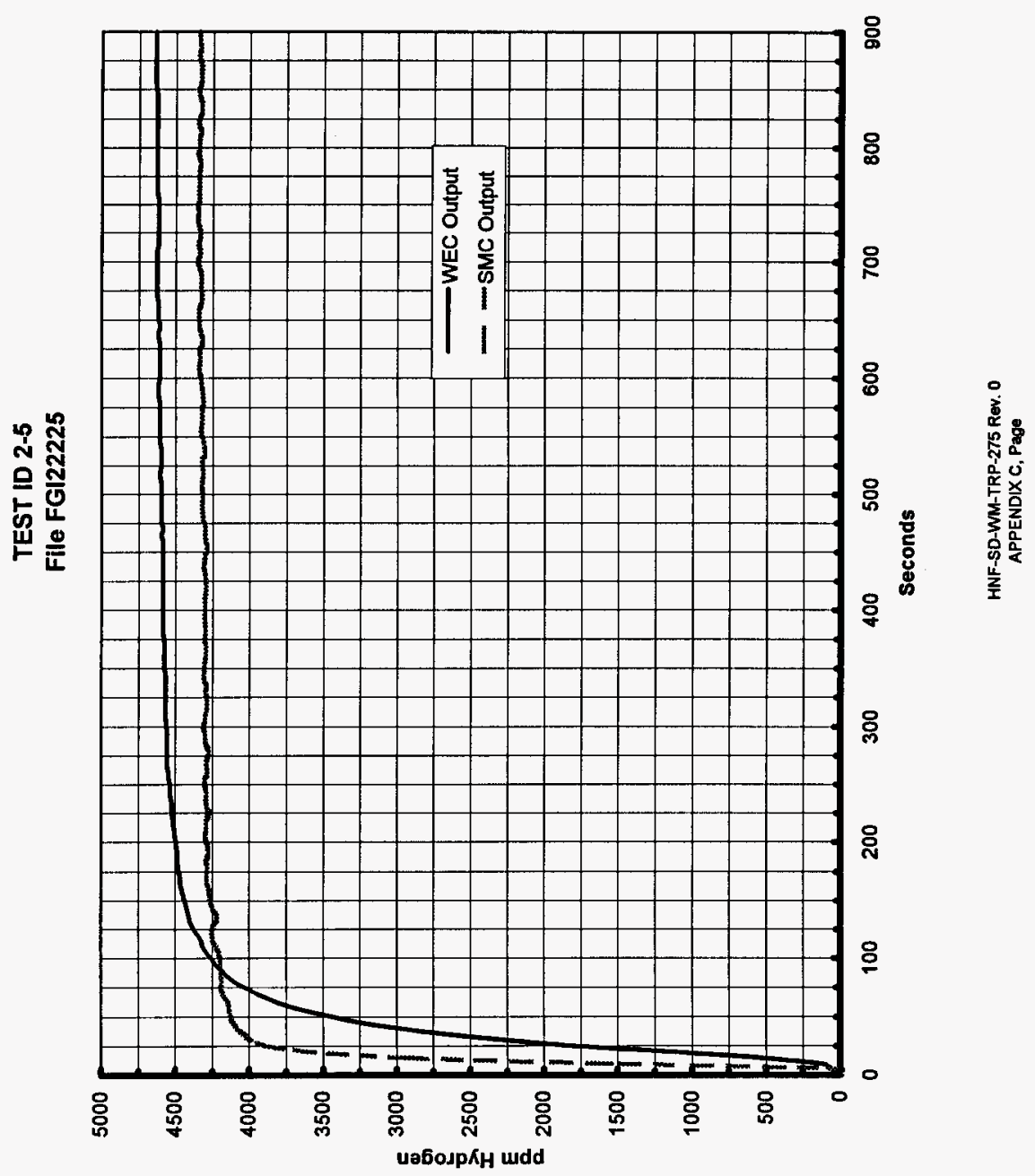




\section{FGI FLOW TEST DATA SHEET}

TEST ID. No. $2-6$

SMC ID. No. 96-6-44-55
DATA FILE No.

GC $022272-6$

WHITTAKER IO NO. GK5=1226

MAIN DUCT FLOW PARAMETERS:

REQUIRED FLOW 200 CFM

DIFF. PRESS. $3.74 " \mathrm{H}_{2} \mathrm{O}$

DUCT LENGTH 14.17 FT.

TRANSPORT TIME $2.32 \mathrm{SEC}$.
CALCULATED FLOW $/ 99.6 \mathrm{CFM}$

ABS. PRESS. 1475 PSIA TEMP. $69^{\circ} \mathrm{F}$

FLOW VELOCITY C./ FT/SEC

INJECTION FLOW PARAMETERS:

Colculutad Hzcud $=4 / 38$

DESIRED $\mathrm{H}_{2}$ CONCENTRATION Y̧OCO PPM MEASURED $\mathrm{H}_{2}$ CONCENTRATION $1377 \mathrm{pPM}$

DIFF. PRESS. 1.979 " $\mathrm{H}_{2} \mathrm{O}$ ABS. PRESS. $\angle 4, G$ GPSIA

TEMP.

$70^{\circ} \mathrm{F}$

SENSOR CONCENTRATION OUTPUT:

2 MINUTE CONCENTRATION VALUES:

SMC Y267 pPm WHITTAKER 4365 ppm

14 MINUTE 30 SECOND CONCENTRATION VALUES:

SMC YZS7 PPM WHITTAKER YG55 ppm.

15 MINUTE CONCENTRATION VALUES:

SMC 4304pPm WHITTAKER Y656 ppm

TEST PERFORMER

TEST DIRECTOR

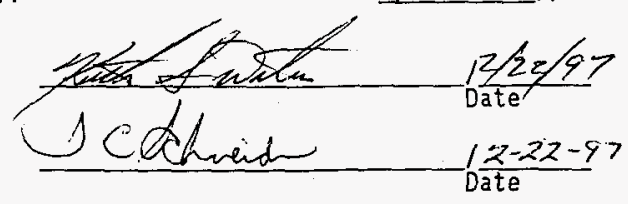




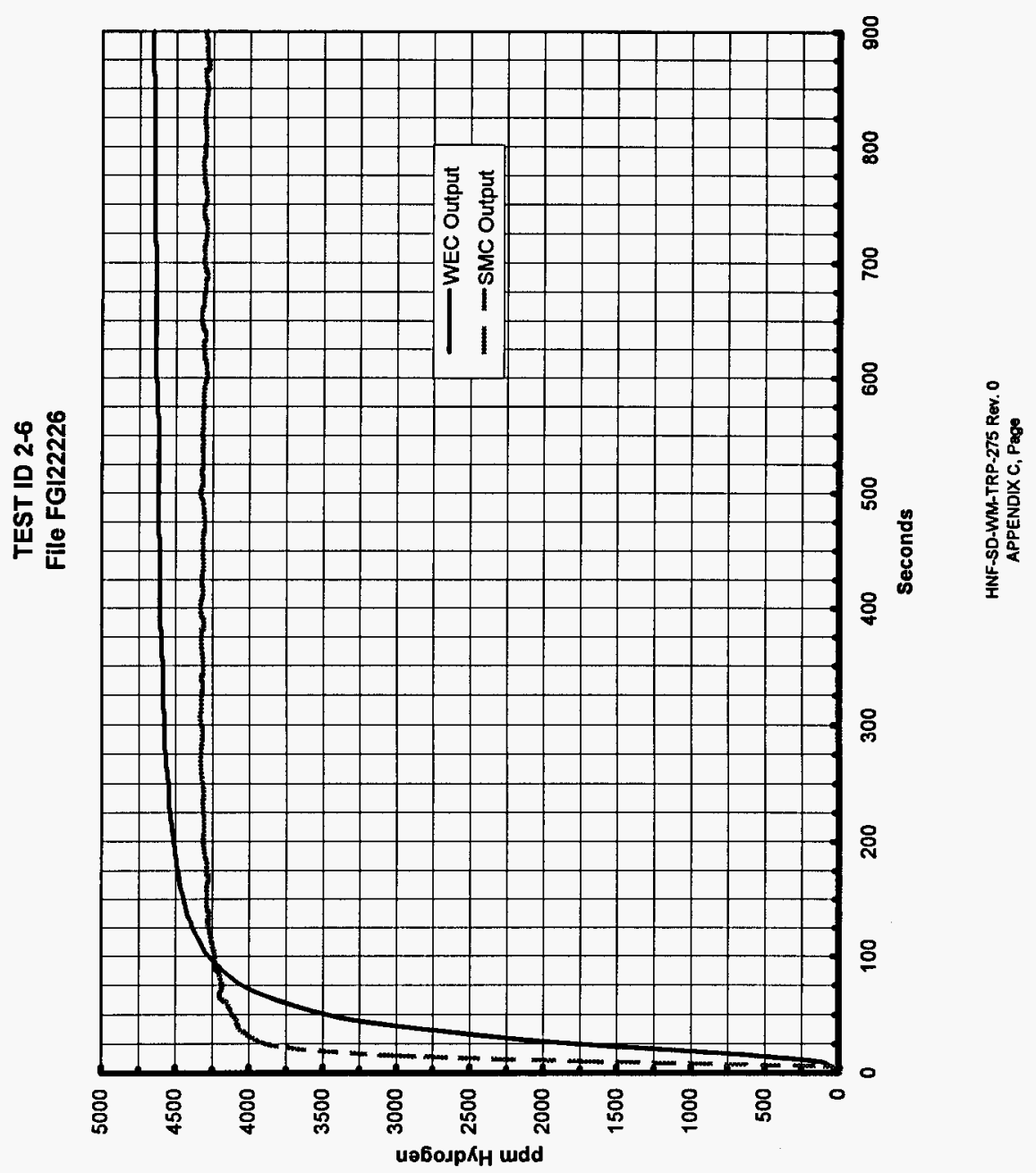


FGI FLOH TEST DATA SHEET

TEST I0. No. 2-7 DATA FILE NO. GC 0222Z72-7 DLFG SMC ID. NO. 96-B-YYSS WHITTAKER IO NO. $195-7226$

MAIN DUCT FLOW PARAMETERS:

REQUIRED FLOW 200 CFM

CALCULATED FLOW $\angle 99.6 \mathrm{CFM}$

DIFF. PRESS. $3.7 Y " \mathrm{H}_{2} \mathrm{O}$ ABS. PRESS. 4.75 PSIA TEMP. $\quad 69{ }^{\circ} \mathrm{F}$

DUCT LENGTH $\ 4.17$ FT. FLOW VELOCITY G./ FT/SEC

TRANSPORT TIME Z.32SEC.

INJECTION FLOW PARAYETERS:

Colculated $\mathrm{H}_{2}$ Cirncendrodis $=6.235 \mathrm{pp}$

DESIRED $\mathrm{H}_{2}$ CONCENTRATION GOOOPPA MEASURED $\mathrm{H}_{2}$ CONCENTRATION $6432 \mathrm{pPm}$

DIFF. PRESS. $\underline{2.97} " \mathrm{H}_{2} \mathrm{O}$ ABS. PRESS. 4.75 PSIA TEMP. $70{ }^{\circ} \mathrm{F}$

SENSOR CONCENTRATION OUTPUT:

2 MINUTE CONCENTRATION VALUES:

SMC $6373 \mathrm{pPm} \quad$ WHITTAKER $6466 \mathrm{pPm}$

14 MINUTE 30 SECOND CONCENTRATION VALUES:

SMC 6YY7_ppm WHITTAKER $69 / 8 \mathrm{ppm}$.

15 MINUTE CONCENTRATION VALUES:

SMC $6485 \mathrm{ppm}$ WHITTAKER $6928 \mathrm{ppm}$

TEST PERFORMER

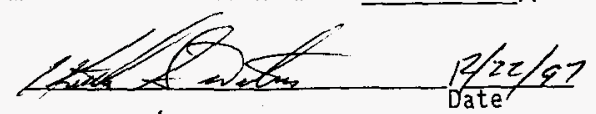

TEST DIRECTOR

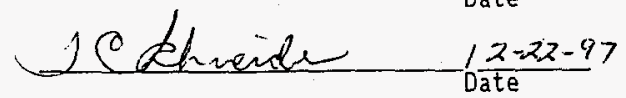




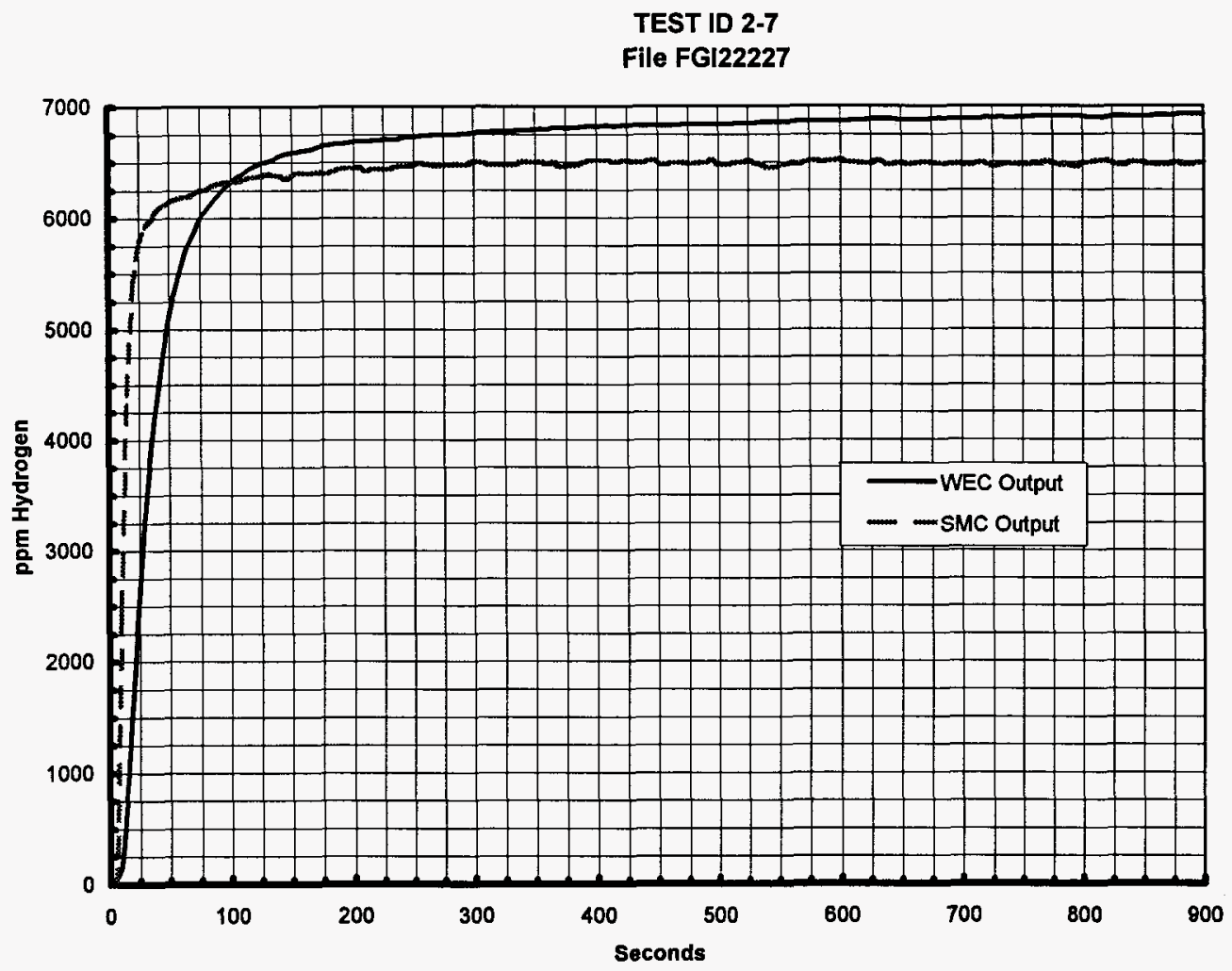

HNF-SD-WM-TRP-275 Rev. 0 APPENDIX C, Page 
TEST ID. No. $2-8$

SMC ID. No. $96-\beta-4455$
DATA FILE No.

GC $022272-8$

DL FGZZ2228

MAIN DUCT FLOW PARAMETERS:

REQUIRED FLOW ZCC C CFM

DIFF. PRESS. $3.74{ }^{\mathrm{n}} \mathrm{H}_{2} \mathrm{O}$

DUCT LENGTH 14,17 FT.

TRANSPORT TIME 2.33 SEC.
CALCULATED FLOW 199.2 CFM ABS. PRESS. 14.75 PSIA TEMP. $70^{\circ} \mathrm{F}$

FLOW VELOCITY $\underline{C}$, FT/SEC

INJECTION FLOW PARAMETERS:

DESIRED $\mathrm{H}_{2}$ CONCENTRATION $600 \mathrm{O}$ PPM MEASURED Hz CONCENTRATION $6665 \mathrm{pPm}$ DIFF. PRESS. $2.98 " \mathrm{H}_{2} \mathrm{O}$ ABS. PRESS. 14.75 PSIA TEMP. $700^{\circ} \mathrm{F}$

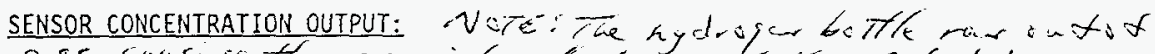

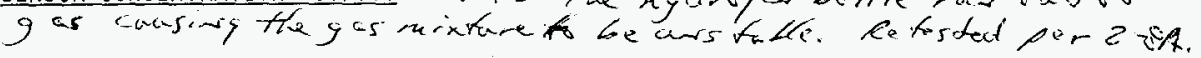
2 MINUTE CONCENTRATION VALUES:

SMC 6322 WPm WHITTAKER $6506 \mathrm{ppm}$

14 MINUTE 30 SECOND CONCENTRATION VALUES:

SMC N/A ppm WHITTAKER Yiezprie

15 MINUTE CONCENTRATION VALUES:

SMC Nétppm WHITTAKER

TEST PERFORMER

TEST DIRECTOR
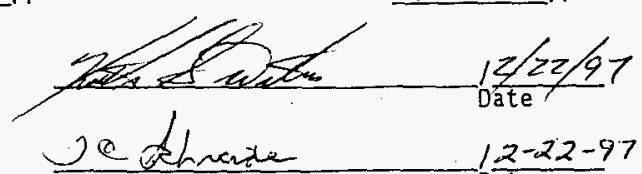
$\frac{12-22-97}{\text { Date }}$ 


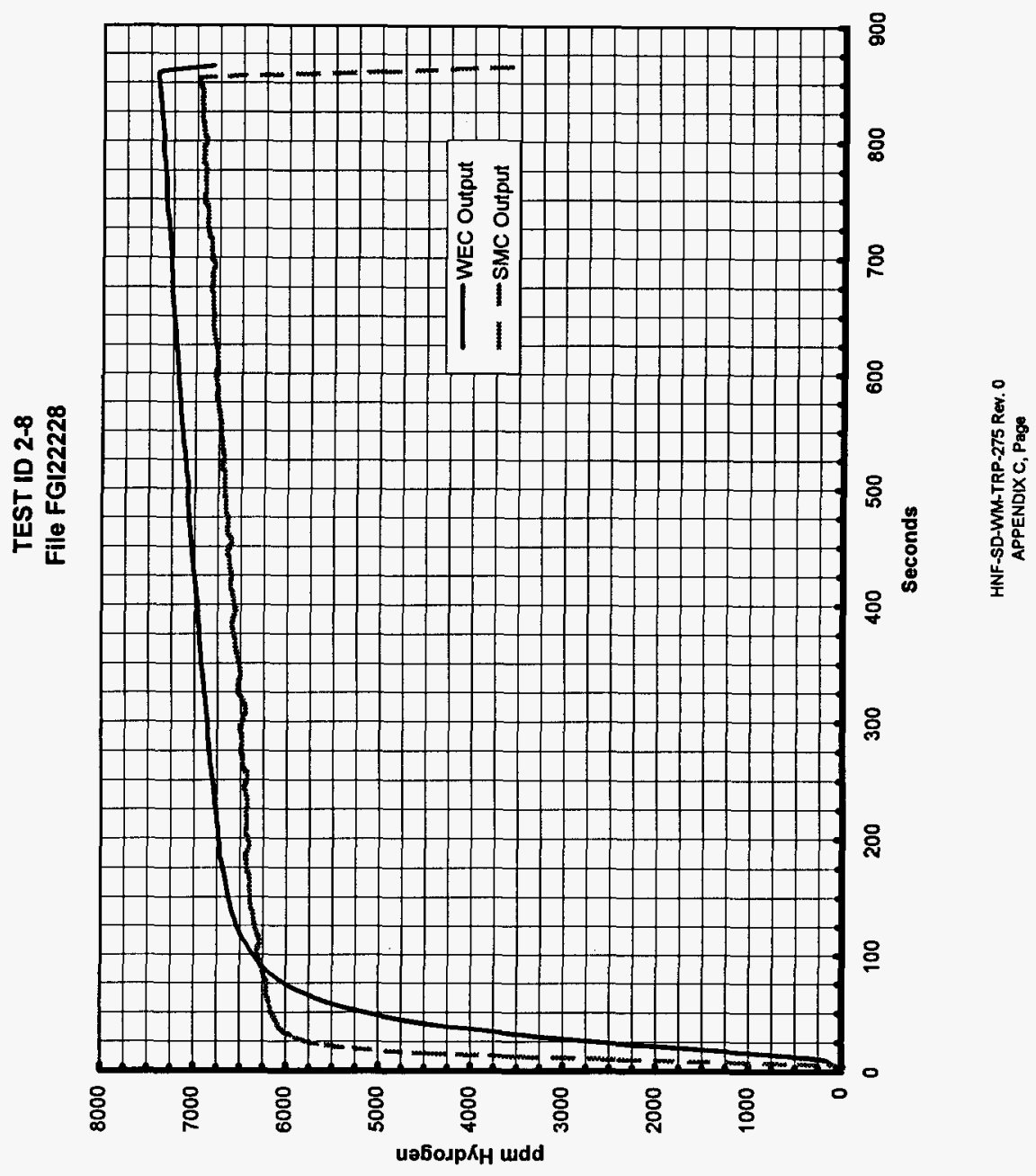




\section{FGI FLOW TEST DATA SHEET}

TEST ID. No. $2-8 A$

SMC ID. No. 96-6-4455
DATA FILE Na.

GC 02227284

OL FG 22228A

\section{MAIN DUCT FLOW PARAMETERS:}

REQUIRED FLOW 200 CFM

DJFF. PRESS, $3,76 " \mathrm{H}_{2} \mathrm{O}$

DUCT LENGTH 14.17 FT.

TRANSPORT TIME 2.32 SEC.
CALCULATED FLOW 200.2CFM

ABS. PRESS. 14.74 PSIA TEMP. $70{ }^{\circ} \mathrm{F}$

FLOW VELOCITY C. I FT/SEC

INJECTION FLOW PARAMETERS:

Cilculated HzCine $=6279 \mathrm{prm}$

DESIRED $\mathrm{H}_{2}$ CONCENTRATION GCEOPPM

MEASURED $\mathrm{H}_{2}$ CONCENTRATION 6.523 Ppm

DIFF. PRESS. 3. COC $" \mathrm{H}_{2} \mathrm{O}$

ABS. PRESS. 14.75 PSIA

TEMP. $\quad 70^{\circ} \mathrm{F}$

\section{SENSOR CONCENTRATION OUTPUT:}

2 MINUTE CONCENTRATION VALUES:

SMC $6437 \mathrm{pPM} \quad$ WHITTAKER Q566 PPM

14 MINUTE 30 SECOND CONCENTPATION VALUES:

SMC 6534ppm

WHITTAKER $7068 \mathrm{ppm}$

15 MINUTE CONCENTRATION VALUES:

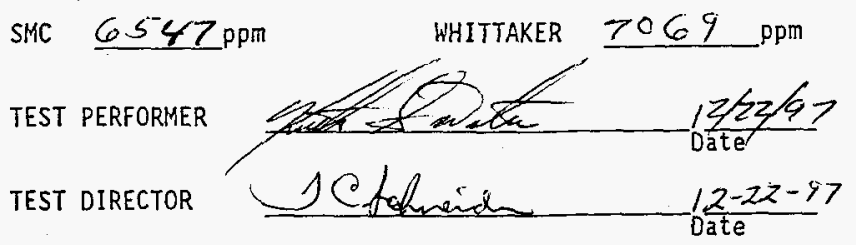




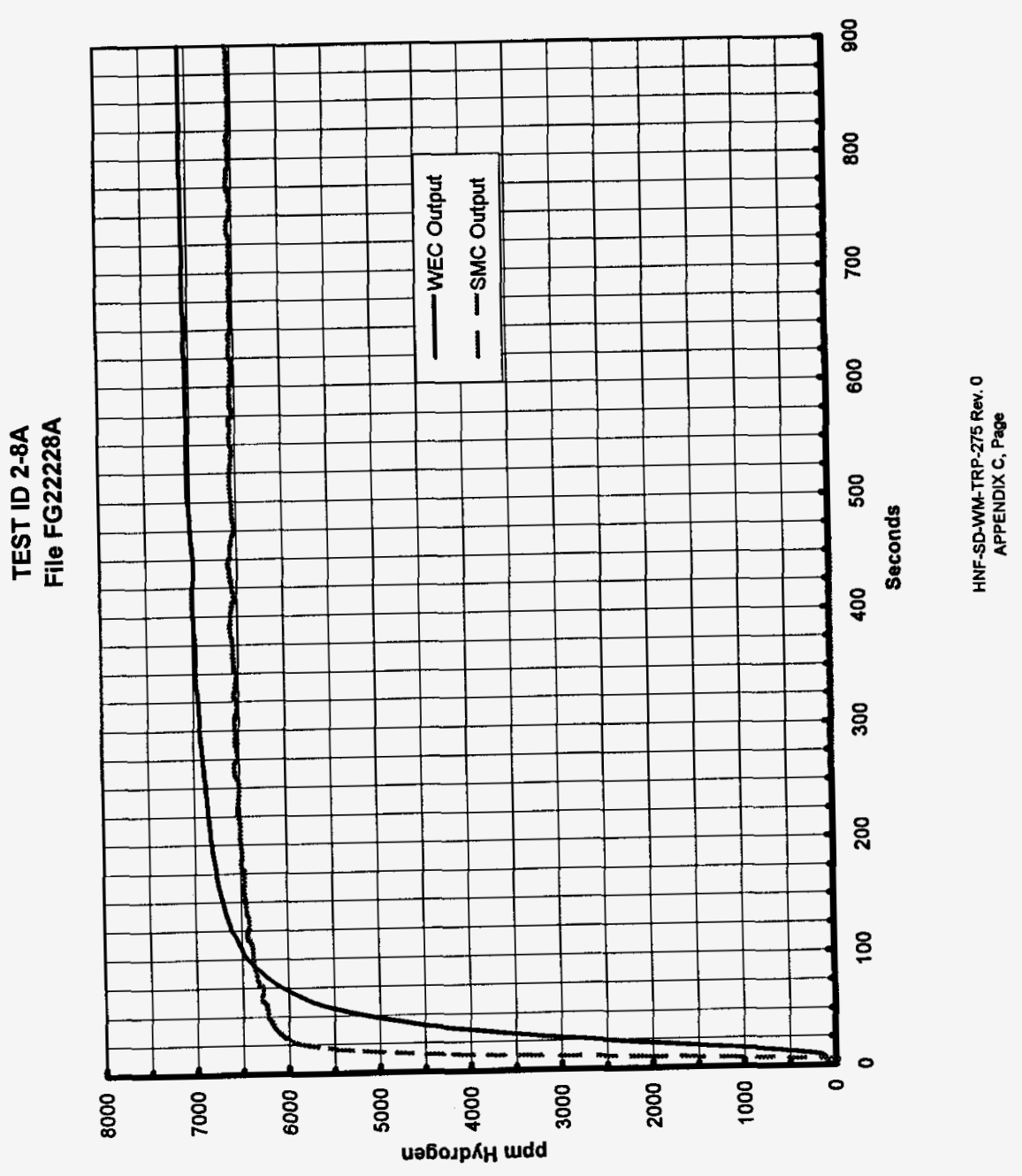




\section{FGI FLOH TEST DATA SHEET}

TEST ID. No, $2-9$ DATA FILE NO. GC 02z27z-9 DL FGI2Z229 SMC ID. NO. $96-B-4455-$ WHITTAKER ID No. $45-1226$

MAIN DUCT FLOW PARAMETERS:

REQUIRED FLOW Z2CO CFM

DIFF. PRESS. $3.744 " \mathrm{H}_{2} \mathrm{O}$

DUCT LENGTH 14.17 FT.

TRANSPORT TIME 2.33 SEC.
CALCULATED FLOW 1993 CFM

ABS. PRESS. 14.74 PSIA TEMP. $70^{\circ}{ }^{\circ}$

FLOW VELOCITY G.I FT/SEC

INJECTION FLOW PARAMETERS:

Culculated tiz Cinre. $=6289$ popen

DESIREO $\mathrm{H}_{2}$ CONCENTRATION $6000 \mathrm{ppm}$ MEASURED $\mathrm{H}_{2}$ CONCENTRATION $6480 \mathrm{ppm}$

DIFF. PRESS. $2.991 " \mathrm{H}_{2} \mathrm{O}$ ABS. PRESS. 14.75 PSIA TEMP. $70^{\circ} \mathrm{F}$

SENSOR CONCENTRATION OUTPUT:

2 MINUTE CONCENTRATION VALUES:

SMC $6388 \mathrm{pPm} \quad$ WHITTAKER $658 \mathrm{G} \mathrm{ppm}$

14 MINUTE 30 SECOND CONCENTRATION VALUES:

SMC GYS8PPM WHITTAKER $7008 \mathrm{ppm}$.

15 MINUTE CONCENTRATION VALUES:

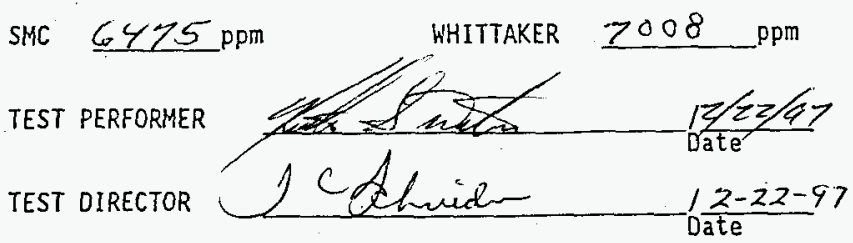


HNF-SD-WM-TRP- 275

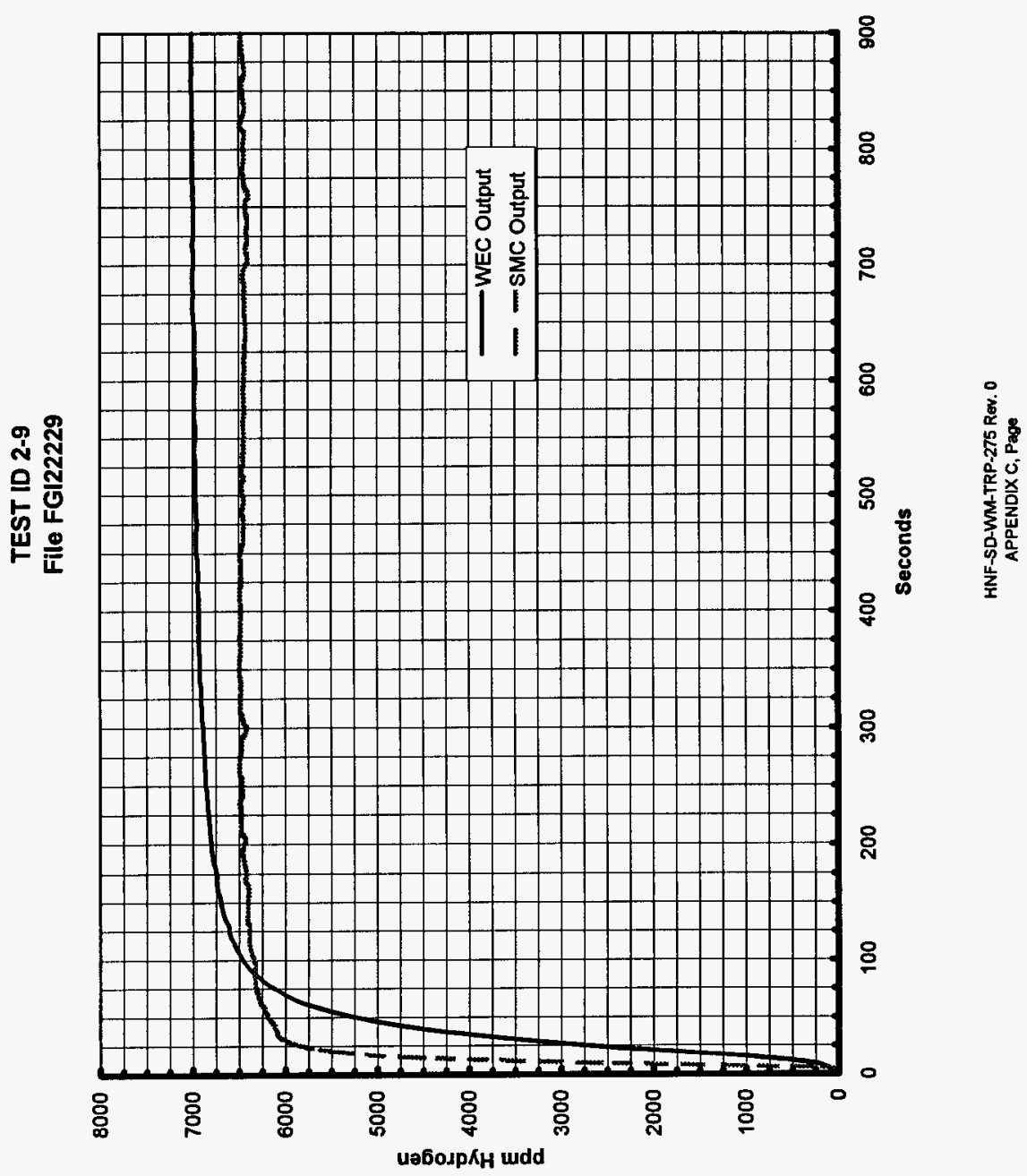




\section{FGI FLOW TEST DATA SHEET}

TEST ID. No. EXS-

SMC ID. No. $96-B-4455$
DATA FILE No.

GC 0 2Z27EX/

WHITTAKER ID NO. HCS-1226

MAIN DUCT FLOW PARAMETERS:

REQUIRED FLOH $/ 80$ CFM

DIFF. PRESS. $3,39 " \mathrm{H}_{2} \mathrm{O}$

DUCT LENGTH 14,17 FT.

TRANSPORT TIME $2.56 \mathrm{SEC}$.
CALCULATED FLOW

ABS. PRESS. 14.74 PSIA

FLOW VELOCITY 5.52 FT/SEC

INJECTION FLOW PARAMETERS:

C.lcolvodedt/2 carre. $=4149 \mathrm{frm}$

DESIRED $\mathrm{H}_{2}$ CONCENTRATION $4,0 \mathrm{O} \mathrm{O}$ pPM MEASURED $\mathrm{H}_{2}$ CONCENTRATION $4348 \mathrm{PPM}$ DIFF. PRESS. 180 " ${ }^{2} \mathrm{O}$ ABS. PRESS. 4.67 PSIA TEMP. $70{ }^{\circ} \mathrm{F}$

SENSOR CONCENTRATION OUTPUT:

2 MINUTE CONCENTRATION VALUES:

SMC YO/2 WPM WHITTAKER YY75 pPm

14 MINUTE 30 SECOND CONCENTRATION VALUES:

SMC YOY/ WPM WHITTAKER Y776 ppm.

15 MINUTE CONCENTRATION VALUES:

SMC $4072 \mathrm{ppm} \quad$ WHITTAKER $4776 \mathrm{ppm}$

TEST PERFORMER

TEST DIRECTOR

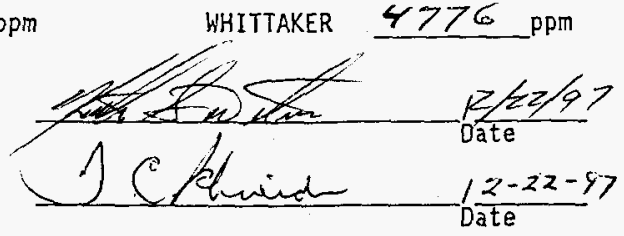




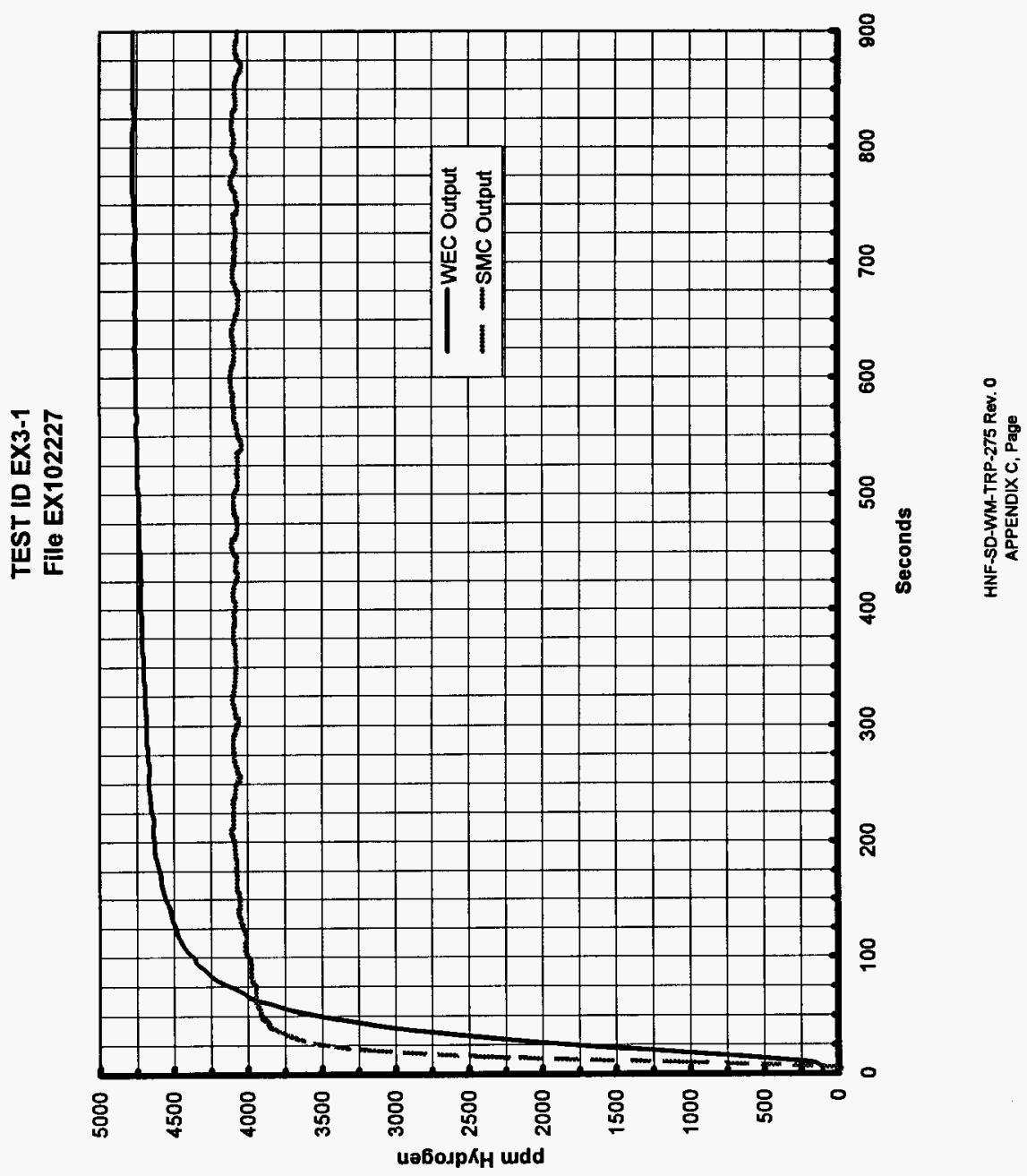


HNF-SD-WM-TRP-275

Rev. 0

Page 83

GI FLOW TEST DATA SHEET

TEST ID. No. EX 3-2 DATA FILE No. GC02227EX2 DLEX202227 SIC ID. NO. $96 \times 3-4455$ WHITTAKER ID No. 4 $45-1226$

MAIN DUCT FLOW PARAMETERS:

REQUIRED FLOW $\triangle 80$ CF

DIFF. PRESS. $3.40 \mathrm{OH}_{2} \mathrm{O}$

DUCT LENGTH $14.17 \mathrm{FT}$.

TRANSPORT TIME $2,56 \mathrm{SEC}$.
CALCULATED FLOW 180.9 CF

ABS. PRESS. 14.73 PSIA TEMP. $70^{\circ} \mathrm{F}$

FLOW VELOCITY 5.53 FT/SEC

INJECTION FLOW PARAMETERS:

Gilcullited Hz cire. $=4 / 35 \mathrm{ppm}$

DESIRED $\mathrm{H}_{2}$ CONCENTRATION $400 \mathrm{O}$ PPM MEASURED $\mathrm{H}_{2}$ CONCENTRATION $\angle 3 \& \mathrm{CPPM}$

DIFF. PRESS. $1.7955^{10} \mathrm{H}_{2}$ ABS. PRESS. 14.67 PSIA TEMP. $70^{\circ} \mathrm{F}$

SENSOR CONCENTRATION OUTPUT:

2 MINUTE CONCENTRATION VALUES:

SIC $40,3 \mathrm{pPm} \quad$ WHITAKER $4985 \mathrm{pPm}$

14 MINUTE 30 SECOND CONCENTRATION VALUES:

SAC $Y 026 \mathrm{PPm} \quad$ WHITTAKER Y766 Ppm

15 MINUTE CONCENTRATION VALUES:

MC $4030 \mathrm{pPm} \quad$ WHITAKER $4766 \mathrm{ppm}$

TEST PERFORMER

TEST DIRECTOR

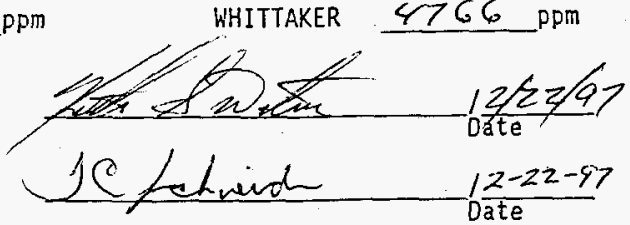




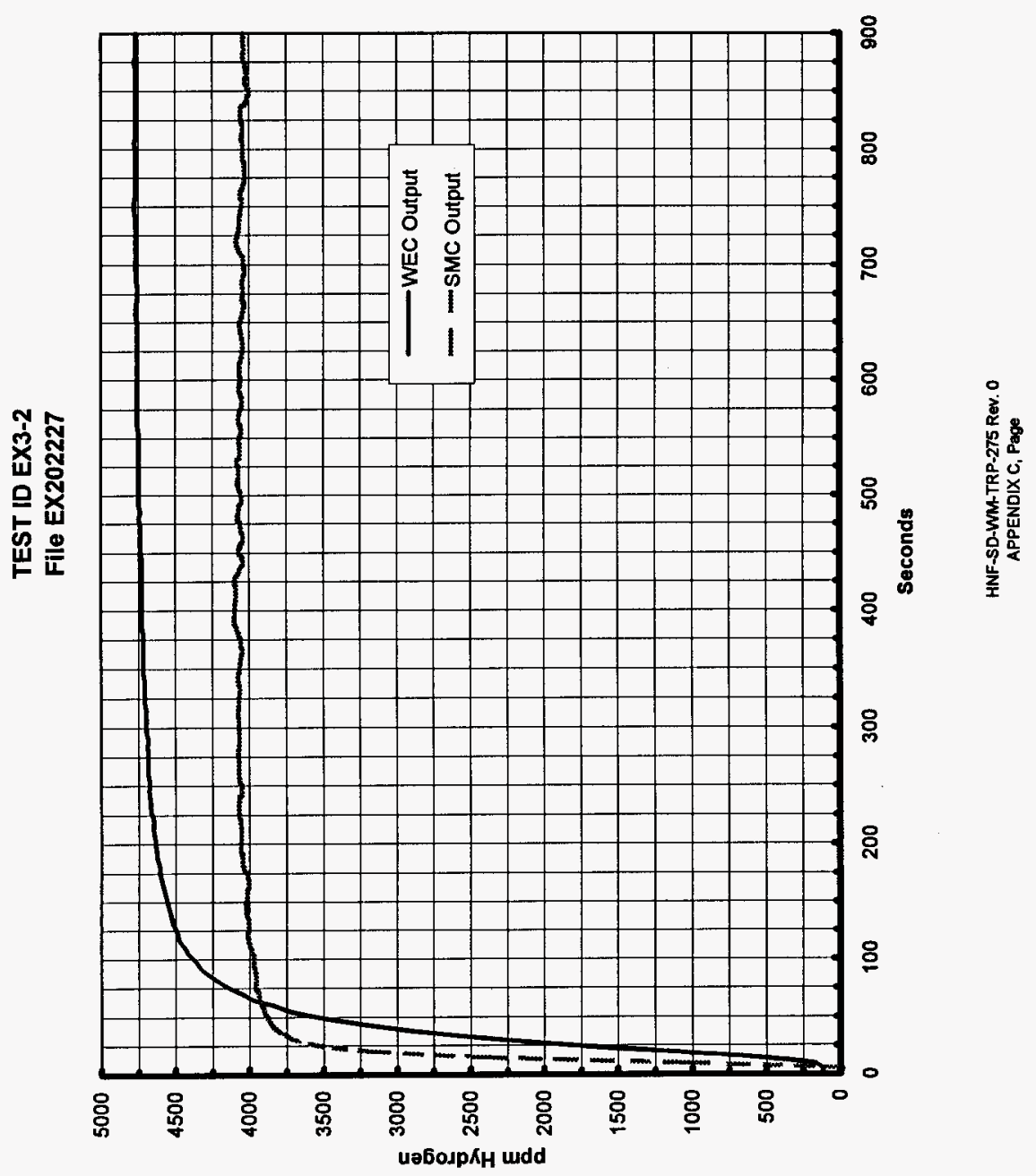


HNF-SD-WM-TRP- 275

Rev. 0

Page Es 5

GI FLOW TEST DATA SHEET

TEST ID. No. EX X3-3 DATA FILE No. GCOZ2Z7EX3 DLEX30ZZZ7

SMC ID. No. 96-B-4Y5S WHITTAKER 10 No. $145-1226$

MAIN DUCT FLOW PARAMETERS:

REQUIRED FLOW $\angle 80 \quad C F M$

DIFF. PRESS. $3.396 " \mathrm{H}_{2} \mathrm{O}$

DUCT LENGTH $14.17 \mathrm{FT}$.

TRANSPORT TIME 2,57 SEC.
CALCULATED FLOW $/ 80.7$ CF

ABS. PRESS. 1473 PSIA TEMP. $70^{\circ} \mathrm{F}$

FLOW VELOCITY 5.52 FT/SEC

INJECTION FLOW PARAMETERS:

DESIRED $\mathrm{H}_{2}$ CONCENTRATION $4 \mathrm{OOO}$ PPM MEASURED $\mathrm{H}_{2}$ CONCENTRATION $433 \mathrm{O}$ pPm DIFF. PRESS. $1.787^{12} \mathrm{H}_{2} \mathrm{O}$ ABS. PRESS. 14.6I PSIA TEMP. $20^{\circ} \mathrm{F}$

SENSOR CONCENTRATION OUTPUT:

2 MINUTE CONCENTRATION VALUES:

SAC $3973 \mathrm{ppm} \quad$ WHITAKER $446.5 \mathrm{pPm}$

14 MINUTE 30 SECOND CONCENTRATION VALUES:

SMC YO WPM WHITAKER $\$ 756 \mathrm{ppm}$

15 MINUTE CONCENTRATION VALUES:

SAC $4026 \mathrm{ppm} \quad$ WHITAKER 4766 ppm

TEST PERFORMER

TEST DIRECTOR

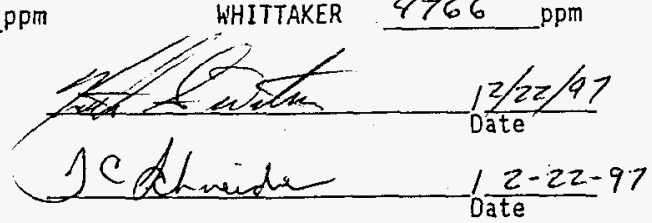




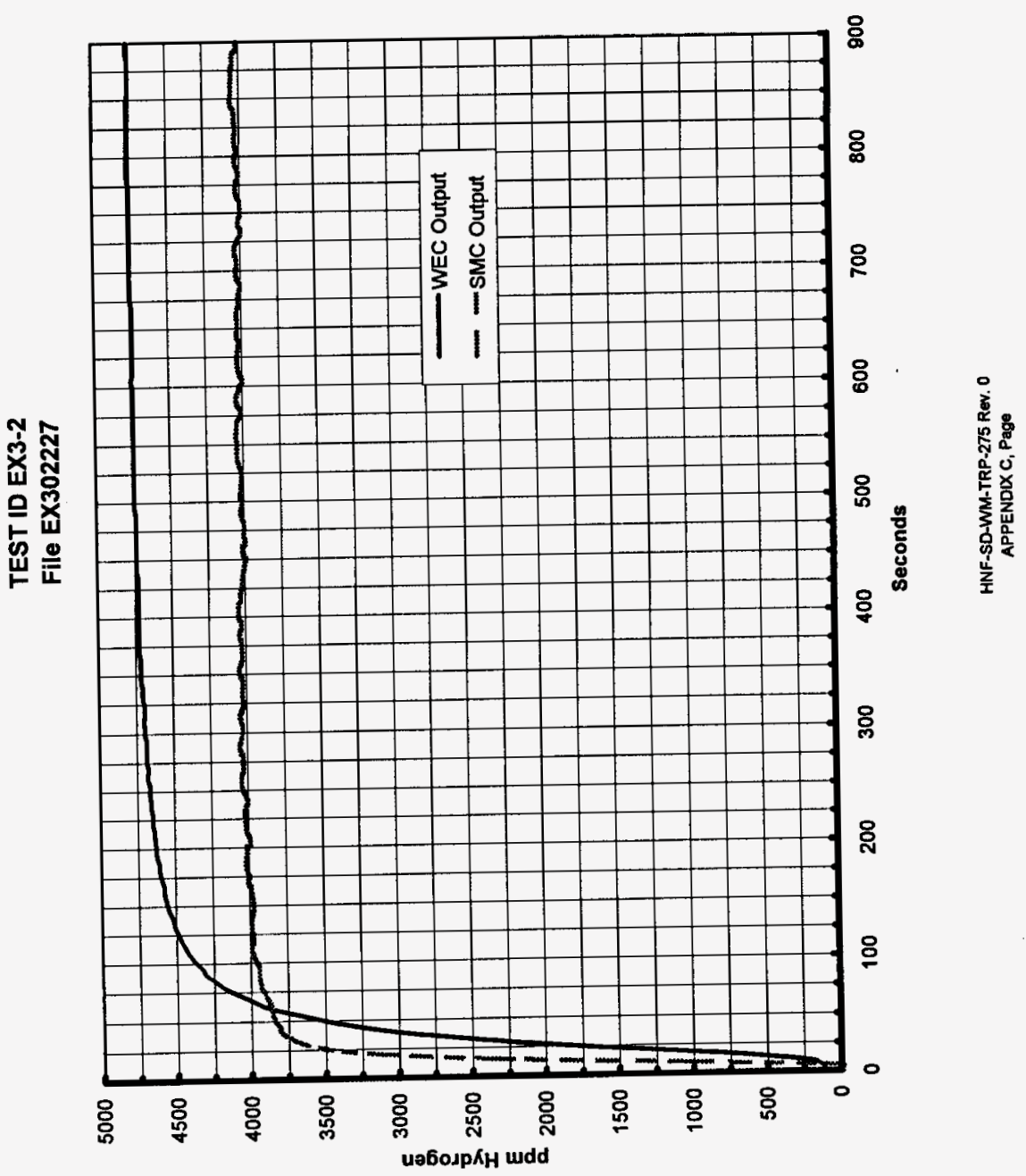




\section{FGI FLOW TEST DATA SHEET}

TEST ID. No. EXYL SMC ID. No. 96-8-4455
DATA FILE No. GC 02257 EX/ $D L E x / 02257$ WHITTAKER ID No. HSE-1226

\section{MAIN DUCT FLOW PARAMETERS:}

REQUIRED FLOH $2 \mathrm{NO}$ CFM

DIFF. PRESS. $3.805{ }^{\prime \prime} \mathrm{H}_{2} \mathrm{O}$

DUCT LENGTH $\angle 4.17 \mathrm{FT}$.

\section{CALCULATED FLOW $198.97 \mathrm{CFM}$}

ABS. PRESS. 14.56 PSIA TEMP. $73^{\circ} \mathrm{F}$

FLOW VELOCITY 6./ FT/SEC

TRANSPORT TIME 2.33 SEC.

INUECTION FLOW PARAMETERS:

DESIRED $\mathrm{H}_{2}$ CONCENTRATION $4,00 \mathrm{O}$ PPM MEASURED $\mathrm{H}_{2}$ CONCENTRATION 3800 pPM

DIFF. PRESS, 1,798 " $\mathrm{H}_{2} \mathrm{O}$ ABS. PRESS. 14.48 PSIA TEMP. $73^{\circ}{ }^{\circ} \mathrm{F}$

SENSOR CONCENTRATION OUTPUT:

2 MINUTE CONCENTRATION VALUES:

SMC $3667 \mathrm{pPM} \quad$ WHITTAKER $3745 \mathrm{ppm}$

14 MINUTE 30 SECOND CONCENTRATION VALUES:

SHC $3706 \mathrm{pPm} \quad$ WHITTAKER $3976 \mathrm{ppm}$

15 MINUTE CONCENTRATION VALUES:

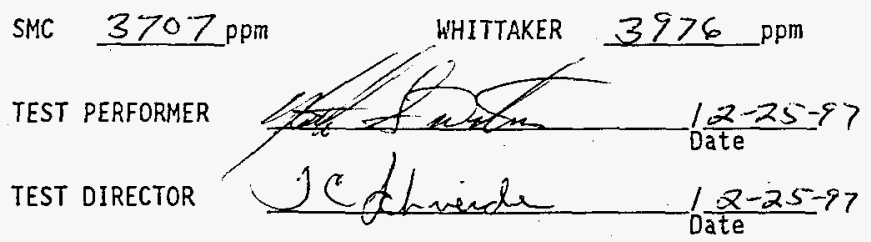




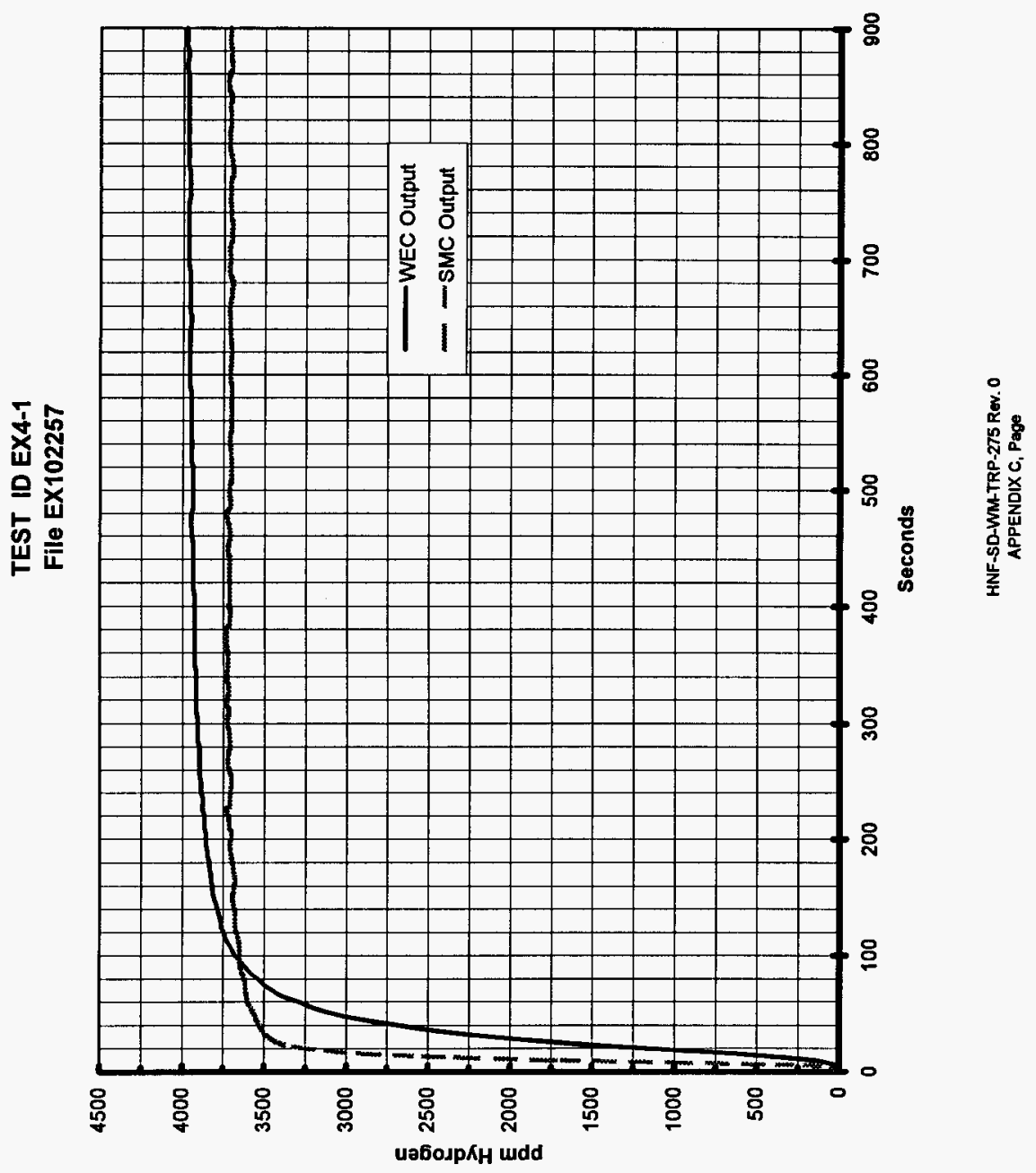


FGI FLOW TEST DATA SHEET

TEST ID. No. Ex<4-2 SMC ID. No. $96 \times 5-4 \times \leq 5$
DATA FILE No.

GC O2257Ex WHITTAKER IO NO. $4<5-1226$

\section{MAIN DUCT FLOW PARAMETERS:}

REQUIRED FLOW 200 CFM

DIFF. PRESS. $3.827{ }^{~} \mathrm{H}_{2} \mathrm{O}$

DUCT LENGTH $/ E . / ?$ FT.

TRANSPORT TIME 2.32 SEC.

\section{CALCULATEO FLOW $99.98 \mathrm{CFH}$}

ABS. PRESS. $/ 5.55$ PSIA TEMP. $73{ }^{\circ} \mathrm{F}$

FLOW VELOCITY G./ FT/SEC

INJECTION FLOH PARAMETERS:

Calcalusfeltre cinve $=4074 \mathrm{ppm}$ DESIRED $\mathrm{H}_{2}$ CONCENTRATION YOOOPPM MEASURED $\mathrm{H}_{2}$ CONCENTRATION 4217 pPm DIFF. PRESS. Z.OE $" \mathrm{H}_{2} \mathrm{O}$ ABS. PRESS. 4.49 PSIA TEMP. $73{ }^{\circ} \mathrm{F}$

SENSOR CONCENTRATION OUTPUT:

2 MINUTE CONCENTRATION VALUES:

SMC $\$ 067$ pPm WHITTAKER 4195

14 MINUTE 30 SECOND CONCENTRATION VALUES:

SMC $4 / 20 \mathrm{ppm}$ WHITTAKER $4 \mathbf{3 6} \mathrm{ppm}$

15 MINUTE CONCENTRATION VALUES:

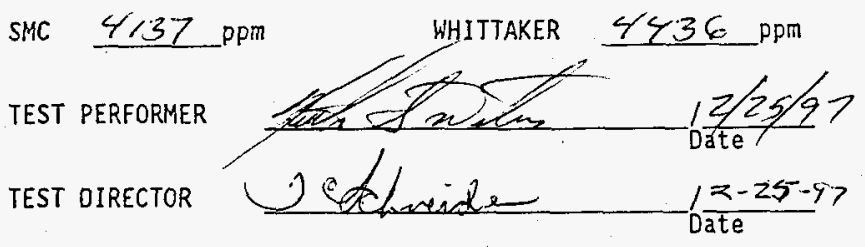




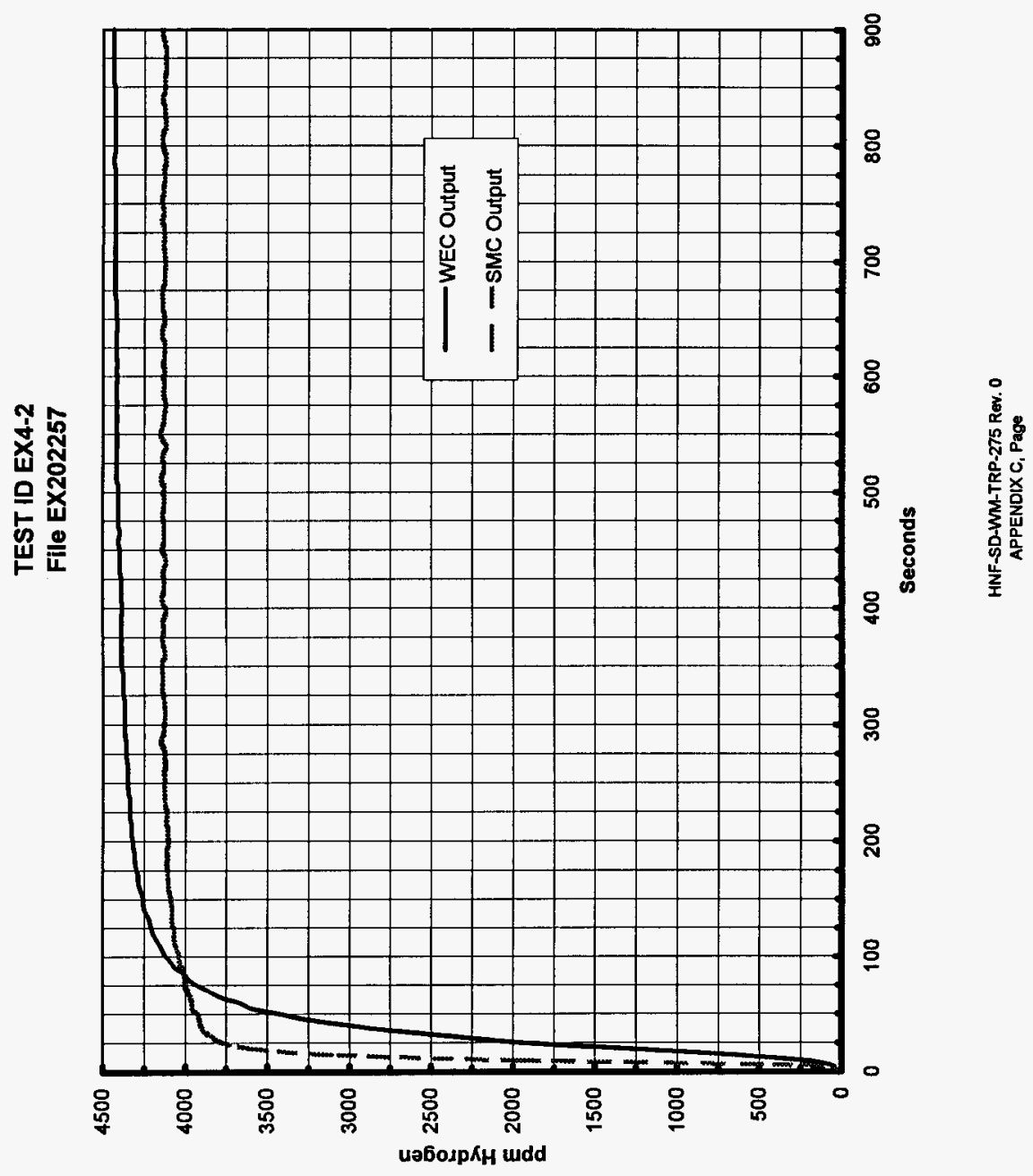




\section{FGI FLOW TEST DATA SHEET}

TEST ID. No. EXY $=3$

DATA FILE NO.

GC 0225TEX3

$D L E \times 302357$

SMC ID. No. $96 B-4455$ WHITTAKER ID NO. $\angle E S-1226$

MAIN DUCT FLOW PARAMETERS:

REQUIRED FLOW 200 CFM CALCULATED FLOW $/ 99.05$ CFM

DIFF, PRESS. $3.802{ }^{~} \mathrm{H}_{2} \mathrm{O}$ ABS. PRESS. $4.55^{\text {PSIA }}$ TEMP. $\ 2^{\circ} \mathrm{F}$

DUCT LENGTH $14 . \angle Z F T$. FLOW VELOCITY 6./ FT/SEC

TRANSPORT TIME Z.3.2 SEC.

INJECTION FLOW PARAMETERS:

Colculated H/2 Civer $=4120 \mathrm{ppm}$

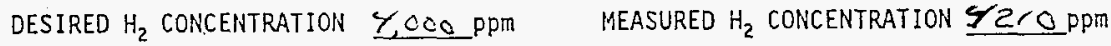
DIFF. PRESS. $Z .005^{-1} \mathrm{H}_{2} \mathrm{O}$ ABS. PRESS. $Y 4 . Y Q$ PSIA TEMP. $Y Z^{\circ} \mathrm{F}$

SENSOR CONCENTRATION OUTPUT:

2 MINUTE CONCENTRATION VALUES:

SMC $\angle 054 \mathrm{ppm} \quad$ WHITTAKER Y236 $\mathrm{ppm}$

14 MINUTE 30 SECOND CONCENTRATION VALUES:

SMC $4 / 35 \mathrm{ppm}$

WHITTAKER YY $Y 66 \mathrm{ppm}$

15 MINUTE CONCENTRATION VALUES:

SMC 4/28 PPM WHITTAKER $4466 \mathrm{ppm}$

TEST PERFORMER

TEST DIRECTOR
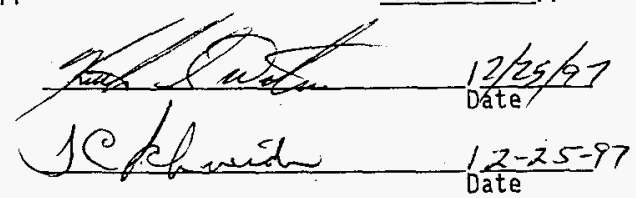


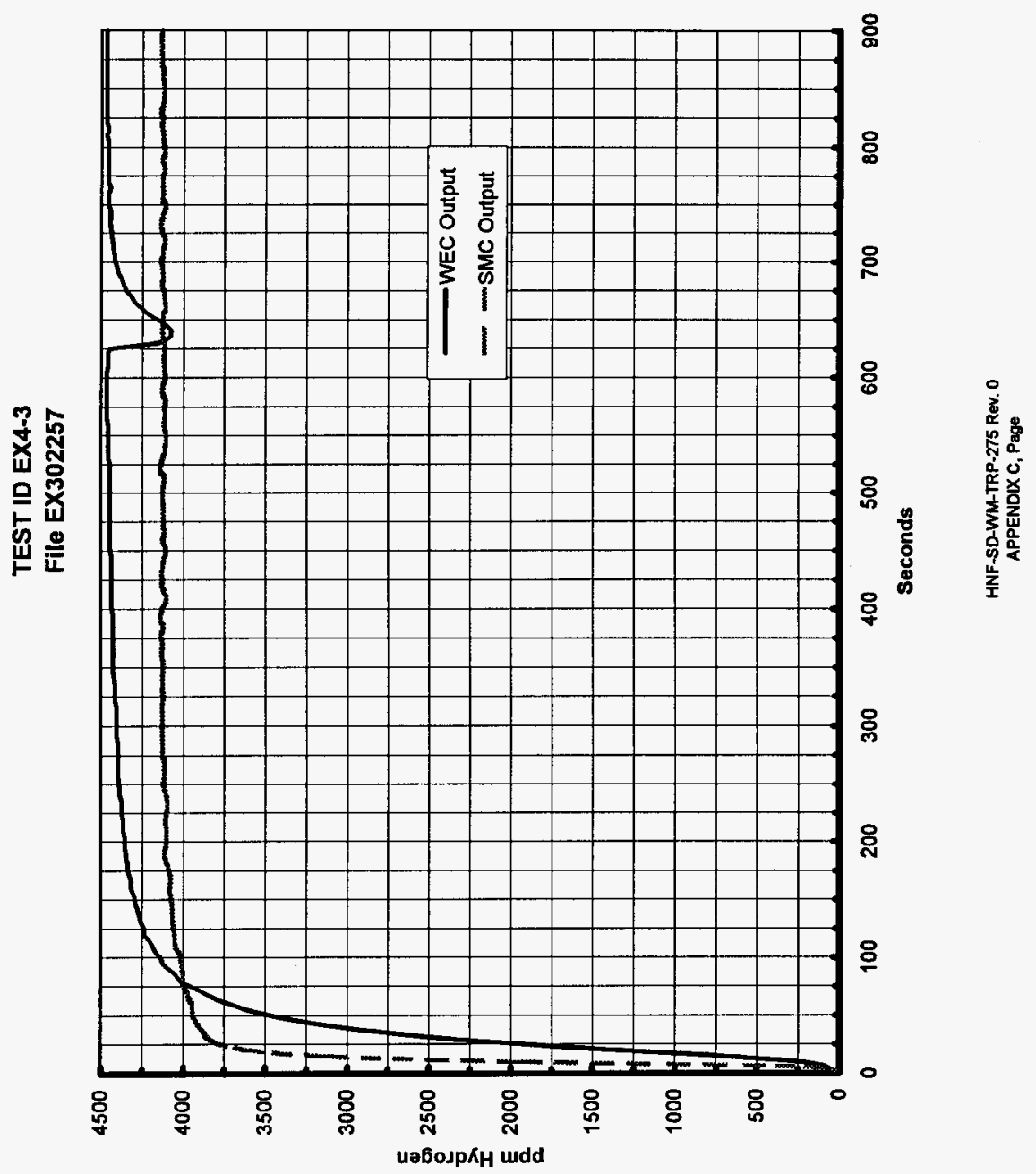




\section{FGI FLOH TEST DATA SHEET}

TEST ID. NO. EXY-Y DATA FILE No. GCOZZ57EX4 OL EXYO2257 SMC ID. No. $86-\beta-8455$ WHITTAKER ID No. $6-5 \gamma 226$

MAIN DUCT FLOW PARAMETERS:

REQUIRED FLOW 200 CFM CALCULATED FLOW $200.3 \mathrm{CFM}$

DIFF. PRESS. $3.836 \quad " \mathrm{H}_{2} \mathrm{O}$

ABS. PRESS. $14,5 Y$ PSIA

TEMP. $\quad 73^{\circ} \mathrm{F}$

DUCT LENGTH $\triangle Y, C Z$ FT.

FLOW VELOCITY 6./2FT/SEC

TRANSPORT TIME $2.3 /$ SEC.

INJECTION FLOW PARAMETERS:

C.lculated He cire. $=4105 \mathrm{rpm}$

DESIRED $\mathrm{H}_{2}$ CONCENTRATION $4 \mathrm{COC}$ PPM MEASURED $\mathrm{H}_{2}$ CONCENTRATION $42 / O \mathrm{PPM}$

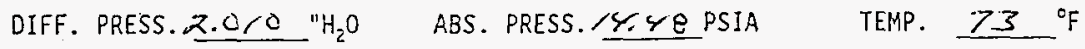

SENSOR CONCENTRATION OUTPUT:

2 MINUTE CONCENTRATION VALUES:

SMC LOY E ppm

WHITTAKER SススS PPm

14 MINUTE 30 SECOND CONCENTRATION VALUES:

SMC 4127 Ppm HHITTAKER YY86 ppm

15 MINUTE CONCENTRATION VALUES:

SMC 4/36 WPITTAKER Y486

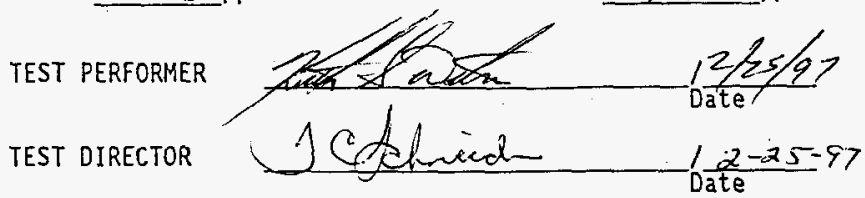




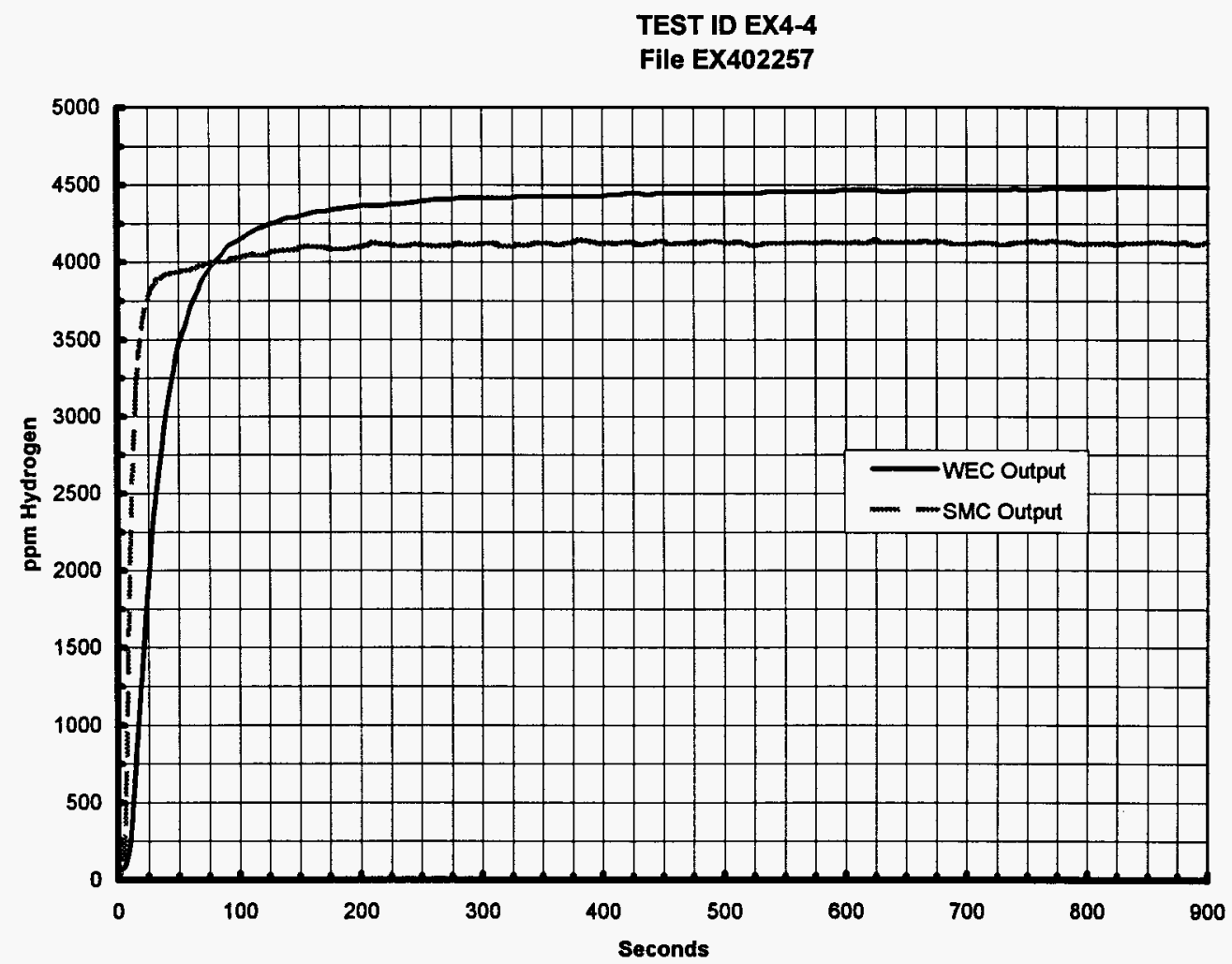

HNF-SD-WM-TRP-275 Rev. 0 APPENDIX C, Page 
The following data provides listing of the gas chromatograph (GC) calibration and test concentration data. The second file listed identifies a validation test of the sensor calibration gas. The $\mathrm{GC}$ file number can be referenced to the data sheets in APPENDIX $C$. The concentrations listed on the individual data sheets are not averaged concentrations, but concentrations that the GC read nominally 6 minutes into the 15 minute test.

\begin{tabular}{|c|c|c|c|c|c|}
\hline $\begin{array}{l}\text { Feb 13, } 1997 \\
\text { Feb 13, } 1997 \\
\text { Feb 13, } 1997 \\
\text { Feb 13, } 1997 \\
\text { Feb 13, } 1997\end{array}$ & $\begin{array}{l}12: 52: 32 \\
12: 51: 32 \\
12: 53: 34 \\
12: 55: 36 \\
12: 57: 38\end{array}$ & $\begin{array}{l}0 \\
1 \\
2 \\
3 \\
4\end{array}$ & $\begin{array}{l}\text { Hydrogen } \\
1009.355 \\
1008.977 \\
1009.561 \\
1009.179\end{array}$ & 02137cal.dif & eric_shp. \\
\hline $\begin{array}{l}\text { Feb 13, } 1997 \\
\text { Feb 13, } 1997 \\
\text { Feb 13, } 1997 \\
\text { Feb 13, } 1997 \\
\text { Feb 13, } 1997\end{array}$ & $\begin{array}{l}13: 01: 49 \\
13: 00: 49 \\
13: 02: 51 \\
13: 04: 53 \\
13: 06: 55\end{array}$ & $\begin{array}{l}0 \\
1 \\
2 \\
3 \\
4\end{array}$ & $\begin{array}{l}\text { Hydrogen } \\
3372.827 \\
8055.043 \\
8040.089 \\
8032.270\end{array}$ & 8070tst.dif & eric_shp. \\
\hline $\begin{array}{l}\text { Feb 13, } 1997 \\
\text { Feb 13, 1997 } \\
\text { Feb 13, 1997 } \\
\text { Feb 13, 1997 } \\
\text { Feb 13, 1997 } \\
\text { Feb 13, } 1997 \\
\text { Feb 13, } 1997 \\
\text { Feb 13, } 1997 \\
\text { Feb 13, 1997 } \\
\text { Feb 13, 1997 } \\
\text { Feb 13, } 1997\end{array}$ & $\begin{array}{l}15: 36: 05 \\
15: 35: 05 \\
15: 37: 07 \\
15: 39: 09 \\
15: 41: 11 \\
15: 43: 13 \\
15: 45: 15 \\
15: 47: 17 \\
15: 49: 20 \\
15: 51: 22 \\
15: 53: 24\end{array}$ & $\begin{array}{l}0 \\
1 \\
2 \\
3 \\
4 \\
5 \\
6 \\
7 \\
8 \\
9 \\
10\end{array}$ & $\begin{array}{c}\text { Hydrogen } \\
0.000 \\
239.844 \\
237.123 \\
236.914 \\
237.646 \\
238.797 \\
237.863 \\
238.328 \\
239.220 \\
0.000\end{array}$ & 021371-1.dif & eric_shp. \\
\hline $\begin{array}{l}\text { Feb 13, } 1997 \\
\text { Feb 13, 1997 } \\
\text { Feb 13, 1997 } \\
\text { Feb 13, 1997 } \\
\text { Feb 13, } 1997 \\
\text { Feb 13, } 1997 \\
\text { Feb 13, } 1997 \\
\text { Feb 13,1 } 1997 \\
\text { Feb 13, 1997 } \\
\text { Feb 13, } 1997 \\
\text { Feb 13, } 1997\end{array}$ & $\begin{array}{l}16: 37: 45 \\
16: 36: 45 \\
16: 38: 47 \\
16: 40: 49 \\
16: 42: 51 \\
16: 44: 53 \\
16: 46: 55 \\
16: 48: 57 \\
16: 50: 59 \\
16: 53: 00 \\
16: 55: 03\end{array}$ & $\begin{array}{l}0 \\
1 \\
2 \\
3 \\
4 \\
5 \\
6 \\
7 \\
8 \\
9 \\
10\end{array}$ & $\begin{array}{c}\text { Hydrogen } \\
0.000 \\
232.394 \\
237.027 \\
235.787 \\
236.225 \\
236.727 \\
237.220 \\
236.609 \\
236.613 \\
0.000\end{array}$ & 021371-2.dif & eric_shp. \\
\hline
\end{tabular}




\begin{tabular}{|c|c|c|c|c|c|c|}
\hline $\begin{array}{ll}\text { Feb } & 13 \\
\text { Feb } & 13 \\
\text { Feb } & 13 \\
\text { Feb } & 13 \\
\text { Feb } & 13 \\
\text { Feb } & 13 \\
\text { Feb } & 13 \\
\text { Feb } & 13 \\
\text { Feb } & 13 \\
\text { Feb } & 13 \\
\text { Feb } & 13\end{array}$ & $\begin{array}{l}1997 \\
1997 \\
1997 \\
1997 \\
1997 \\
1997 \\
1997 \\
1997 \\
1997 \\
1997 \\
1997\end{array}$ & $\begin{array}{l}19: 24: 00 \\
19: 23: 00 \\
19: 25: 02 \\
19: 27: 04 \\
19: 29: 06 \\
19: 31: 08 \\
19: 33: 10 \\
19: 35: 12 \\
19: 37: 14 \\
19: 39: 16 \\
19: 41: 18\end{array}$ & $\begin{array}{l}0 \\
1 \\
2 \\
3 \\
4 \\
5 \\
6 \\
7 \\
8 \\
9 \\
10\end{array}$ & $\begin{array}{c}\text { Hydrogen } \\
0.000 \\
234.535 \\
235.240 \\
234.585 \\
234.680 \\
234.548 \\
235.114 \\
235.391 \\
235.654 \\
0.000\end{array}$ & 021371-3.dif & eric_shp. \\
\hline $\begin{array}{ll}\text { Feb } & 13 \\
\text { Feb } & 13 \\
\text { Feb } & 13 \\
\text { Feb } & 13 \\
\text { Feb } & 13 \\
\text { Feb } & 13 \\
\text { Feb } & 13 \\
\text { Feb } & 13 \\
\text { Feb } & 13 \\
\text { Feb } & 13 \\
\text { Feb } & 13\end{array}$ & $\begin{array}{l}1997 \\
1997 \\
1997 \\
1997 \\
1997 \\
1997 \\
1997 \\
1997 \\
1997 \\
1997 \\
1997\end{array}$ & $\begin{array}{l}20: 49: 41 \\
20: 48: 41 \\
20: 50: 43 \\
20: 52: 45 \\
20: 54: 47 \\
20: 56: 50 \\
20: 58: 52 \\
21: 00: 54 \\
21: 02: 57 \\
21: 04: 59 \\
21: 07: 01\end{array}$ & $\begin{array}{l}0 \\
1 \\
2 \\
3 \\
4 \\
5 \\
6 \\
7 \\
8 \\
9 \\
10\end{array}$ & $\begin{array}{c}\text { Hydrogen } \\
0.000 \\
4084.144 \\
4064.679 \\
4060.621 \\
4054.331 \\
4039.324 \\
4047.384 \\
4032.408 \\
4034.537 \\
0.000\end{array}$ & 021372-4.dif & eric_shp. \\
\hline $\begin{array}{ll}\text { Feb } & 13 \\
\text { Feb } & 13 \\
\text { Feb } & 13 \\
\text { Feb } & 13 \\
\text { Feb } & 13 \\
\text { Feb } & 13 \\
\text { Feb } & 13 \\
\text { Feb } & 13 \\
\text { Feb } & 13 \\
\text { Feb } & 13 \\
\text { Feb } & 13 \\
\text { Feb } & 13\end{array}$ & $\begin{array}{l}1997 \\
1997 \\
1997 \\
1997 \\
1997 \\
1997 \\
1997 \\
1997 \\
1997 \\
1997 \\
1997 \\
1997\end{array}$ & $\begin{array}{l}21: 45: 58 \\
21: 44: 58 \\
21: 47: 00 \\
21: 49: 02 \\
21: 51: 05 \\
21: 53: 08 \\
21: 55: 10 \\
21: 57: 12 \\
21: 59: 14 \\
22: 01: 15 \\
22: 03: 18 \\
22: 05: 20\end{array}$ & $\begin{array}{l}0 \\
1 \\
2 \\
3 \\
4 \\
5 \\
6 \\
7 \\
8 \\
9 \\
10 \\
11\end{array}$ & $\begin{array}{c}\text { Hydrogen } \\
0.000 \\
0.000 \\
0.000 \\
4054.654 \\
4038.196 \\
4026.650 \\
4028.207 \\
4012.841 \\
4003.602 \\
4001.193 \\
5.988\end{array}$ & 02137exl.dif & eric_shp. \\
\hline $\begin{array}{ll}\text { Feb } & 13 \\
\text { Feb } & 13 \\
\text { Feb } & 13 \\
\text { Feb } & 13 \\
\text { Feb } & 13 \\
\text { Feb } & 13 \\
\text { Feb } & 13 \\
\text { Feb } & 13 \\
\text { Feb } & 13 \\
\text { Feb } & 13 \\
\text { Feb } & 13 \\
\text { Feb } & 13\end{array}$ & $\begin{array}{l}1997 \\
1997 \\
1997 \\
1997 \\
1997 \\
1997 \\
1997 \\
1997 \\
1997 \\
1997 \\
1997 \\
1997\end{array}$ & $\begin{array}{l}22: 54: 21 \\
22: 53: 20 \\
22: 55: 22 \\
22: 57: 24 \\
22: 59: 26 \\
23: 01: 28 \\
23: 03: 30 \\
23: 05: 32 \\
23: 07: 34 \\
23: 09: 36 \\
23: 11: 38 \\
23: 13: 40\end{array}$ & $\begin{array}{l}0 \\
1 \\
2 \\
3 \\
4 \\
5 \\
6 \\
7 \\
8 \\
9 \\
10 \\
11\end{array}$ & $\begin{array}{c}\text { Hydrogen } \\
0.000 \\
0.000 \\
4165.151 \\
4115.762 \\
4104.827 \\
4071.867 \\
4064.729 \\
4059.202 \\
4049.874 \\
3901.815 \\
0.000\end{array}$ & 02137ex2.dif & eric_shp. \\
\hline
\end{tabular}


$\begin{array}{llll}\text { Feb 14, 1997 } & 14: 53: 56 & 0 & \text { Hydrogen } \\ \text { Feb 14, 1997 } & 14: 52: 56 & 1 & 0.000 \\ \text { Feb 14, 1997 } & 14: 54: 58 & 2 & 0.000 \\ \text { Feb 14, 1997 } & 14: 57: 00 & 3 & 0.000 \\ \text { Feb 14, 1997 } & 14: 59: 02 & 4 & 3931.989 \\ \text { Feb 14, 1997 } & 15: 01: 04 & 5 & 4173.215 \\ \text { Feb 14, 1997 } & 15: 03: 06 & 6 & 4166.864 \\ \text { Feb 14, 1997 } & 15: 05: 08 & 7 & 4155.838 \\ \text { Feb 14, 1997 } & 15: 07: 10 & 8 & 4147.488 \\ \text { Feb 14, 1997 } & 15: 09: 12 & 9 & 4141.784 \\ \text { Feb 14, 1997 } & 15: 11: 14 & 10 & 4124.018\end{array}$

Feb 22, 1997 06:09:54

Feb 22, 1997 06:08:53

Feb 22, 1997 06:10:55

Feb 22, 1997

Feb 22, 1997

Feb 22, 1997

Feb 22, 1997

Feb 22, 1997

Feb 22, 1997

Feb 22, 1997

Feb 22, 1997

Feb 22, 1997

Feb 22, 1997

Feb 22, 1997

Feb 22, 1997

Feb 22, 1997

Feb 22, 1997

Feb 22, 1997

Feb 22, 1997

Feb 22, 1997

Feb 22, 1997

Feb 22, 1997

Feb 22, 1997

Feb 22, 1997

Feb 22, 1997

Feb 22, 1997

Feb 22, 1997

Feb 22, 1997

Feb 22, 1997

Feb 22, 1997

Feb 22, 1997

Feb 22, 1997

Feb 22, 1997

Feb 22, 1997
06:12:57

06:14:59

06:17:01

07:39:31

$07: 38: 30$

$07: 40: 33$

$07: 42: 35$

$07: 44: 37$

$07: 46: 39$

07:48:41

$07: 50: 43$

$07: 52: 45$

08:01:05

08:00:05

08:02:07

08:04:09

08:06: 12

08:08:14

08:10:16

08:12:18

$08: 21: 44$

$08: 20: 44$

$08: 22: 46$

$08: 24: 48$

$08: 26: 50$

08:28:52

08:30:54

08:32:56

$08: 34: 58$

08:37:00
08:14:20
Hydrogen

1009.363

1009.599

1010.336

1009.090

1010.709

Hydrogen

0.000

219.714

219.018

219.921

219.373

219.898

220.156

220.417

Hydrogen

0.000

218.815

218.121

218.178

218.737

219.049

218.768

219.358

Hydrogen

0.000

0.000

216.933

216.803

216.617

216.377

216.655

216.375

215.919
02147EX1.dif eric_shp.

02227cal.dif eric_shp.

022271-1.dif eric_shp.

022271-2.dif eric_shp.

022271-3.dif eric_shp. 
Feb 22, 1997

Feb 22, 1997

Feb 22, 1997

Feb 22, 1997

Feb 22, 1997

Feb 22, 1997

Feb 22, 1997

Feb 22, 1997

Feb 22, 1997

Feb 22, 1997

Feb 22, 1997

Feb 22, 1997

Feb 22, 1997

Feb 22, 1997

Feb 22, 1997

Feb 22, 1997

Feb 22, 1997

Feb 22, 1997

Feb 22, 1997

Feb 22, 1997

Feb 22, 1997

Feb 22, 1997

Feb 22, 1997

Feb 22, 1997

Feb 22, 1997

Feb 22, 1997

Feb 22, 1997

Feb 22, 1997

Feb 22, 1997

Feb 22, 1997

Feb 22, 1997

Feb 22, 1997

Feb 22, 1997

Feb 22, 1997

Feb 22, 1997

Feb 22, 1997

Feb 22, 1997

Feb 22, 1997

Feb 22, 1997
09:15:01 0

09:14:01

$09: 16: 03$

09:18:05

09:20:08

$09: 22: 10$

$09: 24: 126$

$09: 26: 147$

09:28:16

09:36:15 0

09:35:15 1

09:37:17

09:39:19 3

$09: 41: 22 \quad 4$

$09: 43: 24$

$09: 45: 26$

09:47:28

09:49:30 8

09:51:32

09:59:04

09:58:03

10:00:05

10:02:07

10:04:09

10:06:11

10:08:13

$10: 10: 15$

$10: 12: 18 \quad 8$

$10: 14: 20$

10:29:05

$10: 28: 04$

$10: 30: 06$

10:32:08

$10: 34: 10 \quad 4$

10:36:12 5

$10: 38: 14 \quad 6$

$10: 40: 167$

$10: 42: 18 \quad 8$

$10: 44: 20 \quad 9$
Hydrogen

0.000

2039.065

2029.069

2021.438

2017.879

2008.246

2002.091

1994.316

Hydrogen

4.887

4.507

2176.016

2171.520

2166.213

2169.162

2163.635

2165.513

2152.860

Hydrogen

5.678

2106.223

2158.866

2149.787

2148.808

2151.172

2147.346

2146.195

2144.823

H2

7.733

4346.532

4358.887

4354.556

4358.121

4353.721

4366.371

4352.970

4352.487 022272-1.dif eric_shp.

022272-2.dif eric_shp.

022272-3.dif eric_shp.

022272-4.dif eric_shp. 


\begin{tabular}{|c|c|c|c|c|c|c|}
\hline $\begin{array}{l}\text { Feb 22, } \\
\text { Feb 22, } \\
\text { Feb 22,, } \\
\text { Feb 22, } \\
\text { Feb 22, } \\
\text { Feb 22, } \\
\text { Feb 22, } \\
\text { Feb 22, } \\
\text { Feb 22, } \\
\text { Feb 22, } \\
\text { Feb 22, }\end{array}$ & $\begin{array}{l}1997 \\
1997 \\
1997 \\
1997 \\
1997 \\
1997 \\
1997 \\
1997 \\
1997 \\
1997 \\
1997\end{array}$ & $\begin{array}{l}10: 53: 18 \\
10: 52: 17 \\
10: 54: 20 \\
10: 56: 22 \\
10: 58: 24 \\
11: 00: 26 \\
11: 02: 27 \\
11: 04: 30 \\
11: 06: 32 \\
11: 08: 34 \\
11: 10: 36\end{array}$ & $\begin{array}{l}0 \\
1 \\
2 \\
3 \\
4 \\
5 \\
6 \\
7 \\
8 \\
9 \\
10\end{array}$ & $\begin{array}{l}\text { Hydrogen } \\
10.768 \\
10.627 \\
4037.397 \\
4373.104 \\
4366.323 \\
4369.537 \\
4353.324 \\
4365.395 \\
4350.768 \\
4366.129\end{array}$ & 022272-5.dif & eric_shp. \\
\hline $\begin{array}{l}\text { Feb } 22, \\
\text { Feb } 22, \\
\text { Feb } 22, \\
\text { Feb } 22, \\
\text { Feb } 22, \\
\text { Feb } 22, \\
\text { Feb } 22, \\
\text { Feb } 22, \\
\text { Feb } 22, \\
\text { Feb } 22, \\
\text { Feb } 22,\end{array}$ & $\begin{array}{l}1997 \\
1997 \\
1997 \\
1997 \\
1997 \\
1997 \\
1997 \\
1997 \\
1997 \\
1997 \\
1997\end{array}$ & $\begin{array}{l}11: 22: 17 \\
11: 21: 17 \\
11: 23: 19 \\
11: 25: 21 \\
11: 27: 23 \\
11: 29: 26 \\
11: 31: 28 \\
11: 33: 30 \\
11: 35: 32 \\
11: 37: 34 \\
11: 39: 36\end{array}$ & $\begin{array}{l}0 \\
1 \\
2 \\
3 \\
4 \\
5 \\
6 \\
7 \\
8 \\
9 \\
10\end{array}$ & $\begin{array}{l}\text { Hydrogen } \\
12.067 \\
11.164 \\
4359.464 \\
4376.943 \\
4365.416 \\
4367.537 \\
4369.850 \\
4373.277 \\
4363.086 \\
4373.491\end{array}$ & 022272-6.dif & eric_shp. \\
\hline $\begin{array}{l}\text { Feb 22, } \\
\text { Feb 22, } \\
\text { Feb 22, } \\
\text { Feb 22, } \\
\text { Feb 22, } \\
\text { Feb 22, } \\
\text { Feb 22, } \\
\text { Feb 22, } \\
\text { Feb 22, } \\
\text { Feb 22, }\end{array}$ & $\begin{array}{l}1997 \\
1997 \\
1997 \\
1997 \\
1997 \\
1997 \\
1997 \\
1997 \\
1997 \\
1997\end{array}$ & $\begin{array}{l}11: 57: 06 \\
11: 56: 05 \\
11: 58: 07 \\
12: 00: 09 \\
12: 02: 11 \\
12: 04: 13 \\
12: 06: 16 \\
12: 08: 18 \\
12: 10: 20 \\
12: 12: 22\end{array}$ & $\begin{array}{l}0 \\
1 \\
2 \\
3 \\
4 \\
5 \\
6 \\
7 \\
8 \\
9\end{array}$ & $\begin{array}{l}\text { Hydrogen } \\
14.257 \\
6445.277 \\
6432.761 \\
6452.556 \\
6444.227 \\
6452.016 \\
6446.407 \\
6464.080 \\
6477.664\end{array}$ & 022272-7.dif & eric_shp. \\
\hline $\begin{array}{l}\text { Feb } 22 \text {, } \\
\text { Feb } 22, \\
\text { Feb 22, } \\
\text { Feb 22, } \\
\text { Feb 22, } \\
\text { Feb 22, } \\
\text { Feb 22, } \\
\text { Feb 22, } \\
\text { Feb 22, } \\
\text { Feb 22, }\end{array}$ & $\begin{array}{l}1997 \\
1997 \\
1997 \\
1997 \\
1997 \\
1997 \\
1997 \\
1997 \\
1997 \\
1997\end{array}$ & $\begin{array}{l}12: 28: 31 \\
12: 27: 31 \\
12: 29: 33 \\
12: 31: 36 \\
12: 33: 38 \\
12: 35: 40 \\
12: 37: 42 \\
12: 39: 44 \\
12: 41: 47 \\
12: 43: 49\end{array}$ & $\begin{array}{l}0 \\
1 \\
2 \\
3 \\
4 \\
5 \\
6 \\
7 \\
8 \\
9\end{array}$ & $\begin{array}{l}\text { Hydrogen } \\
16.176 \\
6464.012 \\
6464.790 \\
6510.901 \\
6588.073 \\
6674.932 \\
6767.710 \\
6853.703 \\
470.631\end{array}$ & 022272-8.dif & eric_shp. \\
\hline
\end{tabular}




\begin{tabular}{|c|c|c|c|c|c|c|}
\hline $\begin{array}{l}\text { Feb 22, } \\
\text { Feb 22, } \\
\text { Feb 22, } \\
\text { Feb 22, } \\
\text { Feb 22, } \\
\text { Feb 22, } \\
\text { Feb 22, } \\
\text { Feb 22, } \\
\text { Feb 22, } \\
\text { Feb 22, }\end{array}$ & $\begin{array}{l}1997 \\
1997 \\
1997 \\
1997 \\
1997 \\
1997 \\
1997 \\
1997 \\
1997 \\
1997\end{array}$ & $\begin{array}{l}12: 59: 29 \\
12: 58: 28 \\
13: 00: 30 \\
13: 02: 32 \\
13: 04: 34 \\
13: 06: 36 \\
13: 08: 39 \\
13: 10: 41 \\
13: 12: 43 \\
13: 14: 45\end{array}$ & $\begin{array}{l}0 \\
1 \\
2 \\
3 \\
4 \\
5 \\
6 \\
7 \\
8 \\
9\end{array}$ & $\begin{array}{l}\text { Hydrogen } \\
17.330 \\
30.399 \\
6504.745 \\
6522.693 \\
6552.434 \\
6559.853 \\
6563.333 \\
6545.603 \\
6570.091\end{array}$ & 0222728A.dif & eric_shp. \\
\hline $\begin{array}{ll}\text { Feb } & 22, \\
\text { Feb } & 22, \\
\text { Feb } & 22, \\
\text { Feb } & 22, \\
\text { Feb } & 22, \\
\text { Feb } & 22, \\
\text { Feb } & 22, \\
\text { Feb } & 22, \\
\text { Feb } & 22, \\
\text { Feb } & 22 \text {, }\end{array}$ & $\begin{array}{l}1997 \\
1997 \\
1997 \\
1997 \\
1997 \\
1997 \\
1997 \\
1997 \\
1997 \\
1997\end{array}$ & $\begin{array}{l}13: 37: 35 \\
13: 36: 35 \\
13: 38: 37 \\
13: 40: 39 \\
13: 42: 40 \\
13: 44: 43 \\
13: 46: 45 \\
13: 48: 47 \\
13: 50: 49 \\
13: 52: 51\end{array}$ & $\begin{array}{l}0 \\
1 \\
2 \\
3 \\
4 \\
5 \\
6 \\
7 \\
8 \\
9\end{array}$ & $\begin{array}{l}\text { Hydrogen } \\
18.006 \\
6506.151 \\
6489.973 \\
6473.920 \\
6481.234 \\
6475.514 \\
6471.816 \\
6464.026 \\
6447.447\end{array}$ & 022272-9.dif & eric_shp. \\
\hline $\begin{array}{ll}\text { Feb } & 22, \\
\text { Feb } & 22, \\
\text { Feb } & 22, \\
\text { Feb } & 22, \\
\text { Feb } & 22, \\
\text { Feb } & 22, \\
\text { Feb } & 22, \\
\text { Feb } & 22, \\
\text { Feb } & 22, \\
\text { Feb } & 22,\end{array}$ & $\begin{array}{l}1997 \\
1997 \\
1997 \\
1997 \\
1997 \\
1997 \\
1997 \\
1997 \\
1997 \\
1997\end{array}$ & $\begin{array}{l}14: 13: 26 \\
14: 12: 26 \\
14: 14: 28 \\
14: 16: 30 \\
14: 18: 32 \\
14: 20: 35 \\
14: 22: 37 \\
14: 24: 39 \\
14: 26: 41 \\
14: 28: 43\end{array}$ & $\begin{array}{l}0 \\
1 \\
2 \\
3 \\
4 \\
5 \\
6 \\
7 \\
8 \\
9\end{array}$ & $\begin{array}{l}\text { Hydrogen } \\
19.338 \\
4349.394 \\
4359.913 \\
4347.964 \\
4357.109 \\
4352.464 \\
4349.592 \\
4338.969 \\
4349.167\end{array}$ & 02227EX1.dif & eric_shp. \\
\hline $\begin{array}{ll}\text { Feb } & 22, \\
\text { Feb } & 22, \\
\text { Feb } & 22, \\
\text { Feb } & 22, \\
\text { Feb } & 22, \\
\text { Feb } & 22, \\
\text { Feb } & 22, \\
\text { Feb } & 22, \\
\text { Feb } & 22, \\
\text { Feb } & 22, \\
\text { Feb } & 22,\end{array}$ & $\begin{array}{l}1997 \\
1997 \\
1997 \\
1997 \\
1997 \\
1997 \\
1997 \\
1997 \\
1997 \\
1997 \\
1997\end{array}$ & $\begin{array}{l}14: 43: 47 \\
14: 42: 46 \\
14: 44: 48 \\
14: 46: 50 \\
14: 48: 52 \\
14: 50: 54 \\
14: 52: 57 \\
14: 54: 59 \\
14: 57: 01 \\
14: 59: 03 \\
15: 01: 06\end{array}$ & $\begin{array}{l}0 \\
1 \\
2 \\
3 \\
4 \\
5 \\
6 \\
7 \\
8 \\
9 \\
10\end{array}$ & $\begin{array}{l}\text { Hydrogen } \\
18.225 \\
4357.292 \\
4347.703 \\
4331.313 \\
4335.274 \\
4325.595 \\
4336.448 \\
4331.169 \\
4340.922 \\
21.342\end{array}$ & 02227EX2.dif & eric_shp. \\
\hline
\end{tabular}


$\begin{array}{llll}\text { Feb 22, } 1997 & 15: 14: 46 & 0 \\ \text { Feb 22, } 1997 & 15: 13: 46 & 1 \\ \text { Feb 22, } 1997 & 15: 15: 48 & 2 \\ \text { Feb 22, } 1997 & 15: 17: 50 & 3 \\ \text { Feb 22, } 1997 & 15: 19: 52 & 4 \\ \text { Feb 22, } 1997 & 15: 21: 54 & 5 \\ \text { Feb 22, } 1997 & 15: 23: 56 & 6 \\ \text { Feb 22, } 1997 & 15: 25: 58 & 7 \\ \text { Feb 22, } 1997 & 15: 28: 00 & 8 \\ \text { Feb 22, } 1997 & 15: 30: 02 & 9\end{array}$

Feb 25, 1997 12:04:06

Feb 25, 1997 12:03:06

Feb 25, 1997 12:05:08

Feb 25, 1997 12:07:10

Feb 25, 1997 12:09:12

Feb 25, 1997 12:11:14 5

Feb 25, 1997 13:46:04

Feb 25, 1997 13:45:03

Feb 25, $1997 \quad 13: 47: 05$

Feb 25, 1997 13:49:07

Feb 25, 1997 13:51:10

Feb 25, 1997 13:53:12

Feb 25, 1997 13:55:14

Feb 25, 1997 13:57:16

Feb 25, 1997 13:59:18

Feb 25, $1997 \quad 14: 01: 20$

Feb 25, $1997 \quad 14: 12: 56$

Feb 25, 1997 14:11:55

Feb 25, 1997 14:13:57

Feb 25, $1997 \quad 14: 15: 59$

Feb 25, 1997 14:18:01

Feb 25, $1997 \quad 14: 20: 03$

Feb 25, 1997 14:22:05

Feb 25, $1997 \quad 14: 24: 07$

Feb 25, 1997 14:26:09

Feb 25, $1997 \quad 14: 42: 18$

Feb 25, $1997 \quad 14: 41: 18 \quad 1$

Feb 25, $1997 \quad 14: 43: 20$

Feb 25, $1997 \quad 14: 45: 22$

Feb 25, $1997 \quad 14: 47: 24$

Feb 25, $1997 \quad 14: 49: 26$

Feb 25, $1997 \quad 14: 51: 28$

Feb 25, $1997 \quad 14: 53: 30$

Feb 25, $1997 \quad 14: 55: 32$

Feb 25, $1997 \quad 14: 57: 35$
Hydrogen

18.629

4336.479

4323.214

4326.033

4322.145

4312.876

4313.123

4316.208

4304.706

Hydrogen

1009.299

1008.832

1009.226

1008.699

1008.425

Hydrogen

0.000

2447.853

3809.017

3805.322

3799.938

3796.007

3793.973

3784.658

3801.212

Hydrogen

0.000

4216.762

4217. 365

4213.579

4208.123

4204.204

4208.516

4206.871

Hydrogen

4.639

4206.640

4211.951

4212.225

4208.801

4215.577

4211.121

4215.286

4212.332
02227EX3.dif eric_shp.

02257cal.dif eric_shp.

02257EX1.dif eric_shp.

02257EX2.dif eric_shp.

02257EX3.dif eric_shp. 


\section{APPENDIX C}

Feb 25, $1997 \quad 15: 15: 26$

Feb 25, 1997 15:14:26

Feb 25, 1997 15:16:28

Feb 25, 1997 15:18:30

Feb 25, 1997 15:20:33

Feb 25, $1997 \quad 15: 22: 35 \quad 5$

Feb 25, $1997 \quad 15: 24: 376$

Feb 25, 1997 15:26:39 7

Feb 25, $1997 \quad 15: 28: 428$

Feb 25, 1997 15:30:44 9
Hydrogen
9.041
27.646
4223.193
4211.308
4224.475
4209.358
4209.040
4222.687
4221.587

HNF-SD-WM-TRP-275 Rev. 0 Page 102 


\begin{tabular}{|c|c|c|c|c|c|c|c|c|c|c|c|}
\hline \multicolumn{12}{|c|}{$\begin{array}{l}\text { TEST: EX102227 } 22 \text { Feb } 1997 \text { WEC-SPOOL } \\
\text { Measured Hydrogen Concentration }=4348 \mathrm{ppm}\end{array}$} \\
\hline & DIFF (IN H2O) & ABS (PSIA) & DIFF (IN H2O) & ABS (PSIA) & (PPM) & (PPM) & TEMP.(F) & TEMP. (F) & TEMP. (F) & TEMP. $(F)$ & (VOLTS) \\
\hline 0.0200195 & 3.40222258 & 14.7419885 & 0.612388064 & 14.68425 & 111.225 & 3.349758 & 70.5 & 69.8 & 69.3 & 69.5 & 0.000247 \\
\hline 5.0200195 & 3.39784569 & 14.7434878 & 1.79597072 & 14.67825 & 111.35 & -3.225604 & 70.5 & 69.7 & 69.2 & 69.5 & 0.000248 \\
\hline 10.02002 & 3.39409407 & 14.7427381 & 1.7972216 & 14.6775 & 181.5 & 1040.41292 & 70.5 & 69.9 & 69.3 & 69.5 & 0.000276 \\
\hline 15.02002 & 3.39409407 & 14.7427381 & 1.7972216 & 14.67825 & 644.5 & 2366.87426 & 70.5 & 69.9 & 69.3 & 69.5 & 0.000349 \\
\hline 20.02002 & 3.38971718 & 14.7419885 & 1.79784704 & 14.67825 & 1230.25 & 3108.57025 & 70.5 & 69.9 & 69.3 & 6.5 & 0.000439 \\
\hline 25.02002 & 3.39409407 & 14.7434878 & 1.7972216 & 14.6775 & 1782 & 3461.66423 & 70.3 & 69.9 & 69.1 & 69,4 & 0.000522 \\
\hline 30.02002 & 3.39159299 & 14.7412389 & 1.7972216 & 14.6775 & 2253.75 & 3649.38508 & 70.3 & 69.9 & 69.1 & 69.5 & 0.000593 \\
\hline 35.02002 & 3.38659083 & 14.7427381 & 1.79909792 & 14.6775 & 2681.5 & 3756.90244 & 70.3 & 69.9 & 69.2 & 69.5 & 0.00065 \\
\hline 40.02002 & 3.42285649 & 14.7412389 & 1.79972336 & 14.6775 & 3023.5 & 3836.85762 & 70.2 & 69.9 & 69.3 & 69.5 & 0.000696 \\
\hline 45.02002 & 3.39847096 & 14.7427381 & 1.79972336 & 14.6775 & 3281.5 & 3869.88258 & 70.3 & 69.9 & 69.3 & 69.5 & 0.000733 \\
\hline 50.02002 & 3.38534029 & 14.7419885 & 1.79972336 & 14.67675 & 3504 & 3907.37709 & 70.3 & 69.9 & 69.2 & 69.5 & 0.000764 \\
\hline 55.02002 & 3.39409407 & 14.7419885 & 1.79847248 & 14.6775 & 3704.25 & 3917.30941 & 70.4 & 69.9 & 69.1 & 69.5 & 0.000789 \\
\hline 60.02002 & 3.40222258 & 14.7404893 & 1.79909792 & 14.6775 & 3833.75 & 3937.91897 & 70.3 & 69.9 & 69.2 & 69.5 & 0.000808 \\
\hline 65.02002 & 3.3997215 & 14.7419885 & 1.8003488 & 14.67825 & 3971.5 & 3945.36821 & 70.3 & 69.9 & 69.3 & 69.5 & 0.000825 \\
\hline 70.02002 & 3.41597852 & 14.7427381 & 1.8003488 & 14.6775 & 4035 & 3944.37498 & 70.4 & 69.9 & 69.3 & 69.6 & 0.000838 \\
\hline 75.02002 & 3.39534461 & 14.7419885 & 1.80285056 & 14.67675 & 4115 & 3945.86483 & 70.4 & 69.9 & 69.3 & 69.5 & 0.000849 \\
\hline 80.02002 & 3.40222258 & 14.7419885 & 1.80285056 & 14.6775 & 4194.75 & 3966.47439 & 70.4 & 69.9 & 69.2 & 69.5 & 0.000859 \\
\hline 85.02002 & 3.40409939 & 14.7434878 & 1.80159968 & 14.67825 & 4254.5 & 3975.9101 & 70.4 & 69.9 & 69.3 & 69.5 & 0.000867 \\
\hline 90.02002 & 3.39284353 & 14.7404893 & 1.80097424 & 14.67825 & 4294.75 & 3979.1381 & 70.3 & 69.9 & 69.3 & 69.6 & 0.000873 \\
\hline 95.02002 & 3.3809634 & 14.7419885 & 1.80159968 & 14.67825 & 4344.75 & 3974.66856 & 70.3 & 69.9 & 69.3 & 69.5 & 0.000879 \\
\hline 100.02002 & 3.3934688 & 14.7427381 & 1.80159968 & 14.67825 & 4365.25 & 3989.31873 & 70.3 & 69.9 & 69.3 & 69.5 & 0.000884 \\
\hline 105.02002 & 3.3997215 & 14.7427381 & 1.80222512 & 14.67825 & 4405.25 & 4011.91476 & 70.3 & 70 & 69.2 & 69.5 & 0.000888 \\
\hline 110.02002 & 3.38846664 & 14.7419885 & 1.80222512 & 14.6775 & 4435 & 4020.85384 & 70.3 & 69.9 & 69.3 & 69.5 & 0.000892 \\
\hline 115.02002 & 3.39784569 & 14.7419885 & 1.80159968 & 14.67825 & 4455 & 4008.43844 & 70.4 & 69.9 & 69.3 & 69.5 & 0.000895 \\
\hline 120.02002 & 3.40847528 & 14.7419885 & 1.80097424 & 14.6775 & 4475.25 & 4012.65968 & 70.4 & 69.9 & 69.3 & 69.5 & 0.000898 \\
\hline 125.02002 & 3.39471934 & 14.7427381 & 1.803476 & 14.6775 & 4485 & 4026.06831 & 70.5 & 69.9 & 69.2 & 69.5 & 0.000901 \\
\hline 130.02002 & 3.40785001 & 14.7419885 & 1.80097424 & 14.6775 & 4505 & 4044.4431 & 70.4 & 70 & 69.2 & 6.6 & 0.000901 \\
\hline 135.02002 & 3.3997215 & 14.7412389 & 1.80159968 & 14.67825 & 4515.5 & 4053.38219 & 70.3 & 70 & 69.3 & 69.5 & 0.000904 \\
\hline 140.02002 & 3.3997215 & 14.7427381 & 1.80159968 & 14.67675 & 4525.5 & 4061.57636 & 70.3 & 69.9 & 69.2 & 69.5 & 0.000906 \\
\hline 145.02002 & 3.40159731 & 14.7419885 & 1.80159968 & 14.67675 & 4535.25 & 4048.16772 & 70.3 & 70 & 69.2 & 69.6 & 0.000908 \\
\hline
\end{tabular}




\begin{tabular}{|c|c|c|c|c|c|c|c|c|c|c|c|}
\hline SECONDS & MAIN DUCT & MAIN DUCT & GAS INJECT & GAS INJECT & NEWPORT & SMC & AMBIENT & GAS INJECT & MAIN DUCT & WEC CELL & WEC CELL \\
\hline & DIFF (IN H2O) & ABS (PSIA) & DIFF (IN H2O) & ABS (PSIA) & (PPM) & (PPM) & TEMP.(F) & TEMP. (F) & TEMP. (F) & TEMP. (F) & (VOLTS) \\
\hline 0.0200195 & 3.402222258 & 14.7419885 & 0.612388064 & 14.68425 & 111.225 & -3.349758 & 70.5 & 69.8 & 69.3 & 69.5 & 0.000247 \\
\hline 150.02002 & 3.39534461 & 14.7412389 & 1.80159968 & 14.67825 & 4555.5 & 4052.88558 & 70.4 & 70 & 69.3 & 69.6 & 0.00091 \\
\hline 155.02002 & 3.40159731 & 14.7427381 & 1.80222512 & 14.67825 & 4565 & 4060.33482 & 70.3 & 70 & 69.4 & 69.5 & 0.000911 \\
\hline 160.02002 & 3.4372377 & 14.7404893 & 1.80097424 & 14.6775 & 4575.75 & 4072.75022 & 70.4 & 70 & 69.3 & 69.5 & 0.000911 \\
\hline 165.02002 & 3.38659083 & 14.7427381 & 1.80472688 & 14.6775 & 4585.5 & 4074.73668 & 70.4 & 70 & 69.3 & 69.6 & 0.000913 \\
\hline 170.02002 & 3.38971718 & 14.7427381 & 1.80222512 & 14.6775 & 4585 & 4070.01883 & 70.3 & 70 & 69.2 & 69.6 & 0.000914 \\
\hline 175.04001 & 3.38283921 & 14.7412389 & 1.80222512 & 14.6775 & 4595.25 & 4075.4816 & 70.3 & 70 & 69.3 & 69.6 & 0.000916 \\
\hline 180.02002 & 3.39784569 & 14.7404893 & 1.80097424 & 14.6775 & 4605.5 & 4072.50191 & 70.3 & 70 & 69.3 & 69.6 & 0.000916 \\
\hline 185.02002 & 3.38846664 & 14.7419885 & 1.80159968 & 14.6775 & 4615.25 & 4075.4816 & 70.3 & 70 & 69.2 & 69.5 & 0.000917 \\
\hline 190.02002 & 3.39159299 & 14.7404893 & 1.802202512 & 14.6775 & 4625.5 & 4082.18592 & 70.3 & 70 & 69.2 & 69.5 & 0.000918 \\
\hline 195.02002 & 3.39784569 & 14.7412389 & 1.80159968 & 14.6775 & 4625.5 & 4087.15208 & 70.3 & 70 & 69.2 & 69.6 & 0.000919 \\
\hline 200.02002 & 3.39722042 & 14.7412389 & 1.80159968 & 14.6775 & 4625.5 & 4088.64193 & 70.3 & 70 & 6.3 & 69.6 & 0.00092 \\
\hline 205.02002 & 3.39722042 & 14.7412389 & 1.80159968 & 14.67825 & 4635.25 & 4100.80902 & 70.3 & 70 & 69.3 & 69.6 & 0.00092 \\
\hline 210.03 & 3.3997215 & 14.7419885 & 1.80159968 & 14.6775 & 4635.5 & 4111.48626 & 70.3 & 70 & 69.2 & 69.5 & 0.00092 \\
\hline 215.02002 & 3.39784569 & 14.7412389 & 1.80222512 & 14.67675 & 4635.75 & 4094.1047 & 70.3 & 70 & 69.2 & 69.6 & 0.000921 \\
\hline 220.02002 & 3.38909191 & 14.7404893 & 1.80097424 & 14.6775 & 4634.75 & 4098.07763 & 70.3 & 70 & 69.2 & 69.5 & 0.000921 \\
\hline 225.02002 & 3.4059742 & 14.7412389 & 1.80159968 & 14.6775 & 4645.75 & 4092.61486 & 70.3 & 70 & 69.3 & 69.5 & 0.000922 \\
\hline 230.02002 & 3.38659083 & 14.7404893 & 1.80159968 & 14.67675 & 4654.75 & 4093.8564 & 70.3 & 70 & 69.3 & 69.5 & 0.000923 \\
\hline 235.02002 & 3.40034677 & 14.7419885 & 1.80159968 & 14.67675 & 4655.75 & 4099.31917 & 70.4 & 70 & 69.2 & 69.5 & 0.000923 \\
\hline 240.02002 & 3.3872161 & 14.7419885 & 1.80159968 & 14.67675 & 4655.5 & 4086.15885 & 70.4 & 70 & 69.2 & 69.6 & 0.000924 \\
\hline 245.02002 & 3.39784569 & 14.7412389 & 1.80159968 & 14.6775 & 4665.5 & 4080.94438 & 70.4 & 70 & 69.3 & 69.6 & 0.000924 \\
\hline 250.02002 & 3.41535325 & 14.7412389 & 1.80159968 & 14.67675 & 4665.5 & 4076.72314 & 70.3 & 70 & 69.3 & 69.6 & 0.000924 \\
\hline 255.02002 & 3.40659947 & 14.7427381 & 1.80159968 & 14.6775 & 4665.5 & 4048.91265 & 70.4 & 70 & 69.3 & 69.6 & 0.000924 \\
\hline 260.02002 & 3.39722042 & 14.7412389 & 1.80159968 & 14.6775 & 4665.25 & 4064.80436 & 70.3 & 70 & 69.2 & 69.6 & 0.000925 \\
\hline 265.02002 & 3.39159299 & 14.7412389 & 1.80097424 & 14.67825 & 4665.25 & 4072.99852 & 70.3 & 70 & 69.3 & 69.6 & 0.000926 \\
\hline 270.03 & 3.40722474 & 14.7419885 & 1.80159968 & 14.6775 & 4675.5 & 4089.63516 & 70.3 & 70 & 69.2 & 69.6 & 0.000926 \\
\hline 275.02002 & 3.39659515 & 14.7412389 & 1.80222512 & 14.6775 & 4675.75 & 4089.63516 & 70.3 & 70 & 69.2 & 69.6 & 0.000927 \\
\hline 280.02002 & 3.39596988 & 14.7419885 & 1.80097424 & 14.67675 & 4675.25 & 4098.07763 & 70.3 & 70 & 69.2 & 69.6 & 0.000926 \\
\hline 285.03 & 3.4122269 & 14.7419885 & 1.80159968 & 14.6775 & 4685.75 & 4096.58778 & 70.4 & 70 & 69.3 & 69.6 & 0.000927 \\
\hline 290.02002 & 3.3934688 & 14.7419885 & 1.80159968 & 14.67825 & 4685 & 4097.0844 & 70.3 & 70 & 69.2 & 69.6 & 0.000928 \\
\hline 295.02002 & 3.38784137 & 14.7412389 & 1.80159968 & 14.6775 & 4685.75 & 4083.17915 & 70.4 & 70.1 & 69.3 & 69.6 & 0.000928 \\
\hline 300.02002 & 3.38909191 & 14.7427381 & 1.79909792 & 14.6775 & 4685.75 & 4076.72314 & 70.4 & 70.1 & 69.3 & 69.6 & 0.000928 \\
\hline 305.03 & 3.40034677 & 14.7404893 & 1.79972336 & 14.6775 & 4685.25 & 4059.09328 & 70.4 & 70.1 & 69.2 & 69.6 & 0.000927 \\
\hline 310.02002 & 3.39221826 & 14.7427381 & 1.79909792 & 14.67675 & 4686 & 4086.90377 & 70.4 & 70 & 69.2 & 69.6 & 0.000928 \\
\hline
\end{tabular}




\begin{tabular}{|c|c|c|c|c|c|c|c|c|c|c|c|}
\hline SECONDS & MAIN DUCT & MAIN DUCT & GAS INJECT & GAS INJECT & NEWPORT & SMC & AMBIENT & GAS INJECT & MAIN DUCT & WEC CELL & WEC CELL \\
\hline & DIFF (IN H2O) & ABS (PSIA) & DIFF (IN H2O) & ABS (PSIA) & (PPM) & (PPM) & TEMP.(F) & TEMP. (F) & TEMP. (F) & TEMP.(F) & (VOLTS) \\
\hline 0.0200195 & 3.40222258 & 14.7419885 & 0.612388064 & 14.68425 & 111.225 & -3.349758 & 70.5 & 69.8 & 69.3 & 69.5 & 0.000247 \\
\hline 315.02002 & 3.40159731 & 14.7427381 & 1.79909792 & 14.67825 & 4692.5 & 4085.91054 & 70.5 & 70 & 69.3 & 69.6 & 0.000929 \\
\hline 320.02002 & 3.39722042 & 14.7427381 & 1.79972336 & 14.6775 & 4695.25 & 4098.82256 & 70.5 & 70 & 69.2 & 69.6 & 0.000920 \\
\hline 325.02002 & 3.39784569 & 14.7404893 & 1.79847248 & 14.6775 & 4695.5 & 4102.29887 & 70.5 & 70 & 69.3 & 69.6 & 0.000920 \\
\hline 330.02002 & 3.38971718 & 14.7427381 & 1.79847248 & 14.67675 & 4695.75 & 4088.89024 & 70.5 & 70.1 & 69.3 & 69.6 & 0.000929 \\
\hline 335.02002 & 3.40159731 & 14.7427381 & 1.79972336 & 14.6775 & 4695.25 & 4090.8767 & 70.5 & 70.1 & 69.2 & 69.6 & 0.00093 \\
\hline 340.02002 & 3.3872161 & 14.7427381 & 1.79909792 & 14.6775 & 4705.75 & 4082.43423 & 70.5 & 70.1 & 69.2 & 69.6 & 0.00093 \\
\hline 345.02002 & 3.39596988 & 14.7404893 & 1.79909792 & 14.67675 & 4706 & 4081.93761 & 70.5 & 70.1 & 69.3 & 69.6 & 0.00093 \\
\hline 350.02002 & 3.40847528 & 14.7427381 & 1.79909792 & 14.6775 & 4705 & 4076.22653 & 70.6 & 70.1 & 69.3 & 69.6 & 0.00093 \\
\hline 355.02002 & 3.39159299 & 14.7412389 & 1,79972336 & 14.67675 & 4705.75 & 4078.21299 & 70.6 & 70.1 & 69.3 & 69.6 & 0.00093 \\
\hline 360.02002 & 3.36971718 & 14.7427381 & 1.79972336 & 14.6775 & 4705.5 & 4081.93761 & 70.6 & 70.1 & 69.2 & 69.6 & 0.000931 \\
\hline 365.02002 & 3,39784569 & 14.7412389 & 1.79909792 & 14.6775 & 4714.75 & 4081.441 & 70.6 & 70.1 & 69.2 & 69.6 & 0.000932 \\
\hline 370.02002 & 3.39159299 & 14.7434878 & 1.79847248 & 14.6775 & 4716 & 4085.16562 & 70.6 & 70.1 & 69.3 & 69.6 & 0.000931 \\
\hline 375.02002 & 3.39847096 & 14.7427381 & 1.79972336 & 14.6775 & 4716 & 4098.07763 & 70.6 & 70.1 & 69.4 & 69.6 & 0.000933 \\
\hline 380.02002 & 3.40284785 & 14.7427381 & 1.79909792 & 14.67675 & 4715.5 & 4093.11147 & 70.5 & 70.1 & 69.4 & 69.6 & 0.000932 \\
\hline 385.02002 & 3,41910487 & 14.7412389 & 1.79909792 & 14.6775 & 4716 & 4096.58778 & 70.5 & 70.1 & 69.3 & 69.6 & 0.000932 \\
\hline 390.05002 & 3,38596556 & 14.7419885 & 1.79972336 & 14.67675 & 4715.75 & 4087.40039 & 70.5 & 70.1 & 69.4 & 69.6 & 0.000933 \\
\hline 395.02002 & 3.40284785 & 14.7419885 & 1.7972216 & 14.6775 & 4715.5 & 4092.61486 & 70.6 & 70.1 & 69.4 & 69.6 & 0.000933 \\
\hline 400.02002 & 3.39596988 & 14.7412389 & 1.79847248 & 14.6775 & 4726 & 4090.13178 & 70.6 & 70.1 & 69.3 & 69.6 & 0.000933 \\
\hline 405.02002 & 3.39034245 & 14.7419885 & 1.7972216 & 14.6775 & 4725.75 & 4080.69607 & 70.6 & 70.2 & 69.2 & 69.6 & 0.000932 \\
\hline 410.03 & 3.39722042 & 14.7412389 & 1.7972216 & 14.67825 & 4725.75 & 4094.84963 & 70.5 & 70.1 & 69.2 & 69.6 & 0.000933 \\
\hline 415.02002 & 3.39784569 & 14.7419885 & 1.79972336 & 14.67825 & 4725.75 & 4100.0641 & 70.5 & 70.2 & 69.4 & 69.6 & 0.000934 \\
\hline 420.07001 & 3.402222258 & 14.7419885 & 1.7972216 & 14.67675 & 4724.5 & 4093.11147 & 70.5 & 70.1 & 69.4 & 69.6 & 0.000933 \\
\hline 425.02002 & 3.42348176 & 14.7412389 & 1.79784704 & 14.67675 & 4717.25 & 4082.18592 & 70.6 & 70.2 & 69.4 & 69.6 & 0.000933 \\
\hline 430.02002 & 3.3872161 & 14.7412389 & 1.79659616 & 14.67675 & 4726 & 4067.28744 & 70.6 & 70.1 & 69.3 & 69.6 & 0,000933 \\
\hline 435.02002 & 3.40284785 & 14.7412389 & 1.7972216 & 14.6775 & 4726 & 4082.68254 & 70.6 & 70.2 & 69.3 & 69.6 & 0.000933 \\
\hline 440.08002 & 3.40347312 & 14.7412389 & 1.7972216 & 14.67675 & 4725.75 & 4079.95115 & 70.5 & 70.2 & 69.4 & 69.6 & 0.000933 \\
\hline 445.02002 & 3.39221826 & 14.7404893 & 1.79784704 & 14.6775 & 4726 & 4073.24683 & 70.4 & 70.2 & 69.3 & 69.6 & 0.000934 \\
\hline 450.02002 & 3.39847096 & 14.7412389 & 1.79659616 & 14.6775 & 4726 & 4096.83609 & 70.2 & 70.2 & 69.3 & 69.6 & 0.000934 \\
\hline 455.02002 & 3.39784569 & 14.7404893 & 1.79659616 & 14.6775 & 4735.75 & 4110.24472 & 70.1 & 70.2 & 69.2 & 69.6 & 0.000934 \\
\hline 460.11002 & 3.39784569 & 14.7412339 & 1.7972216 & 14.6775 & 4735.75 & 4106,5201 & 70.1 & 70.2 & 69.3 & 69.6 & 0.000934 \\
\hline 465.02002 & 3.40159731 & 14.7419885 & 1.7972216 & 14.6775 & 4736 & 4080.69607 & 70 & 70.2 & 69.4 & 69.6 & 0.000934 \\
\hline 470.02002 & 3.3872161 & 14.7412389 & 1.79659616 & 14.6775 & 4735.75 & 4074.24006 & 70.1 & 70.2 & 69.3 & 69.6 & 0.000934 \\
\hline 475.02002 & 3.43348608 & 14.7412389 & 1.79659616 & 14.6775 & 4736 & 4069.0256 & 70.2 & 70.2 & 69.3 & 69.6 & 0.000934 \\
\hline
\end{tabular}




\begin{tabular}{|c|c|c|c|c|c|c|c|c|c|c|c|}
\hline SECONDS & MAIN DUCT & MAIN DUCT & GAS INJECT & GAS INJECT & NEWPORT & SMC & AMBIENT & GAS INJECT & MAIN DUCT & WEC CELL & WEC CELL \\
\hline & DIFF (IN H2O) & ABS (PS|A) & DIFF (IN H2O) & ABS (PS|A) & (PPM) & (PPM) & TEMP.(F) & TEMP. (F) & TEMP. (F) & TEMP. (F) & (VOLTS) \\
\hline 0.0200195 & 3.40222258 & 14.7419885 & 0.612388064 & 14.68425 & 111.225 & -3.349758 & 70.5 & 69.8 & 69.3 & 69.5 & 0.000247 \\
\hline 480.02002 & 3.39659515 & 14.7412389 & 1.7972216 & 14.67675 & 4736 & 4076.47484 & 70.3 & 70.2 & 69.2 & 69.6 & 0.000934 \\
\hline 485.02002 & 3.3997215 & 14.7412389 & 1.7972216 & 14.6775 & 4735.75 & 4091.37332 & 70.3 & 70.2 & 69.3 & 69.6 & 0.000934 \\
\hline 490.02002 & 3.39596988 & 14.7412389 & 1.7972216 & 14.6775 & 4736 & 4093.35978 & 70.3 & 70.2 & 69.4 & 69.6 & 0.000935 \\
\hline 495.02002 & 3.40722474 & 14.7427381 & 1.7972216 & 14.6775 & 4736 & 4101.55394 & 70.3 & 70.2 & 69.4 & 69.6 & 0.000935 \\
\hline 500.02002 & 3.38534029 & 14.7419885 & 1.79784704 & 14.6775 & 4735.75 & 4094.35301 & 70.4 & 70.2 & 69.4 & 69.6 & 0.000935 \\
\hline 505.02002 & 3.3934688 & 14.7412389 & 1.7972216 & 14.6775 & 4746 & 4076.97145 & 70.5 & 70.3 & 69.3 & 69.6 & 0.000935 \\
\hline 510.05002 & 3.41660379 & 14.7412389 & 1.79659616 & 14.67675 & 4746 & 4064.80436 & 70.5 & 70.2 & 69.4 & 69.6 & 0.000936 \\
\hline 515.02002 & 3.39847096 & 14.7427381 & 1.79784704 & 14.67825 & 4745.75 & 4068.77729 & 70.5 & 70.3 & 69.3 & 69.6 & 0.000936 \\
\hline 520.02002 & 3.39784569 & 14.7419885 & 1.79659616 & 14.6775 & 4746 & 4075.2333 & 70.5 & 70.2 & 69.3 & 69.6 & 0.000936 \\
\hline 525.02002 & 3.39596988 & 14.7427381 & 1.79659616 & 14.6775 & 4746 & 4062.07297 & 70.6 & 70.2 & 69.3 & 69.6 & 0.000937 \\
\hline 530.02002 & 3.40847528 & 14.7419885 & 1.79784704 & 14.6775 & 4745.75 & 4066.0459 & 70.6 & 70.2 & 69.3 & 69.6 & 0.000937 \\
\hline 535.02002 & 3.39784569 & 14.7419885 & 1.7972216 & 14.67825 & 4746 & 4072.00529 & 70.6 & 70.2 & 69.2 & 69.6 & 0.000937 \\
\hline 540.06 & 3.40722474 & 14.7427381 & 1.79659616 & 14.67675 & 4745.75 & 4040.47018 & 70.6 & 70.2 & 69.3 & 69.6 & 0.000938 \\
\hline 545.03 & 3.39847096 & 14.7427381 & 1.79659616 & 14.6775 & 4745.5 & 4044.93972 & 70.6 & 70.2 & 69.3 & 69.6 & 0,000938 \\
\hline 550.02002 & 3.42098068 & 14.7434878 & 1.7972216 & 14.67675 & 4755.75 & 4061.82466 & 70.6 & 70.2 & 69.4 & 69.6 & 0.000937 \\
\hline 555.02002 & 3.39159299 & 14.7419885 & 1.7972216 & 14.67825 & 4755.5 & 4074.24006 & 70.6 & 70.2 & 69.5 & 69.6 & 0.000938 \\
\hline 560.02002 & 3.41785433 & 14.7427381 & 1.79784704 & 14.6775 & 4756 & 4079.45453 & 70.6 & 70.2 & 69.4 & 69.6 & 0.000938 \\
\hline 565.02002 & 3.39159299 & 14.7412389 & 1.7972216 & 14.67825 & 4745.75 & 4092.11824 & 70.7 & 70.2 & 69.4 & 69.6 & 0.000938 \\
\hline 570.05002 & 3.39847096 & 14.7419885 & 1.7972216 & 14.6775 & 4755.75 & 4089.63516 & 70.7 & 70.2 & 69.3 & 69.6 & 0.000938 \\
\hline 575.02002 & 3.41097636 & 14.7397397 & 1.79784704 & 14.6775 & 4755.75 & 4095.34624 & 70.8 & 70.2 & 69.5 & 69.6 & 0.000938 \\
\hline 580.02002 & 3.39096772 & 14.7412389 & 1.7972216 & 14.6775 & 4755.75 & 4103.04379 & 70.8 & 70.2 & 69.3 & 69.6 & 0,000938 \\
\hline 585.03 & 3.40222258 & 14.7419885 & 1.79659616 & 14.67825 & 4756 & 4095.59455 & 70.7 & 70.3 & 69.4 & 69.7 & 0.000938 \\
\hline 590,02002 & 3.39596988 & 14.7412389 & 1.7972216 & 14.6775 & $\begin{array}{r}4755.5 \\
\end{array}$ & 4108.75488 & 70.7 & 70.3 & 69.4 & 69.6 & 0.000938 \\
\hline 595.02002 & 3.38346448 & 14.7419885 & 1.79659616 & 14.6775 & 4755.75 & 4113.47273 & 70.6 & 70.3 & 69.4 & 69.6 & 0.000938 \\
\hline 600.05002 & 3.40659947 & 14.7419885 & 1.7972216 & 14.67675 & 4756 & 4118.9355 & 70.6 & 70.3 & 69.3 & 69.6 & 0.000938 \\
\hline 605.02002 & 3.40785001 & 14.7427381 & 1.7972216 & 14.6775 & 4756 & 4114.71427 & 70.6 & 70.3 & 69.4 & 69.6 & 0.000938 \\
\hline 610.02002 & 3.3872161 & 14.7427381 & 1.79784704 & 14.67675 & 4756 & 4102.79548 & 70.6 & 70.2 & 69.3 & 69.6 & 0.000938 \\
\hline 615.02002 & 3.40847528 & 14.7427381 & 1.7972216 & 14.6775 & 4755.5 & 4093.8564 & 70.6 & 70.3 & 69.3 & 69.6 & 0.000938 \\
\hline 620.02002 & 3.39784569 & 14.7427381 & 1.79784704 & 14.6775 & 4756 & 4088.14531 & 70.6 & 70.3 & 69.4 & 69.6 & 0.000939 \\
\hline 625.02002 & 3.40910055 & 14.7419885 & 1.79784704 & 14.6775 & 4766 & 4089.88347 & 70.5 & 70.3 & 69.4 & 69.6 & 0.00094 \\
\hline 630.04001 & 3.41722906 & 14.7434878 & 1.79659616 & 14.6775 & 4756 & 4095.09794 & 70.5 & 70.3 & 69.4 & 69.6 & 0.000938 \\
\hline 635.02002 & 3.40472366 & 14.7412389 & 1.79784704 & 14.67825 & 4756 & 4102.79548 & 70.5 & 70.3 & 69.3 & 69.6 & 0.000938 \\
\hline 640.02002 & 3.38471502 & 14.7397397 & 1.7972216 & 14.6775 & 4755.75 & 4111.23796 & 70.5 & 70.3 & 69.3 & 69.6 & 0.000938 \\
\hline
\end{tabular}




\begin{tabular}{|c|c|c|c|c|c|c|c|c|c|c|c|}
\hline SECONDS I & MAIN DUCT & MAIN DUCT & GAS INJECT & GAS INJECT & NEWPORT & SMC & AMBIENT & GAS INJECT & MAIN DUCT & WEC CELL & WEC CELL \\
\hline & DIFF (IN H2O) & ABS (PS|A) & DIFF (IN H2O) & ABS (PS|A) & (PPM) & (PPM) & TEMP.(F) & TEMP. (F) & TEMP. $(F)$ & TEMP. (F) & (VOLTS) \\
\hline 0.0200195 & 3.40222258 & 14.7419885 & 0.612388064 & 14.68425 & 111.225 & -3.349758 & 70.5 & 69.8 & 69.3 & 69.5 & 0.000247 \\
\hline 645.06 & 3.39221826 & 14.7412389 & 1.7972216 & 14.6775 & 4756 & 4096.58778 & 70.5 & 70.3 & 69.2 & 69.6 & 0.000938 \\
\hline 650.02002 & 3.38784137 & 14.7404893 & 1.7972216 & 14.679 & 4756 & 4087.15208 & 70.5 & 70.3 & 69.2 & 69.6 & 0.000937 \\
\hline 655.02002 & 3.3872161 & 14.7412389 & 1.79847248 & 14.67675 & 4762.5 & 4075.4816 & 70.5 & 70.3 & 69.2 & 69.6 & 0.000938 \\
\hline 660.03 & 3.40910055 & 14.7412389 & 1.7972216 & 14.6775 & 4760 & 4063.81113 & 70.5 & 70.3 & 69.2 & 69.6 & 0.000938 \\
\hline 665.02002 & 3.40472366 & 14.7419885 & 1.79597072 & 14.6775 & 4756 & 4066.54252 & 70.5 & 70.3 & 69.3 & 69.6 & 0.000938 \\
\hline 670.02002 & 3.39722042 & 14.7412389 & 1.79784704 & 14.6775 & 4756 & 4058.10004 & 70.5 & 70.3 & 69.1 & 69.7 & 0.000938 \\
\hline 675.02002 & 3.39221826 & 14.7419885 & 1.79534528 & 14.67825 & 4756 & 4069.52221 & 70.5 & 70.3 & 69.4 & 69.7 & 0.000938 \\
\hline 680.02002 & 3.39784569 & 14.7412389 & 1.79534528 & 14.6775 & 4756 & 4084.17238 & 70.5 & 70.3 & 69.2 & 69.7 & 0.000938 \\
\hline 685.02002 & 3.38471502 & 14.7419885 & 1.7972216 & 14.67825 & 4755.75 & 4099.81579 & 70.5 & 70.3 & 69.3 & 69.6 & 0.000937 \\
\hline 690.03 & 3.40409839 & 14.7412389 & 1.79534528 & 14.6775 & 4756 & 4101.55394 & 70.5 & 70.4 & 69.3 & 69.6 & 0.000938 \\
\hline 695.05002 & 3.39159299 & 14.7412389 & 1.79784704 & 14.6775 & 4756 & 4098.82256 & 70.6 & 70.4 & 69.4 & 69.7 & 0.000938 \\
\hline 700.02002 & 3.39784569 & 14.7419885 & 1.79847248 & 14.6775 & 4756 & 4087.40039 & 70.6 & 70.4 & 69.4 & 69.7 & 0.000938 \\
\hline 705.07001 & 3.39096772 & 14.7412389 & 1.79597072 & 14.67825 & 4766 & 4092.61486 & 70.5 & 70.4 & 69.2 & 69.7 & 0.000938 \\
\hline 710.02002 & 3.41973014 & 14.7419885 & 1.79534528 & 14.6775 & 4766 & 4082.93084 & 70.6 & 70.4 & 69.4 & 69.7 & 0.000938 \\
\hline 715.02002 & 3.40034677 & 14.7419885 & 1.79534528 & 14.6775 & 4766 & 4091.62162 & 70.6 & 70.3 & 69.3 & 69.6 & 0.000938 \\
\hline 720.02002 & 3.40659947 & 14.7412389 & 1.79471984 & 14.6775 & 4755.5 & 4082.18592 & 70.5 & 70.3 & 69.2 & 69.7 & 0.000939 \\
\hline 725.02002 & 3.40847528 & 14.7419885 & 1.79534528 & 14.6775 & 4756 & 4077.71638 & 70.5 & 70.4 & 69.3 & 69.7 & 0.000939 \\
\hline 730.02002 & 3.39722042 & 14.7419885 & 1.79471984 & 14.6775 & 4756 & 4084.42069 & 70.5 & 70.4 & 69.4 & 69.7 & 0.000939 \\
\hline 735.03 & 3.39784569 & 14.7412389 & 1.79534528 & 14.6775 & 4766.25 & 4088.89024 & 70.5 & 70,4 & 69.4 & 69.7 & 0.000939 \\
\hline 740.08002 & 3.38909191 & 14.7412389 & 1.79597072 & 14.6775 & 4766 & 4098.07763 & 70.4 & 70.4 & 69.4 & 69.7 & 0.000939 \\
\hline 745.02002 & 3.40159731 & 14.7412389 & 1.79597072 & 14.6775 & 4766.25 & 4068.28067 & 70.3 & 70.4 & 69.4 & 69.6 & 0.000939 \\
\hline 750.02002 & 3.40847528 & 14.7397397 & 1.79534528 & 14.6775 & 4765.5 & 4064,30774 & 70.1 & 70.4 & 69.3 & 69.6 & 0.00094 \\
\hline 755.02002 & 3.38534029 & 14.7412389 & 1.79534528 & 14.67825 & 4766 & 4079.70284 & 70.1 & 70.4 & 69.3 & 69.7 & 0.000939 \\
\hline 760.02002 & 3.39159299 & 14.7419885 & 1.79597072 & 14.67825 & 4776 & 4083.92408 & 70.1 & 70.4 & 69.2 & 69.6 & 0.00094 \\
\hline 765.05002 & 3.39159299 & 14.7404893 & 1.79534528 & 14.6775 & 4776 & 4102.29887 & 70.1 & 70.4 & 69.3 & 69.7 & 0.00094 \\
\hline 770.02002 & 3.42348176 & 14.7397397 & 1.79471984 & 14.6775 & 4776 & 4115.21088 & 70.2 & 70.4 & 69.3 & 69.7 & 0.00094 \\
\hline 775.02002 & 3.39534461 & 14.7412389 & 1.79534528 & 14.6775 & 4776 & 4108.25826 & 70.1 & 70.4 & 69.3 & 69.7 & 0.000941 \\
\hline 780.05002 & 3.39596988 & 14.7419885 & 1.79597072 & 14.67825 & 4776 & 4085.91054 & 70.2 & 70,3 & 69.3 & 69.7 & 0.00094 \\
\hline 785.02002 & 3.39159299 & 14.7419885 & 1.79534528 & 14.67825 & 4776 & 4075.4816 & 70.3 & 70.4 & 69.4 & 69.7 & 0.00094 \\
\hline 790.02002 & 3.38659083 & 14.7419885 & 1.79534528 & 14.6775 & 4776 & 4082.43423 & 70.3 & 70.4 & 69.3 & 69.7 & 0.00094 \\
\hline 795.03 & 3.40284765 & 14.7419885 & 1.79534528 & 14.67825 & 4776 & 4104.03702 & 70.3 & 70.4 & 69.3 & 69.7 & 0.000942 \\
\hline 800.02002 & 3.40034677 & 14.7397397 & 1.79659616 & 14.67825 & 4776 & 4104.53364 & 70.3 & 70.4 & 69.4 & 69.7 & 0.00094 \\
\hline 805.02002 & 3.40220258 & 14.7404893 & 1.79597072 & 14.67825 & 4776.25 & 4090.13178 & 70.3 & 70.4 & 69.3 & 69.7 & 0.000941 \\
\hline
\end{tabular}




\begin{tabular}{|c|c|c|c|c|c|c|c|c|c|c|c|}
\hline SECONDS & MAIN DUCT & MAIN DUCT & GAS INJECT & GAS INJECT & NEWPORT & SMC & AMBIENT & GAS INJECT & MAIN DUCT & WEC CELL & WEC CELL \\
\hline & DIFF (IN H2O) & ABS (PSIA) & DIFF (IN H2O) & ABS (PSIA) & (PPM) & (PPM) & TEMP.(F) & TEMP. (F) & TEMP. (F) & TEMP. (F) & (VOLTS) \\
\hline 0.0200195 & 3.40222258 & 14.7419885 & 0.612388064 & 14.68425 & 111.225 & -3.349758 & 70.5 & 69.8 & 69.3 & 69.5 & 0.000247 \\
\hline 810.05002 & 3.39409407 & 14.7419885 & 1.79471984 & 14.67825 & 4776 & 4094.84953 & 70.4 & 70.4 & 69.3 & 69.7 & 0.00094 \\
\hline 815.02002 & 3.38909191 & 14.7419885 & 1.79471984 & 14.67825 & 4776 & 4105.77518 & 70.4 & 70.4 & 69.3 & 69.7 & 0.000939 \\
\hline 820.02002 & 3.40034677 & 14.7412389 & 1.79471984 & 14.6775 & 4766 & 4108.25826 & 70.3 & 70.4 & 69.3 & 69.7 & 0.00094 \\
\hline 825.03 & 3.39847096 & 14.7419885 & 1.79471984 & 14.6775 & 4765.75 & 4103.78872 & 70.4 & 70.4 & 69.4 & 69.7 & 0.00094 \\
\hline 830.02002 & 3.40284785 & 14.7397397 & 1.79534528 & 14.67825 & 4766 & 4102.79548 & 70.3 & 70.4 & 69.3 & 69.7 & 0.000939 \\
\hline 835.02002 & 3.38534029 & 14.7404893 & 1.79534528 & 14.6775 & 4776.25 & 4083.42746 & 70.4 & 70.4 & 69.3 & 69.7 & 0.00094 \\
\hline 840.04001 & 3.402222258 & 14.7404893 & 1.79471984 & 14.67825 & 4776 & 4077.96468 & 70.4 & 70.4 & 69.3 & 69.7 & 0.000941 \\
\hline 845.020002 & 3.39409407 & 14.7397397 & 1.79534528 & 14.6775 & 4776 & 4090.62839 & 70.4 & 70.4 & 69.3 & 69.7 & 0.00094 \\
\hline 850.02002 & 3.39596988 & 14,7412389 & 1.79597072 & 14.67825. & 4776 & 4084.91731 & 70.4 & 70.4 & 69.3 & 69.7 & 0.00094 \\
\hline 855.03 & 3.40972582 & 14.7419885 & 1.79534528 & 14.6775 & 4776 & 4088.39362 & 70.4 & 70.4 & 69.3 & 69.7 & 0.000041 \\
\hline 660.02002 & 3.40785001 & 14.7404893 & 1.79471984 & 14.6775 & 4776 & 4075.4816 & 70.4 & 70.4 & 69.3 & 69.7 & 0.00094 \\
\hline 865.02002 & 3.40659947 & 14.7412389 & 1.79534528 & 14.67675 & 4776 & 4060,08651 & 70.5 & 70.4 & 69.3 & 69.7 & 0.000939 \\
\hline 870.04001 & 3.38846664 & 14.7404893 & 1.79471984 & 14.6775 & 4776 & 4041.45341 & 70.4 & 70.4 & 69.3 & 69.7 & 0.000941 \\
\hline 875.02002 & 3.39847096 & 14.7404893 & 1.79597072 & 14.67675 & 4776 & 4055.12035 & 70.4 & 70.4 & 69.4 & 69.7 & 0.000942 \\
\hline 880.02002 & 3.38909191 & 14.7412389 & 1.79471984 & 14.67675 & 4776 & 4080.19946 & 70.4 & 70.4 & 69.4 & 69.7 & 0.000041 \\
\hline 885.08002 & 3.38346448 & 14.7404893 & 1.79346896 & 14.6775 & 4776 & 4087.40039 & 70.4 & 70.4 & 69.4 & 69.7 & 0.000942 \\
\hline 890.04001 & 3.39221826 & 14.7404893 & 1.79471984 & 14.6775 & 4776 & 4084.17238 & 70.4 & 70.4 & 69.3 & 69.6 & 0.000941 \\
\hline 895.02002 & 3.40409839 & 14.7412389 & 1.79534528 & 14.6775 & 4776 & 4071.01206 & 70.3 & 70.4 & 69.3 & 69.6 & 0.00094 \\
\hline 900.06 & 3.39534461 & 14.7412389 & 1.79534528 & 14.67675 & 4776 & 4071.75698 & 70.3 & 70.4 & 69.3 & 69.7 & 0.000941 \\
\hline 905.02002 & 3.38909191 & 14.7419885 & 1.79471984 & 14.676 & 4776 & 4083.92408 & 70.4 & 70.4 & 69.4 & 69.7 & 0.00094 \\
\hline 910.02002 & 3.3872161 & 14.7419885 & 1.79534528 & 14.67675 & 4776 & 4081.93761 & 70.4 & 70.4 & 69.3 & 69.6 & 0.000941 \\
\hline 915.07001 & 3.39221826 & 14.7412389 & 1.79346896 & 14.6775 & 4775.75 & 4069.52201 & 70.4 & 70.4 & 69.2 & 69.7 & 0.000941 \\
\hline 920.02002 & 3.38971718 & 14.7419885 & 1.7940944 & 14.6775 & 4776 & \begin{tabular}{|l|}
4065.54928 \\
\end{tabular} & 70.4 & 70.4 & 69.4 & 69.7 & 0.000941 \\
\hline
\end{tabular}




\begin{tabular}{|c|c|c|c|c|c|c|c|c|c|c|c|}
\hline \multicolumn{12}{|c|}{$\begin{array}{l}\text { TEST: EX202227 } 22 \text { Feb } 1997 \text { WEC-SPOOL } \\
\text { Measured Hydrogen Concentration }=4340 \mathrm{ppm}\end{array}$} \\
\hline & DIFF (IN H2O) & ABS (PSIA) & DIFF (IN H2O) & ABS (PSIA) & (PPM) & (PPM) & TEMP.(F) & TEMP. (F) & TEMP. (F) & TEMP. (F) & (VOLTS) \\
\hline 0.0099793 & 3.43848824 & 14.73974 & 0.542526 & 14.6625 & 120.95 & -3.59807 & 70.3 & 69.6 & 69.5 & 69.5 & 0.00025 \\
\hline 5.019989 & 3.38534029 & 14.73899 & 1.786589 & 14.6775 & 121.55 & 3.47391 & 70.3 & 69.7 & 69.4 & 69.5 & 0.000249 \\
\hline 10.019989 & 3.44661675 & 14.73899 & 1.789716 & 14.6775 & 191.525 & 1049.352 & 70.3 & 69.8 & 69.6 & 69.5 & 0.000278 \\
\hline 15.019989 & 3.39847096 & 14.73899 & 1.790342 & 14.6775 & 601.5 & 2403.624 & 70.4 & 69.8 & 69.5 & 69.5 & 0.000352 \\
\hline 20.019989 & 3.39221826 & 14.73899 & 1.791593 & 14.67675 & 1188.75 & 3136.132 & 70.4 & 69.8 & 69.5 & 69.5 & 0.00044 \\
\hline 25.019989 & 3,3934688 & 14.74049 & 1.791593 & 14.6775 & 1773 & 3506.36 & 70.4 & 69.8 & 69.6 & 69.5 & 0.000523 \\
\hline 30.019989 & 3.39221826 & 14.73974 & 1.793469 & 14.6775 & 2280 & 3688.866 & 70.4 & 69.7 & 69.5 & 69.5 & 0.000593 \\
\hline 35.019989 & 3.38596556 & 14.73899 & 1.793469 & 14.67825 & 2689.5 & 3764.848 & 70.5 & 69.7 & 69.4 & 69.5 & 0.000651 \\
\hline 40.019989 & 3.40284785 & 14.73974 & 1.794094 & 14.6775 & 3012.5 & 3824.194 & 70.5 & 69.7 & 69.4 & 69.5 & 0.000697 \\
\hline 45.019989 & 3.42098068 & 14.73899 & 1.794094 & 14.67825 & 3304 & 3856.722 & 70.6 & 69.8 & 69.5 & 69.5 & 0.000735 \\
\hline 50.019989 & 3.41660379 & 14.74049 & 1.793469 & 14.6775 & 3524.25 & 3877.58 & 70.5 & 69.8 & 69.6 & 69.5 & 0.000766 \\
\hline 55.019989 & 3.39847096 & 14.73899 & 1.795345 & 14.67675 & 3723.75 & 3900.424 & 70.5 & 69.8 & 69.5 & 69.5 & 0.00079 \\
\hline 60.019989 & 3.39847096 & 14.73824 & 1.795345 & 14.67675 & 3834.5 & 3912.592 & 70.5 & 69.8 & 69.5 & 69.5 & 0.000811 \\
\hline 65.019989 & 3.41722906 & 14.73899 & 1.795971 & 14.6775 & 3968.25 & 3929.973 & 70.4 & 69.8 & 69.5 & 69.6 & 0.000827 \\
\hline 70.019989 & 3.40159731 & 14.73899 & 1.795345 & 14.67825 & 4044 & 3938.167 & 70.3 & 69.7 & 69.5 & 69.5 & 0.00084 \\
\hline 75.019989 & 3.40409839 & 14.74049 & 1.795345 & 14.6775 & 4134.5 & 3957.287 & 70.4 & 69.8 & 69.4 & 69.5 & 0.000852 \\
\hline 80.019989 & 3.39847096 & 14.73974 & 1.795971 & 14.6775 & 4194.75 & 3957.784 & 70.3 & 69.8 & 69.4 & 69.6 & 0.000861 \\
\hline 85.019989 & 3.38784137 & 14.73974 & 1.795345 & 14.67675 & 4254.5 & 3953.314 & 70.4 & 69.8 & 69.5 & 69.5 & 0.000869 \\
\hline 90.019989 & 3.39847096 & 14.73974 & 1.795971 & 14,676 & 4314.75 & 3960.267 & 70.5 & 69.8 & 69.4 & 69.6 & 0.000876 \\
\hline 95.019989 & 3.39409407 & 14.73974 & 1.795345 & 14.6775 & 4344.75 & 3969.702 & 70.4 & 69.8 & 69.4 & 69.5 & 0.000882 \\
\hline 100.03 & 3.402222258 & 14.74049 & 1.795345 & 14.6775 & 4374.5 & 3966.723 & 70.3 & 69.8 & 69.3 & 69.5 & 0.000885 \\
\hline 105.01999 & 3.3997215 & 14.73899 & 1.797847 & 14.6775 & 4415 & 3974.172 & 70.4 & 69.8 & 69.5 & 69.5 & 0.00089 \\
\hline 110.01999 & 3.40847528 & 14.73974 & 1.795345 & 14.6775 & 4435.25 & 3987.332 & 70.4 & 69.8 & 69.5 & 6.5 & 0.000893 \\
\hline 115.22 & 3.39847096 & 14.73899 & 1.795971 & 14.6775 & 4465.25 & 4003.721 & 70.5 & 69.8 & 69.5 & 69.5 & 0.000897 \\
\hline 120.01999 & 3.40409839 & 14.73974 & 1.795971 & 14.6775 & $\begin{array}{r}4485 \\
\end{array}$ & 4013.156 & 70.5 & 69.8 & 69.5 & 69.5 & 0.000898 \\
\hline 125.13001 & 3.402222258 & 14.73899 & 1.795345 & 14.67825 & 4495.25 & 4008.19 & 70.6 & 69.8 & 69.5 & 69.5 & 0.000901 \\
\hline 130.01999 & 3.402222258 & 14.73824 & 1.797222 & 14.67825 & 4515.25 & 4000,493 & 70.6 & 69.8 & 69.5 & 69.5 & 0.000903 \\
\hline 135.06 & 3.40284785 & 14.73749 & 1.797222 & 14.6775 & 4525.5 & 4009.68 & 70.6 & 69.8 & 69.4 & 69.5 & 0.000904 \\
\hline 140.01999 & 3.39534461 & 14.73899 & 1.797222 & 14.6775 & 4535.25 & 4021.847 & 70.6 & 69.9 & 69.4 & 69.5 & 0.000006 \\
\hline 145.01999 & 3.39221826 & 14.73824 & 1.795345 & 14.67675 & 4545.25 & 4020.109 & 70.6 & 69.9 & 69.4 & 69.5 & 0.000908 \\
\hline
\end{tabular}




\begin{tabular}{|c|c|c|c|c|c|c|c|c|c|c|c|}
\hline SECONDS & MAIN DUCT & MAIN DUCT & GAS INJECT & GAS INJECT & NEWPORT & SMC & AMBIENT & GAS INJECT & MAIN DUCT & WEC CELL & WEC CELL \\
\hline & DIFF (IN H2O) & ABS (PS|A) & DIFF (IN H2O) & ABS (PSIA) & (PPM) & $(\mathrm{PPM})$ & TEMP.(F) & TEMP. (F) & TEMP. (F) & TEMP. (F) & (VOLTS) \\
\hline 0.0099793 & 3.43848824 & 14.73974 & 0.542526 & 14.6625 & 120.95 & -3.59807 & 70.3 & 69.6 & 69.5 & 69.5 & 0.00025 \\
\hline 150.01999 & 3.40847528 & 14.73899 & 1.797222 & 14.6775 & 4555 & 4025.075 & 70.6 & 69.8 & 69,4 & 69.5 & 0.000908 \\
\hline 155.01999 & 3.39847096 & 14.73899 & 1.797222 & 14.6775 & 4565.5 & 4010.425 & 70.6 & 69.8 & 69.5 & 69.6 & 0.00091 \\
\hline 160.01999 & 3.40472366 & 14.73974 & 1.795345 & 14.67675 & 4575.5 & 4014.398 & 70.6 & 69.9 & 69.5 & 69.5 & 0.000912 \\
\hline 165.03 & 3.39847096 & 14.73899 & 1.796596 & 14.67675 & 4585.5 & 4002.479 & 70.6 & 69.8 & 69.3 & 69.6 & 0.000914 \\
\hline 170.01999 & 3.39596988 & 14.74049 & 1.797847 & 14.6775 & 4595.25 & 4007.197 & 70.7 & 69.8 & 69.5 & 69.5 & 0.000915 \\
\hline 175.06 & 3.40534893 & 14.73899 & 1.795345 & 14.6775 & 4605.75 & 4017.626 & 70.7 & 69.9 & 69.5 & 69.6 & 0.000916 \\
\hline 180.03 & 3.40222258 & 14.73899 & 1.795971 & 14.67675 & 4605.75 & 4039.477 & 70.7 & 69.9 & 69.4 & 69.6 & 0.000918 \\
\hline t85.01999 & 3.40034677 & 14.74049 & 1.796596 & 14.6775 & 4615.5 & 4047.671 & 70.6 & 69.9 & 69.5 & 69.5 & 0.000918 \\
\hline 190.01999 & 3.40722474 & 14.74049 & 1.797222 & 14.6775 & 4625.5 & 4044.94 & 70.6 & 69.9 & 69.4 & 69.5 & 0.000918 \\
\hline 195.07001 & 3.3934688 & 14.73749 & 1.79472 & 14.67675 & 4625.75 & 4058.348 & 70.6 & 69.9 & 69.5 & 69.5 & 0.000919 \\
\hline 200.01999 & 3.39534461 & 14.73824 & 1.795345 & 14.6775 & 4635.25 & 4059.838 & 70.5 & 69.9 & 69.5 & 69.5 & 0.00092 \\
\hline 205.06 & 3.40659947 & 14.73899 & 1.795345 & 14.6775 & 4635.75 & 4052.637 & 70.6 & 69.9 & 69.5 & 69.5 & 0.000921 \\
\hline 210.01999 & 3.39409407 & 14.73899 & 1.795345 & 14.67675 & 4645.25 & 4050.899 & 70.5 & 69.9 & 69.6 & 69.6 & 0.000922 \\
\hline 215.01990 & 3.38971718 & 14.73974 & 1.795345 & 14.67675 & 4645.75 & 4054.872 & 70.5 & 69.9 & 69.5 & 69.6 & 0.000922 \\
\hline 220.01990 & 3.39409407 & 14.73824 & 1.795345 & 14.6775 & 4645.75 & 4061.328 & 70.5 & 69.9 & 69.5 & 69.6 & 0.000923 \\
\hline 225.01999 & 3.39784569 & 14.73899 & 1.795345 & 14.6775 & 4655.75 & 4070.267 & 70.5 & 69.9 & 69.5 & 69.6 & 0.000924 \\
\hline 230.01999 & 3.39784569 & 14.73824 & 1.795345 & 14.67675 & 4665.5 & 4073.247 & 70.6 & 69.9 & 69.5 & 69.6 & 0.000924 \\
\hline 235.01999 & 3.4247323 & 14.73749 & 1.795971 & 14.67675 & 4665.5 & 4071.757 & 70.7 & 69.9 & 69.5 & 69.6 & 0.000926 \\
\hline 240.07001 & 3.40222258 & 14.73824 & 1.795971 & 14.6775 & 4675.25 & 4066.543 & 70.7 & 70 & 69.4 & 69.6 & 0.000926 \\
\hline 245.03 & 3.40284785 & 14.73974 & 1.795345 & 14.6775 & 4675.75 & 4049.906 & 70.7 & 70 & 69.4 & 69.6 & 0.000926 \\
\hline 250.10999 & 3.40222258 & 14.73899 & 1.795345 & 14.67675 & 4675.5 & 4051.892 & 70.6 & 70 & 69.4 & 69.6 & 0.000927 \\
\hline 255.01999 & 3.39221826 & 14.73899 & 1.79472 & 14.67675 & 4675.75 & 4067.287 & 70.6 & 70 & 69.4 & 69.6 & 0.000927 \\
\hline 260.01999 & 3.40910055 & 14.73974 & 1.79472 & 14.6775 & 4685.25 & 4072.254 & 70.6 & 70 & 69.5 & 69.6 & 0.000928 \\
\hline 265.39999 & 3.39034245 & 14.73974 & 1.795345 & 14.6775 & 4685 & 4069.522 & 70.6 & 70 & 69.4 & 69.6 & 0.000928 \\
\hline 270.01999 & 3.39722042 & 14.73899 & 1.795345 & 14.67675 & 4685.75 & 4071.26 & 70.5 & 70 & 69.4 & 69.5 & 0.000926 \\
\hline 275.01999 & 3.39159299 & 14.73899 & 1.794094 & 14.6775 & 4685.5 & 4073.247 & 70.5 & 70 & 69.4 & 69.6 & 0.000927 \\
\hline 280.01999 & 3.41660379 & 14.73824 & 1.795345 & 14.6775 & 4685.5 & 4074.985 & 70.5 & 70 & 69.4 & 69.6 & 0.000927 \\
\hline 285.01999 & 3.39034245 & 14.74049 & 1.795345 & 14.67575 & 4686 & 4070.019 & 70.5 & 70 & 69.5 & 69.6 & 0.000928 \\
\hline 290.01999 & 3.38659083 & 14.73974 & 1.795345 & 14.676 & 4685.25 & 4064.308 & 70.5 & 70 & 69.4 & 69.6 & 0.000928 \\
\hline 295.01999 & 3.38971718 & 14.73974 & 1.793469 & 14.6775 & 4695.25 & 4064.804 & 70.4 & 70 & 69.4 & 69.6 & 0.000929 \\
\hline 300.01999 & 3.39596988 & 14.74124 & 1.792844 & 14.6775 & 4696 & 4068.529 & 70.4 & 70 & 69.4 & 69.6 & 0.000929 \\
\hline 305.03 & 3.39722042 & 14.73899 & 1.792844 & 14.67675 & 4695.75 & 4069.771 & 70.4 & 70.1 & 69.4 & 69.6 & 0.000929 \\
\hline 310.01999 & 3.40847528 & 14.74049 & 1.792844 & 14.676 & 4695.25 & 4076.723 & 70.4 & 70.1 & 69.4 & 69.6 & 0.000929 \\
\hline
\end{tabular}




\begin{tabular}{|c|c|c|c|c|c|c|c|c|c|c|c|}
\hline SECONDS & MAIN DUCT & MAIN DUCT & GAS INJECT & GAS INJECT & NEWPORT & SMC & AMBIENT & GAS INJECT & MAIN DUCT & WEC CELL & WEC CELLL \\
\hline & DIFF (IN H2O) & ABS (PSIA) & DIFF (IN H2O) & ABS (PSIA) & (PPM) & (PPM) & TEMP.(F) & TEMP. (F) & TEMP. (F) & TEMP. (F) & (VOLTS) \\
\hline 0.0099793 & 3.43848824 & 14.73974 & 0.542526 & 14.6625 & 120.95 & -3.59807 & 70.3 & 69.6 & 69.5 & 69.5 & 0,00025 \\
\hline 315.03 & 3.42223122 & 14.73899 & 1.792218 & 14.67675 & 4695.75 & 4077.468 & 70.4 & 70 & 69.4 & 69.6 & 0.00093 \\
\hline 320.01999 & 3.3872161 & 14.73899 & 1.792218 & 14.6775 & 4705.25 & 4070.019 & 70.5 & 70.1 & 69.4 & 69.6 & 0.00093 \\
\hline 325.01999 & 3.39847096 & 14.73899 & 1.792844 & 14.6775 & 4706 & 4068.529 & 70.4 & 70.1 & 69.4 & 69.6 & 0.000931 \\
\hline 330.01999 & 3.39722042 & 14.73899 & 1.792844 & 14.67675 & 4705.25 & 4064.556 & 70.4 & 70.1 & 69.5 & 69.6 & 0.000931 \\
\hline 335.01999 & 3.3872161 & 14.73824 & 1.793469 & 14.67825 & 4705.5 & 4063.563 & 70.4 & 70.1 & 69.4 & $@ .6$ & 0.000931 \\
\hline 340.01999 & 3.39596988 & 14.73824 & 1.793469 & 14.6775 & 4705.75 & 4077.468 & 70.4 & 70.1 & 69.4 & 69.6 & 0.000931 \\
\hline 345.01999 & 3.39534461 & 14.73899 & 1.792844 & 14.6775 & 4716 & 4075.482 & 70.5 & 70.1 & 69.4 & 69.6 & 0.000931 \\
\hline 350.01999 & 3.39784569 & 14.73824 & 1.792844 & 14.67675 & 4715.75 & 4061.08 & 70.5 & 70.1 & 69.5 & 69.6 & 0.000932 \\
\hline 355.01999 & 3.42660811 & 14.73899 & 1.792218 & 14.67825 & 4716 & 4063.315 & 70.5 & 70.1 & 69.5 & 69.6 & 0.000932 \\
\hline 360.01999 & 3.402222258 & 14.73824 & 1.793469 & 14.6775 & 4716 & 4055.12 & 70.4 & 70.1 & 69.4 & 69.6 & 0.000932 \\
\hline 365.01999 & 3.39471934 & 14.73974 & 1.794094 & 14.6775 & 4715.5 & 4046.678 & 70.5 & 70.1 & 69.3 & 69.6 & 0.000932 \\
\hline 370.01999 & 3.40910055 & 14.73824 & 1.792844 & 14.6775 & 4715.75 & 4057.107 & 70.5 & 70.1 & 69.4 & 69.6 & 0.000932 \\
\hline 375.01999 & 3.40472366 & 14.73974 & 1.793469 & 14.6775 & 4716 & 4057.852 & 70.5 & 70.1 & 69.6 & 69.7 & 0.000932 \\
\hline 380.01999 & 3.402222258 & 14.73899 & 1.794094 & 14.67825 & 4715.75 & 4073.743 & 70.6 & 70.1 & 69.6 & 69.6 & 0.000933 \\
\hline 385.01999 & 3.39221826 & 14.74049 & 1.793469 & 14.6775 & 4726 & 4087.649 & 70.6 & 70.1 & 69.5 & 69.6 & 0.000933 \\
\hline 390.01999 & 3.40534893 & 14.73974 & 1.793469 & 14.67825 & 4726 & 4103.789 & 70.7 & 70.1 & 69.4 & 69.6 & 0.000932 \\
\hline 395.01999 & 3.39221826 & 14.73899 & 1.792844 & 14.67825 & 4725.75 & 4098.326 & 70.6 & 70.1 & 69.4 & 69.6 & 0.000933 \\
\hline 400.09 & 3.40284785 & 14.73974 & 1.794094 & 14.6775 & 4726 & 4096.091 & 70.6 & 70.1 & 69.5 & 69.6 & 0.000932 \\
\hline 405.01999 & 3.41973014 & 14.73974 & 1.792844 & 14.67825 & 4726 & 4095.098 & 70.6 & 70.2 & 69.5 & 69.6 & 0.000933 \\
\hline 410.09 & 3.39534461 & 14.74049 & 1.792844 & 14.67675 & 4725.75 & 4088.642 & 70.6 & 70.1 & 69.6 & 69.6 & 0.000932 \\
\hline 415.01999 & 3.40222258 & 14.73974 & 1.793469 & 14.67675 & 4726 & 4088.89 & 70.6 & 70.2 & 69.5 & 69.6 & 0.000933 \\
\hline 420.10999 & 3.39784569 & 14.73974 & 1.792218 & 14.6775 & 4725.75 & 4090.38 & 70.6 & 70.1 & 69.5 & 69.6 & 0.000933 \\
\hline 425.01999 & 3.40222258 & 14.73974 & 1.793469 & 14.67675 & 4725.5 & 4099.319 & 70.5 & 70.2 & 69.6 & 69.6 & 0.000934 \\
\hline 430.01999 & 3.43786297 & 14.74049 & 1.792844 & 14.67825 & 4725.75 & 4086.407 & 70.4 & 70.2 & 69.6 & 69.6 & 0.000933 \\
\hline 435.01999 & 3.39590988 & 14.73824 & 1.793469 & 14.6775 & 4726 & 4066.046 & 70.5 & 70.2 & 69.6 & 69.6 & 0.000934 \\
\hline 440.01999 & 3.39847096 & 14.73899 & 1.793469 & 14.6775 & 4725.5 & 4046.678 & 70.5 & 70.2 & 69.5 & 69.6 & 0.000934 \\
\hline 445.01999 & 3.39534461 & 14.73824 & 1.792218 & 14.67825 & 4726 & 4045.436 & 70.5 & 70.2 & 69.6 & 69.6 & 0.000034 \\
\hline 450.01999 & 3.39596988 & 14.73824 & 1.790967 & 14.6775 & 4736 & 4071.757 & 70.4 & 70.2 & 69.6 & 69.6 & 0.000934 \\
\hline 455.01999 & 3.40847528 & 14.73974 & 1.791593 & 14.6775 & 4735.5 & 4067.039 & 70.4 & 70.2 & 69.5 & 69.6 & 0.000934 \\
\hline 460.01999 & 3.38784137 & 14.74124 & 1.792844 & 14.6775 & 4736 & 4046.678 & 70.4 & 70.2 & 69.5 & 69.6 & 0.000934 \\
\hline 465.01999 & 3.39284353 & 14.73974 & 1.791593 & 14.6775 & 4736 & 4040.47 & 70.3 & 70.2 & 69.5 & 69.6 & 0.000935 \\
\hline 470.01999 & 3.4059742 & 14.73699 & 1.790342 & 14.67825 & 4735.5 & 4055.369 & 70.3 & 70.2 & 69.6 & 69.6 & 0.000936 \\
\hline 475.01999 & 3.39784569 & 14.73899 & 1.791593 & 14.6745 & 4736 & 4063.315 & 70.4 & 70.2 & 69.6 & 69.6 & 0.000935 \\
\hline
\end{tabular}




\begin{tabular}{|c|c|c|c|c|c|c|c|c|c|c|c|}
\hline SECONDS & MAIN DUCT & MAIN DUCT & GAS INJECT & GAS INJECT & NEWPORT & SMC & AMBIENT & GAS INJECT & MAIN DUCT & WEC CELL & WEC CELL \\
\hline & DIFF (IN H2O) & ABS (PS|A) & DIFF (IN H2O) & $A B S$ (PSIA) & (PPM) & (PPM) & TEMP.(F) & TEMP. (F) & TEMP. (F) & TEMP. $(F)$ & (VOLTS) \\
\hline 0.0099793 & 3.43848824 & 14.73974 & 0.542526 & 14.6625 & 120.95 & -3.59807 & 70.3 & 69.6 & 69.5 & 69.5 & 0.00025 \\
\hline 480.01999 & 3,41785433 & 14.73974 & 1.791593 & 14.67675 & 4745.75 & 4079.455 & 70.4 & 70.2 & 69.5 & 69.6 & 0.000936 \\
\hline 485.01999 & 3.40034677 & 14.73899 & 1.790967 & 14.67675 & 4735.5 & 4072.999 & 70.4 & 70.2 & 69.5 & 69.6 & 0.000935 \\
\hline 490.01999 & 3.39409407 & 14.73974 & 1.791593 & 14.67675 & 4745.75 & 4068.777 & 70.5 & 70.3 & 69.5 & 69.6 & 0.000936 \\
\hline 495.01999 & 3.39221826 & 14.73974 & 1.790967 & 14.67675 & 4746 & 4050.899 & 70.5 & 70.3 & 69.6 & 69.6 & 0.000936 \\
\hline 500.01999 & 3.38909191 & 14.73899 & 1.791593 & 14.6775 & 4745.5 & 4050.651 & 70.4 & 70.3 & 69.6 & 69.6 & 0.000935 \\
\hline 505.01999 & 3.39409407 & 14.73899 & 1.790342 & 14.67675 & 4745.75 & 4065.798 & 70.4 & 70.2 & 69.5 & 69.6 & 0.000936 \\
\hline 510.07001 & 3.39722042 & 14.74049 & 1.790342 & 14.676 & 4745.25 & 4079.951 & 70.5 & 70.3 & 69.5 & 69.6 & 0.000935 \\
\hline 515.03998 & 3.39847096 & 14.73899 & 1.790967 & 14.67675 & 4746 & 4072.005 & 70.6 & 70.3 & 69.5 & 69.6 & 0.000936 \\
\hline 520.01999 & 3.39409407 & 14.73899 & 1.789716 & 14.676 & 4746 & 4071.509 & 70.6 & 70.3 & 69.6 & 69.6 & 0.000936 \\
\hline 525.07001 & 3.40659947 & 14.73974 & 1.791593 & 14.67525 & 4746 & 4072.254 & 70.6 & 70.3 & 69.7 & 69.6 & 0.000936 \\
\hline 530.01999 & 3.40409839 & 14.73899 & 1.791593 & 14.67675 & 4745.5 & 4083.179 & 70.6 & 70.3 & 69.6 & 69.6 & 0.000936 \\
\hline 535.01999 & 3.39159299 & 14.73974 & 1.790967 & 14.67675 & 4745.75 & 4062.321 & 70.7 & 70.3 & 69.5 & 69.6 & 0.000936 \\
\hline 540.03 & 3.40847528 & 14.73749 & 1.789091 & 14.67675 & 4746 & 4061.825 & 70.7 & 70.3 & 69.6 & 69.6 & 0.000936 \\
\hline 545.09 & 3.39659515 & 14.74124 & 1.791593 & 14.6775 & 4745.5 & 4066.791 & 70.7 & 70.3 & 69.6 & 69.7 & 0.000336 \\
\hline 550.01999 & 3.38534029 & 14.73824 & 1.790967 & 14.6775 & 4745.75 & 4070.515 & 70.7 & 70.3 & 69.5 & 69.6 & 0.000936 \\
\hline 555.01999 & 3.39096772 & 14.73749 & 1.789716 & 14.6775 & 4746 & 4052.141 & 70.6 & 70.3 & 69.5 & 69.6 & 0.000936 \\
\hline 560.01999 & 3.40659947 & 14.73899 & 1.790957 & 14.6745 & 4755.5 & 4058.597 & 70.6 & 70.3 & 69.5 & 69.6 & 0.000936 \\
\hline 565.01999 & 3,41097636 & 14.73899 & 1.789091 & 14.67675 & 4756 & 4071.757 & 70.6 & 70.3 & 69.5 & 69.7 & 0.000937 \\
\hline 570.03998 & 3.39159299 & 14.73824 & 1.789091 & 14.676 & 4755.75 & 4069.771 & 70.6 & 70.3 & 69.5 & 69.6 & 0.000938 \\
\hline 575.01999 & 3.39596988 & 14.73899 & 1.791593 & 14.67675 & 4755.5 & 4057.852 & 70.6 & 70.3 & 69.5 & 69.6 & 0.000937 \\
\hline 580.01999 & 3.41722906 & 14.73974 & 1.789716 & 14.6775 & 4756 & 4050.154 & 70.6 & 70.3 & 69.5 & 69.6 & 0.000937 \\
\hline 585.07001 & 3.40284785 & 14.73974 & 1.788465 & 14.6775 & 4755.5 & 4051.644 & 70.6 & 70.3 & 69.5 & 69.6 & 0.000537 \\
\hline 590.07001 & 3.4059742 & 14.74049 & 1.789716 & 14.6775 & 4756 & 4066.046 & 70.6 & 70.3 & 69.6 & 69.6 & 0.000937 \\
\hline 595.01999 & 3.40659947 & 14.73974 & 1.789001 & 14.6775 & 4756 & 4070.764 & 70.6 & 70.3 & 69.5 & 69.7 & 0.000938 \\
\hline 600.01999 & 3.43223554 & 14.73899 & 1.789091 & 14.67675 & 4756 & 4060.583 & 70.6 & 70.3 & 69.5 & 69.7 & 0.000938 \\
\hline 605.01999 & 3.40472366 & 14.73824 & 1.789091 & 14.67825 & 4755.75 & 4064.308 & 70.6 & 70.3 & 69.5 & 69.7 & 0.000937 \\
\hline 610.01999 & 3.40222258 & 14.74049 & 1.788465 & 14.67675 & 4756 & 4051.576 & 70.6 & 70.3 & 69.5 & 69.7 & 0.000937 \\
\hline 615.04999 & 3.38471502 & 14.74049 & 1.788465 & 14.6775 & 4756 & 4066.791 & 70.6 & 70.3 & 69.5 & 69.7 & 0.000939 \\
\hline 620.01999 & 3.3997215 & 14.73974 & 1.788465 & 14.67675 & 4755.5 & 4051.396 & 70.6 & 70.3 & 69.5 & 69.6 & 0.000839 \\
\hline 625.01999 & 3.38534029 & 14.73974 & 1.788465 & 14.6775 & 4756 & 4048.913 & 70.6 & 70.4 & 69.5 & 69.7 & 0.000938 \\
\hline 630.04999 & 3.39784569 & 14.74049 & 1.788465 & 14.6775 & 4756 & 4041.96 & 70.6 & 70.3 & 69.4 & 69.7 & 0.000939 \\
\hline 635.01999 & 3.3934688 & 14.73899 & 1.789091 & 14.6775 & 4755.75 & 4050.154 & 70.6 & 70.4 & 69.5 & 69.7 & 0.000938 \\
\hline 640.01999 & 3.38408975 & 14.74049 & 1.78784 & 14.67675 & 4755.75 & 4056.61 & 70.6 & 70.4 & 69.3 & 69.7 & 0.000939 \\
\hline
\end{tabular}




\begin{tabular}{|c|c|c|c|c|c|c|c|c|c|c|c|}
\hline SECONDS I & MAIN DUCT & MAIN DUCT & GAS INJECT & GAS INJECT & NEWPORT & SMC & AMBIENT & GAS INJECT & MAIN DUCT & WEC CELL & WEC CELL \\
\hline & DIFF (IN H2O) & ABS (PSIA) & DIFF (IN H2O) & ABS (PSIA) & (PPM) & (PPM) & TEMP.(F) & TEMP. (F) & TEMP. (F) & TEMP. (F) & NOLTS) \\
\hline 0.0099793 & 3.43848824 & 14.73974 & 0.542526 & 14.6625 & 120.95 & 3.59807 & 70.3 & 69.6 & 69.5 & 69.5 & 0.00025 \\
\hline 645.03 & 3.3872161 & 14.73974 & 1.788465 & 14.676 & 4756 & 4064.059 & 70.5 & 70.4 & 69.3 & 69.7 & 0.000938 \\
\hline 650.01999 & 3.3934688 & 14.74049 & 1.788465 & 14.6775 & 4755.75 & 4065.549 & 70.6 & 70.3 & 69.5 & 69.7 & 0.000938 \\
\hline 655.01999 & 3.4184796 & 14.73899 & 1.789091 & 14.67675 & 4765.75 & 4051.147 & 70.6 & 70.4 & 69.5 & 69.7 & 0.000938 \\
\hline 660.07001 & 3.40659947 & 14.73974 & 1.78784 & 14.67675 & 4766 & 4046.926 & 70.7 & 70.4 & 69,5 & 69.7 & 0.000939 \\
\hline 665.01999 & 3.39784569 & 14.73899 & 1.788465 & 14.6775 & 4765.75 & 4031.779 & 70.6 & 70.4 & 69.5 & 69.7 & 0.000938 \\
\hline 670.01999 & 3.40659947 & 14.73824 & 1.788465 & 14.6775 & 4756 & 4047.671 & 70.7 & 70.4 & 69.5 & 69.7 & 0.000938 \\
\hline 675.04999 & 3.39784569 & 14.73824 & 1.789091 & 14.67675 & 4756 & 4041.96 & 70.6 & 70.4 & 69.6 & 69.7 & 0.000938 \\
\hline 680.01999 & 3.39159299 & 14.73824 & 1.788465 & 14.6775 & 4756 & 4045.436 & 70.6 & 70.4 & 69.5 & 69.7 & 0.000939 \\
\hline 685.01999 & 3.39159299 & 14.74049 & 1.789091 & 14.6775 & 4762.75 & 4050.154 & 70.6 & 70.4 & 69.4 & 69.7 & 0.000938 \\
\hline 690.03998 & 3.38909191 & 14.74049 & 1.788465 & 14.6775 & 4766 & 4041.215 & 70.6 & 70.4 & 69.4 & 69.7 & 0.00094 \\
\hline 695.06 & 3.39784569 & 14.73899 & 1.788465 & 14.6775 & 4766 & 4033.518 & 70.6 & 70.4 & 69.4 & 69.7 & 0.000939 \\
\hline 700.01999 & 3.39722042 & 14.74049 & 1.789091 & 14.6775 & 4773.75 & 4040.47 & 70.6 & 70.4 & 69.5 & 69.7 & 0.00094 \\
\hline 705.01999 & 3.38471502 & 14.73824 & 1.786589 & 14.6775 & 4766 & 4037.739 & 70.5 & 70.4 & 69.4 & 69.7 & 0.000941 \\
\hline 710.03998 & 3.3934688 & 14.73899 & 1.788465 & 14.67675 & 4766 & 4058.597 & 70.5 & 70.4 & 69.4 & 69.7 & 0.00094 \\
\hline 715.01999 & 3.41973014 & 14.73824 & 1.789091 & 14.67675 & 4766 & 4079.703 & 70.5 & 70.5 & 69.5 & 69.7 & 0.00094 \\
\hline 720.04999 & 3.40785001 & 14.74049 & 1.787215 & 14,6745 & 4766 & 4088.394 & 70.5 & 70.4 & 69.4 & 69.7 & 0.000939 \\
\hline 725.01999 & 3.4184796 & 14.73899 & 1.788465 & 14.67675 & 4765.75 & 4079.703 & 70.5 & 70.4 & 69.4 & 69.7 & 0.00094 \\
\hline 730.01999 & 3.40222258 & 14.73749 & 1.786589 & 14.67675 & 4766 & 4074.24 & 70.6 & 70.4 & 69.5 & 69.7 & 0.000939 \\
\hline 735.01999 & 3.39847096 & 14.74049 & 1.787215 & 14.67675 & 4766 & 4066.046 & 70.6 & 70.4 & 69.5 & 69.7 & 0.000939 \\
\hline 740.01999 & 3.41910487 & 14.73899 & 1.786589 & 14.67675 & 4766 & 4066.791 & 70.6 & 70.5 & 69.4 & 69.7 & 0.000939 \\
\hline 745.01999 & 3.38596556 & 14.73899 & 1.787215 & 14.67375 & 4776 & 4061.825 & 70.6 & 70.5 & 69.6 & 69.7 & 0.000939 \\
\hline 750.03998 & 3.39847096 & 14.73899 & 1.787215 & 14.6775 & 4776 & 4053.134 & 70.7 & 70.4 & 69.5 & 69.7 & 0.00094 \\
\hline 755.01999 & 3.39159299 & 14.73899 & 1.787215 & 14.6775 & 4774.5 & 4048.913 & 70.7 & 70.5 & 69.6 & 69.7 & 0.000939 \\
\hline 760.01999 & 3.39784569 & 14.73974 & 1.785964 & 14.6775 & 4766 & 4062.321 & 70.7 & 70.5 & 69.4 & 69.6 & 0.000937 \\
\hline 765.03998 & 3.44474094 & 14.74049 & 1.786589 & 14.67675 & 4766.25 & 4052.389 & 70.7 & 70.5 & 69.5 & 69.7 & 0.000939 \\
\hline 770.01999 & 3.39596988 & 14.73899 & 1.787215 & 14.676 & 4765 & 4040.222 & 70.7 & 70.4 & 69.6 & 69.7 & 0.000939 \\
\hline 775.01999 & 3.40284785 & 14.74049 & 1.786589 & 14.6775 & 4766.25 & 4031.034 & 70.6 & 70.5 & 69.6 & 69.7 & 0,000938 \\
\hline 780.04999 & 3.40847528 & 14.73974 & 1.785964 & 14.6775 & 4766 & 4035.256 & 70.6 & 70.5 & 69.6 & 69.7 & 0.00094 \\
\hline 785.01999 & 3.41660379 & 14.73974 & 1.787215 & 14.6775 & 4765.75 & 4032.773 & 70.7 & 70.5 & 69.5 & 69.8 & 0.000941 \\
\hline 790.01999 & 3.40159731 & 14.73824 & 1.785964 & 14.67675 & 4765.75 & 4038.98 & 70.7 & 70.5 & 69.7 & 69.8 & 0.00094 \\
\hline 795.01999 & 3.39596988 & 14.73899 & 1.787215 & 14.67825 & 4766 & 4049.409 & 70.7 & 70.5 & 69.6 & 69.7 & 0.000939 \\
\hline 800.01999 & 3.42285649 & 14.73974 & 1.785964 & 14.6775 & 4767.5 & 4041.463 & 70.7 & 70.5 & 69.5 & 69.7 & 0.000939 \\
\hline 805.01999 & 3.38596556 & 14.73974 & 1.787215 & 14.67675 & 4776 & 4043.45 & 70.7 & 70.5 & 69.5 & 69.7 & 0.00094 \\
\hline
\end{tabular}




\begin{tabular}{|c|c|c|c|c|c|c|c|c|c|c|c|}
\hline SECONDS & MAIN DUCT & MAIN DUCT & GAS INJECT & GAS INJECT & NEWPORT & SMC & AMBIENT & GAS INJECT & MAIN DUCT & WEC CELL & WEC CELL \\
\hline & DIFF (IN H2O) & ABS (PSIA) & DIFF (IN H2O) & ABS (PSIA) & (PPM) & (PPM) & TEMP.(F) & TEMP. (F) & TEMP. (F) & TEMP. (F) & (VOLTS) \\
\hline 0.0099793 & 3.43848824 & 14.73974 & 0.542526 & 14.6625 & 120.95 & -3.59807 & 70.3 & 69.6 & 69.5 & 69.5 & 0.00025 \\
\hline 810.07001 & 3.40222258 & 14.73824 & 1.787215 & 14.6775 & 4766.25 & 4053.879 & 70.6 & 70.4 & 6.4 & 69.7 & 0.00094 \\
\hline 815.01999 & 3.39847096 & 14.73974 & 1.787215 & 14.6775 & 4766 & 4046.181 & 70.5 & 70.5 & 6.4 & 69.7 & 0.000938 \\
\hline 820.01999 & 3.402222258 & 14.73749 & 1.787215 & 14.67675 & 4766 & 4045.188 & 70.6 & 70.5 & 69.4 & 69.7 & 0.000939 \\
\hline 825.03998 & 3.42160595 & 14.73974 & 1.78784 & 14.67675 & 4766 & 4054.127 & 70.6 & 70.5 & 69.4 & 69.7 & 0.000939 \\
\hline 830.01999 & 3.39847096 & 14.73974 & 1.786589 & 14.67675 & 4766 & 4058.845 & 70.6 & 70.5 & 69.6 & 69.7 & 0,000939 \\
\hline 835.01999 & 3.40847528 & 14.73824 & 1.786589 & 14.67825 & 4766 & 4056.362 & 70.6 & 70.5 & 69.6 & 69.7 & 0.000939 \\
\hline 840.07001 & 3.39722042 & 14.74124 & 1.787215 & 14.6775 & 4765.75 & 4023.585 & 70.7 & 70.5 & 6.4 & 69.7 & 0,000939 \\
\hline 845.09 & 3.41097636 & 14.73899 & 1.786589 & 14.6775 & 4766 & 4016.136 & 70.7 & 70.5 & 69.5 & 69.7 & 0.000939 \\
\hline 850.01999 & 3.39847096 & 14.73974 & 1.787215 & 14.6775 & 4766.25 & 3996.271 & 70.7 & 70.5 & 69.6 & 69.7 & 0.000939 \\
\hline 855.01999 & 3.44661675 & 14.73899 & 1.786589 & 14.6775 & 4766.25 & 4021.847 & 70.6 & 70.5 & 69.5 & 69.7 & 0.00094 \\
\hline 860.06 & 3.39596988 & 14.73899 & 1.787215 & 14.6775 & 4766 & 4029.048 & 70.6 & 70.5 & 6.5 & 69.7 & 0.000939 \\
\hline 865.01999 & 3.39034245 & 14.73974 & 1.786589 & 14.67675 & 4766 & 4011.915 & 70.7 & 70.5 & 69.5 & 69.7 & 0.00094 \\
\hline 870.04999 & 3.40847528 & 14.73899 & 1.787215 & 14.6775 & 4766 & 4026.068 & 70.7 & 70.6 & 69.6 & 69.7 & 0.000941 \\
\hline 875.01999 & 3.40034677 & 14.73974 & 1.787215 & 14.67825 & 4776 & 4032.028 & 70.7 & 70.5 & 69.6 & 69.7 & 0.00094 \\
\hline 880.01999 & 3.38971718 & 14.73899 & 1.786589 & 14.67675 & 4766.25 & 4042.208 & 70.7 & 70.5 & 69.6 & 69.7 & 0.000939 \\
\hline 885.03998 & 3.41097636 & 14.73899 & 1.786589 & 14.6775 & 4766 & 4035.504 & 70.7 & 70.5 & 69.7 & 69.7 & 0.000939 \\
\hline 890.01999 & 3.43348608 & 14.74049 & 1.786589 & 14.6775 & 4765.75 & 4037.987 & 70.7 & 70.5 & 69.6 & 69.7 & 0.000939 \\
\hline 895.01999 & 3.3997215 & 14.74049 & 1.786589 & 14.6775 & 4766 & 4043.202 & 70.7 & 70.5 & 69.7 & 69.8 & 0.000939 \\
\hline 900,04999 & 3.39909623 & 14.73899 & 1.786589 & 14.6775 & 4766 & 4030.29 & 70.6 & 70.6 & 69.7 & 69.7 & 0.000939 \\
\hline 905.01999 & 3.402222258 & 14.73899 & 1.786589 & 14.67675 & 4766.25 & 4029.545 & 70.7 & 70.6 & 69.7 & 69.7 & 0.00094 \\
\hline 910.01999 & 3.40097204 & 14.73899 & 1.786589 & 14.67825 & 4766 & 4032.524 & 70.7 & 70.5 & 69.7 & 69.7 & 0.00094 \\
\hline 915.03998 & 3.39909623 & 14.73899 & 1.785964 & 14.6775 & 4766.25 & 4039.974 & 70.7 & 70.5 & 6.7 & 69.7 & 0.000939 \\
\hline 920.01999 & 3.40097204 & 14,73824 & 1.786589 & 14.6775 & 4766 & 4044.195 & 70.7 & 70.5 & 69.7 & 69.7 & 0.00094 \\
\hline 925.01999 & 3.40284785 & 14.74124 & 1.787215 & 14.6775 & 4766 & 4048.913 & 70.7 & 70.5 & 69.7 & 69.7 & 0.00094 \\
\hline 930.03 & 3.39409407 & 14.73899 & 1.785338 & 14.67525 & 4766 & 4046.678 & 70.7 & 70.6 & 69.6 & 69.7 & 0.000939 \\
\hline 935.01999 & 3.38971718 & 14.73824 & 10.16686 & 17.118 & 4776 & 4029.048 & 70.7 & 70.8 & 69.7 & 69.7 & 0.000939 \\
\hline
\end{tabular}




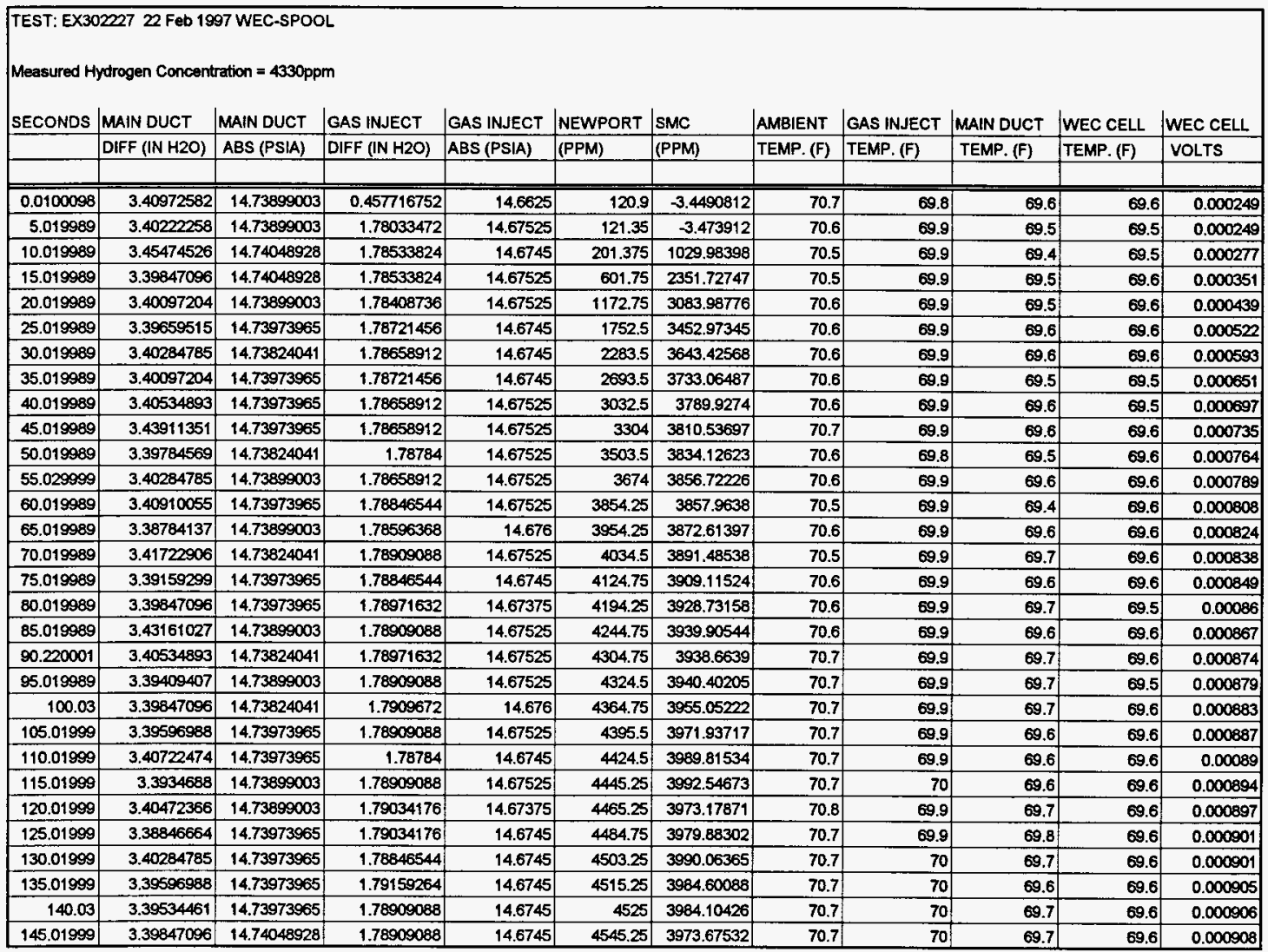




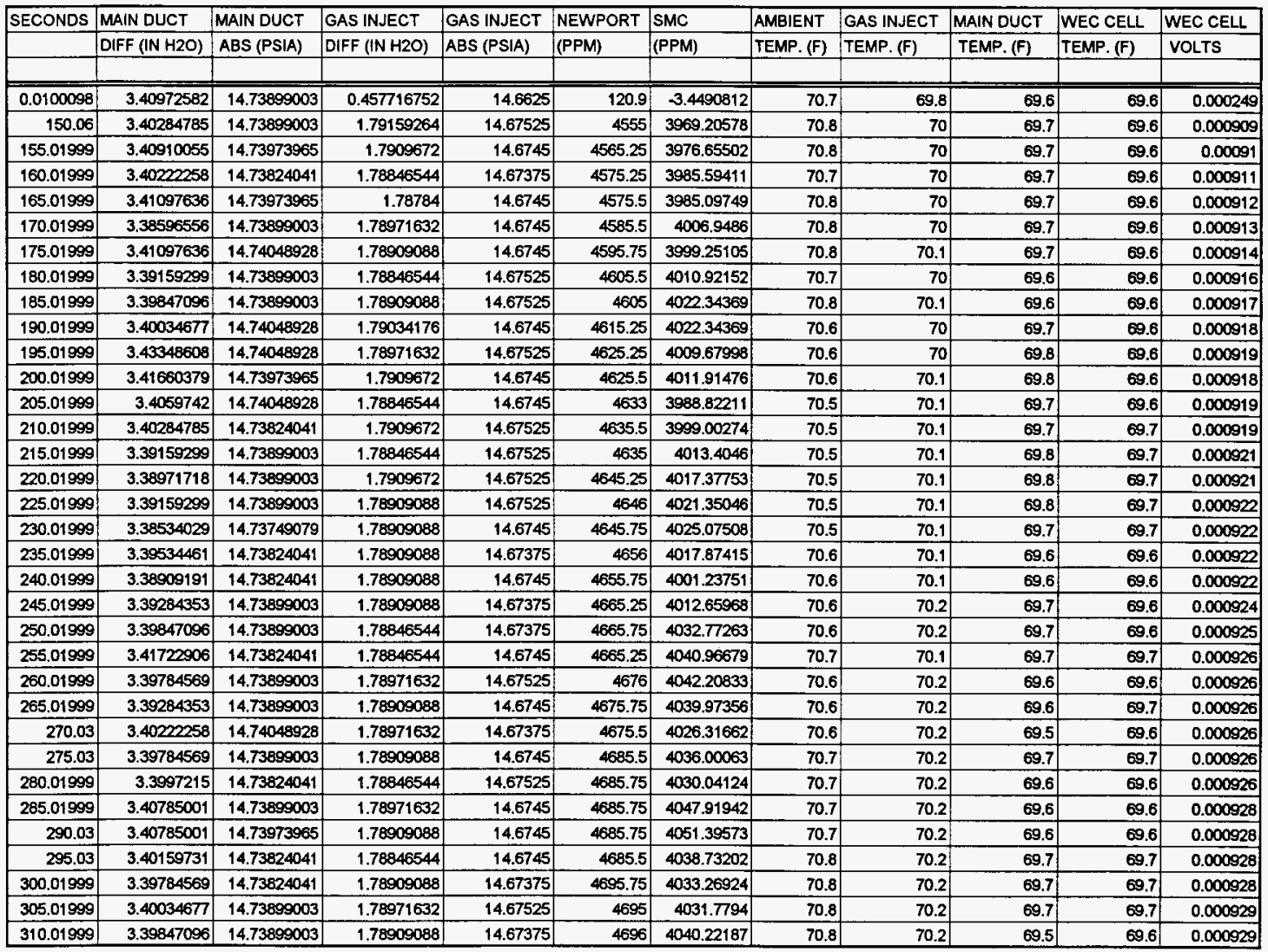




\begin{tabular}{|c|c|c|c|c|c|c|c|c|c|c|c|}
\hline SECONDS 1 & MAIN DUCT & MAIN DUCT & GAS INJECT & GAS INJECT & NEWPORT & SMC & AMBIENT & GAS INJECT & MAIN DUCT & WEC CELL & WEC CELL \\
\hline & DIFF (IN H2O) & ABS (PSIA) & DIFF (IN H2O) & ABS (PSIA) & $(P P M)$ & (PPM) & TEMP. (F) & TEMP.(F) & TEMP. (F) & TEMP. ( $F$ ) & VOLTS \\
\hline 0.0100098 & 3.40972582 & 14.73890003 & 0.457716752 & 14.6625 & 120.9 & -3.4490812 & 70.7 & 69.8 & 69.6 & 69.6 & 0.000249 \\
\hline 315.01999 & 3.39847096 & 14.73899003 & 1.78646544 & 14.67375 & 4695.5 & 4049.16096 & 70.8 & 70.2 & 69.6 & 69.7 & 0.000929 \\
\hline 320.01999 & 3.40847528 & 14.73824041 & 1.78596368 & 14.67525 & 4695.75 & 4033.02094 & 70.8 & 70.2 & 69.6 & 69.7 & 0.00093 \\
\hline 325.01999 & 3.40222258 & 14.73899003 & 1.78721456 & 14.676 & 4695.75 & 4020.35723 & 70.8 & 70.2 & 69.8 & 69.7 & 0.00093 \\
\hline 330.01999 & 3.41722906 & 14.73899003 & 1.78784 & 14.6745 & 4705.25 & 4028.30308 & 70.8 & 70.2 & 69.7 & 69.7 & 0.00093 \\
\hline 340.01999 & 3.38659083 & 14.73899003 & 1.78658912 & 14.67525 & 4705.5 & 4025.5717 & 70.8 & 70.2 & 69.6 & 69.7 & 0.000932 \\
\hline 345.01999 & 3.39847096 & 14.73973965 & 1.78721456 & 14.6745 & 4715.75 & 4024.57846 & 70.8 & 70.2 & 69.7 & 69.7 & 0.000932 \\
\hline 350.01999 & 3.40910055 & 14.73899003 & 1.78721456 & 14.6745 & 4716 & 4046.42957 & 70.8 & 70.3 & 69.7 & 69.7 & 0.000931 \\
\hline 355.01999 & 3.40034677 & 14.73824041 & 1.78721456 & 14.6745 & 4716 & 4048.66434 & 70.8 & 70.2 & 69.7 & 69.7 & 0.000931 \\
\hline 360.01999 & 3.40034677 & 14.73899003 & 1.78658912 & 14.673 & 4715.75 & 4035.0074 & 70.8 & 70.2 & 69.7 & 69.6 & 0.000931 \\
\hline 365.01999 & 3.39909623 & 14.73824041 & 1.78721456 & 14.67375 & 4715.5 & 4023.58523 & 70.8 & 70.3 & 69.8 & 69.7 & 0.000931 \\
\hline 370.01999 & 3.39471934 & 14.74048928 & 1.78721456 & 14.67375 & 4726 & 4030.04124 & 70.8 & 70.3 & 69.7 & 69.7 & 0.000932 \\
\hline 380.01999 & 3.38784137 & 14.73973965 & 1.78721456 & 14.6745 & 4725.75 & 4036.49725 & 70.7 & 70.3 & 69.6 & 69.7 & 0.000933 \\
\hline 385.01999 & 3.40034677 & 14.73973965 & 1.78721456 & 14.67375 & 4726 & 4044.69141 & 70.7 & 70.2 & 69.5 & 69.7 & 0.000933 \\
\hline 390.01999 & 3.40284785 & 14.73899003 & 1.78658912 & 14.67375 & 4725.75 & 4030.78616 & 70.7 & 70.3 & 69.7 & 69.7 & 0.000933 \\
\hline 395.01999 & 3.40284785 & 14.73973965 & 1.78596368 & 14.6745 & 4725.5 & 4030.53786 & 70.7 & 70.3 & 69.6 & 69.7 & 0.000932 \\
\hline 400.01999 & 3.39847096 & 14.73973965 & 1.78658912 & 14.6745 & 4726 & 4047.67111 & 70.7 & 70.3 & 69.7 & 69.7 & 0.000932 \\
\hline 405.06 & 3.40284785 & 14.74048928 & 1.78658912 & 14.67375 & 4726 & 4046.92618 & 70.7 & 70.3 & 69.6 & 69.7 & 0.000933 \\
\hline 410.01999 & 3.38971718 & 14.73899003 & 1.78658912 & 14.6745 & 4725.75 & 4049.90588 & 70.7 & 70.3 & 69.7 & 69.7 & 0.000933 \\
\hline 415.09 & 3.40347312 & 14.73899003 & 1.78658912 & 14.6745 & 4725.75 & 4032.52432 & 70.7 & 70.3 & 69.6 & 69.7 & 0.000933 \\
\hline 420.01999 & 3.3997215 & 14.73973965 & 1.78596368 & 14.67375 & 4726 & 4033.76586 & 70.7 & 70.3 & 69.8 & 69.7 & 0.000933 \\
\hline 425.09 & 3.39096772 & 14.73899003 & 1.78658912 & 14.67375 & 4726 & 4026.31662 & 70.7 & 70.3 & 69.7 & 69.7 & 0.000935 \\
\hline 430.01999 & 3.40722474 & 14.73749079 & 1.78658912 & 14.67375 & 4725,75 & 4021.10215 & 70.7 & 70.3 & 69.8 & 69.7 & 0.000934 \\
\hline 435.07001 & 3.39847096 & 14.73899003 & 1.78596368 & 14.67375 & 4725.5 & 4019.364 & 70.7 & 70.3 & 69.7 & 69.7 & 0.000935 \\
\hline 440.01999 & 3.40034677 & 14.73973965 & 1.78596368 & 14.6745 & 4735.75 & 4006.70029 & 70.7 & 70.3 & 69.6 & 69.7 & 0.000934 \\
\hline 445.01999 & 3.39909623 & 14.73973965 & 1.78721456 & 14.67525 & 4736 & 4005.45875 & 70.8 & 70.3 & 69.7 & 69.7 & 0.000935 \\
\hline 450.01999 & 3.39847096 & 14.73824041 & 1.78658912 & 14.6745 & 4735.5 & 3992.54673 & 70.8 & 70.3 & 69.8 & 69.7 & 0.000934 \\
\hline 465.01999 & 3.39909623 & 14.74048928 & 1.78658912 & 14.67525 & 4735.5 & 3994.7815 & 70.7 & 70.4 & 69.7 & 69.7 & 0.000936 \\
\hline 470.01999 & 3.40910055 & 14.73899003 & 1.78658912 & 14.6745 & 4735.75 & 4007.1969 & 70.7 & 70.3 & 69.8 & 69.8 & 0.000935 \\
\hline 475.01999 & 3.39284353 & 14.73749079 & 1.78721456 & 14.6745 & 4736 & 4004.71382 & 70.6 & 70.4 & 69.8 & 69.7 & 0.000936 \\
\hline
\end{tabular}




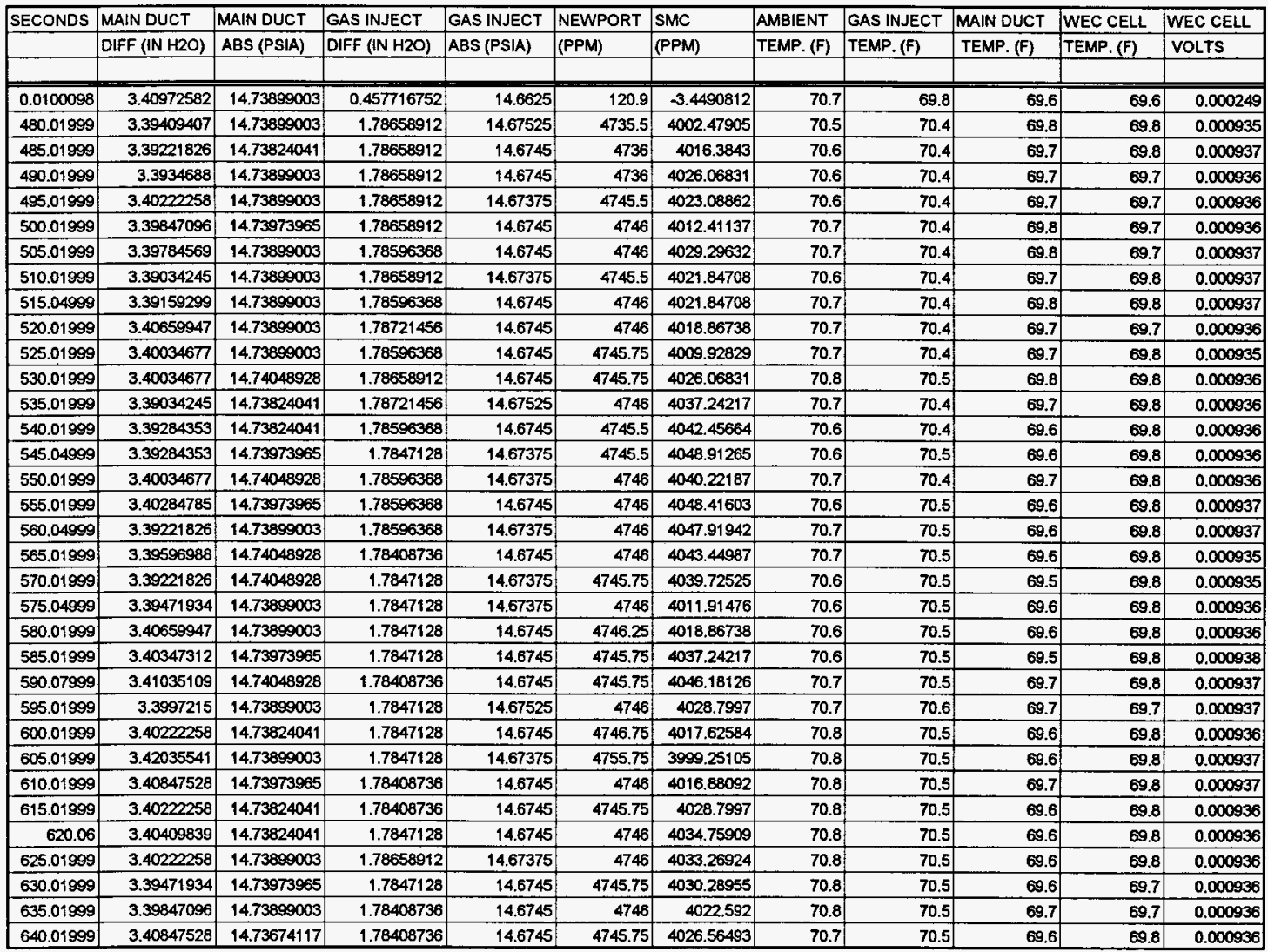




\begin{tabular}{|c|c|c|c|c|c|c|c|c|c|c|c|}
\hline SECONDS & MAIN DUCT & MAIN DUCT & GAS INJECT & GAS INJECT & NEWPORT & SMC & AMBIENT & GAS INJECT & MAIN DUCT & WEC CELL & WEC CELL \\
\hline & DIFF (IN H2O) & ABS (PSIA) & DIFF (IN H2O) & ABS (PSIA) & (PPM) & (PPM) & TEMP. (F) & TEMP. (F) & TEMP. (F) & TEMP. (F) & VOLTS \\
\hline 0.0100098 & 3.40972582 & 14.73899003 & 0.457716752 & 14.6625 & 120.9 & -3.4490812 & 70.7 & 69.8 & 69.6 & 69.6 & 0.000249 \\
\hline 645.01999 & 3.40284785 & 14.73899003 & 1.78408736 & 14.6745 & 4745.75 & 4036.49725 & 70.7 & 70.6 & 69.6 & 69.8 & 0.000936 \\
\hline 650.06 & 3.38971718 & 14.73824041 & 1.78408736 & 14.67375 & 4746 & 4029.54462 & 70.7 & 70.5 & 69.7 & 69.8 & 0.000937 \\
\hline 655.01999 & 3.39784569 & 14.73899003 & 1.7847128 & 14.67525 & 4746.25 & 4027.06154 & 70.7 & 70.6 & 69.7 & 69.8 & 0.000937 \\
\hline 660.01999 & 3.40159731 & 14.73899003 & 1.7847128 & 14.6745 & 4745.75 & 4036.74556 & 70.7 & 70.6 & 69.8 & 69.8 & 0.000937 \\
\hline 670.01999 & 3.40347312 & 14.74048928 & 1.7847128 & 14.6745 & 4746 & 4021.84708 & 70.8 & 70.6 & 69.7 & 69.8 & 0.000937 \\
\hline 675.01999 & 3.39847096 & 14.74048928 & 1.78408736 & 14.67525 & 4754.75 & 4025.5717 & 70.7 & 70.6 & 69.7 & 69.8 & 0.000937 \\
\hline 680.06 & 3.40284785 & 14.73899003 & 1.78408736 & 14.67375 & 4756 & 4019.6123 & 70.7 & 70.6 & 69.7 & 69.8 & 0.000938 \\
\hline 685.01999 & 3.42285649 & 14.73973965 & 1.78346192 & 14.6745 & 4756 & 4003.72059 & 70.8 & 70.6 & 69.6 & 69.8 & 0.000937 \\
\hline 690.01999 & 3.40034677 & 14.73973965 & 1.78408736 & 14.67375 & 4755.75 & 4012.65968 & 70.7 & 70.6 & 69.7 & 69.8 & 0.000938 \\
\hline 695.04999 & 3.40972582 & 14.73973065 & 1.7847128 & 14.6745 & 4756 & 4030.78616 & 70.8 & 70.6 & 69.7 & 69.8 & 0.000337 \\
\hline 700.01999 & 3.39847096 & 14.73899003 & 1.78346192 & 14.6745 & 4756 & 4035.25571 & 70.7 & 70.6 & 69.6 & 69.8 & 0.000936 \\
\hline 710.01999 & 3.39596988 & 14.73899003 & 1.7847128 & 14.6745 & 4756 & 4027.80647 & 70.8 & 70.6 & 69.7 & 69.8 & 0.000937 \\
\hline 715.01999 & 3.417222906 & 14.73973965 & 1.7847128 & 14.673 & 4756 & 4042.20833 & 70.8 & 70.6 & 69.7 & 69.8 & 0.000538 \\
\hline 720.01999 & 3.40034677 & 14.73824041 & 1.78408736 & 14.6745 & 4755.75 & 4037.49048 & 70.8 & 70.6 & 69.6 & 69.8 & 0.000939 \\
\hline 725.04001 & 3.39596988 & 14.73899003 & 1.7847128 & 14.67375 & 4756 & 4026.81324 & 70.8 & 70.6 & 69.6 & 69.8 & 0.000939 \\
\hline 730.01999 & 3.39596988 & 14.73973965 & 1.7847128 & 14.6715 & 4756 & 4005.45875 & 70.7 & 70.6 & 69.6 & 69.8 & 0.000938 \\
\hline 735.01999 & 3.41035109 & 14.73899003 & 1.7847128 & 14.67225 & 4756 & 3995.77474 & 70.7 & 70.6 & 69.6 & 69.8 & 0.000938 \\
\hline 740.01999 & 3.40910055 & 14.73899003 & 1.7847128 & 14.67375 & 4756 & 4013.4046 & 70.6 & 70.6 & 69.6 & 69.8 & 0.000938 \\
\hline 745.01999 & 3.40472366 & 14.73973905 & $1.7847 \nmid 28$ & 14.6715 & 4756 & 4010.92152 & 70.7 & 70.6 & 69.6 & 69.8 & 0.000939 \\
\hline 750.01999 & 3.41035109 & 14.73973965 & 1.78408736 & 14.67225 & 4756 & 3997.51289 & 70.6 & 70.6 & 69.6 & 69.8 & 0.000939 \\
\hline 755.04999 & 3.41973014 & 14.74048928 & 1.7847128 & 14.67375 & 4756 & 4001.73413 & 70.6 & 70.6 & 69.6 & 69.8 & 0.000939 \\
\hline 760.01999 & 3.38971718 & 14.73973965 & 1.7847128 & 14.6715 & 4756 & 4000.49259 & 70.6 & 70.6 & 69.7 & 69.8 & 0.000939 \\
\hline 765.01999 & 3.41097636 & 14.73899003 & 1.78408736 & 14.6745 & 4756 & 4021.10215 & 70.7 & 70.6 & 69.7 & 69.8 & 0.000938 \\
\hline 770.01999 & 3.40534893 & 14.73899003 & 1.78221104 & 14.67375 & 4756 & 4032.52432 & 70.7 & 70.6 & 69.6 & 69.8 & 0.000938 \\
\hline 775.01999 & 3.39784569 & 14.73824041 & 1.78408736 & 14.6745 & 4756 & 4029.29632 & 70.7 & 70.6 & 69.6 & 69.8 & 0.000939 \\
\hline 780.01999 & 3.39847096 & 14.73824041 & 1.78408736 & 14.67375 & 4766 & 4018.86738 & 70.7 & 70.6 & 69.7 & 69.8 & 0.000989 \\
\hline 795.01999 & 3.41285217 & 14.73899003 & 1.78408736 & 14.6745 & 4765.75 & 4035.0074 & 70.7 & 70.7 & 69.6 & 69.8 & 0.000939 \\
\hline 800.04001 & 3.3934688 & 14.73899003 & 1.78221104 & 14.6715 & 4765.75 & 4033.51755 & 70.7 & 70.6 & 69.6 & 69.8 & 0.000939 \\
\hline 805.01999 & 3.42848392 & 14.73973965 & 1.7815856 & 14.6745 & 4766 & 4017.87415 & 70.7 & 70.6 & 69.6 & 69.8 & 0.000938 \\
\hline
\end{tabular}




\begin{tabular}{|c|c|c|c|c|c|c|c|c|c|c|c|}
\hline SECONDS & MAIN DUCT & MAIN DUCT & GAS INJECT & GAS INJECT & NEWPORT & SMC & AMBIENT & GAS INJECT & MAIN DUCT & WEC CELL & WEC CELL \\
\hline & DIFF (IN H2O) & ABS (PS|A) & DIFF (IN H2O) & ABS (PS|A) & (PPM) & (PPM) & TEMP. (F) & TEMP. (F) & TEMP. (F) & TEMP. (F) & VOLTS \\
\hline & & & & & & & & & & & \\
\hline 0.0100098 & 3.40972582 & 14.73899003 & 0.457716752 & 14.6625 & 120.9 & -3.4490812 & 70.7 & 69.8 & 69.6 & 69.6 & 0.000249 \\
\hline 810.01999 & 3.41285217 & 14.73973965 & 1.78408736 & 14.6715 & 4766 & 4031.53109 & 70.7 & 70.6 & 69.6 & 69.8 & 0.000939 \\
\hline 815.07001 & 3.39096772 & 14.73899003 & 1.78221104 & 14.6745 & 4766.25 & 4036.49725 & 70.6 & 70.7 & 69.7 & 69.8 & 0.000938 \\
\hline 820.01999 & 3.39847096 & 14.73899003 & 1.7815856 & 14.67225 & 4766 & 4032.0277 & 70.5 & 70.6 & 69.6 & 69.8 & 0.000939 \\
\hline 825.01999 & 3.40910055 & 14.73824041 & 1.78221104 & 14.6715 & 4766 & 4030.04124 & 70.7 & 70.6 & 69.6 & 69.8 & 0.00094 \\
\hline 830.01999 & 3.40534893 & 14.73899003 & 1.78283648 & 14.6715 & 4766 & 4035.75232 & 70.7 & 70.6 & 69.6 & 69.8 & 0.000939 \\
\hline 835.01999 & 3.39847096 & 14.73824041 & 1.78283648 & 14.6715 & 4766 & 4033.51755 & 70.7 & 70.6 & 69.7 & 69.8 & 0.000938 \\
\hline 840.01999 & 3.40159731 & 14.73824041 & 1.78221104 & 14.6715 & 4766 & 4039.97356 & 70.7 & 70.7 & 69.6 & 69.6 & 0.000939 \\
\hline 845.06 & 3.40347312 & 14.73824041 & 1.78283648 & 14.67225 & 4766 & 4057.85174 & 70.8 & 70.7 & 69.7 & 69.8 & 0.000939 \\
\hline 850.01999 & 3.39221826 & 14.73749079 & 1.78221104 & 14.67225 & 4766 & 4063.0662 & 70.8 & 70.7 & 69.8 & 69.8 & 0.000938 \\
\hline 855.01999 & 3.40910055 & 14.73973965 & 1.78346192 & 14.6715 & 4756 & 4052.88558 & 70.9 & 70.7 & 69.8 & 69.8 & 0.000937 \\
\hline 860.01999 & 3.39409407 & 14.73899003 & 1.78283648 & 14.67225 & 4756 & 4056.11358 & 70.9 & 70.7 & 69.8 & 69.8 & 0.000937 \\
\hline 865.01999 & 3.39596988 & 14.73973965 & 1.78221104 & 14.67225 & 4756 & 4056.11358 & 70.9 & 70.7 & 69.7 & 69.7 & 0.000937 \\
\hline 875.01999 & 3.38784137 & 14.73899003 & 1.7815856 & 14.67375 & 4756.25 & 4054.62373 & 70.9 & 70.7 & 69.8 & 69.8 & 0.000939 \\
\hline 880.01999 & 3.40659947 & 14.73824041 & 1.78221104 & 14.6745 & 4756 & 4041.96002 & 70.8 & 70.7 & 69.8 & 69.8 & 0.000938 \\
\hline 885.01999 & 3.41347744 & 14.73899003 & 1.78221104 & 14.67375 & 4756 & 4044.4431 & 70.9 & 70.7 & 69.7 & 69.8 & 0.000939 \\
\hline 890.04999 & 3.39847096 & 14.73973965 & 1.78221104 & 14.6715 & 4766 & 4038.73202 & 70.8 & 70.7 & 69.7 & 69.8 & 0.000938 \\
\hline 895.04999 & 3.39909623 & 14.74048928 & 1.7815856 & 14.6715 & 4766.25 & 4016.63261 & 70.9 & 70.7 & 69.7 & 69.8 & 0.000938 \\
\hline 900.01999 & 3.39596988 & 14.73973965 & 1.7815856 & 14.6715 & 4765.75 & 4023.58523 & 70.9 & 70.7 & 69.7 & 69.8 & 0.000939 \\
\hline 905.04999 & 3.41660379 & 14.7412389 & 1.7815856 & 14.67225 & 4766 & 4026.06831 & 70.8 & 70.7 & 69.8 & 69.8 & 0.00094 \\
\hline 910.01999 & 3.39409407 & 14.73973965 & 1.7815856 & 14.6745 & 4765.75 & 4026.81324 & 70.7 & 70.7 & 69.7 & 69.8 & 0.000939 \\
\hline 915.01999 & 3.40097204 & 14.73973965 & 1.78221104 & 14.673 & 4766.25 & 4029.29632 & 70.7 & 70.7 & 69.6 & 69.8 & 0.000939 \\
\hline 920.04999 & 3.40097204 & 14.73824041 & 1.78221104 & 14.6715 & 4766 & 4024.08185 & 70.7 & 70.7 & 69.6 & 69.8 & 0.000939 \\
\hline 925.01999 & 3.40910055 & 14.73899003 & 1.78033472 & 14.6715 & 4766.25 & 4021.10215 & 70.8 & 70.7 & 69.6 & 69.8 & 0.000939 \\
\hline 930.01999 & 3.39221826 & 14.73824041 & 1.77970928 & 14.6715 & 4766.25 & 4031.7794 & 70.8 & 70.7 & 69.6 & 69.8 & 0.000939 \\
\hline 935.01999 & 3.39847096 & 14.73824041 & 1.78096016 & 14.6715 & 4766 & 4055.86527 & 70.8 & 70.7 & 69.7 & 69,8 & 0.000939 \\
\hline 940.03 & 3.39847096 & 14.73973965 & 1.78096016 & 14.6715 & 4766 & 4059.09328 & 70.8 & 70.7 & 69.6 & 69.8 & 0.000939 \\
\hline 945.01999 & 3.41660379 & 14.73749079 & 0.213419888 & 14.604 & 4765.5 & 4065.30098 & 70.7 & 70.7 & 69.7 & 69.8 & 0.000938 \\
\hline
\end{tabular}




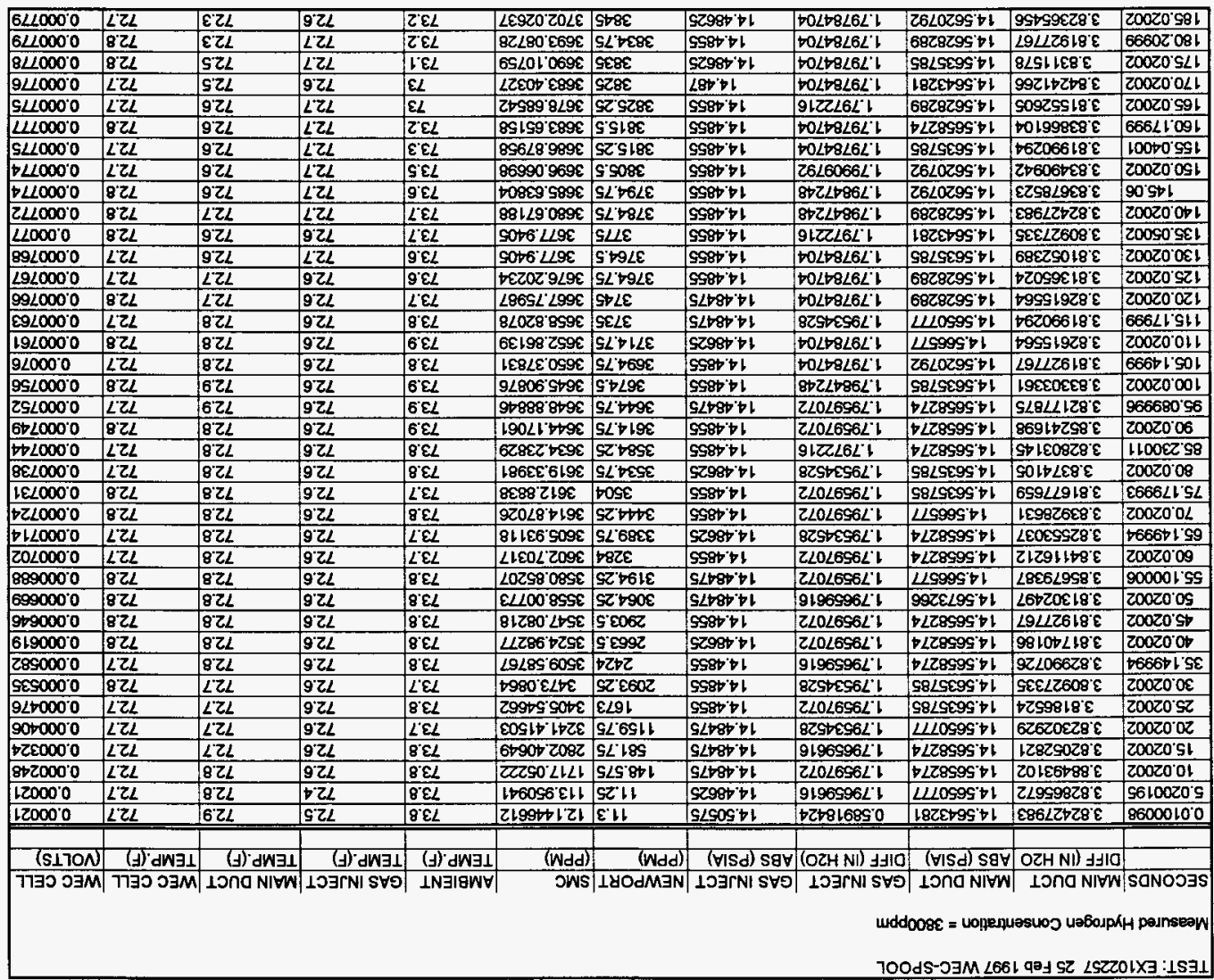




\begin{tabular}{|c|c|c|c|c|c|c|c|c|c|c|c|}
\hline SECONDS & \begin{tabular}{|l|} 
MAIN DUCT \\
DIFF ( (N H2O \\
\end{tabular} & $\begin{array}{l}\text { MAIN DUCT } \\
\text { ABS (PSIA) }\end{array}$ & \begin{tabular}{|l} 
GAS INJECT \\
DIFF (IN H2O)
\end{tabular} & $\begin{array}{l}\text { GAS INJECT } \\
\text { ABS (PSIA) } \\
\end{array}$ & $\begin{array}{l}\text { NEWPORT } \\
\text { (PPM) } \\
\end{array}$ & $\begin{array}{l}\text { SMC } \\
\text { (PPM) }\end{array}$ & $\begin{array}{l}\text { AMBIENT } \\
\text { TEMP.(F) }\end{array}$ & \begin{tabular}{|l|} 
GAS INJECT \\
TEMP.(F) \\
\end{tabular} & \begin{tabular}{|l|} 
MAIN DUCT \\
TEMP.(F) \\
\end{tabular} & \begin{tabular}{|l|} 
WEC CELL \\
TEMP.(F) \\
\end{tabular} & $\begin{array}{l}\text { WEC CELL } \\
\text { (NOLTS) } \\
\end{array}$ \\
\hline 190.02002 & 3.81052389 & 145628289 & 1.79784704 & 14.4855 & 3845,25 & 3704.75776 & 73.2 & 727 & 723 & 727 & 0,00078 \\
\hline 195.02002 & 3.81990294 & 14.5628289 & 1.79784704 & 14.48625 & 3854.75 & 3712.45531 & 73.2 & 72.7 & 72.4 & 72.7 & 0.00078 \\
\hline 200.02002 & 3.80864808 & 14.5620792 & 1.7972216 & 14.4855 & 3355 & 3701.77806 & 73.2 & 72.7 & 72.4 & 72.7 & 0.000781 \\
\hline 205.08002 & 3.8123997 & 14.5620792 & 1.79784704 & 14.4855 & 3855.25 & 3700.28822 & 73.2 & 72.7 & 72.4 & 72.7 & 0.000782 \\
\hline 210.16 & 3.82553037 & 14.5628289 & 1.79784704 & 14.4855 & 3865 & 3713.20023 & 73.2 & 72.7 & 72.5 & 72.7 & 0.000782 \\
\hline 215.02002 & 3.806147 & 14.5635785 & 1.79847248 & 14.4855 & 3864.75 & 3714.69008 & 73.3 & 72.7 & 72.5 & 72.7 & 0.000783 \\
\hline 220.12 & 3.811774443 & 14.5635785 & 1.79784704 & 14.4855 & 3865.25 & 3721.14609 & 73.4 & 72.7 & 72.4 & 72.7 & 0.000783 \\
\hline 225.02002 & 3.81740186 & 14.5635785 & 1.79784704 & 14.4855 & 3875.5 & 3728.09871 & 73.4 & 72.7 & 72.5 & 72.8 & 0.000784 \\
\hline 230.02002 & 3.83053253 & 14.566577 & 1.79784704 & 14.4855 & 3875 & 3707.98576 & 73.5 & 72.7 & 72.4 & 72.7 & 0.000784 \\
\hline 235.04001 & 3.80489646 & 14.5635785 & 1.79847248 & 14.48475 & 3874.75 & 3707.48915 & 73.4 & 72.7 & 72.4 & 72.8 & 0.000784 \\
\hline 240.14999 & 3.80802281 & 14.5628289 & 1.79784704 & 14.4855 & 3885.5 & 3708.73069 & 73.4 & 72.7 & 72.3 & 72.7 & 0.000784 \\
\hline 245.02002 & 3.84303793 & 14.5635785 & 1.79847248 & 14.48625 & 3885.25 & 3705.9993 & 73.3 & 72.7 & 72.3 & 72.8 & 0.000784 \\
\hline 250.09 & 3.81490078 & 14.5635785 & 1.79784704 & 14.48625 & 3885.5 & 3702.02637 & 73.3 & 72.7 & 72.3 & 72.8 & 0.000786 \\
\hline 255.02002 & 3.81802713 & 14.5635785 & 1.79847248 & 14.4855 & 3895.5 & 3704.75776 & 73.3 & 72.7 & 72.3 & 72.8 & 0.000786 \\
\hline 260.02002 & 3.80239538 & 14.5635785 & 1.79784704 & 14.48475 & 3895.5 & 3714.19346 & 73.2 & 72.7 & 72.2 & 72.8 & 0.000786 \\
\hline 265.02002 & 3.83178307 & 14.5635785 & 1.79784704 & 14.4855 & 3895.5 & 3722.88424 & 73.1 & 72.7 & 72.2 & 72.7 & 0.000786 \\
\hline 270.17999 & 3.82115348 & 14.5643281 & 1.79784704 & 14.4855 & 3895.25 & 3719.40793 & 73.1 & 72.7 & 72.1 & 72.8 & 0.000785 \\
\hline 275.02002 & 3.81490078 & 14.5643281 & 1.79847248 & 14.48475 & 3895.25 & 3720.15286 & 73.1 & 72.7 & 72.1 & 72.7 & 0.000786 \\
\hline $280.0 \$ 002$ & 3.81114916 & 14.5628289 & 1.79847248 & 14.48475 & 3895.25 & 3709.72392 & 73.1 & 72.7 & 72.1 & 72.8 & 0.000788 \\
\hline 285.02002 & 3.83240834 & 14.5635785 & 1.79597072 & 14.48475 & 3905.5 & 3710.96546 & 73.2 & 72.7 & 72.3 & 72.8 & 0.000786 \\
\hline 290.02002 & 3.83240834 & 14.5620792 & 1.7972216 & 14.4855 & 3905.5 & 3715.1867 & 73.2 & 72.7 & 72.3 & $\overline{72.8}$ & 0.000787 \\
\hline 295.02002 & 3.80802281 & 14.5643281 & 1.79784704 & 14.48475 & 3905.5 & 3716.17993 & 73.2 & 72.7 & 72.3 & 72.8 & 0.000788 \\
\hline 300.02002 & 3.80927335 & 14.5635785 & 1.79659616 & 14.4855 & 3905.5 & 3726.85717 & 73.2 & 72.7 & 72.3 & 72.8 & 0.000788 \\
\hline 305.02002 & 3.81740186 & 14.5628289 & 1.79784704 & 14.4855 & 3905.5 & 3727.10548 & 73.2 & 72.7 & 72.2 & 72.7 & 0.000788 \\
\hline 310.08002 & 3.80927335 & 14.5628289 & 1.79659616 & $14 . \overline{4855}$ & 3915.5 & 3714.69008 & 73.1 & 72.7 & 72.1 & 72.7 & 0.000789 \\
\hline 315.14001 & 3.83365888 & 14.5658274 & 1.79784704 & 14.48625 & 3915.5 & 3730.58179 & 73 & 72.7 & 72 & 72.8 & 0.000788 \\
\hline 320.02002 & 3.83803577 & 14.5635785 & 1.7972216 & 14.48475 & 3915.5 & 3729.09194 & 72.9 & 72,7 & 71.9 & 72.7 & 0.000788 \\
\hline 325.09 & 3.8186524 & 14.5658274 & 1.7972216 & 14.48475 & 3915.25 & 3721.6427 & 72.8 & 72.7 & 71.9 & 72.8 & 0.000789 \\
\hline 330.02002 & 3.80802281 & 14.5635785 & 1.7972216 & 14.48625 & 3915.5 & 3717.17316 & 72.9 & 72.7 & 71.9 & 72.8 & 0.000789 \\
\hline 335.02002 & 3.82177875 & 14.5628289 & 1.79784704 & 14.48625 & 3915.5 & 3723.38086 & 72.9 & 72.7 & 71.9 & 72.8 & 0.000789 \\
\hline 340.02002 & 3.80239538 & 14.5628289 & 1.79784704 & 14.48175 & 3915.5 & 3733.06487 & 72.8 & 72.7 & 71.8 & 72.8 & 0.000789 \\
\hline 345.03 & 3.81677659 & 14.5635785 & 1.79784704 & 14.4825 & 3915.75 & 3726.85717 & 72.8 & 72.7 & 74.9 & 72.8 & 0.000789 \\
\hline 350.02002 & 3.80427119 & 14.5635785 & 1.79784704 & 14.484 & 3925.5 & 3723.13255 & 72.9 & 72.7 & 74.9 & 72.8 & 0.00079 \\
\hline 355.03 & 3.80239538 & 14.5635785 & 1.79597072 & 14.48175 & 3925.5 & 3720.15286 & 72.8 & 72.7 & 71.9 & 72.8 & 0,000791 \\
\hline 360.02002 & 3.81552605 & 14.5628289 & 1.7972216 & $14.48 \div 75$ & 3925 & 3724.37409 & 72.8 & 72.7 & 72 & 72.8 & 0.000791 \\
\hline 365.02002 & 3.81927767 & 14.5628289 & 1.79784704 & 14.4825 & 3925.5 & 3729.34025 & 72.8 & 72.7 & 72 & 72.8 & 0.000791 \\
\hline 370.06 & 3.80239538 & 14.5628289 & 1.79659616 & 14.4825 & 3925.25 & 3728.84364 & 72.8 & 72.7 & 72 & 72.8 & 0.000792 \\
\hline 375.02002 & 3.80864808 & 14.5628289 & 1.7972216 & 14.48475 & 3925.25 & 3729.09194 & 72.8 & 72.7 & 72 & 72.8 & 0.000791 \\
\hline 380.02002 & 3.82052821 & 14.5643281 & 1.79534528 & 14.48475 & 3925.5 & 3731.82333 & 72.9 & 72.7 & 72 & 72.8 & 0.00079 \\
\hline 385.03 & 3.80427119 & 14.5620792 & 1.79597072 & 14.4825 & 3925.5 & 3714.19346 & 72.8 & 72.7 & 71.9 & 72.8 & 0.000791 \\
\hline 390.14001 & 3.83178307 & 14.5635785 & 1.79534528 & 14.4825 & 3925.5 & 3711.46208 & 72.8 & 72.7 & 71.9 & 72.8 & 0.000791 \\
\hline 395.02002 & 3.81427551 & 14.5643281 & 1.79597072 & 14.4825 & 3925.5 & 3705.9993 & 72.8 & 72.7 & 71.9 & 72.8 & 0,000792 \\
\hline
\end{tabular}




\begin{tabular}{|c|c|c|c|c|c|c|c|c|c|c|c|}
\hline SECONDS & MAIN DUCT & MAIN DUCT & GAS INJECT & GAS INJECT & \begin{tabular}{l|} 
NEWPORT \\
PPM
\end{tabular} & SMC & \begin{tabular}{|l} 
AMBIENT \\
TEMP(F)
\end{tabular} & \begin{tabular}{|l|} 
GAS INJECT \\
TEMP.(F)
\end{tabular} & \begin{tabular}{|l|} 
MAIN DUCT \\
TEMP (F)
\end{tabular} & \begin{tabular}{|l|} 
WEC CELL \\
TEMP.(F)
\end{tabular} & $\begin{array}{l}\text { WEC CELL } \\
\text { (NOLTS) }\end{array}$ \\
\hline & DIFF ( 1 N H2O & ABS (PS|A) & & & & & & & & & \\
\hline 400.02002 & 3.80864808 & 14.5643281 & 1.7972216 & 14.484 & 3925.5 & 3715.93162 & 72.7 & 72.7 & 71.9 & 72.8 & 0.000791 \\
\hline 405.02002 & 3.8186524 & 14.5635785 & 1.79597072 & 14.4825 & 3925.25 & 3712.45531 & 72.7 & 72.7 & 71,9 & 72.8 & 0.000792 \\
\hline 410.02002 & $3.8 \div 86524$ & 14.5635785 & 1.79597072 & 14.48325 & 3925.5 & 3712.45531 & 72.8 & 72.7 & 72 & 72.8 & 0.000792 \\
\hline 415.02002 & 3.806147 & 14.5635785 & 1.79597072 & 14.48175 & 3935 & 3712.207 & 72.8 & 72.7 & 71,9 & 72.8 & 0.000792 \\
\hline 420.02002 & 3.82615564 & 14.5635785 & 1.7972216 & 14,48325 & 3935.25 & 3712.45531 & 72.8 & 72.7 & 71.9 & 72.8 & 0.000792 \\
\hline 425.04001 & 3.80927335 & 14.5635785 & 1.79659616 & 14.48325 & 3935.25 & 3714.69008 & 72.7 & 72.7 & 71.9 & 72.8 & 0.000792 \\
\hline 430.02002 & 3.81552605 & 14.5643281 & 1.79534528 & 14.48325 & 3935.5 & 3711.21377 & 72.8 & 72.8 & 71.8 & 72.7 & 0.000791 \\
\hline 435.14001 & 3.81927767 & 14.5643281 & 1.79659616 & 14.48325 & 3935.5 & 3714.93839 & 72.8 & 72.8 & 71.8 & 72.7 & 0.000792 \\
\hline 440.17001 & 3.80864808 & 14.5643281 & 1.79597072 & 14.48325 & 3935.5 & 3708.979 & 72.8 & 72.7 & 71.9 & 72.8 & 0.000792 \\
\hline 445.17999 & 3.81552605 & 14.5650777 & 1.79784704 & 14.48325 & 3935.5 & 3719.90455 & 72.9 & 72.7 & 72 & 72.8 & 0.000793 \\
\hline 450.02002 & 3.82553037 & 14.5643281 & $1.7947 ! 984$ & 14.48325 & 3935 & 3724.37409 & 73 & 72.8 & 72 & 72.8 & 0.000793 \\
\hline 455.12 & 3.81740186 & 14.5650777 & 1.79784704 & 14.48325 & 3935.25 & 3716.92485 & 73 & 72.7 & 72 & 72.8 & 0.000793 \\
\hline 460.02002 & 3.83490942 & 14.5650777 & 1.79634528 & 14.4825 & 3945 & 3706.99253 & 73 & 72.7 & 72.1 & 72.8 & 0.000793 \\
\hline 465.02002 & 3.81490078 & 14.5658274 & 1.79597072 & 14.48325 & 3944.25 & 3710.96546 & 73 & 72.7 & 72.1 & 72.7 & 0.000793 \\
\hline 470.02002 & 3.81740186 & 14.5643281 & 1.79471984 & 14.48325 & 3945.75 & 3718.66301 & 72.9 & 72.7 & 72.1 & 72.8 & 0.000794 \\
\hline 475.05002 & 3.81677659 & 14.5628289 & 1.79534528 & 14.4825 & 3945.75 & 3727.8504 & 72.9 & 72.8 & 72 & 72.8 & 0.000792 \\
\hline 480.02002 & 3.821153448 & 14.5628289 & 1.79471984 & 14.4825 & 3945.5 & 3732.81656 & 72.8 & 72.7 & 71.9 & 72.8 & 0.000793 \\
\hline 485.17001 & 3.81927767 & 14.5643281 & 1.79634528 & 14.4825 & 3935.5 & 3712.70362 & 73 & 72.7 & 72 & 72.7 & 0.000793 \\
\hline 490.02002 & 3.81740186 & 14.5635785 & 1.79534528 & 14.4825 & 3935.5 & 3706.24761 & 73 & 72.7 & 72 & 72.8 & 0.000793 \\
\hline 495.02002 & 3.83240834 & 14.5635785 & 1.79534528 & 14.4825 & 3935.5 & 3713.69685 & 73 & 72.7 & 72.1 & 72.8 & 0.000792 \\
\hline 500.02002 & 3.83240834 & 14.5620792 & 1.79659616 & 14.4825 & 3935.5 & 3711.46208 & 73 & 72.8 & 72 & 72.8 & 0.000792 \\
\hline 505.08002 & 3.82302929 & 14.5635785 & 1.79697072 & 14.48325 & 3935.25 & 3712.70362 & 72.9 & 72.8 & 71.9 & 72.8 & 0.000792 \\
\hline 510.02002 & 3.825533037 & 14.5635785 & 1.79597072 & 14.4825 & 3935.5 & 3699.04668 & 72.9 & 72.7 & 72 & 72.8 & 0.000792 \\
\hline 515.02002 & 3.825533037 & 14.5650777 & 1.79597072 & 14.4825 & 3935.5 & 3709.72392 & 72.9 & 72.7 & 72 & 72.8 & 0.000792 \\
\hline 520.02002 & 3.82302929 & 14.5643281 & 1.79597072 & 14.48175 & 3935.5 & 3703.51622 & 72.9 & 72.7 & 72 & 72.8 & 0.000793 \\
\hline 525.04001 & 3,81490078 & 14.56058 & 1.79634528 & 14.4825 & 3945.25 & 3701.77806 & 73 & 72.7 & 72 & 72.8 & 0.000793 \\
\hline 530.08002 & 3.82177875 & 14.5635785 & 1.79534528 & 14.48325 & 3945.5 & 3705.25438 & 72.9 & 72.7 & 71.9 & 72.8 & 0.000794 \\
\hline 535.03 & 3.81615132 & 14.5643281 & 1.79597072 & 14.4825 & 3945.25 & 3705.50268 & 72.9 & 72.7 & 72 & 72.8 & 0.000794 \\
\hline 540.03 & 3.83240834 & 14.5643281 & 1.79534528 & 14.4825 & 3945 & 3710.22054 & 72.9 & 72.7 & 72 & 72.8 & 0.000794 \\
\hline 545.02002 & 3.8123997 & 14.5643281 & 1.79471984 & 14.48325 & 3945.75 & $\mathbf{3 7 1 1 . 9 5 8 6 9}$ & 72.9 & 72.7 & 72 & 72.8 & 0.000793 \\
\hline 550.03 & 3.81052389 & 14.5643281 & 1.79534528 & 14.48325 & 3945.25 & 3707.73746 & 72.9 & 72.7 & 72 & 72.8 & 0.000793 \\
\hline 555.02002 & 3.82615564 & 14.5643281 & 1.79534528 & 14.4825 & 3946 & 3703.26791 & 72.9 & 72.7 & 72 & 72.8 & $0 . \overline{000794}$ \\
\hline 560.03 & 3.81927767 & 14.5643281 & 1.79471984 & 14.4825 & 3945.5 & 3704.75776 & 72.9 & 72.7 & 72 & 72.8 & 0.000794 \\
\hline 565.02002 & 3.8374105 & 14.5628289 & 1.79534528 & 14.4825 & 3945.5 & 3701.77806 & 72.9 & 72.7 & 72 & 72.8 & 0.000794 \\
\hline 570.02002 & 3.82177875 & 14.5620792 & 1.79534528 & 14.4825 & 3945.5 & 3703.26791 & 72.9 & 72.8 & 72.1 & 72.8 & 0.000794 \\
\hline 575.02002 & 3.80864808 & 14.56058 & 1.79534528 & 14.48175 & 3945.5 & 3707.48915 & 72.9 & 72.7 & 72.1 & 72.8 & 0.000793 \\
\hline 580.02002 & 3.82115348 & 14.5598304 & 1.79534528 & 14.481 & 3945.5 & 3702.27468 & 72.9 & 72.8 & 72.2 & 72.8 & 0.000795 \\
\hline 585.02002 & 3.80927335 & 14.56058 & 1.79597072 & 14.48175 & 3945.5 & 3703.26791 & 72.9 & 72.8 & 72.2 & 72.8 & 0.000794 \\
\hline 590.02002 & 3.83428415 & 14.5635785 & 1.79597072 & 14.48175 & 3955.5 & 3701.52976 & 72.9 & 72.8 & 72.1 & 72.7 & 0.000795 \\
\hline 595.03 & 3.81927767 & 14.5643281 & 1.79534528 & 14.4825 & 3955 & 3707.24084 & .73 & 72.8 & 72.1 & 72.7 & 0.000795 \\
\hline 600.02002 & 3.81552605 & 14.5628289 & 1.79597072 & 14.4825 & 3955.75 & 3707.24084 & 73 & 72.7 & 72.1 & 72.8 & 0.000795 \\
\hline 605.02002 & 3.81927767 & 14.5620792 & 1.79534528 & 14.48175 & 3955 & 3706.99253 & 73 & 72.8 & 72.1 & 72.7 & 0.000795 \\
\hline
\end{tabular}




\begin{tabular}{|c|c|c|c|c|c|c|c|c|c|c|c|}
\hline SECONNDSI & MAIN DUCT & MAIN DUCT & GAS INJECT & GAS INJECT & NEWPORT & SMC & AMEIENT & GAS INJECT & \begin{tabular}{|l|} 
MAIN DUCT \\
TEM
\end{tabular} & \begin{tabular}{|l|l} 
WEC CELL & \\
TEMP &
\end{tabular} & \begin{tabular}{|l|} 
WEC CELLL \\
NOLTS
\end{tabular} \\
\hline & DIFF (IN H2O & ABS (PSIA) & DIFF (1N H2O) & $A B S$ (PSIA) & (PPM) & (PPM) & & & & & \\
\hline 610.02002 & 3.82365456 & 14.5620792 & 1.79597072 & 14,48325 & 3955.25 & 3706.24761 & 73.1 & 72.8 & 72.1 & 72.7 & 0.000795 \\
\hline 615.05002 & 3.82990726 & 14.5628289 & 1.79534528 & 14.4825 & 3955.5 & 3704.50945 & 73.2 & 72.8 & 72.2 & 72.7 & 0.000794 \\
\hline 620.08002 & 3.81927767 & 14.5643281 & 1.79597072 & 14.48325 & 3955.75 & 3700.78483 & 73.2 & 72.8 & 72.2 & 72.7 & 0.000795 \\
\hline 625.02002 & 3.81490078 & 14.5635785 & 1.79534528 & 14.48325 & 3956 & \begin{tabular}{|l|}
3703.76453 \\
\end{tabular} & 73.3 & 72.8 & 72.1 & 72.7 & 0.000795 \\
\hline 630.03 & 3.82553037 & 14.5643281 & 1.79534528 & 14.48325 & 3955.5 & 3708.979 & 73.3 & 72.8 & 72.1 & 72.7 & 0.000795 \\
\hline 635.07001 & 3.82177875 & 14.5643281 & 1.79534528 & 14.4825 & 3955.5 & 3707.24084 & 73.3 & 72.8 & 72.1 & 72.7 & 0.000794 \\
\hline 640.02002 & 3.80864808 & 14.5643281 & 1.79534528 & 14.48325 & 3955.5 & 3712.207 & 73.3 & 72.8 & 72.2 & 72.7 & 0.000795 \\
\hline 645.04001 & 3.81552605 & 14.5643281 & 1.79471984 & 14.4825 & 3948.75 & 3710.71715 & 73.2 & 72.8 & 72.2 & 72.8 & 0.000794 \\
\hline 650.09 & 3.81927767 & 14.5643281 & 1.79597072 & 14.48325 & 3945.25 & 3710.22054 & 73.2 & 72.8 & 72.2 & 72.7 & 0.000794 \\
\hline 655.02002 & 3.81490078 & 14.5650777 & 1.79597072 & 14.4825 & 3955.5 & 3718.91132 & 73.3 & 72.8 & 72.2 & 72.8 & 0.000794 \\
\hline 660.02002 & 3.83178307 & 14.5635785 & 1.79471984 & 14.4825 & 3955.5 & 3716.92485 & 73.2 & 72.8 & 72.1 & 72.8 & 0.000795 \\
\hline 665.08002 & 3.81365024 & 14.5643281 & 1.79534528 & 14.4825 & 3955.75 & 3709.2273 & 73.2 & 72.8 & 72 & 72.8 & 0,000795 \\
\hline 670.02002 & 3.80927335 & 14.5643281 & 1.79471984 & 14.4825 & 3955.75 & 3701.52976 & 73.1 & 72.8 & 72 & 72.8 & 0.000795 \\
\hline 675.06 & 3.80864808 & 14.5650777 & 1.79534528 & 14.4825 & 3955.25 & 3705.50268 & 73.1 & 72.8 & 72.1 & 72.8 & 0.000795 \\
\hline 680.05002 & 3.806147 & 14.5643281 & 1.79534528 & 14.4825 & 3955.25 & 3693.08728 & 73.1 & 72.8 & 72.2 & 72.8 & 0.000797 \\
\hline 685.02002 & 3.80864808 & 14.5598304 & 1.79534528 & 14.4825 & 3956 & 3708.73069 & 73.1 & 72.8 & 72.1 & 72.8 & 0.000796 \\
\hline 690.07001 & 3.80864808 & 14.5643281 & 1.79597072 & 14.4825 & 3955.5 & 3717.17316 & 73.1 & 72.8 & 72.2 & 72.8 & 0.000796 \\
\hline 695.02002 & 3.81302497 & 14.5635785 & 1.79534528 & 14.4825 & 3965.25 & 3716.92485 & 73 & 72.8 & 72.1 & 72.7 & 0.000796 \\
\hline 700.02002 & 3.81490078 & $14.5650 \mathrm{Tt7}$ & 1.79597072 & 14.48175 & 3965.5 & 3710.96546 & 73 & 72.8 & 72.1 & 72.7 & 0.000795 \\
\hline 705.05002 & 3.83428415 & 14.5650777 & 1.79597072 & 14.4825 & 3965.5 & 3712.95192 & 73 & 72.8 & 72.1 & 72.7 & 0.000796 \\
\hline 710.02002 & 3.81302497 & 14.5628289 & 1.79597072 & 14.4825 & 3965.75 & 3718.4147 & 73 & 72.9 & 72.1 & 72.8 & 0.000796 \\
\hline 715.02002 & 3.86117076 & 14.5635785 & 1.79534528 & 14.4825 & 3965.5 & 3707.98576 & 73.1 & 72.9 & 72.1 & 72.7 & 0.000796 \\
\hline 720.02002 & 3.81740186 & 14.5643281 & 1.79534528 & 14.48175 & 3965.75 & 3702.27468 & 73 & 72.9 & 72.1 & 72.7 & 0.000795 \\
\hline 725.08002 & 3.8123999 & 14.5635785 & 1.79597072 & 14.4825 & 3965.75 & 3706.49592 & 73.1 & 72.9 & 72.1 & 72.8 & 0.000797 \\
\hline 730.02002 & 3.81052389 & 14.5635785 & t.79659616 & 14.48325 & 3966 & 3699.04668 & 73 & 72.9 & 72.2 & 72.8 & 0.000796 \\
\hline 735.02002 & 3.82990726 & 14.5620792 & 1.79597072 & 14.4825 & 3965.75 & 3709.2273 & 73 & 72.9 & 72.1 & 72.7 & 0.000797 \\
\hline 740.02002 & 3.82928199 & 14.5628289 & 1.79597072 & 14.4825 & 3965.5 & 3709.2273 & 73 & 72.9 & 72.2 & 72.8 & 0,000797 \\
\hline 745.02002 & 3.81990294 & 14.5643281 & 1.79597072 & 14.48175 & 3965.75 & 3709.47561 & 73 & 72.9 & 72.1 & 72.8 & 0.000797 \\
\hline 750.06 & 3.81427551 & 14.5613296 & 1.79534528 & 14.481 & 3966 & \begin{tabular}{|c|}
3715.1867 \\
\end{tabular} & 73 & 72.9 & 72.2 & 72.7 & 0.000795 \\
\hline 755.02002 & 3.82803145 & 14.5613296 & 1.79597072 & 14.4825 & 3957 & 3711.21377 & 73 & 72.9 & 72.2 & $\overline{72.7}$ & 0.000794 \\
\hline 760.02002 & 3.84491374 & 14.5628289 & 1.79471984 & 14.48175 & 3960.25 & 3708.979 & 73.1 & 72.9 & 72.2 & 72.8 & 0.000796 \\
\hline 765.03 & 3.82678091 & 14.5635785 & 1.79597072 & 14.4825 & 3955.75 & \begin{tabular}{|l|}
3712.207 \\
\end{tabular} & 73.2 & 72.9 & 72.2 & 72.8 & 0.000796 \\
\hline 770.06 & 3.83178307 & 14.5620792 & 1.79471984 & 14.4825 & 3955.5 & 3705.25438 & 73.2 & 72.9 & 72.2 & 72.8 & 0.000796 \\
\hline 775.02002 & 3.82803145 & 14,56058 & 1.79597072 & 14.4825 & 3955.75 & 3691.59744 & 73.3 & 72.9 & 72.2 & 72.8 & 0.000796 \\
\hline 780,04001 & 3.82553037 & 14.5628289 & 1.79534528 & 14.4825 & 3966 & 3698.30175 & 73.3 & 72.9 & 72.2 & 72.7 & 0.000797 \\
\hline 785.02002 & 3.87117508 & 14.5628289 & 1.79597072 & 14.48175 & 3965.75 & 3702.52299 & 73.3 & 72.9 & 72.2 & 72.8 & 0.000797 \\
\hline 790.02002 & 3.80989862 & 14.5590808 & 1.79597072 & 14.48175 & 3965.75 & 3707.48915 & 73.3 & 72.9 & 72.2 & 72.7 & 0.000797 \\
\hline 795.02002 & 3.80864808 & 14.56058 & 1.79659616 & 14.4825 & 3965.5 & 3716.67654 & 73.3 & 72.9 & 72.2 & 72.7 & 0.000796 \\
\hline 800.04001 & 3.8249051 & 4.5643281 & 1.79659616 & 14.48325 & 3965.25 & 3718.4147 & 73.4 & 72.9 & 72.2 & 72.7 & 0.000797 \\
\hline 805.02002 & 3.83615996 & 14.5635785 & 1.79659616 & 14.4825 & 3965.5 & 3713.69685 & 73.4 & 72.9 & 72.2 & 72.7 & 0.000797 \\
\hline 810,02002 & 3.81552605 & 14.5613296 & 1.79597072 & 14.48175 & 3965.5 & 3716.67654 & 73.4 & 72.9 & 72.2 & 72.7 & 0.000797 \\
\hline 815.10001 & 3.83178307 & 14.56058 & 1.79659616 & 14.4825 & 3965.5 & 3711.46208 & 73.4 & 72.9 & 72.2 & 72.7 & 0.000797 \\
\hline
\end{tabular}




\begin{tabular}{|c|c|c|c|c|c|c|c|c|c|c|c|}
\hline SECONDS & \begin{tabular}{|l|} 
MAIN DUCT \\
DIFF (iN H2O
\end{tabular} & $\begin{array}{l}\text { MAIN DUCT } \\
\text { ABS (PSIA) }\end{array}$ & \begin{tabular}{|l|} 
GAS INJECT \\
DIFF (INH2O) \\
\end{tabular} & \begin{tabular}{|l|} 
GAS INJECT \\
$A B S$ (PSIA)
\end{tabular} & \begin{tabular}{|l|} 
NEWPORT \\
(PPM)
\end{tabular} & \begin{tabular}{|l|} 
SMC \\
(PPM)
\end{tabular} & \begin{tabular}{|l} 
AMBIENT \\
TEMP.(F)
\end{tabular} & \begin{tabular}{|l|} 
GAS INJECT \\
TEMP.(F)
\end{tabular} & \begin{tabular}{|l|} 
MAIN DUCT \\
TEMP,(F)
\end{tabular} & $\begin{array}{l}\text { WEC CELL } \\
\text { TEMP.(F) }\end{array}$ & \begin{tabular}{|l|} 
WEC CELL \\
(VOLTS)
\end{tabular} \\
\hline & & & & & & & & & & & \\
\hline 820.02002 & 3.81677659 & 14.56058 & 1.79597072 & 14.48175 & 3965.5 & 3706.99253 & 73.4 & 72.9 & 72.2 & 72.7 & 0.000797 \\
\hline 825.07001 & 3.81490078 & 14.56058 & 1.79597072 & 14.48325 & 3965.5 & 3706.74422 & 73.5 & 72.9 & 72.3 & 72.7 & 0.000797 \\
\hline 830.08002 & 3.8186524 & 14.5620792 & 1.79534528 & 14.4825 & 3965.75 & 3713.44854 & 73.4 & 72.9 & 72.2 & 72.7 & 0.000797 \\
\hline 835.02002 & 3.8123997 & 14.5613296 & 1.79597072 & 14.4825 & 3965.75 & 3711.21377 & 73.4 & 72.9 & 72.3 & 72.7 & 0.000796 \\
\hline 840.03 & 3.84303793 & 14.5635785 & 1.79597072 & 14.48325 & 3965.75 & 3701.28145 & 73.3 & 73 & 72.3 & 72.7 & 0.000797 \\
\hline 845.02002 & 3.81677659 & 14.5598304 & 1.79597072 & 14.48175 & 3065.5 & 3718.4147 & 73.3 & 72.9 & 72.4 & 72.7 & 0.000796 \\
\hline 850.02002 & 3.83803577 & 14.56058 & 1.79534528 & 14.4825 & 3965.75 & 3719.40793 & 73.2 & 72.9 & 72.4 & 72.7 & 0.000796 \\
\hline 855.03 & 3.80864808 & 14.5613296 & 1.79597072 & 14.4825 & 3965.5 & 3724.12578 & 73.3 & 72.9 & 72.3 & 72.7 & 0.000797 \\
\hline 860.02002 & 3.81490078 & 14.5598304 & 1.79534528 & 14.4825 & 3965.75 & 3715.93162 & 73.3 & 73 & 72.4 & 72.7 & 0.000797 \\
\hline 865.02002 & 3.8374105 & 14.5613296 & 1.79534528 & 14.4825 & 3975 & 3701.52976 & 73.3 & 73 & 72.4 & 72.7 & 0.000798 \\
\hline 870.06 & 3.81927767 & 14.5620792 & 1.795997072 & 14.4825 & 3975.5 & 3706.49592 & 73.4 & 72.9 & 72.3 & 72.8 & 0.000797 \\
\hline 875.11002 & 3.82740618 & 14.5613296 & 1.79534528 & 14.4825 & 3965.5 & 3709.47561 & 73.4 & 73 & 72.4 & 72.8 & 0.000797 \\
\hline 880.02002 & 3.83178307 & 14.5613296 & 1.79534528 & 14.4825 & 3965.25 & 3706.24761 & 73.4 & 72.9 & 72.3 & 72.7 & 0.000797 \\
\hline 885.02002 & 3.82427983 & 14.5628289 & 1.79534528 & 14.4825 & 3975.75 & 3711.71038 & 73.3 & 73 & 72.4 & 72.7 & 0.000798 \\
\hline 890.02002 & 3.82553037 & 14.5613296 & 1.79597072 & 14.4825 & 3975.5 & 3715.93162 & 73.3 & 72.9 & 72.3 & 72.8 & 0.000798 \\
\hline 895.02002 & 3.81302497 & 14.5613296 & 1.79697072 & 14.4825 & 3975.25 & 3711.46208 & 73.3 & 73 & 72,3 & 72.8 & 0.000798 \\
\hline 900.02002 & 3.81677659 & 14.5613296 & 1.79534528 & 14.4825 & 3975.75 & 3706.99253 & 73.4 & 73 & 72.3 & 72.8 & 0.000799 \\
\hline 905.04001 & 3.80802281 & 14.5620792 & 1.79597072 & 14.4825 & 3975.5 & 3697.30852 & 73.4 & 73 & 72.3 & 72.7 & 0.000798 \\
\hline 910.02002 & 3.82553037 & 14.5613296 & 1.79659616 & 14.4825 & 3975.5 & 3701.28145 & 73.4 & 73 & 72.3 & 72.8 & 0.000799 \\
\hline 915.02002 & 3.80677227 & 145613296 & 1.79534528 & 14.48175 & 3975.5 & 3704.50945 & 73.3 & 73 & 72.3 & 72.8 & 0.000798 \\
\hline
\end{tabular}




\begin{tabular}{|c|c|c|c|c|c|c|c|c|c|c|c|c|}
\hline \multicolumn{12}{|c|}{$\begin{array}{l}\text { TEST: EX202257 } 25 \text { Feb } 1997 \text { WEC-SPOOL } \\
\text { Measured Hydrogen Concentration }=4217 \mathrm{ppm}\end{array}$} & \\
\hline SECOND & MAIN DUCT & MAIN DUCT & GAS INJECT & GAS INJECT & NEWPORT & SMC & AMBIENT & GAS INJECT & MAIN DUCT & WEC CELL & |WEC CELL & \\
\hline & DIFF (IN H2O & ABS (PSIA) & DIFF (IN H2O & ABS (PSIA) & (PPM) & (PPM) & TEMP.(F) & TEMP.(F) & TEMP.(F) & TEMP.(F) & (VOLTS) & \\
\hline & & & & & & & & & & & & \\
\hline 0.01001 & 3.8249051 & 14.55908075 & 0.661922912 & 14.499 & $\overline{51.5}$ & -0.0224308 & 73.3 & 72.6 & 72.5 & 72.7 & 0.000214 & \\
\hline 5.019989 & 3.81490078 & 14.55908075 & 1.99611152 & 14.49225 & 51.4 & 136.10001 & 73.3 & 72.6 & 72.5 & 72.8 & 0.000216 & \\
\hline 10.01999 & 3.84866536 & 14.558333113 & 1.99548608 & 14.4915 & 224.925 & 1936.8048 & 73.3 & 72.8 & 72.3 & 72.7 & 0.000259 & \\
\hline 15.01999 & 3.83428415 & 14.55908075 & 1.99548608 & 14.49 & 702.25 & 3107.577 & 73.2 & 72.8 & 72.3 & 72.8 & 0.000343 & \\
\hline 20.01999 & 3.83803577 & 14.55983037 & 1.99923872 & 14.4885 & 1302.25 & 3582.8385 & 73.3 & 72.8 & 72.3 & 72.7 & 0.000433 & \\
\hline 25.01999 & 3.83928631 & 14.55833113 & 1.99923872 & 14.49 & 1920 & 3760.6271 & 73.4 & 72.8 & 72.3 & 72.7 & 0.000512 & \\
\hline 30.01999 & 3.80552173 & 14.55908075 & 1.99923872 & 14.49075 & 2328.75 & 3835.1195 & 73.4 & 72.8 & 72.3 & 72.7 & 0.000576 & \\
\hline 35.01999 & 3.82302929 & 14.55833113 & 1.99923872 & 14.48925 & 2723.75 & 3883.7878 & 73.5 & 72.8 & 72.3 & 72.7 & 0.000627 & \\
\hline 40.01999 & 3.83991158 & 14.55983037 & 1.99986416 & 14.49075 & 2994.25 & 3905.1423 & 73.4 & 72.8 & 72.4 & 72.7 & 0.000669 & \\
\hline 45.01999 & 3.8249051 & 14.55833113 & 2.0004896 & 14.49 & 3254.25 & 3913.0882 & 73.4 & 72.8 & 72.4 & 72.8 & 0.0007 & \\
\hline 50.01999 & 3.82115348 & 14.55908075 & 1.99923872 & 14.48925 & 3414.5 & 3922.7722 & 73.3 & 72.8 & 72.4 & 72.7 & 0.000725 & \\
\hline 55.01999 & 3.81490078 & 14.56058 & 1.99986416 & 14.49 & 3596.25 & 3957.7836 & 73.3 & 72.8 & 72.4 & 72.8 & 0.000746 & \\
\hline 60.01999 & 3.81114916 & 14.55983037 & 2.00111504 & 14.49 & 3674.75 & 3955.0522 & 73.3 & 72.8 & 72.4 & 72.7 & 0.000761 & \\
\hline 65.01999 & 3.82302929 & 14.55983037 & 2.0004896 & 14.49 & 3784.75 & 3967.7159 & 73.3 & 72.8 & 72.4 & 72.7 & 0.000774 & \\
\hline 70.01999 & 3.82115348 & 14,55983037 & 2.00174048 & 14.49 & 3855.25 & 3984.6009 & 73.3 & 72.8 & 72.4 & 72.7 & 0.000786 & \\
\hline 75.01999 & 3.81552605 & 14.55908075 & 2.00174048 & 14.48925 & 3925 & 4003.9689 & 73.3 & 72.8 & 72.3 & 72.8 & 0.000794 & \\
\hline 80.01999 & 3.80864808 & 14.55908075 & 2.00174048 & 14.49 & 3974.5 & 3996.5197 & 73.2 & 72.8 & 72.3 & 72.8 & 0.000802 & \\
\hline 85.01999 & 3.81740186 & 14.56058 & 2.00174048 & 14.49 & 4005.5 & 4009.9283 & 73.2 & 72.8 & 72.3 & 72.7 & 0.000809 & \\
\hline 90.01999 & 3.80864808 & 14.55983037 & 2.00111504 & 14.49 & 4060.25 & 4013.1563 & 73.2 & 728 & 72.4 & 72.7 & 0.000813 & \\
\hline 95.01999 & 3.8186524 & 14.55983037 & 200174048 & 14.48925 & 4085.25 & 4036.4972 & 73.3 & 72.8 & 72.4 & 72.7 & 0.000818 & \\
\hline 100.02 & 3.83178307 & 14.55983037 & 2.00174048 & 14.48925 & 4115.25 & 4034.0142 & 73.3 & 72.8 & 72.5 & 72.7 & 0.000822 & \\
\hline 105.02 & 3.82115348 & 14.55983037 & 2.00111504 & 14.48925 & 4135.25 & 4045.933 & 73.3 & 72.8 & 72.4 & 72.7 & 0.000825 & \\
\hline 110.02 & 3.8186524 & 14.56058 & 20036168 & 14.4885 & 4155 & 4057.3551 & 73.4 & 72.9 & 72.3 & 72.8 & 0.000828 & \\
\hline 115.02 & 3.81927767 & 14.56058 & 2.0036168 & 14.48925 & 4175.5 & 4068.2807 & 73.3 & 72.8 & 72.4 & 72.8 & 0.000829 & \\
\hline 120.02 & 3.81052389 & 14.56207924 & 2.00424224 & 14.49 & 4195.25 & 4067.2874 & 73.4 & 72.8 & 72.3 & 72.7 & 0.000831 & \\
\hline 125.02 & 3.81427551 & 14.55983037 & 2.00424224 & 14.49 & 4205.5 & 4068.2807 & 73.3 & 72.8 & 72.3 & 72.8 & 0.000833 & \\
\hline 130.02 & 3.81677659 & 14.55833113 & 2.00486768 & 14.48925 & 4215.25 & 4081.6893 & 73.3 & 72.8 & 72.3 & 72.7 & 0.000836 & \\
\hline 135.02 & 3.8186524 & 14.55833113 & 2.00236592 & 14.49 & 4225.75 & 4080.6961 & 73.3 & 72.8 & 72.3 & $\overline{72.7}$ & 0.000837 & \\
\hline 140.02 & 3.81677659 & 14.55908075 & 2.00424224 & 14.48925 & 4245.5 & 4078.7096 & 73.2 & 72.9 & 72.3 & 72.7 & 0.000838 & \\
\hline 145.02 & 3.80802281 & 14.55983037 & 2.0036468 & 14.49 & 4255.75 & 4084.1724 & 73.2 & 72.9 & 72.3 & 72.7 & 0.00084 & \\
\hline 150.02 & 3.83178307 & 14.55983037 & 2.00299136 & 14.49 & 4255.25 & 4087.897 & 73.1 & 72.8 & 72.3 & 72.7 & 0.000841 & \\
\hline 155.02 & 3.83178307 & 14.56132962 & 2.0036168 & 14.49 & 4265.75 & 4095.3462 & 73.1 & 72.9 & 72.3 & 72.7 & 0.000842 & \\
\hline 160.02 & 3.82678091 & 14.55983037 & 2.00424224 & 14.48925 & 4275.5 & 4102.0506 & 73.1 & 72.9 & 72.3 & 72.7 & 0.000843 & \\
\hline 165.02 & 3.81052389 & 14.56058 & 2.00299136 & 14.4885 & 4285.5 & 4102.2989 & 73.2 & 72.9 & 72.2 & 72.7 & 0.000844 & \\
\hline 170.02 & 3.80802281 & \begin{tabular}{|l|}
14.56058 \\
\end{tabular} & 2.00424224 & 14.48925 & 4285.75 & 4108.5066 & 73.2 & 72.9 & 72.2 & 72.8 & 0.000846 & \\
\hline 175.02 & 3.80989862 & 14.55983037 & 2.0036168 & 14.48925 & 4295.75 & 4107.0167 & 73.2 & 72.8 & 72.2 & 72.8 & 0.000846 & \\
\hline 180.02 & 3.83490942 & 14.55983037 & 2.00299136 & 14.48925 & 4305.75 & 4116.2041 & 73.2 & 72.9 & 72.3 & 72.8 & 0.000847 & \\
\hline 185.02 & 3.8123997 & 14.55608226 & 2.00424224 & 14.48925 & 4306 & 4105.7752 & 73.2 & 72.9 & 72.2 & 72.8 & 0.000848 & \\
\hline
\end{tabular}




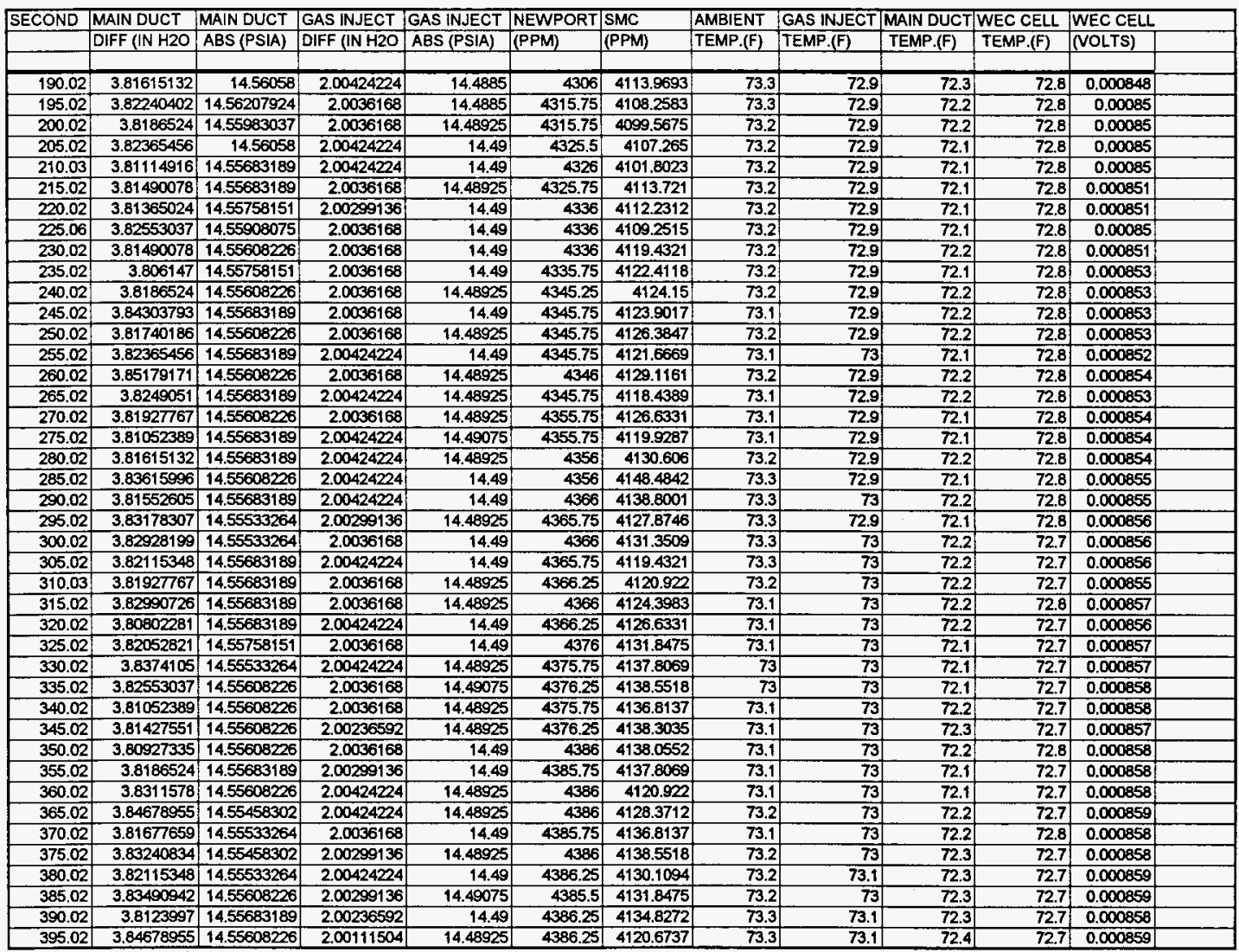




\begin{tabular}{|c|c|c|c|c|c|c|c|c|c|c|c|}
\hline SECOND & MAIN DUCT & MAIN DUCT & GAS INJECT & GAS INJECT & NEWPORT & SMC & AMBIENT & GAS INJECT & MAIN DUCT & WEC CELL & WEC CELL \\
\hline & DIFF (IN H2O & ABS (PSIA) & DIFF (IN H2O & ABS (PS|A) & (PPM) & (PPM) & TEMP.(F) & TEMP.(F) & TEMP.(F) & TEMP.(F) & (VOLTS) \\
\hline 400.02 & 3.6311578 & 14.55683189 & 2.0036168 & 14.49 & 4385.25 & 4120.922 & 73.3 & 73.1 & 72.3 & 727 & 0,000858 \\
\hline 405.02 & 3.85679387 & 14.55683189 & 2.00174048 & 14.49075 & 4386.25 & 4135.5721 & 73.4 & 73.1 & 72.3 & 72.7 & 0.000858 \\
\hline 410.02 & 3.81927767 & 14.55683189 & 2.00111504 & 14.49 & 4386 & 4141.2832 & 73.3 & 73.1 & 72.3 & 72.7 & 0.000859 \\
\hline 415.02 & 3.8186524 & 14.55683189 & 2.00111504 & 14.48925 & 4386 & 4131.1026 & 73.4 & 73.1 & 72.2 & 72.7 & 0.000859 \\
\hline 420.02 & 3.82740618 & 14.55683189 & 2.0004896 & 14.49 & 4386 & 4132.0958 & 73.3 & 73.1 & 72.3 & 72.7 & 0.000859 \\
\hline 425.02 & 3.81615132 & 14.55608226 & 2.0004896 & 14.49 & 4385.75 & 4125.8881 & 73.3 & 73.1 & 72.4 & 72.7 & 0.000859 \\
\hline 430.02 & 3.82052821 & 14.55683189 & 2.00174048 & 14.48925 & 4395.75 & 4134.8272 & 73.3 & 73.1 & 72.3 & 72.7 & 0.000859 \\
\hline 435.02 & 3.83553469 & 14.55758151 & 2.00111504 & 14.48925 & 4395.75 & 4127.378 & 73.3 & 73.1 & 72.3 & 72.7 & 0.000861 \\
\hline 440.02 & 3.83178307 & 14.55608226 & 2.00111504 & 14.48925 & 4396.25 & 4118.4389 & 73.3 & 73.2 & 72.2 & 72.7 & 0.00086 \\
\hline 445.02 & 3.81802713 & 14.55683189 & 2.00174048 & 14.48925 & 4395.75 & 4124.3983 & 73.3 & 73.1 & 72.3 & 72.7 & 0.000861 \\
\hline 450.02 & 3.81552605 & 14.55758151 & 2.00174048 & 14.49 & 4396.25 & 4142.2765 & 73.4 & 73.1 & 72.3 & 72.8 & 0.00086 \\
\hline 455.07 & 3.8123997 & 14.55683189 & 2.00111504 & 14.48925 & 4406 & 4132.8408 & 73.3 & 73.2 & 72.2 & 72.7 & 0.000861 \\
\hline 460.02 & 3.80864808 & 14.55608226 & 2.00111504 & 14.48925 & 4406.25 & 4129.1161 & 73.3 & 73.1 & 72.2 & 72.7 & 0.00086 \\
\hline 465.05 & 3.82553037 & 14.55758151 & 2.00174048 & 14.48925 & 4396 & 4128.6195 & 73.3 & 73.2 & 72.2 & 72.8 & 0.000861 \\
\hline 470.02 & 3.81490078 & 14.55758151 & 2.00236592 & 14.49 & 4405.5 & 4130.8543 & 73.3 & 73.2 & 72.2 & 72.7 & 0.000862 \\
\hline 475.04 & 3.81677659 & 14.55533264 & 2.00174048 & 14.49 & 4405.75 & 4133.3374 & 73.4 & 73.1 & 72.3 & 72.8 & 0.000862 \\
\hline 480.02 & 3.84178739 & 14.55833113 & 2.00174048 & 14.49 & 4406 & 4136.0688 & 73.3 & 73.1 & 72.2 & 72.8 & 0.000862 \\
\hline 485.02 & 3.8186524 & 14.55683189 & 2.00111504 & 14.48925 & 4406.25 & 4134.0823 & 73.3 & 73.2 & 72.1 & 72.8 & 0.000862 \\
\hline 490.02 & 3.82615564 & \begin{tabular}{|l|}
14.55908075 \\
\end{tabular} & 2.00174048 & 14.48925 & 4406.25 & 4130.1094 & 73.3 & 73.2 & 72.1 & 72.8 & 0.000862 \\
\hline 495.1 & 3.81302497 & 14.55683189 & 2.00174048 & 14.49 & 4406.25 & 4127.378 & 73.4 & 73.2 & 72.2 & 72.8 & 0.000862 \\
\hline 500.02 & 3.81740186 & 14.55683189 & 2.00111504 & 14.48925 & 4406.25 & 4135.8204 & 73.3 & 73.1 & 72.1 & 72.7 & 0.000861 \\
\hline 505.02 & 3.80864808 & 14.55683189 & 2.00111504 & 14.4885 & 4405.75 & 4126.8814 & 73.3 & 73.1 & 72.2 & 72.7 & 0.000863 \\
\hline 510.02 & 3.81802713 & 14.55608226 & 2.00111504 & 14.4885 & 4416.25 & 4144.7595 & 73.3 & 73.2 & 72.1 & 72.7 & 0.000863 \\
\hline 515.02 & 3.82553037 & 14.556833189 & 2.00174048 & 14,48925 & 4416 & 4143.0214 & 73.3 & 73.2 & 72.1 & 72.7 & 0.000862 \\
\hline 520.02 & 3.84178739 & 14.55608226 & 2.00174048 & 14.4885 & 4416 & 4129.8611 & 73.3 & 73.2 & 72.2 & 72.7 & 0.000863 \\
\hline 525.02 & 3.81740186 & 14.55608226 & 2.00174048 & 14.48925 & 4416 & 4130.1094 & 73.3 & 73.2 & 72.1 & 72.7 & 0.000863 \\
\hline 530.04 & $3.8142755 t$ & 14.55608226 & 2.00174048 & 14.48925 & 4416.25 & 4139.0485 & 73.3 & 73.2 & 72.1 & 72.7 & 0.000862 \\
\hline 535.02 & 3.81927767 & 14.55533264 & 2.00236592 & 14.48925 & 4416 & 4129.3644 & 73.3 & 73.2 & 72.1 & 72.8 & 0.000863 \\
\hline 540.02 & 3.82553037 & 14.55608226 & 2.00111504 & 14.4885 & 4415.75 & 4115.4592 & 73.3 & 73.2 & 72.2 & 72.8 & 0.000863 \\
\hline 545,02 & 3.8311578 & 14.55533264 & 2.00174048 & 14.48925 & 4415.75 & 4136.0688 & 73.3 & 73.2 & 72.2 & 72.8 & 0.000863 \\
\hline 550.02 & 3.8186524 & 14.55608226 & 2.00174048 & 14.48925 & 4415.75 & 4149.974 & 73.3 & 73.2 & 72.2 & 72.8 & 0.000863 \\
\hline 555.02 & 3.83178307 & 14.55533264 & 2.00174048 & 14.49 & 4416 & 4143.2697 & 73.3 & 73.2 & 72.2 & 72.8 & 0.000863 \\
\hline 560.04 & 3.8311578 & 14.55683189 & 2.00174048 & 14.49 & 4416.25 & 4133.3374 & 73.3 & 73.2 & 72.2 & 72.7 & 0.000862 \\
\hline 565.02 & 3.8249051 & 14.55533264 & 2.00111504 & 14,48925 & 4415.75 & 4129.3644 & 73.3 & 73.2 & 72.3 & 72.7 & 0.000861 \\
\hline 570.02 & 3.8374105 & 14.55608226 & 2.00111504 & 14.48925 & 4416 & 4122.9084 & 73.3 & 73.2 & 72.2 & 72.7 & 0.000861 \\
\hline 575.03 & 3.81990294 & 14.55608226 & 2.0004896 & 14.49 & 4415.75 & 4121.4186 & 73.3 & 73.2 & 72.2 & 72.7 & 0.000862 \\
\hline 580.02 & 3.83991158 & 14.55533264 & 2.00111504 & 14.48925 & 4416.25 & 4125.1432 & 73.3 & 73.2 & 72.3 & 72.7 & 0.000862 \\
\hline 585.02 & 3.84241266 & 14.55683189 & 2.00174048 & 14.49075 & 4416 & 4133.634 & 73.3 & 73.2 & 72,3 & 72.8 & 0.000863 \\
\hline 590.07 & 3.80927335 & 14.55608226 & 2.0004896 & 14.49075 & 4416.25 & 4129.1161 & 73.3 & 73.3 & 72.3 & 72.7 & 0,000863 \\
\hline 595.05 & 3.82865672 & 14.55608226 & 2.00111504 & 14.48925 & 4416 & 4126.8814 & 73.4 & 73.3 & 72.3 & 72.7 & 0.000863 \\
\hline 600.02 & 3.83303361 & 14.55533264 & 2.00174048 & 14.49 & 4415.75 & 4134.8272 & 73.3 & 73.3 & 72.3 & 72.8 & 0.000864 \\
\hline 605.02 & 3.81490078 & 14.55758151 & 2.00111504 & 14.49 & 4416.25 & 4124.3983 & 73.4 & 73.3 & 72.4 & 72.8 & 0.000862 \\
\hline
\end{tabular}




\begin{tabular}{|c|c|c|c|c|c|c|c|c|c|c|c|c|}
\hline SECOND & MAIN DUCT & MAIN DUCT & GAS INJECT & GAS INJECT & NEWPORT & SMC & AMBIENT & GAS INJECT & MAIN DUCT & WEC CELL & WEC CELL & \\
\hline & DIFF (IN H2O & ABS (PSIA) & DIFF (IN H2O & ABS (PSIA) & (PPM) & (PPM) & TEMP.(F) & TEMP.(F) & TEMP.(F) & TEMP.(F) & (VOLTS) & \\
\hline 61002 & 383053253 & 145500870 & 200111504 & 144885 & & 41358704 & & & & & & \\
\hline 610.02 & 3.83053253 & 14.55608226 & 2.00111504 & 14.4835 & 4415.75 & 4135.8204 & 73.5 & 73.3 & 72.4 & 72.7 & 0.000863 & \\
\hline 615.02 & 3.83178307 & 14.55683189 & 2.00111504 & 14.48925 & 4416.25 & 4128.1229 & 73.5 & 73.3 & 72.4 & 72.7 & 0.000863 & \\
\hline 620.06 & 3.84241266 & 14.55608226 & 2.00111504 & 14.48925 & 4416.25 & 4130.8543 & 73.5 & 73.3 & 72.4 & 72.8 & 0.000864 & \\
\hline 625,02 & 3.82928199 & 14.55683189 & 2.00236592 & 14.48925 & 4416 & 4125.1432 & 73.5 & 73.3 & 72.4 & 72.7 & 0.000864 & \\
\hline 630.07 & 3.81927767 & 14.55533264 & 2.00111504 & 14.48925 & 4416 & 4128.3712 & 73.5 & 73.3 & 72.4 & 72.7 & 0.000864 & \\
\hline 635.02 & 3.83490942 & 14.55458302 & 2.00174048 & 14.49 & 4416 & 4138.5518 & 73.5 & 73.3 & 72.4 & 72.7 & 0.000863 & \\
\hline 640.02 & 3.81365024 & 14.55458302 & 2.00174048 & 14.48925 & 4416.25 & 4143.7663 & 73.4 & 73.3 & 72.5 & 72.7 & 0.000864 & \\
\hline 645.02 & 3.83053253 & 14.55608226 & 2.00111504 & 14.49 & 4415.75 & 4133.834 & 73.4 & 73.3 & 72.5 & 72.8 & 0.000864 & \\
\hline 650.02 & 3.84241266 & 14.55608226 & 2.00111504 & 14,48925 & 4416.25 & 4123.1567 & 73.4 & 73.3 & 72.5 & 72.8 & 0.000864 & \\
\hline 655.02 & 3.81677659 & 14.55533264 & 2.00111504 & 14.49 & 4416 & 4133.3374 & 73.5 & 73.3 & 72.5 & 72.7 & 0.000865 & \\
\hline 660.07 & 3.80864808 & 14.55683189 & 2.0004896 & 14.49 & 4416 & $4 \uparrow 39.7934$ & 73.5 & 73.3 & 72.5 & 72.7 & 0.000865 & \\
\hline 665.02 & 3.81427551 & 14.55608226 & 2.00111504 & 14.49 & 4416 & 4135.8204 & 73.5 & 73.3 & 72.4 & 72.7 & 0.000864 & \\
\hline 670.02 & 3.83240834 & 14.55608226 & 2.00174048 & 14.49 & 4425.75 & 4141,0349 & 73.5 & 73.3 & 72.5 & 72.7 & 0,000865 & \\
\hline 675.04 & 3.81365024 & 14.55608226 & 2.00236592 & 14.49 & 4426.25 & 4139.0485 & 73.6 & 73.3 & 72.5 & 72.8 & 0.000865 & \\
\hline 680.02 & 3.81177443 & 14.55608226 & 2.00174048 & 14.49075 & 4426 & 4130.606 & 73.6 & 73.3 & 72.5 & 72.7 & 0.000865 & \\
\hline 685.02 & 3.81427551 & 14.55233415 & 2.00174048 & 14.49075 & 4426.25 & 4123.6534 & 73.6 & 73.4 & 72.4 & 72.7 & 0.000864 & \\
\hline 700.02 & 3.8186524 & 14.55533264 & 2.00174048 & 14.49 & 4426.25 & 4122.1635 & 73.7 & 73.4 & 72.5 & 72.7 & 0.000864 & \\
\hline 705.07 & 3.81677659 & 14.55758151 & 2.00111504 & 14.49 & 4426 & 4125.8881 & 73.6 & 73.4 & 72.5 & 72.7 & 0.000864 & \\
\hline 710.02 & 3.8249051 & 14.5538334 & 1.99986416 & 14.49075 & 4426 & 4129.1161 & 73.6 & 73.4 & 72.4 & 72.7 & 0.000865 & \\
\hline 715.02 & 3.81927767 & 14.55683189 & 2.0004896 & 14.49 & 4426 & 4136.5654 & 73.5 & 73.4 & 72.4 & 72.8 & 0.000866 & \\
\hline 720.02 & 3.83178307 & 14.55683189 & 2.0004896 & 14.49 & 4426.25 & 4126.6331 & 73.3 & 73.4 & 72.3 & 72.7 & 0.000865 & \\
\hline 725.02 & 3.82678091 & 14.55608226 & 2.00174048 & 14.49 & 4426.25 & 4132.8408 & 73.4 & 73.4 & 72.3 & 72.7 & 0.000865 & \\
\hline 730.02 & 3.80989862 & 14.55308378 & 2.00111504 & 14.49 & 4426.25 & 4130.606 & 73.4 & 73.4 & 72.3 & $\overline{72.7}$ & 0.000865 & \\
\hline 735.03 & 3.82177875 & 14.55608226 & 2.00111504 & 14.49075 & 4426.25 & 4144.2629 & 73.4 & 73.4 & 72.4 & 72.8 & 0.000865 & \\
\hline 740.05 & 3.84053685 & 14.55608226 & 2.00174048 & 14.48925 & 4426 & 4137.3103 & 73.3 & 73.4 & 72.3 & 72.7 & 0,000865 & \\
\hline 745.02 & 3.8186524 & 14.55308378 & 2.00111504 & 14.49 & 4426.25 & 4134.3306 & 73.3 & 73,4 & 72.4 & 72.7 & 0.000865 & \\
\hline 750.02 & 3.80864808 & 14.55158453 & 2.00111504 & 14.49075 & 4426.25 & 4142.0281 & 73.3 & 73.4 & 72.4 & 72.7 & 0,000865 & \\
\hline 755.02 & 3.81927767 & 14.55308378 & 2.00174048 & 14.4915 & 4426.25 & 4146.0011 & 73.3 & 73.4 & 72.4 & 72.7 & 0.000866 & \\
\hline 760.02 & 3.82553037 & 14.5538334 & 2.00236592 & 14.49 & 4426.25 & 4133.3374 & 73.2 & 73.4 & 72.3 & 72.7 & 0.000865 & \\
\hline 765.06 & 3.81490078 & 14.55308378 & 2.00174048 & 14.49 & 4426.25 & 4137.062 & 73.3 & 73.4 & 72.3 & 72.7 & 0.000865 & \\
\hline 770.02 & 3.8186524 & 14.55308378 & 2.0004896 & 14.4885 & 4426.25 & 4125.6398 & 73.2 & 73.4 & 72.3 & 72.7 & 0.000865 & \\
\hline 775.02 & 3.81427551 & 14.5538334 & 2.00111504 & 14.487 & 4425.75 & 4128.3712 & 73.2 & 73.4 & 72.3 & 72.7 & 0.000865 & \\
\hline 780.03 & 3.81990294 & 14.5538334 & 2.00174048 & 14.487 & 4429.5 & 4133.5857 & 73.3 & 73.4 & 72.3 & 72.7 & 0.000865 & \\
\hline 785.02 & 3.81740186 & 14.55533264 & 2.00111504 & 14.49 & 4436.25 & 4121.4186 & 73.2 & 73.4 & 72.3 & 72.7 & 0.000866 & \\
\hline 800.02 & 3.83178307 & 14.55533264 & 2.00174048 & 14.48925 & 4425.75 & 4138.3035 & 73.2 & 73.4 & 72.3 & 72.7 & 0.000865 & \\
\hline 805.02 & 3.81927767 & 14.55233415 & 2.00111504 & 14.48925 & 4426.25 & 4141.0349 & 73.1 & 73.4 & 72.3 & 72.7 & 0.000865 & \\
\hline 810.07 & 3.84741482 & 14.55533264 & 2.00174048 & 44.49075 & 4426.25 & 4140.29 & 73.2 & 73.5 & 72.4 & 72.8 & 0.000864 & \\
\hline 815.02 & 3.82115348 & 14.55683189 & 2.00111504 & 14.4885 & 4426.25 & 4124.3983 & 73.2 & 73.5 & 72.3 & 72.7 & 0.000865 & \\
\hline
\end{tabular}




\begin{tabular}{|c|c|c|c|c|c|c|c|c|c|c|c|}
\hline \multirow[t]{2}{*}{ SECOND } & MAIN DUCT & MAIN DUCT & GAS INJECT & GAS INJECT & NEWPORT & SMC & AMBIENT & GAS INJECT & MAIN DUCT & WEC CELL & WEC CELLL \\
\hline & DIFF (IN H2O & ABS (PSIA) & DIFF (IN H2O & ABS (PS|A) & (PPM) & (PPM) & TEMP.(F) & TEMP.(F) & TEMP.(F) & TEMP.(F) & (VOLTS) \\
\hline 820.02 & 3.81677659 & 14.5538334 & 200111504 & 14.48775 & 4426.25 & 41224118 & 732 & 73.5 & 723 & 727 & \\
\hline 825.06 & 3.80114484 & 14.55683189 & 2.00111504 & 14.49 & 4426 & 4123.9017 & 73.2 & 73.5 & 72.2 & $\frac{12.8}{72.8}$ & $\begin{array}{l}0.000864 \\
0.000864\end{array}$ \\
\hline 830.02 & 3.82302929 & 14.55683189 & 2.0004896 & 14.48925 & 4426 & 4127.6263 & 73.3 & 73.5 & 72.3 & 72.7 & 0.000864 \\
\hline 835.02 & 3.84303793 & 14.55308378 & 2.00111504 & 14.49 & 4426.25 & 4129.1161 & 73.2 & 73.5 & 72.2 & 72.8 & 0.000865 \\
\hline 840.04 & 3.8186524 & 14.55608226 & 2.00174048 & 14.48625 & 4426.25 & 4140.29 & 73.2 & 73.5 & 72.2 & 72.8 & 0.000865 \\
\hline 845.03 & 3.81802713 & 14.55308378 & 2.00111504 & 14.48625 & 4425.75 & 4131.5992 & 73.2 & 73.5 & 72.2 & 72.8 & 0.000866 \\
\hline 850.02 & 3.82865672 & 14.55683189 & 2.00174048 & 14.48625 & 4426.25 & 4122.6601 & 73.2 & 73.5 & 72.3 & 72.8 & 0.000867 \\
\hline 855.03 & 3.81365024 & 14.55308378 & 2.00174048 & 14.48625 & 4436 & 4124.3983 & 73.4 & 73.5 & 72.2 & 72.7 & 0.000867 \\
\hline 860.02 & 3.81552605 & 14.5538334 & 2.00111504 & 14.49 & 4436.5 & 4124.15 & 73.4 & 73.5 & 72.3 & 72.7 & 0.000866 \\
\hline 865.02 & 3.8311578 & 14.5538334 & 2.00236592 & 14.487 & 4436.25 & 4118.4389 & 73.4 & 73.5 & 72.3 & 72.7 & 0.000867 \\
\hline 870.02 & 3.82553037 & 14.55383334 & 2.00111504 & 14.487 & 4436 & 4120.4254 & 73.4 & 73.5 & 72.4 & 72.7 & 0.000867 \\
\hline 875.02 & 3.84866536 & 14.55458302 & 2.00174048 & 14.48925 & 4436.5 & 4120.4254 & 73.3 & 73.5 & 72.4 & 72.7 & 0.000866 \\
\hline 880.02 & 3.83053253 & 14.55458302 & 2.00174048 & 14.487 & 4436.25 & 4117.9423 & 73.4 & 73.6 & 72.4 & 72.7 & 0.000866 \\
\hline 885.03 & 3.83615996 & 14.5538334 & 2.00111504 & 14.487 & 4436 & 4121,6669 & 73.4 & 73.5 & 72.4 & 72.7 & 0.000867 \\
\hline 890.02 & 3.81052389 & 14.5538334 & 2.00174048 & 14.487 & 4436.25 & 4127.6263 & 73.3 & 73.5 & 72.3 & 72.8 & 0.000867 \\
\hline 895.02 & 3.8123997 & 14.55458302 & 2.00174048 & 14.487 & 4436.25 & 4138.3035 & 73.3 & 73.6 & 72.3 & 72.8 & 0.000867 \\
\hline 900.05 & 3.80989862 & 14.55308378 & 2.00111504 & 14.487 & 4436.25 & 4137.062 & 73.4 & 73.5 & 72.4 & 72.8 & 0.000868 \\
\hline 905.02 & 3.81677659 & 14.55458302 & 2.00236592 & 14.48775 & 4436.25 & 4129.1161 & 73.4 & 73.6 & 72.4 & 72.7 & 0.000667 \\
\hline 910.02 & 3.88242994 & 14.55533264 & 2.00174048 & 14.487 & 4436 & 4127.6263 & 73.4 & 73.6 & 72.4 & 72.8 & 0.000866 \\
\hline 915.04 & 3.84553901 & 14.55608226 & 2.00174048 & 14.48925 & 4436.5 & 4134.3306 & 73.4 & 73.5 & 72.3 & 72.7 & 0.000866 \\
\hline 920.02 & 3.81552605 & 14.5538334 & 2.00174048 & 14,48625 & 4436.25 & 4132.8408 & 73.3 & 73.6 & 72.3 & 72.8 & 0,000867 \\
\hline 925.02 & 3.81427551 & 14.5538334 & 2.00111504 & 14.48625 & 4435.75 & 4122.6601 & 73.2 & 73.6 & 72.3 & 72.8 & 0.000866 \\
\hline
\end{tabular}




\begin{tabular}{|c|c|c|c|c|c|c|c|c|c|c|c|}
\hline \multicolumn{12}{|c|}{$\begin{array}{l}\text { TEST: EX302257 } 25 \text { Feb } 1997 \text { WEC-SPOOL } \\
\text { Measured Hydrogen Concentration = 4210ppm }\end{array}$} \\
\hline SECONDS & \begin{tabular}{|l|} 
MAIN DUCT \\
DIFF (IN H2O
\end{tabular} & \begin{tabular}{|l|} 
MAIN DUCT \\
ABS (PSIA) \\
\end{tabular} & $\begin{array}{l}\text { GAS INJECT } \\
\text { DIFF (IN H2O }\end{array}$ & \begin{tabular}{|l|} 
GAS INJECT \\
ABS (PSIA) \\
\end{tabular} & \begin{tabular}{|l|} 
NEWPORT \\
(PPM)
\end{tabular} & $\begin{array}{l}\text { SMC } \\
\text { (PPM) }\end{array}$ & \begin{tabular}{|l} 
AMBIENT \\
TEMP. (F)
\end{tabular} & \begin{tabular}{|l|} 
GAS INJECT \\
TEMP.(F) \\
\end{tabular} & $\mid$ MAIN DUCT & \begin{tabular}{|l|} 
WEC CELL \\
TEMP. (F)
\end{tabular} \mid & $\begin{array}{l}\text { WEC CELL } \\
\text { (VOLTS) }\end{array}$ \\
\hline & & & & & & & & & & & \\
\hline 0.0100098 & 3.82240402 & 14.5500853 & 0.55478504 & 14.4555 & 71.3 & -0.370062 & 72.5 & 72.5 & 71.5 & 72.6 & 0.000217 \\
\hline 5.0200195 & 3.81927767 & 14.5508349 & 1.99923872 & 14.484 & 71.275 & 107.966718 & 72.6 & 72.6 & 71.6 & 72.7 & 0.00022 \\
\hline 10.02002 & 3.85366752 & 14.5515845 & 1.99923872 & 14.484 & 231.575 & 1924.3894 & 72.5 & 72.6 & 71.6 & 72.6 & 0.000266 \\
\hline 15.02002 & 3.82553037 & 14.5523342 & 1.99986416 & 14.48475 & 732.5 & 3127.44166 & 72.5 & 72.6 & 71.6 & 72.6 & 0.000349 \\
\hline 20.02002 & 3.81052389 & 14.5523342 & 2.0004896 & 14.48475 & 1390 & 3602.45486 & 72.6 & 72.5 & 71.6 & 72.6 & 0.000439 \\
\hline 25.02002 & 3.81802713 & 14.5515845 & 2.00236592 & 14.484 & 1904.75 & 3765.84153 & 72.6 & 72.6 & 71.5 & 72.6 & 0.000518 \\
\hline 30.02002 & 3.82865672 & 14.5508349 & 2.00111504 & 14.48475 & 2391.75 & 3845.05178 & 72.6 & 72.6 & 71.5 & 72.6 & 0.000582 \\
\hline 35.02002 & 3.8186524 & 14.5515845 & 2.00111504 & 14.4855 & 2763.75 & 3873.6072 & 72.7 & 72.5 & 71.5 & 72.6 & 0.000631 \\
\hline 40.02002 & 3.81615132 & 14.5523342 & 2.0036168 & 14.48475 & 3045.25 & 3893.96846 & 72.7 & 72.5 & 71.5 & 72.6 & 0.000673 \\
\hline 45.02002 & 3.86992454 & 14.5506349 & 2.00299136 & 14.48475 & 3284 & 3923.2688 & 72.6 & 72.6 & 71.5 & 72.6 & 0.000706 \\
\hline 50.02002 & 3.82115348 & 14.5515845 & 2.00424224 & 14.48475 & 3454.5 & 3940.65036 & 72.6 & 72.5 & 71.5 & 72.6 & 0.00073 \\
\hline 55.02002 & 3.81490078 & 14.5508349 & 2.0036168 & 14.48475 & 3614.5 & 3943.63006 & 72.6 & 72.5 & 71.5 & 72.6 & 0.00075 \\
\hline 60.02002 & 3.81114916 & 14.5508349 & 2.00424224 & 14.484 & 3712.75 & 3944.62329 & 72.6 & 72.6 & 71.6 & 72.6 & 0.000766 \\
\hline 65.02002 & 3.80802281 & 14.5508349 & 2.00611856 & 14,48475 & 3805 & 3963.4947 & 72.6 & 72.6 & 71.6 & 72.6 & 0.000779 \\
\hline 70.02002 & 3.82990726 & 14.5515845 & 2.00549312 & 14,4855 & 3875.25 & 3975.66179 & 72.6 & 72.5 & 71.6 & 726 & 0.000789 \\
\hline 75.02002 & 3.8123997 & 14.5515845 & 2.00549312 & 14.4855 & 3945.5 & 3995.52643 & 72.6 & 72.6 & 71.6 & 72.6 & 0.000798 \\
\hline 80.02002 & 3.82302929 & 14.5508349 & 2.00549312 & 14.4855 & 4015 & 4000.9892 & 72.7 & 72.6 & 71.7 & 72.6 & 0.000806 \\
\hline 85.02002 & 3.82865672 & 14.5508349 & 2.00549312 & 14.4855 & 4045.25 & 3998.25782 & 72.8 & 72.6 & 71.6 & 72.6 & 0.000813 \\
\hline 90.02002 & 3.8311578 & 14.5515845 & 2.00611856 & 14.4825 & 4085.5 & 4007.1969 & 72.8 & 72.6 & 71.6 & 72.6 & 0.000818 \\
\hline 95.02002 & 3.82928199 & 14.5508349 & 2.006744 & 14.4855 & 4125.25 & 4008.68675 & 73 & 72.5 & 71.7 & 72.6 & 0.000822 \\
\hline 100.02002 & 3.81740186 & 14.5515845 & 2.00549312 & 14.4855 & 4135.5 & 4018.37076 & 72.9 & 72.6 & 71.7 & 72.6 & 0.000826 \\
\hline 105.02002 & 3.80864808 & 44.5515845 & 2.00611856 & 14.4855 & 4165.5 & 4027.30985 & 72.8 & 72.6 & 71.7 & 72.6 & 0.000829 \\
\hline 110.02002 & 3.81552605 & 14.5515845 & 2.00549312 & 14.4855 & 4185.25 & 4048.16772 & 72.8 & 72.6 & 71.6 & 72.6 & 0.000832 \\
\hline 115.02002 & 3.83803577 & 14.5523342 & 2.00549312 & 14.484 & 4205 & 4051.89234 & 72.9 & 72.6 & 71.6 & 72.6 & 0.000834 \\
\hline 120.02002 & 3.8186524 & 14.5523342 & 2.00486768 & 14,48475 & 4235.75 & 4054.37542 & 72.8 & 72.6 & 71.7 & 72.6 & 0.000837 \\
\hline 125.02002 & 3.84241266 & 14.5515845 & 2.00549312 & 14.48475 & 4235.75 & 4056.11358 & 72.8 & 72.6 & 71.7 & 72.6 & 0.000839 \\
\hline 130.02002 & 3.8186524 & \begin{tabular}{|l|}
14.5500853 \\
\end{tabular} & 2.00486768 & 14.4855 & 4255.25 & 4062.8179 & 72.8 & 72.6 & 71.7 & 72.6 & 0.000841 \\
\hline 135.02002 & 3.83178307 & 14.5523342 & 2.00611856 & 14.48475 & 4266 & 4063.81113 & 72.7 & 72.6 & 71.7 & 72.6 & 0.000843 \\
\hline 140.02002 & 3.83240834 & 14.5508349 & 2.00486768 & 14.48475 & 4275 & 4065.79759 & 72.7 & 72.6 & 71.6 & 72.6 & 0.000845 \\
\hline 145.02002 & 3.82990726 & 14.5515845 & 2.00736944 & 14.4855 & 4285.5 & 4069.0256 & 72.8 & 72.6 & 71.6 & 72.6 & 0.000846 \\
\hline 150.02002 & 3.81052389 & 14.5515845 & 2.00549312 & 14,484 & 4296 & 4073.24683 & 72.9 & 72.6 & 71.7 & 72.6 & 0.000848 \\
\hline 155.02002 & 3.8123997 & 14.5508349 & 2.00611856 & 14.48475 & 4305.75 & 4076.72314 & 72.9 & 72.6 & 71.7 & 72.6 & 0.000848 \\
\hline 160.02002 & 3.8123997 & 14.5493357 & 2.00611856 & 14.48475 & 4316 & 4088.64193 & 72.9 & 72.6 & 71.8 & 72.6 & 0.00085 \\
\hline 165.06 & 3.81615132 & 14.5503349 & 2.00611856 & 14.4855 & 4315.25 & 4074.73668 & 72.9 & 72.6 & 71.8 & 72.6 & 0.000851 \\
\hline 170.02002 & 3.8186524 & 14.5503349 & 2.00736944 & 14.48475 & 4325.5 & 4076.72314 & 73 & 72.6 & 71.7 & 72.6 & 0.000852 \\
\hline 175.02002 & 3.83303361 & 14.5515845 & 2.00486768 & 14.4855 & 4335.5 & 4087.40039 & 73 & 72.7 & 71.7 & 72.6 & 0.000852 \\
\hline 180.02002 & 3.8249051 & 14.5508349 & 2.00611856 & 14.48625 & 4335.75 & 4094.84963 & 73 & 72.7 & 71.7 & 72.6 & 0.000852 \\
\hline 185.02002 & 3.82052821 & 14.5508349 & 2.00611856 & 14,48625 & 4345.75 & 4108.00995 & 72.9 & 72.7 & 71.8 & 72.5 & 0.000853 \\
\hline
\end{tabular}




\begin{tabular}{|c|c|c|c|c|c|c|c|c|c|c|c|}
\hline SECONDS & \begin{tabular}{|l|} 
MAIN DUCT \\
DIFF (IN H2O
\end{tabular} & $\frac{\text { MAIN DUCT }}{\text { ABS (PSIA) }}$ & $\frac{\text { GAS INJECT }}{\text { DIFF (IN H2O }}$ & $\begin{array}{l}\text { GAS INJECT } \\
\text { ABS (PSIA) }\end{array}$ & \begin{tabular}{|l|} 
NEWPORT \\
(PPM)
\end{tabular} & \begin{tabular}{|l} 
SMC \\
(PPM)
\end{tabular} & \begin{tabular}{|l|} 
AMBIENT \\
TEMP (F)
\end{tabular} & \begin{tabular}{|l} 
GAS INJECT \\
TEMP (F)
\end{tabular} & \begin{tabular}{|l} 
MAIN DUCT \\
TEMP (F)
\end{tabular} & $\begin{array}{l}\text { TWEC CELL } \\
\text { TEMP (F) }\end{array}$ & $\begin{array}{l}\text { WEC CELL } \\
\text { NOLTS) }\end{array}$ \\
\hline & & & & & & & & & & & \\
\hline 190.02002 & 3.82115348 & 14.5515845 & 2.00549312 & 14.4855 & 4345.75 & 4115.7075 & 72.8 & 72.7 & 71.8 & 72.6 & 0.000855 \\
\hline 195.02002 & 3.81427551 & 14.5515845 & 2.00549312 & 14.4855 & 4355.75 & 4108.00995 & 72.8 & 72.7 & 71.8 & 72.6 & 0.000854 \\
\hline 200.02002 & 3.82302929 & 14.5523342 & 2.00486768 & 14.4855 & 4355.5 & 4103.54041 & 72.8 & 72.7 & 71.7 & 72.5 & 0.000854 \\
\hline 205.02002 & 3.83803577 & 14.5515845 & 2.00549312 & 14.4855 & 4355.75 & 4101.30564 & 72.7 & 72.7 & 71.6 & 72.5 & 0.000854 \\
\hline 210.02002 & 3.8186524 & 14.5508349 & 2.00549312 & 14.4855 & 4366 & 4100.0641 & 72.6 & 72.7 & 71.6 & 72.6 & 0.000856 \\
\hline 215.02002 & 3.81052389 & 14.5515845 & 2.00549312 & 14.4855 & 4366 & 4107.76164 & 72.6 & 72.7 & 71.6 & 72.5 & 0.000855 \\
\hline 220.02002 & 3.81052389 & 14.5508349 & 2.00549312 & 14.4855 & 4366 & 4108.00995 & 72.7 & 72.7 & 71.6 & 72.5 & 0.000857 \\
\hline 225.02002 & 3.80927335 & 14.5523342 & 2.00549312 & 14.4855 & 4375.75 & 4101.30564 & 72.7 & 72.7 & 71.7 & 72.5 & 0.000857 \\
\hline 230.02002 & 3.8123997 & 14.5508349 & 2.00486768 & 14.4855 & 4375.75 & 4097.0844 & 72.8 & 72.7 & 71.6 & 72.6 & 0.00086 \\
\hline 235.02002 & 3.81990294 & 14.5515845 & 2.00549312 & 14.48475 & 4375.75 & 4097.33271 & 72.8 & 72.7 & 71.6 & 72.5 & 0.000859 \\
\hline 240.02002 & 3.83178307 & 14.5523342 & 2.00549312 & 14.48475 & 4386.25 & 4103.2921 & 72.8 & 72.8 & 71.6 & 72.5 & 0.000859 \\
\hline 245.02002 & 3.81677659 & 14.5508349 & 2.00549312 & 14.48475 & 4385.5 & 4113.22442 & 72.8 & 72.8 & 71.7 & 72.5 & 0.000861 \\
\hline 250.02002 & 3.82553037 & 14.5515845 & 2.00611856 & 14.484 & 4393.5 & 4124.64659 & 72.8 & 72.7 & 71.7 & 72.6 & 0.00086 \\
\hline 255.02002 & 3.82115348 & 14.5515845 & 2.00486768 & 14.4855 & 4395.75 & 4118.6872 & 72.8 & 72.8 & 71.7 & 72.5 & 0.00086 \\
\hline 260.02002 & 3.82302929 & 14.5515845 & 2.00549312 & 14.4855 & 4395 & 4115.7075 & 72.9 & 72.8 & 71.8 & 72.5 & 0.00086 \\
\hline 265.02002 & 3.82052821 & 14.5523342 & 2.00611856 & 14.48475 & 4396 & 4116.45242 & 72.8 & 72.8 & 71.7 & 72.5 & 0.00086 \\
\hline 270.02002 & 3.8186524 & 14.5515845 & 2.00611856 & 14.48475 & 4395.75 & 4114.96258 & 72.8 & 72.8 & 71.7 & 72.5 & 0.00086 \\
\hline 275.02002 & 3.81552605 & 14.5515845 & 2.00486768 & 14.48475 & 4396 & 4125.88813 & 72.8 & 72.8 & 71.7 & 72.5 & 0.000861 \\
\hline 280.02002 & 3.81365024 & 14.5515845 & 2.00611856 & 14.48475 & 4396 & 4123.90166 & 72.9 & 72.8 & 71.7 & 72.5 & 0.000861 \\
\hline 285.02002 & 3.82365456 & 14.5508349 & 2.00549312 & 14.484 & 4395.75 & 4124.64659 & 73 & 72.8 & 71.7 & 72.5 & 0.000861 \\
\hline 290.02002 & 3.81615132 & 14.5530838 & 2.006744 & 14.484 & 4406 & 4129.61275 & 72.9 & 72.8 & 71.7 & 72.5 & 0.000861 \\
\hline 295.02002 & 3.82240402 & 14.5515845 & 2.00611856 & 14.48475 & 4405.75 & 4127.87459 & 72.9 & 72.8 & 71.7 & 72.5 & 0.000862 \\
\hline 300.02002 & 3.8186524 & 14.5508349 & 2.00611856 & 14.4855 & 4406.25 & 4129.86106 & 72.8 & 72.8 & 71.7 & 72.5 & 0.000863 \\
\hline 305.02002 & 3.81427551 & 14.5523342 & 2.00549312 & 14.48475 & 4405.75 & 4126.38474 & 72.8 & 72.8 & 71.7 & 72.5 & 0.000862 \\
\hline 310.02002 & 3.81427551 & 14.5515845 & 2.00549312 & 14.48625 & 4406 & 4128.37121 & 72.8 & 72.8 & 71.8 & 72.6 & 0.000863 \\
\hline 315.02002 & 3.81302497 & 14.5515845 & 2.00549312 & 14.48475 & 4406 & 4129.36444 & 72.7 & 72.8 & 71.7 & 72.6 & 0.000863 \\
\hline 320.02002 & 3.82240402 & 14.5515845 & 2.00549312 & 14.48475 & 4415.5 & 4128.86782 & 72.7 & 72.8 & 71.7 & 72.5 & 0.000864 \\
\hline 325.02002 & 3.8123997 & 14.5515845 & 2.00486768 & 14.48475 & 4416.25 & 4120.42535 & 72.7 & 72.8 & 71.7 & 72.6 & 0.000864 \\
\hline 330.02002 & 3.82928199 & 14.5515845 & 2.006744 & 14.48475 & 4415.75 & 4130.60598 & 72.7 & 72.8 & 71.7 & 72.5 & 0.000864 \\
\hline 335.02002 & 3.8374105 & 14.5515845 & 2.00549312 & 14.4855 & 4416 & 4122.66012 & 72.7 & 72.8 & $\overline{71.7}$ & $\overline{72.5}$ & 0.000865 \\
\hline 340.02002 & 3.8249051 & 14.5523342 & 2.00549312 & 14,484 & 4416 & 4126.63305 & 72.7 & 72.9 & 71.8 & 72.6 & 0.000865 \\
\hline 345.02002 & 3.81365024 & 14.5508349 & 2.00611856 & 14.4855 & 4425.75 & 4125.63982 & 72.7 & 72.8 & 71.8 & 72.5 & 0.000864 \\
\hline 350.02002 & 3.82052821 & 14.5508349 & 2.00611856 & 14.4855 & 4426 & 4120.67366 & 72.7 & 72.9 & 71.8 & 72.5 & 0.000865 \\
\hline 355.02002 & 3.82865672 & 14.5515845 & 2.00549312 & 14.4855 & 4425.5 & 4123.90166 & 72.7 & 72.9 & 71.8 & 72.6 & 0.000865 \\
\hline 360.02002 & 3.8311578 & 14.5515845 & 2.00549312 & 14.4855 & 4425.75 & 4133.83398 & 72.8 & 72.9 & 71.8 & 72.5 & 0.000865 \\
\hline 365.02002 & 3.82302929 & 14.5538334 & 2.00549312 & 14.48475 & 4425.5 & 4132.59244 & 72.9 & 72.9 & 71.9 & 72.6 & 0.000865 \\
\hline 370.02002 & 3.8186524 & 14.5523342 & 2.00549312 & 14,4855 & 4426.25 & 4125.39151 & 72.9 & 72.9 & 72 & 72.5 & 0.000865 \\
\hline 375,02002 & 3.81615132 & 14.5508349 & 2.00611856 & 14.4855 & 4425.75 & 4135.82045 & 72.7 & 72.9 & 71.9 & 72.5 & 0.000865 \\
\hline 380.02002 & 3.81490078 & 14.5523342 & 2.00611856 & 14.48625 & 4426.25 & 4131.84752 & 72.7 & 72.9 & 71.9 & 72.5 & 0.000865 \\
\hline 385.02002 & 3.82740618 & 14.5523342 & 2.00611856 & 14.4855 & 4426.25 & 4121.17028 & 72.7 & 72.9 & 71.9 & 72.5 & 0.000865 \\
\hline 390.02002 & 3.85679387 & 14.5515845 & 2.00611856 & 14.4855 & 4425.25 & 4136.31706 & 72.7 & 72.9 & 71.9 & 72.6 & 0.000866 \\
\hline 395,02002 & 3.8186524 & 14.5508349 & 2.00549312 & 14,48475 & 4426.25 & 4138.05522 & 72.6 & 72.9 & 71.8 & 72.5 & 0.000866 \\
\hline
\end{tabular}




\begin{tabular}{|c|c|c|c|c|c|c|c|c|c|c|c|}
\hline SECONDS & \begin{tabular}{|l|} 
MAIN DUCT \\
DIFF (IN H2O \\
\end{tabular} & \begin{tabular}{|l|} 
MAIN DUCT \\
ABS (PSIA) \\
\end{tabular} & \begin{tabular}{|l|} 
GAS INJECT \\
DIFF (IN H2O \\
\end{tabular} & \begin{tabular}{|l|} 
GAS INJECT \\
$A B S$ (PSIA)
\end{tabular} & \begin{tabular}{|l|} 
NEWPORT \\
(PPM) \\
\end{tabular} & \begin{tabular}{|l} 
SMC \\
(PPM)
\end{tabular} & \begin{tabular}{|l|} 
AMBIENT \\
TEMP. (F) \\
\end{tabular} & \begin{tabular}{|l} 
GAS INJECT \\
TEMP. (F)
\end{tabular} & \begin{tabular}{|l|} 
MAIN DUCT \\
TEMP. (F) \\
\end{tabular} & \begin{tabular}{|l|} 
WEC CELL \\
TEMP. (F) \\
\end{tabular} & \begin{tabular}{|l} 
WEC CELL \\
(VOLTS)
\end{tabular} \\
\hline 40002002 & 38249051 & 145530838 & 2006744 & 14,48475 & 443625 & 413085429 & 726 & 729 & 717 & 775 & 0.000866 \\
\hline 405.02002 & 3.83803577 & 14.5515845 & 2.00486768 & 14.4855 & 4436 & 4111.48626 & 72.4 & 73 & 71.6 & 72.5 & 0.000865 \\
\hline 410.02002 & 3.81365024 & 14.5508349 & 2.00549312 & 14.484 & 4436.25 & 4104.03702 & 72.5 & 72.9 & 71.6 & 72.5 & 0.000866 \\
\hline 415.03 & 3.81802713 & 14.5515845 & 2.00486768 & 14.48475 & 4436.25 & 4115.95581 & 72.5 & 73 & 71.5 & 72.5 & 0.000867 \\
\hline 420.02002 & 3.83490942 & 14.5515845 & 2.00549312 & 14.484 & 4436.25 & 4131.84752 & 72.4 & 73 & 71.6 & 72.5 & 0.000867 \\
\hline 425.02002 & 3.81302497 & 14.5523342 & 2.00486768 & 14.48475 & 4436.25 & 4124.8949 & 72.4 & 73 & 74.6 & 72.5 & 0.000866 \\
\hline 430,02002 & 3.82553037 & 14.5508349 & 2.00549312 & 14.48475 & 4436.25 & 4132.84075 & 72.4 & 73 & 71.6 & 72.5 & 0.000867 \\
\hline 435.09003 & 3.82803145 & 14.5508349 & 2.006744 & 14.4855 & 4436 & 4124.14997 & 72.5 & 73 & 71.7 & 72.5 & 0.000868 \\
\hline 440.02002 & 3.82177875 & 14.5500853 & 2.00611856 & 14.4855 & 4436.25 & 4125.1432 & 72.6 & 73 & 71.7 & 72.5 & 0.000867 \\
\hline 445.04001 & 3.82115348 & 14.5508349 & 2.00549312 & 14.4855 & 4436.25 & 4114.21765 & 72.6 & 73 & 71.8 & 72.5 & 0.000866 \\
\hline 450.02002 & 3.83490942 & 14.5508349 & 2.00549312 & 14.48475 & 4445.75 & 4106.76841 & 72.6 & 73 & 71.7 & 72.5 & 0.000867 \\
\hline 455.02002 & 3.81400078 & 14.5508349 & 2.00611856 & 14.4855 & 4446.25 & 4114.96258 & 72.6 & 73 & 71.7 & 72.5 & 0.000868 \\
\hline 460.02002 & 3.81615132 & 14.5508349 & 2.00486768 & 14.48475 & 4446 & 4126.13644 & 72.6 & 73 & 71.6 & 72.5 & 0.000868 \\
\hline 465.09003 & 3.83303361 & 14.5523342 & 2.00549312 & 14.4855 & 4446 & 4115.9558 & 72.6 & 73 & 71.6 & 72.5 & 0.000867 \\
\hline 470.02002 & 3.8436632 & 14.5515845 & 2.00549312 & 14.4855 & 4446.25 & 4122.41182 & 72.5 & 73 & 71.6 & 72.5 & 0.000867 \\
\hline 475.08002 & 3.82052821 & 14.5523342 & 2.00549312 & 14.4855 & 4446.25 & 4120.17704 & 72.5 & 73 & 71.6 & 72.5 & 0.000867 \\
\hline 480.02002 & 3.82928199 & 14.5515845 & 2.00611856 & 14.48475 & 4446 & 4120.67366 & 72.5 & 73 & 71.6 & 72.5 & 0.000867 \\
\hline 485.02002 & 3.81177443 & 14.5508349 & 2.00611856 & 14.48475 & 4446.25 & 4120.42535 & 72.5 & 73 & 71.4 & 72.5 & 0.000867 \\
\hline 490.02002 & 3.8374105 & 14.5500853 & 2.00549312 & 14.4855 & 4446 & 4121.9152 & 72.5 & 73 & 71.4 & 72.5 & 0.000867 \\
\hline 495.02002 & 3.81990294 & 14.5515845 & 2.00549312 & 14.4855 & 4445.75 & 4123.40505 & 72.5 & 73 & 71.4 & 72.5 & 0.000867 \\
\hline 500.02002 & 3.81615132 & 14.5508349 & 2.00611856 & 14.4855 & 4446.25 & 4122.66012 & 72.5 & 73 & 71.3 & 72.5 & 0.000868 \\
\hline 505.02002 & 3.82052821 & 14.5508349 & 2.00549312 & 14.48475 & 4446 & 4122.66012 & 72.5 & 73.1 & 71.4 & 72.5 & 0.000868 \\
\hline 510.02002 & 3.81677659 & 14.5515845 & 2.00611856 & 14.4855 & 4446 & 4119.18381 & 72.5 & 73 & 71.5 & 72.5 & 0.000868 \\
\hline 515.03 & 3.81052389 & 14.5500853 & 2.00486768 & 14.48475 & 4446.25 & 4116.94904 & 72.5 & 73.1 & 71.5 & 72.5 & 0.000869 \\
\hline 520.02002 & 3.81927767 & 14.5508349 & 2.00549312 & 14.48475 & 4446 & 4142.27646 & 72.5 & 73 & 71.5 & 72.4 & 0.000868 \\
\hline 525.02002 & 3.8249051 & 14.5508349 & 2.00611856 & 14.4855 & 4446 & 4135.32383 & 72.5 & 73.1 & 71.5 & 72.4 & 0.00087 \\
\hline 530.04001 & 3.81677659 & 14.5508349 & 2.00549312 & 14.4855 & 4456.25 & 4131.3509 & 72.5 & 73.1 & 71.6 & 72.4 & 0.00087 \\
\hline 535.02002 & 3.81552605 & 14.5508349 & 2.00549312 & 14.484 & 4455.75 & 4115.95581 & 72.5 & 73.1 & 71.6 & 72.4 & 0.00087 \\
\hline 540.02002 & 3.81552605 & 14.5508349 & 2.00424224 & 14.48475 & 4456 & 4111.48626 & 72.5 & 73.1 & 71.6 & 72.5 & 0.00087 \\
\hline 545.05002 & 3.82052821 & 14.5508349 & 2.00549312 & 14.4855 & 4456 & 4107.01672 & 72.6 & 73.1 & 71.7 & 72.5 & 0.00087 \\
\hline 550.03 & 3.81677659 & 14.5508349 & 2.00611856 & 14.4855 & 4456 & 4105.77518 & 72.7 & 73.1 & 71.7 & 72.5 & 0.000871 \\
\hline 555.02002 & 3.8123997 & 14.5523342 & 2.00611856 & 14.4855 & 4456.25 & 4110.74134 & 72.6 & 73.1 & 71.7 & 72.4 & 0.00087 \\
\hline 560.06 & 3.8186524 & 14.5508349 & 2.00611856 & 14.48475 & 4456.25 & 4121.47028 & 72.6 & 73.1 & 71.6 & 72.4 & 0.00087 \\
\hline 565.04001 & 3.82553037 & 14.5515845 & 2.00549312 & 14.4855 & 4466.25 & 4114.21765 & 72.5 & 73.1 & 71.5 & 72.5 & 0.00087 \\
\hline 570.02002 & 3.84428847 & 14.5508349 & 2.00611856 & 14.48475 & 4466 & 4110.24472 & 72.5 & 73.1 & 71.5 & 72.5 & 0.000871 \\
\hline 575.04001 & 3.84741482 & 14.5508349 & 2.00611856 & 14.48475 & 4466.25 & 4116.94904 & 72.5 & 73.1 & 71.5 & 72.5 & 0.000871 \\
\hline 580.02002 & 3.80864808 & 14.5530838 & 2.00611856 & 14.4855 & 4466 & 4106.76841 & 72.6 & 73 & 71.5 & 72.5 & 0.000871 \\
\hline 585.02002 & 3.80864808 & 14.5515845 & 2.00611856 & 14.4855 & 4466 & 4125.39151 & 72.6 & 73.1 & 71.4 & 72.5 & 0.000871 \\
\hline 590.03 & 3.82302929 & 14.5515845 & 2.00549312 & 14.4855 & 4466.25 & 4127.37798 & 72.6 & 73.1 & 71.6 & 72.4 & 0.000871 \\
\hline 595.02002 & 3.81427551 & 14.5515845 & 2.00549312 & 14.4855 & 4466.25 & 4122.90843 & 72.6 & 73.1 & 71.6 & 72.5 & 0.000871 \\
\hline 600.02002 & 3.82365456 & 14.5515845 & 2.00611856 & 14.48475 & 4466.25 & 4107.51334 & 72.6 & 73.1 & 71.5 & 72.5 & 0.00087 \\
\hline 605.02002 & 3.8249051 & 14.5508349 & 2.00611856 & 14.48475 & 4466.25 & 4115.7075 & 72.6 & 73.1 & 71.6 & 72.5 & 0,00087 \\
\hline
\end{tabular}




\begin{tabular}{|c|c|c|c|c|c|c|c|c|c|c|c|}
\hline SECONDS & MAIN DUCT & MAIN DUCT & GAS INJECT & GAS INJECT & NEWPORT & SMC & AMBIENT & GAS INJECT & MAIN DUCT & WEC CELL & WEC CELL \\
\hline & DIFF (IN H2O & ABS (PSIA) & DIFF (1N H2O & ABS (PS|A) & (PPM) & (PPM) & TEMP. (F) & TEMP. (F) & TEMP. (F) & TEMP. (F) & (VOLTS) \\
\hline 610.04001 & 3.8186524 & 14.5515845 & 2.00549312 & 14.4855 & 4456.25 & 4117.44566 & 72.6 & 73.1 & 71.6 & 72.5 & 0.00087 \\
\hline 615.02002 & 3.8249051 & 14.5508349 & 2.00486768 & 14.4855 & 4456.25 & 4119.43212 & 72.6 & 73.1 & 71.7 & 72.4 & 0.000867 \\
\hline 620.05002 & 3.82115348 & 14.5515845 & 2.00611856 & 14.48475 & 4456.25 & 4119.92874 & 72.6 & 73.1 & 71.6 & 72.4 & 0.000868 \\
\hline 625.02002 & 3.82365456 & 14.5523342 & 2.00611856 & 14.48475 & 4446 & 4110.98965 & 72.6 & 73.1 & 71.7 & 72.5 & 0,000857 \\
\hline 630.02002 & 3.83991158 & 14.5493357 & 2.00549312 & 14.48475 & 4164.75 & 4118.19058 & 72.7 & 73.1 & 71.6 & 72.4 & 0.000818 \\
\hline 635.02002 & 3.81740186 & 14.5515845 & 2.00549312 & 14.48475 & 4086 & 4123.90166 & 72.7 & 73.1 & 71.7 & 72.5 & 0.00081 \\
\hline 640.09003 & 3.82115348 & 14.5508349 & 2.00549312 & 14.4855 & 4074.75 & 4130.85429 & 72.7 & 73.1 & 71.6 & 72.4 & 0.000815 \\
\hline 645.02002 & 3.8186524 & 14.5515845 & 2.006744 & 14.48475 & 4102.75 & 4127.87459 & 72.8 & 73.1 & 71.7 & 72.5 & 0.000822 \\
\hline 650.05002 & 3.81990294 & 14.5508349 & 2.00549312 & 14.484 & 4156 & 4124.39828 & 72.7 & 73.1 & 71.6 & 72.5 & 0.000832 \\
\hline 655.02002 & 3.81427551 & 14.5515845 & 2.00611856 & 14.4855 & 4215.75 & 4123.40505 & 72.7 & 73.1 & 71.5 & 72.4 & 0.000839 \\
\hline 660.02002 & 3.83615996 & 14.5515845 & 2.00611856 & 14.4855 & 4256 & 4108.25826 & 72.7 & 73.2 & 71.6 & 72.5 & 0.000844 \\
\hline 665.02002 & 3,81427551 & 14.5500853 & 2.00549312 & 14.484 & 4296.5 & 4110.24472 & 72.8 & 73.2 & 71.7 & 72.5 & 0.000849 \\
\hline 670.05002 & 3.82365456 & 14.5515845 & 2.00611856 & 14.48475 & 4316.25 & 4116.70073 & 72.8 & 73.1 & 71.8 & 72.5 & 0.000853 \\
\hline 675.02002 & 3.84616428 & 14.5508349 & 2.00611856 & 14.4855 & 4346 & 4119.43212 & 72.8 & 73.1 & 71.8 & 72.5 & 0,000857 \\
\hline 680.04001 & 3.83240834 & 14.5508349 & 2.00611856 & 14.4855 & 4366.5 & 4133.58568 & 72.8 & 73.2 & 71.8 & 72.5 & 0.000859 \\
\hline 685.02002 & 3.81677659 & 14.5515845 & 2.00549312 & 14.48475 & 4375.5 & 4123.40505 & 72.8 & 73.2 & 71.8 & 72.5 & 0.00086 \\
\hline 690.02002 & 3.81740186 & 14.5515845 & 2.00611856 & 14.4855 & 4396.5 & 4127.37798 & 72.9 & 73.2 & 71.9 & 72.4 & 0.000861 \\
\hline 695.02002 & 3.8186524 & 14,5508349 & 2.00611856 & 14.48475 & 4405.25 & 4129.61275 & 72.7 & 73.2 & 71.9 & 72.4 & 0.000863 \\
\hline 700.04001 & 3.83803577 & 14.5508349 & 2.00611856 & 14.48475 & 4416 & 4136.06876 & 72.4 & 73.2 & 71.9 & 72.5 & 0.000865 \\
\hline 705.02002 & 3.81990294 & 14.5508349 & 2.00611856 & 14.48325 & 4416.25 & 4125.63982 & 72.3 & 73.2 & 71.8 & 72.5 & 0.000865 \\
\hline 710.02002 & 3.83991158 & 14.5508349 & 2.00549312 & 14.4855 & 4426 & 4123.15674 & 72.2 & 73.2 & 71.7 & 72.4 & 0.000866 \\
\hline 715.04001 & 3.81615132 & 14.5515845 & 2.00549312 & 14.48475 & 4425.75 & 4122.16351 & 72.2 & 73.2 & 71.7 & 72,4 & 0.000867 \\
\hline 720.02002 & 3.8123997 & 14.5515845 & 2.00611856 & 14.48475 & 4436.25 & 4112.7278 & 72.3 & 73.2 & 71.6 & 72.4 & 0.000867 \\
\hline 725.05002 & 3.8311578 & 14.5508349 & 2.006744 & 14.4855 & 4436.25 & 4130.10936 & 72.3 & 73.2 & 71.6 & 72.4 & 0.000867 \\
\hline 730.02002 & 3.84616428 & 14.5515845 & 2.00486768 & 14,48325 & 4446.25 & 4136.31706 & 72.3 & 73.2 & 71.6 & 72.4 & 0.000868 \\
\hline 735.02002 & 3.81427551 & 14.5523342 & 2.00486768 & 44.48475 & 4446.25 & 4135.32383 & 72.3 & 73.2 & 71.6 & 72.4 & 0.000868 \\
\hline 740.02002 & 3.83603577 & 14.5508349 & 2.00486768 & 14.48475 & 4446.25 & 4126.63305 & 72.3 & 73.2 & 71.7 & 72.4 & 0.000869 \\
\hline 745.10001 & 3.84491374 & 14.5523342 & 2.00424224 & 14.48475 & 4446.5 & 4116.45242 & 72.3 & 73.2 & 71.7 & 72.4 & 0.000869 \\
\hline 750.02002 & 3.81490078 & 14.5508349 & 2.0036168 & 14.484 & 4456.25 & 4108.75488 & 72.4 & 73.2 & 71.8 & 72.4 & 0.000868 \\
\hline 755.04001 & 3.8123997 & 14.5508349 & 2.00549312 & 14.4825 & 4456.25 & 4129.61275 & 72.6 & 73.2 & 71.7 & 72.4 & 0.000868 \\
\hline 760.02002 & 3.81427551 & 14.5500853 & 2.00486768 & 14.48325 & 4456.25 & 4124.8949 & 72.6 & 73.2 & 71.8 & 72.4 & 0.000869 \\
\hline 765.02002 & 3.82740618 & 14.5508349 & 2.00549312 & 14.48475 & 4446.5 & 4128.37121 & 72.7 & 73.2 & 71.8 & 72.5 & 0.000869 \\
\hline 770.02002 & 3.82240402 & 14.5508349 & 2.00611856 & 14.4855 & 4456 & 4128.37121 & 72.7 & 73.2 & 71.9 & 72.4 & 0.00087 \\
\hline 775.02002 & 3.82365456 & 14.5515845 & 2.00611856 & 14.48475 & 4456.25 & 4127.37798 & 72.8 & 73.2 & 71.9 & 72.4 & 0.00087 \\
\hline 780.02002 & 3.81427551 & 14.5515845 & 2.00549312 & 14.48175 & 4456.25 & 4115.7075 & 72.8 & 73,3 & 71.8 & 72.5 & 0.00087 \\
\hline 785.02002 & 3.82553037 & 14.5500853 & 2.00549312 & 14.4825 & 4456 & 4119.92874 & 72.8 & 73.3 & 71.7 & 72.4 & 0.000869 \\
\hline 790.02002 & 3.83803577 & 14.5508349 & 2.00424224 & 14.4825 & 4456.25 & 4129.11613 & 72.8 & 73.2 & 71.7 & 72.5 & 0.00087 \\
\hline 795.02002 & 3.8186524 & 14.5515845 & 2.00486768 & 14.4825 & 4456.25 & 4112.97611 & 72,8 & 73.3 & $71 . \overline{7}$ & 72.4 & 0.00087 \\
\hline 800.02002 & 3.81615132 & 14.5515845 & 2.00611856 & 14.4825 & 4456 & 4118.9355 & 72.8 & 73.3 & 71.7 & 72.4 & 0.00087 \\
\hline 805.02002 & 3.83866104 & 14.5530838 & 2.00549312 & 14.48325 & 4456.25 & 4124.39828 & 72.8 & 73.3 & 71.7 & 72.5 & 0.00087 \\
\hline 810.02002 & 3.8374105 & 14.5515845 & 2.00611856 & 14.4825 & 4466.25 & 4125.1432 & 72.8 & 73.3 & 71.8 & 72.5 & $0 . \overline{000871}$ \\
\hline 815.06 & 3.84241266 & 14.5508349 & 2.00424224 & 14.48175 & 4456.25 & 4127.12967 & 72.9 & 73.3 & 71.8 & 72.4 & 0.000869 \\
\hline
\end{tabular}




\begin{tabular}{|c|c|c|c|c|c|c|c|c|c|c|c|}
\hline SECONDS & MAIN DUCT & MAIN DUCT & GAS INJECT & GAS INJECT & NEWPORT & SMC & AMBIENT & GAS INJECT & MAIN DUCT & WEC CELL & $\begin{array}{l}\text { WEC CELL } \\
\text { NOLTS }\end{array}$ \\
\hline & DIFF (IN H2O & ABS (PSIA) & DIFF (IN H2O & ABS (PSIA) & (PPM) & (PPM) & TEMP. (F) & & & & \\
\hline 820,03 & 3.84866536 & 14.5515845 & 2,00486768 & 14,481 & 4465.75 & 4134.82722 & 72.9 & 73.3 & 71.9 & 72.5 & 0.000871 \\
\hline 825.02002 & 3.82740618 & 14.5508349 & 2.00611856 & 14,4825 & 4466.25 & 4124.64659 & 72.9 & 73.3 & 71.9 & 72.5 & 0.000871 \\
\hline 830.06 & 3.81552605 & 14.5515845 & 2.0036168 & 14.48175 & 4466.25 & 4138.80014 & 72.9 & 73.3 & 71.9 & 72.5 & 0.000872 \\
\hline 835.02002 & 3.81802713 & 14.5508349 & 2.00549312 & 14.48325 & 4466.25 & 4128.1229 & 72.9 & 73.2 & 71.8 & 72.5 & 0.000871 \\
\hline 840.02002 & 3.82928199 & 14.5508349 & 2.00549312 & 14.4825 & 4466.25 & 4121,41858 & 72.9 & 73.3 & 71.9 & 72.4 & 0.000871 \\
\hline 845.03 & 3.82678091 & 14.5508349 & 2.00424224 & 14.4825 & 4466 & 4120.42535 & 72.9 & 73.3 & 72.1 & 72.5 & 0.000871 \\
\hline 850.02002 & 3.82427983 & 14.5500853 & 2.0036168 & 14,4825 & 4466.25 & 4119.18381 & 73 & 73.3 & 72 & 72.5 & 0.000872 \\
\hline 855.02002 & 3.81740186 & 14.5523342 & 2.0036168 & 14.4825 & 4466.25 & 4121.41858 & 72.9 & 73.3 & 71.9 & 72.5 & 0.000872 \\
\hline 860.06 & 3.8249051 & 14.5500853 & 2.00549312 & 14.48175 & 4466.25 & 4127.37798 & 72.8 & 73.3 & 71.9 & 72.4 & 0.000869 \\
\hline 865.02002 & 3.81927767 & 14.5515845 & 2.00549312 & 14.48325 & 4466.25 & 4122.41182 & 72.7 & 73.3 & 71.8 & 72.4 & 0,00087 \\
\hline 870.02002 & 3.8311578 & 14.5515845 & 2.00549312 & 14.4825 & 4466 & 4134.57891 & 72.7 & 73.3 & 71.8 & 72.4 & 0.000871 \\
\hline 875.06 & 3.83053253 & 14.5500853 & 2.00549312 & 14.4825 & 4466.25 & 4130.35767 & 72.8 & 73.3 & 71.7 & 72.4 & 0.000872 \\
\hline 880.02002 & 3.81052389 & 14.5515845 & 2.00486768 & 14.4825 & 4466.25 & 4132.09583 & 72.7 & 73.3 & 71.8 & 72.4 & 0.000871 \\
\hline 885.02002 & 3.81490078 & 14.5500853 & 2.00486768 & 14.48325 & 4466.25 & 4134.57891 & 72.7 & 73.3 & 71.8 & 72.4 & 0.00087 \\
\hline 890.03 & 3.81490078 & 14.5500853 & 2.00424224 & 14.48325 & 4466 & 4131.84752 & 72.7 & 73.3 & 71.8 & 72.4 & 0.00087 \\
\hline 895.02002 & 3.83303361 & 14.5500853 & 2.00549312 & 14.484 & 4466.25 & 4137.3103 & 72.7 & 73.3 & 71.7 & 72.4 & 0.000872 \\
\hline 900.02002 & 3.84052389 & 14.5493357 & 2.00611856 & 14.4825 & 4466 & 4127.62628 & 72.7 & 73.3 & 71.7 & 72.4 & 0.000872 \\
\hline 905.02002 & 3.81615132 & 14.5500853 & 2.00486768 & 14.4825 & 4465.75 & 4109.4998 & 72.7 & 73.3 & 71.8 & 72.4 & 0.000872 \\
\hline 910.05002 & 3.82740618 & 14.5508349 & 2.00611856 & 14.4825 & 4466.25 & 4109.00318 & 72.8 & 73.3 & 71.9 & 72.4 & 0.000872 \\
\hline 915.02002 & 3.82678091 & 14.5515845 & 2.00549312 & 14.484 & 4476 & 4121.17028 & 72.8 & 73.3 & 71.9 & 72.4 & 0.000874 \\
\hline 920.02002 & 3.82177875 & 14.5523342 & 2.0036168 & 14.48325 & 4476 & 4124.39828 & 72.8 & 73.4 & 71.9 & 72.4 & 0.000872 \\
\hline 925.03 & 3.833658888 & 14.5493357 & 2.00486768 & 14,48325 & 4476 & 4121.41858 & 72.8 & 73.3 & 71.8 & 72.4 & 0.000873 \\
\hline 930.02002 & 3.82553037 & 14.5515845 & 2,006744 & 14.484 & 4475.75 & 4117.44566 & 72.7 & 73.3 & 71.9 & 72.4 & 0.000874 \\
\hline 935.02002 & 3.81490078 & 14.548586 & 2.00611856 & 14.48325 & 4475.75 & 4121.66689 & 72.7 & 73.3 & 71.9 & 72.5 & 0.000872 \\
\hline 940.02002 & 3.82740618 & 14.5515845 & 2.00549312 & 14.48325 & 4476 & 4128.61952 & 72.7 & 73.3 & 71.9 & 72.4 & 0.000872 \\
\hline 945.02002 & 3.84303793 & 14.5515845 & 2.00549312 & 14.48325 & 4475.75 & 4134.57891 & 72.8 & 73.3 & 71.9 & 72.4 & 0.000872 \\
\hline 950.02002 & 3.82115348 & 14.5508349 & 2.006744 & 14.4825 & 4475.75 & 4120,42535 & 72.8 & 73.3 & 71.9 & 72.4 & 0.000873 \\
\hline 955.02002 & 3.82990726 & 14.5508349 & 2.00549312 & 14.4825 & 4476 & 4129.61275 & 72.9 & 73.3 & 71.9 & 72.4 & 0.000873 \\
\hline 960.02002 & 3.81490078 & 14.5508349 & 2.00549312 & 14.4825 & 4476 & 4132.34414 & 72.8 & 73.3 & 71.9 & 72.5 & 0.000873 \\
\hline
\end{tabular}




\begin{tabular}{|c|c|c|c|c|c|c|c|c|c|c|c|}
\hline \multicolumn{12}{|c|}{$\begin{array}{l}\text { TEST: EX402257 } 25 \text { Feb } 1997 \text { WEC-SPOOL } \\
\text { Measured Hydrogen Concentration }=4210 \mathrm{ppm}\end{array}$} \\
\hline SECONDS & \begin{tabular}{|l|} 
MAIN DUCT \\
DIFF (IN H2O \\
\end{tabular} & \begin{tabular}{|l|} 
MAIN DUCT \\
ABS (PSIA)
\end{tabular} & $\begin{array}{l}\text { GAS INJECT } \\
\text { DIFF (IN H2O } \\
\end{array}$ & \begin{tabular}{|l|} 
GAS INJECT \\
ABS (PSIA)
\end{tabular} & \begin{tabular}{|l|} 
NEWPORT \\
(PPM) \\
\end{tabular} & \begin{tabular}{|l} 
SMC \\
(PPM)
\end{tabular} & \begin{tabular}{|l|} 
AMBIENT \\
TEMP. (F \\
\end{tabular} & \begin{tabular}{|l|} 
GAS INJECT \\
TEMP. (F) \\
\end{tabular} & \begin{tabular}{|l|} 
MAIN DUCT \\
TEMP. (F) \\
\end{tabular} & \begin{tabular}{|l} 
WEC CEL \\
TEMP. (F)
\end{tabular} & $\frac{\mid \text { WEC CELL }}{\text { (VOLTS) }}$ \\
\hline 792 & 384678955 & 45453372 & 07902632 & 1446525 & 81.025 & -0.61837 & 73.5 & 728 & 727 & 72.5 & 0,000218 \\
\hline 5.019989 & 3.83490942 & 14.5478364 & $\begin{array}{r}0.99986416 \\
\end{array}$ & 14.4825 & $\begin{array}{r}01.020 \\
81.15\end{array}$ & 173.19723 & 73.5 & $-\frac{12.0}{72.9}$ & 72.6 & 72.5 & 0.000221 \\
\hline 10.209991 & 3.8249051 & 14.5478364 & 1.99861328 & 14.4825 & 247.475 & 2111.117 & 73.4 & 72.8 & 72.6 & 72.5 & 0,000271 \\
\hline 15.019989 & 3.82553037 & 14.5478364 & 1.99986416 & 14,48325 & 780.25 & 3166.6743 & 73.4 & 72.8 & 72.6 & 72.5 & 0.000352 \\
\hline 20.019989 & 3.83178307 & 14.548586 & 1.99986416 & 14.4825 & 1382.75 & 3609.6558 & 73.4 & 72.8 & 72.6 & 72.5 & 0.00044 \\
\hline 25,019989 & 3.84428847 & 14.548586 & 2.00174048 & 14.48325 & 1903 & 3770.8077 & 73.4 & 72.8 & 72.6 & 72.5 & 0.000517 \\
\hline 30.179993 & 3.84303793 & 14.548586 & 2.00174048 & 14.4825 & 2393.5 & 3861.4401 & 73.4 & 72.9 & 72.6 & 72.5 & 0.000583 \\
\hline 35.019989 & 3.84053685 & 14.5493357 & 2.00424224 & 14.4825 & 2736 & 3896.9346 & 73.5 & 72.8 & 72.6 & 72.4 & 0.000632 \\
\hline 40.019989 & 3.82740618 & 14.5478364 & 2.0036168 & 14.4825 & 3043.5 & 3919.0476 & 73.5 & 72.8 & 72.8 & 72.5 & 0.000672 \\
\hline 45.189972 & 3.85491806 & 14.5463372 & 2.00424224 & 14.4825 & 3263.25 & 3929.2282 & 73.5 & 72.9 & 72.8 & 72.4 & 0.000705 \\
\hline 50.039978 & 3.83178307 & 14.5478364 & 2.00611856 & 14.48325 & 3465.25 & 3938,4156 & 73.5 & 72.8 & 72.7 & 72.5 & 0.000728 \\
\hline 55.029999 & 3.81740186 & 14.548586 & 2.00611856 & 14.48325 & 3584.25 & 3948.3479 & 73.5 & 72.8 & 72.6 & 72.5 & 0.00075 \\
\hline 60.019989 & 3.83240834 & 14.5493357 & 2.00549312 & 14.48325 & 3714.75 & 3949.0928 & 73.5 & 72.8 & 72.6 & 72.5 & 0.000766 \\
\hline 65.089996 & 3.82365456 & 14.5478364 & 2.00549312 & 14.4825 & 3804.75 & 3968.2125 & 73.5 & 72.8 & 72.6 & 72.5 & 0.000779 \\
\hline 70.019989 & 3.8311578 & 14.548586 & 2.00611856 & 14.4825 & 3895 & 3978.6415 & 73.4 & 72.9 & 72.6 & 72.5 & 0.00079 \\
\hline 75.029999 & 3.87367616 & 14.5478364 & 2.00611856 & 14.4825 & 3955.25 & 3996.023 & 73.5 & 72.9 & 72.6 & 72.5 & 0.0008 \\
\hline 80.029999 & 3.82990726 & 14.5478364 & 2.00549312 & 14.4825 & 4005.25 & 4001.2375 & 73.4 & 72.9 & 72.6 & 72.5 & 0.000807 \\
\hline 85.019989 & 3.82740618 & 14.548586 & 2.00549312 & 14.48175 & 4045 & 4000.7409 & 73.4 & 72.9 & 72.6 & 72.5 & 0.000812 \\
\hline 90.019989 & 3.84929063 & 14.548586 & 2.00549312 & 14.4825 & 4095.25 & 4006.452 & 73.5 & 72.9 & 72.6 & 72.5 & 0.000819 \\
\hline 95.089996 & 3.82427983 & 14.5478364 & 2.00611856 & 14.4825 & 4125 & 4025.82 & 73.5 & 72.9 & 72.6 & 72.5 & 0.000823 \\
\hline 100.01999 & 3.84116212 & 14.548586 & 2.00736944 & 14.4825 & 4145.5 & 4031.0345 & 73.5 & 72.9 & 72.7 & 72.5 & 0.000827 \\
\hline 105.01999 & 3.83803577 & 14.548586 & 2.00799488 & 14.48175 & 4165.5 & 4037.4905 & 73.5 & 72.9 & 72.7 & 72.5 & 0.00083 \\
\hline 110.20999 & 3.8249051 & 14.548586 & 2.00862032 & 14.4825 & 4195.25 & 4052.389 & 73.5 & 73 & 72.7 & 72.5 & 0.000833 \\
\hline 115.15997 & 3.82177875 & 14.5478364 & 2.006744 & 14.48325 & 4215.5 & 4045.188 & 73.6 & 72.9 & 72.7 & 72.5 & 0.000835 \\
\hline 120.01999 & 3.8311578 & \begin{tabular}{|l|l|} 
& 14.548586 \\
\end{tabular} & 200799488 & 14.48325 & 4225.5 & 4046.9262 & 73.6 & 72.9 & 72.7 & 72.5 & 0.000837 \\
\hline 125.34 & 3.8249051 & 14.548586 & 2.00862032 & 14.4825 & 4246 & 4056.1136 & 73.7 & 73 & $\mathbf{7 2 . 7}$ & 72.5 & 0.00084 \\
\hline 130.01999 & 3.82740618 & \begin{tabular}{|l|l|}
34.5470868 \\
\end{tabular} & 2.00736944 & 14.4825 & 4255.25 & 4071.757 & 73.7 & 73 & 72.6 & 72.5 & 0.000842 \\
\hline 135.01999 & 3.82177875 & 14.5478364 & 2.0098712 & 14.4825 & 4273.25 & 4073.4951 & 73.7 & 73 & 72.6 & 72.5 & 0.000843 \\
\hline 140.01999 & 3.82678091 & 14.5455876 & 2.00799488 & 14.48325 & 4285.75 & 4074.4884 & 73.7 & 73 & 72.6 & 72.5 & 0.000845 \\
\hline 145.01999 & 3.84741482 & 14.5493357 & 2.00924576 & 14.4825 & 4285.25 & 4076.7231 & 73.7 & 73 & 72.6 & 72.5 & 0.000846 \\
\hline 150.06998 & 3.83803577 & 14.5455876 & 2.00799488 & 14.48325 & 4295.25 & 4091.8699 & 73.7 & 73 & 72.6 & 72.5 & 0.000846 \\
\hline 155.20999 & 3.83178307 & 14.5455876 & 2.0098712 & 14.48175 & 4306 & 4104.2853 & 73.6 & 73 & 72.6 & 72.5 & 0.000848 \\
\hline 160.01999 & 3.82615564 & 14.5463372 & 2.00799488 & 14.484 & 4316 & 4100.809 & 73.7 & 73 & 72.6 & 72.5 & 0.000849 \\
\hline 165.01999 & 3.84241266 & 14.5463372 & 2.00799488 & 14.48325 & 4326 & 4100.809 & 73.8 & 73 & 72.6 & 72.5 & 0.00085 \\
\hline 170.01999 & 3.84678955 & 14.5455876 & 2.00862032 & 14.4825 & 4325.75 & 4096.0912 & 73.8 & 73 & 72.7 & 72.5 & 0.000852 \\
\hline 175.01999 & 3.85241698 & 14.5470868 & 2.0098712 & 14.48325 & 4335.5 & 4085.6622 & 73.8 & 73 & 72.7 & 72.5 & 0.000853 \\
\hline 180.01999 & 3.84491374 & 14.5448379 & 201049664 & 14.4825 & 4345.5 & 4083.4275 & 73.8 & 73 & 72.7 & 72.5 & 0.000854 \\
\hline 185.01999 & 3.83803577 & 14.5478364 & 2.00799488 & 14.48325 & 4345.25 & 4083.4275 & 73.8 & 73 & 72.7 & 72.6 & 0.000855 \\
\hline
\end{tabular}




\begin{tabular}{|c|c|c|c|c|c|c|c|c|c|c|c|}
\hline SECONDS & $\begin{array}{l}\text { MAIN DUCT } \\
\text { DIFF (IN H2O }\end{array}$ & \begin{tabular}{|l} 
MAIN DUCT \\
ABS (PSIA)
\end{tabular} & $\begin{array}{l}\text { GAS INJECT } \\
\text { DIFF (IN H2O }\end{array}$ & $\frac{\text { GAS INJECT }}{\text { ABS (PS|A) }}$ & $\begin{array}{l}\text { NEWPORT } \\
\text { (PPM) } \\
\end{array}$ & \begin{tabular}{|l} 
SMC \\
(PPM)
\end{tabular} & $\begin{array}{l}\text { AMBIENT } \\
\text { TEMP. (F }\end{array}$ & \begin{tabular}{|l|} 
GAS INJECT \\
TEMP. (F)
\end{tabular} & \begin{tabular}{|l|} 
MAIN DUCT \\
TEMP. (F)
\end{tabular} & \begin{tabular}{|l} 
WEC CEL \\
TEMP. (F)
\end{tabular} & $\begin{array}{l}\text { TWEC CELL } \\
\text { (VOLTS) }\end{array}$ \\
\hline & & & & & & & & & & & \\
\hline 190.01999 & 3.83178307 & 14.5463372 & 2.00799488 & 14.4825 & 4355.75 & 4087.897 & 73.8 & 73.1 & 72.6 & 72.6 & 0.000855 \\
\hline 195.01999 & 3.82052821 & 14.5463372 & 2.0098712 & 14.4825 & 4356 & 4094.6013 & 73.7 & 73.1 & 72.8 & 72.6 & 0.000855 \\
\hline 200.01999 & 3.82740618 & 14.5463372 & 2.00862032 & 14.4825 & 4365.75 & 4098.3259 & 73.7 & 73.1 & 72.6 & 72.6 & 0.000855 \\
\hline 205.01999 & 3.83615996 & 14.5455876 & 2.01049664 & 14.4825 & 4366.25 & 4107.0167 & 73.6 & 73.1 & 72.6 & 72.5 & 0.000856 \\
\hline 210.01999 & 3.82115348 & 14.5463372 & 2.00924576 & 14.4825 & 4366.25 & 4125.8881 & 73.6 & 73.1 & 72.7 & 72.6 & 0.000856 \\
\hline 215.01999 & 3.8249051 & 14.5463372 & 2.0096712 & 14.48325 & 4365.5 & 4120.922 & 73.7 & 73.1 & 72.7 & 72.5 & 0.000857 \\
\hline 220.01999 & 3.83803577 & 14.5448379 & 2.00799488 & 14.48325 & 4366.25 & 4113.721 & 73.6 & 73.1 & 72.7 & 72.5 & 0.000857 \\
\hline 225.01999 & 3.83803577 & 14.5463372 & 2.00862032 & 14,48325 & 4375.5 & 4116.2041 & 73.7 & 73.1 & 72.7 & 72.5 & 0.000858 \\
\hline 230.01999 & 3.82302929 & 14.5448379 & 2.00924576 & 14.48175 & 4375.5 & 4104.5336 & 73.6 & 73.1 & 72.7 & 72.6 & 0.000859 \\
\hline 235.01999 & 3.83365888 & 14.5455876 & 2.00862032 & 14.48325 & 4376.25 & 4104.7819 & 73.6 & 73.1 & 72.7 & 72.6 & 0.00086 \\
\hline 240.01999 & 3.83178307 & 14.5455876 & 2.00862032 & 14.48325 & 4385.75 & 4105.0303 & 73.7 & 73.2 & 72.7 & 72.6 & 0.00086 \\
\hline 245.01999 & 3.8249051 & 14.5455876 & 2.00799488 & 14.48325 & 4386 & 4115.9558 & 73.7 & 73.2 & 72.8 & 72.5 & 0.00086 \\
\hline 250.01999 & 3.83490942 & 14.5455876 & 2.00736944 & 14.4825 & 4395.75 & 4114.2177 & 73.6 & 73.2 & 72.8 & 72.5 & 0,000861 \\
\hline 255.01999 & 3.84178739 & 14.5448379 & 2.0098712 & 14.4825 & 4396 & 4110.7413 & 73.6 & 73.2 & 72.8 & 72.6 & 0.000861 \\
\hline 260.01999 & 3.82615564 & 14.5463372 & 2.00862032 & 14.4825 & 4405.75 & 4104.037 & 73.6 & 73.2 & 72.8 & 72.6 & 0.000862 \\
\hline 265.01999 & 3.82740618 & 14.5455876 & 2.0098712 & 14.48325 & 4405.75 & 4107.7616 & 73.6 & 73.2 & 72.8 & 72.6 & 0.000862 \\
\hline 270.01999 & 3.83803577 & 14.5455876 & 2.00799488 & 14.48325 & 4406 & 4114.2177 & 73.7 & 73.2 & 72.8 & 72.6 & 0.000863 \\
\hline 275.01999 & 3.82365456 & 14.5463372 & 2.0098712 & 14.48325 & 4405.75 & 4104.037 & 73.6 & 73.2 & 72.8 & 72.6 & 0.000863 \\
\hline 280.01999 & 3.83240834 & 14.5448379 & 2.00862032 & 14.4825 & 4416.25 & 4122.6601 & 73.7 & 73.2 & 72.8 & 72.6 & 0.000863 \\
\hline 285.01999 & 3.83365888 & 14.5470868 & 2.00736944 & 14.4825 & 4416 & $4112 . \overline{4795}$ & 73.6 & 73.3 & 72.7 & 72.6 & 0.000863 \\
\hline 290.01999 & 3.84116212 & 14,5463372 & 2.00799488 & 14.4825 & 4416.25 & 4112.2312 & 73.6 & 73.3 & 72.7 & 72.5 & 0.000863 \\
\hline 295.01999 & 3.84616428 & 14.5448379 & 2.00862032 & 14.48325 & 4416 & 4117.1973 & 73.6 & 73,3 & 72.7 & 72.5 & .000864 \\
\hline 300.01999 & 3.8311578 & 14.5463372 & 2.00862032 & 14,4795 & 4415.75 & 4119.1838 & 73.6 & 73.3 & 72.7 & 72.5 & 0.000862 \\
\hline 305.01999 & 3.83240834 & 14.5455876 & 2.00799488 & 14.4825 & 4416.25 & 4119.1838 & 73.5 & 73.3 & 72.7 & 72.5 & 0.000863 \\
\hline 310.03 & 3.84553901 & 14.5455876 & 2.00799488 & 14.48175 & 4416 & 4124.8949 & 73.6 & 73.3 & 72.7 & 72.5 & 0.000863 \\
\hline 315.01999 & 3.82740618 & 14.5440883 & 2.00611856 & 14.4825 & 4415.75 & 4108.01 & 73.4 & 73.3 & 72.6 & 72.6 & 0.000863 \\
\hline 320.01999 & 3.83803577 & 14.5455876 & 2.00486768 & 14.4795 & 4415.75 & 4103.5404 & 73.4 & 73.3 & 72.7 & 72.6 & 0,000863 \\
\hline 325.03 & 3.85054117 & 14.5463372 & 2.006744 & 14.48025 & 4420 & 4103.7887 & 73.5 & 73.3 & 72.7 & 72.6 & 0.000864 \\
\hline 330.01999 & $\mathbf{3 . 8 3 1 7 8 3 0 7}$ & 14.5463372 & 2.00799488 & 14.48025 & 4425.5 & 4119.1838 & 73.5 & 73.3 & 72.7 & 72.6 & 0.000865 \\
\hline 335.01999 & 3.83365688 & 14.5448379 & 2.00799488 & 14.481 & 4425.5 & 4107.265 & 73.5 & 73.3 & 72.6 & 72.6 & 0.000865 \\
\hline 340.01999 & 3.87242562 & 14.5470868 & 2.00611856 & 14.481 & 4426 & 4112.9761 & 73.6 & 73.3 & 72.7 & 72.6 & 0.000865 \\
\hline 345.01999 & 3.82740618 & 14.5463372 & 2.00549312 & 14.48025 & 4425.25 & 4125.8881 & 73.6 & 73.4 & 72.7 & 72.6 & 0.000865 \\
\hline 350.01999 & 3.8436632 & 14.5448379 & 2.00611856 & 14.48025 & 4426.25 & 4118.6872 & 73.6 & 73.3 & 72.8 & 72.6 & 0.000866 \\
\hline 355.03 & 3.82928199 & 14.5448379 & 2.00799488 & 14.48025 & 4426 & 4123.9017 & 73.6 & 73.3 & 72.7 & 72.6 & 0.000865 \\
\hline 350.01999 & 3.83428415 & 14.5463372 & 2.00549312 & 14.48025 & 4425.75 & 4113.9693 & 73.7 & 73.3 & 72.7 & 72.5 & 0.000866 \\
\hline 365.01999 & 3.83553469 & 14.5455876 & 2.006744 & 14.48025 & 4426 & 4114.7143 & 73.7 & 73.4 & 72.6 & 72.6 & 0.000865 \\
\hline 370.01999 & 3.82427983 & 14.5463372 & 2.00862032 & 14.48025 & 4425.75 & 4123.9017 & 73.7 & 73.4 & 72.7 & 72.6 & 0.000866 \\
\hline 375.01999 & 3.84116212 & 14.5463372 & 2.006744 & 14.48025 & 4425.75 & 4128.1229 & 73.6 & 73.3 & 72.7 & 72.6 & 0.000865 \\
\hline 380,01999 & 3.83428415 & 14.5455876 & 2.00549312 & 14.481 & 4425.75 & 4145.0078 & 73.6 & 73.4 & 72.7 & 72.6 & 0.000866 \\
\hline 385.01999 & 3.83803577 & 14.5455876 & 2.00799488 & 14.48025 & 4426.25 & 4140.29 & 73.6 & 73.4 & 72.7 & 72.6 & 0.000866 \\
\hline 390.01999 & 3.82615564 & 14.5440883 & 2.00736944 & 14.48025 & 4425.75 & 4134.0823 & 73.6 & 73.4 & 72.8 & 72.6 & 0.000866 \\
\hline 395.01999 & 3.86179603 & 14.5463372 & 2.00549312 & 14.48025 & 4426.25 & 4126.8814 & 73.6 & 73.4 & 72.7 & 72.6 & 0.000866 \\
\hline
\end{tabular}




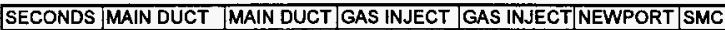
DIFF (IN H2O ABS (PSIA) DIFF (IN H2O ABS (PSIA) (PPM)

AMBIENT GAS INJECT MAIN DUCT TWEC CEL WEC CELL

\begin{tabular}{|c|c|c|c|c|c|c|c|c|c|c|c|}
\hline & DiFF (IN HLO & $\operatorname{MBS}(\mathrm{RSIA})$ & 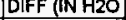 & AOS (PSIA) & (IPM) & $(\mathbf{P} \mathbf{T M})$ & IEMF. (F & IEMP. (r) & IEMT. (r) & IEMA. (r) & (VOLIS) \\
\hline 400.01999 & 3.8624213 & 14.5470868 & 2.00611856 & 14.4795 & 4426 & 4117.4457 & 73.6 & 73.4 & 72.8 & 72.6 & 0.000866 \\
\hline 405.01999 & 3.82803145 & 14.5463372 & 2.00611856 & 14.48025 & 4436.25 & 4127.1297 & 73.6 & 73.4 & 72.8 & 72.6 & 0.000867 \\
\hline 410,01999 & 3.82365456 & 14.5455876 & 2.006744 & 14.48025 & 4436.5 & 4117.9423 & 73.6 & 73.4 & 72.8 & 72.6 & 0.000868 \\
\hline 415.01999 & 3.82803145 & 14.5463372 & 2.006744 & 14.481 & 4436.25 & 4126.6331 & 73.5 & 73.4 & 72,8 & 72.6 & 0.000868 \\
\hline 420.03998 & 3.84428847 & 14.5455876 & 2.00549312 & 14.4795 & 4446.25 & 4129.8611 & 73.5 & 73.4 & 72.8 & 72.6 & 0.000868 \\
\hline 425.01999 & 3.82365456 & 14.5440883 & 2.00549312 & 14.48025 & 4446.25 & 4131.1026 & 73.6 & 73.4 & 72.7 & 72.6 & 0.000868 \\
\hline 430.01999 & 3.83178307 & 14.5448379 & 2.00611856 & 14.48025 & 4446.25 & 4112.9761 & 73.6 & 73.5 & 72.7 & 72.6 & 0.000867 \\
\hline 435.01999 & 3.82803145 & 14.5448379 & 2.00611856 & 14.48025 & 4435.75 & 4121.6669 & 73.6 & 73.4 & 72.7 & 72.6 & 0.000867 \\
\hline 440.01999 & 3.83240834 & 14.5455876 & 2.00611856 & 14,48025 & 4436.25 & 4120.177 & 73.6 & 73.5 & 72.7 & 72.6 & 0.000867 \\
\hline 445.01999 & 3.8311578 & 14.5455876 & 2.00549312 & 14.48025 & 4445.5 & 4132.8408 & 73.6 & 73.5 & 72.7 & 72.6 & 0.000867 \\
\hline 450.01999 & 3.84553901 & 14.5455876 & 2.00486768 & 14.48025 & 4446 & 4132.5924 & 73.6 & 73.5 & 72.6 & 72.6 & 0.000867 \\
\hline 455.01999 & 3.82365456 & 14.5448379 & 2.00611856 & 14.4795 & 4446.25 & 4117.4457 & 73.7 & 73.5 & 72.6 & 72.6 & 0.000868 \\
\hline 460.03998 & 3.85804441 & 14.5448379 & 2.00549312 & 14.48025 & 4446.25 & 4120.4254 & 73.7 & 73.5 & 72.6 & 72.6 & 0,000868 \\
\hline 465.01990 & 3.83428415 & 14.5463372 & 2.00611856 & 14.48025 & 4446.25 & 4119.1838 & 73.6 & 73.5 & 72.6 & 72.6 & 0.000867 \\
\hline 470.01999 & 3.82865672 & 14.5448379 & 2.00511856 & 14.48025 & 4446.25 & 4120.177 & 73.6 & 73.5 & 72.6 & 72.6 & 0.000867 \\
\hline 475.01999 & 3.83803577 & 14.5448379 & 2.00611856 & 14.48025 & 4446.25 & 4134.3306 & 73.6 & 73.5 & 72.6 & 72.6 & 0.000868 \\
\hline 480,06998 & 3.83428415 & 14.5455876 & 2.00549312 & 14.481 & 4446.25 & 4118.6872 & 73.6 & 73.5 & 72.5 & 72.6 & 0.000869 \\
\hline 485.01999 & 3.83178307 & 14.5455876 & 2.00611856 & 14.48175 & 4446.25 & 4133,834 & 73.6 & 73.5 & 72.6 & 72.6 & 0.000868 \\
\hline 490.10999 & 3.83991158 & 14,5455876 & 2.006744 & 14,48025 & 4446.25 & 4133.0891 & 73.6 & 73.5 & 72.6 & 72.6 & 0.000869 \\
\hline 495.01990 & 3.83803577 & 14.5455876 & 2.00549312 & 14.481 & 4446 & 4134.3306 & 73.4 & 73.5 & 72.5 & 72.6 & 0.000868 \\
\hline 500.10999 & 3.85116644 & 14.5448379 & 2.00611856 & 14.481 & 4446.25 & 4128.8678 & 73.5 & 73.5 & 72.6 & 72.6 & 0.000868 \\
\hline 505.01999 & 3.82553037 & 14.5455876 & 2.00549312 & 14.481 & 4445.75 & 4121.6669 & 73.5 & 73.6 & 72.5 & 72.6 & 0.000868 \\
\hline 510.06 & 3.85304225 & 14.5455876 & 2.00549312 & 14.48025 & 4446.25 & 4132.3441 & 73.5 & 73.5 & 72.5 & 72.6 & 0.000868 \\
\hline 515.01999 & 3.82803145 & 14.5455876 & 2.00549312 & 14.48025 & 4446.25 & 4122.6601 & 73.5 & 73.5 & 72.6 & 72.6 & 0.000868 \\
\hline 520.01999 & 3.83678523 & 14.5463372 & 2.00549312 & 14.481 & 4445.75 & 4117.9423 & 73.6 & 73.6 & 72.6 & 72.6 & 0.000869 \\
\hline 525.03 & 3.84241266 & 14.5463372 & 2.00486768 & 14.481 & 4446.25 & 4106.5201 & 73.6 & 73.5 & 72.6 & 72.6 & 0.000869 \\
\hline 530.01999 & 3.82427983 & 14.5455876 & 2.00611856 & 14.48025 & 4446.25 & 4116.2041 & 73.5 & 73.6 & 72.6 & 72.6 & 0.00087 \\
\hline 535.01999 & 3.84116212 & 14.5470868 & 2.00611856 & 14.48025 & 4455 & 4120.922 & 73.6 & 73.6 & 72.6 & 72.6 & 0.00087 \\
\hline 540.07999 & 3.84866536 & 14.5463372 & 2.00611856 & 14.481 & 4456 & 4122.6601 & 73.5 & 73.6 & 72.6 & 72.6 & 0.000869 \\
\hline 545.01999 & 3.84428847 & 14.5470868 & 2.00549312 & 14.48025 & 4456.5 & 4120.177 & 73.5 & 73.5 & 72.6 & 72.6 & 0.000869 \\
\hline 550.01999 & 3.83428415 & 14.5463372 & 2.00611856 & 14.48025 & 4456 & 4123.405 & 73.5 & 73.6 & 72.6 & 72.6 & 0.00087 \\
\hline 555.04999 & 3.83803577 & 14,5463372 & 2.00549312 & 14.48025 & 4455.75 & 4122,6601 & 73.5 & 73.6 & 72.5 & 72.6 & 0.00087 \\
\hline 560.01999 & 3.82990726 & 14.5440883 & 2.00486768 & 14.4795 & 4456 & 4127.1297 & 73.4 & 73.6 & 72.5 & 72.6 & 0.000871 \\
\hline 565.01999 & 3.85679387 & 14.5455876 & 2.00549312 & 14.48025 & 4456.25 & 4126.6331 & 73.3 & 73.6 & 72.4 & 72.6 & 0.00087 \\
\hline 570.01999 & 3.82740618 & 14.5448379 & 2.006744 & 14.48025 & 4456.25 & 4126.3847 & 73.3 & 73.6 & 72.5 & 72.6 & 0.000871 \\
\hline 575.01999 & 3.8249051 & 14.5455876 & 2.00611856 & 14.4795 & 4456.25 & 4124.3983 & 73.3 & 73.6 & 72.5 & 72.6 & 0.00087 \\
\hline 580.01999 & 3.82740618 & 14.5455876 & 2.00611856 & 14.481 & 4456 & 4128.6195 & 73.3 & 73.6 & 72.5 & 72.6 & 0.00087 \\
\hline 585.04999 & 3.8311578 & 14.5448379 & 200611856 & 14.48025 & 4456 & 4130.1094 & 73.3 & 73.6 & 72.6 & 72.6 & 0.000871 \\
\hline 590.06998 & 3.86617292 & 14.5455876 & 2.00486768 & 14.481 & 4456 & 4126.8814 & 73.3 & 73.6 & 72.5 & 72.6 & 0.000871 \\
\hline 595.01999 & 3.83240834 & 14.5455876 & 2.00549312 & 14.481 & 4466 & 4127.378 & 73.3 & 73.6 & 72.5 & 72.6 & 0.000872 \\
\hline 600.03998 & 3.83365888 & 14.5455876 & 2.00549312 & 14.481 & 4466 & 4122.6601 & 73.3 & 73.6 & 72.5 & 72.6 & 0.000871 \\
\hline 605.01999 & 3.8374105 & 14.5440883 & 200611856 & 14.48025 & 4466.25 & 4127,378 & 73.3 & 73.6 & $\overline{72.5}$ & 72.6 & 0.000871 \\
\hline
\end{tabular}




\begin{tabular}{|c|c|c|c|c|c|c|c|c|c|c|c|}
\hline SECONDS & \begin{tabular}{|l} 
MAIN DUCT \\
DIFF (IN H2O
\end{tabular} & $\begin{array}{l}\text { MAIN DUCT } \\
\text { ABS (PSIA) }\end{array}$ & \begin{tabular}{|l|} 
GAS INJECT \\
DIFF (IN H2O
\end{tabular} & \begin{tabular}{|l|} 
GAS INJECT \\
ABS (PSIA)
\end{tabular} & $\begin{array}{l}\text { NEWPORT } \\
\text { (PPM) }\end{array}$ & $\begin{array}{l}\text { SMC } \\
\text { (PPM) }\end{array}$ & $\begin{array}{l}\text { AMBIENT } \\
\text { TEMP. F }\end{array}$ & $\begin{array}{l}\text { GAS INJECT } \\
\text { TEMP. (F) } \\
\end{array}$ & \begin{tabular}{|l|} 
MAIN DUCT \\
TEMP. (F)
\end{tabular} & $\frac{\text { WEC CEL }}{\text { TEMP.(F) }}$ & $\begin{array}{l}\text { WEC CELL } \\
\text { (VOLTS) }\end{array}$ \\
\hline 610.01999 & 3.85241698 & 14.5448379 & 2.00549312 & 14.48025 & 4466 & 4131.1026 & 73.3 & 73.6 & 72.6 & 72.7 & 0.000872 \\
\hline 615.01999 & 3.85491806 & 14.5448379 & 2.00611856 & 14.48025 & 4466.25 & 4120.177 & 73.4 & 73.6 & 72.6 & 72.6 & 0.000872 \\
\hline 620.01999 & 3.84616428 & 14.5440883 & 2.00611856 & 14.48025 & 4466.25 & 4131.8475 & 73.4 & 73.6 & 72.6 & 72.6 & 0.000871 \\
\hline 625.01999 & 3.83428415 & 14.5448379 & 2.00611856 & 14.481 & 4466 & 4142.5248 & 73.5 & 73.6 & 72.6 & 72.6 & 0.000871 \\
\hline 630.04999 & 3.8374105 & 14.5455876 & 2.00611856 & 14.47725 & 4456.75 & 4128.3712 & 73.5 & 73.7 & 72.6 & 72.6 & 0.00087 \\
\hline 635.01999 & 3.83803577 & 14.5455876 & 2.00611856 & 14.478 & 4456.25 & 4129.8611 & 73.4 & 73.7 & 72.5 & 72.6 & 0.000869 \\
\hline 640.01999 & 3.83991158 & 14.5455876 & 2.00611856 & 14,481 & 4456.25 & 4127.378 & 73.5 & 73.6 & 72.6 & 72.6 & 0.00087 \\
\hline 645.01999 & 3.8249051 & 14.5448379 & 2.00611856 & 14.47725 & 4456.25 & 4125.6398 & 73.5 & 73.7 & 72.6 & 72.6 & 0.00087 \\
\hline 650.01909 & 3.8374105 & 14.5448379 & 2.00549312 & 14.48025 & 4456.25 & 4125.8881 & 73.4 & 73.7 & 72.6 & 72.6 & 0.00087 \\
\hline 655.01999 & 3.83803577 & 14.5455876 & 2.00611856 & 14.48025 & 4466.25 & 4128.8678 & 73.5 & 73.7 & 72.6 & 72.6 & 0.00087 \\
\hline 660.04999 & 3.87117508 & 14.5455876 & 2.00549312 & 14.481 & 4465.75 & 4128.1229 & 73.5 & 73.7 & 72.6 & 72.6 & 0.00087 \\
\hline 665.01999 & 3.81802713 & 14.5463372 & 200611856 & 14.48025 & 4466.25 & 4136.3171 & 73.5 & 73.7 & 72.6 & 72.7 & 0.00087 \\
\hline 670.01999 & 3.84178739 & 14.5455876 & 2.00611856 & 14,48025 & 4465.75 & 4126.8814 & 73.5 & 73.7 & 72.6 & 72.7 & 0.00087 \\
\hline 675.03998 & 3.83928631 & 14.5455876 & 2.00549312 & 14.48025 & 4466.25 & 4131.5992 & 73.5 & 73.7 & 72.6 & 72.6 & 0.00087 \\
\hline 680.01999 & 3.8311578 & 14.5455876 & 2.006744 & 14.481 & 4466 & 4137.8069 & 73.5 & 73.7 & 72.6 & 72.7 & 0.00087 \\
\hline 685.01999 & 3.82928199 & 14.5463372 & 2.00611856 & 14.48025 & 4466 & 4126.1364 & 73.4 & 73.7 & 72.5 & 72.7 & 0.000872 \\
\hline 690.03998 & 3.8311578 & 14.5455876 & 2.00611856 & 14.481 & 4466.5 & 4115.9558 & 73.5 & 73.7 & 72.5 & 72.7 & 0.00087 \\
\hline 695.04999 & 3.82740618 & 14.5470868 & 2.00611856 & 14.48025 & 4466 & 4118.1906 & 73.4 & 73.7 & 72.5 & 72.7 & 0.000874 \\
\hline 700.01999 & 3.84178739 & 14.5448379 & 2.006744 & 14.478 & 4465.75 & 4118.1906 & 73.4 & 73.7 & 72.6 & 72.7 & 0.000871 \\
\hline 705.01999 & 3.82928199 & 14.5463372 & 2.00611856 & 14.478 & 4466.25 & 4117.1973 & 73.4 & 73.7 & 72.6 & $\overline{72.7}$ & 0.00087 \\
\hline 710.06998 & 3.82052821 & 14.5448379 & 2.00549312 & 14.48025 & 4465.75 & 4128.1229 & 73.4 & 73.7 & 72.6 & 72.7 & 0.00087 \\
\hline 715.01999 & 3.82553037 & 14.5455876 & 2.00549312 & 14.478 & 4466.25 & 4122.4118 & 73.4 & 73.7 & 72.6 & 72.7 & 0.000871 \\
\hline 720.06998 & 3.82865672 & 14.5448379 & 2.00611856 & 14,4795 & 4466.25 & 4123.9017 & 73.4 & 73.7 & 72.6 & 72.7 & 0.000871 \\
\hline 725.01999 & 3.83303361 & 14.5455876 & 2.00611856 & 14.48025 & 4466.25 & 4114.2177 & 73.4 & 73.7 & 72.6 & 72.7 & 0.000871 \\
\hline 730.01999 & 3.83053253 & 14.5455876 & 200611856 & 14.47725 & 4466.25 & 4114.466 & 73.4 & 73.7 & 72.5 & 72.7 & 0.000871 \\
\hline 735.06998 & 3.82928199 & 14.5455876 & 2.00549312 & 14.47725 & 4456.25 & 4117.1973 & 73.4 & 73.7 & 72.5 & 72.7 & 0.000871 \\
\hline 740.01999 & 3.82678091 & 14.5470868 & 2.00611856 & 14.478 & 4476.25 & 4126.8814 & 73.4 & 73.7 & 72.5 & 72.6 & 0.000871 \\
\hline 745.01999 & 3.8249051 & 14.5448379 & 2.00611856 & 14.48025 & 4466.25 & 4128.1229 & 73.4 & 73.7 & 72.6 & 72.7 & 0.000871 \\
\hline 750.04999 & 3.83240834 & 14.5455876 & 2.00549312 & 14.47875 & 4466.25 & 4132.0958 & 73.4 & 73.7 & 72.6 & 72.6 & 0.000871 \\
\hline 755.01999 & 3.83178307 & 14.5463372 & 2.006744 & 14.48025 & 4466 & 4136.3171 & 73.5 & 73.8 & 72.7 & 72.6 & 0.000872 \\
\hline 760.01999 & 3.82553037 & 14.5455876 & 2.00486768 & 14.4795 & 4466.25 & 4125.3915 & 73.5 & 73.8 & 72.6 & 72.7 & 0.000872 \\
\hline 765.03998 & 3.83615996 & 14.5463372 & 2.00549312 & 14.47725 & 4476.25 & 4129.1161 & 73.5 & 73.8 & 72.6 & 72.6 & 0.000873 \\
\hline 770.01999 & 3.8249051 & 14.5470868 & 2.00486768 & 14.48025 & 4476 & 4138.0552 & 73.5 & 73.7 & 72.6 & 72.7 & 0.000873 \\
\hline 775.01999 & 3.8311578 & 14.5455876 & 2.00611856 & 14.47725 & 4476.25 & 4135.3238 & 73.4 & 73.7 & 72.5 & 72.6 & 0.000874 \\
\hline 780.04999 & 3.83053253 & 14.5455876 & 2.00611856 & 14.478 & 4476.5 & 4129.8611 & 73.5 & 73.7 & 72.6 & 72.7 & 0.000873 \\
\hline 785.01999 & 3.82990726 & 14.5463372 & 2.00549312 & 14.478 & 4476 & 4129.8611 & 73.5 & 73.8 & 72.6 & 72.7 & 0.000872 \\
\hline 790.01999 & 3.84866536 & 14.5455876 & 2.00611856 & 14.478 & 4476.25 & 4119.9287 & 73.5 & 73.8 & 72.6 & 72.7 & 0.000872 \\
\hline 795.01999 & 3.84303793 & 14.5448379 & 2.00549312 & 14.47725 & 4476 & 4117.4457 & 73.5 & 73.7 & 72.6 & 72.7 & 0.000872 \\
\hline 800.01999 & 3.8311578 & 14.5440883 & 2.00549312 & 14.478 & 4476 & 4115.2109 & 73.4 & 73.8 & 72.6 & 72.7 & 0.000873 \\
\hline 805.01999 & 3.84053685 & 14.5455876 & 2.00611856 & 14.478 & 4476.25 & 4122.4118 & 73.4 & 73.8 & 72.6 & 72.7 & 0.000873 \\
\hline 810.03998 & 3.82240402 & 14.5448379 & 2.00611856 & 14.47725 & 4476.25 & 4118.4389 & 73.5 & 73.8 & 72.6 & 72.7 & 0.000872 \\
\hline 815.04999 & 3.83490942 & 14.5463372 & 2.00611856 & 14.478 & 4476.25 & 4122.1635 & 73.5 & 73.8 & 72.6 & 72.7 & 0.000872 \\
\hline
\end{tabular}




\begin{tabular}{|c|c|c|c|c|c|c|c|c|c|c|c|}
\hline SECONDS & \begin{tabular}{|l|} 
MAIN DUCT \\
DIFF (IN H2O \\
\end{tabular} & \begin{tabular}{|l|} 
MAIN DUCT \\
ABS (PSIA)
\end{tabular} & $\begin{array}{l}\text { GAS INJECT } \\
\text { DIFF (IN H2O }\end{array}$ & \begin{tabular}{|l|} 
GAS INJECT \\
ABS (PSIA)
\end{tabular} & $\begin{array}{l}\text { NEWPORT } \\
\text { (PPM) }\end{array}$ & $\frac{\text { SMC }}{\text { (PPM) }}$ & \begin{tabular}{|l|} 
AMBIENT \\
TEMP. $\mathbf{F}$ \\
\end{tabular} & \begin{tabular}{|l|} 
GAS INJECT \\
TEMP. (F)
\end{tabular} & \begin{tabular}{|l|} 
MAIN DUCT \\
TEMP. (F)
\end{tabular} & \begin{tabular}{|l} 
WEC CEL \\
TEMP. (F)
\end{tabular} & $\begin{array}{l}\text { WEC CELL } \\
\text { (VOLTS) }\end{array}$ \\
\hline & & & & & & & & & & & \\
\hline 820.01999 & 3.82678091 & 14.5463372 & 2.00549312 & 14.4765 & 4486.25 & 4121.6669 & 73.5 & 73.8 & 72.6 & 72.7 & 0.000872 \\
\hline 825.03 & 3.83053253 & 14.54555876 & 2.00611856 & 14.481 & 4486.25 & 4114.2177 & 73.5 & 73.8 & 72.6 & 72.7 & 0.000874 \\
\hline 830,07999 & 3.82052821 & 14.5455876 & 2.00611856 & 14.47725 & 4486.25 & 4122.1635 & 73.5 & 73.8 & 72.6 & 72.7 & 0.000874 \\
\hline 835.01999 & 3.83240834 & 14.5455876 & 2.00611856 & 14.4765 & 4486.25 & 4121.6669 & 73.5 & 73.8 & 72.6 & 72.7 & 0.000874 \\
\hline 840.03 & 3.82865672 & 14.5455876 & 2.00611856 & 14.47725 & 4486.25 & 4125.1432 & 73.5 & 73.8 & 72.6 & 72.7 & 0.000874 \\
\hline 845.01999 & 3.81427551 & 14.5433387 & 2.00549312 & 14.478 & 4486 & 4127.1297 & 73.5 & 73.8 & 72.6 & 72.7 & 0.000874 \\
\hline 850,01999 & 3.82553037 & 14.5463372 & 2.00549312 & 14.47875 & 4486.25 & 4128.3712 & 73.5 & 73.8 & 72.6 & 72.7 & 0.000874 \\
\hline 855.01999 & 3.82115348 & 14.5455876 & 2.00611856 & 14.478 & 4486.25 & 4130.1094 & 73.4 & 73.8 & 72.6 & 72.7 & 0.000874 \\
\hline 860.07999 & 3.84116212 & 14.5463372 & 2.00549312 & 14.478 & 4486.25 & 4130.1094 & 73.5 & 73.8 & 72.6 & 72.7 & 0.000873 \\
\hline 665.01999 & 3.82553037 & 14.5455876 & 2.00549312 & 14.478 & 4486.25 & 4127.8746 & 73.5 & 73.8 & 72.7 & 72.7 & 0.000874 \\
\hline 870.04999 & 3.84178739 & 14.5463372 & 2.00611856 & 14.478 & 4486.25 & 4126.8814 & 73.4 & 73.8 & 72.6 & 72.7 & 0.000874 \\
\hline 875.03998 & 3.84929063 & 14.5455876 & 2.00486768 & 14.4795 & 4486.25 & 4120.177 & 73.3 & 73.8 & 72.7 & 72.7 & 0.000874 \\
\hline 880.01999 & 3.83178307 & 14.5470868 & 2.00611856 & 14.478 & 4486.25 & 4132.5924 & 73.3 & 73.8 & 72.6 & 72.7 & 0.000874 \\
\hline 885.01999 & 3.83178307 & 14.5455876 & 2.00549312 & 14.478 & 4486.25 & 4118.6872 & 73.4 & 73.8 & 72.6 & 72.7 & 0.000874 \\
\hline 890.01999 & 3.82115348 & 14.5455876 & 2.00486768 & 14.48025 & 4486 & 4114.466 & 73.4 & 73.8 & 72.6 & 72.7 & 0.000873 \\
\hline 895.01999 & 3.83866104 & 14.5463372 & 2.00549312 & 14.478 & 4486.25 & 4121.1703 & 73.4 & 73.8 & 72.6 & 72.7 & 0.000873 \\
\hline 900.04999 & 3.84428847 & 14.5463372 & 2.00549312 & 14.47725 & 4486.25 & 4135.5721 & 73.4 & 73.8 & 72.6 & 72.7 & 0.000874 \\
\hline 905.01999 & 3.82803145 & 14.5463372 & 2.00549312 & 14.48025 & 4486.25 & 4139.5451 & 73.5 & 73.8 & 72.7 & 72.7 & 0.000873 \\
\hline 910.01999 & 3.82803145 & 14.5470868 & 2.00611856 & 14.478 & 4476.25 & 4140.5383 & 73.5 & 73.8 & 72.6 & 72.7 & 0.000873 \\
\hline 915.04999 & 3.83553469 & 14.5455876 & 2.00611856 & 14.478 & 4476.25 & 4141.7798 & 73.5 & 73.8 & 72.7 & 72.7 & 0.000872 \\
\hline 920.01999 & 3.82177875 & 14.5455876 & 2.00549312 & 14.47725 & 4476.5 & 4141.2832 & 73.5 & 73.9 & 72.6 & 72.7 & 0.000873 \\
\hline 925.01999 & 3.82803145 & 14.5455876 & 2.00486768 & 14.47875 & 4476.5 & 4130.1094 & 73.5 & 73.8 & 72.7 & 72.7 & 0.000872 \\
\hline 930.03 & 3.8186524 & 14.5448379 & 2.00549312 & 14.47725 & 4476.5 & 4125.3915 & 73.6 & 73.8 & 72.7 & 72.7 & 0.000872 \\
\hline 935.01999 & 3.84678955 & 14.5448379 & 2.00611856 & 14.478 & 4476.5 & 4134.0823 & 73.6 & 73.8 & 72.7 & 72.7 & 0.000873 \\
\hline
\end{tabular}




\begin{tabular}{|c|c|c|c|c|c|c|c|c|c|c|c|}
\hline \multicolumn{12}{|c|}{$\begin{array}{l}\text { TEST: FGI21311 } 13 \text { Feb } 1997 \text { WEC-SPOOL } \\
\text { Measured Hydrogen Concentration }=237.8 \mathrm{ppm}\end{array}$} \\
\hline & & & & & & & & & & & \\
\hline 0.01000977 & 3.78551309 & 14.8034575 & 0.79401584 & 15.0015 & 0.1725 & 3.6773584 & 70.6 & 70.4 & 78 & 74.5 & 0.000208 \\
\hline 5.02999878 & 3.79114052 & 14.8012087 & 6.9383384 & 15.885 & 0.17 & 3.1807424 & 70.5 & 70.5 & 78 & 74.6 & 0.000208 \\
\hline 10.0200195 & 3.77425823 & 14.8027079 & 6.95835248 & 15.88875 & 0.135 & -0.2210772 & 70.6 & 70.5 & 78 & 74.6 & 0.000207 \\
\hline 15.0200195 & 3.7686308 & \begin{tabular}{|l|l|} 
& 14,8034575 \\
\end{tabular} & 6.96585776 & 15.891 & 0.1675 & 32.4810864 & 70.5 & 70.6 & 78 & 74.5 & 0.000207 \\
\hline 20.0200195 & 3.76925607 & 14.8042071 & 6.97461392 & 15.89175 & 0.1275 & 58.7769036 & 70.5 & 70.6 & 78 & 74.6 & 0.000208 \\
\hline 25.0200195 & 3.76800553 & 14.8034575 & 6.97649024 & 15.891 & 0.16 & 72.260028 & 70.5 & 70.7 & 78 & 74.6 & 0.000209 \\
\hline 30.0200195 & 3.75174851 & 14.8034575 & 6.97961744 & 15.894 & 10.125 & 77.5738192 & 70.6 & 70.7 & 78 & 74.5 & 0.000209 \\
\hline 35.0200195 & 3.75987702 & 14.8049568 & 6.98086832 & 15.89325 & 10.1625 & 79.8582528 & 70.6 & 70.8 & 77.9 & 74.6 & 0.00021 \\
\hline 40.0200195 & 3.76988134 & 14.8049568 & 6.98086832 & 15.89325 & 20.165 & 81.0501312 & 70.6 & 70.8 & 78 & 74.6 & 0.000211 \\
\hline 45.0200195 & 3.76800553 & 14.8042071 & 6.98086832 & 15.894 & 20.12 & 81.1742852 & 70.6 & 70.9 & 77.9 & 74.6 & 0.000212 \\
\hline 50.0200195 & 3.77488 .35 & 14.8042071 & 6.98024288 & 15.894 & 30.15 & 81.0997928 & 70.5 & 70.9 & 78 & 74.6 & 0.000212 \\
\hline 55.0200195 & 3.77300769 & 14.8049568 & 6.98024288 & 15.8925 & 30.15 & 81.0997928 & 70.5 & 70.9 & 77.9 & 74.6 & 0.000213 \\
\hline 60.0200195 & 3.77425823 & 14.8049568 & 6.98086832 & 15.89325 & 40.125 & 81.1246236 & 70.5 & 71 & 78 & 74.5 & 0.000214 \\
\hline 65.0200195 & 3.77238242 & 14.8049568 & 6.98024288 & 15.894 & 40.175 & 81.2487776 & 70.5 & 71 & 78 & 74.5 & 0.000214 \\
\hline 70.0200195 & 3.79551741 & 14.8049568 & 6.98086832 & 15.897 & 50.2 & 80.6776692 & 70.5 & 71 & 78 & 74.6 & 0.000217 \\
\hline 75.0200195 & 3.79676795 & 14.8034575 & 6.98086832 & 15.8955 & 50.175 & 80.9011464 & 70.5 & 71 & 78 & 74.6 & 0.000217 \\
\hline 80.0200195 & 3.79864376 & 14.8049568 & 6.97961744 & 15.89625 & 60.2 & 80.578346 & 70.5 & 71.1 & 78 & 74.5 & 0.000218 \\
\hline 85.0200195 & 3.78488782 & 14.8034575 & 6.98086832 & 15.8955 & 60.175 & 80.3548688 & 70.5 & 71.1 & 78 & 74.6 & 0.000217 \\
\hline 90.0200195 & 3.79614268 & 14.8042071 & 6.98149376 & 15.8955 & 70.2 & 80.4293612 & 70.5 & 71.1 & 78 & 74.6 & 0.000219 \\
\hline 95.0200195 & 3.79676795 & 14.8034575 & 6.978992 & 15.8955 & 70.175 & 80.7025 & 70.5 & 71.1 & 77.9 & 74.6 & 0.000219 \\
\hline 100.02002 & 3.79614268 & 14.8042071 & 6.978992 & 15.897 & 80.2 & 80.47902228 & 70.5 & 71.1 & 78 & 74.6 & 0.00022 \\
\hline 105.02002 & 3.60864808 & 14.8042071 & 6.978992 & 15.89625 & 80.2 & 80.5038536 & 70.5 & 71.1 & 78 & 74.6 & 0.000221 \\
\hline 110.02002 & 3.81490078 & 14.8049568 & 6.97649024 & 15.8955 & 90.15 & 80.5038536 & 70.5 & 71.1 & 78 & $\overline{74.6}$ & 0.000222 \\
\hline 115.02002 & 3.78676363 & 14.8042074 & 6.97461392 & 15.89475 & 90.225 & 80.0568992 & 70.5 & 71.1 & 78 & 74.6 & 0.000222 \\
\hline 120.02002 & 3.75862648 & 14.8012087 & 6.97461392 & 15.897 & 100.25 & 80.205884 & 70.5 & 71.1 & 78 & 74.6 & 0.000223 \\
\hline 125.02002 & 3.76175283 & 14.8019583 & 6.97523936 & 15.897 & 100.175 & 80.6280076 & 70.5 & 71.1 & 78 & 74.6 & 0.000222 \\
\hline 130.02002 & 3.7686308 & 14.7997094 & 6.97461392 & 15.89475 & 100.25 & 81.8695476 & 70.5 & 71.1 & 78 & 74.6 & 0.000223 \\
\hline 135.02002 & 3.77738458 & 14,8042071 & 6.9727376 & 15.89325 & 110.25 & 82.2420096 & 70.5 & 71.1 & 78 & 74.6 & 0.000224 \\
\hline 140.02002 & 3.79114052 & 14.8042071 & 6.97336304 & 15.89325 & $\$ 10.25$ & 82.3413328 & 70.4 & 71.1 & 77.9 & 74.7 & 0.000225 \\
\hline 145.02002 & 3.76988134 & 14.8034575 & 6.97211216 & 15.8955 & 120.275 & 81.7950552 & 70.4 & 71.1 & 77.9 & 74.6 & 0.000226 \\
\hline 150,02002 & 3.76738026 & 14.8042071 & 6.9727376 & 15.89625 & 120.25 & 84.94404 & 70.2 & 71.1 & 77.8 & 74.7 & 0.000227 \\
\hline 155.02002 & 3.76925607 & 14.8034575 & 6.97211216 & 15.89625 & 128.9 & 82.66413332 & 70.1 & 71.1 & 77.7 & 74.6 & 0.000226 \\
\hline 160.02002 & 3.77425823 & 14.8049568 & 6.9695104 & 15.8955 & 130.225 & 82.4654868 & 70 & 71.2 & 77.7 & 74.7 & 0.000228 \\
\hline 165.02002 & 3.75862648 & 14.8034575 & 6.96835952 & 15.89625 & 130.25 & 82.3661636 & 70 & 71.1 & 77.6 & 74.5 & 0.000227 \\
\hline 170.02002 & 3.75487486 & 14.8042071 & 6.96898496 & 15.89625 & 130.25 & 81.2487776 & 70 & 71.2 & 77.6 & 74.6 & 0.000220 \\
\hline 175.02002 & 3.77738458 & 14.8042071 & 6.96835952 & 15.89625 & 140.275 & 81.4722548 & 70 & 71.2 & 77.6 & 74.6 & 0.000228 \\
\hline 180.02002 & 3.76487918 & 14.8049568 & 6.9664832 & 15.89625 & 140.275 & 81.2487776 & 70.1 & 71.2 & 77.6 & 74.7 & 0.000229 \\
\hline 185.02002 & 3.77926039 & 14.8034575 & 6.9664832 & 15.8955 & 140.275 & 81.2984392 & 70.1 & 71.2 & 77.6 & 74.5 & 0.000229 \\
\hline
\end{tabular}




\begin{tabular}{|c|c|c|c|c|c|c|c|c|c|c|c|}
\hline SECONDS & $\begin{array}{l}\text { MAIN DUCT } \\
\text { DIFF (IN H2O }\end{array}$ & \begin{tabular}{|l|} 
MA|N DUCT \\
ABS (PSIA)
\end{tabular} & \begin{tabular}{|l|} 
GAS INJECT \\
DIFF (IN H2O)
\end{tabular} & $\begin{array}{l}\text { GAS INJECT } \\
\text { ABS (PSIA) }\end{array}$ & $\begin{array}{l}\text { NEWPORT } \\
\text { (PPM) }\end{array}$ & $\begin{array}{l}\text { SMC } \\
\text { (PPM) }\end{array}$ & $\begin{array}{l}\text { AMBIENT } \\
\text { TEMP.(F) }\end{array}$ & $\begin{array}{l}\text { GAS INJECT } \\
\text { TEMP.(F) }\end{array}$ & \begin{tabular}{|l|} 
MAIN DUCT \\
TEMP.(F) \\
\end{tabular} & \begin{tabular}{|l|} 
WEC CELL \\
TEMP.(F)
\end{tabular} & $\begin{array}{l}\text { WEC CELL } \\
\text { (VOLTS) }\end{array}$ \\
\hline & & & & & & & & & & & \\
\hline 190.179993 & 3.7748835 & 14.8027079 & 6.9664832 & 15.8955 & 140.25 & 81.199116 & 70.2 & 71.2 & 77.6 & 74.6 & 0.000229 \\
\hline 195.02002 & 3.77050661 & 14.8042071 & 6.9664832 & 15.69625 & 150.3 & 81.5467472 & 70.3 & 71.2 & 77.6 & 74.5 & 0.00023 \\
\hline 200.02002 & 3.76925607 & 14.8027079 & 6.96710864 & 15.8955 & 150.275 & 81.3481008 & 70.3 & 71.2 & 77.6 & 74.6 & 0.000231 \\
\hline 205.02002 & 3.76612972 & 14.8027079 & 6.96460688 & 15.89625 & 150.275 & 80.9011464 & 70.2 & 71.2 & 77.6 & 74.5 & 0.000231 \\
\hline 210.02002 & 3.78238674 & 14.8027079 & 6.96523232 & 15.89625 & 150.3 & 81.6212396 & 70.2 & 71.2 & 77.6 & 74.6 & 0.000232 \\
\hline 215.149994 & 3.76988134 & 14.8034575 & 6.96210512 & 15.8955 & 160.275 & 80.7521616 & 70.2 & 71.2 & 77.6 & 74.6 & 0.000231 \\
\hline 220,02002 & 3.77238242 & 14.8042071 & 6.96273056 & 15.897 & 160.275 & 80.8763156 & 70.2 & 71.2 & 77.6 & 74.6 & 0.000233 \\
\hline 225.02002 & 3.7748835 & 14.8034575 & 6.96273056 & 15.89625 & 160.3 & 80.4293612 & 70.2 & 71.2 & 77.6 & 74.6 & 0.000232 \\
\hline 230.02002 & 3.77300769 & 14.8042071 & 6.96273056 & 15.89625 & 160.275 & 80.7521616 & 70.2 & 71.2 & 77.6 & 74.6 & 0.000233 \\
\hline 235.02002 & 3.76362864 & 14.8049568 & 6.96147968 & 15.89625 & 170.3 & 80.8763156 & 70.3 & 71.2 & 77.6 & 74.6 & 0.000232 \\
\hline 240.02002 & 3.75862648 & 14.8042071 & 6.96210512 & 15.89625 & 470.35 & 81.32327 & 70.2 & 71.2 & 77.6 & 74.5 & 0.000233 \\
\hline 245.02002 & 3.77050661 & 14.8019583 & 6.96273056 & 15.89625 & 170.3 & 81.2487776 & 70.3 & 71.2 & 77.7 & 74.6 & 0.000234 \\
\hline 250.02002 & 3.77363296 & 14.8027079 & 6.96273056 & 15.897 & 170.325 & 81.447424 & 70.2 & 71.2 & 77.6 & 74.6 & 0.000234 \\
\hline 255.02002 & 3.77926039 & 14.8012087 & 6.96273056 & 15.89775 & 170.275 & 81.3977624 & 70.2 & 71.2 & 77.6 & 74.5 & 0.000233 \\
\hline 260.029999 & 3.78301201 & 14.8034575 & 6.96273056 & 15.89625 & 180.35 & 81.3977624 & 70.2 & 71.2 & 77.6 & 74.6 & 0.000234 \\
\hline 265.02002 & 3.76612972 & 14.8019583 & 6.9602288 & 15.89625 & 180.325 & 80.6280076 & 70.2 & 71.2 & 77.6 & 74.5 & 0.000235 \\
\hline 270.02002 & 3.77300769 & 14.8027079 & 6.9602288 & 15.897 & 180.325 & 80.6528384 & 70.2 & 71.2 & 77.6 & 74.5 & 0.000235 \\
\hline 275.02002 & 3.75737594 & 14.8042071 & 6.959600336 & 15.89625 & 480.3 & 80.7273308 & 70.3 & 71.2 & 77.6 & 74.6 & 0.000235 \\
\hline 280.029999 & 3.76300337 & 14.8012087 & 6.9602288 & 15.89625 & 180.325 & 80.6528384 & 70.2 & 71.2 & 77.6 & 74.6 & 0.000235 \\
\hline 285.02002 & 3.77238242 & 14.8034575 & 6.95960336 & 15.89625 & 180.35 & 80.8514848 & 70.3 & 71.2 & 77.6 & 74.6 & 0.000237 \\
\hline 290.02002 & 3.77238242 & 14.8019583 & 6.96147968 & 15.89625 & 190.325 & 80.8763156 & 70.4 & $7 \overline{71.2}$ & 77.6 & 74.5 & 0.000237 \\
\hline 295.029999 & 3.77738458 & 14.8019583 & 6.95772704 & 15.89625 & 190.3 & 80.7273308 & 70.3 & 71.2 & 77.7 & 74.6 & 0.000236 \\
\hline 300.02002 & 3.77613404 & 14.8042071 & 6.95835248 & 15.89625 & 190.35 & 80.7521616 & 70.3 & 71.2 & 77.6 & 74.5 & 0.000237 \\
\hline 305.02002 & 3.77113188 & 14.8027079 & 6.95835248 & 15.8955 & 190.325 & 80.5535152 & 70.3 & 71.2 & 77.6 & 74.5 & 0.000237 \\
\hline 310.02002 & 3.77050661 & 14.8027079 & 6.95585072 & 15.89625 & 190.3 & 80.7521616 & 70.3 & 71.2 & 77.7 & 74.6 & 0.000237 \\
\hline 315.02002 & 3.77238242 & 14.8012087 & 6.95960336 & 15.897 & 200.3 & 81.32327 & 70.3 & 71.2 & 77.6 & 74.5 & 0.000235 \\
\hline 320.02002 & 3.76425391 & 14.8027079 & 6.9602288 & 15.89625 & 200,325 & 80.7273308 & 70.3 & 71.2 & 77.6 & 74.6 & 0.000237 \\
\hline 325.029999 & 3.77800985 & 14.8027079 & 6.95772704 & 15.89625 & 200.35 & 80.9011464 & 70.3 & 71.2 & 77.6 & 74.6 & 0.000237 \\
\hline 330.02002 & 3.75362432 & 14.8012087 & 6.95772704 & 15.89625 & 200.325 & 81.2239468 & 70.3 & 71.3 & 77.7 & 74.5 & 0.000237 \\
\hline 335.040009 & 3.77926039 & 14.8027079 & 6.95585072 & 15.8955 & 200.325 & 81.074962 & 70.3 & 71.3 & $\overline{77.7}$ & 74.6 & 0.000237 \\
\hline 340.02002 & 3.76675499 & 14.8027079 & 6.95772704 & 15.89525 & 200.325 & 81.32327 & $70 . \overline{3}$ & 71.2 & $\overline{77.7}$ & $\overline{74.5}$ & 0.000238 \\
\hline 345.02002 & 3.77675931 & 14.8019583 & 6.95522528 & 15.897 & 200.325 & 81.2736084 & 70.3 & 71.3 & 77.6 & 74.5 & 0.000237 \\
\hline 350.02002 & 3.77363296 & 14.8042079 & 6.95585072 & 15.89625 & 200.325 & 81.3481008 & 70.3 & 71,3 & $\overline{77.6}$ & 74.5 & 0.000238 \\
\hline 355.02002 & 3.77300769 & 14.8027079 & 6.9571016 & 15.8985 & 200.325 & 80.7273308 & 70.3 & 71.3 & 77.6 & 74.5 & 0.000238 \\
\hline 360.02002 & 3.78176147 & 14.8019583 & 6.95585072 & 15.89625 & 210.275 & 81.7205628 & 70.3 & 71.2 & 77.6 & 74.5 & 0.000238 \\
\hline 365.070007 & 3.77175715 & 14.8019583 & 6.95522528 & 15.89625 & 210.325 & 81.9937016 & 70.3 & 71.2 & 77.6 & 74.6 & 0.000238 \\
\hline 370.02002 & 3.7811362 & 14.800459 & 6.9571016 & 15.897 & 210.35 & 81.3729316 & 70.3 & 71.3 & 77.6 & 74.5 & 0.000238 \\
\hline 375.070007 & 3.77926039 & 14.8049568 & 6.95585072 & 15.89625 & 210.35 & 81.8695476 & 70.3 & 71.3 & 77.7 & 74.6 & 0.000239 \\
\hline 380.02002 & 3.7561254 & 14.8019583 & 6.95647616 & 15.89925 & 210.35 & 81.6212396 & 70.4 & 71.2 & 77.6 & 74.5 & 0.000239 \\
\hline 385.070007 & 3.77113188 & 14.8042071 & 6.96585072 & 15.8985 & 210.325 & 81.32327 & 70.4 & 71.2 & 77.7 & 74.6 & 0.000239 \\
\hline 390.02002 & 3.7561254 & 14.8034575 & 6.95585072 & 15.8985 & 210.3 & 81.2487776 & 70.4 & 71.2 & 77.7 & 74.5 & 0.000239 \\
\hline 395.050018 & 3.7685308 & 14.7989598 & 6.95522528 & 15.89775 & 210.35 & 81.2984392 & 70.3 & 71.2 & 77.6 & 74.6 & 0.00024 \\
\hline
\end{tabular}




\begin{tabular}{|c|c|c|c|c|c|c|c|c|c|c|c|}
\hline SECONDS & \begin{tabular}{|l|} 
MAIN DUCT \\
DIFF (IN H2O \\
\end{tabular} & \begin{tabular}{|l|} 
MAIN DUCT \\
ABS (PSIA) \\
\end{tabular} & \begin{tabular}{|l|} 
GAS INJECT \\
DIFF (IN H2O) \\
\end{tabular} & \begin{tabular}{|l|} 
GAS INJECT \\
ABS (PSIA) \\
\end{tabular} & $\begin{array}{l}\text { NEWPORT } \\
\text { (PPM) }\end{array}$ & \begin{tabular}{|l|} 
SMC \\
(PPM)
\end{tabular} & \begin{tabular}{|l|} 
AMBIENT \\
TEMP.(F)
\end{tabular} & \begin{tabular}{|l|} 
GAS INJECT \\
TEMP.(F)
\end{tabular} & \begin{tabular}{|l|} 
MAIN DUCT \\
TEMP.(F)
\end{tabular} & \begin{tabular}{|l|} 
WEC CELL \\
TEMP.(F)
\end{tabular} & \begin{tabular}{|l|} 
WEC CELL \\
(VOLTS)
\end{tabular} \\
\hline & & & & & & & & & & & \\
\hline 400.02002 & 3.76550445 & 14.7997094 & 6.95522528 & 15.89775 & 219.525 & 81.94404 & 70.3 & 71.2 & 77.6 & 74.5 & 0.000239 \\
\hline 405.02002 & 3.77300769 & 14,8034575 & 6.95647616 & 15.89925 & 220.35 & 81.2487776 & 70.3 & 71.3 & 77.7 & 74.6 & 0.000241 \\
\hline 410.02002 & 3.76925607 & 14.8034575 & 6.95647616 & 15.8985 & 220.35 & 81.6460704 & 70.4 & 71.3 & 77.7 & 74.6 & 0.00024 \\
\hline 415.02002 & 3.78363728 & 14.8034575 & 6.95585072 & 15.89775 & 220.35 & 81.8447168 & 70.3 & 71.3 & 77.7 & 74.6 & 0.000241 \\
\hline 420.02002 & 3.77113188 & 14.8027079 & 6.9539744 & 15.8985 & 220.375 & 81.1742852 & 70.4 & 71.2 & 77.6 & 74.6 & 0.00024 \\
\hline 425.02002 & 3.77926039 & 14.8019583 & 6.95459984 & 15.9 & 220.325 & 80.4045304 & 70.4 & 71.3 & 77.7 & 74.6 & 0.00024 \\
\hline 430.02002 & 3.76988134 & 14.8034575 & 6.95459984 & 15.8985 & 220.35 & 81.0004696 & 70.4 & 71.3 & 77.7 & 74.6 & 0.00024 \\
\hline 435.02002 & 3.7686308 & 14.8027079 & 6.955855072 & 15.89775 & 220.35 & 80.6280076 & 70.4 & 71.2 & 77.7 & 74,6 & 0.000241 \\
\hline 440.02002 & 3.76800553 & 14.7997094 & 6.95647616 & 15.8985 & 220.35 & 80.5535152 & 70.4 & 71.3 & 77.6 & 74.5 & 0.000241 \\
\hline 445.02002 & 3.80489646 & 14.8019583 & 6.95647616 & 15.9 & 220.35 & 80.4790228 & 70.4 & 71.2 & 77.7 & 74.6 & 0.00024 \\
\hline 450.02002 & 3.77113188 & 14.8027079 & 6.9539744 & 15.8985 & 220.35 & 80.3052072 & 70.3 & 71.2 & 77.6 & 74.5 & 0.000241 \\
\hline 455,02002 & 3.76925607 & 14.8042071 & 6.95272352 & 15.9 & 220.35 & 80.1810532 & 70.3 & 71.2 & 77.7 & 74.6 & 0.00024 \\
\hline 460.02002 & 3.78988998 & 14.8027079 & 6.95334896 & 15.89775 & 220.35 & 80.1810532 & 70.3 & 71.2 & 77.6 & 74.6 & 0.000241 \\
\hline 465.02002 & 3.76050229 & 14.8034575 & 6.9539744 & 15.897 & 230.375 & 80.7769924 & 70.4 & 71.3 & 77.7 & 74.6 & 0.00024 \\
\hline 470.02002 & 3.77738458 & 14.8034575 & 6.95209308 & 15.90075 & 220.375 & 80.6776692 & 70.4 & 71.2 & 77.7 & 74.6 & 0.000241 \\
\hline 475.02002 & 3.7748835 & 14.8049568 & 6.9508472 & 15.89775 & 230.325 & 80.578346 & 70.4 & 71.2 & 77.6 & 74.5 & 0.00024 \\
\hline 480.02002 & 3.77738458 & 14.8049568 & 6.95334896 & 15.8985 & 230.4 & 80.3796996 & 70.3 & 71.2 & 77.7 & 74.6 & 0.000241 \\
\hline 485.02002 & 3.75550013 & 14.8034575 & 6.95209808 & 15.90075 & 220.675 & 80.2307148 & 70.4 & 71.2 & 77.7 & 74.5 & 0.00024 \\
\hline 490.02002 & 3.77926039 & 14.8027079 & 6.95147264 & 15.90075 & 230.35 & 80.6031768 & 70.3 & 71.2 & 77.7 & 74,6 & 0.000241 \\
\hline 495.02002 & 3.76487918 & 14.8027079 & 6.95147264 & 15.90075 & 230.35 & 80.578346 & 70.3 & 71.2 & 77.7 & 74.5 & 0.000242 \\
\hline 500.02002 & 3.77113188 & 14.8034575 & 6.95147264 & 15.90075 & 230.35 & 80.4045304 & 70.4 & 71.3 & 77.8 & 74.6 & 0.000241 \\
\hline 505.02002 & 3.76800553 & 14.8034575 & 6.95209808 & 15.90075 & 230.35 & 80.3052072 & 70.4 & 71.2 & 77.7 & 74.5 & 0.000242 \\
\hline 510.02002 & 3.77425823 & 14.8034575 & 6.95209808 & 15.9 & 230.35 & 80.8018232 & 70.4 & 71.2 & 77.8 & 74.6 & 0.000241 \\
\hline 515.029999 & 3.75800121 & 14.8027079 & 6.95209808 & 15.90075 & 230.375 & 80.330038 & 70.4 & 71.3 & 77.7 & 74.6 & 0.000241 \\
\hline 520.02002 & 3.75987702 & 14.8019583 & 6.95147264 & 15.90075 & 230.35 & 80.3052072 & 70.4 & 71.2 & 77.8 & 74.6 & 0.000242 \\
\hline 525.02002 & 3.76550445 & 14.8019583 & 6.95272352 & 15.90075 & 230.4 & 79.833422 & 70.4 & 71.2 & 77.7 & 74.5 & 0.000243 \\
\hline 530.070007 & 3.75237378 & 14.8034575 & 6.95209808 & 15.90075 & 230.375 & 80.1562224 & 70.4 & 71.2 & 77.7 & 74.6 & 0.000242 \\
\hline 535.02002 & 3.7686308 & 14.8027079 & 6.95209308 & 15.9 & 230.35 & 80.4045304 & 70.4 & 71.2 & 77.7 & 74.6 & 0.000243 \\
\hline 540.02002 & 3.7623781 & 14.8027079 & 6.95209808 & 15.90075 & 230.35 & 80.3548688 & 70.4 & 71.2 & 77.8 & 74.6 & 0.000242 \\
\hline 545.02002 & 3.7748835 & 14.8034575 & 6.95147264 & 15.9 & 230.35 & 80.1562224 & 70.4 & 71.2 & 77.7 & 74.7 & 0.000242 \\
\hline 550.02002 & 3.7623781 & 14.8027079 & 6.95147264 & 15.90075 & 230.4 & 79.9079144 & 70.4 & 71.2 & 77.7 & 74.5 & 0.000241 \\
\hline 555.02002 & 3.76925607 & 14.8042071 & 6.95147264 & 15.90075 & 230.3 & 80.2555456 & 70.4 & 71.2 & 77.7 & 74.7 & 0.000243 \\
\hline 560.059998 & 3.75550013 & 14.8034575 & 6.95209808 & 15.90075 & 230.3 & 80.4293612 & 70.4 & 71.2 & 77.8 & 74.6 & 0.000242 \\
\hline 565.02002 & 3.77675931 & 14.8049568 & 6.9508472 & 15.90075 & 230.325 & 80.6776692 & 70.4 & 71.2 & 77.8 & 74.6 & 0.000242 \\
\hline 570.02002 & 3.7686308 & 14.8034575 & 6.95147264 & 15.9015 & 230.325 & 81.0253004 & 70.4 & 71.2 & 77.7 & 74.5 & 0.000242 \\
\hline 575.029999 & 3.7685308 & 14.8027079 & 6.9508472 & 15,90075 & 230.375 & 80.5286844 & 70.4 & 71.2 & 77.7 & 74.5 & 0.000243 \\
\hline 580.02002 & 3.7811362 & 14.8042071 & 6.95022176 & 15,9015 & 230.35 & 80.6776692 & 70.5 & 71.2 & 77.7 & 74.5 & 0.000242 \\
\hline 585.02002 & 3.77926039 & 14.8019583 & 6.9508472 & 15.90075 & 230.4 & 80.6528384 & 70.5 & 71.2 & 77.8 & 74.7 & 0.000242 \\
\hline 590.02002 & 3.77863512 & 14.8042071 & 6.95209808 & 15.90075 & 240.15 & 80.8514848 & 70.4 & 71.2 & 77.7 & 74.5 & 0.000242 \\
\hline 595.02002 & 3.78363728 & 14.8034575 & 6.94897088 & 15.90075 & 239.25 & 80.9259772 & 70.4 & 71.2 & 77.8 & 74.7 & 0.000243 \\
\hline 600.02002 & 3.77300769 & 14.8042071 & 6.94959632 & 15.903 & 240.4 & 81.2239468 & 70.5 & 71.2 & 77.7 & 74.5 & 0.000242 \\
\hline 605.070007 & 3.77926039 & 14.8034575 & 6.95147264 & 15.9015 & 240.375 & 81.074962 & 70.5 & 71.2 & 77.8 & 74.7 & 0.000243 \\
\hline
\end{tabular}




\begin{tabular}{|c|c|c|c|c|c|c|c|c|c|c|c|}
\hline SECONDS & \begin{tabular}{|l|} 
MAIN DUCT \\
DiFF (IN H2O \\
\end{tabular} & \begin{tabular}{|l|} 
MAIN DUCT \\
ABS (PSIA)
\end{tabular} & \begin{tabular}{|l|} 
GAS INJECT \\
DIFF (IN H2O) \\
\end{tabular} & \begin{tabular}{|l} 
GAS INJECT \\
ABS (PS|A)
\end{tabular} & \begin{tabular}{|l|} 
NEWPORT \\
(PPM)
\end{tabular} & \begin{tabular}{|l|} 
SMC \\
(PPM)
\end{tabular} & \begin{tabular}{|l|} 
AMBIENT \\
TEMP.(F)
\end{tabular} & \begin{tabular}{|l|} 
GAS INJECT \\
TEMP.(F)
\end{tabular} & \begin{tabular}{|l|} 
MAIN DUCT \\
TEMP.(F) \\
\end{tabular} & \begin{tabular}{|l|} 
WEC CELL \\
TEMP.(F)
\end{tabular} & \begin{tabular}{|l|} 
WEC CELL \\
NOLTS)
\end{tabular} \\
\hline & & & & & & & & & & & \\
\hline 610.02002 & 3.76738026 & $14.804207 i$ & 6.94959632 & 15.9015 & 240.325 & 80,330038 & 70.5 & 71.2 & 77.7 & 74.5 & 0.000243 \\
\hline 615.02002 & 3.775500877 & 14.8027079 & 6.95147264 & 15.9045 & 240.35 & 79.9327452 & 70.5 & 71.2 & 77.7 & 74.6 & 0.000243 \\
\hline 620.040009 & 3.7623781 & 14.8034575 & 6.9508472 & 15.9045 & 240.375 & 80.2307148 & 70.5 & 71.2 & 77.8 & 74.7 & 0.000243 \\
\hline 625.059998 & 3.77675931 & 14.8027079 & 6.94897088 & 15.90375 & 240.375 & 80.2307148 & 70.5 & 71.2 & 77.8 & 74.5 & 0.000243 \\
\hline 630.02002 & 3.76800553 & 14.8027079 & 6.94959632 & 15.90375 & 240.375 & 80.2803764 & 70.6 & 71.2 & 77.7 & 74.6 & 0.000243 \\
\hline 635.059998 & 3.76300337 & 14.8019583 & 6.94897088 & 15.90375 & 240.375 & 80.5286844 & 70.5 & 71.2 & 77.7 & 74.6 & 0.000243 \\
\hline 640.02002 & 3.77550877 & 14.8019583 & 6.95022176 & 15.903 & 240.35 & 80.6776692 & 70.6 & 71.2 & 77.8 & 74.6 & 0.000243 \\
\hline 645,02002 & 3.76112756 & 14.8042071 & 6.9508472 & 15.90375 & 240.35 & 80.6031768 & 70.6 & 71.2 & 77.7 & 74.5 & 0.000243 \\
\hline 650.040009 & 3.77363296 & 14.8049568 & 6.95022176 & 15.90375 & 240.4 & 80.4293612 & 70.7 & 71.2 & 77.7 & 74.5 & 0.000243 \\
\hline 655.02002 & 3.7686308 & 14.8042071 & 6.94959632 & 15.90375 & 240.4 & 80.0568992 & 70.7 & 71.2 & 77.7 & 74.5 & 0.000243 \\
\hline 660.02002 & 3.79114052 & 14.8042071 & 6.94709456 & 15.90375 & 240.35 & 79.9327452 & 70.7 & 71.2 & 77.8 & 74.6 & 0.000243 \\
\hline 665.059998 & 3.76925607 & 14.8049568 & 6.94959532 & 15.90375 & 240.375 & 80.3052072 & 70.6 & 71.2 & 77.8 & 74.6 & 0.000243 \\
\hline 670.02002 & 3.76675499 & 14.8034575 & 6.94772 & 15.903 & 240.4 & 79.833422 & 70.6 & 71.2 & 77.8 & 74.6 & 0.000243 \\
\hline 675.02002 & 3.77238242 & 14.8042071 & 6.94646912 & 15.903 & 240,35 & 80.1313916 & 70.6 & 71.2 & 77.7 & 74.5 & 000242 \\
\hline 680.02002 & 3.78676363 & 14.8042071 & 6.949596332 & 15.903 & 240.4 & 80.6031768 & 70.6 & 71.2 & 77.8 & 74.6 & 0.000243 \\
\hline 685.070007 & 3.77113188 & 14.8042071 & 6.94709456 & 15.903 & 240.425 & 80.5286844 & 70.5 & 71.2 & 77.7 & 74.6 & 0.000243 \\
\hline 690.02002 & 3.75550013 & 14.8034575 & 6.94897088 & 15.903 & 240.4 & 80.7025 & 70.5 & 71.2 & 77.7 & 74.4 & 0.000242 \\
\hline 695.050018 & 3.77050661 & 14.8027079 & 6.94646912 & 15.90225 & 240.4 & 80.454192 & 70.5 & 71.2 & 77.7 & 74.5 & 0.000243 \\
\hline 700.02002 & 3.77926039 & 14.8034575 & 6.94396736 & 15.90375 & 240.375 & 79.8582528 & 70.5 & 71.2 & 77.8 & 74.5 & 0.000242 \\
\hline 705.02002 & 3.76925607 & 14.8034575 & 6.94897083 & 15.90375 & 240.4 & 80.1562224 & 70.5 & 712 & 77.8 & 74.6 & 0.000244 \\
\hline 710.02002 & 3.77050661 & 14.8027079 & 6.94521824 & 15.90375 & 240.325 & 80.3548688 & 70.5 & 71.2 & 77.8 & 74.5 & 0.000243 \\
\hline 715.02002 & 3.77300769 & 14.8027079 & 6.94521824 & 15.903 & 240.4 & 80.08173 & 70.4 & 71.2 & 77.8 & 74.6 & 0.000244 \\
\hline 720.02002 & 3.76425391 & 14.8019583 & 6.9445928 & 15.90375 & 240.4 & 80.2307148 & 70.4 & 71.2 & 77.9 & 74.6 & 0.000243 \\
\hline 725.02002 & 3.76425391 & 14.8027079 & 6.94521824 & 15.903 & 240.35 & 80.3548688 & 70.5 & 71.2 & 77.8 & 74.6 & 0.000243 \\
\hline 730.02002 & 3.76800553 & 14.8034575 & 6.95022176 & 15.9045 & 240.4 & 80.5535152 & 70.5 & 71.2 & 77.7 & 74.5 & 0.000243 \\
\hline 735.02002 & 3.7748835 & 14.8034575 & 6.94521824 & 15.90375 & 240.325 & 80.7769924 & 70.4 & 71.2 & 77.8 & 74.6 & 0.000244 \\
\hline 740.050018 & 3.77926039 & 14.8034575 & 6.94521824 & 15.903 & 240.375 & 80.5286844 & 70.4 & 71.2 & 77.8 & 74.6 & 0.000243 \\
\hline 745.02002 & 3.77613404 & 14.8027079 & 6.94584368 & 15.9045 & 240.375 & 80.2555456 & 70.5 & 71.1 & 77.8 & 74.5 & 0.000243 \\
\hline 750.02002 & 3.7686308 & 14.8027079 & 6.94521824 & 15.903 & 240.375 & 79.7837604 & 70.4 & 71.2 & 77.8 & 74.6 & 0.000243 \\
\hline 755.029999 & 3.76675499 & 14.8019583 & 6.94521824 & 15.90375 & 240.375 & 80.1810532 & 70.4 & 71.2 & 77.8 & 74.6 & 0.000244 \\
\hline 760.02002 & 3.77300769 & 14.8034575 & 6.94521824 & 15.90375 & 240.35 & 80.1562224 & 70.4 & 71.2 & 77.9 & 74.6 & 0.000244 \\
\hline 765.02002 & 3.75800121 & 14.8034575 & 6.94521824 & 15.90375 & 240.425 & 80.1313916 & 70.4 & 71.2 & 77.8 & 74.5 & 0.000243 \\
\hline 770.050018 & 3.77300769 & 14.8027079 & 6.94521824 & 15.9045 & 240.375 & 80.205884 & 70.4 & 71.2 & 77.8 & 74.5 & 0.000243 \\
\hline 775.02002 & 3.76988134 & 14.8027079 & 6.94521824 & 15.90375 & 240.375 & 80.0568992 & 70.4 & 71.2 & 77.9 & 74.5 & 0.000243 \\
\hline 780.02002 & 3.7811362 & 14.8049568 & 6.94271648 & 15.90375 & 240.375 & 79.8085912 & 70.4 & 71.2 & 77.8 & 74.5 & 0.000244 \\
\hline 785.02002 & 3.75925175 & 14.8027079 & 6.94209104 & 15.90375 & 240.4 & 80,0568992 & 70.5 & 71.1 & 77.8 & 74.6 & 0.000244 \\
\hline 790.02002 & 3.76800553 & 14.8042071 & 6.9445928 & 15.903 & 240.375 & 80.0320684 & 70.4 & 71.2 & 77.8 & 74.5 & 0.000242 \\
\hline 795.02002 & 3.75987702 & 14.8034575 & 6.9445928 & 15.90375 & 240.35 & 79.8085912 & 70.4 & 71.2 & 77.8 & 74.6 & 0.000244 \\
\hline 800.070007 & 3.78551309 & 14.8042071 & 6.94334192 & 15.90375 & 240.35 & 79.957576 & 70.4 & 71.2 & 77.8 & 74.5 & 0.000244 \\
\hline 805.110016 & 3.7748835 & 14.8049568 & 6.94334192 & 15.903 & 240.375 & 80.3548688 & 70.4 & 71.1 & 77.8 & 74.5 & 0.000244 \\
\hline 810.02002 & 3.77238242 & 14.8042071 & 6.94334192 & 15.90375 & 240.425 & 80.578346 & 70.4 & 71.1 & 77.8 & 74.5 & 0.000244 \\
\hline 815.040009 & 3.76675499 & 14.8034575 & 6.94271648 & 15.90375 & 240.4 & 80.2803764 & 70.4 & 71.1 & 77.8 & 74.5 & 0.000245 \\
\hline
\end{tabular}




\begin{tabular}{|c|c|c|c|c|c|c|c|c|c|c|c|}
\hline SECONDS & \begin{tabular}{|l|} 
MAIN DUCT \\
DIFF (IN H2O \\
\end{tabular} & \begin{tabular}{|l|} 
MAIN DUCT \\
ABS (PSIA) \\
\end{tabular} & \begin{tabular}{|l} 
GAS INJECT \\
DIFF (IN H2O)
\end{tabular} & \begin{tabular}{|l|} 
GAS INJECT \\
ABS (PSIA)
\end{tabular} & \begin{tabular}{|l|} 
NEWPORT \\
(PPM)
\end{tabular} & $\begin{array}{l}\text { SMC } \\
\text { (PPM) }\end{array}$ & $\frac{\text { AMBIENT }}{\text { TEMP.(F) }}$ & $\frac{\text { GAS INJECT }}{\text { TEMP(F) }}$ & $\begin{array}{l}\text { MAIN DUCT } \\
\text { TEMP (F) }\end{array}$ & \begin{tabular}{|l|} 
WEC CELL \\
TEMP (F)
\end{tabular} & $\frac{\text { WEC CELL }}{\text { (NOLTS) }}$ \\
\hline & & & & & & & & & & & \\
\hline 820.02002 & 3.7686308 & 14.8034575 & 6.94271648 & 15.903 & 240.375 & 80.08173 & 70.3 & 71.1 & 77.8 & 74.5 & 0.000244 \\
\hline 825.02002 & 77238242 & 14.8027079 & 6.94334192 & 15.90225 & 240.4 & 79.4361292 & 70.4 & 71.1 & 77.9 & 74.6 & 0.000244 \\
\hline 830.040009 & 3.77238242 & 14.8034575 & 6.94271648 & 15.903 & 240.4 & 80.1313916 & 70.4 & 71.1 & 77.8 & 74.5 & 0.000243 \\
\hline 835.02002 & 3.76362864 & 14.8034575 & 6.94021472 & 15.903 & 240.4 & 79,6844372 & 70.6 & 71.1 & 77.8 & 74.6 & 0.000244 \\
\hline 840.02002 & 3.76925607 & 14.8027079 & 6.9414656 & 15.9045 & 240.375 & 80.3796996 & 70.5 & 71.1 & 77.9 & 74.6 & 0.000245 \\
\hline 845.029999 & 3.77050661 & 14.8034575 & 6.94334192 & 15.90375 & 240.4 & 80.4293612 & 70.5 & 71.1 & 77.8 & 74.6 & 0.000244 \\
\hline 850.02002 & 3.76800553 & 14.8034575 & 6.9414656 & 15.9045 & 240.4 & 80.454192 & 70.5 & 71.1 & 77.9 & 74.6 & 0.000244 \\
\hline 855.02002 & 3.7686308 & 14.8034575 & 6.9414656 & 15.9045 & 240.325 & 80.0072376 & 70.5 & 71.1 & 77.8 & 74.5 & 0.000244 \\
\hline 860,059998 & 3.77988566 & 14.8042071 & 6.9414656 & 15.90375 & 240.35 & 80.8018232 & 70.5 & 71.1 & 77.9 & 74.5 & 0.000244 \\
\hline 865.02002 & 3.76800553 & 14.8019583 & 6.94209104 & 15.90375 & 240.375 & 79.9327452 & 70.5 & 71.1 & 77.8 & 74.5 & 0.000244 \\
\hline 870.02002 & 3.76800553 & 14.8027079 & 6.94084016 & 15.9045 & 240.375 & 79.336806 & 70.6 & 71.1 & 77.9 & 74.6 & 0.000244 \\
\hline 875.059998 & 3.77613404 & 14.8027079 & 6.94021472 & 15.903 & 240.375 & 79.7837604 & 70.5 & 71.1 & 77.9 & 74.6 & 0.000244 \\
\hline 880.02002 & 3.7685308 & 14.8034575 & 6.93896384 & 15.90375 & 240.325 & 79.709268 & 70.5 & 71.1 & 77.9 & 74.5 & 0.000245 \\
\hline 885.02002 & 3.77050661 & 14.8027079 & 6.9414656 & 15.903 & 240.4 & 79.585114 & 70.5 & 71.1 & 77.9 & 74.5 & 0.000243 \\
\hline 890.050018 & 3.76175283 & 14.8034575 & 6.9414656 & 15.903 & 240.4 & 79,709268 & 70.5 & 71.1 & 77.9 & 74.5 & 0.000243 \\
\hline 895.02002 & 3.76112756 & 14.8042071 & 6.94209104 & 15.903 & 240.4 & 79.6347756 & 70.5 & 71.1 & 77.9 & 74.5 & 0.000243 \\
\hline 900.02002 & 3.76738026 & 14.8027079 & 6.93958928 & 15.90375 & 240.4 & 79.6844372 & 70.5 & 71.1 & 77.9 & 74.6 & 0.000244 \\
\hline 905.059998 & 3.76800553 & 14.8034575 & 6.93833384 & 15.90375 & 240.375 & 79.4112984 & 70.4 & 71.1 & 78 & 74.6 & 0.000245 \\
\hline 910.02002 & 3.77300769 & 14.8034575 & 6.94084016 & 15.90375 & 250.4 & 79.9079144 & 70.5 & 71.1 & $\pi .9$ & 74.5 & 0.000244 \\
\hline
\end{tabular}




\begin{tabular}{|c|c|c|c|c|c|c|c|c|c|c|c|}
\hline Measured H & tydrogen Conce & 1997 WEC-SP & OOL & & & & & & & & \\
\hline SECONDS & MAIN OUCT & MAIN DUCT & GAS INJECT & GAS INJECT & |NEWPORT & SMC & AMBIENT & GAS INJECT & MAIN DUCT & WEC CELL & WEC CEL \\
\hline & DIFF(IN H2O & ABS (PSIA) & DIF F(IN H2O) & ABS (PSIA) & (PPM) & (PPM) & TEMP.(F) & \begin{tabular}{|l|} 
TEMP.(F) \\
\end{tabular} & TEMP.(F) & TEMP.(F) & (VOLTS) \\
\hline & & & & & & & & & & & \\
\hline 0.019989 & 3.76925607 & 14.8102041 & 0.604069712 & 15.03 & 0.1525 & 12.7406 & 70.5 & 70.4 & 78 & 74.7 & 0.000206 \\
\hline 5.019989 & 3.76175283 & 14.8094545 & 6.90519008 & 15.8835 & 0.1475 & 11.921184 & 70.6 & 70.4 & 78 & 74.7 & 0.000206 \\
\hline 10.160004 & 3.76362864 & 14.8087049 & 6.9258296 & 15.88725 & 0.135 & 8.9911496 & 70.5 & 70.4 & 77.9 & 74.7 & 0.000206 \\
\hline 15.019989 & 3.77050661 & 14.8102041 & 6.93708752 & 15.89025 & 0.135 & 37.397585 & 70.6 & 70.5 & 77.9 & 74.8 & 0.000207 \\
\hline 20.049988 & 3.7686308 & 14.8079553 & 6.94209104 & 15.89025 & 0.1475 & 64.785957 & 70.7 & 70.6 & 77.9 & 74.7 & 0.000207 \\
\hline 25.019989 & 3.7811362 & 14.8087049 & 6.9445928 & 15.888 & 0.1125 & 77.747635 & 70.7 & 70.6 & 77.9 & 74.7 & 0.000208 \\
\hline 30.019989 & 3.76800553 & 14.8079553 & 6.94709456 & 15.89025 & 0.1575 & 81.993702 & 70.6 & 70.7 & 77.9 & 74.7 & 0.000207 \\
\hline 35.380005 & 3.76612972 & 14.8087049 & 6.94709456 & 15.8865 & 0.14 & 84.42712 & 70.6 & 70.7 & 77.9 & 74.8 & 0.000209 \\
\hline 40.019989 & 3.77300769 & 14.8094545 & 6.9508472 & 15.8665 & 10.16 & 84.923736 & 70.6 & 70.7 & 77.9 & 74.7 & 0.000209 \\
\hline 45.019989 & 3.7811362 & 14.8094545 & 6.94897088 & 15.88875 & 10.1225 & 84.625766 & 70.6 & 70.7 & 77.9 & 74.7 & 0.000211 \\
\hline 50.029999 & 3.75800121 & 14.8102041 & 6.9508472 & 15.891 & 20.115 & 88.573864 & 70.6 & 70.8 & 77.9 & 74.7 & 0.000211 \\
\hline 55.109985 & 3.7561254 & 14.8087049 & 6.95022176 & 15.8895 & 20.15 & 87.381985 & 70.7 & 70.8 & 77.9 & 74.7 & 0.000212 \\
\hline 60.040009 & 3.76612972 & 14.8079553 & 6.94897088 & 15.89025 & 30.15 & 85.246536 & 70.6 & 70.9 & 78 & 74.7 & 0.000213 \\
\hline 65.070007 & 3.7623781 & 14.8087049 & 6.94959632 & 15.89025 & 40.15 & 84.799582 & 70.6 & 70.9 & 78 & 74.7 & 0.000214 \\
\hline 70.019989 & 3.75362432 & 14.8087049 & 6.95147264 & 15.89025 & 40.125 & 86.339092 & 70.6 & 70.9 & 78 & 74.7 & 0.000215 \\
\hline 75.029999 & 3.77863512 & 14.806456 & 6.95147264 & 15.8895 & 50.175 & 88.424879 & 70.6 & 70.9 & 77.9 & 74.6 & 0.000214 \\
\hline 80.019989 & 3.7686308 & 14.8087049 & 6.94897088 & 15.8895 & 50.2 & 85.172044 & 70.6 & 70.9 & 77.9 & 74.7 & 0.000216 \\
\hline 85.119995 & 3.77300769 & 14.8072056 & 6.9508472 & 15.89325 & 60.2 & 86.463246 & 70.7 & 71 & 78 & 74.6 & 0.000216 \\
\hline 90.169983 & 3.75987702 & 14.8087049 & 6.95147264 & 15.89325 & 60.2 & 86.935031 & 70.6 & 71 & 77.9 & 74.7 & 0.000217 \\
\hline 95.019989 & 3.75487486 & 14.8057064 & 6.94959632 & 15.89025 & 70.175 & 87.332324 & 70.6 & 71 & 77.9 & 74.6 & 0.000217 \\
\hline 100.22 & 3.7811362 & 14.8094545 & 6.94772 & 15.8925 & 70.2 & 87.108846 & 70.6 & 71 & 77.9 & 74.7 & 0.000218 \\
\hline 105.04001 & 3.77113188 & 14.8072056 & 6.94396736 & 15,89025 & 80.225 & 87.183339 & 70.6 & 71 & 78 & 74.7 & 0.00022 \\
\hline 110.01999 & 3.77863512 & 14.8079553 & 6.94834544 & 15.8925 & 80.2 & 86.786046 & 70.6 & 71 & 77.9 & 74.7 & 0.00022 \\
\hline 115.03 & 3.78301201 & 14.8087049 & 6.94897088 & 15.89175 & 90.2 & 88.573864 & 70.6 & 71 & 77.9 & 74.7 & 0.000221 \\
\hline 120.01999 & 3.76425391 & 14.8072056 & 6.9445928 & 15.89175 & 90.2 & 88.275894 & 70.5 & 71 & 78 & 74.7 & 0.00022 \\
\hline 125.01999 & 3.77238242 & 14.8079553 & 6.9445928 & 15.8925 & 90.225 & 88.549033 & 70.6 & 71 & 77.9 & 74.7 & 0.000223 \\
\hline 130.01999 & 3.75925175 & 14.806456 & 6.94396736 & 15.89175 & 100.2 & 84.501612 & 70.6 & 71 & 78 & 74.6 & 0.000222 \\
\hline 135.01999 & 3.76800553 & 14.8072056 & 6.94396736 & 15.8925 & 100.225 & 86.363922 & 70.6 & 71.1 & 78 & 74.7 & 0.000223 \\
\hline 140.01999 & 3.77238242 & 14.8094545 & 6.94271648 & 15.8925 & 110.225 & 86.736384 & 70.6 & 71 & 78 & 74.7 & 0.000224 \\
\hline 145.07001 & 3.77238242 & 14.8094545 & 6.94271648 & 15.89325 & 110.25 & 86.28943 & 70.6 & 71 & 78 & 74.8 & 0,000225 \\
\hline 150.01999 & 3.76800553 & 14.8094545 & 6.94334192 & 15.89175 & 110.2 & 88.052417 & 70.6 & 71 & 78 & 74.7 & 0.000226 \\
\hline 155.01999 & 3.76988134 & 14.8087049 & 6.94021472 & 15.89325 & 120.275 & 88.77251 & 70.6 & 71.1 & 77.9 & 74.6 & 0.000226 \\
\hline 160.01999 & 3.77800985 & 14.8094545 & 6.94021472 & 15.8925 & 120.225 & 87.729616 & 70.7 & 71.1 & 77.9 & 74.8 & 0.000226 \\
\hline 165.06 & 3.77050661 & 14.8087049 & 6.94021472 & 15.89325 & 130.275 & 88.275894 & 70.7 & 71.1 & 77.9 & 74.6 & 0.000226 \\
\hline 170.01999 & 3.77300769 & 14.8102041 & 6.9383384 & 15.89325 & 130.3 & 86.363922 & 70.7 & 71.1 & 77.9 & 74.7 & 0.000228 \\
\hline 175.10999 & 3.76800553 & 14.8057064 & 6.93896384 & 15.89175 & 130.275 & 87.158508 & 70.7 & 71.1 & 77.9 & 74.6 & 0.000227 \\
\hline 180.01999 & 3.7686308 & 14.8087049 & 6.93896384 & 15.89475 & 130.275 & 86.661892 & 70.8 & 71.1 & 77.9 & 74.6 & 0.000227 \\
\hline 185.10999 & 3.75737594 & 14.8072056 & 6.9383384 & 15.89325 & 140.25 & 87.630293 & 70.7 & 71.1 & 78 & 74.7 & 0.000228 \\
\hline
\end{tabular}




\begin{tabular}{|c|c|c|c|c|c|c|c|c|c|c|c|}
\hline SECONDS & \begin{tabular}{|l|} 
MAIN DUCT \\
DIFF(IN H2O \\
\end{tabular} & $\begin{array}{l}\text { MAIN DUCT } \\
\text { ABS (PS|A) }\end{array}$ & $\begin{array}{l}\text { GAS INJECT } \\
\text { DIFF(IN H2O) } \\
\end{array}$ & $\begin{array}{l}\text { GAS INJECT } \\
\text { ABS (PSIA) }\end{array}$ & \begin{tabular}{|l|} 
NEWPORT \\
(PPM)
\end{tabular} & \begin{tabular}{|l|} 
SMC \\
(PPM) \\
\end{tabular} & $\begin{array}{l}\text { AMBIENT } \\
\text { TEMP.(F) } \\
\end{array}$ & \begin{tabular}{|l|} 
GAS INJECT \\
TEMP.(F) \\
\end{tabular} & \begin{tabular}{|l|} 
MAIN DUCT \\
TEMP.(F) \\
\end{tabular} & \begin{tabular}{|l|} 
WEC CELL \\
TEMP.(F) \\
\end{tabular} & $\begin{array}{l}\text { WEC CEL } \\
\text { (VOLTS) }\end{array}$ \\
\hline 190.01999 & 3.7623781 & 14.8072056 & 6.93771296 & 15.89475 & 140.25 & 86.363922 & 70.7 & 71.1 & 78 & 74.7 & 0.000229 \\
\hline 195.10999 & 3.78488782 & 14.8102041 & 6.93896384 & 15.89475 & 140.275 & 85,470014 & 70.7 & 71.1 & 78 & 74.7 & 0.00023 \\
\hline 200.01999 & 3.78488782 & 14.8087049 & 6.9383384 & 15.89475 & 140.225 & 86.835708 & 70.7 & 71.1 & 77.9 & 74.6 & 0.000229 \\
\hline 205.23001 & 3.7623781 & 14.8079553 & 6.93771296 & 15.8955 & 150.3 & 87.133677 & 70.7 & 71.1 & 77.9 & 74.6 & 0.00023 \\
\hline 210.01999 & 3.77863512 & 14.8072056 & 6.93583664 & 15.8955 & 150.3 & 87.481308 & 70.6 & 71.1 & 78 & 74.7 & 0.00023 \\
\hline 215.01999 & 3.7873889 & 14.8087049 & 6.9352112 & 15.89475 & 150.225 & 86.736384 & 70.7 & 71.2 & 77.9 & 74.7 & 0.00023 \\
\hline 220.01999 & 3.77675931 & 14.8079553 & 6.93396032 & 15.8955 & 150.275 & 86.363922 & 70.6 & 71.1 & 78 & 74.8 & 0.000231 \\
\hline 225.26001 & 3.79239106 & 14.8072056 & 6.93396032 & 15.89625 & 155.625 & 85.817645 & 70.7 & 71.1 & 78 & 74.8 & 0.000231 \\
\hline 230.01999 & 3.76925607 & 14.8072056 & 6.9352112 & 15.8955 & 160.3 & 85.594168 & 70.8 & 71.2 & 78 & 74.8 & 0.000232 \\
\hline 235.01999 & 3.75800121 & 14.8079553 & 6.93396032 & 15.8955 & 160.275 & 86.935031 & 70.9 & 71.1 & 78 & 74.8 & 0.000232 \\
\hline 240.01999 & 3.77863512 & 14.8087049 & 6.93145856 & 15.8955 & 160.225 & 87.282662 & 70.9 & 71.1 & 77.9 & 74.7 & 0.000232 \\
\hline 245.01999 & 3.7686308 & 14.8057064 & 6.932084 & 15.89475 & 160.3 & 86.090784 & 70.9 & 71.1 & 78 & 74.8 & 0.000232 \\
\hline 250.01999 & 3.77926039 & 14.8079553 & 6.932084 & 15.89475 & 160.275 & 86,885369 & 71 & 71.2 & 77.9 & 74.8 & 0.000232 \\
\hline 255.01980 & 3.78488782 & 14.8072056 & 6.93083312 & 15.8955 & 170.35 & 87.481308 & 71 & 71.1 & 77.9 & 74.7 & 0.000232 \\
\hline 260.01999 & 3.75987702 & 14.8094545 & 6.93145856 & 15.89475 & 170.275 & 86.090784 & 71 & 71.2 & 78 & 74.7 & 0.000234 \\
\hline 265.01999 & 3.76988134 & 14.806456 & 6.93145856 & 15.89625 & 170.3 & 86.537738 & 71 & 71.2 & 78 & 74.7 & 0.000233 \\
\hline 270.01999 & 3.76988134 & 14.806456 & 6.932084 & 15.8955 & 170.35 & 86,388753 & 71 & 71.1 & 78 & 74.8 & 0.000234 \\
\hline 275.01999 & 3.77238242 & 14.8087049 & 6.93083312 & 15.8955 & 180.3 & 86.016291 & 71 & 71.2 & 78 & 74.7 & 0.000234 \\
\hline 280.01999 & 3.7686308 & 14.8079553 & 6.93145856 & 15.89475 & 180.25 & 85.892137 & 70.9 & 71.2 & 78 & 74.7 & 0.000235 \\
\hline 285.01999 & 3.78426255 & 14.8079553 & 6.93083312 & 15.89625 & 180.275 & 86.463246 & 70.9 & 71.2 & 78 & 74.6 & 0.000234 \\
\hline 290.01999 & 3.76925607 & 14.8087049 & 6.932084 & 15.8955 & 180.325 & 84,501612 & 70.9 & 71.2 & 78 & 74.7 & 0.000235 \\
\hline 295.04001 & 3.76050229 & 14.8072056 & 6.92958224 & 15,89475 & 180.3 & 84.029827 & 70.9 & 71.2 & 78 & 74.7 & 0,000235 \\
\hline 300.01999 & 3.76175283 & 14.8072056 & 6.92958224 & 15.8955 & 180.325 & 83.731858 & 70.9 & 71.2 & 7.9 & 74.7 & 0.000235 \\
\hline 305.01999 & 3.77050661 & 14.8079553 & 6.93020768 & 15.89475 & 180.25 & 82.86278 & 70.9 & 71.2 & 78 & 74.8 & 0.000236 \\
\hline 310.01999 & 3.78176147 & 14.8079553 & 6.93020768 & 15.8955 & 190.325 & 85.544506 & 71 & 71.2 & 78 & 74.7 & 0.000235 \\
\hline 315.01999 & 3.78926471 & 14.8087049 & 6.93083312 & 15.89475 & 190.325 & 85.420352 & 70.9 & 71.2 & 78 & 74.7 & 0.000237 \\
\hline 320.01999 & 3.75550013 & 14.8079553 & 6.92770592 & 15.89475 & 190.325 & 84.774751 & 71 & 71.2 & 77.9 & 74.7 & 0.000236 \\
\hline 325.01999 & 3.75800121 & 14.8079553 & 6.93020768 & 15.8955 & 190.35 & 84.625766 & 71 & 71.2 & 78 & 74.8 & 0.000237 \\
\hline 330.01999 & 3.77050661 & 14.8087049 & 6.93020768 & 15.89475 & 190.325 & 85.867306 & 70.9 & 71.2 & 78 & 74.7 & 0.000236 \\
\hline 335.01999 & 3.77926039 & 14.8094545 & 6.92770592 & 15.8955 & 190.325 & 85.34586 & 71 & 71.3 & 78 & 74.8 & 0.000236 \\
\hline 340.01999 & 3.7811362 & 14.8087049 & 6.9289568 & 15.8955 & 200.35 & 85.916968 & 70.9 & 71.2 & 77.9 & 74.7 & 0.000236 \\
\hline 345.01999 & 3.77738458 & 14.8072056 & 6.93145856 & 15.89625 & 190.4 & 86.214938 & 70.9 & 71.2 & 77.9 & 74.7 & 0.000237 \\
\hline 350.01999 & 3.7873869 & 14.8087049 & 6.92833136 & 15.8955 & 200.325 & 86.661892 & 70.9 & 71.3 & 78 & 74.8 & 0.000237 \\
\hline 355.01999 & 3.77300769 & 14.8087049 & 6.92833136 & 15.89475 & 200.275 & 86.090784 & 70.8 & 71.2 & 77.9 & 74.7 & 0.000237 \\
\hline 360.01999 & 3.78051093 & 14.8087049 & 6.9289568 & 15.8955 & 200.325 & 85.172044 & 70.8 & 71.3 & 78 & 74.7 & 0.000237 \\
\hline 365.01999 & 3.7811362 & 14.8079553 & 6.92645504 & 15.8955 & 200.35 & 85.420352 & 70.8 & 71.2 & 77.9 & 74.7 & 0.000238 \\
\hline 370.01999 & 3.76675499 & 14.8087049 & 6.92833136 & 15.6955 & 200.3 & 85.99146 & 70.7 & 71.3 & 78 & 74.8 & 0.000237 \\
\hline 375.01999 & 3.7561254 & 14.8087049 & 6.9258296 & 15.89475 & 200.375 & 84.352628 & 70.8 & 71.3 & 77.9 & 74.6 & 0.000237 \\
\hline 380.01999 & 3.76300337 & 14.8087049 & 6.92833136 & 15.8955 & 200,3 & 84.10432 & 70.9 & 71.2 & 78 & 74.8 & 0.000238 \\
\hline 385.01999 & 3.77113188 & 14.8087049 & 6.9258296 & 15.89475 & 208.65 & 84.302966 & 70.9 & 71.2 & 78 & 74.7 & 0.000238 \\
\hline 390.01999 & 3.76487918 & 14.8079553 & 6.92770592 & 15.89775 & 200.375 & 84.576105 & 70.9 & 71.3 & 78 & 74.8 & 0.000238 \\
\hline 395.01999 & 3.78051093 & 14.8102041 & 6.9289568 & 15.897 & 200.3 & 84.625766 & 70.9 & 71.2 & 78 & 74.7 & 0.000238 \\
\hline
\end{tabular}




\begin{tabular}{|c|c|c|c|c|c|c|c|c|c|c|c|}
\hline SECONDS & \begin{tabular}{|l} 
MAIN DUCT \\
DIFF/N H2O
\end{tabular} & MAIN DUCT & GAS INJECT & \begin{tabular}{|l|} 
GAS INJECT \\
ABS PSIA
\end{tabular} & NEWPORT & SMC & \begin{tabular}{|l} 
AMBIENT \\
TF (F)
\end{tabular} & GAS INJECT & MAIN DUCT & WEC CELL & WEC CEL \\
\hline & & & & & & & & & & & I(VOLTS) \\
\hline 400.01999 & 3.79426687 & 14.8087049 & 6.92645504 & 15.89475 & 210.325 & 85.122382 & 70.9 & 71.3 & 78.1 & 74.8 & 0.000239 \\
\hline 405.01999 & 3.7686308 & 14.8102041 & 6.92645504 & 15.897 & 210.3 & 84.625766 & 70.9 & 79.3 & 78 & 74.7 & 0.000238 \\
\hline 410.01999 & 3.77863512 & 14.8094545 & 6.92833136 & 15.897 & 210.325 & 84.451951 & 70.9 & 71.3 & 78 & 74.8 & 0.000239 \\
\hline 415.01999 & 3.77613404 & 14.8102041 & 6.92645504 & 15.89775 & 210.35 & 84.203643 & 70.8 & 71.2 & 78 & 74.8 & 0.000239 \\
\hline 420.01999 & 3.77613404 & 14.8079553 & 6.9258296 & 15.897 & 210.3 & 84.178812 & 70.8 & 71.3 & 78 & 74.8 & 0.000238 \\
\hline 425.01999 & 3.76675499 & 14.8102041 & 6.92708048 & 15.89775 & 210.325 & 83.781519 & 70.8 & 71.3 & 78 & 74.8 & 0.000239 \\
\hline 430.01999 & 3.7686308 & 14.8094545 & 6.9258296 & 15.89775 & 210.325 & 84.799582 & 70.7 & 71.3 & 78 & 74.8 & 0.00024 \\
\hline 435.01999 & 3.77988566 & 14.8102041 & 6.9258296 & 15.897 & 210.3 & 84,675428 & 70.8 & 71.3 & 78 & 74.8 & 0.000239 \\
\hline 440.01999 & 3.76112756 & 14.8087049 & 6.92708048 & 15.8985 & 210.35 & 84.874074 & 70.8 & 71.3 & 78 & 74.7 & 0.000239 \\
\hline 445.01999 & 3.76925607 & 14.8087049 & 6.92645504 & 15.89925 & 210.325 & 84.253304 & 70.8 & 71.3 & 78 & 74.8 & 0.00024 \\
\hline 450.01999 & 3.77926039 & 14.8079553 & 6.92645504 & 15.89625 & 210.3 & 84.551274 & 70.8 & 71.3 & 78 & 74.7 & 0.000239 \\
\hline 455.01999 & 3.77613404 & 14.8087049 & 6.92395328 & 15.897 & 210.325 & 84.029827 & 70.7 & 71.3 & 78 & 74.8 & 0.000239 \\
\hline 460.01999 & 3.77613404 & 14.8094545 & 6.92645504 & 15.897 & 220.325 & 84.576105 & 70.7 & 71.3 & 78 & 74.7 & 0.000241 \\
\hline 465.01999 & 3.76675499 & 14.8087049 & 6.92457872 & 15.89775 & 220.35 & 84.774751 & 70.7 & 71.3 & 78 & 74.8 & 0.00024 \\
\hline 470.01999 & 3.77738458 & 14,8079553 & 6.92457872 & 15.89775 & 220.35 & 84.352628 & 70.7 & 71.3 & 78 & 74.7 & 0.00024 \\
\hline 475.01999 & 3.77863512 & 14.8072056 & 6.92457872 & 15.897 & 220.35 & 84.476782 & 70.7 & 71.3 & 78 & 74.8 & 0.00024 \\
\hline 480.01999 & 3.77363296 & 14.8087049 & 6.92457872 & 15.89775 & 220.375 & 84.625766 & 70.7 & 71.3 & 78 & 74.7 & 0.000241 \\
\hline 485.01999 & 3.7686308 & 14.8087049 & 6.92395328 & 15.89775 & 220.325 & 84.675428 & 70.6 & 71.3 & 78 & 74.8 & 0.000241 \\
\hline 490.01999 & 3.76925607 & 14.8094545 & 6.92395328 & 15.89775 & 220.35 & 84.551274 & 70.6 & 71.3 & 78 & 74.7 & 0.000242 \\
\hline 495.01999 & 3.78988998 & 14.8094545 & 6.92457872 & 15.89775 & 220.35 & 84.501612 & 70.6 & 71.3 & 78 & 74.8 & 0.000241 \\
\hline 500.01999 & 3.76300337 & 14.8087049 & 6.92395328 & 15.89775 & 220.325 & 83.905673 & 70.6 & 71.3 & 78 & 74.7 & 0.00024 \\
\hline 505.01999 & 3.77300769 & 14.8079553 & 6.92457872 & 15.89775 & 220.375 & 84.576105 & 70.5 & 71.3 & 78 & 74.8 & 0.000241 \\
\hline 510.01999 & 3.77675931 & 14.8087049 & 6.92395328 & 15.8985 & 220.375 & 84.42712 & 70.5 & 71.3 & 78 & 74.7 & 0.000241 \\
\hline 515.01999 & 3.76175283 & 14.8079553 & 6.92395328 & 15.89625 & 220.35 & 84.153981 & 70.5 & 71.3 & 77.9 & 74.8 & 0.000241 \\
\hline 520.01999 & 3.7811362 & 14.8087049 & 6.92332784 & 15.89775 & 220.375 & 84.029827 & 70.5 & 71.3 & 77.9 & 74.7 & 0.000241 \\
\hline 525.01999 & 3.78488782 & 14.8094545 & 6.92332784 & 15.89775 & 220.325 & 84.451951 & 70.5 & 71.3 & 78 & 74.8 & 0.000241 \\
\hline 530.06 & 3.77300769 & 14.8102041 & 6.92520416 & 15.89775 & 220.35 & 84.526443 & 70.5 & 71.3 & 78 & 74.7 & 0.000241 \\
\hline 535,01999 & 3.77238242 & 14.8094545 & 6.92332784 & 15.8985 & 230.35 & 84.476782 & 70.6 & 71.3 & 78 & 74.7 & 0.000241 \\
\hline 540.01999 & 3.78801417 & 14.8087049 & 6.92332784 & 15.897 & 230.325 & 84.625766 & 70.6 & 71.3 & 78 & 74.7 & 0.000242 \\
\hline 545.06 & 3.76487918 & 14.8087049 & 6.92332784 & 15.897 & 230.325 & 84.476782 & 70.6 & 71.3 & 77.9 & 74.8 & 0.000242 \\
\hline 550.01999 & 3.77113188 & 14.8094545 & 6.92145152 & 15.89775 & 230.35 & 84.402289 & 70.6 & 71.3 & 78 & 74.7 & 0.000243 \\
\hline 555.01999 & 3.76800553 & 14.8087049 & 6.92332784 & 15.89775 & 230.4 & 86.5874 & 70.6 & 71.3 & 78 & 74.7 & 0.000241 \\
\hline 560.01999 & 3.77050661 & 14.8079553 & 6.92457872 & 15.897 & 230.325 & B7.282662 & 70.5 & 71.3 & 78 & 74.6 & 0.000241 \\
\hline 565.01999 & 3.77675931 & 14.8102041 & 6.92395328 & 15.89775 & 230.325 & 84.898905 & 70.5 & 71.3 & 77.9 & 74.7 & 0.00024 \\
\hline 570.01999 & 3.77675931 & 14.8087049 & 6.92207696 & 15.8985 & 230.35 & 85.34586 & 70.5 & 71.3 & 77.9 & 74.6 & 0.00024 \\
\hline 575.01999 & 3.7873889 & 14.8094545 & 6.92145152 & 15.8985 & 230.35 & 85.792814 & 70.5 & 71.3 & 78 & 74.7 & 0.000243 \\
\hline 580.01999 & 3.76487918 & 14.8079553 & 6.92395328 & 15.89775 & 230.4 & 85.96663 & 70.5 & 71.3 & 78 & 74.7 & 0,000241 \\
\hline 585.01999 & 3,77050661 & 14.8087049 & 6.92457872 & 15.8985 & 230.35 & 85.04789 & 70.5 & 71.3 & 78 & 74.7 & 0.000242 \\
\hline 590.03 & 3.78738889 & 14.8102041 & 6.92395328 & 15.8985 & 230.35 & 85.37069 & 70.5 & 71.3 & 78 & 74.8 & 0.000242 \\
\hline 595.01999 & 3.77926039 & 14.8079553 & 6.92207696 & 15.8985 & 230.35 & 84.302966 & 70.5 & 71.3 & 78 & 74.7 & 0.000242 \\
\hline 600.01999 & 3.7686308 & 14.8087049 & 6.92145152 & 15.89775 & 230.375 & 84.700259 & 70.5 & 71.3 & 78 & 74.7 & 0.000241 \\
\hline 605.07001 & 3.77300769 & 14.8094545 & 6.92207696 & 15.89775 & 230.35 & 84.302966 & 70.5 & 71.3 & 78 & 74.8 & 0.000242 \\
\hline
\end{tabular}




\begin{tabular}{|c|c|c|c|c|c|c|c|c|c|c|c|}
\hline SECONDS & \begin{tabular}{|l} 
MAIN DUCT \\
DIFF(IN H2O \\
\end{tabular} & \begin{tabular}{|l|} 
MAIN DUCT \\
ABS (PSIA)
\end{tabular} & \begin{tabular}{|l|} 
GAS INJECT \\
DIFF(IN H2O) \\
\end{tabular} & \begin{tabular}{|l|} 
GAS INJECT \\
ABS (PSIA) \\
\end{tabular} & \begin{tabular}{|l|} 
NEWPORT \\
(PPM) \\
\end{tabular} & \begin{tabular}{|l|} 
SMC \\
(PPM) \\
\end{tabular} & $\begin{array}{l}\text { AMBIENT } \\
\text { TEMP.(F) }\end{array}$ & $\begin{array}{l}\text { GAS INJECT } \\
\text { TEMP.(F) } \\
\end{array}$ & \begin{tabular}{|l} 
MAIN DUCT \\
TEMP.(F) \\
\end{tabular} & \begin{tabular}{|l|} 
WEC CELL \\
TEMP.(F) \\
\end{tabular} & \begin{tabular}{|l} 
WEC CEL \\
(VOLTS)
\end{tabular} \\
\hline 610.01999 & 3.78551309 & 14.8094545 & 6.92082608 & 15.89775 & 230.35 & 84.451951 & 70.6 & 71.3 & 77.9 & $\mathbf{7 4 . 7}$ & 0.000242 \\
\hline 615.01999 & 3.77738458 & 14.8072056 & 6.92082608 & 15.897 & 230.375 & 83.80635 & 70.5 & 71.3 & 77.9 & 74.7 & 0.000241 \\
\hline 620.01999 & 3.76988134 & 14.8079553 & 6.92145152 & 15.89775 & 230.35 & 83.756688 & 70.6 & 71.3 & 77.9 & 74.6 & 0.000242 \\
\hline 625.01999 & 3.7686308 & 14.8087049 & 6.92145152 & 15.89775 & 230.35 & 84.302966 & 70.5 & 71.3 & 78 & 74.8 & 0.000242 \\
\hline 630.01999 & 3.7686308 & 14.8094545 & 6.92082608 & 15.89775 & 230.325 & 84.476782 & 70.5 & 71.3 & 77.9 & 74.7 & 0.000242 \\
\hline 635.06 & 3.77675931 & 14.8094545 & 6.92145152 & 15.8985 & 230.375 & 84.551274 & 70.6 & 71.3 & 78 & 74.8 & 0.000243 \\
\hline 640.01999 & 3.77113188 & 14.8079553 & 6.92145152 & 15,897 & 230.375 & 83.781519 & 70.6 & 71.2 & 77.9 & 74.7 & 0.000243 \\
\hline 645.01999 & 3.78301201 & 14.8094545 & 6.92145152 & 15.89775 & 230.325 & 83.80635 & 70.6 & 71.3 & 78 & 74.8 & 0.000242 \\
\hline 650.04999 & 3.76675499 & 14.8094545 & 6.92145152 & 15.89775 & 230.3 & 84.029827 & 70.6 & 71.3 & 78 & 74.8 & 0.000241 \\
\hline 655.01999 & 3.76925607 & 14.8087049 & 6.92145152 & 15.897 & 230.3 & 84.526443 & 70.6 & 71.3 & 78 & 74.8 & 0.000243 \\
\hline 660.01999 & 3.77238242 & 14.8079553 & 6.92145152 & 15.8985 & 230.35 & 84.501612 & 70.6 & 71.3 & 77.9 & 74.7 & 0.000242 \\
\hline 665.06 & 3.76925607 & 14.8079553 & 6.92145152 & 15.89775 & 230.375 & 84.352628 & 70.6 & 71.3 & 77.9 & 74.8 & 0.000241 \\
\hline 670.01999 & 3.76575499 & 14.8087049 & 6.92020064 & 15.8985 & 230.325 & 84.650597 & 70.6 & 71.3 & 77.9 & 74.8 & 0.000241 \\
\hline 675.01999 & 3.79176579 & 14.8079553 & 6.91894976 & 15.89775 & 230.375 & 84.153981 & 70.6 & 71.3 & 77.9 & 74.7 & 0.000243 \\
\hline 680.04001 & 3.79426687 & 14.8079553 & 6.9195752 & 15.89775 & 230.35 & 84.402289 & 70.5 & 71.3 & 78 & 74.7 & 0.000241 \\
\hline 685.01999 & 3.77300769 & 14.8087049 & 6.9195752 & 15.89775 & 230.35 & 84.72500 & 70.5 & 71.3 & 78 & 74.7 & 0.000242 \\
\hline 690.01999 & 3.76988134 & 14.8079553 & 6.92020064 & 15.89775 & 230.35 & 84.377458 & 70.5 & 71.2 & 78 & 74.8 & 0.000242 \\
\hline 695.04001 & 3.77425823 & 14.8087049 & 6.92082608 & 15.89775 & 230.35 & 84.178812 & 70.5 & 71.3 & 77.9 & 74.8 & 0.000243 \\
\hline 700.01999 & 3.76738026 & 14.8087049 & 6.91894976 & 15.89775 & 240.4 & 83.657365 & 70.5 & 71.3 & 78 & 74.8 & 0.000243 \\
\hline 705.01999 & 3.77113188 & 14.8094545 & 6.9195752 & 15.897 & 240.35 & 83.334565 & 70.6 & 71.3 & 77.9 & 74.7 & 0.000243 \\
\hline 710.04999 & 3.77300769 & 14.8087049 & 6.91769888 & 15.89775 & 236.075 & 83.334565 & 70.4 & 71.3 & 77.9 & 74.7 & 0.000242 \\
\hline 715.01999 & 3.76425391 & 14.8079553 & 6.91894976 & 15.89775 & 240.375 & 83.48355 & 70.4 & 71.3 & 78 & 74.8 & 0.000243 \\
\hline 720.01999 & 3.77988566 & 14.8087049 & 6.91832432 & 15.89775 & 240.375 & 83.111088 & 70.4 & 71.3 & 77.9 & 74.7 & 0.000243 \\
\hline 725.01999 & 3.77675931 & 14.8087049 & 6.9195752 & 15.89775 & 240.4 & 83.458719 & 70.5 & 71.3 & 77.9 & 74.8 & 0.000243 \\
\hline 730.01999 & 3.76925607 & 14.8087049 & 6.9195752 & 15.897 & 230.375 & 83.856012 & 70.5 & 71.2 & 77.9 & 74.7. & 0.000242 \\
\hline 735.01999 & 3.7623781 & 14.8072056 & 6.9195752 & 15.89775 & 240.15 & 83.284903 & 70.6 & 71.3 & 77.9 & 74.8 & 0.000243 \\
\hline 740.01999 & 3.77800985 & 14.8072056 & 6.91832432 & 15.897 & 240.35 & 84.973398 & 70.6 & 71,3 & 77.9 & 74.7 & 0.000242 \\
\hline 745.01999 & 3.77363296 & 14.8079553 & 6.920220064 & 15.89775 & 240.4 & 84.998228 & 70.6 & 71,3 & 77.9 & 74.8 & 0.000242 \\
\hline 750.01999 & 3.78426255 & 14.8094545 & 6.9195752 & 15.8985 & 240.4 & 85.147213 & 70.6 & 71.3 & 77.9 & 74.7 & 0.000242 \\
\hline 755.06 & 3.77300769 & 14.8079553 & 6.916448 & 15.89775 & 230.925 & 84.526443 & 70.6 & 71.3 & 77.9 & 74.8 & 0.000243 \\
\hline 760.01999 & 3.76988134 & 14.8087049 & 6.91769888 & 15.8985 & 240.375 & 84.650597 & 70.6 & 71.3 & 77.9 & 74.7 & 0.000243 \\
\hline 765.01999 & 3.77300769 & 14.8087049 & 6.9195752 & 15.89775 & 240.375 & 83.905673 & 70.7 & 71.3 & 77.9 & 74.8 & 0.000242 \\
\hline 770.07001 & 3.77988566 & 14.8087049 & 6.91707344 & 15.89775 & 230.9 & 83.359396 & 70.7 & 71.3 & $\pi .9$ & 74.7 & 0.000242 \\
\hline 775.01999 & 3.78613836 & 14.806456 & 6.91707344 & 15.8985 & 240.375 & 83.930504 & 70.7 & 71.2 & 78 & 74.8 & 0.000243 \\
\hline 780.01999 & 3.7686308 & 14.8079553 & 6.91894976 & 15.89775 & 240.375 & 83.930504 & 70.7 & 71.2 & 77.9 & 74.7 & 0.000243 \\
\hline 785.03 & 3.77300769 & 14.8087049 & 6.91769888 & 15,89775 & 240.4 & 83.260072 & 70.7 & 71.2 & 77.9 & 74.7 & 0.000242 \\
\hline 790.01999 & 3.77425823 & 14.8087049 & 6.916448 & 15.89775 & 240.325 & 82.763456 & 70.7 & 71.2 & 77.9 & 74.7 & 0.000243 \\
\hline 795.01999 & 3.77238242 & 14.8087049 & 6.91769888 & 15.89775 & 240.4 & 82.614472 & 70.7 & 71.2 & 77.9 & 74.8 & 0.000242 \\
\hline 800.07001 & 3.77300769 & 14.8079553 & 6.91769888 & 15.897 & 230.475 & 83.036595 & 70.8 & 71.2 & 77.9 & 74.7 & 0.000242 \\
\hline 805.01999 & 3.78551309 & 14.8094545 & 6.91769888 & 15.897 & 240.4 & 83.682196 & 70.8 & 71.2 & 78 & 74.8 & 0.000243 \\
\hline 810.01999 & 3.77363296 & 14.8094545 & 6.91894976 & 15.89775 & 240.375 & 83.235242 & 70.7 & 71.2 & $\overline{78}$ & 74.7 & 0.000243 \\
\hline 815.04999 & 3.77363296 & 14.8087049 & 6.91707344 & 15.89775 & 240.4 & 83.856012 & 70.7 & 71.2 & 77.9 & 74.7 & 0.000243 \\
\hline
\end{tabular}




\begin{tabular}{|c|c|c|c|c|c|c|c|c|c|c|c|}
\hline SECONDS & MAIN DUCT & MAIN DUCT & GAS INJECT & GAS INJECT & NEWPORT & SMC & AMBIENT & GAS INJECT & MAIN DUCT & \begin{tabular}{|l|} 
WEC CELL \\
TEMP(F)
\end{tabular} & WEC CEL \\
\hline & DIFF(IN H2O & ABS (PSIA) & DIFF(N H2O) & $A B S$ (PSIA) & (PPM) & (PPM) & & & & & \\
\hline 820.01999 & 3.76050229 & 14.8079553 & 6.91707344 & 15.89775 & 240.4 & 83.731858 & 70.7 & 71.3 & 77.9 & 74.7 & 0.000243 \\
\hline 825.01999 & 3.76550445 & 14.8079553 & 6.91707344 & 15.897 & 231.125 & 84.849244 & 70.6 & 71.2 & 77.9 & 74.8 & 0.000242 \\
\hline 830.04999 & 3.77550877 & 14.8049568 & 6.91769888 & 15.89775 & 239.575 & 85.221706 & 70.8 & 71.3 & 77.9 & 74.8 & 0.000243 \\
\hline 835.01999 & 3.7686308 & 14.8079553 & 6.91707344 & 15.89775 & 236.675 & 82.713795 & 70.7 & 71.2 & 77.9 & 74.7 & 0.000242 \\
\hline 840.01999 & 3.78176147 & 14.8057064 & 6.91582256 & 15.89775 & 230.375 & 85.867306 & 70.7 & 71.3 & 77.9 & 74.8 & 0.000242 \\
\hline 845.03 & 3.77550877 & 14.8087049 & 6.91519712 & 15.8985 & 230.4 & 83.582873 & 70.7 & 71.3 & 77.9 & 74.7 & 0.000242 \\
\hline 850.01999 & 3.77613404 & 14.8087049 & 6.91519712 & 15.89775 & 240.35 & 83.955335 & 70.6 & 71.2 & 77.9 & 74.7 & 0.000242 \\
\hline 855.01999 & 3.75800121 & 14.8049568 & 6.91582256 & 15.89775 & 240.375 & 83.48355 & 70.8 & 71.2 & 77.9 & 74.7 & 0.000242 \\
\hline 860.04001 & 3.77550877 & 14.8087049 & 6.91519712 & 15.897 & 240.4 & 83.756688 & 70.8 & 71.2 & 78 & 74.8 & 0.000243 \\
\hline 865.01999 & 3.77113188 & 14.8057064 & 6.91519712 & 15.8985 & 240.4 & 83.731858 & 70.7 & 71.2 & 77.9 & 74.8 & 0.000242 \\
\hline 870.01999 & 3.75987702 & 14.8079553 & 6.91457168 & 15.89775 & 240.4 & 84.079489 & 70.8 & 71.2 & 77.9 & 74.7 & 0.000242 \\
\hline 875.04001 & 3.77363296 & 14.8057064 & 6.91519712 & 15.897 & 240.35 & 83.50838 & 70.7 & 71.2 & 77.9 & 74.7 & 0.000242 \\
\hline 880.01999 & 3.75362432 & 14.8057064 & 6.9133208 & 15.89775 & 240.375 & 83.657365 & 70.7 & 71.2 & 77.9 & 74.8 & 0.000243 \\
\hline 885.03 & 3.77113188 & 14.8087049 & 6.91519712 & 15.89775 & 240.4 & 83.682196 & 70.7 & 71.2 & 77.9 & 74.7 & 0.000243 \\
\hline 890.07999 & 3.78613836 & 14.8057064 & 6.91519712 & 15.89775 & 240.35 & 83.80635 & 70.7 & 71.2 & 78 & 74.8 & 0.000243 \\
\hline 895.01999 & 3.78176147 & 14.8087049 & 6.9133208 & 15.8985 & 240.375 & 83.607704 & 70.6 & 71.2 & 78 & 74.8 & 0.000243 \\
\hline 900.01999 & 3.76425391 & 14.8072056 & 6.91582256 & 15.89775 & 240.4 & 84.377458 & 70.7 & 71.2 & 77.9 & 74.8 & 0.000243 \\
\hline 905.04001 & 3.76675499 & 14.8094545 & 6.91457168 & 15.8985 & 240.375 & 83.80635 & 70.6 & 71.2 & 77.9 & 74.7 & 0.000243 \\
\hline 910.01999 & 3.75800121 & 14.8072056 & 6.91394624 & 15.8955 & 240.375 & 83.781519 & 70.6 & 71.2 & 77.9 & 74.8 & 0.000243 \\
\hline 915.01999 & 3.78301201 & 14.8057064 & 6.9133208 & 15.89625 & 240.4 & 84.278135 & 70.6 & 71.2 & 77.9 & 74.8 & 0.000243 \\
\hline
\end{tabular}




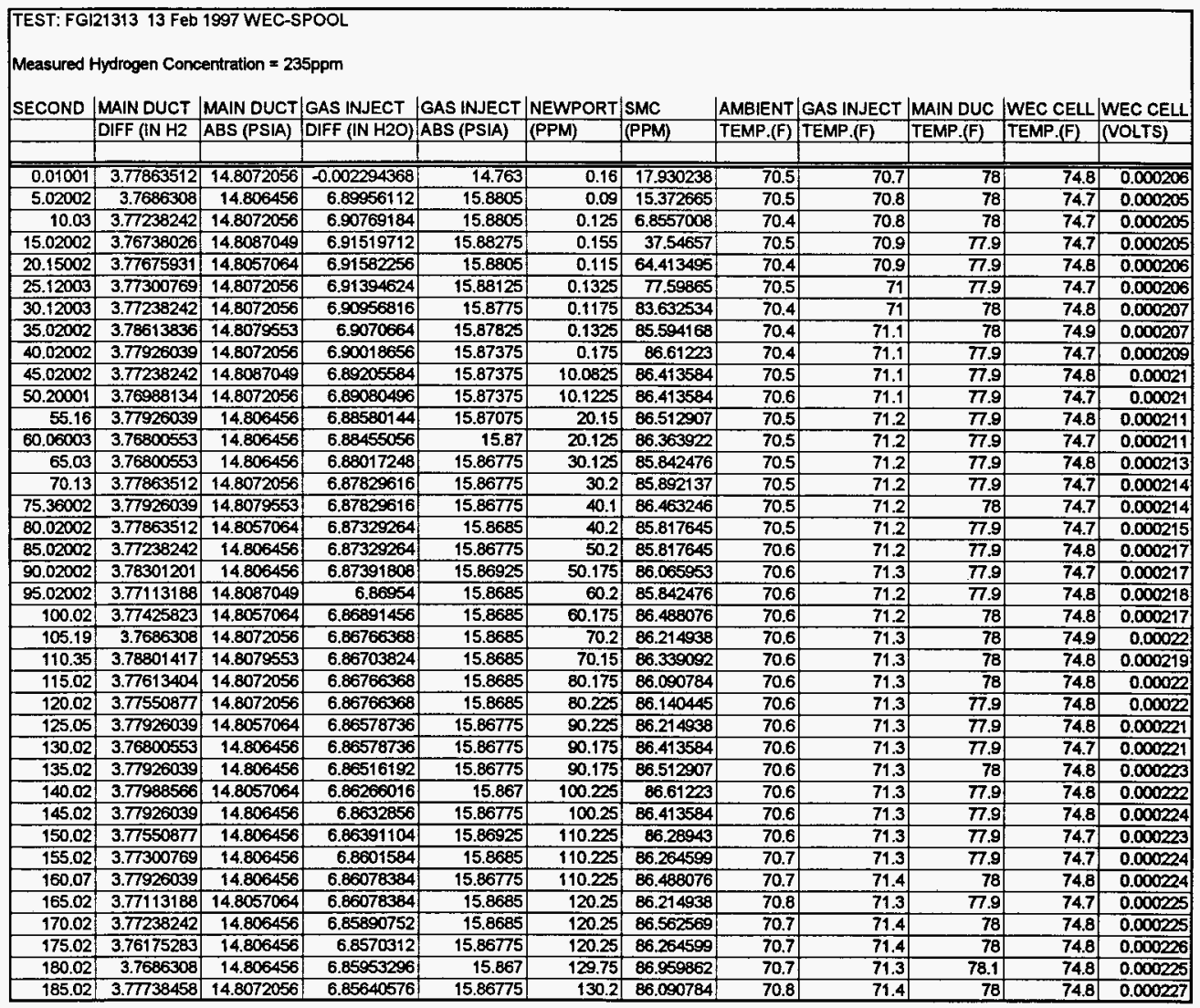




\begin{tabular}{|c|c|c|c|c|c|c|c|c|c|c|c|}
\hline SECOND & \begin{tabular}{|l|} 
MAIN DUCT \\
DIFF (IN H2
\end{tabular} & $\begin{array}{l}\text { MAIN DUCT } \\
\text { ABS (PSIA) }\end{array}$ & \begin{tabular}{|l|} 
GAS INJECT \\
DIFF (IN H2O) \\
\end{tabular} & $\frac{\text { GAS INJECT }}{\text { ABS (PSIA) }}$ & \begin{tabular}{|l|} 
NEWPORT \\
(PPM) \\
\end{tabular} & $\begin{array}{l}\text { SMC } \\
\text { (PPM) }\end{array}$ & \begin{tabular}{|} 
AMBIENT \\
TEMP.(F) \\
\end{tabular} & $\begin{array}{l}\text { GAS INJECT } \\
\text { TEMP.(F) }\end{array}$ & \begin{tabular}{|l|} 
MAIN DUC \\
TEMP.(F) \\
\end{tabular} & $\begin{array}{l}\text { WEC CELL } \\
\text { TEMP.(F) }\end{array}$ & \begin{tabular}{|l} 
WEC CELL \\
NOLTS) \\
\end{tabular} \\
\hline & & & & & & & & & & & \\
\hline 190.02 & 3.78613836 & 14.8057064 & 6.8570312 & 15.867 & 130.275 & 86.165276 & 70.7 & 71.4 & 78. & 74.8 & 0.000226 \\
\hline 195.02 & 3.79051525 & 14.8079553 & 6.85640576 & 15.8685 & 130.25 & 86.388753 & 70.8 & 71.4 & 78 & 74.8 & 0.000227 \\
\hline 200.02 & 3.78551309 & 14.8072056 & 6.85640576 & 15.867 & 140.25 & 86.761215 & 70.7 & 71.4 & 78 & 74.8 & 0.000228 \\
\hline 205.02 & 3.78238674 & 14.8072056 & 6.85640576 & 15.8685 & 140.225 & 85.867306 & 70.7 & 71.4 & 78 & 74.8 & 0.000229 \\
\hline 210.02 & 3.77863512 & 14.8072056 & 6.85640576 & 15.867 & 140.275 & 85.618998 & 70.7 & 71.4 & 77.9 & 74.8 & 0.000229 \\
\hline 215.02 & 3.77675931 & 14.8079553 & 6.8570312 & 15.867 & 140.25 & 85.941799 & 70.8 & 71.4 & 78 & 74.8 & 0.000229 \\
\hline 220.02 & 3.77863512 & 14.806456 & 6.85452944 & 15.8685 & 141.075 & 87.381985 & 70.9 & 71.4 & 77.9 & 74.7 & 0.000229 \\
\hline 225.02 & 3.76988134 & 14.8057064 & 6.85452944 & 15.86775 & 150.275 & 88.251063 & 70.9 & 71.4 & 78 & 74.8 & 0.00023 \\
\hline 230.02 & 3.77675931 & 14.8087049 & 6.85515488 & 15.86775 & 150.275 & 87.977924 & 70.9 & 71.4 & 77.9 & 74.8 & 0.000229 \\
\hline 235.02 & 3.77300769 & 14.8072056 & 6.85327856 & 15.86775 & 150.275 & 86.463246 & 70.9 & 71.4 & 77.9 & 74.8 & 0.00023 \\
\hline 240.02 & 3.76988134 & 14.806456 & 6.85265312 & 15.86775 & 150.275 & 86.090784 & 71 & 71.4 & 78 & 74.7 & 0.000231 \\
\hline 245.04 & 3.7811362 & 14.8079553 & 6.853904 & 15.86775 & 150.3 & 86.388753 & 71 & 71.4 & 78 & 74.9 & 0.000232 \\
\hline 250.09 & 3.78551309 & 14.8079553 & 6.84952592 & 15.867 & 160.275 & 86.239768 & 70.9 & 71.4 & 77.9 & 74.7 & 0.000231 \\
\hline 255.02 & 3.788639944 & 14.8079553 & 6.85202768 & 15.867 & 160.275 & 86.090784 & 70.8 & 71.4 & 77.9 & 74.8 & 0.000231 \\
\hline 260.02 & 3.77613404 & 14.8072056 & 6.85140224 & 15.8685 & 160.275 & 86.090784 & 70.9 & 71.4 & 78 & 74.8 & 0.000232 \\
\hline 265.02 & 3.77863512 & 14.8087049 & 6.8507768 & 15.8685 & 160.3 & 88.524202 & 70.8 & 71.4 & 78 & 74.8 & 0.000232 \\
\hline 270.02 & 3.7748835 & 14.8072056 & 6.85015136 & 15.86775 & 160.2 & 88.598694 & 70.8 & 71.4 & 78 & 74.8 & 0.000233 \\
\hline 275.02 & 3.76112756 & 14.806456 & 6.8507768 & 15.86775 & 170.3 & 88.400048 & 70.9 & 71.4 & 77.9 & 74.8 & 0.000233 \\
\hline 280.02 & 3.77988566 & 14.806456 & 6.85015136 & 15.86775 & 170.275 & 85.693491 & 70.8 & 71.5 & 78 & 74.8 & 0.000233 \\
\hline 285.03 & 3.7748835 & 14.8079553 & 6.85202768 & 15.8685 & 170.25 & 85.867306 & 70.8 & 71.5 & 78 & 74.8 & 0.000233 \\
\hline 290.02 & 3.77863512 & 14.8087049 & 6.85015136 & 15.86775 & 170.275 & 85.867306 & 70.8 & 71.5 & 78 & 74.8 & 0.000233 \\
\hline 295.02 & 3.78488782 & 14.8049568 & 6.85327856 & 15.86625 & 170.275 & 86.810877 & 70.8 & 71.4 & 77.9 & 74.7 & 0.000233 \\
\hline 300.02 & 3.78051093 & 14.806456 & 6.8507768 & 15.86775 & 170.35 & 86.562569 & 70.8 & 71.5 & 77.9 & 74.8 & 0,000234 \\
\hline 305.02 & 3.77300769 & 14.8087049 & 6.8507768 & 15.86775 & 170.275 & 86.761215 & 70.8 & 71.5 & $\pi 7.9$ & 74.8 & 0.000233 \\
\hline 310.02 & 3.7811362 & 14.8049568 & 6.85015136 & 15.867 & 180.3 & 85.941799 & 70.8 & 71.4 & 78 & 74.8 & 0.000234 \\
\hline 315.02 & 3.78488782 & 14.8072056 & 6.85015136 & 15.86775 & 180.325 & 85.99146 & 70.9 & 71.5 & 77.9 & 74.8 & 0.000233 \\
\hline 320.02 & 3.77926039 & 14.8057064 & 6.8507768 & 15.86775 & 180.275 & 86.860538 & 70.8 & 71.5 & 78 & 74,8 & 0.000235 \\
\hline 325.02 & 3.77926039 & 14.806456 & 6.84890048 & 15.86775 & 180.3 & 86.686723 & 70.9 & 71.5 & 78 & 74.7 & 0,000234 \\
\hline 330.02 & 3.78613836 & 14.8057064 & 6.84890048 & 15.8685 & 180.3 & 85.96663 & 71 & 71.5 & 78 & 74.8 & 0,000235 \\
\hline 335.02 & 3.7811362 & 14.8057064 & 6.8476496 & 15.86775 & 180.25 & 86.041122 & 71.1 & 71.5 & 78 & 74.8 & 0.000235 \\
\hline 340.02 & 3.76425391 & 14.8057064 & 6.84827504 & 15.8685 & 180.325 & 86.165276 & 71 & 71.5 & 77.9 & 74.8 & 0.000236 \\
\hline 345.02 & 3.77800985 & 14.805456 & 6.84890048 & 15.86775 & 180.325 & 86.339092 & 71 & 71.5 & 78 & 74.8 & 0.000235 \\
\hline 350.02 & 3.77300769 & 14.806456 & 6.85202768 & 15.86775 & 190.275 & 86.314261 & 71 & 71.5 & 78 & 74.8 & 0.000235 \\
\hline 355.02 & 3.77863512 & 14.8072056 & 6.85140224 & 15.8685 & 190.325 & 86.016291 & 71 & 71.5 & 7.9 & 74.8 & 0.000235 \\
\hline 360.02 & 3.77175715 & 14.806456 & 6.84890048 & 15.8685 & 190.325 & 86.190107 & 71 & 71.5 & 78 & 74.8 & 0.000236 \\
\hline 365.02 & 3.7811362 & 14.806456 & 6.84827504 & 15.8685 & 190.3 & 86.190107 & 71 & 71.5 & 78 & 74.8 & 0.000236 \\
\hline 370.00 & 3.77988566 & 14.8049568 & 6.84952592 & 15.86775 & 190.325 & 86.935031 & 70.9 & 71.5 & 78 & 74.8 & 0.000236 \\
\hline 375.02 & 3.77863512 & 14.8049568 & 6.84890048 & 15.867 & 190.3 & 86.363922 & 71 & 71.5 & 78 & 74.8 & 0.000235 \\
\hline 380.02 & 3.7873889 & 14.8072056 & 6.84827504 & 15.86775 & 190.325 & 85.96663 & 71 & 71.5 & 78 & 74.8 & 0.000236 \\
\hline 385.02 & 3.77175715 & 14.8057064 & 6.84952592 & 15.86775 & 190.325 & 86.810877 & 71.1 & 71.5 & 78 & 74.8 & 0.000236 \\
\hline 390.02 & 3.78176147 & 14.8057064 & 6.84827504 & 15.867 & 190.325 & 86.5874 & 71.2 & 71.5 & 78 & 74.8 & 0.000236 \\
\hline 395.02 & 3.7811362 & 14.8079553 & 6.84952592 & 15.867 & 190.325 & 86.438415 & 71.1 & 71.5 & 78.1 & 74.9 & 0.000237 \\
\hline
\end{tabular}




\begin{tabular}{|c|c|c|c|c|c|c|c|c|c|c|c|}
\hline SECOND & MAIN DUCT & MAIN DUCT & GAS INJECT & \begin{tabular}{|l|l|} 
GAS INJECT \\
\end{tabular} & NEWPORT & SMC & AMBIENT & GAS INJECT & MAIN DUC & WEC CELLI & WEC CELL \\
\hline & DIFF (IN H2 & ABS (PSIA) & DIFF (IN H2O) & ABS (PSIA) & \begin{tabular}{|l|l} 
(PPM) \\
\end{tabular} & (PPM) & TEMP.(F) & TEMP.(F) & TEMP.(F) & TEMP.(F) & \\
\hline 400.07 & 3.77425823 & 14.8057064 & 6.84952592 & 15.867 & 200.25 & 86.5874 & 71.1 & 71.5 & 78 & 74.8 & 0.000237 \\
\hline 405.02 & 3.78488782 & 14.8072056 & 6.84702416 & 15.857 & 200.3 & 86.5874 & 71.1 & 71.5 & 78 & 74.8 & 0.000237 \\
\hline 410.08 & 3.77926039 & 14.8079553 & 6.84890048 & 15.86775 & 200.3 & 86.140445 & 71.1 & 71.5 & 78 & 75.6 & 0.000237 \\
\hline 415.02 & 3.77238242 & 14.8072056 & 6.84952592 & 15.867 & 200.275 & 85.66866 & 71 & 71.5 & 78 & 75.4 & 0.000237 \\
\hline 420.02 & 3.79051525 & 14.8079553 & 6.84639872 & 15.867 & 200.325 & 86.463246 & 71 & 71.5 & 78 & 75.2 & 0.000238 \\
\hline 425.02 & 3.7811362 & 14.8072056 & 6.84702416 & 15.8685 & 200.3 & 86.115614 & 71.1 & 71.5 & 78 & 75 & 0.000237 \\
\hline 430.02 & 3.7748835 & 14.806456 & 6.84639872 & 15.86775 & 200.325 & 85.941799 & 71.1 & 71.5 & 78 & 75 & 0.000238 \\
\hline 435.02 & 3.78551309 & 14.8049568 & 6.84639872 & 15.86775 & 200.35 & 86.041122 & 71.2 & 71.5 & 78 & 74,9 & 0.000238 \\
\hline 440.02 & 3.77675931 & 14.8072056 & 6.84952592 & 15.86775 & 200.275 & 86.140445 & 71.2 & 71.5 & 78 & 74.9 & 0.000238 \\
\hline 445.02 & 3.77050661 & 14.8072056 & 6.8476496 & 15.8685 & 200.35 & B6.041122 & 71.2 & 71.5 & 78 & 74.9 & 0.000238 \\
\hline 450.02 & 3.76112756 & 14.8057064 & 6.84827504 & 15.8685 & 200.325 & 85.817645 & 71.2 & 71.5 & 78 & 74.8 & 0.000238 \\
\hline 455.02 & 3.78551309 & 14.8057064 & 6.84639872 & 15.8685 & 200.3 & 86.214938 & 71.2 & 71.5 & 78.1 & 74.8 & 0.000238 \\
\hline 460.02 & 3.78551309 & 14.806456 & 6.8476496 & 15.86775 & 200.3 & 86.661892 & 71.1 & 71.5 & 77.9 & 74.7 & 0.000238 \\
\hline 465.02 & 3.7746835 & 14.8072056 & 6.84639872 & 15.86775 & 200.3 & 85.892137 & 71.1 & 71.5 & 78.1 & 74.9 & 0.000238 \\
\hline 470.02 & 3.77613404 & 14.8072056 & 6.84639872 & 15.867 & 210.3 & 86.686723 & 71.2 & 71.5 & 78 & 74.8 & 0.000238 \\
\hline 475.02 & 3.7811362 & 14.806456 & 6.84702416 & 15.8685 & 210.325 & 86.786046 & 71.3 & 71.5 & 78.1 & 74.9 & 0.000238 \\
\hline 480.02 & 3.77300769 & 14.8087049 & 6.84639872 & 15.86775 & 210.3 & 86.637061 & 71,5 & 71.5 & 78 & 74.8 & 0.000238 \\
\hline 485.02 & 3.78301201 & 14.8079553 & 6.84890048 & 15.86775 & 210.35 & 86.736384 & 71.4 & 71.5 & 78 & 74.8 & 0.000238 \\
\hline 490.02 & 3.7748835 & 14.8072056 & 6.84827504 & 15.86775 & 210.35 & 86.835708 & 71.4 & 71.5 & 78.1 & 74.8 & 0.000239 \\
\hline 495.02 & 3.77050661 & 14.8072056 & 6.8507768 & 15.86775 & 210.275 & 86.711554 & 71.3 & 71.5 & 78 & 74.8 & 0.000238 \\
\hline 50002 & 3.77675931 & 14.806456 & 6.84702416 & 15.86775 & 210.3 & 86.239768 & 71.3 & 71.5 & 78.1 & 74.9 & 0.000239 \\
\hline 505.02 & 3.77926039 & 14.806456 & 6.8507768 & 15.86775 & 210.35 & 86.214938 & 71.3 & 71.5 & 78 & 74.9 & 0.000238 \\
\hline 510.05 & 3.78238674 & 14.8409537 & 6.84639872 & 15.86775 & 210.3 & 86.214938 & 71.2 & 71.5 & 78 & 74.9 & 0.00024 \\
\hline 515.02 & 3.77238242 & 14.8072056 & 6.84890048 & 15.867 & 210.325 & 86.065953 & 71.2 & 79.5 & 78.1 & 74.8 & 0.00024 \\
\hline 520.02 & 3.77300769 & 14.8079553 & 6.84827504 & 15.87 & 210.275 & 85.792814 & 71.2 & 71.5 & 78 & 74.8 & 0.000238 \\
\hline 525.02 & 3.7748835 & 14.8057064 & 6.84827504 & 15,87 & 210.325 & 85.96663 & 71.2 & 71.5 & 78.1 & 74.9 & 0.000239 \\
\hline 530.02 & 3.77738458 & 14.8094545 & 6.84827504 & 15.87 & 210.325 & 86.438415 & 71.2 & 71.5 & 78 & 74.8 & 0.000238 \\
\hline 535.02 & 3.7811362 & 14.8079553 & 6.84827504 & 15.87075 & 210.3 & 86.140445 & 71.3 & 71.5 & 78 & 74.8 & 0.000239 \\
\hline 540.05 & 3.77800985 & 14.8087049 & 6.84827504 & 15.87075 & 210.325 & 85.99146 & 71.2 & 75.6 & 78 & 74.9 & 0.00024 \\
\hline 545.02 & 3.77300769 & 14.8057064 & 6.85015136 & 15.87 & 210.3 & 86.065953 & 71.1 & 71.5 & 78 & 74.8 & 0.00024 \\
\hline 550.02 & 3.77050661 & 14.8072056 & 6.84890048 & 15.87 & 210.35 & 86.28943 & 71.1 & 71.5 & 78 & 74.8 & 0.000239 \\
\hline 555.03 & 3.77425823 & 14.8087049 & 6.8507768 & 15.87075 & 210.3 & 85.817645 & 71.2 & 71.5 & 78.1 & 74.9 & 0.000239 \\
\hline 560.2 & 3.76425391 & 14.8079553 & 6.8507768 & 15.87075 & 210.35 & 86.140445 & 71.3 & 71.5 & 78 & 74.8 & 0.000239 \\
\hline 565.02 & 3.77675931 & 14.8079553 & 6.8507768 & 15.8715 & 210.325 & 86,140445 & 71.4 & 71.5 & 78.1 & 74.9 & 0.000239 \\
\hline 570.02 & 3.79051525 & 14.8087049 & 6.8507768 & 15.87 & 210.3 & 88.77251 & 71.3 & 71.5 & 78.1 & 74.8 & 0.00024 \\
\hline 575.06 & 3.78676363 & 14.8087049 & 6.85140224 & 15.87075 & 210.35 & 68.722848 & 71.2 & 71.5 & 78 & 74.8 & 0.00024 \\
\hline 580.02 & 3.77738458 & 14.8079553 & 6.85015136 & 15.87 & $2 \div 0.35$ & 88.499371 & 71.1 & 71.5 & 78.1 & 74.9 & 0.00024 \\
\hline 585.02 & 3.77050661 & 14.8057064 & 6.85327856 & 15.87075 & 220.3 & 68.226232 & 71.1 & 71.5 & 78 & 74.9 & 0.000239 \\
\hline 590.05 & 3.77050661 & 14.8072056 & 6.85140224 & 15.87075 & 220.325 & 85.817645 & 71.1 & 71.5 & 78 & 74.9 & 0.000239 \\
\hline 595.02 & 3.7686308 & 14.806456 & 6.85202768 & 15.87075 & 210.325 & 86.28943 & 71.1 & 71.5 & 78 & 74.8 & 0.000239 \\
\hline 600.02 & 3.7748835 & 14.8072056 & 6.8507768 & 15.87375 & 210.325 & 86.314261 & 71 & 74.5 & 78 & 74.8 & 0.00024 \\
\hline 605.02 & 3.77926039 & 14.8072056 & 6.85140224 & 15.873 & 220.35 & 86.562569 & 71.1 & 71.5 & 78.1 & 74.9 & 0.000239 \\
\hline
\end{tabular}




\begin{tabular}{|c|c|c|c|c|c|c|c|c|c|c|c|}
\hline SECOND & MAIN DUCT & MAIN DUCT & GAS INJECT & GAS INJECT & NEWPORT & SMC & AMBIENT & GAS INJECT & MAIN DUC & WEC CELL & WEC CELLL \\
\hline & DIFF (IN H2 & $A B S$ (PS|A) & DIFF (IN H2O) & ABS (PSIA) & $(\mathrm{PPM})$ & (PPM) & TEMP.(F) & TEMP.(F) & TEMP.(F) & TEMP.(F) & \\
\hline 610.02 & 3.79114052 & 14.806456 & 6.85202768 & 15.873 & 211.9 & 86.363922 & 71.1 & 71.5 & 78 & 74.8 & 0.00024 \\
\hline 615.02 & 3,77238242 & 14.8087049 & 6.85265312 & 15.873 & 220.35 & 86.5874 & 71.1 & 71.5 & 77.9 & 74.8 & 0.00024 \\
\hline 620.02 & 3.78488782 & 14.8087049 & 6.85452944 & 15.873 & 220.35 & 86.61223 & 71 & 71.5 & 78 & 74,8 & 0.00024 \\
\hline 625.02 & 3.77175715 & 14.8087049 & 6.85265312 & 15.873 & 220.35 & 85.96663 & 71 & 71.5 & 77.9 & 74.8 & 0.00024 \\
\hline 630.02 & 3.77238242 & 14.8072056 & 6.85515488 & 15.87375 & 220.35 & 85.916968 & 70.9 & 71.5 & 78 & 74.8 & 0.000239 \\
\hline 635.02 & 3.77675931 & 14.8079553 & 6.85515488 & 15.87375 & 220.3 & 86.041122 & 71 & 71.5 & 77.9 & 74.8 & 0.00024 \\
\hline 640.02 & 3.78551309 & 14.8087049 & 6.85452944 & 15.873 & 220.35 & 86.115614 & 70.9 & 71.5 & 78 & 74.8 & 0.000239 \\
\hline 645.02 & 3.77363296 & 14,806456 & 6.85578032 & 15.873 & 220.35 & 86.016291 & 70.9 & 71.5 & 78 & 74.9 & $0.0002 \overline{41}$ \\
\hline 650.04 & 3.79051525 & 14.8102041 & 6.85515488 & 15.87225 & 220.35 & 85.494844 & 71 & 71.5 & 77.9 & 74.8 & 0.00024 \\
\hline 655.02 & 3.77550877 & 14.8094545 & 6.85640576 & 15.87375 & 220.35 & 85.693491 & 71 & 71.5 & 78.1 & 74.9 & 0.000239 \\
\hline 660.02 & 3.77238242 & 14.8094545 & 6.85640576 & 15.873 & 220.35 & 86.041122 & 71 & 71.5 & 78 & 74.9 & 0.000241 \\
\hline 665.05 & 3.77238242 & 14.8102041 & 6.85640576 & 15.873 & 220.35 & 86.041122 & 70.9 & 71.5 & 78 & 74.8 & 0.00024 \\
\hline 670.02 & 3.77926039 & 14.8102041 & 6.85765664 & 15.8745 & 220.35 & 86.165276 & 70.9 & 71.5 & 78 & 74,8 & 0.000241 \\
\hline 675.02 & 3.77926039 & 14.8087049 & 6.85765664 & 15.873 & 220.325 & 86.28943 & 70.9 & 71.5 & 78 & 74.8 & 0.00024 \\
\hline 680.11 & 3.77238242 & 14.8087049 & 6.85890752 & 15.873 & 220.325 & 86.264599 & 71 & 71.5 & 78.1 & 74.9 & 0.000239 \\
\hline 685.02 & 3.76675499 & 14.8057064 & 6.85890752 & 15.873 & 220.35 & 86.339092 & 71 & 71.5 & 78 & 74.8 & 0.000241 \\
\hline 690.02 & 3.77863512 & 14.806456 & 6.85890752 & 15.87375 & 220.35 & 86.363922 & 71 & 71.5 & 78 & 74.9 & 0.00024 \\
\hline 695.03 & 3.76800553 & 14.8087049 & 6.86140928 & 15.873 & 220.35 & 86.264599 & 71 & 71.5 & 78 & 74.8 & 0.00024 \\
\hline 700.02 & 3.77300769 & 14.8094545 & 6.86078384 & 15.876 & 220.325 & 86.438415 & 71 & 71.5 & 78 & 74.8 & 0.000241 \\
\hline 705.02 & 3.78176147 & 14.8094545 & 6.86266016 & 15.87525 & 220.35 & 86.686723 & 71 & 71.5 & 78 & 74.8 & 0.000239 \\
\hline 710.05 & 3.7748835 & 14.8087049 & 6.86266016 & 15.876 & 220.325 & 86.5874 & 71 & 71.5 & 78 & 74.9 & 0.00024 \\
\hline 715.02 & 3.77863512 & 14.8087049 & 6.8632856 & 15.87525 & 220.325 & 86.438415 & 71 & 71.5 & 77.9 & 74.8 & 0.00024 \\
\hline 720.02 & 3.76487918 & 14.8087049 & 6.86516192 & 15.876 & 220.35 & 86.339092 & 71 & 71.5 & 78.1 & 74.9 & 0.000241 \\
\hline 725.02 & 3.7748835 & 14.8087049 & 6.86578736 & 15.87525 & 220.35 & 86.61223 & 71.2 & 71.5 & 78 & 74.8 & 0.000241 \\
\hline 730.02 & 3.7686308 & 14.8087049 & 6.86703824 & 15.87525 & 220.325 & 86.512907 & 71.2 & 71.5 & 78.1 & 74.9 & 0.00024 \\
\hline 735.02 & 3.78926471 & 14.8087049 & 6.86766368 & 15.879 & 220.35 & 86.28943 & 71.1 & 71.5 & 78 & 74.8 & 0.000241 \\
\hline 740.02 & 3.77050661 & 14.8087049 & 6.86766368 & 15.876 & 220.325 & 86.214938 & 71.1 & 71.5 & 78.1 & 74.9 & 0.000241 \\
\hline 745.02 & 3.76487918 & 14.8087049 & 6.86954 & 15.879 & 220.35 & 86.339092 & 71.1 & 71.5 & 78 & 74.8 & 0.00024 \\
\hline 750.02 & 3.77926039 & 14.8094545 & 6.86954 & 15.879 & 220.35 & 86.339092 & 71.1 & 71.5 & 78 & 74.9 & 0.00024 \\
\hline 755.06 & 3.76800553 & 14.8087049 & 6.87079088 & 15.87825 & 220.35 & 86.761215 & 71.1 & 71.5 & 78 & 74.8 & 0.00024 \\
\hline 760.02 & 3.77550877 & 14.8087049 & 6.87329264 & 15.87875 & 220.35 & 86.28943 & 71 & 71.5 & 78 & 74.8 & 0.000241 \\
\hline 765.02 & 3.78426255 & 14.8094545 & 6.87329264 & 15.8805 & 220.35 & 86.463246 & $\overline{71}$ & 71.5 & 78 & 74.9 & 0.000241 \\
\hline 770.22 & 3.7873889 & 14.8102041 & 6.8757944 & 15.8805 & 220.35 & 86.860538 & 71 & 71.5 & 78 & 74.9 & 0.000241 \\
\hline 775.06 & 3.78863944 & 14.806456 & 6.87767072 & 15.8805 & 220.35 & 87.307493 & 70.9 & 71.5 & 78 & 74.9 & 0.00024 \\
\hline 780.02 & 3.78613836 & 14.8094545 & 6.87954704 & 15,8805 & 220.325 & 86.835708 & 70.9 & 71.5 & 78 & 74.8 & 0.000241 \\
\hline 785.02 & 3.77800985 & 14,8087049 & 6.87954704 & 15.88125 & 220.375 & 86.562569 & 70.8 & 71.5 & 78 & 74.9 & 0.00024 \\
\hline 790.02 & 3.78176147 & 14.8094545 & 6.8820488 & 15.8805 & 220.35 & 86.463246 & 70.8 & 71.5 & 78 & 74.8 & 0.000242 \\
\hline 795.02 & 3.77738458 & 14.8094545 & 6.88392512 & 15.8835 & 220.35 & 86.835708 & 70.8 & 71.5 & 78 & 74.9 & 0.00024 \\
\hline 800.02 & 3.77926039 & 14.8094545 & 6.88455056 & 15.8835 & 220.35 & 86.935031 & 70.8 & 71.5 & 78 & 74.8 & 0.000242 \\
\hline 805.02 & 3.77863512 & 14.8087049 & 6.88642688 & 15.8835 & 230.35 & 87.059185 & 70.8 & 71.5 & 78.1 & 74,8 & 0.000241 \\
\hline 810.02 & 3.77675931 & 14.8094545 & 6.88767776 & 15.88275 & 220.35 & 89.641588 & 70.8 & 71.5 & 78 & 74.8 & 0.000242 \\
\hline 815.02 & 3.77926039 & 14.8109537 & 6.89205584 & 15.88575 & 230.375 & 86.512907 & 70.8 & 71.6 & 78.1 & 74.9 & 0.000242 \\
\hline
\end{tabular}




\begin{tabular}{|c|c|c|c|c|c|c|c|c|c|c|c|}
\hline SECOND & \begin{tabular}{|l|} 
MAIN DUCT \\
DIFF (IN H2
\end{tabular} & $\frac{\text { MAIN DUCT }}{\text { ABS (PSIA) }}$ & $\begin{array}{l}\text { GAS INJECT } \\
\text { DIFF (IN H2O) }\end{array}$ & \begin{tabular}{|l|} 
GAS INJECT \\
ABS (PSIA)
\end{tabular} & \begin{tabular}{|l} 
NEWPORT \\
(PPM)
\end{tabular} & $\frac{\text { SMC }}{\text { (PPM) }}$ & \begin{tabular}{|l|} 
AMBIENT \\
TEMP.(F)
\end{tabular} & \begin{tabular}{|l|} 
GAS INJECT \\
TEMP.(F)
\end{tabular} & \begin{tabular}{|l|} 
MAIN DUC \\
TEMP.(F)
\end{tabular} & $\begin{array}{l}\text { WEC CELL } \\
\text { TEMP.(F) }\end{array}$ & $\begin{array}{l}\text { WEC CELL } \\
\text { (VOLTS) }\end{array}$ \\
\hline & & & & & & & & & & & \\
\hline 820.02 & 3.79239106 & 14.8094545 & 6.8914304 & 15.88575 & 230.35 & 86.637061 & 70.8 & 71.5 & 78 & 74.9 & 0.00024 \\
\hline 825.02 & 3.78551309 & 14.8094545 & 6.89643392 & 15.88875 & 230.35 & 86.885369 & 70.8 & 71.5 & 78.1 & 74.9 & 0.000241 \\
\hline 830.02 & 3.79176579 & 14.8102041 & 6.89893568 & 15.888 & 230.4 & 86.388753 & 70.8 & 71.5 & 78 & 74.8 & 0.000242 \\
\hline 835.03 & 3.80864808 & 14.8087049 & 6.90331376 & 15.888 & 230.3 & 88.300725 & 70.8 & 71.5 & 78.1 & 74.8 & 0.00024 \\
\hline 840.02 & 3.80302065 & 14.8102041 & 6.90268832 & 15.88875 & 230.375 & 86.264599 & 70.7 & 71.5 & 78 & 74.9 & 0.000241 \\
\hline 845.02 & 3.79239106 & 14.8094545 & 6.9070664 & 15.89025 & 230.35 & 86.239768 & 70.7 & 71.5 & 78 & 74.8 & 0.000241 \\
\hline 850.03 & 3.77926039 & 14,8094545 & 6.90894272 & 15.891 & 230.325 & 86.314261 & 70.7 & 71.5 & 78.1 & 74.9 & 0.000242 \\
\hline 855.02 & 3.79676795 & 14.8087049 & 6.91144448 & 15.89325 & 230.4 & 86.810877 & 70.7 & 71.5 & 78 & 74.8 & 0.000241 \\
\hline 860.02 & 3.78926471 & 14.8109537 & 6.916448 & 15.89325 & 230.35 & 86.959862 & 70.6 & 71.5 & 78 & 74.8 & 0.000241 \\
\hline 865.02 & 3.78551309 & 14.8094545 & 6.916448 & 15.89475 & 230.375 & 86.562569 & 70.6 & 71.5 & 78.1 & 74.8 & 0.000241 \\
\hline 870.02 & 3.80364592 & 14.8094545 & 6.92145152 & 15.8956 & 230.3 & 86.935031 & 70.7 & 71.5 & 78 & 74.8 & 0.000241 \\
\hline 875.02 & 3.79489214 & 14.8087049 & 6.92207696 & 15.8955 & 230.35 & 86.661892 & 70.6 & 71.5 & 78 & 74.8 & 0.000241 \\
\hline 880.04 & 3.79176579 & 14.8094545 & 6.9258296 & 15.89475 & 230.4 & 86.711554 & 70.6 & 71.5 & 78 & 74.8 & 0.000242 \\
\hline 885.02 & 3.79239106 & 14.8087049 & 6.92833136 & 15.8985 & 230.325 & 86.512907 & 70.6 & 71.5 & 78 & 74.8 & 0,000242 \\
\hline 890.02 & 3.80302065 & 14.8087049 & 6.932084 & 15.90075 & 230.375 & 86.537738 & 70.5 & 71.5 & 78 & 74.9 & 0.000242 \\
\hline 895.02 & 3.77926039 & 14.8094545 & 6.9352112 & 15.90375 & 230.325 & 87.456478 & 70.5 & 71.5 & 77.9 & 74.8 & 0.000242 \\
\hline 900.02 & 3.78613836 & 14.8094545 & 6.9383384 & 15.9045 & 230.35 & 87.804109 & 70.4 & 71.5 & 77.8 & 74.9 & 0.000242 \\
\hline 905.02 & 3.78176147 & 14.8094545 & 6.9445928 & 15.9045 & 230.35 & 87.903432 & 70.3 & 71.5 & 77.8 & 74.8 & 0.000241 \\
\hline 910.07 & 3.7748835 & 14.8087049 & 6.9445928 & 15.903 & 230.35 & 87.754447 & 70.3 & 71.5 & 77.7 & 74.9 & 0.000241 \\
\hline 915.02 & 3.78801417 & 14.8102041 & 6.9508472 & 15.906 & 230.4 & 87.704786 & 70.2 & 71.5 & 77.7 & 74.9 & 0.000242 \\
\hline
\end{tabular}




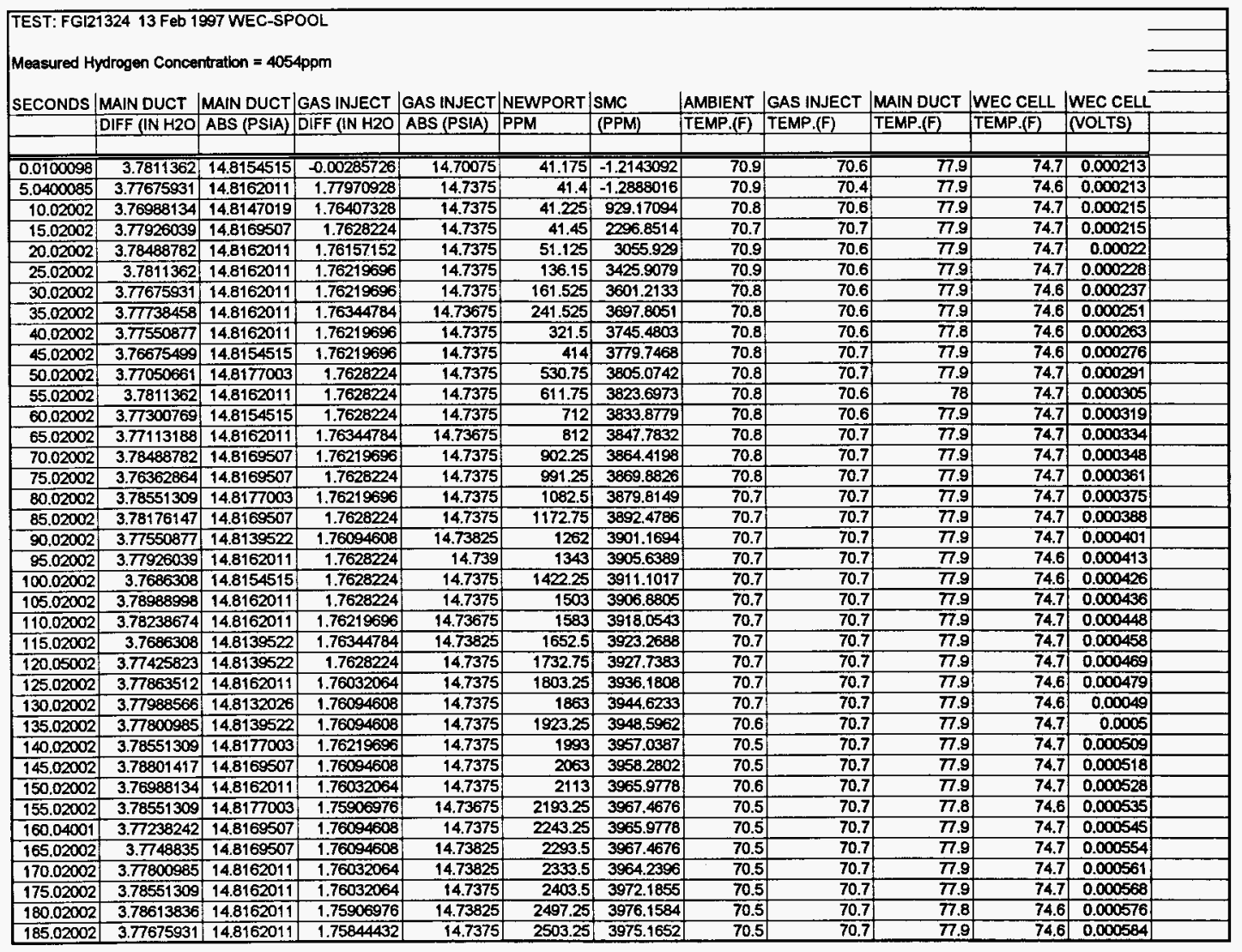




\begin{tabular}{|c|c|c|c|c|c|c|c|c|c|c|c|}
\hline SECONDS & $\begin{array}{l}\text { MAIN DUCT } \\
\text { DIFF (IN H2O }\end{array}$ & \begin{tabular}{|l|} 
MAIN DUCT \\
ABS (PSIA) \\
\end{tabular} & $\begin{array}{l}\text { GAS INJECT } \\
\text { OIFF (IN H2O }\end{array}$ & \begin{tabular}{|l|} 
GAS INJECT \\
ABS (PSIA) \\
\end{tabular} & $\begin{array}{l}\text { NEWPORT } \\
\text { PPM }\end{array}$ & \begin{tabular}{|l|} 
SMC \\
(PPM) \\
\end{tabular} & \begin{tabular}{|l|} 
AMBIENT \\
TEMP.(F) \\
\end{tabular} & \begin{tabular}{|l|} 
GAS INJECT \\
TEMP.(F) \\
\end{tabular} & \begin{tabular}{|l|} 
MAIN DUCT \\
TEMP.(F) \\
\end{tabular} & $\begin{array}{l}\text { WEC CELL } \\
\text { TEMP.(F) }\end{array}$ & $\begin{array}{l}\text { WEC CELL } \\
\text { (VOLTS) }\end{array}$ \\
\hline 190.02002 & 3.78551309 & 14.8177003 & 1.75844432 & 14.739 & 2544 & 3970.6956 & 70.5 & 70.7 & 779 & 746 & \\
\hline 195.02002 & 3.7811362 & 14.8162011 & 1.75844432 & 14.7375 & 2583 & 3970.9439 & 70.5 & 70.7 & 77.8 & $\begin{array}{l}74.6 \\
74.7\end{array}$ & $\begin{array}{r}0.00059 \\
0.000598\end{array}$ \\
\hline 200.02002 & 3.79176579 & 14.8169507 & 1.76032064 & 14.7375 & 2633.5 & 3973.6753 & 70.5 & 70.7 & 77.8 & 74.7 & 0.000604 \\
\hline 205,02002 & 3.77363296 & 14.8162011 & 1.75906976 & 14.7375 & 2673.75 & 3975.9101 & 70.5 & 70.7 & 77.8 & 74.7 & 0.000611 \\
\hline 210.02002 & 3.77738458 & 14.8154515 & 1.75781888 & 14.73825 & 2743.75 & 3976.9033 & 70.5 & 70.7 & 77.8 & 74.7 & 0.000617 \\
\hline 215.02002 & 3.77988566 & 14.8162011 & 1.75844432 & 14.73825 & 2783.75 & 3982.6144 & 70.5 & 70.7 & 77.9 & 74.6 & 0.000623 \\
\hline 220.02002 & 3.77238242 & 14.8147019 & 1.75781888 & 14.73825 & 2814 & 3974.9169 & 70.5 & 70.7 & 77.9 & 74.6 & 0.000629 \\
\hline 225.02002 & 3.77926039 & 14.8162011 & 1.75844432 & 14.73825 & 2843.75 & 3977.6483 & 70.6 & 70.7 & 77.9 & 74.7 & 0.000635 \\
\hline 230.02002 & 3.78488782 & 14.8154515 & 1.75844432 & 14.73825 & 2894.25 & 3980.3796 & 70.5 & 70.7 & 77.9 & 74.8 & 0.000641 \\
\hline 235.02002 & 3.78801417 & 14.8169507 & 1.75906976 & 14.73825 & 2924 & 3969.9507 & 70.5 & 70.7 & 77.9 & 74.7 & 0.000646 \\
\hline 240.02002 & 3.7748835 & 14.8169507 & 1.75594256 & 14.73825 & 2953.5 & 3976.4067 & 70.5 & 70.7 & 77.9 & 74.6 & 0.00065 \\
\hline 245.02002 & 3.77425823 & 14.8169507 & 1.75906976 & 14.7375 & 2993.75 & 3972.1855 & 70.5 & 70.7 & 77.8 & 74.7 & 0.000655 \\
\hline 250.02002 & 3.77800985 & 14.8139522 & 1.75594256 & 14.73825 & 3024.25 & 3982.1178 & 70,4 & 70.7 & $\pi 7.8$ & 74.7 & 0.000661 \\
\hline 255.02002 & 3.77300769 & 14.8162011 & 1.756568 & 14.7375 & 3063.75 & 3981.1246 & 70.4 & 70.7 & 77.9 & 74.7 & 0.000666 \\
\hline 260.02002 & 3.78551309 & 14.8169507 & 1.75719344 & 14.73825 & 3094.25 & 3981.3729 & 70.4 & 70.7 & 77.9 & 74.7 & 0.00067 \\
\hline 265.02002 & 3.77238242 & 14.8177003 & 1.75594256 & 14.73825 & 3124.25 & 3983.3593 & 70.4 & 70.7 & 77.9 & 74.7 & 0.000675 \\
\hline 270.02002 & 3.78551300 & 14.8169507 & 1.756568 & 14.7375 & 3153.75 & 3981.8695 & 70.4 & 70.7 & 77.9 & 74.7 & 0.00058 \\
\hline 275.02002 & 3.77550877 & 14.8169507 & 1.756568 & 14.7375 & 3184.5 & 3976.9033 & 70.5 & 70.8 & 77.9 & 74.7 & 0.000682 \\
\hline 280.02002 & 3.77863512 & 14.81770003 & 1.756568 & 14.73825 & 3214.25 & 3980.6279 & 70.5 & 70.7 & 77.9 & 74.7 & 0.000688 \\
\hline 285.02002 & 3.77238242 & 14.8177003 & 1.75594256 & 14.7375 & 3234.75 & 3988.5738 & 70.6 & 70.7 & 77.9 & 74.7 & 0.000692 \\
\hline 290.02002 & 3.77175715 & 14.8169507 & 1.756568 & 14.7375 & 3264.5 & 3992.5467 & 70.7 & 70.7 & 77.9 & 74.7 & 0.000695 \\
\hline 295.02002 & 3.77113188 & 14.81845 & 1.756568 & 14.7375 & 3293.75 & 3983.6076 & 70.6 & 70.8 & 77.9 & 74.7 & 0.0007 \\
\hline 300.02002 & 3.76925607 & 14.8162011 & 1.75719344 & 14.7375 & 3314.5 & 3989.8153 & 70.6 & 70.7 & 77.9 & 74.7 & 0.000702 \\
\hline 305.02002 & 3.7748835 & 14.8177003 & 1.75594256 & 14.736 & 3344.5 & 3994.5332 & 70.6 & 70.8 & 77.9 & 74.7 & 0.000706 \\
\hline 310.02002 & 3.7686308 & 14.8147019 & 1.75781888 & 14.7375 & 3364.25 & 3999.996 & 70.6 & 70.8 & 77.9 & 74.7 & 0.00071 \\
\hline 315.02002 & 3.77425823 & 14.8169507 & 1.756568 & 14.73675 & 3384.5 & 3990.5603 & 70.7 & 70.8 & 77.9 & 74.7 & 0.000713 \\
\hline 320.02002 & 3.77675931 & 14.8162011 & 1.75594256 & 14.73675 & 3416.75 & 3984.6009 & 70.7 & 70.7 & 77.9 & 74.7 & 0.000717 \\
\hline 325.02002 & 3.77050661 & 14.8147019 & 1.756568 & 14.7345 & 3434.75 & 3978.8898 & 70.7 & 70.8 & 77.9 & 74.7 & 0.00072 \\
\hline 330.02002 & 3.79239106 & 14.8139522 & 1.75531712 & 14.73375 & 3454.5 & 3981.6212 & 70.7 & 70.7 & 77.9 & 74.7 & 0.000723 \\
\hline 335.02002 & 3.77613404 & 14.8147019 & 1.75469168 & 14.73675 & $\mathbf{3 4 7 4 . 7 5}$ & 3979.1381 & 70.6 & 70.8 & 77.9 & 74.7 & 0.000726 \\
\hline 340,02002 & 3.77113188 & 14.8132026 & 1.75594256 & 14.73675 & 3494.75 & 3977.6483 & 70.7 & 70.8 & 77.9 & 74.7 & 0.000729 \\
\hline 345.02002 & 3.78176147 & 14.8169507 & 1.756568 & 14.73825 & 3514.75 & 3981.6212 & 70.6 & 70.8 & 77.9 & 74.7 & 0.000733 \\
\hline 350.02002 & 3.77238242 & 14.8154515 & 1.756568 & 14.7375 & 3535 & 3976.1584 & 70.6 & 70.8 & 77.9 & 74.7 & 0.000736 \\
\hline 355.02002 & 3.77238242 & 14.8139522 & 1.75594256 & 14.73825 & 3553.25 & 3980.1313 & 70.6 & 70.8 & 77.9 & 74.7 & 0.000738 \\
\hline 360,02002 & 3.77550877 & 14.8169507 & 1.75594256 & 1473675 & 3574.75 & 3981.3729 & 70.6 & 70.8 & 77.9 & 74.7 & 0.00074 \\
\hline 365.05002 & 3.78551309 & 14.8154515 & 1.75594256 & 14.73675 & 3584.75 & 3988.5738 & 70.5 & 70.8 & 77.9 & 74.7 & 0.000743 \\
\hline 370.02002 & 3.77675931 & 14.8132026 & 1.756568 & 14.73375 & 3605 & 3986.5873 & 70.5 & 70,8 & 77.9 & 74.7 & 0.000746 \\
\hline 375.04001 & 3.77863512 & 14.8147019 & 1.75594256 & 14.736 & 3614.75 & 3986.339 & 70.6 & 70.8 & 77.9 & 74.7 & 0.000747 \\
\hline 380.02002 & 3.78551309 & 14.8154515 & 1.75531712 & 14.736 & 3535 & 3988.5738 & 70.6 & 70.8 & 77.9 & 74.7 & 0.000751 \\
\hline 385.06 & 3.77926039 & 14.8169507 & 1.75531712 & 14.7375 & 3655 & 3990.8086 & 70.6 & 70.8 & $\pi .9$ & 74.7 & 0.000753 \\
\hline 390.02002 & 3.77863512 & 14.8177003 & 1.756568 & 14.7375 & 3665 & 3983.3593 & 70.5 & 70.8 & 77.9 & 74.7 & 0.000755 \\
\hline 395.05002 & 3.77550877 & 14.8169507 & 1.756568 & 14.7375 & 3685 & 3981.1246 & 70.5 & 70.8 & 77.9 & 74.7 & 0.000757 \\
\hline
\end{tabular}




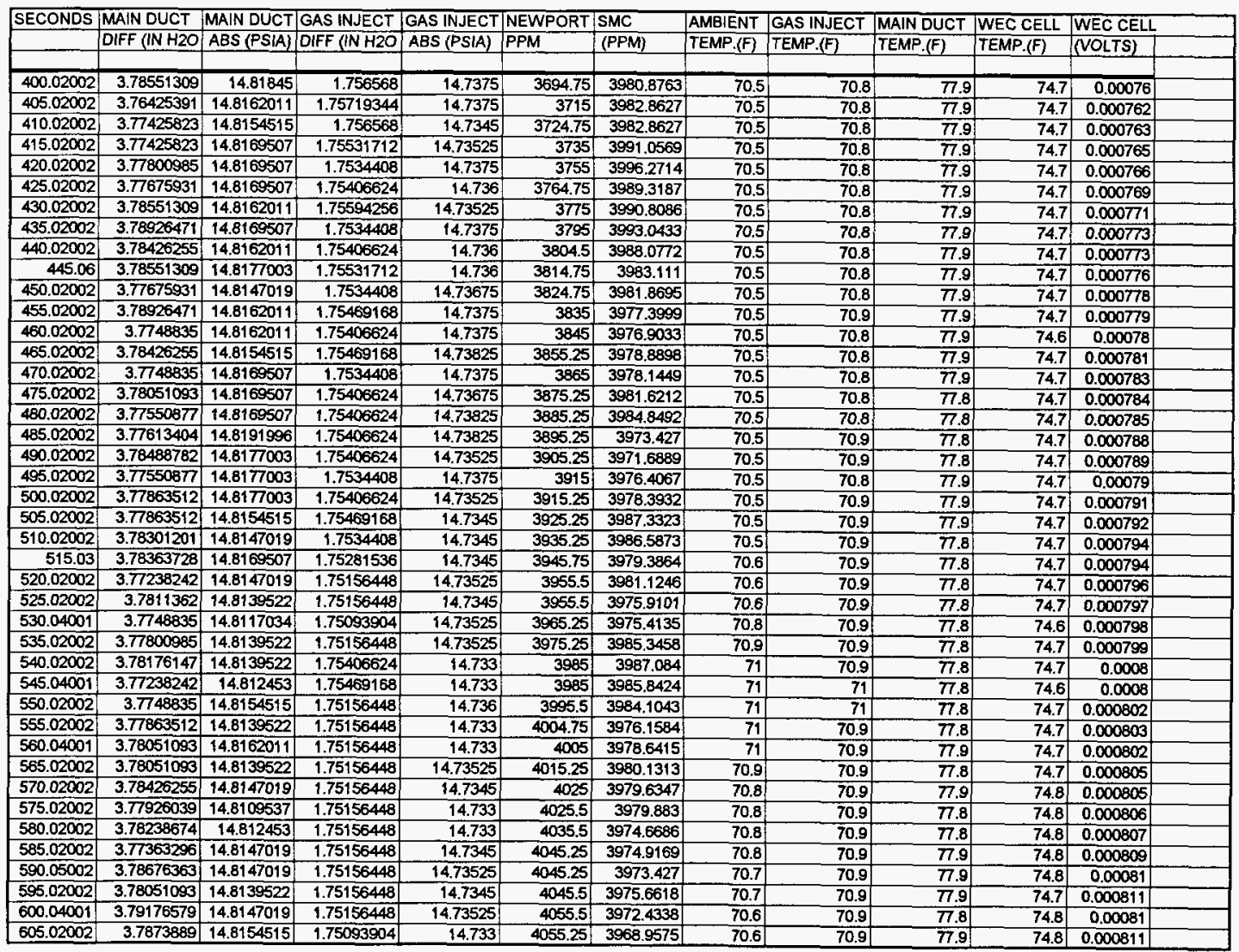




\begin{tabular}{|c|c|c|c|c|c|c|c|c|c|c|c|c|}
\hline SECONDS & MAIN DUCT & MAIN DUCT & GAS INJECT & GAS INJECT & NEWPORT & SMC & AMBIENT & GAS WNECT & MAIN DUCT & WEC CELL & WEC CELL & \\
\hline & DIFF (IN H2O & ABS (PSIA) & DIFF (IN H2O & ABS (PSIA) & PPM & (PPM) & TEMP.(F) & TEMP.(F) & TEMP.(F) & TEMP.(F) & (VOLTS) & \\
\hline 610.05002 & 3.78488782 & 14.8154515 & 1.75218992 & 14.733 & 4065.75 & 3969.7024 & 70.6 & 71 & 77.8 & 74.8 & 0.000811 & \\
\hline 615.02002 & 3.76800553 & 14.8109537 & 1.75156448 & 14.73225 & 4075.25 & 3973.1787 & 70.6 & 71 & 77.9 & 74.8 & 0.000812 & \\
\hline 620.02002 & 3.77863512 & 14.8154515 & 1.749688816 & 14.73525 & 4075.25 & 3973.1787 & 70.6 & 70.9 & 77.9 & 74.8 & 0.000813 & \\
\hline 625.05002 & 3.77363296 & 14.8139522 & 1.75156448 & 14.736 & 4085.75 & 3968.2125 & 70.7 & 70.9 & $\pi 7.8$ & 74.7 & 0.000814 & \\
\hline 630.06 & 3.7811362 & 14.8139522 & 1.7503136 & 14.73525 & 4085.5 & 3970.6956 & 70.7 & 71 & 77.9 & 74.7 & 0.000814 & \\
\hline 635.02002 & 3.77738458 & 14.8139522 & 1.74968816 & 14.7345 & 4095.75 & 3969.2058 & 70.7 & 70.9 & 77.8 & 74.8 & 0.000816 & \\
\hline 640.05002 & 3.78301201 & 14.8154515 & 1.7503136 & 14.736 & 4095.5 & 3969.4541 & 70.6 & 71 & 77.9 & 74.8 & 0.000817 & \\
\hline 645.02002 & 3.77736458 & 14.8147019 & 1.7503136 & 14.7345 & 4105.75 & 3970.4473 & 70.6 & 71 & 77.8 & 74.7 & 0.000817 & \\
\hline 650.02002 & 3.77800985 & 14.8139522 & 1.7503136 & 14.73525 & 4105.5 & 3972.6821 & 70.6 & 71 & 77.8 & 74.8 & 0.000817 & \\
\hline 655.02002 & 3.77050661 & 14.8139522 & 1.74968816 & 14.733 & 4105.5 & 3974.4202 & 70.5 & 71 & 77.9 & 74.8 & 0.000819 & \\
\hline 660.02002 & 3.78488782 & 14.8139522 & 1.74968816 & 14.733 & 4115.5 & 3969.9507 & 70.5 & 71 & 77.9 & 74.8 & 0.000819 & \\
\hline 665.02002 & 3.76988134 & 14.8147019 & 1.75093904 & 14.73525 & 4115.75 & 3973.1787 & 70.5 & 71 & 77.9 & 74.8 & 0.000821 & \\
\hline 670.02002 & 3.77675931 & 14.8139520 & 1.7503136 & 14.733 & 4125.5 & 3972.9304 & 70.5 & 71 & 77.9 & 74.7 & 0.000821 & \\
\hline 675.04001 & 3.77363296 & 14.8117034 & 1.7503136 & 14.733 & 4125.25 & 3972.43338 & 70.5 & 71 & 77.9 & 74.8 & 0.000821 & \\
\hline 680.02002 & 3.7686308 & 14.812453 & 1.7503136 & 14.73225 & 4125.75 & 3971.6889 & 70.5 & 71 & 77.9 & 74.8 & 0.000821 & \\
\hline 685.02002 & 3.7748835 & 14.8139522 & 1.7503136 & 14.733 & 4135.25 & 3971.1922 & 70.6 & 70.9 & 77.9 & 74.8 & 0.000822 & \\
\hline 700.03 & 3.77238242 & 14.812453 & 1.74906272 & 14.733 & 4144.75 & 3982.6144 & 70.6 & 71 & 77.9 & 74.8 & 0.000823 & \\
\hline 705.02002 & 3.7811362 & 14.8117034 & 1.74968816 & 14.73225 & 4145.5 & 3976.655 & 70.6 & 71 & 77.9 & 74.8 & 0.000823 & \\
\hline 710.02002 & 3.77613404 & 14.8147019 & 1.74968816 & 14.7315 & 4145 & 3972,4338 & 70.6 & 71 & 77.9 & 74.8 & 0.000825 & \\
\hline 715.04001 & 3.77926039 & 14.8102041 & 1.7503136 & 14.73225 & 4155.5 & 3970.4473 & 70.6 & 71 & 77.9 & 74.8 & 0.000825 & \\
\hline 720.02002 & 3.77050661 & 14.8117034 & 1.7503136 & 14.733 & 4155.5 & 3972.4338 & 70.5 & 71 & 77.9 & 74.8 & 0.000826 & \\
\hline 725.03 & 3.7748835 & 14,812453 & 1.74781184 & 14.73225 & 4155.75 & 3974.1719 & 70.5 & 71 & 77.9 & 74.8 & 0.000825 & \\
\hline 730.05002 & 3.77675931 & 14.8117034 & 1.74843728 & 14.733 & 4165.75 & 3967.2193 & 70.6 & 71 & 77.9 & 74.7 & 0.000825 & \\
\hline 735.02002 & 3.76800553 & 14.812453 & 1.74781184 & 14.73225 & 4165.5 & 3975,4135 & 70.6 & 71 & 77.9 & 74.8 & 0.000826 & \\
\hline 740.06 & 3.78426255 & 14.8117034 & 1.7503136 & 14.733 & 4165.25 & 3976.655 & 70.6 & 71 & 77.9 & 74.8 & 0.000826 & \\
\hline 745.02002 & 3.77050661 & 14.8139522 & 1.74843728 & 14.733 & 4165 & 3978.8898 & 70.6 & 71 & 77.9 & 74.8 & 0.000826 & \\
\hline 750.02002 & 3.77863512 & 14.812453 & 1.74781184 & 14.73225 & 4175.75 & 3979.883 & 70.5 & 71 & 77.9 & 74.8 & 0.000828 & \\
\hline 755.02002 & 3.77988566 & 14.8139522 & 1.74843728 & 14.73225 & 4175.75 & 3979.6347 & 70.5 & 71 & 77.9 & $74 . \overline{8}$ & 0.000827 & \\
\hline 760.04001 & 3.77613404 & 14.8147019 & 1.74781184 & 14.73225 & 4175.75 & 3970.199 & 70.5 & 71 & 77.9 & 74.8 & 0.000827 & \\
\hline 765.02002 & 3.78551309 & 14.8139522 & 1.74781184 & 14.733 & 4175.5 & 3972.4338 & 70.5 & 71 & 77.9 & 74.8 & 0.000828 & \\
\hline 770.04001 & 3.77863512 & 14.8117034 & 1.74781184 & 14.733 & 4185.5 & 3968.9575 & 70.5 & 71 & 77.8 & 74.8 & 0.000829 & \\
\hline 775.02002 & 3.78488782 & 14.8139522 & 1.74781184 & 14.73225 & 4185.25 & 3969.4541 & 70.5 & 71 & 77.9 & 74.8 & 0.000828 & \\
\hline 780.02002 & 3.77238242 & 14.8132026 & 1.74843728 & 14.73225 & 4185.5 & 3962.9981 & 70.5 & 71.1 & 77.8 & 74.7 & 0.000829 & \\
\hline 785.08002 & 3.78551309 & 14.812453 & 1.7503136 & 14.733 & 4185.25 & 3965.9778 & 70.5 & 71 & 77.8 & 74.7 & 0.000831 & \\
\hline 800.02002 & 3.78551309 & 14.8154515 & 1.7471864 & 14.733 & 4195.75 & 3962.5015 & 70.5 & 71 & 77.9 & 74.8 & 0.00083 & \\
\hline 805.02002 & 3.78301201 & 14.8139522 & 1.7471864 & 14.73225 & 4195.5 & 3963.743 & 70.5 & 71 & 77.9 & 74.8 & 0.000832 & \\
\hline 810.02002 & 3.78051093 & 14.812453 & 1.7471864 & 14.733 & 4195.5 & 3964.9845 & 70.4 & 71 & 77.9 & 74.8 & 0.000831 & \\
\hline 815.02002 & 3.79551741 & 14.8147019 & 1.74781184 & 14.73225 & 4205.25 & 3973.427 & 70.4 & 71 & 77.9 & 74.8 & 0.000831 & \\
\hline
\end{tabular}




\begin{tabular}{|c|c|c|c|c|c|c|c|c|c|c|c|c|}
\hline SECONDS & MAIN DUCT & MAIN DUCT & GAS INJECT & GAS INJECT & NEWPORT & SMC & AMBIENT & GAS INJECT & MAIN DUCT & WEC CELL & WEC CELL & \\
\hline & DIFF (IN H2O & ABS $(P S \mid A)$ & DIFF (IN H2O & ABS (PS|A) & PPM & (PPM) & TEMP.(F) & TEMP.(F) & TEMP.(F) & TEMP.(F) & (VOLTS) & \\
\hline 820.02002 & 3.78551309 & 14.812453 & 1.7471864 & 14.733 & 4205.5 & 3973.6753 & 70.4 & 79 & 77.9 & 74.7 & 0,000833 & \\
\hline 825.02002 & 3.78051093 & 14.8102041 & 1.7471864 & 14,73225 & 4205.5 & 3964.2396 & 70.5 & 71 & 77.9 & 74.7 & 0.000832 & \\
\hline 830.02002 & 3.77800985 & 14.8117034 & 1.74656096 & 14,73225 & 4205 & 3959.0252 & 70.5 & 71 & 77.8 & 74.8 & 0.000833 & \\
\hline 835.04001 & 3.78301201 & 14.8117034 & 1.7471864 & 14.733 & 4205.5 & 3957.7836 & 70.5 & 71 & 77.8 & 74.8 & 0.000833 & \\
\hline 840.02002 & 3.7811362 & 14.8117034 & 1.7471864 & 14.7315 & 4215.75 & 3960.2667 & 70.5 & 71.1 & 77.9 & 74.8 & 0.000834 & \\
\hline 845.03 & 3.77675931 & 14,812453 & 1.74781184 & 14,73225 & 4215.25 & 3955.7971 & 70.5 & 71 & 77.8 & 74.7 & 0.000834 & \\
\hline 850.02002 & 3.77675931 & 14.8109537 & 1.7471864 & 14.73225 & 4215.5 & 3955.0522 & 70.5 & 71 & 77.8 & 74.7 & 0.000834 & \\
\hline 855.02002 & 3.77863512 & 14.8117034 & 1.7471864 & 14.733 & 4215.5 & 3957.287 & 70.5 & 71 & 77.8 & 74.7 & 0.000834 & \\
\hline 860.04001 & 3.77926039 & 14.8139522 & 1.74656096 & 14.73225 & 4215.5 & 3960.7633 & 70.5 & 71.1 & 77.9 & 74.7 & 0.000834 & \\
\hline 865.02002 & 3.78488782 & 14.8117034 & 1.7471864 & 14.7315 & 4215.5 & 3960.515 & 70.5 & 71.1 & 77.8 & 74.7 & 0.000834 & \\
\hline 870.02002 & 3.79114052 & 14.8132026 & 1.74781184 & 14.73225 & 4226 & 3957.7836 & 70.6 & 71 & 77.8 & 74.7 & 0.000835 & \\
\hline 875.03 & 3.76800553 & 14.8117034 & 1.74781184 & 14.73225 & 4225 & 3955.5488 & 70.6 & 71 & 77.8 & 74.8 & 0.000834 & \\
\hline 880.02002 & 3.78488782 & 14.812453 & 1.74656096 & 14.7315 & 4225.75 & 3956.5421 & 70.8 & 71.1 & 77.9 & 74.7 & 0.000835 & \\
\hline 885.02002 & 3.77675931 & 14.8117034 & 1.74468464 & 14,73225 & 4225.5 & 3955.5488 & 70.7 & 74.1 & 77.8 & 74.7 & 0,000835 & \\
\hline 890.04001 & 3.7811362 & 14.812453 & 1.74656096 & 14.7315 & 4225.5 & 3963.9913 & 70.8 & 71.1 & 77.9 & 74.7 & 0.000836 & \\
\hline 895.02002 & 3.77863512 & 14.8147019 & 1.7471864 & 14.73225 & 4226.25 & 3966.4744 & 70.7 & 71.1 & 77.9 & 74.7 & 0.000836 & \\
\hline 900.02002 & 3.7811362 & 14.812453 & 1.74531008 & 14.73225 & 4225.75 & 3962.7498 & 70.7 & 71.1 & 77.9 & 74.8 & 0.000835 & \\
\hline 905.04001 & $\mathbf{3 . 7 8 7 3 8 8 9}$ & 14.8117034 & 1.7471864 & 14.7315 & 4235.25 & 3959.0252 & 70.8 & 71.1 & 77.9 & 74.7 & 0.000835 & \\
\hline 910.02002 & 3.78426255 & 14.8117034 & 1.74468464 & 14.7315 & 4235.25 & 3947.603 & 70.7 & 71.1 & 77.9 & 74.7 & 0.000836 & \\
\hline 915.02002 & 3.78051093 & 14.8109537 & 1.74468464 & 14.733 & 4235.75 & 3950.3344 & 70.7 & 71.1 & 77.9 & 74.8 & 0.000836 & \\
\hline 920.02002 & 3.77613404 & 14.8109537 & 1.74468464 & 14.73225 & 4235.5 & 3951.8242 & 70.6 & 71 & 77.9 & 74.7 & 0.000836 & \\
\hline 925.02002 & 3.77238242 & 14.8102041 & 1.74531008 & 14.73225 & 4235.5 & 3960.515 & 70.6 & 71 & 77.9 & 74.7 & 0,000837 & \\
\hline 930.02002 & 3.77300769 & 14.8117034 & 1.74531008 & 14.73225 & 4236 & 3965.7295 & 70.6 & 71.1 & 77.9 & 74.6 & 0.000838 & \\
\hline 935.04001 & 3.76925607 & 14.8109537 & 1.74531008 & 14.73225 & 4235.25 & 3966.4744 & 70.7 & 71.1 & 77.8 & 74.7 & 0.000837 & \\
\hline 940.02002 & 3.7748835 & 14.812453 & 1.74531008 & 14.733 & 4236 & 3966.4744 & 70.7 & 71.1 & 77.9 & 74.7 & 0.000837 & \\
\hline 945.02002 & 3.7873889 & 14.8117034 & 1.74593552 & 14.73225 & 4245.75 & 3967.2193 & 70.8 & 71.1 & 77.9 & 74.7 & 0.000838 & \\
\hline 950.06 & 3.77675931 & 14.8402041 & 6.5630744 & 15.993 & 4245.5 & 3965.2329 & 70.9 & 71.2 & 77.9 & 74.8 & 0.000838 & \\
\hline 955.02002 & 3.76988134 & 14.8117034 & 8.37184688 & 16.43175 & 4245.75 & 3772.5458 & 70.8 & 71.3 & 77.9 & 74.7 & 0.000838 & \\
\hline 960.02002 & 3.78238674 & 14.8109537 & 8.35621088 & 16.42725 & 4245.75 & 2342.5401 & 70.8 & 71.4 & 77.8 & 74.8 & 0.000838 & \\
\hline 965.07001 & 3.77926039 & 14.8139522 & 8.3737232 & 16.4325 & 4245.5 & 1218.9464 & 70.8 & 71.5 & 77.9 & 74.8 & 0.000836 & \\
\hline 970.02002 & 3.7873889 & 14.8132026 & 8.36934512 & 16.4325 & 4205.75 & 652.55582 & 70.8 & 71.5 & 77.9 & 74.7 & 0.00082 & \\
\hline 975.02002 & 3.77863512 & 14.8132026 & 8.38185392 & 16.43475 & 3845.25 & 380.16195 & 70.9 & 71.6 & 77.9 & 74.8 & 0.000752 & \\
\hline
\end{tabular}




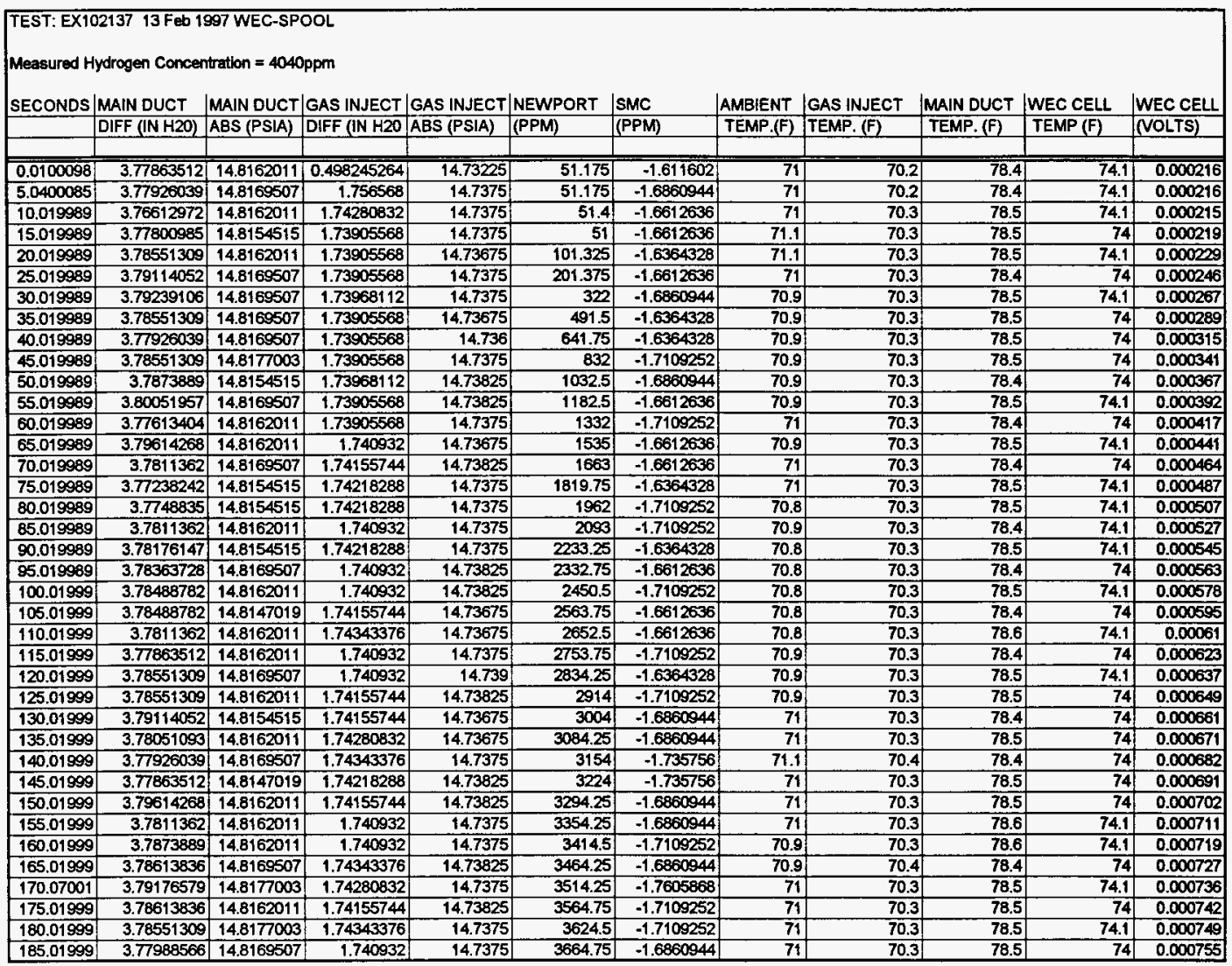

HNF-SD-WM-TRP-275 Rev. 0

APPENDIX C, Page

Engineering Testing Laboratory 3/3/9/ 


\begin{tabular}{|c|c|c|c|c|c|c|c|c|c|c|c|}
\hline SECONDS & MAIN DUCT & MAIN DUCT & GAS INJECT & GASINJECT & NEWPORT & SMC & AMBIENT & GAS INJECT & MAIN DUCT & WEC CELL & WEC CELL \\
\hline & DIFF (IN H2O) & ABS (PSIA) & DIFF (IN H2O & $\mathrm{ABS}$ (PS|A) & (PPM) & (PPM) & TEMP.(F) & TEMP. (F) & TEMP. (F) & TEMP $(F)$ & (VOLTS) \\
\hline 190.04001 & 3.78551309 & 14.8162011 & 1.74280832 & 14.73675 & 3694.75 & -1.6860944 & 71 & 70.3 & 78.6 & 74 & 0.000761 \\
\hline 195.01999 & 3.77800985 & 14.8169507 & 1.74280832 & 14.7375 & 3735 & -1.6860944 & 71 & 70.3 & 78.4 & 74 & 0.000767 \\
\hline 200.01999 & 3.78613836 & 14.8162011 & 1.74218288 & 14.73675 & 3774.5 & -1.7109252 & 71 & 70.3 & 78.5 & 74.1 & 0.000771 \\
\hline 205.01999 & 3.79176579 & 14.8154515 & 1.74280832 & 14.7375 & 3804.5 & -1.7605868 & 71 & 70.4 & 78.4 & 74 & 0.000778 \\
\hline 210.01999 & 3.78551309 & 14.8162011 & 1.74343376 & 14.7375 & 3844.75 & -1.6612636 & 71 & 70.4 & 78.5 & 74.1 & 0.00078 \\
\hline 215.01999 & 3.77863512 & 14.8177003 & 1.740932 & 14.73675 & 3864.75 & -1.735756 & 71.1 & 70.3 & 78.5 & 74.1 & 0.000786 \\
\hline 220.01999 & 3.78238674 & 14.8162011 & 1.74155744 & 14.736 & 3895 & -1.7109252 & 71 & 70.4 & 78.4 & 74.1 & 0.000789 \\
\hline 225.01999 & 3.77363296 & 14.8162011 & 1.740932 & 14.73675 & 3915 & -1.7854176 & 71.1 & 70.4 & 78.5 & 74.1 & 0.000794 \\
\hline 230.01999 & 3.78426255 & 14.8162011 & 1.74280832 & 14.7375 & 3945 & -1.7605868 & 71 & 70.4 & 78.4 & 74 & 0.000797 \\
\hline 235.01999 & 3.7936416 & 14.8154515 & 1.740932 & 14.73825 & 3974.75 & -1.7854176 & 71.1 & 70.3 & 78.5 & 74.1 & 0.000801 \\
\hline 240.01999 & 3.77300769 & 14.8177003 & 1.74155744 & 14,73675 & 3994.75 & -1.735756 & 71.1 & 70.4 & 78.3 & 74 & 0.000804 \\
\hline 245.01999 & 3.78051093 & 14.8169507 & 1.740932 & 14.7375 & 4015 & -1.7605868 & 71.1 & 70.4 & 78.4 & 74 & 0.000807 \\
\hline 250.01999 & 3.78426255 & 14.8169507 & 1.74155744 & 14.7375 & 4035.25 & -1.8102484 & 71 & 70.4 & 78.4 & 74 & 0.00081 \\
\hline 255.01999 & 3.78551309 & 14.8154515 & 1.74155744 & 14.73675 & 4065.5 & -1.7605868 & 71.1 & 70.4 & 78.4 & 74 & 0.000813 \\
\hline 260.01999 & 3.77425823 & 14.8169507 & 1.74030656 & 14.7375 & 4075 & -1.8102484 & 71 & 70.4 & 78.6 & 74.1 & 0.000815 \\
\hline 265.01999 & 3.78488782 & 14.8169507 & 1.74155744 & 14.7375 & 4085.5 & -1.7854176 & 70.9 & 70.4 & 78.4 & 74 & 0.000818 \\
\hline 270.01999 & 3.78551309 & 14.8162011 & 1.73968112 & 14.73675 & 4105.5 & -1.7854176 & 70.9 & 70.4 & 78.5 & 74.1 & 0.00082 \\
\hline 275.01999 & 3.7811362 & 14.8162011 & 1.74155744 & 14.7375 & 4122.5 & -1.7854176 & 70.8 & 70.4 & 78.4 & 74 & 0.000823 \\
\hline 280.04001 & 3.77863512 & 14.8162011 & 1.74030656 & 14.7375 & 4135.25 & -1.7605868 & 70.8 & 70.4 & 78.3 & 74 & 0.000824 \\
\hline 285.01999 & 3.78613836 & 14.8162011 & 1.74155744 & 14.7375 & 4155.5 & -1.7605868 & 70.8 & 70.5 & 78.4 & 74 & 0,000826 \\
\hline 290.01999 & 3.79426687 & 14.8162011 & 1.73843024 & 14.7375 & 4165.5 & -1.7854176 & 70.7 & 70.4 & 78.6 & 74.1 & 0.000828 \\
\hline 295.01999 & 3.7748835 & 14.8169507 & 1.740932 & 14.73825 & 4175 & -1.7605868 & 70.7 & 70.4 & 78.4 & 74 & 0.00083 \\
\hline 300.01999 & 3.79239106 & 14.8177003 & 1.74155744 & 14.73825 & 4185.25 & -1.8350792 & 70.7 & 70.4 & 78.5 & 74.1 & 0.000831 \\
\hline 305.03 & 3.76738026 & 14.8177003 & 1.73968112 & 14.7375 & 4495.5 & -1.7854176 & 70.7 & 70.4 & 78.5 & 74.1 & 0.000833 \\
\hline 310.01999 & 3.78051093 & 14.8162011 & 1.73843024 & 14.7375 & 4205.25 & -1.7854176 & 70.7 & 70.5 & 78.5 & 74.1 & 0.000834 \\
\hline 315.01999 & 3.77926039 & 14.8177003 & 1.73968112 & 14.7375 & 4215.5 & -1.7854176 & 70.8 & 70.5 & 78.5 & 74 & 0.000836 \\
\hline 320.01999 & 3.79614268 & 14.8169507 & 1.73905568 & 14.7375 & 4225.5 & -1.7605868 & 70.8 & 70.4 & 78.4 & 74 & 0.000837 \\
\hline 325.04001 & 3.78801417 & 14.8169507 & 1.73905568 & 14.7375 & 4235.25 & -1.7854176 & 70.9 & 70.5 & 78.4 & 74 & 0.000839 \\
\hline 330.01999 & 3.79051525 & 14.8154515 & 1.73968112 & 14.73825 & 4245.75 & -1.735756 & 70.8 & 70.5 & 78.5 & 74.1 & 0.00084 \\
\hline 335.01999 & 3.7873889 & 14.8169507 & 1.73905568 & 14.73825 & 4255.5 & -1.7109252 & 70.7 & 70.5 & 78.5 & 74 & 0.00084 \\
\hline 340.01999 & 3.78863944 & 14.81845 & 1.73905568 & 14.7375 & 4265 & -1.7605868 & 70.7 & 70.5 & 78.5 & 74.1 & 0.000842 \\
\hline 345.01999 & 3.78488782 & 14.8162011 & 1.73905568 & 14.7375 & 4265.5 & -1.8102484 & 70.7 & 70.5 & 78.5 & 74.1 & 0.000843 \\
\hline 350.01999 & 3.77675931 & 14.8147019 & 1.73905568 & 14.73825 & 4275.25 & -1.7605868 & 70.7 & 70.5 & 78.4 & 74.1 & 0.000844 \\
\hline 355.01999 & 3.78488782 & 14.8162011 & 1.73843024 & 14.73825 & 4285.5 & -1.7854176 & 70.7 & 70.5 & 78.5 & 74.1 & 0.000844 \\
\hline 360.01999 & 3.78238674 & 14.8169507 & 1.73905568 & 14.73675 & 4295.5 & -1.7605868 & 70.7 & 70.5 & 78.4 & 74.1 & 0.000847 \\
\hline 365.07990 & 3.78551309 & 14.81845 & 1.73843024 & 14.7375 & 4295 & -1.7605868 & 70.7 & 70.5 & 78.5 & 74.1 & 0.000847 \\
\hline 370.01999 & 3.78176147 & 14.8191996 & 1.73968112 & 14.73675 & 4305.75 & -1.7605868 & 70.7 & 70.5 & 78.4 & 74.1 & 0.000847 \\
\hline 375.09 & 3.78801417 & 14.8169507 & 1.73905568 & 14.7375 & 4305.5 & -1.735756 & 70.7 & 70.5 & 78.3 & 74 & 0.000849 \\
\hline 380.01999 & 3.77738458 & 14.8154515 & 1.73655392 & 14.73675 & 4315.5 & -1.735756 & 70.6 & 70.5 & 78.4 & 74 & 0.000849 \\
\hline 385.10999 & 3.78051093 & 14.8177003 & 1.73717936 & 14.7375 & 4315.5 & -1.8102484 & 70.5 & 70.5 & 78.5 & 74.1 & 0.00085 \\
\hline 390.01999 & 3.77675931 & 14.8169507 & 1.73905568 & 14.73675 & 4315.5 & -1.7605868 & 70.5 & 70.5 & 78.5 & 74.1 & 0.000851 \\
\hline 395.01999 & 3.78926471 & 14.8199492 & 1.73905568 & 14.73825 & 4325 & -1.8350792 & 70.5 & 70.5 & 78.3 & 74 & 0.00085 \\
\hline
\end{tabular}

HNF-SD-WM-TRP-275 Rev, 0

APPENDIX C, Page 


\begin{tabular}{|c|c|c|c|c|c|c|c|c|c|c|c|}
\hline SECONDS & \begin{tabular}{|l|} 
MAIN DUCT \\
DIFF (IN H20) \\
\end{tabular} & \begin{tabular}{|l|} 
MAIN DUCT \\
ABS (PS|A)
\end{tabular} & \begin{tabular}{|l|} 
GAS INJECT \\
DIFF (IN H2O
\end{tabular} & \begin{tabular}{|l|} 
GAS INJECT \\
ABS (PSIA)
\end{tabular} & $\begin{array}{l}\text { NEWPORT } \\
\text { (PPM) }\end{array}$ & \begin{tabular}{|l|} 
SMC \\
(PPM) \\
\end{tabular} & \begin{tabular}{|l} 
AMBIENT \\
TEMP (F)
\end{tabular} & $\begin{array}{l}\text { GAS INJECT } \\
\text { TEMP (F) }\end{array}$ & $\begin{array}{l}\text { MAIN DUCT } \\
\text { TEMP (F) }\end{array}$ & $\begin{array}{l}\text { WEC CELL } \\
\text { TEMP (F) }\end{array}$ & \begin{tabular}{|l} 
WEC CELL \\
NOLTS)
\end{tabular} \\
\hline & & & & & & & & & & & \\
\hline 400.01999 & 3.79176579 & 14.81845 & 1.73843024 & 14.73675 & 4325.5 & -1.7605868 & 70.6 & 70.5 & 78.5 & 74.1 & 0.000852 \\
\hline 405.01999 & 3.7811362 & 14.8191996 & 1.73905568 & 14.7375 & 4335.75 & -1.7605868 & 70.6 & 70.6 & 78.4 & 74.1 & 0.000853 \\
\hline 410.01999 & 3.78176147 & 14.81845 & 1.73655392 & 14.73675 & 4335.5 & -1.735756 & 70.7 & 70.5 & 78.5 & 74.1 & 0.000853 \\
\hline 415.01999 & 3.7748835 & 14.8154515 & 1.736555392 & 14.7375 & 4335.5 & -1.735756 & 70.7 & 70.5 & 78.5 & 74 & 0.000853 \\
\hline 420.01999 & 3.78426255 & 14.8191996 & 1.73717936 & 14.7375 & 4345.25 & -1.735756 & 70.8 & 70.5 & 78.4 & 74 & 0.000853 \\
\hline 425.01999 & 3.78051093 & 14.8199492 & 1.73655392 & 14.7375 & 4345 & 9 & 70.9 & 70.5 & 78.5 & 74.1 & 0.000854 \\
\hline 430.01999 & 3.78488782 & 14.81845 & 1.736553992 & 14.73675 & 4355.75 & -1.7109252 & 79 & 70.6 & 78.3 & 74 & 0.000855 \\
\hline 435.01999 & 3.77675931 & 14.81845 & 1.736553992 & 14.73825 & 4355.75 & -1.735756 & 71.1 & 70.6 & 78.3 & 74 & 0.000855 \\
\hline 440.01999 & 3.77863512 & 14.8191996 & 1.73717936 & 14.73825 & 4355.5 & -1.7109252 & 71.1 & 70.6 & 78.5 & 74 & 0,000855 \\
\hline 445.01999 & 3.77863512 & 14.8177003 & 1.73655392 & 14.7375 & 4365.75 & -1.8102484 & 71.2 & 70.6 & 78.4 & 74 & 0.000856 \\
\hline 450.01999 & 3.79176579 & 14.8199492 & 1.73592848 & 14.73675 & 4366 & -1.8350792 & 71.2 & 70.6 & 78.5 & 74.1 & 0.000857 \\
\hline 455.01999 & 3.7811362 & 14.8191996 & 1.73717936 & 14.736 & 4365 & -1.7605868 & 71.2 & 70.6 & 78.4 & 74 & 0.000856 \\
\hline 460.01999 & 3.78676363 & 14.8199492 & 1.73717936 & 14.73675 & 4366 & -1.7605868 & 71.1 & 70.6 & 78.4 & 74.1 & 0.000857 \\
\hline 465.01999 & 3.789889998 & 14.8191996 & 1.7378048 & 14.7375 & 4365.75 & -1.7605868 & 71 & 70.6 & 78.5 & 74.1 & 0.000856 \\
\hline 470.01999 & 3.78238674 & 14.8191996 & 1.7378048 & 14.7375 & 4375.5 & -1.7605868 & 71 & 70.6 & 78.3 & 74 & 0.000856 \\
\hline 475.01999 & 3.77988566 & 14.8191996 & 1.73717936 & 14.7375 & 4375.75 & -1.7854176 & 70.9 & 70.6 & 78.4 & 74.1 & 0.000857 \\
\hline 480.01999 & 3.77300769 & 14.81845 & 1.73717936 & 14.7375 & 4375.5 & -1.8102484 & 70.9 & 70.6 & 78.3 & 74 & 0.000858 \\
\hline 485.01999 & 3.77550877 & 14.8191996 & 1.73655392 & 14.73675 & 4375.75 & -1.7605868 & 70.8 & 70.6 & 78.4 & 74.1 & 0.000858 \\
\hline 490.01999 & 3.78488782 & 14.81845 & 1.73717936 & 14.73675 & 4375.75 & -1.7854176 & 70.8 & 70.6 & 78.3 & 74 & 0.000858 \\
\hline 495.01999 & 3.7873889 & 14.8191996 & 1.73655392 & 14.7375 & 4386 & -1.7854176 & 70.7 & 70.6 & 78.4 & 74 & 0.000859 \\
\hline 500.01999 & 3.78551309 & 14.8191996 & 1.7378048 & 14.73825 & 4385.25 & -1.8102484 & 70.7 & 70.6 & 78.3 & 74 & 0.000858 \\
\hline 505.01999 & 3.78551309 & 14.8191996 & 1.73655392 & 14.7375 & 4386 & -1.735756 & 70.8 & 70.6 & 78.3 & 74 & 0.000859 \\
\hline 510.01999 & 3.77300769 & 14.8177003 & 1.736555392 & 14.73675 & 4386 & -1.8102484 & 70.8 & 70.6 & 78.4 & 74.1 & 0.000859 \\
\hline 515.03 & $3.786+3836$ & 14.81845 & 1.7346776 & 14.7375 & 4385.5 & -1.735756 & 70.8 & 70.6 & 78.4 & 74.1 & 0.00086 \\
\hline 520.01999 & 3.78863944 & 14.8199492 & 1.73530304 & 14.7375 & 4386 & -1.735756 & 70.8 & 70.6 & 78.4 & 74.1 & 0.00086 \\
\hline 525.01999 & 3.77300769 & 14.8162011 & 1.73530304 & 14.73675 & 4395.25 & -1.735756 & 70.8 & 70.7 & 78.3 & 74.1 & 0.00086 \\
\hline 530.07001 & 3.79239106 & 14.8169507 & 1.73592848 & 14.7375 & 4395.5 & -1.7854176 & 70.9 & 70.6 & 78.3 & 74 & 0.00086 \\
\hline 535.01999 & 3.78926471 & 14.8177003 & 1.7346776 & 14.7375 & 4396 & -1.7605868 & 70.8 & 70.6 & 78.2 & 74 & 0.000861 \\
\hline 540.01999 & 3.7811362 & 14.8177003 & 1.7346776 & 14.7375 & 4395.75 & -1.7605868 & 70.7 & 70.6 & 78.4 & 74.1 & 0.000861 \\
\hline 545.03 & 3.78551309 & 14.8177003 & 1.73530304 & 14.7375 & 4395.5 & -1.7605868 & 70.7 & 70.7 & 78.3 & 74 & 0.000859 \\
\hline 550.01999 & 3.78551309 & 14.8169507 & 1.7346776 & 14.73675 & 4395.5 & -1.7854176 & 70.6 & 70.7 & 78.3 & 74 & 0.000861 \\
\hline 555.01999 & 3.78176147 & 14.8162011 & 1.7346776 & 14.73675 & 4396 & -1.735756 & 70.6 & 70.7 & 78.4 & 74 & 0.000861 \\
\hline 560.07001 & 3.78551309 & 14.8169507 & 1.7346776 & 14.7375 & 4396 & -1.7854176 & 70.6 & 70.7 & 78.4 & 74 & 0.000861 \\
\hline 565.01999 & 3.77988566 & 14.8162011 & 1.73530304 & 14.7375 & 4396 & -1.8350792 & 70.6 & 70.7 & 78.4 & 74 & 0.000862 \\
\hline 570.01999 & 3.77300769 & 14.8177003 & 1.73530304 & 14.73675 & 4405.75 & -1.8102484 & 70.5 & 70.7 & 78.5 & 74.1 & 0.000861 \\
\hline 575.03 & 3.7811362 & 14.8177003 & 1.7346776 & 14.7375 & 4405.25 & -1.7854176 & 70.5 & 70.7 & 78.3 & 74.1 & 0.000862 \\
\hline 580.01999 & 3.78676363 & 14.8162011 & 1.73405216 & 14.7375 & 4406 & -1.7605868 & 70.5 & 70.7 & 78.4 & 74 & 0.000861 \\
\hline 585.01999 & 3.79176579 & 14.8154515 & 1.73217584 & 14.7375 & 4405 & -1.735756 & 70.5 & 70.7 & 78.5 & 74.1 & 0.000861 \\
\hline 590,07001 & 3.78488782 & 14.8162011 & 1.73217584 & 14.73825 & 4405.5 & -1.8350792 & 70.6 & 70.7 & 78.4 & 74.1 & 0.000863 \\
\hline 595.01999 & 3.77988566 & 14.8162011 & 1.73530304 & 14.73675 & 4406 & -1.7605868 & 70.6 & 70.7 & 78.5 & 74.1 & 0.000863 \\
\hline 600.01999 & 3.78426255 & 14.8177003 & 1.73530304 & 14.7375 & 4405.75 & -1.7854176 & 70.6 & $70 . \overline{7}$ & 78.3 & 74 & 0.000862 \\
\hline 605.04999 & 3.77988566 & 14.8162011 & 1.73342672 & 14.7375 & 4406 & -1.8102484 & 70.6 & 70.7 & 78.3 & 74 & 0.000863 \\
\hline
\end{tabular}

HNF-SD-WM-TRP-275 Rev. 0

APPENDIX C, Page 


\begin{tabular}{|c|c|c|c|c|c|c|c|c|c|c|c|}
\hline SECONDS & MAIN DUCT & MAIN DUCT & GAS INJECT & GAS INJECT & NEWPORT & SMC & AMBIENT & GAS INJECT & MAIN DUCT & WEC CELL & WEC CELL \\
\hline & DIFF (IN H2O) & ABS (PS|A) & DIFF ( 1 N H2O & ABS (PSIA) & (PPM) & (PPM) & TEMP,(F) & TEMP, $(F)$ & TEMP. (F) & TEMP (F) & (VOLTS) \\
\hline 610.01999 & 3.78863944 . & 14.8169507 & 1.7346776 & 14.73675 & 4405.5 & -1.7605868 & 70.6 & 70.7 & 78.4 & 74 & 0.000862 \\
\hline 615.01999 & 3.78863944 & 14.81845 & 1.73405216 & 14.7375 & 4406 & -1.7854176 & 70.6 & 70.7 & 78.4 & 74.1 & 0.000862 \\
\hline 620.04001 & 3.79239106 & 14.8169507 & 1.73217584 & 14.736 & 4405.25 & -1.8102484 & 70.6 & 70.7 & 78.3 & 74 & 0.000862 \\
\hline 625.10001 & 3.78551309 & 14.8169507 & 1.73217584 & 14.73675 & 4406 & -1.735756 & 70.5 & 70.7 & 78.4 & 74.1 & 0.000863 \\
\hline 630.01999 & 3.78488782 & 14.8169507 & 1.73280128 & 14.73675 & 4405.25 & -1.8102484 & 70.6 & 70.7 & 78.5 & 74.1 & 0.000863 \\
\hline 635.04999 & 3.7811362 & 14.8169507 & 1.73217584 & 14.73675 & 4415 & -1.7605868 & 70.6 & 70.7 & 78.4 & 74.1 & 0.000864 \\
\hline 640.01999 & 3.79239106 & 14.8154515 & 1.73280128 & 14.7375 & 4415.75 & -1.7854176 & 70.6 & 70.7 & 78.4 & 74 & 0.000864 \\
\hline 645.01999 & 3.77425823 & 14.8191996 & 1.73217584 & 14.73675 & 4415 & -1.8102484 & 70.6 & 70.8 & 78.4 & 74.1 & 0.000863 \\
\hline 650.01999 & 3.77738458 & 14.8169507 & 1.73217584 & 14.7375 & 4416 & -1.8102484 & 70.6 & 70.7 & 78.4 & 74 & 0.000863 \\
\hline 655.01999 & 3.78551309 & 14.8162011 & 1.73217584 & 14.7375 & 4415.5 & -1.735756 & 70.7 & 70.7 & 78.4 & 74.1 & 0.000863 \\
\hline 660.01999 & 3.78238674 & 14.8154515 & 1.73280128 & 14.7375 & 4415.75 & -1.7605868 & 70.8 & 70.8 & 78.5 & 74.1 & 0.000864 \\
\hline 665.01999 & 3.78738889 & 14.8154515 & 1.73217584 & 14,73675 & 4415.75 & -1.7854176 & 70.8 & 70.8 & 78.4 & 74 & 0,000863 \\
\hline 670.01999 & 3.77675931 & 14.8162011 & 1.73280128 & 14.7375 & 4415.5 & -1.735756 & 70.9 & 70.8 & 78.5 & 74.1 & 0.000864 \\
\hline 675.01999 & 3.78988998 & 14.8177003 & 1.73280128 & 14.73675 & 4415.5 & -1.7109252 & 70.9 & 70.8 & 78.5 & 74 & 0.000863 \\
\hline 680.04001 & 3.79239106 & 14.8177003 & 1.73217584 & 14.73825 & 4415.25 & -1.7854176 & 70.9 & 70.8 & 78.5 & 74.1 & 0.000864 \\
\hline 685.01999 & 3.79239106 & 14.8191996 & 1.73280128 & 14.7375 & 4416 & -1.7605868 & 70.9 & 70.8 & 78.6 & 74.1 & 0.000863 \\
\hline 690.01999 & 3.78613836 & 14.8169507 & 1.73217584 & 14.73825 & 4415.5 & -1.7854176 & 70.9 & 70.8 & 78.4 & 74 & 0.000865 \\
\hline 695.01999 & 3.77863512 & 14.8191996 & 1.73280128 & 14.73675 & 4416 & -1.7605868 & 70.8 & 70.8 & 78.5 & 74.1 & 0.000864 \\
\hline 700.01999 & 3.78551309 & 14.8177003 & 1.73342672 & 14.7375 & 4416 & -1.735756 & 70.8 & 70.8 & 78.4 & 74.1 & 0.000865 \\
\hline 705.01999 & 3.77926039 & 14.8169507 & 1.73217584 & 14.73825 & 4415.5 & -1.735756 & 70.7 & 70.8 & 78.5 & 74.1 & 0.000864 \\
\hline 710.04999 & 3.79051525 & 14.8177003 & 1.7315504 & 14.7375 & 4415.5 & -1.7109252 & 70.7 & 70.8 & 78.5 & 74.1 & 0.000864 \\
\hline 715.01999 & 3.77988566 & 14.8162011 & 1.73217584 & 14.73825 & 4415.5 & -1.8350792 & 70.7 & 70.8 & 78.4 & 74 & 0.000864 \\
\hline 720.01999 & 3.79176579 & 14.8199492 & 1.73280128 & 14.7375 & 4415.75 & -1.7605868 & 70.8 & 70.8 & 78.5 & 74.1 & 0.000864 \\
\hline 725.01999 & 3.78551309 & 14.8177003 & 1.73217584 & 14.73825 & 4415.75 & -1.8350792 & 70.7 & 70.8 & 78.4 & 74 & 0.000864 \\
\hline 730.01999 & 3.77800985 & 14.8169507 & 1.7315504 & 14.73825 & 4415.75 & $-1,7605868$ & 70.7 & 70.8 & 78.6 & 74.1 & 0.000864 \\
\hline 735.01999 & 3.78551309 & 14.8191996 & 1.7315504 & 14.73825 & 4426 & -1.7854176 & 70.8 & 70.8 & 78.5 & 74 & 0.000863 \\
\hline 740.04999 & 3.78176147 & 14.81845 & 1.73217584 & 14.73825 & 4425.5 & -1.8102484 & 70.8 & 70.8 & 78.5 & 74.1 & 0.000864 \\
\hline 745.01999 & 3.78551309 & 14.81845 & 1.73280128 & 14.73825 & 4425.75 & -1.7605868 & 70.7 & 70.9 & 78.6 & 74.1 & 0.000865 \\
\hline 750.01999 & 3.78988998 & 14.81845 & 1.73217584 & 14.73675 & 4425.25 & -1.7605868 & 70.8 & 70.8 & 78.4 & 74 & 0.000864 \\
\hline 755.03 & 3.77238242 & 14.8177003 & 1.7315504 & 14.73675 & 4425.75 & -1.7854176 & 70.7 & 70.8 & 78.5 & 74.1 & 0.000864 \\
\hline 760.01999 & 3.78926471 & 14.8191996 & 1.73217584 & 14.73675 & 4425.75 & -1.8350792 & 70.8 & 70.8 & 78.4 & 74 & 0.000864 \\
\hline 765.01999 & 3.7811362 & 14.8191996 & 1.73217584 & 14.73675 & 4426 & -1.8102484 & 70.7 & 70.8 & 78.4 & 74 & 0.000864 \\
\hline 770.04999 & 3.78926471 & 14.8191996 & 1.73280128 & 14.7375 & 4425.75 & -1.7605868 & 70.6 & 70.9 & 78.4 & 74 & 0.000865 \\
\hline 775.01999 & 3.79239106 & 14.8199492 & 1.73280128 & 14.7375 & 4425.25 & -1.735756 & 70.6 & 70.9 & 78.4 & 74 & 0.000864 \\
\hline 780.01999 & 3.79239106 & 14.8199492 & 1.73280128 & 14.7375 & 4425.5 & -1.8102484 & 70.7 & 70.9 & 78.5 & 74.1 & 0.000865 \\
\hline 785.07001 & 3.77988566 & 14.81845 & 1.73280128 & 14.73675 & 4426 & -1.7854176 & 70.7 & 70.9 & 78.4 & 74 & 0.000864 \\
\hline 790.01999 & 3.78363728 & 14.8169507 & 1.73217584 & 14.7375 & 4425.75 & -1.7854176 & 70.6 & 70.9 & 78.5 & 74.1 & 0.000864 \\
\hline 795.01999 & 3.78176147 & 14.8191996 & 1.73280128 & 14.73825 & 4425.5 & -1.7605868 & 70.6 & 70.9 & 78.4 & 74 & 0.000864 \\
\hline 800,07001 & 3.78801417 & 14.8191996 & 1.7315504 & 14.7375 & 4426 & -1.8350792 & 70.5 & 70.9 & 78.5 & 74.1 & 0.000864 \\
\hline 805,01999 & 3.77926039 & 14.8199492 & 1.73217584 & 14.73825 & 4426 & -1.7605868 & 70.5 & 70.9 & 78.4 & 74 & 0.000864 \\
\hline 810.01999 & 3.77926039 & 14.81845 & 1.73217584 & 14.7375 & 4425.5 & -1.7854176 & 70.6 & 70.9 & 78.5 & 74.1 & 0.000865 \\
\hline 815.04001 & 3.78426255 & 14.8177003 & 1.73280128 & 14.7375 & 4426 & -1.7605868 & 70.6 & 70.9 & 78.5 & 74.1 & 0.000865 \\
\hline
\end{tabular}




\begin{tabular}{|c|c|c|c|c|c|c|c|c|c|c|c|}
\hline SECONDS & \begin{tabular}{|l|} 
MAIN DUCT \\
DIFF (IN H2O)
\end{tabular} & \begin{tabular}{|l|} 
MAIN DUCT \\
ABS (PSIA) \\
\end{tabular} & \begin{tabular}{|l|} 
GAS INJECT \\
DIFF (IN H20 \\
\end{tabular} & \begin{tabular}{|l|} 
GAS INJECT \\
ABS (PSIA)
\end{tabular} & \begin{tabular}{|l} 
NEWPORT \\
(PPM)
\end{tabular} & \begin{tabular}{|l|} 
SMC \\
(PPM)
\end{tabular} & \begin{tabular}{|c|} 
AMBIENT \\
TEMP.(F)
\end{tabular} & \begin{tabular}{|l} 
GAS INJECT \\
TEMP. (F)
\end{tabular} & \begin{tabular}{|l|} 
MAIN DUCT \\
TEMP. (F)
\end{tabular} & $\begin{array}{l}\text { WEC CELL } \\
\text { TEMP (F) }\end{array}$ & \begin{tabular}{|l|} 
WEC CELL \\
NOLTS)
\end{tabular} \\
\hline 820.01999 & 3.78863944 & 14.8191996 & 1.73280128 & 14.73825 & 4426 & -1.7605868 & 70.7 & 70.9 & 78.4 & 74 & 0.000864 \\
\hline 825.01999 & 3.7748335 & 14.8206988 & 1.73280128 & 14.7375 & 4426.25 & -1.7854176 & 70.6 & 70.9 & 78.4 & 74.1 & 0.000865 \\
\hline 830.03 & 3.7811362 & 14.81845 & 1.73217584 & 14.73675 & 4426 & -1.735756 & 70.6 & 70.9 & 78.4 & 74 & 0.000865 \\
\hline 835.01999 & 3.79176579 & 14.8177003 & 1.73280128 & 14.7375 & 4425.5 & -1.8350792 & 70.6 & 70.9 & 78.5 & 74.1 & 0.000864 \\
\hline 840.01999 & 3.775508977 & 14.8199492 & 1.72967408 & 14.73675 & 4426 & -1.8350792 & 70.6 & 70.9 & 78.4 & 74 & 0.000865 \\
\hline 845.01999 & 3.79114052 & 14.8199492 & 1.73217584 & 14.73675 & 4425.75 & -1.7854176 & 70.7 & 70.9 & 78.5 & 74.1 & 0.000865 \\
\hline 850.01999 & 3.78676363 & 14.8191996 & 1.73217584 & 14.7375 & 4425.5 & -1.8102484 & 70.7 & 70.9 & 78.4 & 74 & 0.000865 \\
\hline 855.01999 & 3.77926039 & 14.8191996 & 1.73280128 & 14.7375 & 4425.75 & -1.8350792 & 70.7 & 71 & 78.6 & 74.1 & 0.000864 \\
\hline 860.01999 & 3.77613404 & 14.8477003 & 1.73217584 & 14.7375 & 4426 & $-1,8350792$ & 70.7 & 71 & 78.6 & 74.1 & 0.000865 \\
\hline 865.01999 & 3.7811362 & 14.8191996 & 1.73029952 & 14.739 & 4426 & -1.7854176 & 70.7 & 71 & 78.6 & 74.1 & 0.000865 \\
\hline 870.01999 & 3.78988998 & 14.8177003 & 1.73217584 & 14.73825 & 4425.5 & -1.8847408 & 70.8 & 71 & 78.6 & 74.1 & 0.000865 \\
\hline 875.06 & 3.79551741 & 14.8206988 & 1.7315504 & 14.7375 & 4425 & -1.8102484 & 70.7 & 71 & 78.5 & 74.1 & 0.000865 \\
\hline 880,09 & 3.77675931 & 14.81845 & 1.73092496 & 14.73825 & 4425.5 & -1.7854176 & 70.7 & 71 & 78.5 & 74.1 & 0.000866 \\
\hline 885.01999 & 3.77675931 & 14.8199492 & 1.73217584 & 14.73825 & 4426 & -1.8102484 & 70.8 & 71 & 78.5 & 74 & 0.000864 \\
\hline 890.04999 & 3.78676363 & 14.8177003 & 1.73029952 & 14.7375 & 4426 & -1.8102484 & 70.8 & 71 & 78.6 & 74.1 & 0.000865 \\
\hline 895.01999 & 3.78176147 & 14.8177003 & 1.73029952 & 14.7375 & 4425.5 & -1.8102484 & 70.7 & 71 & 78.6 & 74.2 & 0.000866 \\
\hline 900.01999 & 3.77926039 & 14.8191996 & 1.73029952 & 14.7375 & 4426 & -1.7854176 & 70.8 & 71 & 78.5 & 74.1 & 0.000866 \\
\hline 905.01999 & 3.77425823 & 14.8191996 & 1.73029952 & 14.739 & 4425.5 & -1.7854176 & 70.8 & 71 & 78.6 & 74.2 & 0.000866 \\
\hline 910.01999 & 3.7873889 & 14.8199492 & 1.73092496 & 14.73825 & 4425.5 & -1.8102484 & 70.9 & 71 & 78.5 & 74 & 0.000865 \\
\hline 915.01999 & 3.78051093 & 14.8199492 & 1.73029952 & 14.73675 & 4425.75 & -1.8102484 & 70.9 & 71 & 78.5 & 74 & 0.000866 \\
\hline
\end{tabular}




\begin{tabular}{|c|c|c|c|c|c|c|c|c|c|c|c|}
\hline $\begin{array}{l}\text { TEST: EX202 } \\
\text { Measured Hyc }\end{array}$ & $\begin{array}{l}213713 \text { Feb } 199 \\
\text { drogen Concent }\end{array}$ & $\begin{array}{l}297 \text { WEC-SPO } \\
\text { tration }=4100 p\end{array}$ & pol & & & & & & \multicolumn{3}{|c|}{ Measured Hydrogen Concentration $=4100 \mathrm{ppm}$} \\
\hline SECONDS & MAIN DUCT & MAIN DUCT & GAS INJECT & GAS INJECT I & NEWPORT & SMC & AMBIENT & GAS INJECT & MAIN DUCT & WEC CELL & WEC CELL \\
\hline & DIFF (IN H2O & ABS (PSIA) & DIFF (IN H2O & ABS (PSIA) & (PPM) & (PPM) & TEMP.(F) & TEMP.(F) & TEMP.(F) & TEMP.(F) & (VOLTS) \\
\hline & & & & & & & & & & & \\
\hline 0.0099792 & 3.7686308 & 14.8169507 & 0.57010832 & 14.75625 & 31.175 & -1.4626172 & 71.5 & 68.2 & $\overline{78.4}$ & \begin{tabular}{l|l|}
74.2 \\
\end{tabular} & 0.000212 \\
\hline 5.0299988 & 3.77238242 & 14.8154515 & 1.79972336 & 14.74275 & 31.05 & -1.4129556 & 71.5 & 68 & 78.5 & 74.2 & 0.000213 \\
\hline 10.019989 & 3.7623781 & 14.8169507 & 1.78971632 & 14.742 & 131.35 & 956.484816 & 71.6 & 68.1 & 78.5 & 74.2 & 0.000248 \\
\hline 15.019989 & 3.77988566 & 14.8154515 & 1.78408736 & 14.7405 & 622 & 2372.08872 & 71.7 & 68.2 & 78.5 & 74.2 & 0.000331 \\
\hline 20.019989 & 3.76550445 & 14.8162011 & 1.77970928 & 14.73975 & 1262.25 & 3186.53896 & 71.9 & 68.1 & 78.4 & 74.2 & 0.000425 \\
\hline 25.019989 & 3.7623781 & 14.8147019 & 1.77595664 & 14,73975 & 1852.5 & 3578.12068 & 72 & 68.1 & 78.3 & 74.2 & 0.00051 \\
\hline 30.019989 & 3.76050229 & \begin{tabular}{|l|}
14.8169507 \\
\end{tabular} & 1.77345488 & 14.73975 & 2333.5 & \begin{tabular}{|l|}
3748.95658 \\
\end{tabular} & 72 & 68.1 & 78.3 & 74.3 & 0.000578 \\
\hline 35.019989 & 3.77300769 & 14.8162011 & 1.76970224 & 14.739 & 2763.5 & 3836.361 & 71.9 & 68.1 & 78.3 & 74.3 & 0.000632 \\
\hline 40.019989 & 3.77800985 & 14.8162011 & 1.76657504 & 14.739 & 3064 & 3894.46507 & 72 & 68.1 & 78.3 & 74.3 & 0.000675 \\
\hline 45.019989 & 3.77113188 & 14.8169507 & 1.76469872 & 14.73975 & 3294.5 & 3921.28234 & 74.8 & 68.1 & 78.4 & 74.2 & 0.000709 \\
\hline 50.019989 & 3.77300769 & 14.8169507 & 1.7628224 & 14.7375 & 3464 & 3944.8716 & 71.9 & 68.1 & 78.3 & 74.2 & 0.000735 \\
\hline 55.019989 & 3.77050661 & 14.8169507 & 1.7628224 & 14.7375 & 3624.25 & 3952.56914 & 71.9 & 68.1 & 78.3 & 74.2 & 0.000756 \\
\hline 60.019989 & 3.75800121 & \begin{tabular}{|l|}
14.8169507 \\
\end{tabular} & 1.76032064 & 14.7375 & 3754.75 & 3955.54884 & 71,9 & 68.1 & 78.3 & 74.4 & 0.000772 \\
\hline 65.019989 & 3.78238674 & \begin{tabular}{|l|}
14.8162011 \\
\end{tabular} & 1.75906976 & 14.7375 & 3844.5 & 3963.743 & 72 & 68.1 & 78.4 & 74.3 & 0.000785 \\
\hline 70.019989 & 3.77733458 & 14.8162011 & 1.75594256 & 14.73825 & 3914.75 & 3972.68209 & 72 & 68.1 & 78.4 & 74.3 & 0.000795 \\
\hline 75.019989 & 3.78051093 & 14.8162011 & 1.75594256 & 14.7375 & 3980.75 & 3974.66856 & 72 & 68.1 & 78.3 & 74.2 & 0.000805 \\
\hline 80.019989 & 3.77113188 & 14.8162011 & 1.75594256 & 14.73825 & 4034.5 & 3976.40671 & 72 & 68.1 & 78.3 & 74.2 & 0.000812 \\
\hline 85.019989 & 3.77988566 & 14.8177003 & 1.75719344 & 14.7375 & 4084.75 & 3964.73624 & 71.8 & 68.1 & 78.3 & 74.3 & 0.000818 \\
\hline 90.019989 & 3.77863512 & 14.8169507 & 1.75406624 & 14.7375 & 4125 & 3971.44055 & 71.9 & 68.2 & 78.4 & 74.3 & 0.000822 \\
\hline 95.019989 & 3.77863512 & 14.8169507 & 1.75156448 & 14.7375 & 4155 & 3974.17194 & 71.9 & 68.1 & 78.4 & 74.2 & 0.000827 \\
\hline 100.01999 & 3.77050661 & 14.8169507 & 1.74968816 & 14.73675 & 4175.25 & 3980.37964 & 71.9 & 68.2 & 78.3 & 74.2 & 0.000831 \\
\hline 105.01999 & 3.77926039 & 14.81845 & 1.75218992 & 14.7375 & 4195.25 & 3985.09749 & 72 & 68.2 & 78.3 & 74.2 & 0.000834 \\
\hline 110.01999 & 3.77738458 & 14.8169507 & 1.7503136 & 14.7375 & 4214.75 & 3989.07042 & 71.8 & 68.2 & 78.4 & 74.3 & 0.000836 \\
\hline 115.01999 & 3.76362864 & 14.8162011 & 1.74968816 & 14.7375 & 4235.5 & 3997.51289 & 79.8 & 68.2 & 78.3 & 74.3 & 0.000838 \\
\hline 120.01999 & 3.77238242 & 14.8154515 & 1.74781184 & 14.7375 & 4255 & 4006.9486 & 71.7 & 68.2 & 78.4 & 74.2 & 0.000841 \\
\hline 125.01999 & 3.7748835 & 14.8169507 & 1.74843728 & 14.7375 & 4265.5 & 4024.08185 & 71.7 & 68.2 & 78.4 & 74.3 & 0.000843 \\
\hline 130.07999 & 3.7623781 & 14.8169507 & 1.74781184 & 14.7375 & 4275.25 & 4021.10215 & 71.8 & 68.2 & 78.3 & 74.3 & 0.000844 \\
\hline 135.01999 & 3.76738026 & 14.8177003 & 1.74781184 & 14.7375 & 4285.25 & 4009.18337 & 71.8 & 68.2 & 78.2 & 74.3 & 0.000846 \\
\hline 140.01999 & 3.77988566 & 14.8162011 & 1.74593552 & 14.7375 & 4295.5 & 4013.1563 & 71.8 & 68.2 & 78.2 & 74.3 & 0.000847 \\
\hline 145.01999 & 3.76925607 & 14.8169507 & 1.74593552 & 14.7375 & 4305.5 & 4022.34369 & 71.7 & 68.2 & 78.4 & 74.3 & 0.000849 \\
\hline 150.01999 & 3.77550877 & 14.8169507 & 1.74531008 & 14.73825 & 4315.25 & 4017.12922 & 71.7 & 68.3 & 78,4 & 74.3 & 0.00085 \\
\hline 155.01999 & 3.76925607 & 14.8162011 & 1.7440592 & 14.7375 & 4315.5 & 4015.63938 & 71.7 & 68.3 & 78.4 & 74.3 & 0.00085 \\
\hline 160.01999 & 3.77050661 & 14.6162011 & 1.74343376 & 14.7375 & 4325.5 & 4014.89445 & 71.6 & 68.3 & 78.4 & 74.3 & 0.000851 \\
\hline 165.01999 & 3.77363296 & 14.8162011 & 1.7440592 & 14.73675 & 4325.25 & 4022.34369 & 71.5 & 68.3 & 78.3 & 74.3 & 0.000852 \\
\hline 170.01999 & 3.7623781 & 14.8162011 & 1.74343376 & 14.73675 & 4335.5 & 4020.60554 & 71.4 & 68.3 & 78.3 & 74.3 & 0.000851 \\
\hline 175.01999 & 3.76800553 & 14.8169507 & 1.7440592 & 14.7375 & 4335.5 & 4023.08862 & 71.4 & 68.3 & 78.3 & 74.3 & 0.000852 \\
\hline 180.01999 & 3.76675499 & 14.8169507 & 1.7440592 & 14.7375 & 4345.5 & 4022.09538 & 71,3 & 68.3 & 78.4 & 74,4 & 0.000853 \\
\hline 185.01999 & 3.75800121 & 14.8177003 & 1.74343376 & 14.7375 & 4345.75 & 4013.4046 & 71.2 & 68.3 & 78.4 & 74.4 & 0.000854 \\
\hline
\end{tabular}




\begin{tabular}{|c|c|c|c|c|c|c|c|c|c|c|c|}
\hline SECONDS & \begin{tabular}{|l|} 
MAIN DUCT \\
DIFF (IN H2O \\
\end{tabular} & \begin{tabular}{|l|} 
MAIN DUCT \\
ABS (PSIA)
\end{tabular} & \begin{tabular}{|l|} 
GAS INJECT \\
DIFF (IN H2O \\
\end{tabular} & $\begin{array}{l}\text { GAS INJECT } \\
\text { ABS (PSIA) }\end{array}$ & \begin{tabular}{|l} 
NEWPORT \\
(PPM)
\end{tabular} & $\frac{\text { SMC }}{\text { (PPM) }}$ & \begin{tabular}{|l} 
AMBIENT \\
TEMP.(F)
\end{tabular} & \begin{tabular}{|l|} 
GAS INJECT \\
TEMP.(F)
\end{tabular} & \begin{tabular}{|l|} 
MAIN DUCT \\
TEMP.(F) \\
\end{tabular} & \begin{tabular}{|l|} 
WEC CELL \\
TEMP.(F)
\end{tabular} & \begin{tabular}{|l|} 
WEC CELL \\
(VOLTS)
\end{tabular} \\
\hline & & & & & & & & & & & \\
\hline 190.01999 & 3.76738026 & 14.8169507 & 1.74343376 & 14.73825 & 4345.75 & 4016.13599 & 71.2 & 68.4 & 78.3 & 74.3 & 0.000854 \\
\hline 195.01999 & 3.76050229 & 14.8154515 & 1.74218288 & 14.7375 & 4355.5 & 4023.08862 & 71.1 & 68.4 & 78.4 & 74.3 & 0.000854 \\
\hline 200.01999 & 3.7623781 & 14.8162011 & 1.74155744 & 14.736 & 4355.25 & 4014.64614 & 71.1 & 68.4 & 78.3 & 74.4 & 0.000856 \\
\hline 205.01999 & 3.7686308 & 14.8177003 & 1.74155744 & 14.7375 & 4365.25 & 4018.61907 & 71.1 & 68.4 & 78.3 & 74.5 & 0.000856 \\
\hline 210.01999 & 3.76925607 & 14.8154515 & 1.74155744 & 14.7375 & 4365.5 & 4034.01417 & 71.1 & 68.4 & 78.3 & 74.4 & 0.000856 \\
\hline 215.01999 & 3.77238242 & 14.8162011 & 1.73968112 & 14.73675 & 4365.75 & 4029.04801 & 71 & 68.4 & 78.4 & 74.4 & 0.000855 \\
\hline 220.01999 & 3.77363296 & 14.8169507 & 1.73968112 & 14.73825 & 4366 & 4021.10215 & 71.1 & 68.4 & 78.4 & 74.4 & 0.000855 \\
\hline 225.01999 & 3.76675499 & 14.8154515 & 1.73968112 & 14.7375 & 4365.25 & 4013.65291 & 71.1 & 68,4 & 78.3 & 74.3 & 0.000857 \\
\hline 230,01999 & 3.76675499 & 14.8177003 & 1.73905568 & 14.7375 & 4366 & 4023.08862 & 71.1 & 68.4 & 78.2 & 74.3 & 0.000857 \\
\hline 235.01999 & 3.7748835 & 14.8162011 & 1.73905568 & 14.73825 & 4366 & 4025.32339 & 71.1 & 68.5 & 78.2 & 74.4 & 0.000857 \\
\hline 240.01999 & 3.7686308 & 14.8169507 & 1.73968112 & 14.7375 & 4375 & 4027.30985 & 71.1 & 68.5 & 78.3 & 74.4 & 0.000857 \\
\hline 245.01999 & 3.77113188 & 14.8169507 & 1.73968112 & 14.7375 & 4376 & 4025.07508 & 71 & 68.5 & 78.4 & 74.5 & 0.000858 \\
\hline 250.01999 & 3.77050661 & 14.81845 & 1.73717936 & 14.7375 & 4375.75 & 4022.34369 & 71 & 68.5 & 78.3 & 74.3 & 0.000857 \\
\hline 255.01999 & 3.77738458 & 14.8169507 & 1.7378048 & 14.73825 & 4375.25 & 4026.31662 & 71 & 68.5 & 78.4 & 74.3 & 0.000858 \\
\hline 260.01999 & 3.77926039 & 14.8169507 & 1.73843024 & 14.73825 & 4375.75 & 4021.35046 & 70.9 & 68.5 & 78.3 & 74.3 & 0.000858 \\
\hline 265.06 & 3.7748835 & 14.8177003 & 1.73717936 & 14.7375 & 4375.75 & 4023.08862 & 70.9 & 68.5 & 78.3 & 74.3 & 0.000859 \\
\hline 270.01999 & 3.77113188 & 14.8177003 & 1.7378048 & 14.7375 & 4375.5 & 4023.33692 & 70.9 & 68.5 & 78.3 & 74.4 & 0.000859 \\
\hline 275.01999 & 3.76425391 & 14.8177003 & 1.7378048 & 14.73825 & 4385.5 & 4014.14953 & 71 & 68.5 & 78.4 & 74.4 & 0.000858 \\
\hline 280.03 & 3.77926039 & 14.81845 & 1.73717936 & 14.7375 & 4386 & 4012.41137 & 71 & 68.5 & 78.3 & 74.4 & 0.000858 \\
\hline 285.01999 & 3.77113188 & 14.8169507 & 1.73717936 & 14.7375 & 4385.5 & 4012.65968 & 71 & 68.5 & 78.3 & 74.4 & 0.000859 \\
\hline 290.01999 & 3.7686308 & 14.8162011 & 1.73717936 & 14.73825 & 4385.5 & 4004.46552 & 70.9 & 68.6 & 78.3 & 74.4 & 0.000859 \\
\hline 295.01999 & 3.75675067 & 14.8169507 & 1.73717936 & 14.739 & 4386 & 4010.67322 & 70.9 & 68.5 & 78.2 & 74.4 & 0.000858 \\
\hline 300.01999 & 3.77113188 & 14.8169507 & 1.73717936 & 14.739 & 4385.5 & 4020.60554 & 70.9 & 68.6 & 78.3 & 74.4 & 0.000858 \\
\hline 305.01999 & 3.7623781 & 14.8169507 & 1.73655392 & 14.73825 & 4386 & 4019.86061 & 70.9 & 68.6 & 78.3 & 74.4 & 0.000859 \\
\hline 310.01999 & 3.77550877 & 14.8162011 & 1.73717936 & 14.7375 & 4385.75 & 4017.12922 & 70.9 & 68.6 & 78.4 & 74.4 & 0.000859 \\
\hline 315.01999 & 3.75362432 & 14.8177003 & 1.7378048 & 14.7375 & 4386 & 4012.41137 & 70.9 & 68.6 & 78.4 & 74.4 & 0.000859 \\
\hline 320.01999 & 3.76050229 & 14.8169507 & 1.73655392 & 14.7375 & 4395.75 & 4010.92152 & 70.9 & 68.6 & 78.3 & 74.4 & 0.00086 \\
\hline 325.01999 & 3.76675499 & 14.8169507 & 1.73530304 & 14.73825 & 4395.5 & 4013.1563 & 70.9 & 68.6 & 78.3 & 74.4 & 0.00086 \\
\hline 330.01999 & 3.7811362 & 14.8177003 & 1.73530304 & 14.7375 & 4396 & 4016.3843 & 70.9 & 68.6 & 78.4 & 74.4 & 0.000859 \\
\hline 335.01999 & 3.76988134 & 14.8169507 & 1.73530304 & 14.73825 & 4395.75 & 4014.39784 & 70.9 & 68.6 & 78.4 & 74.4 & 0.000861 \\
\hline 340.01999 & 3.77550877 & 14.8162011 & 1.73530304 & 14.7375 & 4396 & 4011.66645 & 70.9 & 68.6 & 78.4 & 74.4 & 0.000861 \\
\hline 345.01999 & 3.7686308 & 14.8177003 & 1.73530304 & 14.73825 & 4396 & 4012.16306 & 70.9 & 68.6 & 78.3 & 74.4 & 0.00086 \\
\hline 350.06 & 3.76925607 & 14.8177003 & 1.73530304 & 14.739 & 4395.25 & 4015.39107 & 70.9 & 68.6 & 78.4 & 74.4 & 0.000861 \\
\hline 355.01999 & 3.7748835 & 14.8169507 & 1.73530304 & 14.7375 & 4396 & 4017.62584 & 70.9 & 68.7 & 78.4 & 74.5 & 0.000861 \\
\hline 360.03 & 3.77050661 & 14.81845 & 1.7346776 & 14.73825 & 4395.75 & 4011.41814 & 70.9 & 68.6 & 78.4 & 74.5 & 0.000861 \\
\hline 365.01999 & 3.76487918 & 14.8162011 & 1.73530304 & 14.739 & 4396.25 & 4020.60554 & 71 & 68.6 & 78.3 & 74.4 & 0.000861 \\
\hline 370.03998 & 3.75862648 & 14.8177003 & 1.73217584 & 14.73825 & 4396 & 4024.57846 & 71 & 68.7 & 78.3 & 74.5 & 0.000861 \\
\hline 375.01999 & 3.76925607 & 14.8169507 & 1.73280128 & 14.73825 & 4396.25 & 4024,33016 & 71.1 & 68.6 & 78.2 & 74.4 & 0.000859 \\
\hline 380.01999 & 3.75550013 & 14.81845 & 1.73342672 & 14.73825 & 4396 & 4021.10215 & 71.1 & 68.6 & 78.3 & 74.4 & 0.00086 \\
\hline 385.01999 & 3.77988566 & 14.8177003 & 1.73280128 & 14.7375 & 4395.75 & 4016.88092 & 71.1 & 68.6 & 78.3 & 74.4 & 0.000861 \\
\hline 390.01999 & 3.76175283 & 14.8169507 & 1.73342672 & 14.73825 & 4396 & 4011.16983 & 71.2 & 68.7 & 78.4 & 74.5 & 0.000861 \\
\hline 395.01999 & 3.76800553 & 14.8162011 & 1.73342672 & 14.73825 & 4395.5 & 4002.97567 & 71.2 & 68.7 & 78.4 & 74.4 & 0.00086 \\
\hline
\end{tabular}




\begin{tabular}{|c|c|c|c|c|c|c|c|c|c|c|c|}
\hline SECONDS & \begin{tabular}{|l|} 
MAIN DUCT \\
DIFF (IN H2O \\
\end{tabular} & \begin{tabular}{|l|} 
MAIN DUCT \\
ABS (PSIA)
\end{tabular} & \begin{tabular}{|l|} 
GAS INJECT \\
DIEF
\end{tabular} & \begin{tabular}{l|} 
GAS INJECT \\
ABS (PSIA)
\end{tabular} & $\begin{array}{l}\text { NEWPORT } \\
\text { (PPM) }\end{array}$ & $\begin{array}{l}\text { SMC } \\
\text { PPM }\end{array}$ & $\begin{array}{l}\text { AMBIENT } \\
\text { TEMP (F) }\end{array}$ & \begin{tabular}{|l|} 
GAS INJECT \\
TEMP(F)
\end{tabular} & \begin{tabular}{|l|} 
MAIN DUCT \\
TEMP (F)
\end{tabular} & \begin{tabular}{l|} 
WEC CELL \\
TEMP(F)
\end{tabular} & $\frac{\text { WEC CELL }}{\text { (NOLTS) }}$ \\
\hline & & & & & & & & & & & \\
\hline 400.01999 & 3.7811362 & 14.8199492 & 1.73342672 & 14.74125 & 4395.75 & 4010.42491 & 71.3 & 68.7 & 78.3 & 74.4 & 0.000861 \\
\hline 405.01999 & 3.77050661 & 14.8177003 & 1.73280128 & 14.73825 & 4395.5 & 4010.67322 & 71.2 & 68.7 & 78.3 & 74.4 & 0.00086 \\
\hline 410.01999 & 3.7748835 & 14.8162011 & 1.73217584 & 14.73975 & 4396 & 4002.97567 & 71.3 & 68.7 & 78.3 & 74.5 & 0.000861 \\
\hline 415.01999 & 3.76800553 & 14.81845 & 1.73217584 & 14.7375 & 4395.5 & 4005.95536 & 71.2 & 68.8 & 78.3 & 74.5 & 0.000861 \\
\hline 420.01999 & 3.76800553 & 14.81845 & 1.73342672 & 14.73675 & 4406 & 4001.98244 & 71.2 & 68.7 & 78.3 & 74.5 & 0.000862 \\
\hline 425.01999 & 3.7748835 & 14.81845 & 1.73280128 & 14.73975 & 4406 & 4003.72059 & 71.3 & 68.7 & 78.4 & 74.5 & 0.000862 \\
\hline 430,04999 & 3.77238242 & 14.81845 & 1.73280128 & 14.7375 & 4405.75 & 4001.48582 & 71.3 & 68.8 & 78.4 & 74.5 & 0.000862 \\
\hline 435.01999 & 3.7686308 & 14.8169507 & 1.73280128 & 14.7375 & 4406 & 3996.27135 & 71.3 & 68.8 & 78.2 & 74.5 & 0.000862 \\
\hline 440.01999 & 3.77300769 & 14.6191996 & 1.73280128 & 14.7405 & 4405.75 & 3998.25782 & 71.4 & 68.8 & 78.4 & 74.5 & 0.000862 \\
\hline 445.01999 & 3.75737594 & 14.8162011 & 1.73405216 & 14.7375 & 4405.75 & 3994.7815 & 71.4 & 68.8 & 78.4 & 74.5 & 0.000861 \\
\hline 450.03 & 3.7686308 & 14.81845 & 1.73280128 & 14.73825 & 4405.75 & 3993.53996 & 71.3 & 68.8 & 78.2 & 74.4 & 0.000861 \\
\hline 455.01999 & 3.77300769 & 14.8154515 & 1.73280128 & 14.73825 & 4406 & 3999.99597 & 71.4 & 68.8 & 78.3 & 74.4 & 0.000861 \\
\hline 460.06998 & 3.77550877 & 14.8177003 & 1.73342672 & 14.7405 & 4405.5 & 3991.5535 & 71.4 & 68.8 & 78.3 & 74.5 & 0.000862 \\
\hline 465.01999 & 3.76925607 & 14.8177003 & 1.73280128 & 14.7375 & 4405.75 & 3983.60764 & 71.5 & 68.8 & 78.2 & 74.5 & 0.000861 \\
\hline 470.01999 & 3.77050661 & 14.8191996 & 1.73280128 & 14.73825 & 4406.25 & 3982.61441 & 79.5 & 68.8 & 78.3 & 74.5 & 0.000863 \\
\hline 475.01999 & 3.77238242 & 14.8191996 & 1.73217584 & 14.7375 & 4405.75 & 3987.58057 & 71.5 & 68.8 & 78.3 & 74.5 & 0.000863 \\
\hline 480.09998 & 3.77988566 & \begin{tabular}{|l|}
14.8199492 \\
\end{tabular} & 1.73280128 & 14.7375 & 4406 & 4009.67998 & 71.5 & 68.8 & 78.2 & 74.4 & 0.000861 \\
\hline 485.01999 & 3.7686308 & 14.81845 & 1.73217584 & 14.7375 & 4406 & 4012.41137 & 71.5 & 68.8 & 78.2 & 74.4 & 0.000862 \\
\hline 490.01999 & 3.78613836 & 14.81845 & 1.73342672 & 14.73825 & 4406 & 3997.51289 & 71.5 & 68.8 & 78.2 & 74.5 & 0.000862 \\
\hline 495.01999 & 3.76988134 & 14.8177003 & 1.73342672 & 14.7405 & 4396 & 4001.48582 & 71.5 & 68.9 & 78.3 & 74.5 & 0.000862 \\
\hline 500.01999 & 3.77300769 & 14.8199492 & 1.73217584 & 14.73825 & 4395.75 & 4005.70706 & 71.5 & 68.8 & 78.3 & 74.5 & 0.000861 \\
\hline 505.01999 & 3.77113188 & 14.8191996 & 1.73342672 & 14.7375 & 4396.25 & 4000.24428 & 71.5 & 68.8 & 78.3 & 74.5 & 0.000861 \\
\hline 510.06 & 3.77425823 & 14.8177003 & 1.73280128 & 14.7405 & 4395.75 & 3985.09749 & 71.5 & 68.9 & 78.3 & 74.5 & 0.000861 \\
\hline 515.01999 & 3.77300769 & 14.81845 & 1.73217584 & 14.7375 & 4396 & 3991.30519 & 71.6 & 68.9 & 78.2 & 74.6 & 0.000861 \\
\hline 520.01999 & 3.76487918 & 14.8162011 & 1,73280128 & 14.73675 & 4396 & 3992.05012 & 71.6 & 68.9 & 78.3 & 74.5 & 0.000861 \\
\hline 525.03998 & 3.77675931 & 14.81845 & 1.73280128 & 14.7375 & 4395.5 & 3988.3255 & 71.6 & 68.9 & 78.2 & 74.5 & $0.00086 t$ \\
\hline 530.01999 & 3.76925607 & 14.8191996 & 1.73280128 & 14.73975 & 4396.25 & 3991.30519 & 71.7 & 68.9 & 78.2 & 74.5 & 0.00086 \\
\hline 535.01999 & 3.77675931 & 14.8191996 & 1.73217584 & 14.7405 & 4395.75 & 3991.30519 & 71.7 & 68.9 & 78.2 & 74.5 & 0.000861 \\
\hline 540.07999 & 3.7686308 & 14.8191996 & 1.73217584 & 14.7375 & 4396 & 3989.07042 & 71.7 & 68.9 & 78.2 & 74.5 & 0.000861 \\
\hline 545.01999 & 3.76738026 & 14.8191996 & 1.73342672 & 14.7375 & 4406 & 3982.3661 & 71.7 & 68.9 & 78.3 & 74.5 & 0.000861 \\
\hline 550.01999 & 3.7811362 & 14.81845 & 1.73092496 & 14.73975 & 4406 & 3980.62795 & 71.7 & 68.9 & 78.2 & 74.5 & 0.000862 \\
\hline 555.06 & 3.78176147 & 14.81845 & 1.73280128 & 14.7375 & 4405.75 & 3972.43378 & 71.7 & 68.9 & 78.3 & 74.5 & 0.000862 \\
\hline 560.01999 & 3.7686308 & 14.8191996 & 1.73280128 & 14.73825 & 4405.75 & 3971.93717 & 71.8 & 68.9 & 78.3 & 74.6 & 0.000862 \\
\hline 565.01999 & 3.76675499 & 14.8177003 & 1.73217584 & 14.7375 & 4406 & 3972.9304 & 71.8 & 68.9 & 78.3 & 74.6 & 0.000862 \\
\hline 570.06998 & 3.76738026 & 14.8177003 & 1.73092496 & 14.7405 & 4406.25 & 3978.14487 & 71.9 & 68.9 & 78.2 & 74.6 & 0.000862 \\
\hline 575.01999 & 3.77175715 & 14.8191996 & 1.73217584 & 14.74125 & 4406.25 & 3977.15164 & 71.8 & 68.9 & 78.2 & 74.6 & 0.000862 \\
\hline 580.01999 & 3.76675499 & 14.8169507 & 1.73092496 & 14.73825 & 4406.25 & 3985.59411 & 71.8 & 68.9 & 78.2 & 74.6 & 0.000861 \\
\hline 585.03998 & 3.77550877 & 14.8199492 & 1.73092496 & 14.73825 & 4385.75 & 3992.54673 & 71.8 & 68.9 & 78.3 & 74.5 & 0.000858 \\
\hline 590,03998 & 3.76112756 & 14.8169507 & 1.73029952 & 14.7405 & 4386.25 & 3989.07042 & 71.7 & 68.9 & 78.2 & 74.6 & 0.000659 \\
\hline 595.01999 & 3.7623781 & 14.8206988 & 1.7315504 & 14.73825 & 4395.75 & 3989.31873 & 71.6 & 69 & 78.2 & 74.6 & 0.000861 \\
\hline 600.01999 & 3.7686308 & 14.8191996 & 1.73092496 & 14.7405 & 4396 & 3972.18548 & 71.5 & 69 & 78.2 & $\frac{7-.9}{74.6}$ & 0.000861 \\
\hline 605.01999 & 3.76675499 & 14.8191996 & 1.73092496 & 14.7375 & 4405.25 & 3971.44055 & 71.5 & 69 & 78.2 & 74.7 & 0.000862 \\
\hline
\end{tabular}




\begin{tabular}{|c|c|c|c|c|c|c|c|c|c|c|c|}
\hline SECONDS & \begin{tabular}{|l|} 
MAIN DUCT \\
DIFF (IN H2O
\end{tabular} & $\frac{\text { MAIN DUCT }}{\text { ABS (PSIA) }}$ & \begin{tabular}{|l|} 
GAS INJECT \\
DIFF (IN H2O
\end{tabular} & \begin{tabular}{|l|} 
GAS INJECT \\
ABS (PSIA) \\
\end{tabular} & $\begin{array}{l}\text { NEWPORT } \\
\text { (PPM) }\end{array}$ & \begin{tabular}{|l|} 
SMC \\
(PPM)
\end{tabular} & \begin{tabular}{|l} 
AMBIENT \\
TEMP.(F)
\end{tabular} & \begin{tabular}{|l|} 
GAS INJECT \\
TEMP.(F) \\
\end{tabular} & $\frac{\text { MAIN DUCT }}{\mid \text { TEMP.(F) }}$ & \begin{tabular}{|l|} 
WEC CELL \\
TEMP.(F) \\
\end{tabular} & $\begin{array}{l}\text { WEC CELL } \\
\text { VOLTS) }\end{array}$ \\
\hline & & & & & & & & & & & \\
\hline 610.01999 & 3.77050661 & 14.8199492 & 1.73092496 & 14.73825 & 4406 & 3973.67532 & 71.4 & 69 & 78.3 & 74.7 & 0.000862 \\
\hline 615.03 & 3.7686308 & 14.8191996 & 1.73029952 & 14.73825 & 4406.25 & 3981.62118 & 71.4 & 69 & 78.2 & 74.6 & 0.000863 \\
\hline 620.01999 & 3.76675499 & 14.8214485: & 1.73029952 & 14.7405 & 4405.25 & 3982.3661 & 71.3 & 69 & 78.3 & 74.6 & 0.000861 \\
\hline 625.01999 & 3.76925607 & 14.8154515 & 1.73029952 & 14.7405 & 4406 & 3981.86949 & 71.4 & 69.1 & 78.2 & $\mathbf{7 4 . 6}$ & 0.000862 \\
\hline 630.03998 & 3.76612972 & 14.8191996 & 1.73092496 & 14.73825 & 4406 & 3983.85595 & 71.4 & 69.1 & 78.3 & 74.6 & 0.000862 \\
\hline 635.01999 & 3.7686308 & 14.8191996 & 1.73092496 & 14.7405 & 4405.75 & 3982.61441 & 71.4 & 69 & 78.3 & 74.7 & 0.000862 \\
\hline 640.01999 & 3.77238242 & 14.8191996 & 1.73029952 & 14.7375 & 4405.75 & 3983.35934 & 71.4 & 69.1 & 78.3 & 74.6 & 0.000863 \\
\hline 645.01999 & 3.76925607 & 14.8199492 & 1.73092496 & 14.739 & 4405.75 & 3985.09749 & 71.5 & 69 & 78.2 & 74.6 & 0.000863 \\
\hline 650.01999 & 3.75987702 & 14.8162011 & 1.73092496 & 14.73825 & 4416 & 3981.62118 & 71.6 & 69.1 & 78.2 & 74.6 & 0.000863 \\
\hline 655.01999 & 3.76425391 & 14.8199492 & 1.73092496 & 14.73825 & 4415.75 & 3984.84918 & 71.6 & 69.1 & 78.2 & 74.6 & 0.000863 \\
\hline 660.06 & 3.76487918 & 14.8206988 & 1.72967408 & 14.73825 & 4415.5 & 3981,62118 & 71.7 & 69.1 & 78.2 & 74.6 & 0.000862 \\
\hline 665.01999 & 3.76925607 & 14.8206988 & 1.73029952 & 14.73825 & 4405.5 & 3973.92363 & 71.7 & 69.1 & 78.2 & 74.7 & 0.000863 \\
\hline 670.01999 & 3.76800553 & 14.8199492 & 1.73029952 & 14.7375 & 4406 & 3964.23962 & 71.7 & 69.1 & 78.3 & 74.6 & 0.000863 \\
\hline 675.03998 & 3.76487918 & 14.8169507 & 1.7284232 & 14.7375 & 4406.25 & 3961.01162 & 71.7 & 69.1 & 78.1 & 74.7 & 0.000862 \\
\hline 680.01999 & 3.77863512 & 14.81845 & 1.73092496 & 14.739 & 4406 & 3964.98454 & 71.7 & 69.1 & 78.2 & 74.6 & 0.000862 \\
\hline 685.01999 & 3.77425823 & 14.8169507 & 1.73092496 & 14.73825 & 4406.25 & 3969.7024 & 71.7 & 69.1 & 78.2 & 74.6 & 0.000862 \\
\hline 690.03 & 3.76175283 & 14.81845 & 1.73029952 & 14.7375 & 4405.5 & 3972.68200 & 71.6 & 69.1 & 78.2 & 74.7 & 0.000862 \\
\hline 695.07999 & 3.7748835 & 14.81845 & 1.73029952 & 14.7375 & 4406 & 3972.43378 & 71.6 & 69.1 & 78.3 & 74.6 & 0.000861 \\
\hline 700.01999 & 3.77238242 & 14.81845 & 1.73029952 & 14.73825 & 4405.75 & 3972.18548 & 71.4 & 69.1 & 78.4 & 74.7 & 0.000863 \\
\hline 705.06 & 3.77238242 & 14.8191996 & 1.72779776 & 14.7375 & 4405.5 & 3974.42025 & 71.5 & 69.1 & 78.2 & 74.7 & 0.000863 \\
\hline 710.01999 & 3.76738026 & 14.8191996 & 1.72967408 & 14.73825 & 4405.75 & 3976.90333 & 71.5 & 69.1 & 78.2 & 74.6 & 0.000862 \\
\hline 715.01999 & 3.76300337 & 14.81845 & 1.7284232 & 14.7375 & 4406.25 & 3974.91686 & 71.5 & 69.1 & 78.2 & 74.6 & 0.000862 \\
\hline 720.06 & 3.77613404 & 14.8199492 & 1.73029952 & 14.73675 & 4406.25 & 3968.21255 & 71.5 & 69.2 & 78.2 & 74.7 & 0.000862 \\
\hline 725.01999 & 3.7686308 & 14.81845 & 1.72779776 & 14.74125 & 4405.25 & 3953.743 & 71.5 & 69.2 & 78,3 & 74.7 & 0.000862 \\
\hline 730.01999 & 3.77550877 & 14.8199492 & 1.7284232 & 14.73825 & 4406.25 & 3956.79038 & 71.5 & 69.1 & 78.3 & 74.7 & 0.000862 \\
\hline 735.06998 & 3.76050229 & 14.8199492 & 1.7284232 & 14.73825 & 4406 & 3952.56914 & 71.5 & 69.2 & 78.2 & 74.6 & 0.000852 \\
\hline 740.01999 & 3.76300337 & 14.81845 & 1.7284232 & 14.73825 & 4406.25 & 3963.24639 & 71.5 & 69.2 & 78.2 & 74.7 & 0.000362 \\
\hline 745.01999 & 3.76612972 & 14.8214465 & 1.7284232 & 14.73825 & 4406 & 3968.70916 & 71.5 & 6.2 & 78.3 & 74,7 & 0.000862 \\
\hline 750.03998 & 3.77675931 & 14.8199492 & 1.7284232 & 14.7375 & 4405.75 & 3971.44055 & 71.6 & 69.2 & 78.1 & 74.7 & 0.000862 \\
\hline 755.01999 & 3.76925607 & 14.8191996 & 1.72904864 & 14.73825 & 4406.25 & 3970.94394 & 71.5 & 69.2 & 78.1 & 74.6 & 0.000861 \\
\hline 760.01999 & 3.75487486 & 14.8199492 & 1.72904864 & 14.73825 & 4405.5 & 3974.91686 & 71.3 & 6.2 & 78.1 & 74.7 & 0.000862 \\
\hline 765.03998 & 3.76112756 & 14.8191996 & 1.7284232 & 14.7375 & 4405.75 & 3981.12456 & 71.3 & 69.2 & 78.2 & 74.7 & 0.000861 \\
\hline 770.01999 & 3.75424959 & 14.81845 & 1.7284232 & 14.7375 & 4405.75 & 3980.87626 & 71.2 & 69.2 & 78.3 & 74.7 & 0.000862 \\
\hline 775.01999 & 3.76112756 & 14.8177003 & 1.72904864 & 14.739 & 4406.25 & 3983.11103 & 71.2 & 69.2 & 78.3 & 74.7 & 0.000862 \\
\hline 780.04999 & 3.7686308 & 14.81845 & 1.7284232 & 14.7375 & 4406.25 & 3971.19224 & 71.2 & 69.2 & 78.3 & 74.7 & 0.000862 \\
\hline 785.01999 & 3.76800553 & 14.8177003 & 1.7284232 & 14.7375 & 4405.5 & 3972.18548 & 71.1 & 69.2 & 78.1 & 74.7 & 0.000863 \\
\hline 790.01999 & 3.76925607 & 14.81845 & 1.72904864 & 14.739 & 4406.25 & 3969.7024 & 71 & 69.3 & 78.2 & 74.7 & 0.000862 \\
\hline 795.03 & 3.76800553 & 14.81845 & 1.7284232 & 14.7375 & 4406 & 3959.77008 & 71.1 & 69.2 & 78.3 & 74.6 & 0.00086 \\
\hline 800.01999 & 3.77675931 & 14.8199492 & 1.72779776 & 14.73975 & 4406 & 3958.52854 & 71 & 69.2 & 78.3 & 74.6 & 0.000862 \\
\hline 805.01999 & 3.7623781 & 14.8191996 & 1.72904864 & 14.7375 & 4406 & 3958.52854 & 71 & 69.3 & 78.2 & 74.6 & 0.000861 \\
\hline 810.03 & 3.76988134 & 14.8191996 & 1.72779776 & 14.7375 & 4405.5 & 3966.22608 & 74.2 & 69.3 & 78.2 & 74.7 & 0.000851 \\
\hline 815.04999 & 3.7686308 & 14.8199492 & 1.7284232 & 14.7375 & 4395.75 & 3975.16517 & 71.1 & 69.3 & 78.2 & 74.6 & 0.00086 \\
\hline
\end{tabular}




\begin{tabular}{|c|c|c|c|c|c|c|c|c|c|c|c|}
\hline SECONDS & \begin{tabular}{|l|} 
MAIN DUCT \\
\end{tabular} & MAIN DUCT & GAS INJECT & GAS INJECT & NEWPORT & SMC & $\begin{array}{l}\text { AMBIENT } \\
\text { TEMP (F) }\end{array}$ & $\begin{array}{l}\text { GAS INJECT } \\
\text { TEMP (F) }\end{array}$ & \begin{tabular}{|l|} 
MAIN DUCT \\
TEMP(F)
\end{tabular} & \begin{tabular}{|l|} 
WEC CELL \\
TEMP IF
\end{tabular} & $\begin{array}{l}\text { WEC CELL } \\
\text { NOLTS }\end{array}$ \\
\hline & & ABS (PS:A) & DIFF (IN H2O & ABS (PSIA) & & & & & & & \\
\hline 820.01999 & 3.77425823 & 14.81245 & 1.72779776 & 14.7375 & 4405.5 & 3972.68209 & 71.1 & 69.3 & 78.3 & $\mathbf{7 4 . 7}$ & 0.000862 \\
\hline 825.06 & 3.76612972 & 14.8177003 & 1.7284232 & 14.73825 & 4405.75 & 3973.17871 & 71 & 69.3 & 78.2 & 74.6 & 0.000861 \\
\hline 830.03 & 3.76988134 & 14.8191996 & 1.72779776 & 14.73825 & 4406 & 3968.70916 & 70.9 & 69.3 & 78.1 & 74.6 & 0.000862 \\
\hline 835.01999 & 3.76612972 & 14.81845 & 1.72904864 & 14.7375 & 4406.25 & 3961.50823 & 71 & 69.4 & 78.2 & 74.7 & 0.000852 \\
\hline 840.01999 & 3.77238242 & 14.81845 & 1.72904864 & 14.739 & 4405.75 & 3957.78361 & 71.1 & 69.3 & 78.2 & 74.7 & 0.000861 \\
\hline 845.01999 & 3.77175715 & 14.8199492 & 1.7284232 & 14.7375 & 4405.75 & 3964.73624 & 71.2 & 69.3 & 78.3 & 74.6 & 0.000861 \\
\hline 850.01999 & 3.76738026 & 14.8199492 & 1.72904864 & 14.7375 & 4406 & 3964.73624 & 71.2 & 69.3 & 78.3 & 74.6 & 0.000862 \\
\hline 855.01999 & 3.77238242 & 14.8199492 & 1,72904864 & 14.7375 & 4405.25 & 3964.23962 & 71.3 & 69.4 & 78.2 & 74.6 & 0.000861 \\
\hline 860.06998 & 3.77675931 & 14.8199492 & 1.72904864 & 14.73825 & 4406 & 3972.18548 & 71.3 & 69.4 & 78.2 & 74.6 & 0.000861 \\
\hline 865.01999 & 3.7748835 & 14.81845 & 1.72967408 & 14.73825 & 4406 & 3962.25316 & 71.3 & 69.4 & 78.3 & 74.7 & 0.000861 \\
\hline 870.07999 & 3.7686308 & 14.8199492 & 1.72904864 & 14.7375 & 4406 & 3968,46086 & 71.4 & 69.4 & 78.3 & 74.7 & 0.000852 \\
\hline 875.06998 & 3.76487918 & 14.8191996 & 1.72904864 & 14.7375 & 4406 & 3963.24639 & 71.5 & 69.4 & 78.3 & 74.7 & 0.000861 \\
\hline 880.01999 & 3.75800121 & 14.8199492 & 1.72904864 & 14.73825 & 4396.25 & 3956.54207 & 71.5 & 69.4 & 78.3 & 74.7 & 0.00036 \\
\hline 885.03998 & 3.75800121 & 14.8162011 & 1.72904864 & 14.73825 & 4396.25 & 3957.287 & 71.4 & 69.4 & 78.2 & 74.7 & 0.000859 \\
\hline 890.01999 & 3.77988566 & 14.8191996 & 1.72967408 & 14.73825 & 4395.75 & 3955.05222 & 71.4 & 69.4 & 78.1 & 74.7 & 0.00066 \\
\hline 895.01999 & 3.77050661 & 14.8199492 & 1.7284232 & 14.7375 & 4396.25 & 3956.79038 & 71.3 & 69.4 & 78.3 & 74.7 & 0.00086 \\
\hline 900.01999 & 3.76362864 & 14.8199492 & 1.72904864 & 14.7375 & 4385.75 & 3960.76331 & 71.3 & 69.4 & 78.3 & 74.7 & 0.000861 \\
\hline 905.01999 & 3.77926039 & 14.8199492 & 1.72904864 & 14.7375 & 4396.25 & 3963.24639 & 71.3 & 69.4 & 78.2 & 74.6 & 0.00086 \\
\hline 910.01999 & 3.7623781 & 14.8199492 & 1.72904864 & 14.73825 & 4386.25 & 3958.52854 & 71.4 & 69.4 & 78.1 & 74.7 & 0.000861 \\
\hline 915.06 & 3.76800553 & 14.8206988 & 1.72904864 & 14.73825 & 4396 & 3954.3073 & 71.4 & 69.4 & 78.2 & 74.6 & 0.000861 \\
\hline 920.01999 & 3.77113188 & 14.8191996 & 1.72904864 & 14.73825 & 4396.25 & 3959.02515 & 71.5 & 69.4 & 78.2 & 74.7 & 0.000861 \\
\hline
\end{tabular}




\begin{tabular}{|c|c|c|c|c|c|c|c|c|c|c|c|}
\hline \multicolumn{12}{|c|}{$\begin{array}{l}\text { TEST: EX102147 } 14 \text { Feb } 1997 \text { WEC-SPOOL } \\
\text { Measured Hydrogen Concentration = 4150ppm }\end{array}$} \\
\hline SECOND & \begin{tabular}{|l|} 
MAIN DUCT \\
DIFF (IN H2O
\end{tabular} & \begin{tabular}{|l|} 
MAIN DUCT \\
ABS (PSIA)
\end{tabular} & \begin{tabular}{|l|} 
GAS INJECT \\
DIFF (IN H2O)
\end{tabular} & \begin{tabular}{|l|} 
GAS INJECT \\
ABS (PSIA)
\end{tabular} & \begin{tabular}{|l|} 
NEWPORT \\
(PPM)
\end{tabular} & $\frac{\text { SMC }}{\text { (PPM) }}$ & \begin{tabular}{|l|} 
AMBIENT \\
TEMP. (F)
\end{tabular} & \begin{tabular}{|l|} 
GAS INJECT \\
TEMP. (F)
\end{tabular} & \begin{tabular}{|l|} 
MAIN DUCT \\
TEMP. (F)
\end{tabular} & \begin{tabular}{|l|} 
WEC CELL \\
TEMP. (F)
\end{tabular} & $\begin{array}{l}\text { WEC CELL } \\
\text { Nolts) }\end{array}$ \\
\hline & & & & & & & & & & & \\
\hline 0.01001 & 3.75987702 & 14.7524832 & -0.007297888 & 14.5845 & 31.125 & 36.6029992 & 70.9 & 70.7 & 77.7 & 73.8 & 0.000213 \\
\hline 5.019989 & 3.77238242 & 14.7532329 & 1.66150112 & 14.664 & 31.275 & \begin{tabular}{|l|}
37.3230924 \\
\end{tabular} & 70.9 & 70.5 & 77.7 & 73.8 & 0.000213 \\
\hline 10.01999 & 3.76425391 & \begin{tabular}{|l|l|}
14.7532329 \\
\end{tabular} & 1.66462832 & 14.66625 & 137.4 & 827.116348 & 70.9 & 70.7 & 77.7 & 73.8 & 0.000248 \\
\hline 15.01999 & 3.7748835 & 14.750984 & 1.7690768 & 14.67225 & 619.75 & 2092.24561 & 70.9 & 70.8 & $\pi .7$ & 73.8 & 0.000326 \\
\hline 20.01999 & 3.77050661 & 14.7539825 & 1.772204 & 14.6745 & 1198.5 & 2884.84474 & 70.9 & 70.7 & 77.7 & 73.8 & 0.000409 \\
\hline 25.01999 & 3.77425823 & 14.7524832 & 1.772204 & 14.6715 & 1741 & 3354.89179 & 70.8 & 70.7 & 77.6 & 73.8 & 0.000488 \\
\hline 30.01999 & 3.76362864 & 14.7524832 & 1.77157856 & 14.6715 & 2203 & 3622.56781 & 70.8 & 70.8 & 77.7 & 73.8 & 0.000552 \\
\hline 35.29001 & 3.76050220 & 14.7517336 & 1.772204 & 14.6745 & 2543 & 3759.88214 & 70.7 & 70.7 & 77.7 & 73.8 & 0.000607 \\
\hline 40.01999 & 3.77050661 & 14.7517336 & 1.77282944 & 14.6715 & 2862.5 & 3822.95237 & 70.7 & 70.7 & 77.7 & 73.8 & 0.000646 \\
\hline 45.01999 & 3.77238242 & 14.7532329 & 1.77345488 & 14.6715 & 3090.5 & 3865.66134 & 70.8 & 70.8 & $\pi .7$ & 73.8 & 0.000679 \\
\hline 50.01999 & 3.76175283 & 14.7502344 & 1.77282944 & 14.67075 & 3274.75 & 3896.94815 & 70.8 & 70.7 & 77.7 & 73.8 & 0.000706 \\
\hline 55.01999 & 3.76612972 & 14.7524832 & 1.772204 & 14.6715 & 3442.75 & 3923.02049 & 70.8 & 70.7 & $\pi .7$ & 73.8 & 0.000728 \\
\hline 60.01999 & 3.77300769 & 14.7539825 & 1.77282944 & 14.6715 & 3554.5 & 3933.20112 & 70.8 & 70.7 & 77.7 & 73.9 & 0.000744 \\
\hline 65.01999 & 3.75737594 & 14.7494847 & 1.77282944 & 14.6715 & 3664.25 & 3948.34791 & 70.7 & 70.7 & 7.6 & 73.9 & 0.000757 \\
\hline 70.01999 & 3.7686308 & 14.7532329 & 1.77345488 & 14.67075 & 3754.5 & 3962.50146 & 70.7 & 70.8 & 7.7 & 73.8 & 0.00077 \\
\hline 75.01999 & 3.77675931 & 14.7517336 & 1.77282944 & 14.67225 & 3814.75 & 3966.22608 & 70.6 & 70.7 & $\pi .7$ & 73.8 & 0.000778 \\
\hline 80.01999 & 3.77425823 & 14.7494847 & 1.77282944 & $\begin{array}{l}14.6715 \\
\end{array}$ & 3864.25 & 3975.41348 & 70.7 & 70.7 & 77.7 & 73.8 & 0.000786 \\
\hline 85.01999 & 3.76362864 & 14.7494847 & 1.77282944 & 14.67225 & 3914.75 & 3985.59411 & 70.7 & 70.7 & $\pi .7$ & 73.8 & 0.000792 \\
\hline 90.01999 & 3.7686308 & 14.7524832 & 1.77345488 & 14.67225 & 3954.75 & 3998.50612 & 70.7 & 70.8 & $\pi .7$ & 73.9 & 0.000799 \\
\hline 95.01999 & 3.75800121 & 14.750984 & 1.77282944 & 14.67225 & 3974.75 & 4006.45198 & 70.8 & 70.7 & $\pi .6$ & 73.9 & 000803 \\
\hline 100.02 & 3.77300769 & 14.7539825 & 1.77282944 & 14.6715 & 4015 & 4011.41814 & 70.7 & 70.7 & 77.7 & 73.9 & 0.000807 \\
\hline 105.02 & 3.77926039 & 14.7517336 & 1.77282944 & 14.67225 & 4035.25 & 4018.37076 & 70.7 & 70.8 & 77.7 & 73.9 & 0.000809 \\
\hline 110.02 & 3.78988998 & 14.7524832 & 1.77282944 & 14.6745 & 4054.75 & 4024.82677 & 70.8 & 70.7 & 7.8 & 73.8 & 0.000812 \\
\hline 115.02 & 9239106 & 7336 & 1.77282944 & 14.6745 & 4074.5 & 4021.59877 & 70.8 & 70.8 & 7.8 & 73.9 & 0.000815 \\
\hline 120.02 & 3.77738458 & 14.753 & 1.77345488 & 14.6745 & 4095.5 & 4025.82 & 70.7 & 70.8 & 77.8 & 73.9 & 0.000817 \\
\hline 125.02 & 3.78926471 & 14.7524832 & 1.77282944 & 14.6715 & 4104.5 & 4031.03447 & 70.8 & 70.8 & 77.8 & 73.9 & 0.000818 \\
\hline 130.02 & 3.7748835 & 14.7539825 & 1.77345488 & 14.6715 & 4115.25 & 4039.97356 & 70.8 & 70.7 & 77.8 & 73.9 & 0.000819 \\
\hline 135.02 & 3.7748835 & 14.7494847 & 1.77282944 & 14.67225 & 4125 & 4038.2354 & 70.7 & 70.8 & $\pi .7$ & 73.8 & 0.000821 \\
\hline 140.02 & 3.75987702 & 14.750984 & 1.77408032 & 14.67225 & 4135 & 4037.24217 & 70.7 & 70.8 & 7.7 & 74 & 0.000823 \\
\hline 145.02 & 3.7686308 & 14.7502344 & 1.77345488 & 14.673 & 4135.25 & 4045.43634 & 70.7 & 70.8 & 7.8 & 74 & 0.000823 \\
\hline 150.02 & 3.75862648 & 14.7502344 & 1.77408032 & 14.6715 & 4150 & 4046.42957 & 70.7 & 70.8 & 7.8 & 74 & 0.000825 \\
\hline 155.02 & 3.76112756 & 14.7494847 & 1.77345488 & 14.67225 & 4155.25 & 4053.38219 & 70.6 & 70.8 & 77.7 & 73.9 & 0.000825 \\
\hline 160.02 & 3.77050661 & 14.750984 & 1.77282944 & 14.67225 & 4165.5 & 4060.58312 & 70.5 & 70.8 & $\pi .7$ & 73.9 & 0.000827 \\
\hline 165.02 & 3.76800553 & 14.7517336 & 1.77282944 & 14.67225 & 4170.75 & 4062.07297 & 70.4 & 70,8 & 7.7 & 73.9 & 0.000828 \\
\hline 170.02 & 3.75737594 & 14.7502344 & 1.77282944 & 14.673 & 4175 & 4064.80436 & 70.4 & 70.8 & 77.6 & 73.9 & 0.000829 \\
\hline 175.02 & 3.76675499 & 14.7517336 & 1.77408032 & 14.67225 & 4185.5 & 4066.54252 & 70.4 & 70.8 & 77.6 & 73.9 & 0.00083 \\
\hline 180.02 & 3.75424959 & 14.7502344 & 1.77282944 & 14.67225 & 4195.25 & 4069.2739 & 70.4 & 70.8 & 77.5 & 74 & 0.000831 \\
\hline 185.02 & 3.75675067 & 14.7502344 & 1.77345488 & 14.6715 & 4195 & 4070.01883 & 70.4 & 70.8 & 7.5 & 73.9 & 0.000832 \\
\hline
\end{tabular}

HNF-SD-WM-TRP-275 REVO APPENDIX C, Page
Englneering Testing Laboratory 313/97 


\begin{tabular}{|c|c|c|c|c|c|c|c|c|c|c|c|}
\hline SECOND & MAIN DUCT & MAIN DUCT & GAS INJECT & GAS INJECT & NEWPORT & SMC & AMBIENT & GAS INJECT & MAIN DUCT & WEC CELL & WEC CELL \\
\hline & DIFF (IN H2O & ABS (PSIA) & DIFF (IN H2O) & ABS (PSIA) & (PPM) & (PPM) & TEMP. (F) & TEMP. (F) & TEMP. $(F)$ & TEMP. (F) & (Volts) \\
\hline 190.02 & 3.76362864 & 14.7502344 & 1.77345488 & 14.67225 & 4205.25 & 4070.26714 & 70.5 & 70.8 & 77.5 & 74 & 0.000832 \\
\hline 195.02 & 3.76988134 & 14.7502344 & 1.77282944 & 14.67225 & 4204.75 & 4073.74345 & 70.5 & 70.8 & 77.5 & 74 & 0.000833 \\
\hline 200.02 & 3.76612972 & 14.7487351 & 1.77345488 & 14.6715 & 4205.75 & 4081,93761 & 70.5 & 70.8 & 77.6 & 73.9 & 0.000834 \\
\hline 205.02 & 3.75737594 & 14.7524832 & 1,77282944 & 14.67075 & 4215.5 & 4083.92408 & 70.5 & 70.8 & 77.6 & 73.9 & 0.000833 \\
\hline 210.02 & 3.7686308 & 14.7502344 & 1.77345488 & 14.67225 & 4215 & 4086.90377 & 70.5 & 70.8 & 77.5 & 73.9 & 0.000834 \\
\hline 215.02 & 3.7623781 & 14.7524832 & 1.77345488 & 14.6715 & 4225.25 & 4086.65546 & 70.5 & 70.8 & 77.5 & 74 & 0.000835 \\
\hline 220.02 & 3.77300769 & 14.750984 & 1.77345488 & 14.67225 & 4225.25 & 4091.37332 & 70.6 & 70.8 & 77.6 & 73.9 & 0.000836 \\
\hline 225.02 & 3.76800553 & 14.7494847 & 1.77282944 & 14.673 & 4235.25 & 4093.60809 & 70.6 & 70.8 & 77.5 & 74 & 0.000837 \\
\hline 230.02 & 3.76175283 & 14.7502344 & 1.77345488 & 14.67225 & 4235.5 & 4096.58778 & 70.6 & 70.8 & 77.5 & 74 & 0.000837 \\
\hline 235.02 & 3.78488782 & 14.7502344 & 1.77282944 & 14.67225 & 4235.5 & 4092.11824 & 70.6 & 70.8 & 77.6 & 74 & 0.000837 \\
\hline 240.02 & 3.75987702 & 14.750984 & 1.77345488 & 14.67225 & 4245.5 & 4086.15885 & 70.6 & 70.8 & 77.6 & 73.9 & 0.000837 \\
\hline 245.02 & 3.775500877 & 14.7487351 & 1.772204 & 14.67225 & 4245.25 & 4082.68254 & 70.6 & 70.8 & 77.6 & 74 & 0.000837 \\
\hline 250.02 & 3.7686308 & 14.7502344 & 1.77282944 & 14.6715 & 4245.5 & 4081.441 & 70.6 & 70.8 & $\pi 7.6$ & 73.9 & 0,000838 \\
\hline 255.02 & 3.77363296 & 14.7502344 & 1.772204 & 14.67225 & 4245.25 & 4081.19269 & 70.7 & 70.8 & 77.5 & 73.9 & 0.000838 \\
\hline 260.02 & 3.76738026 & 14.7502344 & 1.772204 & 14.6715 & 4245.5 & 4083.67577 & 70.6 & 70.8 & 77.5 & 74 & 0.000838 \\
\hline 265.02 & 3.77988566 & 14.7502344 & 1.77282944 & 14.6715 & 4245.25 & 4084.17238 & 70.7 & 70.8 & 77.5 & 74 & 0.000838 \\
\hline 270.02 & 3.77926039 & 14.7532329 & 1.77282944 & 14.67225 & 4255.25 & 4084.17238 & 70.7 & 70.8 & 77.5 & 74 & 0.000838 \\
\hline 275.02 & 3.7748835 & 14.7517336 & 1.77095312 & 14.67225 & 4255.75 & 4080.69607 & 70.6 & 70.8 & $\pi .6$ & 74 & 0.000839 \\
\hline 280.02 & 3.76988134 & 14.7517336 & 1.77032768 & 14.6715 & 4255.5 & 4078.95792 & 70.7 & 70.8 & 77.6 & 74 & 0.000839 \\
\hline 285.02 & 3.76675499 & 14.750984 & 1.77345488 & 14.67075 & 4255.5 & 4085.41392 & 70.7 & 70.8 & 77.5 & 74 & 0.000841 \\
\hline 290.02 & 3.77800985 & 14.7502344 & 1.77095312 & 14.67225 & 4265.5 & 4092.36655 & 70.7 & 70.8 & 77.6 & 74 & 0.00084 \\
\hline 295.02 & 3.76675499 & 14.7494847 & 1.77095312 & 14.6715 & 4265.5 & 4090.38008 & 70.7 & 70.8 & 77.6 & 74 & 0,00084 \\
\hline 300.02 & 3.78176147 & 14.7502344 & 1.77032768 & 14.6715 & 4256.25 & 4088.89024 & 70.8 & 70.8 & 77.6 & 74 & 0.000839 \\
\hline 305.02 & 3.77550877 & 14.750984 & 1.77157856 & 14.6715 & 4255.5 & 4085.16562 & 70.8 & 70.8 & 77.7 & 74 & 0.00084 \\
\hline 310.03 & 3.75675067 & 14.750984 & 1.77032768 & 14.6715 & 4255.75 & 4081.19269 & 70.8 & 70.8 & 77.6 & 74 & 0.00084 \\
\hline 315.02 & 3.75800121 & 44.7517336 & 1.77095312 & 14.67225 & 4255.25 & 4089.38685 & 70.8 & 70.8 & 77.7 & 74 & 0.000839 \\
\hline 320.02 & 3.76675499 & 14.7502344 & 1.7690768 & 14.67225 & 4265.75 & 4088.14531 & 70.8 & 70.8 & 77.7 & 74 & 0.00084 \\
\hline 325.02 & 3.7561254 & 14.750984 & 1.76845136 & 14.6715 & 4265.5 & 4093.11147 & 70.8 & 70.8 & 77.8 & 74 & 0.000841 \\
\hline 330.02 & 3.76175280 & 14.7494847 & 1.7690768 & 14.6715 & 4265.5 & 4087.897 & 70,8 & 70.8 & 77.7 & 74 & 0,000841 \\
\hline 335.02 & 3.77113188 & 14.7502344 & 1.7690768 & 14.6715 & 4265.75 & 4086.15885 & 70.8 & 70.9 & 77.7 & 74 & 0,000841 \\
\hline 340.02 & 3.7748835 & 14.7494847 & 1.77095312 & 14.6715 & 4265.5 & 4086.65546 & 70.8 & 70.8 & 77.7 & 74 & 0.000842 \\
\hline 345.02 & 3.76675499 & 14.7502344 & 1.7690768 & 14.6715 & 4265.75 & 4089.63516 & 70.8 & 70.8 & 77.7 & 74 & 0.000842 \\
\hline 350.02 & 3.7686308 & 14.7487351 & 1.7690768 & 14.67075 & 4265,5 & 4096.58778 & 70,8 & 70.9 & 77.8 & 74 & 0.000843 \\
\hline 355.03 & 3.75362432 & 14.7494847 & 1.7690768 & 14.67075 & 4275.5 & 4096.83609 & 70.8 & 70.8 & 77.8 & 74 & 0.000842 \\
\hline 360.02 & 3.77363296 & 14.7502344 & 1.76970224 & 14.67075 & 4275.5 & 4098.07763 & 70.8 & 70.8 & 77.8 & 74 & 0.000842 \\
\hline 365.02 & 3.77175715 & 14.7502344 & 1.76970224 & 14.6715 & 4275.5 & 4084.91731 & 70.8 & 70.8 & 77.7 & 74 & 0.000842 \\
\hline 370.02 & 3.77926039 & 14.750984 & 1.7690768 & 14.6715 & 4275.75 & 4084.669 & 70.8 & 70.8 & 77.8 & 74 & 0.000842 \\
\hline 375.02 & 3.76050229 & 14.750984 & 1.76970224 & 14.6715 & 4275.5 & 4085.91054 & 70.8 & 70.8 & 77.7 & 74 & 0.000843 \\
\hline 380.02 & 3.7561254 & 14.7517336 & 1.7690768 & 14.6715 & 4275.75 & 4083.17915 & 70.8 & 70.8 & $\pi .7$ & 74 & 0.000842 \\
\hline 385.02 & 3.77738458 & 14.750984 & 1.7690768 & 14.6715 & 4275.5 & 4086.40716 & 70.8 & 70.8 & $\pi .7$ & 74 & 0.000843 \\
\hline 390.02 & 3.7686308 & 14.7502344 & 1.7690768 & 14.67075 & 4275.25 & 4087.15208 & 70.7 & 70.8 & 77.7 & 74 & 0.000843 \\
\hline 395.02 & 3.76487918 & 14.7502344 & 1.7690768 & 14.67225 & 4285.75 & 4076.22653 & 70.8 & 70.9 & 77.7 & 74 & 0.000844 \\
\hline
\end{tabular}




\begin{tabular}{|c|c|c|c|c|c|c|c|c|c|c|c|}
\hline SECOND & \begin{tabular}{|l|} 
MAIN DUCT \\
DIFF (IN H2O
\end{tabular} & \begin{tabular}{|l|} 
MAIN DUCT \\
ABS (PSIA)
\end{tabular} & \begin{tabular}{|l|} 
GAS INJECT \\
DIFF (IN H2O)
\end{tabular} & \begin{tabular}{|l} 
GAS INJECT \\
ABS (PSIA)
\end{tabular} & \begin{tabular}{|l} 
NEWPORT \\
(PPM)
\end{tabular} & \begin{tabular}{|l|} 
SMC \\
(PPM)
\end{tabular} & \begin{tabular}{|l|} 
AMBIENT \\
TEMP. (F)
\end{tabular} & \begin{tabular}{|l|} 
GAS INJECT \\
TEMP. (F)
\end{tabular} & $\begin{array}{l}\text { MAIN DUCT } \\
\text { TEMP. (F) }\end{array}$ & \begin{tabular}{|l} 
WEC CELL \\
TEMP. (F)
\end{tabular} & $\begin{array}{l}\text { WEC CELL } \\
\text { Nolts) }\end{array}$ \\
\hline & & & & & & & & & & & \\
\hline 400.02 & 3.77363296 & 14.7502344 & 1.7690768 & 14.6715 & 4285 & 4078.4613 & 70.8 & 70.9 & 77.6 & 74 & 0.000844 \\
\hline 405.02 & 3.76988134 & 14.7502344 & 1.7690768 & 14.67225 & 4285.5 & 4081.19269 & 70.8 & 70.9 & 77.6 & 74 & 0.000843 \\
\hline 410.02 & 3.7686308 & 14.750984 & 1.76845136 & 14.67075 & 4285.25 & 4077.46807 & 70.8 & 70.9 & 77.6 & 74 & 0.000843 \\
\hline 415.02 & 3.76675499 & 14.7502344 & 1.7690768 & 14.6715 & 4285.25 & 4073.49514 & 70.7 & 70.8 & 77.6 & 74 & 0.000843 \\
\hline 420.02 & 3.77300769 & 14.7487351 & 1.7690768 & 14.67225 & 4285.5 & 4076.22653 & 70.8 & 70.9 & 77.6 & 74.1 & 0.000845 \\
\hline 425.02 & 3.75862648 & 14.7502344 & 1.7690768 & 14.67225 & 4285.5 & 4079.45453 & 70.8 & 70.9 & 77.7 & 74.1 & 0.000844 \\
\hline 430.02 & 3.77113188 & 14.7517336 & 1.76845136 & 14.6715 & 4285.75 & 4075.72991 & 70.8 & 70.9 & 77.7 & 74 & 0.000844 \\
\hline 435.02 & 3.7686308 & 14.7539825 & 1.76845136 & 14.6715 & 4285.25 & 4081.441 & 70.8 & 70.9 & 77.7 & 74.1 & 0.000843 \\
\hline 440.02 & 3.7686308 & 14.7539825 & 1.76657504 & 14.6715 & 4286 & 4083.67577 & 70.8 & 70.9 & 77.7 & 74.1 & 0.000844 \\
\hline 445.11 & 3.76800553 & 14.750984 & 1.76845136 & 14.67225 & 4285.5 & 4087.40039 & 70.8 & 70.9 & 77.7 & 74 & 0.000843 \\
\hline 450.02 & 3.76362864 & 14.7502344 & 1.76720048 & 14.6715 & 4285.75 & 4095.09794 & 70.8 & 70.9 & $\pi 7.7$ & 74 & 0.000843 \\
\hline 455.02 & 3.77863512 & 14.7539825 & 1.76657504 & 14.6715 & 4285.5 & 4101.05733 & 70.8 & 70.9 & 77.8 & 74 & 0.000844 \\
\hline 460.02 & 3.76425391 & 14.7502344 & 1.76657504 & 14.67225 & 4285.25 & 4100.0641 & 70.8 & 70.9 & 77.8 & 74 & 0.000843 \\
\hline 465.1 & 3.7561254 & 14.750984 & 1.7659496 & 14.6715 & 4285.25 & 4099.31917 & 70.8 & 70.9 & 77.7 & 74.1 & 0.000845 \\
\hline 470.02 & 3.76175283 & 14.7517336 & 1.76657504 & 14.6715 & 4285.5 & 4092.61486 & 70.8 & 70.9 & 77.8 & 74 & 0.000845 \\
\hline 475.11 & 3.77238242 & 14.750984 & 1.76657504 & 14.67075 & 4285.75 & 4090.13178 & 70.9 & 70.9 & 77.8 & 74.1 & 0.000845 \\
\hline 480.02 & 3.76112756 & 14.750984 & 1.76657504 & 14.67225 & 4285.5 & 4100.3124 & 70.9 & 70.9 & 77.8 & 74.1 & 0,000845 \\
\hline 485.02 & 3.75737594 & 14.7502344 & 1.76657504 & 14.67225 & 4285.75 & 4099.81579 & 71 & 70.9 & 77.8 & 74.1 & 0.000845 \\
\hline 490.02 & 3.76925607 & 14.7494847 & 1.76655504 & 14.67225 & 4295.25 & 4095.84286 & 71.1 & 70.9 & 77.8 & 74.1 & 0.000846 \\
\hline 495.02 & 3.76050229 & 14.7502344 & 1.76720048 & 14.67075 & 4295.75 & 4085.91054 & 71.1 & 71 & 77.8 & 74 & 0.000846 \\
\hline 500.02 & 3.76675499 & 14.7494847 & 1.76657504 & 14.6715 & 4295.75 & 4087.15208 & 71.2 & 70.9 & 77.9 & 74.1 & 0.000846 \\
\hline 505.02 & 3.76425391 & 14.750984 & 1.7659496 & 14.6715 & 4295.5 & 4085.16562 & 71.2 & 71 & $\pi 7.9$ & 74.1 & 0.000847 \\
\hline 510.02 & 3.7686308 & 14.750984 & 1.7659496 & 14.6715 & 4296 & 4088.39362 & 71.1 & 70.9 & 77.8 & 74.1 & 0.000846 \\
\hline 515.03 & 3.77113188 & 14.7502344 & 1.76720048 & 14.6715 & 4305.75 & 4092.36655 & 71.1 & 71 & 77.8 & 74.1 & 0.000845 \\
\hline 520.02 & 3.7561254 & 14.750984 & 1.76720048 & 14.67 & 4305.25 & 4084.91731 & 71 & 71 & 77.8 & 74.1 & 0.000847 \\
\hline 525.02 & 3.77363296 & 14.750984 & 1.766557504 & 14.67 & 4303.5 & 4081.19269 & 70.9 & 71 & 77.8 & 74.1 & 0.000846 \\
\hline 530.06 & 3.76050229 & 14.7494847 & 1.76657504 & $\$ 4.6715$ & 4296 & 4085.41392 & 70.8 & 70.9 & 77.7 & 74.1 & 0.000847 \\
\hline 535.02 & 3.76112756 & 14.7524832 & 1.7659496 & 14.6715 & 4296.5 & 4079.70284 & 70.8 & 70.9 & 77.8 & 74.1 & 0.000846 \\
\hline 540,02 & 3.76612972 & 14.7517336 & 1.76782592 & 14.67 & 4306 & 4079.45453 & 70.8 & 71 & 77.7 & 74.1 & 0.000846 \\
\hline 545.02 & 3.76175283 & 14.7502344 & 1.76657504 & 14.6715 & 4296 & 4080.44776 & 70.8 & 71 & 77.7 & 74.1 & 0.000845 \\
\hline 550.05 & 3.7686308 & 14.7517336 & 1.76720048 & 14.66925 & 4295.75 & 4077.96468 & 70.8 & 71 & 77.7 & 74.1 & 0.000846 \\
\hline 555.02 & 3.76800553 & 14.7479855 & 1.76720048 & 14.6715 & 4295.75 & 4078,95792 & 70.7 & 71 & 77.6 & 74.1 & 0.000846 \\
\hline 560.05 & 3.75550013 & 14.7494847 & 1.76782592 & 14.6715 & 4296 & 4079.95115 & 70.8 & 71 & 77.6 & 74.1 & 0.000845 \\
\hline 565.02 & 3.75550013 & 14.7502344 & 1.76782592 & 14.66925 & 4295.75 & 4086.65546 & 70.7 & 71 & 77.7 & 74.1 & 0.000844 \\
\hline 570.02 & 3.75237378 & 94.7502344 & 1.76720048 & 14.6715 & 4295.75 & 4079.95115 & 70.7 & 71 & 77.6 & 74.1 & 0.000845 \\
\hline 575.06 & 3.76175283 & 14.7464863 & 1.76657504 & 14.67075 & 4295.75 & 4079.45453 & 70.7 & 71 & 77.6 & 74.1 & 0.000845 \\
\hline 580.02 & 3.76738026 & 14.750984 & 1.76720048 & 14.6685 & 4296 & 4082.68254 & 70.7 & 71 & 77.6 & 74.1 & 0.000845 \\
\hline 585.02 & 3,75987702 & 14.7502344 & 1.76657504 & 14.67075 & 4296.25 & 4077.46807 & 70.7 & 71 & 77.6 & 74.1 & 0.000845 \\
\hline 590.07 & 3.77175715 & 14.7502344 & 1.76657504 & 14.6715 & 4296 & 4083.42746 & 70.7 & 71 & 77.6 & 74.1 & 0.000844 \\
\hline 595.02 & 3.77300769 & 14.7502344 & 1.7659496 & 14.6715 & 4295.75 & 4086.15885 & 70.7 & 71 & 77.7 & 74.1 & 0.000845 \\
\hline 600.02 & 3.76800553 & 14.7517336 & 1.76657504 & 14.67 & 4295.75 & 4085.41392 & 70.7 & 71 & 77.7 & 74.1 & 0.000845 \\
\hline 605.02 & 3.76175283 & 14.7479355 & 1.7659496 & 14.6715 & 4296 & 4094.84963 & 70.8 & 71 & 77.6 & 74.1 & 0.000845 \\
\hline
\end{tabular}




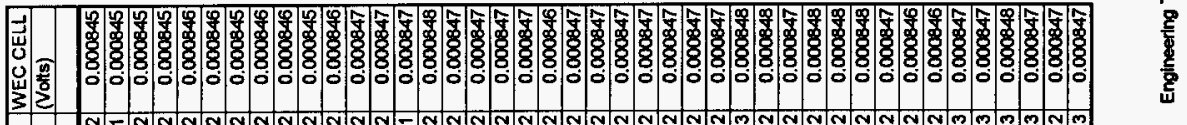

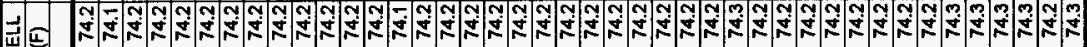

$\vec{c}$

岸育

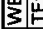

0勿

a

za

$\frac{\mathrm{s}}{\mathrm{z}}$

5

岃

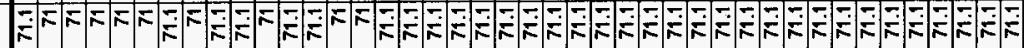

$\frac{z}{\cos }$

ô.

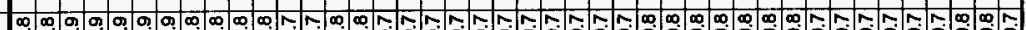

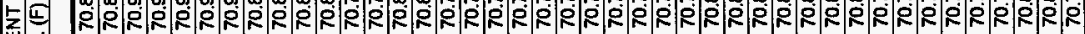

剀

密

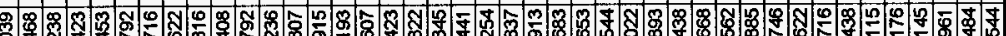

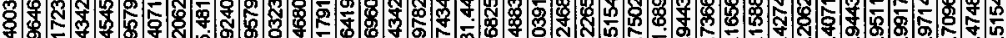

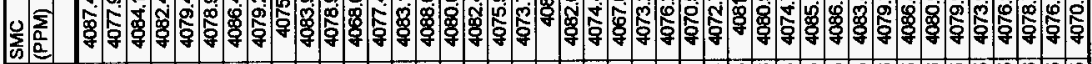

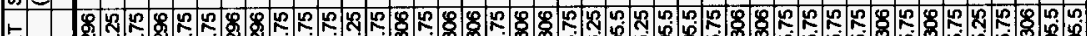

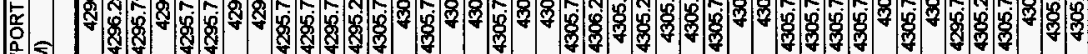

岃郭

宸吾

-

Ł 象

岂至

an

ธิ巛

穴

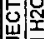

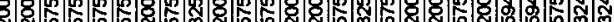

$\underline{\underline{z}} \geqq$

is

合言

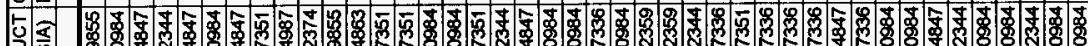

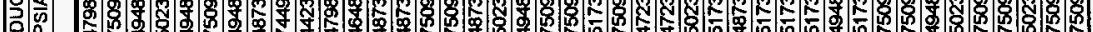

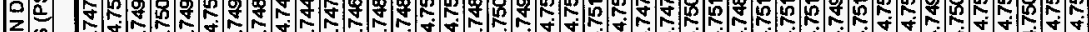

直置

-

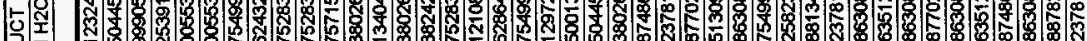

良至

空崔

学

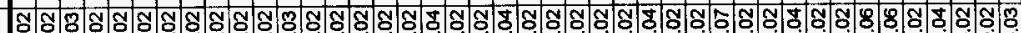

ż 0. 


\begin{tabular}{|c|c|c|c|c|c|c|c|c|c|c|c|}
\hline SECOND & \begin{tabular}{|l|} 
MAIN DUCT \\
DIFF (IN H2O \\
\end{tabular} & \begin{tabular}{|l} 
MAIN DUCT \\
ABS (PSIA)
\end{tabular} & \begin{tabular}{|l|} 
GAS INJECT \\
DIFF (IN H2O) \\
\end{tabular} & $\begin{array}{l}\text { GAS INJECT } \\
\text { ABS (PSIA) }\end{array}$ & $\begin{array}{l}\text { NEWPORT } \\
\text { (PPM) } \\
\end{array}$ & $\begin{array}{l}\text { SMC } \\
\text { (PPM) }\end{array}$ & \begin{tabular}{|l|} 
AMBIENT \\
TEMP. (f) \\
\end{tabular} & \begin{tabular}{|l|} 
GAS INJECT \\
TEMP. (F) \\
\end{tabular} & \begin{tabular}{|l|} 
MAIN DUCT \\
TEMP. (F) \\
\end{tabular} & $\begin{array}{l}\text { WEC CELL } \\
\text { TEMP. (F) } \\
\end{array}$ & $\begin{array}{l}\text { WEC CELL } \\
\text { (Volts) }\end{array}$ \\
\hline & & & & & & & & & & & \\
\hline 820.02 & 3.75737594 & 14.7494847 & 1.76469872 & 14.6715 & 4305.5 & 4069.77052 & 70.7 & 71.1 & 77.8 & 74.3 & 0.000848 \\
\hline 825.02 & 3.75362432 & 14.7517336 & 1.76532416 & 14.673 & 4305.5 & 4074.48837 & 70.8 & 71.1 & 77.9 & 74.3 & 0.000848 \\
\hline 830.04 & 3.76050229 & 14.7524832 & 1.76532416 & 14.6715 & 4305.75 & 4074.24006 & 70.8 & 71.1 & 77.9 & 74.3 & 0.000847 \\
\hline 835.02 & 3.7686308 & 14.750984 & 1.76469872 & 14.6715 & 4305.75 & 4074.24006 & 70.7 & 71.1 & 77.9 & 74.2 & 0.000848 \\
\hline 840.02 & 3.7686308 & 14.7502344 & 1.76469872 & 14.67075 & 4316 & 4077.96468 & 70.8 & 71.1 & 77.9 & 74.2 & 0.000848 \\
\hline 845.03 & 3.76175283 & 14.750984 & 1.76469872 & 14.6715 & 4306.25 & 4075.4816 & 70.8 & 71.1 & 77.9 & 74.2 & 0.000848 \\
\hline 850.02 & 3.76612972 & 14.7479855 & 1.76407328 & 14.67075 & 4316 & 4074.98499 & 70.8 & 71.1 & 78 & 74.3 & 0.000847 \\
\hline 855.02 & 3.76300337 & 14.750084 & 1.76469872 & 14.6715 & 4306 & 4080.69607 & 70.9 & 71.1 & 78 & 74.3 & 0.000848 \\
\hline 860.04 & 3.77113188 & 14.750984 & 1.76532416 & 14.6715 & 4305.75 & 4087.897 & 70.9 & 71.1 & 78 & 74.2 & 0.000846 \\
\hline 865,02 & 3.76487918 & 14.7494847 & 1.76469872 & 14.6715 & 4305.75 & 4081.6893 & 70.8 & 71.2 & 78 & 74.3 & 0.000848 \\
\hline 870.02 & 3.7623781 & 14.7502344 & 1.7659496 & 14.67225 & 4305.75 & 4077.71638 & 70.9 & 71.2 & 78 & 74.3 & 0.000848 \\
\hline 875.05 & 3.76925607 & 14.7502344 & 1.76532416 & 14.6715 & 4305.5 & 4074.73668 & 70.9 & 71.2 & 78 & 74.3 & 0,000847 \\
\hline 880.02 & 3.76612972 & 14.7517336 & 1.76469872 & 14.67075 & 4306 & 4075.97822 & 70.9 & 71.2 & 77.9 & 74.3 & 0.000846 \\
\hline 885.02 & 3.75237378 & 14.7494847 & 1.76532416 & 14.6715 & 4305.5 & 4075.4816 & 70.9 & 71.1 & 77.9 & 74.2 & 0.000846 \\
\hline 890.04 & 3.77675931 & 14.7517336 & 1.76469872 & 14.67075 & 4305.75 & 4085.16562 & 70.9 & 71.2 & 78 & 74.3 & 0.000847 \\
\hline 895.05 & 3.7686308 & 14.7502344 & 1.76407328 & 14.6715 & 4306.25 & 4086.65546 & 70.9 & 71.2 & 78 & 74.2 & 0,000846 \\
\hline 900.02 & 3.76362864 & 14.750984 & 1.76407328 & 14.67075 & 4305.5 & 4081.6893 & 70.9 & 71.2 & 77.9 & 74.3 & 0.000847 \\
\hline 905.04 & 3.76487918 & 14.7532329 & 1.76407328 & 14.67 & 4306 & 4071.50868 & 70.9 & 71.2 & 78 & 74.3 & 0.000847 \\
\hline 910.02 & 3.77550877 & 14.750984 & 1.76469872 & 14.6715 & 4306 & 4071.01206 & 70.8 & 71.2 & 78 & 74.2 & 0.000846 \\
\hline
\end{tabular}




\begin{tabular}{|c|c|c|c|c|c|c|c|c|c|c|c|}
\hline $\begin{array}{l}\text { TEST: FGI2 } \\
\text { Measured H }\end{array}$ & $\begin{array}{l}2221122 \text { Feb } 1 \\
\text { tydrogen Conce }\end{array}$ & sentration $=21$ & Sppm & & & & & & & & \\
\hline SECONDS & MAIN DUCT & MAIN DUCT & GAS INJECT & GAS INJEC & |NEWPORT| & SMC & AMBIENT & GAS INJECT & MAIN DUCT & WEC CELL & -WWEC CELL \\
\hline & DIFF (IN H2O & ABS (PSIA) & DIFF (IN H2O & ABS (PSIA) & (PPM) & (PPM) & TEMP.(F) & TEMP.(F) & TEMP.(F) & TEMP.(F) & (VOLTS) \\
\hline 0.01001 & 3.7686308 & 14.7472359 & 0.327937952 & 14.71875 & 1.125 & 2.8082804 & 69.4 & 68.7 & 68.4 & 69 & 0.00023 \\
\hline 5.02002 & 3.77926039 & 14.7472359 & 3.98751248 & 15.05775 & 1.075 & 1.5915712 & 69.4 & 68.7 & 68.3 & 68.9 & 0.000231 \\
\hline 10.02002 & 3.80739754 & 14.7464863 & 4.00314848 & 15.06075 & 1.125 & 0.2755388 & 69.4 & 68.7 & 68.3 & 69 & 0.000233 \\
\hline 15.02002 & 3.74612108 & 14.7472359 & 4.01628272 & 15.06225 & 10.95 & 28.1605272 & 69.4 & 68.7 & 68.2 & 68.9 & 0.000235 \\
\hline 20.02002 & 3.7686308 & 14.7472359 & 4.0269152 & 15.066 & 40.775 & 46.7091348 & 69.4 & 68.8 & 68.4 & 69 & 0.00024 \\
\hline 25.02002 & 3.74737162 & 14.7464863 & 4.02879152 & 15.0645 & 61.25 & 55.25093 & 69.3 & 68.8 & 68,4 & 69 & 0.000244 \\
\hline 30.02002 & 3.75487486 & 14.7472359 & 4.03191872 & 15.06525 & 91.075 & 58.35478 & 69.3 & 68.8 & 68.3 & 69 & 0.000247 \\
\hline 35.02002 & 3.76050229 & 14.7464863 & 4.03254416 & 15.06525 & 111.075 & 59.472166 & 69.4 & 68.8 & 68.4 & 68.9 & 0.00025 \\
\hline 40.02002 & 3.75862648 & 14.7464863 & 4.03442048 & 15.06525 & 131.025 & 59.2486888 & 69.4 & 68.8 & 68.2 & 68.9 & 0.000252 \\
\hline 45.02002 & 3.77988566 & 14.7479855 & 4.03379504 & 15.0645 & 141.025 & 59.0500424 & 69.5 & 68.8 & 68.4 & 69 & 0.000255 \\
\hline 50.02002 & 3.76050229 & 14.7479855 & 4.03129328 & 15.0645 & 151.475 & 59.6708124 & 69.4 & 68.8 & 68.5 & 69 & 0.000257 \\
\hline 55.02002 & 3.75049797 & 14.7472359 & 4,03129328 & 15.066 & 161.025 & 59.1245348 & 69.5 & 68.8 & 68.4 & 69 & 0.000256 \\
\hline 60.02002 & 3.77926039 & 14.744987 & 4.03504592 & 15.06525 & 171.175 & 59.1245348 & 69.6 & 68.8 & 68.3 & 68.9 & 0.000258 \\
\hline 65.02002 & 3.77300769 & 14.7464863 & 4.03254416 & 15.0645 & 181.075 & 59.0500424 & 69.6 & 68.8 & 68.3 & 68.9 & 0.000259 \\
\hline 70.02002 & 3.75852648 & 14.7457366 & 4.03129328 & 15.0645 & 181.375 & 58.1809644 & 69.6 & 68.8 & 68.5 & 68.9 & 0.000250 \\
\hline 75.02002 & 3.75862648 & 14.7472359 & 4.03129328 & 15.0645 & 191.3 & 58.5782572 & 69.7 & 68.8 & 68.4 & 68.9 & 0.00026 \\
\hline 80.02002 & 3.7623781 & 14.7479855 & 4.02879152 & 15.06525 & 190.9 & 59.2735196 & 69.6 & 68.8 & 68.5 & 69 & 0,000261 \\
\hline 85.02002 & 3.76362864 & 14.7472359 & 4.02879152 & 15.066 & 201.3 & 59.5714892 & 69.7 & 68.8 & 68.5 & 69 & 0.000261 \\
\hline 90.02002 & 3.75675067 & 14.7464863 & 4.02628976 & 15.066 & 201.05 & 58.8017344 & 69.7 & 68.9 & 68.3 & 68.9 & 0.000261 \\
\hline 95.02002 & 3.74549581 & 14.744987 & 4.02754064 & 15.06525 & 201.35 & 58.8265652 & 69.7 & 68.8 & 68.5 & 69 & 0.000261 \\
\hline 100.02 & 3.75424959 & 14.7479855 & 4.02754064 & 15.066 & 201.25 & $59.64598 \div 6$ & 69.7 & 68.9 & 68.3 & 68.9 & 0.000261 \\
\hline 105.02 & 3.75675067 & 14.7464863 & 4.02441344 & 15.066 & 210.825 & 59.4969968 & 69.6 & 68.8 & 68.5 & 69 & 0.000262 \\
\hline 110.02 & 3.76487918 & 14.7464863 & 4.02503888 & 15.06675 & 211.025 & 59.3728428 & 69.6 & 68.8 & 68.5 & 69 & 0.000262 \\
\hline 115.02 & 3.7561254 & 14.7472359 & 4.02441344 & 15.06375 & 211.1 & 59.4473352 & 69.6 & 68.9 & 68.4 & 69 & 0.000263 \\
\hline 120.02 & 3.7561254 & 14.7464863 & 4.02253712 & 15.06675 & 211.425 & 59.1741964 & 69.6 & 68.8 & 68.4 & 69 & 0.000262 \\
\hline 125.02 & 3.7623781 & 14.7479855 & 4.02253712 & 15.063 & 211.025 & 58.851396 & 69.5 & 68.9 & 68.3 & 69 & 0.000263 \\
\hline 130.02 & 3.76300337 & 14.7457366 & 4.02253712 & 15.063 & 210.85 & 59.9936128 & 69.5 & 68.9 & 68.5 & 69 & 0.000263 \\
\hline 135.02 & 3.77363296 & 14.7472359 & 4.02128624 & 15.06375 & 211.15 & 59.3976736 & 69.5 & 68.8 & 68.4 & 69 & 0.000264 \\
\hline 140.02 & 3.75800121 & 14.7464863 & 4.02003536 & 15.06375 & 210.95 & 58.851396 & 69.5 & 68.8 & 68.4 & 69.1 & 0.000263 \\
\hline 145.02 & 3.7561254 & 14.7472359 & 4.02003536 & 15.0645 & 211.425 & 59.6211508 & 69.5 & 68.9 & 68.4 & 69.1 & 0.000263 \\
\hline 150.02 & 3.7561254 & 14.7479855 & 4.02253712 & 15.06675 & 210.875 & 58.6279188 & 69.5 & 68.8 & 68.3 & 69 & 0.000263 \\
\hline 155.02 & 3.75237378 & 14.7479855 & 4.02003536 & 15.00525 & 211.025 & 58.851396 & 69.5 & 68.9 & 68.3 & 68.9 & 0.000263 \\
\hline 160.02 & 3.76175283 & 14.7464863 & 4.02003536 & 15.066 & 211.325 & 59.3231812 & 69.5 & 68.9 & 68.2 & 68.9 & 0.000263 \\
\hline 165.02 & 3.76738026 & 14.7472359 & 4.01940992 & 15.0645 & 210.65 & 59.9439512 & 69.5 & 68.9 & 68.3 & 69.1 & 0.000263 \\
\hline 170.02 & 3.75174851 & 14.7479855 & 4.0206608 & 15.06675 & 221.525 & 59.844628 & 69.4 & 68.9 & 68.2 & 69 & 0.000264 \\
\hline 175.02 & 3.76112756 & 14.7472359 & 4.01940992 & 15.06225 & 221.125 & 59.3231812 & 69.3 & 68.9 & 68.3 & 69.1 & 0.000263 \\
\hline 180.02 & 3.75800121 & 14.7472359 & 4.01940992 & 15.0645 & 221.425 & 60.0184436 & 69.3 & 68.8 & 68.1 & 68.9 & 0.000263 \\
\hline 185.02 & 3.75675067 & 14.7472359 & 4.02253712 & 15.0645 & 221.575 & 59.2486888 & 69.3 & 68.9 & $68 . \overline{2}$ & 68.9 & 0.000263 \\
\hline
\end{tabular}




\begin{tabular}{|c|c|c|c|c|c|c|c|c|c|c|c|}
\hline SECONDS & \begin{tabular}{|l} 
MAIN DUCT \\
DIFF (IN H2O
\end{tabular} & \begin{tabular}{|l|} 
MAIN DUCT \\
ABS (PSIA) \\
\end{tabular} & $\begin{array}{l}\text { GAS INJECT } \\
\text { DIFF (IN H2O }\end{array}$ & $\begin{array}{l}\text { GAS INJEC } \\
\text { ABS (PSIA) }\end{array}$ & $\begin{array}{l}\text { NEWPORT } \\
\text { (PPM) }\end{array}$ & \begin{tabular}{|l|} 
SMC \\
(PPM) \\
\end{tabular} & $\begin{array}{l}\text { AMBIENT } \\
\text { TEMP.(F) }\end{array}$ & \begin{tabular}{|l|} 
GAS INJECT \\
TEMP.(F) \\
\end{tabular} & \begin{tabular}{|l|} 
MAIN DUCT \\
TEMP.(F)
\end{tabular} & $\begin{array}{l}\text { WEC CELL } \\
\text { TEMP.(F) }\end{array}$ & \begin{tabular}{|l} 
WEC CELL \\
(VOLTS)
\end{tabular} \\
\hline & & & & & & & & & & & \\
\hline 190.02 & 3.7748835 & 14.7487351 & 4.02191168 & 15.06525 & 221.175 & 59.720474 & 69.3 & 68.9 & 68.4 & 69 & 0.000263 \\
\hline 195.02 & 3.7498727 & 14.7479855 & 4.02316256 & 15.06375 & 221.475 & 60.0681052 & 69.3 & 68.9 & 68.4 & 68.9 & 0.000264 \\
\hline 200.02 & 3.76425391 & 14,7479855 & 4.02253712 & 15.06525 & 221.35 & 60.4902288 & 69.4 & 68.9 & 68.4 & 69 & 0.000264 \\
\hline 205.02 & 3.7623781 & 14.7464863 & 4.02003536 & 15.0645 & 213 & 60.0184436 & 69.5 & 68.9 & 68.5 & 69 & 0.000264 \\
\hline 210.02 & 3.76112756 & 14.7472359 & 4.0206608 & 15.0645 & 221.375 & 58.9010576 & 69.6 & 68.9 & 68.4 & 68.9 & 0.000264 \\
\hline 215.02 & 3.76175283 & 14.7472359 & 4.02316256 & 15.06525 & 221.05 & 60.21709 & 69.6 & 68.9 & 68.2 & 68.8 & 0.000263 \\
\hline 220.14 & 3.75987702 & 14.7464863 & 4.02316256 & 15.06525 & 221.525 & 59.6956432 & 69.6 & 68.9 & 68.4 & 69 & 0.000264 \\
\hline 225.02 & 3.76487918 & 14.7479855 & 4.02253712 & 15.06525 & 221.575 & 60.713706 & 69.5 & 68.9 & 68.4 & 69 & 0.000264 \\
\hline 230.02 & 3.7561254 & 14.7479855 & 4.02253712 & 15.0645 & 221.15 & 59.2983504 & 69.5 & 68.9 & 68.3 & 69 & 0.000263 \\
\hline 235.02 & 3.74737162 & 14.7479855 & 4.02316256 & 15.06525 & 221.4 & 58.7769036 & 69.6 & 68.9 & 68.4 & 69 & 0.000263 \\
\hline 240.02 & $3.7517485 t$ & 14.7479855 & 4.02253712 & 15.06525 & 221.35 & 59.4225044 & 69.5 & 68,9 & 68.3 & 68.9 & 0.000263 \\
\hline 245.02 & 3.74737162 & 14.7472359 & 4.02503888 & 15.066 & 221.35 & 59.1990272 & 69.4 & 68.9 & 68.2 & 68.9 & 0.000263 \\
\hline 250.02 & 3.75362432 & 14.7457366 & 4,02316256 & 15.066 & 221.575 & 59.1493656 & 69.4 & 68.9 & 68.4 & 68.9 & 0.000264 \\
\hline 255.02 & 3.7561254 & 14.7472359 & 4.02441344 & 15.06525 & 221.325 & 58.1561336 & 69.4 & 68.9 & 68.3 & 69 & 0.000264 \\
\hline 260.02 & 3.76425391 & 14.7464863 & 4.02441344 & 15.066 & 221.375 & 60.4157364 & 69.3 & 68.9 & 68.4 & 69 & 0.000263 \\
\hline 265.02 & 3.76362864 & 14.7472359 & 4.023788 & 15.06525 & 221.475 & 59.1245348 & 69.4 & 68.9 & 68.3 & 69 & 0.000263 \\
\hline 270.02 & 3.77300769 & 14.7472359 & 4,02754064 & 15.06525 & 221.25 & 60.0681052 & 69.4 & 68.9 & 68.3 & 68.9 & 0.000263 \\
\hline 275.02 & 3.75675067 & 14.7464863 & 4.02566432 & 15.066 & 221.5 & 59.5714892 & 69.4 & 68.9 & 68.2 & 68.9 & 0.000263 \\
\hline 280.03 & 3.75362432 & 14.7479855 & 4.0269152 & 15.066 & 221.325 & 59.7701356 & 69.4 & 68.9 & 68.4 & 69 & 0.000264 \\
\hline 285.02 & 3.7748835 & 14.7464863 & 4.0269152 & 15.0645 & 221.4 & 58.851396 & 69.4 & 68.9 & 68.3 & 68.9 & 0.000264 \\
\hline 290.02 & 3.75424959 & 14.7464863 & 4.02754064 & 15.0645 & 221.55 & 58.478934 & 69.5 & 69 & 68.4 & 69.1 & 0,000263 \\
\hline 295.02 & 3.76612972 & 14.7479855 & 4.02754064 & 15.06525 & 221.15 & 58.5037648 & 69.5 & 69 & 68.3 & 68.9 & 0.000264 \\
\hline 300.02 & 3.77300769 & 14.7472359 & 4.0259152 & 15.066 & 221.35 & 58.8017344 & 69.5 & 69 & 68.4 & 69 & 0.000264 \\
\hline 305.03 & 3.7686308 & 14.7464863 & 4.0269152 & 15.06525 & 221.475 & 58.4044416 & 69.5 & 69 & 68.4 & 68.9 & 0.000264 \\
\hline 310.02 & 3.76175283 & 14.7457366 & 4.0269152 & 15.06525 & 221.5 & 58.603088 & 69.5 & 69 & 68.3 & 68.9 & 0.000264 \\
\hline 315.03 & 3.78863944 & 14.7472359 & 4.02628976 & 15.0675 & 221.55 & 58.5782572 & 69.6 & 69 & 68.4 & 69 & 0.000264 \\
\hline 320.02 & 3.7748835 & 14.7464863 & 4.02754064 & 15.06525 & 221.35 & 58.8265652 & 69.6 & 68.9 & 68.2 & 68.9 & 0.000264 \\
\hline 325.02 & 3.76425391 & 14.7479855 & 4.0269152 & 15.06525 & 221.375 & 58.8762268 & 69.5 & 68.9 & 68.3 & 69 & 0.000264 \\
\hline 330.02 & 3.76425391 & 14.7464663 & 4.02754064 & 15.06825 & 221.5 & 58.2802876 & 69.5 & 69 & 68.3 & 68.9 & 0.000263 \\
\hline 335.06 & 3.77926039 & 14.7464863 & 4.03129328 & 15.06525 & 221.4 & 59.0500424 & 69.5 & 6 & 68.3 & 68.9 & 0.000263 \\
\hline 340.02 & 3.76112756 & 14.7479855 & 4.02941696 & 15.0645 & 221.5 & 59.1990272 & 69.5 & 69 & 68.4 & 69 & 0.000264 \\
\hline 345.02 & 3.77300769 & 14.7487351 & 4.03066784 & 15.06525 & 221.35 & 59.5714892 & 69.5 & 69 & 68.3 & 68.9 & 0.000264 \\
\hline 350.02 & 3.79676795 & 14.7464863 & 4.03191872 & 15.066 & 221.45 & 58.5782572 & 69.6 & 69 & 68.4 & 69 & 0.000264 \\
\hline 355.02 & 3.7561254 & 14.7472359 & 4.03066784 & 15.06525 & 221.575 & 58.5285956 & 69.7 & 69 & 68.3 & 68.9 & 0.000264 \\
\hline 360.02 & 3.7561254 & 14.7472359 & 4.03191872 & 15.0675 & 221.35 & 59.1493656 & 69.7 & 69 & 68.2 & 68.9 & 0.000264 \\
\hline 365.08 & 3.7686308 & 14.7464863 & 4.03066784 & 15.06525 & 221.55 & 60.2915824 & 69.7 & 69 & 68.3 & 68.9 & 0.000264 \\
\hline 370.02 & 3.75800121 & 14.7472359 & 4.03254416 & 15.06675 & 221.475 & 59.3231812 & 69.8 & 69 & 68.3 & 68.9 & 0.000263 \\
\hline 375.02 & 3.7686308 & 14.7464863 & 4.0331696 & 15.0675 & 221.525 & 58.4292724 & 69.8 & 69 & 68.4 & 69 & 0.000263 \\
\hline 380.02 & 3.77800985 & 14.7487351 & 4.0331696 & 15.06525 & 221.525 & 59.0500424 & 69.8 & 69 & 68.3 & 68.9 & 0.000264 \\
\hline 385.02 & 3.76300337 & 14.7487351 & 4.03254416 & 15.06675 & 221.15 & 58.9258884 & 69.8 & 69 & 68.3 & 68.9 & 0.000264 \\
\hline 390.02 & 3.77988566 & 14.7479855 & 4,03191872 & 15.06525 & 221.35 & 59.3728428 & 69.7 & 69 & 68.4 & 69 & 0.000265 \\
\hline 395.1 & 3.76988134 & 14.7487351 & 4.03379504 & 15.06675 & 221.35 & 58.851396 & 69.6 & 6 & 68.4 & 69 & 0.000264 \\
\hline
\end{tabular}




\begin{tabular}{|c|c|c|c|c|c|c|c|c|c|c|c|}
\hline SECONDS & \begin{tabular}{|l|} 
MAIN DUCT \\
DIFF (IN H2O
\end{tabular} & \begin{tabular}{|l|} 
MAIN DUCT \\
ABS (PSIA)
\end{tabular} & \begin{tabular}{|l|} 
GAS INJECT \\
DIFF IN H2O
\end{tabular} & $\begin{array}{l}\text { GAS INJEC } \\
\text { ABS (PSIA) }\end{array}$ & \begin{tabular}{|l} 
NEWPORT \\
(PPM)
\end{tabular} & SMC & \begin{tabular}{|l} 
AMBIENT \\
TEMP (F)
\end{tabular} & \begin{tabular}{|l|} 
GAS INJECT \\
TEMP (F)
\end{tabular} & MAIN DUCT & \begin{tabular}{|l|} 
WEC CELL \\
TEMP (F)
\end{tabular} & WEC CELL \\
\hline & & & & & & & & & & & \\
\hline 400.02 & 3.78863944 & 14.7479855 & 4.0331696 & 15,06825 & 221.45 & 59.472166 & 69.5 & 69 & 68.5 & 69 & 0.000264 \\
\hline 405.02 & 3.77363296 & 14.7479855 & 4.0331696 & 15.0675 & 221.375 & 57.9574872 & 69.6 & 69 & 68,4 & 69 & 0.000264 \\
\hline 410.02 & 3.76675499 & 14.7464863 & 4.03066784 & 15.06825 & 221.5 & 59.1741964 & 69.6 & 69 & 68.4 & 68.9 & 0.000264 \\
\hline 415.02 & 3.76112756 & 14.7479855 & 4.03379504 & 15.0675 & 221.575 & 59.1741964 & 69.7 & 69 & 68.3 & 68.9 & 0.000264 \\
\hline 420.02 & 3.77613404 & 14.7464853 & 4.03254416 & 15.0675 & 221.475 & 59.1990272 & 69.7 & 69 & 68.3 & 69 & 0.000264 \\
\hline 425.02 & 3.76925607 & 14.7479855 & 4.03379504 & 15.0675 & 221.1 & \begin{tabular}{|l}
58.35478 \\
\end{tabular} & 69.7 & 69 & 68.4 & 69 & 0.000264 \\
\hline 430.02 & 3.77113188 & 14.7479855 & 4.03254416 & 15.0675 & 221.35 & 58.2554568 & 69.7 & 69 & 68.2 & 68.9 & 0.000263 \\
\hline 435.02 & 3.79239106 & 14.7479855 & 4.03379504 & 15.0675 & 221.325 & 59.0748732 & 69.7 & 69 & 68.2 & 68.9 & 0.000263 \\
\hline 440.02 & 3.76738026 & 14.7479855 & 4.0331696 & 15.06825 & 221.5 & 59.5466584 & 69.7 & 69.1 & 68.3 & 69 & 0.000264 \\
\hline 445.02 & 3.76988134 & 14.7472359 & 4.0331696 & 15.06675 & 221.375 & 58.3796108 & 69.6 & 69 & 68.2 & 68.9 & 0.000265 \\
\hline 450.02 & 3.76612972 & 14.7472359 & 4.0331696 & 15.0675 & 221.05 & 58.35478 & 69.6 & 69 & 68.3 & 69 & 0.000264 \\
\hline 455.02 & 3.7811362 & 14.7472359 & 4.03379504 & 15.0675 & 221.35 & 58,3299492 & 69.6 & 69 & 68.2 & 68.9 & 0.000263 \\
\hline 460.02 & 3.75675067 & 14.7479855 & 4.0331696 & 15.06825 & 221.475 & 58.5534264 & 69.6 & 69 & 68.1 & 68.9 & 0.000264 \\
\hline 465.02 & 3.77113188 & 14.7479855 & 4.03379504 & 15.0675 & 221.2 & 58.6279188 & 69.6 & 69 & 68.2 & 69 & 0.000264 \\
\hline 470.02 & 3.7686308 & 14.7479855 & 4.0331696 & 15.0675 & 221.375 & 58.5782572 & 69.6 & 69 & 68.1 & 68.9 & 0.000264 \\
\hline 475.02 & 3.78676363 & 14.7472359 & 4.03442048 & 15.0675 & 221.25 & 59.1245348 & 69.6 & 6 & 68.2 & 69 & 0.000264 \\
\hline 480.02 & 3.77988566 & 14.7464863 & 4.0331696 & 15.0675 & 221.425 & 59.4969968 & 69.6 & 69 & 68.3 & 68.9 & 0.000264 \\
\hline 485.02 & 3.7811362 & 14.7464863 & 4.0331696 & 15.0675 & 221.575 & 58.5534264 & 69.5 & 69.1 & 68.2 & 68.9 & 0.000264 \\
\hline 490.02 & 3.77988566 & 14.7479855 & 4.03254416 & 15.0675 & 221.05 & 58.3299492 & 69.5 & 69 & 68.3 & 69 & 0.000264 \\
\hline 495.02 & 3.77300769 & 14.7472359 & 4.03254416 & 15.0675 & 221.55 & 58.7520728 & 69.5 & 69 & 68.2 & 69 & 0.000265 \\
\hline 500.02 & 3.79926903 & 44.7472359 & 4.03442048 & 15.069 & 221.375 & 58.5037648 & 69.4 & 69 & 68.1 & 68.9 & 0.000265 \\
\hline 505.02 & 3.77050661 & 14.7479855 & 4.03379504 & 15.0675 & 221.35 & 59.472166 & 69.4 & 69 & 68.1 & 68.9 & 0.000265 \\
\hline 510.02 & 3.77175715 & 14.7487351 & 4.03254416 & 15.06675 & 221.425 & 59.1493656 & 69.5 & 69.1 & 68.3 & 69 & 0.000264 \\
\hline 515.06 & 3.76675499 & 14.7487351 & 4.03504592 & 15.06675 & 221.45 & 58.5782572 & 69.5 & 69 & 68.3 & 69 & 0.000264 \\
\hline 520,02 & 3.779888566 & 14.7472359 & 4.0331696 & 15.06825 & 221.475 & 59.2983504 & 69.5 & 69 & 68.3 & 69 & 0.000264 \\
\hline 525.02 & 3.76425391 & 14.7464863 & 4.03379504 & 15.07125 & 221.35 & 58.2554568 & 69.6 & 69.1 & 68.2 & 68.9 & 0.000263 \\
\hline 530.05 & 3.7811362 & 14.7464863 & 4.03504592 & 15.06675 & 221.575 & 58.3051184 & 69.6 & 69 & 66.3 & 68.9 & 0.000264 \\
\hline 535.02 & 3.76487918 & 14.7479855 & 4.03442048 & 15.06975 & 221.5 & 58.97555 & 69.6 & 69.1 & 68.4 & 69 & 0.000263 \\
\hline 540.02 & 3.76675499 & 44.7487351 & 4.03567136 & 15.0675 & 221.475 & 58.851396 & 69.5 & 69.1 & 68.3 & 6 & 0.000264 \\
\hline 545.04 & 3.80051957 & 14.7479855 & 4.03442048 & 15,06975 & 221.4 & 58.5285956 & 69.5 & 69.1 & 68.5 & 69 & 0.000264 \\
\hline 550.02 & 3.77300769 & 14.7494847 & 4.03504592 & 15.06675 & 221.525 & 58.1561336 & 69.4 & 69.1 & 68.4 & 69 & 0.000265 \\
\hline 555.02 & 3.77363296 & 14.7472359 & 4.03442048 & 15.06975 & 221.1 & \begin{tabular}{|l|}
58.8762268 \\
\end{tabular} & 69.3 & 69.1 & 68,3 & 68.9 & 0.000265 \\
\hline 560.03 & 3.77363296 & 14.7464863 & 4.03442048 & 15.0675 & 221.55 & 58.3796108 & 69.4 & 69.1 & 68.5 & 69 & 0.000264 \\
\hline 565.02 & 3.78988998 & 14.7472359 & 4.03504592 & 15.06675 & 221.6 & 59.4473352 & 69.4 & 69.1 & 68.4 & 69 & 0.000264 \\
\hline 570.02 & 3.7623781 & 14.744987 & 4.03254416 & 15.0705 & 221.175 & 59.3728428 & 69.4 & 69.1 & 68.3 & 69 & 0.000265 \\
\hline 575.07 & 3.77300769 & 14.7472359 & 4.03504592 & 15.06825 & 221.575 & 60.7385368 & 69.4 & 69.1 & 68.4 & 69 & 0.000264 \\
\hline 580.02 & $3.76\{12756$ & 14.7479855 & 4.0331696 & 15.0675 & 221.35 & 59.6211508 & 69.5 & 69.1 & 68.3 & 68.9 & 0.000265 \\
\hline 585.02 & 3.76300337 & 14.7479855 & 4.03504592 & 15.06825 & 221.425 & 60.1425976 & 69.5 & 69.1 & 68.4 & 69 & 0.000264 \\
\hline 590.04 & 3.76738026 & 14.7472359 & 4.03504592 & 15.0705 & 221.35 & 59.968782 & 69.5 & 69.1 & 68.2 & 68.9 & 0.000264 \\
\hline 595.02 & 3.80364592 & 14.7457366 & 4.0362968 & 15.06975 & 221.125 & 58.2057952 & 69.5 & 69.1 & 68.3 & 69 & 0.000265 \\
\hline 600,02 & 3.79676795 & 14.7479855 & 4,03567136 & 15.0675 & 221.575 & 59.099704 & 69.6 & 69.1 & 68.2 & 68.9 & 0.000264 \\
\hline 605.02 & 3.7998943 & 14.7472359 & 4.03504592 & 15.0705 & 221.575 & 59.8197972 & 69.6 & 69.1 & 68.3 & 69 & 0.000264 \\
\hline
\end{tabular}




\begin{tabular}{|c|c|c|c|c|c|c|c|c|c|c|c|}
\hline SECONDS & MAIN DUCT & MAIN DUCT & GAS INJECT & GAS INJEC & NEWPORT & SMC & AMBIENT & GAS INJECT & MAINDUCT & WEC CELL & WEC CELL \\
\hline & DIFF (IN H2O & ABS (PSIA) & DIFF (IN H2O & ABS (PSIA) & (PPM) & (PPM) & TEMP.(F) & TEMP.(F) & TEMP.(F) & TEMP.(F) & (VOLTS) \\
\hline 610.02 & 3.79301633 & 14.7472359 & 4.03504592 & 15.0705 & 221.075 & 60.1922592 & 69.6 & 69.1 & 68.4 & 69 & 0.000264 \\
\hline 615.02 & 3.79301633 & 14.7472359 & 4.03504592 & 15.0705 & 221.35 & 58.9258884 & 69.5 & 69.1 & 68.4 & 68.9 & 0.000264 \\
\hline 620.03 & 3.76050229 & 14.7479855 & 4.03504592 & 15.06975 & 221.5 & 59.7453048 & 69.5 & 69.1 & 68.4 & 69 & 0.000264 \\
\hline 625.03 & 3.76300337 & 14.7472359 & 4.03567136 & 15.0705 & 221.375 & 58.9010576 & 69.5 & 69.1 & 68.3 & 68.9 & 0.000265 \\
\hline 630.2 & 3.77550877 & 14.7472359 & 4.03504592 & 15.069 & 221.15 & 58.7769036 & 69.5 & 69.1 & 68.3 & 69 & 0.000264 \\
\hline 635.02 & 3.77363296 & 14.7472359 & 4.03504592 & 15.00975 & 221.425 & 57.9574872 & 69.4 & 69.1 & 68.4 & 69 & 0.000265 \\
\hline 640.02 & 3.7561254 & 14.7479855 & 4.0331696 & 15.00975 & 221.35 & 58.5534264 & 69.4 & 69.1 & 68.3 & 68.9 & 0.000264 \\
\hline 645.04 & 3.77550877 & 14.7464863 & 4.03567136 & 15.0705 & 221.525 & 59.0748732 & 69.5 & 69.1 & 68.3 & 68.9 & 0.000264 \\
\hline 650.02 & 3.7623781 & 14.7472359 & 4.03504592 & 15.069 & 221.5 & 58.7520728 & 69.5 & 69.1 & 68.3 & 69 & 0.000264 \\
\hline 655.02 & 3.76300337 & 14.7479855 & 4.03754768 & 15.06975 & 221.575 & 58.9010576 & 69.6 & 69.1 & 68.1 & 68.9 & 0.000265 \\
\hline 660.02 & 3.76300337 & 14.7479855 & 4.03504592 & 15.00975 & 221.375 & 58.1313028 & 69.6 & 69.1 & 68.3 & 68,9 & 0.000264 \\
\hline 665.06 & 3.80364592 & 14.7464863 & 4.03567136 & 15.06975 & 221.5 & 57.7588408 & 69.6 & 69.1 & 68.4 & 69 & 0,000264 \\
\hline 670.02 & 3.78176147 & 14.7472359 & 4.03504592 & 15.0705 & 221.425 & 58.3299492 & 69.6 & 69.1 & 68.3 & 69 & 0.000265 \\
\hline 675.04 & 3.76425391 & 14.7457366 & 4.03567136 & 15.0705 & 221.55 & 58.478934 & 69.6 & 69.1 & 68.3 & 68.9 & 0.000264 \\
\hline 680.02 & 3.77675931 & 14.7472359 & 4.03692224 & 15.0705 & 221.575 & 59.3728428 & 69.6 & 69.1 & 68.2 & 68.9 & 0.000265 \\
\hline 685.02 & 3.78426255 & 14.7464863 & 4.03504592 & 15.009 & 221.225 & 57.8829948 & 69.5 & 69.1 & 68.2 & 68,9 & 0.000265 \\
\hline 690.06 & 3.77926039 & 14.7472359 & 4.0362968 & 15.069 & 221.575 & 57.609856 & 69.5 & 69.1 & 68.4 & 68.9 & 0.000265 \\
\hline 695.02 & 3.76738026 & 14.7464863 & 4.03504592 & 15.06975 & 221.55 & 57.3118864 & 69.5 & 69.1 & 68.4 & 69 & 0.000265 \\
\hline 700.02 & 3.7623781 & 14.7457366 & 4.03504592 & 15.06975 & 221.275 & 58.8265652 & 69.6 & 69.1 & 68.4 & 69 & 0.000265 \\
\hline 705.04 & 3.7748835 & 14.7457366 & 4.03504592 & 15.06975 & 221.275 & 57.9078256 & 69.6 & 69.1 & 68.4 & 68.9 & 0.000265 \\
\hline 710.02 & 3.78176147 & 14.7457366 & 4.03567136 & 15.06975 & 221.5 & 58.106472 & 69.7 & 69.1 & 68.3 & 69 & 0.000265 \\
\hline 715.02 & 3.80927335 & 14.7472359 & 4.03567136 & 15.0705 & 221.575 & 58.6279188 & 69.7 & 69.1 & 68.2 & 68.9 & 0.000264 \\
\hline 720.02 & 3.77050661 & 14.7457366 & 4.03817312 & 15.069 & 221.375 & 58.3299492 & 69.7 & 69.1 & 68.3 & 68.9 & 0.000264 \\
\hline 725.02 & 3.78051093 & 14.7457366 & 4.03567136 & 15.06975 & 221.475 & 58.7024112 & 69.7 & 69.1 & 68.3 & 69 & 0.000265 \\
\hline 730.02 & 3.77363296 & 14.7472359 & 4.03504592 & 15.06975 & 221.575 & 57.485702 & 69.7 & 69.1 & 68.4 & 6 & 0.000264 \\
\hline 735.07 & 3.77175715 & 14.7472359 & 4.03504592 & 15.00975 & 221.075 & 57.858164 & 69.7 & 69.1 & 68.4 & 68.9 & 0.000265 \\
\hline 740.02 & 3.76050229 & 14.7479855 & 4.0362968 & 15.0705 & 221.35 & 57.8333332 & 69.8 & 69.1 & 68.3 & 68.9 & 0.000265 \\
\hline 745.02 & 3.77988566 & 14.7472359 & 4.03754768 & 15.07125 & 221.375 & 58.6527496 & 69.8 & 69.1 & 68.4 & 69 & 0.000264 \\
\hline 750.02 & 3.76925607 & 14.7464863 & 4,0362968 & 15.072 & 221.45 & 58.1809644 & 69.8 & 69.1 & 68.3 & 69 & 0.000265 \\
\hline 755.02 & 3.77363296 & 14.7472359 & 4,03817312 & 15.06975 & 221275 & 58,851396 & 69.8 & 69.1 & 68.4 & 69 & 0.000264 \\
\hline 760.02 & 3.76487918 & 14.7472359 & 4,03567136 & 15.0705 & 221.475 & 58.7520728 & 69.8 & 69.1 & 68.5 & 69 & 0.000265 \\
\hline 765.02 & 3.76925607 & 14.7479855 & 4.03567136 & 15.072 & 221.45 & 57.9574872 & 69.7 & 69.1 & 68.3 & 68.9 & 0.000264 \\
\hline 770.02 & 3.79426687 & 14.7464863 & 4.03567136 & 15.07275 & 221.6 & 57.7836716 & 69.7 & 69.1 & 68.3 & 68.9 & 0.000264 \\
\hline 775.07 & 3.79239106 & 14.7464863 & 4,0362968 & 15.0705 & 221.1 & $\mathbf{5 7 . 7 5 8 8 4 0 8}$ & 69.7 & 69.1 & 68.3 & 69 & 0.000265 \\
\hline 780.04 & 3.78488782 & 14.7479855 & 4,0362968 & 15.069 & 221.15 & 57.3118864 & 69.7 & 69.1 & 68.3 & 69 & 0.000265 \\
\hline 785.02 & 3.78551309 & 14.7464863 & 4.0362968 & 15.0705 & 221.475 & 58.0071488 & 69.7 & 69.1 & 68.4 & 69 & 0.000264 \\
\hline 790.07 & 3.77300769 & 14.7464863 & 4.03504592 & 15,06975 & 221.325 & 58.2554568 & 69.6 & 69.1 & 68.4 & 68.9 & 0.000264 \\
\hline 795.02 & 3.76800653 & 14.7464863 & 4.03754768 & 15.06975 & 221.5 & 57.5353636 & 69.7 & 69.1 & 68.4 & 68.8 & 0.000264 \\
\hline 800.02 & 3.7686308 & 14.7457366 & 4.03504592 & 15.06975 & 221.525 & 57.8333332 & 69.7 & 69.1 & 68.3 & 68.9 & 0.000264 \\
\hline 805.07 & 3.76675499 & 14.7464863 & 4.03692224 & 15.07125 & 221,35 & 58.2057952 & 69.8 & 6.1 & 68.4 & 68.9 & 0.000264 \\
\hline 810.02 & 3.79301633 & 14.744987 & 4.03567136 & 15.07125 & 221.35 & 57.3367172 & 69.8 & 69.1 & 68.3 & 68.9 & 0.000265 \\
\hline 815.02 & 3.77113188 & 14.7472359 & 4.03692224 & 15.0705 & 221.4 & 57.5601944 & 69.8 & 69.1 & 68.4 & 68.9 & 0.000265 \\
\hline
\end{tabular}




\begin{tabular}{|c|c|c|c|c|c|c|c|c|c|c|c|}
\hline SECONDS & MAIN DUCT & MAIN DUCT & GAS INJECT & GAS INJEC & NEWPORT & SMC & AMBIENT & GAS INJECT & MAIN DUCT & WEC CELL & WEC CELL \\
\hline & DIFF (IN H2O & ABS (PSIA) & DiFF (IN H2O & ABS (PSIA) & (PPM) & (PPM) & TEMP.(F) & TEMP.(F) & TEMP.(F) & TEMP.(F) & (NOLTS) \\
\hline 820.02 & 3.77550877 & 14.7457366 & 4.03754768 & 15.0705 & 221.175 & 57.1877324 & 69.8 & 69.1 & 68.4 & 68.9 & 0.000264 \\
\hline 825.02 & 3.7748835 & 14.7464863 & 4.03692224 & 15.07275 & 221.55 & 58.0319796 & 69.8 & 69.2 & 68.4 & 68.8 & 0.000263 \\
\hline 830.02 & 3.76925607 & 14.7472359 & 4.03754768 & 15.072 & 221.5 & 57.2622248 & 69.7 & 69.1 & 68.4 & 69 & 0.000264 \\
\hline 835.02 & 3.78363728 & 14.7464863 & 4.03754768 & 15.0735 & 221.15 & 57.9326564 & 69.8 & 69.2 & 68.4 & 68.9 & 0.000265 \\
\hline 840.02 & 3.75675067 & 14.7464863 & 4.03504592 & 15.0735 & 221.375 & 57.7836716 & 69.7 & 69.2 & 68.3 & 68.9 & 0.000265 \\
\hline 845.02 & 3.76800553 & 14.7464863 & 4.03754768 & 15.06975 & 221.3 & 57.6595176 & 69.7 & 69.2 & 68.3 & 68.9 & 0.000264 \\
\hline 850.03 & 3.77926039 & 14.7479855 & 4.03754768 & 15.072 & 221.375 & 57.7588408 & 69.7 & 69.2 & 68.4 & 69 & 0.000265 \\
\hline 855.02 & 3.7686308 & 14.7472359 & 4.03754768 & 15.07275 & 221.35 & 58.2057952 & 69.7 & 69.2 & 68.5 & 68.9 & 0.000265 \\
\hline 860.02 & 3.77988566 & 14.7464863 & 4.03754768 & 15.0735 & 221.375 & 57.4608712 & 69.7 & 69.2 & 68.3 & 68.9 & 0.000264 \\
\hline 865.03 & 3.78988998 & 14.7457366 & 4.03692224 & 15.07275 & 221.525 & 57.5601944 & 69.7 & 69.2 & 68.4 & 68.9 & 0.000265 \\
\hline 870.02 & 3.76612972 & 14.7464863 & 4.03692224 & 15.07275 & 221.05 & 57.9078256 & 69.7 & 69.2 & 68.3 & 68.9 & 0.000265 \\
\hline 875.02 & 3.7748835 & 14.7472359 & 4.03692224 & 15.06975 & 221.375 & 58.3796108 & 69.7 & 69.2 & 68.3 & 68.9 & 0.000264 \\
\hline 880.02 & 3.80927335 & 14.7464863 & 4.03817312 & 15.0735 & 221.5 & 58.0319796 & 69.7 & 69.2 & 68.4 & 68.9 & 0.000265 \\
\hline 885.02 & 3.76300337 & 14.7464863 & 4.03692224 & 15.07275 & 221.25 & 58.3796108 & 69.7 & 69.2 & 68.5 & 68.9 & 0.000264 \\
\hline 890.02 & 3.78988998 & 14.7464863 & 4.0362968 & 15.072 & 221.475 & 58.0816412 & 69.6 & 69.2 & 68.5 & 68.9 & 0.000264 \\
\hline 895.07 & 3.76925607 & 14.7464863 & 4.03754768 & 15.0735 & 221.575 & 57.5105328 & 69.6 & 69.2 & 68.3 & 68.9 & 0.000265 \\
\hline 900.02 & 3.78426255 & 14.7479855 & 4.03504592 & 15.0735 & 221.425 & 57.2870556 & 6.6 & 69.2 & 68.4 & 68.9 & 0.000264 \\
\hline 905.02 & 3.77050661 & 14.7457366 & 4.0362968 & 15.07275 & 221.525 & 57.7588408 & 69.6 & 69.2 & 68.2 & 68.9 & 0.000264 \\
\hline 910.07 & 3.7686308 & 14.7464863 & 4.03754768 & 15.07275 & 221.575 & 57.2622248 & 69.6 & 69.2 & 68.3 & 69 & 0.000265 \\
\hline 915.02 & 3.78301201 & 14.7472359 & 4.03754768 & 15.07275 & 221.5 & 57.9326564 & 69.6 & 69.1 & 68.3 & 69 & 0.000264 \\
\hline 920.02 & 3.77675931 & 14.7464863 & 4.03754768 & 15.07275 & 221.45 & 58.7024112 & 69.6 & 69.2 & 68.3 & 68.9 & 0.000265 \\
\hline
\end{tabular}




\begin{tabular}{|c|c|c|c|c|c|c|c|c|c|c|c|}
\hline \multicolumn{12}{|c|}{$\begin{array}{l}\text { TEST: FGI22212 } 22 \text { Feb } 1997 \text { WEC-SPOOL } \\
\text { Measured Hydrogen Concentration }=218 p p m\end{array}$} \\
\hline & & & & & & & & & & & \\
\hline 0.0100098 & 3.80239538 & 14.7479855 & 0.410683664 & 14.72925 & 1.45 & -1.9592332 & 69.5 & 68.9 & 68.4 & 69 & 0.00023 \\
\hline 5.019989 & 3.79301633 & 14.7494847 & 4.00690112 & 15.06525 & 1.2 & -2.1578796 & 69.5 & 68.9 & 68.1 & 68.9 & 0.00023 \\
\hline 10.019989 & 3.78801417 & 14.7464863 & 4.0206608 & 15.06525 & 1.15 & \begin{tabular}{|c|}
-1.7605868 \\
\end{tabular} & 69.3 & 68.9 & 68.3 & 68.9 & 0.000232 \\
\hline 15.019989 & 3.78613836 & 14.7502344 & 4.02754064 & 15.0675 & 11.025 & 24.162768 & 69.3 & 68.9 & 68.3 & 69 & 0.000236 \\
\hline 20.019989 & 3.79426687 & 14.7487351 & 4.0331696 & 15.0675 & 41.25 & 42.984515 & 69.3 & 68.9 & 68.3 & 69 & 0.00024 \\
\hline 25.019989 & 3.78613836 & 14.7494847 & 4.03442048 & 15.069 & 60.975 & 50.334432 & 69.3 & 68.9 & 68.2 & 68.9 & 0.000244 \\
\hline 30.019989 & 3.76800553 & 14.744987 & 4.03754768 & 15.06975 & 91.275 & 52.569204 & 69.4 & 68.9 & 68.3 & 69 & 0.000247 \\
\hline 35.019989 & 3.78801417 & 14.7472359 & 4.03817312 & 15.06975 & 111.35 & 54.282529 & 69.5 & 68.9 & 68.2 & 68.9 & 0.00025 \\
\hline 40.019989 & 3.78613836 & 14.7494847 & 4.03754768 & 15.06975 & 130.975 & 53.761082 & 69.5 & 68.9 & 68,4 & 68.9 & 0.000253 \\
\hline 45.019989 & 3.78176147 & 14.7494847 & 4.03754768 & 15.06975 & 140.85 & 53.910067 & 69.5 & 68.9 & 68.3 & 68.9 & 0.000254 \\
\hline 50.019989 & 3.76300337 & 14.7464863 & 4.03817312 & 15.0705 & 151.225 & 53.165143 & 69.5 & 68.9 & 68.3 & 68.9 & 0.000255 \\
\hline 55.019989 & 3.78863944 & 14.7479855 & 4.03754768 & 15.06975 & 161.45 & 53.115481 & 69.5 & 68.9 & 68.4 & 68.9 & 0.000257 \\
\hline 60.019989 & 3.78676363 & 14.7472359 & 4.03817312 & 15.0675 & 171 & 53.736251 & 69.6 & 69 & 68.2 & 68.9 & 0.000258 \\
\hline 65.019989 & 3.78613836 & 14.7472359 & 4.03567136 & 15.0675 & 181.175 & 52.718188 & 69.6 & 68.9 & 68.4 & 68.9 & 0.000259 \\
\hline 70.019989 & 3.78426255 & 14.7464863 & 4.03567136 & 15,06675 & 181.225 & 51.476648 & 69.6 & 69 & 68.2 & 68.9 & 0.00026 \\
\hline 75.019989 & 3.78676363 & 14.7487351 & 4.03254416 & 15.0675 & 190.85 & 51.898772 & 69.6 & 68.9 & 68.4 & 69 & 0.000261 \\
\hline 80.019989 & 3.78676363 & 14.7464863 & 4.03442048 & 15.0675 & 191.45 & 53.438282 & 69.6 & 69 & 68.3 & 69 & 0.000261 \\
\hline 85.019989 & 3.78801417 & 14.750984 & 4.0331696 & 15.0675 & 190.875 & 53.264466 & 69.6 & 68.9 & 68.4 & 69 & 0.000261 \\
\hline 90.019989 & 3.78613836 & 14.7494847 & 4.03254416 & 15.06675 & 201.2 & 52.991327 & 69.6 & 69 & 68.2 & 68.9 & 0.000261 \\
\hline 95.019989 & 3.79926903 & 14.7524832 & 4.0331696 & 15.06825 & 201.225 & 52.569204 & 69.5 & 68.9 & 68.3 & 68.9 & 0.000261 \\
\hline 100.01999 & 3.77550877 & 14.7464863 & 4.03191872 & 15.06675 & 200.575 & 53.06582 & 69.5 & 69 & 68.3 & 68.9 & 0.000262 \\
\hline 105.01999 & 3.78676363 & 14.750984 & 4,02941696 & 15.069 & 201.475 & 53.314128 & 69.4 & 68.9 & 68.2 & 68.9 & 0.000261 \\
\hline 110.01999 & 3.77175715 & 14.7502344 & 4.02754064 & 15.06825 & 210.975 & 53,413451 & 69.4 & 68.9 & 68.4 & 69 & 0.000263 \\
\hline 115.01999 & 3.79301633 & \begin{tabular}{|l|}
14.750984 \\
\end{tabular} & 4.0269152 & 15.0675 & 211.25 & 53.761082 & 69.4 & 69 & 68.3 & 68.9 & 0.000264 \\
\hline 120.01999 & 3.81927767 & 14.7479355 & 4.02566432 & 15.06675 & 211.3 & 53.562436 & 69.4 & 68.9 & 68.4 & 68.9 & 0.000263 \\
\hline 125.01999 & 3.80239538 & 14.750984 & 4.02503888 & 15.06825 & 210.525 & 53.314128 & 69.4 & 69 & 68.2 & 68.9 & 0.000262 \\
\hline 130.01999 & 3.79176579 & 14.7502344 & 4.02441344 & 15.06525 & 211.4 & 53.512774 & 69.5 & 69 & 68.3 & 68,9 & 0.000263 \\
\hline 135.01999 & 3.78488782 & 14.7479855 & 4.02503888 & 15.06825 & 211.125 & 53.040989 & 69.4 & 69 & 68.4 & 69 & 0.000262 \\
\hline 140.01999 & 3.79489214 & 14.7502344 & 4.02503888 & 15.0675 & 211.425 & 52.643696 & 69.4 & 69 & 68.3 & 68.9 & 0.000263 \\
\hline 145.01999 & 3.77300769 & 14.7479855 & 4.02754064 & 15.06825 & 211.275 & 53.289297 & 69.4 & 69 & 68.4 & 69 & 0.000263 \\
\hline 150.01999 & 3.77988566 & 14.7494847 & 4.02503888 & 15.0645 & 210.85 & 52.792681 & 69.5 & 69 & 68.3 & 68.9 & 0.000263 \\
\hline 155.01999 & 3.78676363 & 14.7472359 & 4.02503888 & 15.0675 & 211.475 & 54.158375 & 69.5 & 69 & 68.3 & 68.9 & 0.000263 \\
\hline 160,01999 & 3.79239106 & 14.7494847 & 4.02441344 & 15.0675 & 221.275 & 53.860405 & 69.5 & 69 & 68.4 & 68.9 & 0.000262 \\
\hline 165,01999 & 3.79676795 & 14.7524832 & 4.02191168 & 15.06675 & 211.35 & 52.718188 & 69.6 & 69 & 68.3 & 68.9 & 0.000263 \\
\hline 170.01999 & 3.78176147 & 14.7494847 & 4.02253712 & 15.0675 & 221.375 & 53.463112 & 69.6 & 69 & 68.4 & 69 & 0.000264 \\
\hline 175.01999 & 3.79551741 & 14.7494847 & 4.0206608 & 15.06675 & 221.4 & 53.115481 & 69.6 & 69 & 68.4 & 69 & 0.000263 \\
\hline 180.01999 & 3.77113188 & 14.7494847 & 4.0206608 & 15.06675 & 221.475 & 53.636928 & 69.6 & 69 & 68.5 & 69 & 0.000264 \\
\hline 185.01999 & 3.77550877 & 14.7487351 & 4.02003536 & 15.06675 & 214.1 & 53.115481 & 69.6 & 69 & 68.3 & 68.9 & 0.000264 \\
\hline
\end{tabular}




\begin{tabular}{|c|c|c|c|c|c|c|c|c|c|c|c|}
\hline SECONDS & $\begin{array}{l}\text { MAIN DUCT } \\
\text { DIFF (IN H2O }\end{array}$ & $\frac{\text { MAIN DUCT }}{\text { ABS (PSIA) }}$ & $\begin{array}{l}\text { GAS INJECT } \\
\text { DIFF (IN H2O) }\end{array}$ & $\frac{\text { GAS INJECT }}{\text { ABS (PSIA) }}$ & \begin{tabular}{|l|} 
NEWPORT \\
(PPM) \\
\end{tabular} & $\begin{array}{l}\text { SMC } \\
\text { (PPM) }\end{array}$ & \begin{tabular}{|l} 
AMBIENT \\
TEMP.(F)
\end{tabular} & \begin{tabular}{|l|} 
GAS INJECT \\
TEMP.(F) \\
\end{tabular} & \begin{tabular}{|l|} 
MAIN DUCT \\
TEMP.(F)
\end{tabular} & \begin{tabular}{|l|} 
WEC CELL \\
TEMP.(F)
\end{tabular} & $\begin{array}{l}\text { WEC CELL } \\
\text { (VOLTS) }\end{array}$ \\
\hline 190.01999 & 3.76925607 & 14.7502344 & 4.0206608 & 15.0675 & 221.25 & 52.916835 & 69.7 & 69) & 68.3 & 68.9 & 0.000264 \\
\hline 195.01999 & 3.79239106 & 14.7494847 & 4.01940992 & 15.0675 & 221.35 & 53.512774 & 69.6 & 69 & 68.3 & 69 & 0.000264 \\
\hline 200.01999 & 3.79239106 & 14.7487351 & 4.02128624 & 15.066 & 211.4 & 54.63016 & 69.6 & 69 & 68.5 & 69 & 0.000264 \\
\hline 205.01999 & 3.78051093 & 14.7494847 & 4.0206608 & 15.0675 & 221.6 & 53.314128 & 69.6 & 69 & 68.5 & 69 & 0.000264 \\
\hline 210.01999 & 3.77988566 & 14.7487351 & 4.01940992 & 15.0675 & 221.525 & 53.661759 & 69.5 & 69 & 68.4 & 68.9 & 0.000264 \\
\hline 215.01999 & 3.77988566 & 14.7494847 & 4.01815904 & 15.0645 & 221.225 & 54.208036 & 69.6 & 69.1 & 68.4 & 69 & 0.000263 \\
\hline 220.01999 & 3.79239106 & 14.7502344 & 4.01878448 & 15.0645 & 221.35 & 53.463112 & 69.6 & 69 & 68.4 & 68.9 & 0.000264 \\
\hline 225.01999 & 3.78176147 & 14.7487351 & 4.01815904 & 15.0645 & 221.45 & 53.363789 & 69.6 & 69 & 68.4 & 69 & 0.000264 \\
\hline 230.01999 & 3.78613836 & 14.7494847 & 4,01878448 & 15.0675 & 221.225 & 53.289297 & 69.6 & 69 & 68.5 & 69 & 0.000263 \\
\hline 235.01999 & 3.82052821 & 14.7502344 & 4.02003536 & 15.0645 & 221.55 & 53.661759 & 69.5 & 69 & 68.4 & 68.9 & 0.000264 \\
\hline 240.01999 & 3.76988134 & 14.7524832 & 4.01878448 & 15.06525 & 221.275 & 53,38862 & 69.5 & 69.1 & 68.5 & 68.9 & 0.000264 \\
\hline 245.01999 & 3.7873889 & 14.7517336 & 4.0206608 & 15.066 & 221.175 & 52.991327 & 69.6 & 69 & 68.3 & 68.8 & 0.000263 \\
\hline 250.01999 & 3.7748835 & 14.7517336 & 4.01878448 & 15.0675 & 221.45 & 53.587266 & 69.6 & 69 & 68.4 & 69 & 0.000264 \\
\hline 255.04001 & 3.78551309 & 14.7517336 & 4.01940992 & 15.0675 & 221.55 & 53.289297 & 69.6 & 69 & 68.3 & 68.9 & 0.000263 \\
\hline 260.01999 & 3.77738458 & 14.7487351 & 4.0206608 & 15.0675 & 221.575 & 53.140312 & 69.6 & 69 & 68.3 & 68.9 & 0.000264 \\
\hline 265.01999 & 3.77738458 & 14.7494847 & 4.01528272 & 15.0675 & 221.35 & 52.420219 & 69.6 & 69 & 68.4 & 68.9 & 0.000264 \\
\hline 270.01999 & 3.78176147 & 14.7532329 & 4.01690816 & 15.0675 & 221.15 & 52.594034 & 69.6 & 69 & 68.3 & 6 & 0.000264 \\
\hline 275.01999 & 3.77300769 & 14.7494847 & 4.01690816 & 15.0675 & 221.5 & 53.587266 & 69.6 & 69 & 68.4 & 69 & 0.000263 \\
\hline 280.01999 & 3.79801849 & 14.750984 & 4.01940992 & 15,06825 & 221.575 & 53.314128 & 69.6 & 69.1 & 68.3 & 68.9 & 0.000264 \\
\hline 285.01999 & 3.80552173 & 14.7532329 & 4.01878448 & 15.06825 & 221.55 & 52.221572 & 69.6 & 69.1 & 68.4 & 69 & 0.000265 \\
\hline 290.01999 & 3.79176579 & 14.7517336 & 4.01815904 & 15.0675 & 221.375 & 53.636928 & 69.6 & 69 & 68.3 & 68.9 & 0.000264 \\
\hline 295.01999 & 3.79614268 & 14.7524832 & 4.01940992 & 15.0675 & 221.15 & 53.38862 & 69.6 & 69.1 & 68.4 & 68.9 & .000264 \\
\hline 300.01999 & 3.7811362 & 14.7502344 & 4.01690816 & 15.0675 & 221.5 & 53.934898 & 69.6 & 69.1 & 68.3 & 68.8 & 0.000264 \\
\hline 305.01999 & 3.79051525 & 14.7524832 & 4.01628272 & 15.06675 & 221.55 & 52.743019 & 69.5 & 69 & 68.5 & 69 & 0.000264 \\
\hline 340.01999 & 3.77988566 & 14.7524832 & 4.01690816 & 15.06825 & 221.475 & 53.363789 & 69.5 & 69 & 68.4 & 68.8 & 0.000264 \\
\hline 315.01999 & 3.7686308 & 14.7517336 & 4.01815904 & 15.066 & 221.525 & 53.189974 & 69.5 & 69 & 68.2 & 68.8 & 0.000263 \\
\hline 320.01999 & 3.78301201 & 14.7524832 & 4.01565728 & 15.0675 & 221.075 & 53.165143 & 69.5 & 69 & 68.4 & 68.9 & 0.000264 \\
\hline 325.01999 & 3.78363728 & 14.750984 & 4.01878448 & 15.06675 & 221.4 & 53.562436 & 69.5 & 69 & 68.2 & 68.9 & 0.000264 \\
\hline 330.01999 & 3.80051957 & 14.7524832 & 4.0206608 & 15.06675 & 221.475 & 52.916835 & 69.5 & 69 & 68.1 & 68.9 & 0.000264 \\
\hline 335.10001 & 3.806147 & 14.750984 & 4.01815904 & 15.06675 & 221.05 & 53.016158 & 69.5 & 69 & 68.3 & 68.9 & 0.000264 \\
\hline 340.01999 & 3.79176579 & 14.750984 & 4.01565728 & 15.06675 & 221.575 & 52.345726 & 69.5 & 69 & 68.3 & 68.9 & 0.000265 \\
\hline 345.01999 & 3.78801417 & 14.7494847 & 4.01815904 & 15.06675 & 221.35 & 51.675295 & 69.5 & 69.1 & 68.3 & 68.9 & 0.000264 \\
\hline 350.01999 & 3.77113188 & 14.7517336 & 4.01628272 & 15.0675 & 221.25 & 52.594034 & 69.6 & 69 & 68.2 & 68.9 & 0.000264 \\
\hline 355.01999 & 3.77926039 & 14.750934 & 4.01815904 & 15.06675 & 221.525 & 52.271234 & 69.5 & 69 & 68.2 & 68.9 & 0.000264 \\
\hline 360.01999 & 3.77988566 & 14.750984 & 4.01878448 & 15.06825 & 221.475 & 52.594034 & 69.5 & 69 & 68.4 & 69 & 0.000265 \\
\hline 365.07999 & 3.79614268 & 14.7517336 & 4.01878448 & 15.0675 & 221.475 & 53.552436 & 69.5 & 69.1 & 68.4 & 68.9 & 0.000264 \\
\hline 370.01999 & 3.79739322 & 14.7524832 & 4.01690816 & 15.0675 & 221.375 & 52.842342 & 69.6 & 69.1 & 68.3 & 68.9 & 0.000264 \\
\hline 375.10001 & 3.76925607 & 14.750984 & 4.01628272 & 15.06825 & 221.075 & 52.643696 & 69.6 & 69.1 & 68.3 & 68.9 & 0.000264 \\
\hline 380.01999 & 3.78801417 & 14.7502344 & 4.02128624 & 15.0675 & 221.375 & 52.668527 & 69.5 & 69.1 & 68.4 & 68.9 & 0.000264 \\
\hline 385,07999 & 3.79739322 & 14.7517336 & 4.01878448 & 15.06675 & 221.175 & 54.232867 & 69.4 & 69.1 & 68.3 & 68.8 & 0.000263 \\
\hline 390.01999 & 3.77675931 & 14.7517336 & 4.0206608 & 15.06675 & 221.5 & 53.562436 & 69.5 & 69 & 68.5 & 69 & 0.000264 \\
\hline 395.06 & 3.80239538 & 14.7517336 & 4.0206608 & 15.06975 & 221.375 & 52.519542 & 69.5 & 69 & 68.4 & 68.9 & 0.000264 \\
\hline
\end{tabular}




\begin{tabular}{|c|c|c|c|c|c|c|c|c|c|c|c|}
\hline SECONDS & MAIN DUCT & MAIN DUCT & GAS INJECT & GAS INJECT & NEWPORT & SMC & AMBIENT & GAS INJECT & MAIN DUCT & WEC CELL & WEC CELL \\
\hline & DIFF (IN H2O & ABS (PSIA) & DIFF (IN H2O) & $A B S$ (PSIA) & (PPM) & (PPM) & TEMP.(F) & TEMP.(F) & TEMP.(F) & TEMP.(F) & (VOLTS) \\
\hline 400.01999 & 3.78676363 & 14.7517336 & 4.01878448 & 15.0705 & 221.125 & 52.395388 & 69.5 & 69.1 & 68.3 & 69 & 0.000264 \\
\hline 405.01999 & 3.78613836 & 14.7524832 & 4.02003536 & 15.069 & 221.375 & 52.122249 & 69.5 & 69 & 68.5 & 68.9 & 0.000264 \\
\hline 410.01999 & 3.79239106 & 14.750984 & 4.01878448 & 15.06675 & 221.5 & 51.948434 & 69.6 & 69.1 & 68.3 & 68.8 & 0.000265 \\
\hline 415.01999 & 3.79676795 & 14.7502344 & 4.01878448 & 15.06675 & 221.25 & 53.835574 & 69.7 & 69 & 68.5 & 69 & 0.000263 \\
\hline 420.01999 & 3.79676795 & 14.750984 & 4.01878448 & 15.06825 & 221.55 & 54.034221 & 69.7 & 69.1 & 68.4 & 68.9 & 0.000264 \\
\hline 425.01999 & 3.77738458 & 14.750984 & 4.01940992 & 15.06975 & 221.15 & 53.264466 & 69.7 & 69.1 & 68.5 & 68.9 & 0.000265 \\
\hline 430.01999 & 3.78613836 & 14.750984 & 4.01940992 & 15.0705 & 221.45 & 53.289297 & 69.7 & 69.1 & 68.3 & 68.9 & 0.000264 \\
\hline 435.01999 & 3.78613836 & 14.7517336 & 4.0206608 & 15.06975 & 221.55 & 53.761082 & 69.7 & 69.1 & 68.3 & 68.8 & 0.000264 \\
\hline 440.01999 & 3.7811362 & 14.750984 & 4.0206608 & 15.0705 & 221.2 & 53.910067 & 69.7 & 69.1 & 68.5 & 68,9 & 0.000264 \\
\hline 445.01999 & 3.79614268 & 14.7517336 & 4.0206608 & 15.0675 & 221.425 & 53.264466 & 69.7 & 69.1 & 68.4 & 68.9 & 0.000264 \\
\hline 450.01999 & 3.77675931 & 14.7517336 & 4.01940992 & 15.06975 & 221.375 & 53.06582 & 69.6 & 69.1 & 68.5 & 69 & 0.000263 \\
\hline 455.01999 & 3.7748835 & 14.7517336 & 4.0206608 & 15.06975 & 221.4 & 53.09065 & 69.5 & 69.1 & 68.6 & 69 & 0.000264 \\
\hline 460.01999 & 3.7686308 & 14.7517336 & 4.02128624 & 15.06975 & 221.575 & 52.14708 & 69.5 & 69.1 & 68.4 & 68.9 & 0.000265 \\
\hline 465.01999 & 3.77738458 & 14.7524832 & 4.01815904 & 15.06975 & 221.4 & $\mathbf{5 3 . 4 1 5 4 8 1}$ & 69.5 & 69.1 & 68.4 & 68.9 & 0.000263 \\
\hline 470.01999 & 3.77988566 & 14.7524832 & 4.02128624 & 15.06975 & 221.375 & 52.842342 & 69.5 & 69.1 & 68.3 & 68.9 & 0.000263 \\
\hline 475.01999 & 3.7998943 & 14.7517336 & 4.02003536 & 15.0705 & 221.5 & 52.966496 & 69.5 & 69.1 & 68.5 & 68.9 & 0.000264 \\
\hline 480.01999 & 3.77926039 & 14.750984 & 4.0206608 & 15.069 & 221.5 & 52.991327 & 69.5 & 69.1 & 68.4 & 68.9 & 0.000264 \\
\hline 485.01999 & 3.78863944 & 14.7524832 & 4.02003536 & 15.06975 & 221.425 & 52.941666 & 69.5 & 69.1 & 68.5 & 69 & 0.000264 \\
\hline 490.01999 & 3.80677227 & 14.7524832 & 4.02128624 & 15.06975 & 221.35 & 52.345726 & 69.5 & 69.1 & 68.4 & 68.9 & 0.000264 \\
\hline 495.01999 & 3.7873889 & 14.7517336 & 4.0206608 & 15.0705 & 221.375 & 52.991327 & 69.5 & 69.1 & 68.4 & 68.9 & 0.000265 \\
\hline 500.01999 & 3.7811362 & 14.750984 & 4.0206608 & 15.06975 & 221.5 & 52.941666 & 69.5 & 69.1 & 68.5 & 68.9 & 0.000264 \\
\hline 505.01999 & 3.79114052 & 14.7517336 & 4.02128624 & 15.06975 & 221.3 & 52.345726 & 69.5 & 69.1 & 68.5 & 68.9 & 0.000265 \\
\hline 510.01999 & 3.7873889 & 14.750984 & 4.02128624 & 15.06975 & 221.55 & 52.668527 & 69.5 & 69.1 & 68.4 & 68.9 & 0.000264 \\
\hline 515.01999 & 3.79051525 & 14.7517336 & 4.02191168 & 15.06975 & 221.35 & 53.438282 & 69.5 & 69.1 & 68.4 & 68.9 & 0.000265 \\
\hline 520.01999 & 3.77863512 & 14.7524832 & 4.0206608 & 15.06975 & 221.4 & 53.587266 & 69.5 & 69.1 & 68.4 & 68.8 & 0.000264 \\
\hline 525.01999 & 3.78801417 & 14.7517336 & 4.02128624 & 15.06975 & 221.525 & 52.594034 & 69.6 & 69.1 & 68.5 & 68.9 & 0.000265 \\
\hline 530.01999 & 3.7811362 & 14.7517336 & 4.02128624 & 15.06975 & 221.275 & 53.537605 & 69.7 & 69.1 & 68.4 & 68.9 & 0.000264 \\
\hline 535.01999 & 3.77238242 & 14.7524832 & 4.0206608 & 15.0705 & 221.425 & 53.562436 & 69.8 & 69.1 & 68.5 & 68.9 & 0.000264 \\
\hline 540.01999 & 3.80927335 & 14.7502344 & 4.02128624 & 15.069 & 221.375 & 52.966496 & 69.8 & 69.1 & 68.5 & 68.9 & 0.000264 \\
\hline 545.01999 & 3.77988566 & 14.7517336 & 4.0206608 & 15.06975 & 224.275 & 52.867173 & 69.8 & 69.2 & 68.4 & 68.9 & 0.000264 \\
\hline 550.01999 & 3.78176147 & 14.7517336 & 4.0206608 & 15.06975 & 221.55 & 52.668527 & 69.8 & 69.2 & 68.3 & 68.9 & 0.000264 \\
\hline 555.01999 & 3.80239538 & 14.7517336 & 4.0206608 & 15.069 & 221.175 & 53.09065 & 69.7 & 69.1 & 68.4 & 68.9 & 0.000264 \\
\hline 560.04999 & 3.77988566 & 14.7524832 & 4.0206608 & 15.0705 & 221.4 & 52.420219 & 69.7 & 69.2 & 68.3 & 68.9 & 0.000264 \\
\hline 565.11002 & 3.77926039 & 14.7517336 & 4.02128624 & 15.06975 & 221.45 & 53.443451 & 69.7 & 69.1 & 68.3 & 68.9 & 0.000264 \\
\hline 570.01999 & 3.80051957 & 14.7524832 & 4.02003536 & 15.06975 & 221.275 & 53.165143 & 69.7 & 69.2 & 68.4 & 68.9 & 0.000264 \\
\hline 575.03 & 3.7873889 & 14.7524832 & 4.02128624 & 15.06975 & 221.525 & 52.594034 & 69.7 & 69.2 & 68.3 & 68.9 & 0.000264 \\
\hline 580.01999 & 3.7686308 & 14.7524832 & 4.0206608 & 15.06975 & 221.425 & 52.966496 & 69.7 & 69.2 & 68.3 & 68.9 & 0.000264 \\
\hline 585.01999 & 3.78551309 & 14.7524832 & 4.02003536 & 15.06975 & 221.5 & 52.892004 & 69.7 & $69 . \overline{1}$ & 68.4 & 68.9 & 0.000263 \\
\hline 590.06 & 3.79551741 & 14.750984 & 4.01940992 & 15,06975 & 221.275 & 52.618865 & 69.6 & 69.2 & 68.4 & 69 & 0.000264 \\
\hline 595.01999 & 3.77988566 & 14.7517336 & 4.0206608 & 15.06975 & 231.425 & 53.06582 & 6.7 & 69.2 & 68.4 & 68.9 & 0.000264 \\
\hline 600.01999 & 3.7873889 & 14.7524832 & 4.0206608 & 15.069 & 221.575 & 53.661759 & 69.6 & 69.2 & 68.5 & 68.9 & 0.000264 \\
\hline 605.03 & 3.78176147 & 14.7524832 & 4.02128624 & 15.06975 & 221.4 & 53.785913 & 69.7 & 69.2 & 68.3 & 68.9 & 0.000265 \\
\hline
\end{tabular}




\begin{tabular}{|c|c|c|c|c|c|c|c|c|c|c|c|}
\hline SECONDS & \begin{tabular}{|l|} 
MAIN DUCT \\
\end{tabular} & \begin{tabular}{|l|} 
MAIN DUCT \\
$A B S$ PS(A) \\
\end{tabular} & \begin{tabular}{|l|} 
GAS INJECT \\
DIFF
\end{tabular} & \begin{tabular}{|l|} 
GAS INJECT \\
$A B S$ (PSIA)
\end{tabular} & \begin{tabular}{|l|} 
NEWPORT \\
(PPM)
\end{tabular} & SMC & \begin{tabular}{|l|} 
AMBIENT \\
TEMP.(F) \\
\end{tabular} & \begin{tabular}{|l|} 
GAS INJECT \\
TEMP (F)
\end{tabular} & \begin{tabular}{|l} 
MAIN DUCT \\
TEMP(F)
\end{tabular} & \begin{tabular}{|l|} 
WEC CELL \\
TEMP.(F)
\end{tabular} & \begin{tabular}{|l|} 
WEC CELL \\
NOLTS)
\end{tabular} \\
\hline & & & & ABS (PS|A) & & (PPM) & IEMP.(F) & & & & \\
\hline 610.01999 & 3.80239538 & 14.7532329 & 4.0206608 & 15.072 & 221.25 & 53.016158 & 69.7 & 69.2 & 68.4 & 68.9 & 0.000265 \\
\hline 645.01999 & 3,79176579 & 14.7524832 & 4.0206608 & 15.06975 & 221.55 & 52.892004 & 69.7 & 69.2 & 68.3 & 68.9 & 0.000263 \\
\hline 620.04001 & 3.7686308 & 14.7524832 & 4.0206608 & 15.07275 & 221.5 & 52.842342 & 69.7 & 69.2 & 68.3 & 68.9 & 0.000264 \\
\hline 625.01999 & 3.76487918 & 14.7532329 & 4,0206608 & 15.07275 & 221.55 & 52.395388 & 69.6 & 69.1 & 68.4 & 68.9 & 0.000264 \\
\hline 630.01999 & 3.77300769 & 14.750984 & 4.02128624 & 15.0705 & 221.45 & 53.165143 & 69.6 & 69.1 & 68.2 & 68.9 & 0.000264 \\
\hline 635.07999 & 3.80927335 & 14.7517336 & 4.02128624 & 15.07275 & 221.3 & 52.494711 & 69.6 & 69.1 & 68.4 & 69 & 0.000265 \\
\hline 640.01999 & 3.76925607 & 14.7524832 & 4.0206608 & 15.07275 & 221.4 & 52.320896 & 69.5 & 69.1 & 68.3 & 68.9 & 0.000264 \\
\hline 645.01999 & 3.76925607 & 14.7524832 & 4.0206608 & 15.0705 & 221.5 & 52.792681 & 69.6 & 69.2 & 68.3 & 68.9 & 0.000265 \\
\hline 650.04001 & 3.7873889 & 14.7532329 & 4.0206608 & 15.06975 & 221.575 & 52.892004 & 69.6 & 69.1 & 68.3 & 68.9 & 0.000265 \\
\hline 655.01999 & 3.77113188 & 14.7524832 & 4.0206608 & 15.069 & 221.6 & 52.966496 & 69.6 & 69.1 & 68.4 & 68.9 & 0.000264 \\
\hline 660.01999 & 3.77550877 & 14.750984 & 4.02003536 & 15.06975 & 221.5 & 53.810744 & 69.6 & 69.2 & 68.4 & 68.9 & 0.000265 \\
\hline 665.01999 & 3.78488782 & 14.7524832 & 4.02003536 & 15.06975 & 221.45 & 53.636928 & 69.6 & 69.2 & 68.5 & 69 & 0.000264 \\
\hline 670.01999 & 3.78426255 & 14.7524832 & 4.02003536 & 15.072 & 221.475 & 52,320896 & 69.5 & 69.1 & 68.3 & 68.9 & 0.000265 \\
\hline 675.01999 & 3.79176579 & 14.7532329 & 4.02128624 & 15.069 & 221.4 & 52.792681 & 69.6 & 69.1 & 68.4 & 68.9 & 0.000264 \\
\hline 680.03 & 3.7686308 & 14.7539825 & 4.02128624 & 15.0705 & 221.375 & 53.338958 & 69.6 & 69.1 & 68.4 & 69 & 0.000264 \\
\hline 685.01999 & 3.7623781 & 14.7524832 & 4.0206608 & 15.07275 & 221.45 & 53.115481 & 69.6 & 69.1 & 68.5 & 68.9 & 0.000264 \\
\hline 690.01999 & 3.79176579 & 14.7539825 & 4.02128624 & 15.072 & 221.375 & 52.46988 & 69.6 & 69.1 & 68.4 & 68.9 & 0.000265 \\
\hline 695.04999 & 3.77175715 & 14.7532329 & 4.02191168 & 15.07275 & 221.575 & 53.338958 & 69.7 & 69.1 & 68.4 & 68.9 & 0.000265 \\
\hline 700.01999 & 3.79614268 & 14.7532329 & 4.0206608 & 15.072 & 221.2 & 53.612097 & 69.6 & 69.2 & 68.5 & 69 & 0.000264 \\
\hline 705.01999 & 3.79239106 & 14.7532329 & 4.02128624 & 45.06975 & 221.425 & 52.792681 & 69.7 & 69.1 & 68.4 & 69 & 0.000265 \\
\hline 710.01999 & 3.7811362 & 14.7532329 & 4.02128624 & 15.07275 & 221.375 & 53,338958 & 69.7 & 69.1 & 68.5 & 69 & 0.000265 \\
\hline 715.01999 & 3.81615132 & 14.7517336 & 4.02128624 & 15.072 & 231.325 & 53.314128 & 69.7 & 69.1 & 68.4 & 68.9 & 0.000264 \\
\hline 720.01999 & 3.76800553 & 14.7532329 & 4.02128624 & 15.06975 & 231.45 & 53.487943 & 69.8 & 69.2 & 68.6 & 69 & 0.000264 \\
\hline 725.01999 & 3.79614268 & 14.7539825 & 4.0206608 & 15.06975 & 231.475 & 55.201268 & 68.7 & 6.2 & 68.4 & 68.9 & 0.000264 \\
\hline 730.01999 & 3.77300769 & 14.7539825 & 4.0206608 & 15.07275 & 221.4 & 53.810744 & 69.7 & 69.2 & 68.5 & 6 & 0.000265 \\
\hline 735.01999 & 3.79551741 & 14.7539825 & 4.02128624 & 15.06975 & 221.425 & 52.718188 & 69.7 & 69.2 & 68.5 & 68.9 & 0.000264 \\
\hline 740.06 & 3.79551741 & 14.7517336 & 4.02128624 & 15.0705 & 221.55 & 52.916835 & 69.7 & 69.1 & 68.5 & 68.9 & 0.000264 \\
\hline 745.01999 & 3.77926039 & 14.7532329 & 4.02191168 & 15.072 & 221.55 & 53.165143 & 69.7 & 69.2 & 68.6 & 69 & 0.000265 \\
\hline 750.01999 & 3.7686308 & 14.7517336 & 4.02191168 & 15.07275 & 221.55 & 52.494711 & 69.7 & 69.1 & 68.6 & 68.9 & 0.000264 \\
\hline 755.07001 & 3.7811362 & 14.7517336 & 4.02128624 & 15.072 & 221.5 & 53.264466 & 69.6 & 69.1 & 68.4 & 68.9 & 0,000264 \\
\hline 760.01999 & 3.77926039 & 14.7524832 & 4.02128624 & 15.072 & 221.425 & 52.420219 & 69.6 & 69.2 & 68.5 & 68.9 & 0.000264 \\
\hline 765.01999 & 3.77988566 & 14.7517336 & 4.0206608 & 15.07275 & 221.3 & 52.76785 & 69.7 & 69.1 & 68.3 & 68.9 & 0.000265 \\
\hline 770.04999 & 3.77926039 & 14.7524832 & 4.02126624 & 15.07275 & 221.15 & 52.345726 & 69.7 & 69.2 & 68.3 & 68.9 & 0.000264 \\
\hline 775.01999 & 3.79676795 & 14.7524832 & 4.02191168 & 15.072 & 221.425 & 52.867173 & 69.6 & 69.1 & 68.3 & 68.9 & 0.000264 \\
\hline 780.01999 & 3.77113188 & 14.7517336 & 4.02128624 & 15.072 & 221.175 & 52.792681 & 69.6 & 69.2 & 68.5 & 68.9 & 0.000264 \\
\hline 785.07001 & 3.77113188 & 14.7532329 & 4.02191168 & 15.072 & 221.375 & 53.68659 & 69.6 & 69.2 & 68.4 & 68.9 & 0.000264 \\
\hline 790.01999 & 3.7998943 & 14.7517336 & 4.02128624 & 15.06975 & 231.325 & 53.115481 & 69.6 & 69.1 & 68.4 & 68.9 & 0.000265 \\
\hline 795.01999 & 3.78551309 & 14.7524832 & 4.02128624 & 15.072 & 231.275 & 52.842342 & 69.7 & 69.2 & 68.4 & 68.9 & 0.000264 \\
\hline 800.06 & 3.76612972 & 14.7524832 & 4.0206608 & 15.0735 & 21.6 & 52.271234 & 69.7 & 69.2 & 68.4 & 68.9 & 0.000266 \\
\hline 805.01999 & 3.7748835 & 14.7517336 & 4,0206608 & 15.07275 & 221.275 & 52.916835 & 69.6 & 69.1 & 68.5 & 68.9 & 0.000264 \\
\hline 810.01999 & 3.78801417 & 14.7524832 & 4.02128624 & 15.072 & 221.525 & 53.09065 & 69.6 & 69.2 & 68.3 & 68.9 & 0.000266 \\
\hline 815.04999 & 3.76425394 & \begin{tabular}{|l|}
4.7539825 \\
\end{tabular} & 4.0206608 & 15.07275 & 221.175 & 52.370557 & 69.7 & 69.2 & 68.4 & 68.9 & 0.000265 \\
\hline
\end{tabular}




\begin{tabular}{|c|c|c|c|c|c|c|c|c|c|c|c|}
\hline SECONDS & \begin{tabular}{|l|} 
MAIN DUCT \\
DIFF (IN H2O
\end{tabular} & \begin{tabular}{|l|} 
MAIN DUCT \\
ABS (PSIA)
\end{tabular} & \begin{tabular}{|l|} 
GAS INJECT \\
DIFF (IN H2O)
\end{tabular} & \begin{tabular}{|l|} 
GAS INJECT \\
ABS (PSIA)
\end{tabular} & \begin{tabular}{|l} 
NEWPORT \\
(PPM)
\end{tabular} & \begin{tabular}{|l|} 
SMC \\
(PPM)
\end{tabular} & \begin{tabular}{|l|} 
AMBIENT \\
TEMP.(F)
\end{tabular} & \begin{tabular}{|l} 
GAS INJECT \\
TEMP(F)
\end{tabular} & $\begin{array}{l}\text { MAIN DUCT } \\
\text { TEMP (F) }\end{array}$ & $\frac{\mid \text { WEC CELL }}{\text { TEMP.(F) }}$ & $\begin{array}{l}\text { WEC CELL } \\
\text { (VOLTS) }\end{array}$ \\
\hline & & & & & & & & & & & \\
\hline 820.01999 & 3.7748835 & 14.7517336 & 4.02128624 & 15.07275 & 230.75 & 52.693358 & 69.7 & 69.2 & 68.4 & 69 & 0.000265 \\
\hline 825.01999 & 3.77238242 & 14.7532329 & 4.0206608 & 15.07275 & 221.4 & 51.873941 & 69.7 & 69.1 & 68.3 & 68.9 & 0.000265 \\
\hline 830.04001 & 3.79926903 & 14.7524832 & 4.02128624 & 15.072 & 221.6 & 52.693358 & 69.8 & 69.2 & 68.4 & 68.9 & 0.000266 \\
\hline 835.01999 & 3.77363296 & 14.7524832 & 4.02128624 & 15.072 & 221.55 & 52.370557 & 69,8 & 69.2 & 68.4 & 68.9 & 0.000265 \\
\hline 840.04999 & 3.78676363 & 14.7532329 & 4.0206608 & 15.07275 & 231.425 & 52.022926 & 69.8 & 69.2 & 68.4 & 69 & 0.000265 \\
\hline 845.04001 & 3.77175715 & 14.7524832 & 4.02128624 & 15.072 & 221.5 & 53.264466 & 69.8 & 69.2 & 68.4 & 68.9 & 0.000265 \\
\hline 850.01999 & 3.79301633 & 14.7524832 & 4.0206608 & 15.07275 & 221.45 & 52.097418 & 69.8 & 69.2 & 68.3 & 68.8 & 0.000265 \\
\hline 855.01999 & 3.83490942 & 14.7554817 & 4.0206608 & 15.07275 & 221.575 & 52.296065 & 69.7 & 69.2 & 68.5 & 68.9 & 0.000265 \\
\hline 860.03 & 3.77363296 & 14.7532329 & 4.0206608 & 15.07275 & 221,35 & 52,022926 & 69.7 & 69.2 & 68.4 & 68.9 & 0.000265 \\
\hline 865.03 & 3.77363296 & 14.7532329 & 4.02128624 & 15.07275 & 221.5 & 52,14708 & 69.6 & 69.2 & 68.4 & 69 & 0.000265 \\
\hline 870.39999 & 3.78988998 & 14.7532329 & 4.0206608 & 15.07275 & 231.45 & 53.040989 & 69.7 & 69.2 & 68.5 & 68.9 & 0.000264 \\
\hline 875.01999 & 3.78801417 & 14.7524832 & 4.0206608 & 15.07275 & 231.425 & 52.792681 & 69.7 & 69.2 & 68.5 & 68.9 & 0.000264 \\
\hline 880.01999 & 3.81114916 & 14.7517336 & 4.02128624 & 15.07275 & 231.475 & 53.363789 & 69.7 & 69.2 & 68.4 & 68.9 & 0.000265 \\
\hline 885.01999 & 3.78363728 & 14.7532329 & 4.02003536 & 15.07275 & 231.15 & 53.289297 & 69.7 & 69.2 & 68.6 & 69 & 0.000265 \\
\hline 890.01999 & 3.76362864 & 14.7539825 & 4.0175336 & 15.0735 & 231.425 & 52.196742 & 69.6 & 69.2 & 68.6 & 68.9 & 0.000264 \\
\hline 895.01999 & 3.77613404 & 14.7524832 & 4.01878448 & 15.07275 & 231.475 & 53.239635 & 69.7 & 69.2 & 68.5 & 68.9 & 0.000264 \\
\hline 900.01999 & 3.78051093 & 14.7524832 & 4.01878448 & 15.072 & 230 & 52.46988 & 69.8 & 69.2 & 68.6 & 68.9 & 0.000265 \\
\hline 905.01999 & 3.76362864 & 14.7532329 & 4.0206608 & 15.07275 & 221.425 & 52.395388 & 69.7 & 69.2 & 68.4 & 68.9 & 0.000265 \\
\hline 910.01999 & 3.78988998 & 14.7539825 & 4.0206608 & 15.072 & 231.45 & 51.973264 & 69.7 & 69.2 & 68.5 & 69 & 0.000265 \\
\hline 915.01999 & 3.77988566 & 14.7539825 & 4.0206608 & 15.06975 & 231.35 & 52.892004 & 69.7 & 69.2 & 68.3 & 68.9 & 0.000266 \\
\hline 920.04999 & 3.77988566 & 14.7524832 & 4.01878448 & 15.072 & 221.375 & 52.569204 & 69.7 & 69.2 & 68.4 & 68.9 & 0.000264 \\
\hline 925.01999 & 3.79114052 & 14.7532329 & 4.01878448 & 15.072 & 231.45 & 52.693358 & 69.8 & 69.2 & 68.5 & 68.9 & 0.000264 \\
\hline
\end{tabular}




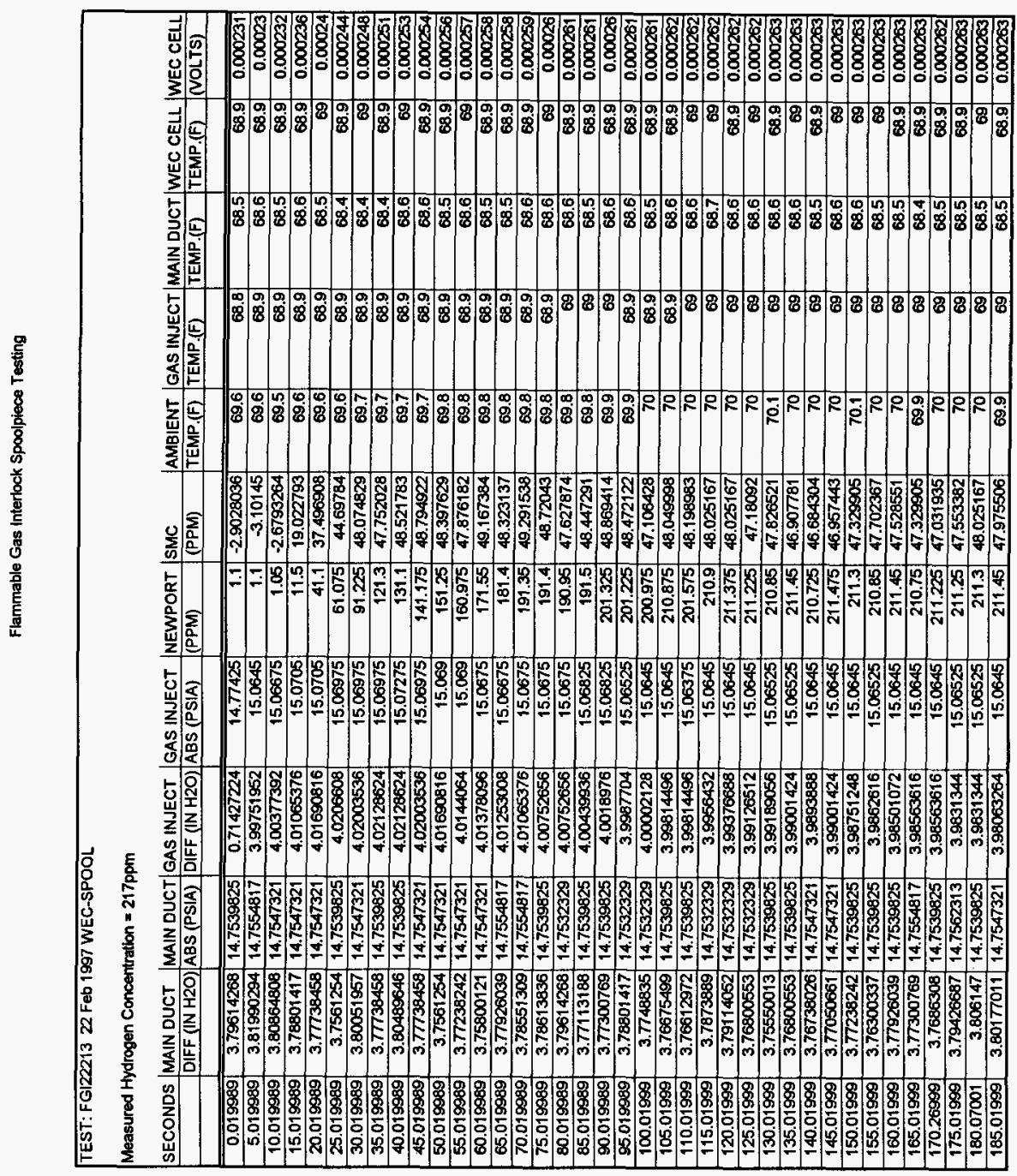




\begin{tabular}{|c|c|c|c|c|c|c|c|c|c|c|c|}
\hline SECONDS & $\begin{array}{l}\text { MAIN DUCT } \\
\text { DIFF (IN H2O) }\end{array}$ & $\begin{array}{l}\text { MAIN DUCT } \\
\text { ABS (PSIA) }\end{array}$ & \begin{tabular}{|l|} 
GAS INJECT \\
DIFF (IN H2O) \\
\end{tabular} & \begin{tabular}{|l} 
GAS INJECT \\
ABS (PSIA)
\end{tabular} & $\begin{array}{l}\text { NEWPORT } \\
\text { (PPM) }\end{array}$ & $\begin{array}{l}\text { SMC } \\
\text { (PPM) }\end{array}$ & \begin{tabular}{|l} 
AMBIENT \\
TEMP.(F) \\
\end{tabular} & \begin{tabular}{|l} 
GAS INJECT \\
TEMP.(F)
\end{tabular} & $\frac{\text { MAIN DUCT }}{\text { TEMP.(F) }}$ & \begin{tabular}{|l} 
WEC CELLL \\
TEMP.(F)
\end{tabular} & \begin{tabular}{|l} 
WEC CELL \\
(VOLTS)
\end{tabular} \\
\hline 190.01999 & 3.77926039 & 14.7532329 & 3.98125808 & 15.06375 & 211.275 & 48.074829 & 69.9 & 69 & 68.5 & 69 & 0.000262 \\
\hline 195.01999 & 3.77926039 & 14.7524832 & 3.98063264 & 15.06525 & 221.025 & 48.273475 & 69.8 & 69 & 68.4 & 69 & 0.000263 \\
\hline 200.01999 & 3.77863512 & 14.7539825 & 3.98125808 & 15.06525 & 211.2 & 47.826521 & 69.8 & 69.1 & 68.4 & 68.9 & 0.000263 \\
\hline 205.01999 & 3.79551741 & 14.7554817 & 3.98188352 & 15.06525 & 211.675 & 48.546614 & 69.9 & 69.1 & 68.5 & 69 & 0.000263 \\
\hline 210.01999 & 3.79239106 & 14.7554817 & 3.98125808 & 15.0645 & 211.475 & 47.404397 & 69.9 & 69.1 & 68.5 & 68.9 & 0.000263 \\
\hline 215.01999 & 3.78988998 & 14.7554817 & 3.97875632 & 15.0645 & 221.175 & 47.205751 & 69.9 & 69 & 68.6 & 69 & 0,000263 \\
\hline 220.01999 & 3.76925607 & 14.7532329 & 3.97875632 & 15.0645 & 221.175 & 48.273475 & 69.8 & 69 & 68.4 & 68.9 & 0.000263 \\
\hline 225.01999 & 3.77238242 & 14.7554817 & 3.97813088 & 15.0645 & 218.55 & 48.447291 & 69.8 & 69.1 & 68.5 & 69 & 0.000263 \\
\hline 230.01999 & 3.79614268 & 14.7547321 & 3.97875632 & 15.0645 & 221.6 & 47.901013 & 69.9 & 69.1 & 68.6 & 68.9 & 0.000264 \\
\hline 235.01999 & 3.76925607 & 14.7554817 & 3.97875632 & 15.063 & 221.6 & 47.280243 & 69.9 & 69.1 & 68.4 & 68.9 & 0.000263 \\
\hline 240.01999 & 3.77300769 & 14.7554817 & 3.97813088 & 15.0645 & 221.375 & 47.752028 & 69.9 & 69.1 & 68.6 & 69.1 & 0.000263 \\
\hline 245.10001 & 3.77800985 & 14.7539825 & 3.97875632 & 15.06525 & 221.375 & 48.273475 & 69.9 & 69.1 & 68.6 & 69 & 0.000263 \\
\hline 250.01999 & 3.78613836 & 14.7532329 & 3.97875632 & 15.066 & 221.575 & 48.596276 & 70 & 69.1 & 68.6 & 69 & 0.000263 \\
\hline 255.01999 & 3.7686308 & 14.7532329 & 3.97813088 & 15.06225 & 211.5 & 48.347968 & 69.9 & 69.1 & 68.5 & 68.9 & 0.000263 \\
\hline 260.01999 & 3.77738458 & 14.7539825 & 3.97688 & 15.06225 & 211.275 & 48.298306 & 69.9 & 69.1 & 68.5 & 68.9 & 0.000263 \\
\hline 265.03 & 3.79676795 & 14.7554817 & 3.97625456 & 15.06225 & 211.025 & 47.429228 & 70 & 69.1 & 68.5 & 68.9 & 0.000263 \\
\hline 270.03 & 3.81114916 & 14.7539825 & 3.97625456 & 15.0615 & 221.575 & 47.50372 & 70 & 69.1 & 68.6 & 69 & 0.000263 \\
\hline 275.01999 & 3.76487918 & 14.7547321 & 3.97688 & 15.0615 & 211 & 47.131258 & 69.8 & 69.1 & 68.7 & 69.1 & 0.000263 \\
\hline 280.01999 & 3.77675931 & 14.7547321 & 3.97500368 & 15.0645 & 211.5 & 47.18092 & 69.8 & 69.1 & 68.5 & 68.9 & 0.000263 \\
\hline 285.03 & 3.78426255 & 14.7532329 & 3.9737528 & 15.0645 & 221.425 & 47.056766 & 69.7 & 69.1 & 68.6 & 68.9 & 0.000263 \\
\hline 290.01999 & 3.77363296 & 14.7539825 & 3.97437824 & 15.0645 & 221.6 & 48.09966 & 69.6 & 69.1 & 68.6 & 69 & 0.000263 \\
\hline 295.03 & 3.77300769 & 14.7532329 & 3.97688 & 15.0615 & 221.45 & 47.578213 & 69.7 & 69.1 & 68.7 & 68.9 & 0.000263 \\
\hline 300.01999 & 3.79114052 & 14.7532329 & 3.97688 & 15.06225 & 221.4 & 47.528551 & 69.6 & 69.1 & 68.5 & 68.9 & 0.000262 \\
\hline 305.03 & 3.78238674 & 14.7539825 & 3.97500368 & 15.06225 & 221.375 & 48.074829 & 69.7 & 69.1 & 68.6 & 69 & 0.000263 \\
\hline 310.01999 & 3.77113188 & 14.7532329 & 3.97688 & 15.0645 & 221.075 & 47.47889 & 69.8 & 69.1 & 68.8 & 69 & 0.000264 \\
\hline 315.03 & 3.78176147 & 14.7532329 & 3.97688 & 15.0615 & 221.55 & 48.695599 & 69.9 & 69.1 & 68.6 & 68.9 & 0.000263 \\
\hline 320.01999 & 3.791765579 & 14.7547321 & 3.97250192 & 15.06225 & 221.6 & 48.12449 & 69.9 & 69.1 & 68.6 & 69 & 0.000263 \\
\hline 325.01999 & 3.77175715 & 14.7524832 & 3.97562912 & 15.06525 & 221.2 & 48.447291 & 69.9 & 69.1 & 68.7 & 68.9 & 0.000264 \\
\hline 330.01999 & 3.77113188 & 14.7547321 & 3.97250192 & 15.06375 & 221.425 & 48.025167 & 70 & 69.1 & 68.6 & 69 & 0.000264 \\
\hline 335.01999 & 3.7811362 & 14.7547321 & 3.9737528 & 15.06225 & 221.325 & 48.298306 & 69.9 & 69.1 & 68.8 & 69 & 0.000263 \\
\hline 340.01999 & 3.77988566 & 14.7562313 & 3.97312736 & 15.06525 & 221.4 & 48.347968 & 69.9 & 69.1 & 68.6 & 69 & 0.000263 \\
\hline 345.01999 & 3.7686308 & 14.7562313 & 3.97250192 & 15.0615 & 221.6 & 48.74526 & 69.9 & 69.1 & 68.6 & 69 & 0.000263 \\
\hline 350.01999 & 3.77363296 & 14.7562313 & 3.97312736 & 15.06225 & 221.275 & 47.925844 & 69.9 & 69.1 & 68.7 & 69.1 & 0.000263 \\
\hline 355.01999 & 3.77300769 & 14.7539825 & 3.97312736 & 15.06225 & 221.3 & 47.454059 & 69.9 & 69.1 & 68.6 & 69 & 0.000263 \\
\hline 360.01999 & 3.77926039 & 14.7539825 & 3.97250192 & 15.06225 & 221.525 & 47.80169 & 69.9 & 69.1 & 68.8 & 69.1 & 0.000263 \\
\hline 365.04001 & 3.77738458 & 14.7539825 & 3.97187648 & 15.06225 & 221.55 & 47.950675 & 70 & 69.2 & 68.7 & 69 & 0.000264 \\
\hline 370.01999 & 3.79176579 & 14.7554817 & 3.97250192 & 15.063 & 221.55 & 48.323137 & 69.9 & 69.2 & 68.7 & 69 & 0.000263 \\
\hline 375.06 & 3.78801417 & 14.7532329 & 3.97250192 & 15.06225 & 221.5 & 48.72043 & 69.9 & 69.2 & 68.8 & 69.1 & 0.000264 \\
\hline 380.01999 & 3.77988566 & 14.7554817 & 3.97250192 & 15.0615 & 221.375 & 48.968738 & 69.8 & 69.1 & 68.7 & 69 & 0.000263 \\
\hline 385.04001 & 3.77863512 & 14.7547321 & 3.97187648 & 15.06075 & 221.575 & 48.223814 & 69.7 & 69.1 & 68.7 & 68.9 & 0.000263 \\
\hline 390.01999 & 3.779885666 & 14.7554817 & 3.97250192 & 15.06225 & 221.35 & 48.993568 & 69.8 & 69.1 & 68.5 & 69 & 0.000264 \\
\hline 395.06 & 3.80427119 & 14.7539825 & 3.97250192 & 15.06225 & 221.325 & 49.887477 & 69.8 & 69.1 & 68.5 & 68.9 & 0.000263 \\
\hline
\end{tabular}




\begin{tabular}{|c|c|c|c|c|c|c|c|c|c|c|c|}
\hline SECONDS & MAIN DUCT & MAIN DUCT & GAS INJECT & GAS INJECT & \begin{tabular}{|l|} 
NEWPORT \\
\end{tabular} & SMC & AMBIENT & GAS INJECT & MAIN DUCT & WEC CELL & WEC CELL \\
\hline & DIFF (IN H2O) & $A B S$ (PSIA) & DIFF (IN H2O) & ABS (PSIA) & \begin{tabular}{|l|} 
(PPM) \\
\end{tabular} & (PPM) & TEMP.(F) & TEMP.(F) & TEMP.(F) & TEMP.(F) & (VOLTS) \\
\hline 400.01999 & 3.79676795 & 14.7539825 & 3.97312736 & 15.0615 & 221.425 & 48.869414 & 69.9 & 69.2 & 68.7 & 69 & 0.000263 \\
\hline 405.01999 & 3.78613836 & 14.7539825 & 3.97187648 & 15.0615 & 221.475 & 50.135785 & 69.8 & 69.2 & 68.7 & 69 & 0.000263 \\
\hline 410.01999 & 3.78613836 & 14.7539825 & 3.97187648 & 15.06225 & 221.575 & 49.241876 & 69.7 & 69.2 & 68.7 & 6 & 0.000263 \\
\hline 415.01999 & 3.77926039 & 14.7539825 & 3.97250192 & 15.06225 & 221.525 & 48.993568 & 69.7 & 69.1 & 68.6 & 68.9 & 0.000263 \\
\hline 420.01999 & 3.77300769 & 14.7539825 & 3.97187648 & 15.06225 & 221.4 & 48.894245 & 69.7 & 69.2 & 68.6 & 68.9 & 0.000263 \\
\hline 425.01999 & 3.77300769 & 14.7547321 & 3.97187648 & 15.063 & 211.275 & 49.589508 & 69.7 & 69.2 & 68.7 & 69 & 0.000263 \\
\hline 430.01999 & 3.77613404 & 14.7554817 & 3.97250192 & 15.063 & 221.45 & 48.695599 & 69.7 & 69.2 & 68.6 & 68.9 & 0.000263 \\
\hline 435.01999 & 3.78863944 & 14.7532329 & 3.97000016 & 15.0615 & 221.475 & 49.241876 & 69.8 & 69.2 & 68.7 & 69 & 0.000263 \\
\hline 440.01999 & 3.78551309 & 14.7524832 & 3.97312736 & 15.063 & 221.325 & 50.781386 & 69.8 & 69.2 & 68.8 & 69.1 & 0.000264 \\
\hline 445.01999 & 3.7686308 & 14.7532329 & 3.97312736 & 15.06225 & 221.35 & 48.149321 & 69.8 & 69.2 & 68.6 & 68.9 & 0.000263 \\
\hline 450.01999 & 3.77238242 & 14.7547321 & 3.97187648 & 15.06225 & 221.375 & 49.639169 & 69.9 & 69.2 & 68.6 & 69 & 0.000263 \\
\hline 455.01999 & 3.77675931 & 14.7539825 & 3.97250192 & 15.06375 & 221.225 & 49.068061 & 69.9 & 69.2 & 68.6 & 68.9 & 0.000264 \\
\hline 460.01999 & 3.77738458 & 14.7539825 & 3.97312736 & 15.06225 & 221.55 & 48.273475 & 69.9 & 69.2 & 68.7 & $\infty$ & 0.000263 \\
\hline 465.01999 & 3.79239106 & 14.7539825 & 3.97250192 & 15.0615 & 211.425 & 49.217046 & 69.9 & 69.2 & 68.7 & 69 & 0.000263 \\
\hline 470.01999 & 3.77926039 & 14.75399825 & 3.97250192 & 15.06225 & 221.075 & 48.571445 & 69.9 & 69.2 & 68.6 & 68.9 & 0.000263 \\
\hline 475.01999 & 3.77926039 & 14.75398825 & 3.97312736 & 15.063 & 221.4 & 48.645937 & 69.9 & 69.2 & 68.5 & 69 & 0.000263 \\
\hline 480.01999 & 3.77550877 & 14.7539825 & 3.97000016 & 15.06375 & 221.5 & 48.496952 & 69.8 & 69.2 & 68.6 & 69 & 0.000263 \\
\hline 485.01999 & 3.76925607 & 14.7539825 & 3.97187648 & 15.06225 & 221.375 & 48.670768 & 69.8 & 69.2 & 68.6 & 69 & 0.000263 \\
\hline 490.01999 & 3.77238242 & 14.7539825 & 3.97250192 & 15.05925 & 221.4 & 49.415692 & 69.8 & 69.2 & 68.6 & 69.1 & 0.000264 \\
\hline 495.01999 & 3.7811362 & 14.7539825 & 3.97250192 & 15.06525 & 221.2 & 49.465354 & 69.8 & 69.2 & 68.5 & 69 & 0.000263 \\
\hline 500.01999 & 3.76425391 & 14.7532329 & 3.97000016 & 15.06225 & 221.5 & 48.273475 & 69.8 & 69.2 & 68.5 & 69 & 0.000263 \\
\hline 505.01999 & 3.77738458 & 14.7547321 & 3.97250192 & 15.063 & 221.55 & 48.372798 & 69.8 & 69.2 & 68.6 & 69 & 0.000263 \\
\hline 510.01999 & 3.77363296 & 14.7524832 & 3.97312736 & 15.063 & 210.55 & 48.447291 & 69.8 & 69.2 & 68.5 & 6 & 0.000263 \\
\hline 515.04001 & 3.77675931 & 14.7547321 & 3.97000016 & 15.0615 & 221.55 & 48.397629 & 69.7 & 69.2 & 68.5 & 69 & 0.000264 \\
\hline 520.01999 & 3.80739754 & 14.7524832 & 3.9706256 & 15.05225 & 221.225 & 48.000336 & 69.7 & 69.2 & 68.5 & 69 & 0.000264 \\
\hline 525.01999 & 3.77926039 & 14.7547321 & 3.96874928 & 15.06225 & 211.475 & 47.80169 & 69.8 & 69.2 & 68.6 & 69 & 0.000263 \\
\hline 530.01999 & 3.79614268 & 14.7539825 & 3.96937472 & 15.0615 & 214.175 & 47.851352 & 69.8 & 69.2 & 68.5 & 69 & 0.000264 \\
\hline 535.01999 & 3.79676795 & 14.7547321 & 3.96812384 & 15.06 & 21.2 & 47.578213 & 69.9 & 69.2 & 68.6 & 69 & 0.000263 \\
\hline 540.01999 & 3.779885566 & 14.7554817 & 3.9674984 & 15.06225 & 221.55 & 47.156089 & 69.9 & 69.2 & 68.6 & 69.1 & 0.000264 \\
\hline 545.08002 & 3.77425823 & 14.7547321 & 3.96874928 & 15.063 & 221.25 & 47.47889 & 69.9 & 69.2 & 68.5 & 68.9 & 0.000262 \\
\hline 550.01999 & 3.78176147 & 14.7539825 & 3.96812384 & 15.05925 & 221.55 & 47.404397 & 69.8 & 69.2 & 68.5 & 6 & 0.000263 \\
\hline 555.01999 & 3.77300769 & 14.7539825 & 3.96812384 & 15.0615 & 221.425 & 47.727198 & 69.9 & 69.2 & 68.6 & 69 & 0.000264 \\
\hline 560.04999 & 3.77926039 & 14.7532329 & 3.96874928 & 15.06 & 221.35 & 48.149321 & 69.9 & 69.2 & 68.6 & 69 & 0.000264 \\
\hline 565.01999 & 3.7748835 & 14.7532329 & 3.96874928 & 15.06225 & 221.475 & 47.627874 & 69.8 & 69.2 & 68.6 & 69 & 0.000264 \\
\hline 570.01999 & 3.77300769 & 14.7532329 & 3.96874928 & 15.06 & 210.9 & 48.074829 & 69.8 & 69.2 & 68.6 & 69 & 0.000263 \\
\hline 575.04001 & 3.77363296 & 14.7539825 & 3.96812384 & 15.06075 & 221.1 & 48.794922 & 69.8 & 69.2 & 68.7 & 69.1 & 0.000264 \\
\hline 580,01999 & 3.806147 & 14.7539825 & 3.96687296 & 15.05925 & 221.525 & 47.901013 & 69.9 & 69.2 & 68.6 & 6 & 0.000263 \\
\hline 585.01999 & 3.77675931 & 14.7539825 & 3.966872296 & 15.05925 & 221.6 & 48.372798 & 69.9 & 69.2 & 68.5 & 69 & 0.000263 \\
\hline 590.01999 & 3.77300769 & 14.7532329 & 3.96562208 & 15.0615 & 221.6 & 48.496952 & 69.8 & 69.2 & 68.6 & 6 & 0.000263 \\
\hline 595.01999 & 3.78926471 & 14.7547321 & 3.96874928 & 15.063 & 221.4 & 48.025167 & 69.9 & 69.2 & 68.6 & 6 & 0.000262 \\
\hline 600.01999 & 3.77175715 & 14.7547321 & 3.96624752 & 15.05925 & 221.3 & 47.50372 & 70 & 69.2 & 68.5 & 68.9 & 0.000263 \\
\hline 605.04999 & 3.78426255 & 14.7539825 & 3.96624752 & 15.06225 & 221.5 & 48.000336 & 70 & 69.2 & 68.5 & 69 & 0.000263 \\
\hline
\end{tabular}




\begin{tabular}{|c|c|c|c|c|c|c|c|c|c|c|c|}
\hline SECONDS I & \begin{tabular}{|l|} 
MAIN DUCT \\
DIFF (IN H2O)
\end{tabular} & \begin{tabular}{|l} 
MAIN DUCT \\
ABS (PS|A)
\end{tabular} & $\begin{array}{l}\text { GAS INJECT } \\
\text { DIFF (IN H2O) }\end{array}$ & $\begin{array}{l}\text { GAS INJECT } \\
\text { ABS (PSIA) }\end{array}$ & \begin{tabular}{|l|} 
NEWPORT \\
(PPM) \\
\end{tabular} & $\begin{array}{l}\text { SMC } \\
\text { (PPM) }\end{array}$ & \begin{tabular}{|l} 
AMBIENT \\
TEMP.(F) \\
\end{tabular} & \begin{tabular}{|l|} 
GAS INJECT \\
TEMP.(F) \\
\end{tabular} & \begin{tabular}{|l|} 
MAIN DUCT \\
TEMP.(F) \\
\end{tabular} & $\begin{array}{l}\text { WEC CELL } \\
\text { TEMP.(F) }\end{array}$ & $\begin{array}{l}\text { WEC CELL } \\
\text { (VOLTS) }\end{array}$ \\
\hline 610.01999 & 3.77300769 & 14.7524832 & 3.96687296 & 15.06 & 221.55 & 48.42246 & 70 & 69.2 & 68.6 & 69.1 & 0.000263 \\
\hline 615.01999 & 3.78176147 & 14.7539825 & 3.96624752 & 15.06 & 221.4 & 47.826521 & 70 & 69.2 & 68.7 & 69.1 & 0.000263 \\
\hline 620.01999 & 3.77863512 & 14.7547321 & 3.96687296 & 15.05925 & 218.175 & 48.819753 & 70.1 & 69.2 & 68.5 & 69 & 0.000253 \\
\hline 625.01999 & 3.77175715 & 14.7547321 & 3.96624752 & 15.05925 & 221.15 & 48.770091 & 70.1 & 69.2 & 68.6 & 69 & 0.000263 \\
\hline 630.01999 & 3.79739322 & 14.7524832 & 3.96562208 & 15.05925 & 221.575 & 48.72043 & 70.1 & 69.2 & 68.7 & 69 & 0.000264 \\
\hline 635.01999 & 3.76925607 & 14.7532329 & 3.96624752 & 15.06 & 221.4 & 47.652705 & 70.1 & 69.2 & 68.7 & 69.1 & 0.000264 \\
\hline 640.07001 & 3.79239106 & 14.7532329 & 3.96624752 & 15.05925 & 221.575 & 48.397629 & 70.1 & 69.3 & 68.6 & 69 & 0.000263 \\
\hline 645.01999 & 3.77550877 & 14.7539825 & 3.96562208 & 15.05925 & 213.5 & 48.447291 & 70.1 & 69.2 & 68.6 & 69 & 0.000263 \\
\hline 650.01999 & 3.77113188 & 14.7547321 & 3.96624752 & 15.06 & 211.025 & 48.621106 & 70.1 & 69.2 & 68.6 & 69.1 & 0.000263 \\
\hline 655.06 & 3.7811362 & 14.7547321 & 3.96562208 & 15.05925 & 211.4 & 49.3412 & 70.1 & 69.2 & 68.7 & 69.1 & 0,000263 \\
\hline 660.01999 & 3.79551741 & 14.7547321 & 3.96687296 & 15.06 & 221.25 & 48.621106 & 70.1 & 69.2 & 68.6 & 69 & 0.000263 \\
\hline 665.01999 & 3.77988566 & 14.7547321 & 3.96374576 & 15.06 & 221.1 & 47.652705 & 70 & 69.2 & 68.7 & 69.1 & 0.000263 \\
\hline 670.04001 & 3.79739322 & 14.7547321 & 3.96499664 & 15.06 & 221.55 & 48.273475 & 69.9 & $69 . \overline{3}$ & 68.5 & 69 & 0.000264 \\
\hline 675.01999 & 3.76925607 & 14.7547321 & 3.96562208 & 15.05925 & 211.275 & 48.819753 & 70 & 69.2 & 68.6 & 69 & 0.000263 \\
\hline 680.01999 & 3.78363728 & 14.7547321 & 3.96186944 & 15.05925 & 210.7 & 47.553382 & 70 & 69.2 & 68.7 & 69.1 & 0.000264 \\
\hline 685.03 & 3.79301633 & 14.7532329 & 3.961244 & 15.06 & 211.475 & 47.702367 & 70 & 69.2 & 68.5 & 69 & 0.000262 \\
\hline 690.01999 & 3.7686308 & 14.7554817 & 3.96186944 & 15.05925 & 212.775 & 48.372798 & 70 & 69.2 & 68.6 & 69.1 & 0.000264 \\
\hline 695.01999 & 3.7686308 & 14.7562313 & 3.96186944 & 15.06 & 219.075 & 47.677536 & 70 & 69.3 & 68.7 & 69.1 & 0.000263 \\
\hline 700.04001 & 3.78551309 & 14.7539825 & 3.96186944 & 15.06 & 211.45 & 48.695599 & 70 & 69.3 & 68.7 & 69.1 & 0.000263 \\
\hline 705.01999 & 3.77738458 & 14.7554817 & 3.96186944 & 15.06 & 221.175 & 48.943907 & $\overline{70.1}$ & 69.2 & 68.7 & 69.1 & 0.000263 \\
\hline 710.01999 & 3.78613836 & 14.7547321 & 3.961244 & 15.0585 & 221.4 & 48.174152 & 70 & 69.3 & 68.5 & 69 & 0.000263 \\
\hline 715.04999 & 3.76738026 & 14.7547321 & 3.96186944 & 15.06 & 211.35 & 47.901013 & 70 & 69.2 & 68.6 & 69 & 0.000264 \\
\hline 720.01999 & 3.77550877 & 14.7532329 & 3.96186944 & 15.0585 & 211.475 & 47.5533382 & 70.1 & 69.2 & 68.7 & 69.1 & 0.000263 \\
\hline 725.01999 & 3.81490078 & 14.7547321 & 3.96186944 & 15.05925 & 216.525 & 48.12449 & 70 & 69.3 & 68.6 & 69.1 & 0.000263 \\
\hline 730.04001 & 3.76675499 & 14.7539825 & 3.96061856 & 15.05925 & 221.5 & 47.50372 & 69.9 & 69.2 & 68.6 & 69 & 0.000263 \\
\hline 735.01999 & 3.76988134 & 14.7539825 & 3.96186944 & 15.06 & 211.4 & 47.553382 & 69.9 & 69.3 & 68.6 & 68.9 & 0.000263 \\
\hline 740.01999 & 3.78051093 & 14.7547321 & 3.96249488 & 15.0585 & 211.1 & 47.553382 & 69.9 & 69.2 & 68.8 & 69.1 & 0,000263 \\
\hline 745.01999 & 3.76739026 & 14.7547321 & 3.96249488 & 15.05775 & 221.6 & 46.982274 & 69.9 & 69.3 & 68.7 & 69 & 0.000262 \\
\hline 750.01999 & 3.78613836 & 14.7547321 & 3.96186944 & 15.06 & 221.5 & 46.659473 & 70 & 69.3 & 68.6 & 69 & 0.000263 \\
\hline 755.01999 & 3.79301633 & 14.7554817 & 3.961244 & 15.05925 & 221.125 & 46.609812 & 69.9 & 69.2 & 68.7 & 69.1 & 0.000223 \\
\hline 760.06 & 3.78613836 & 14.7554817 & 3.95874224 & 15.06 & 221.55 & 48.496952 & 69.9 & 69.3 & 68.7 & 69.1 & 0.000263 \\
\hline 765.01999 & 3.77300769 & 14.7554817 & 3.95936768 & 15.05925 & 221.525 & 47.454059 & 70 & 69.3 & 68.7 & 69.1 & 0.000262 \\
\hline 770.01999 & 3.79239106 & 14.7554817 & 3.96061856 & 15.05925 & 221.3 & 47.578213 & 70 & 69.2 & 68.7 & 69.1 & 0.000262 \\
\hline 775.04999 & 3.8123997 & 14.7539825 & 3.96249488 & 15.06 & 211.075 & 46.808458 & 69.9 & 69.2 & 68.7 & 69.1 & 0.000263 \\
\hline 780.01999 & 3.78988998 & 14.7547321 & 3.95936768 & 15.06 & 211.3 & 46.187688 & 69.9 & 69.3 & 68.7 & 69.1 & 0.000263 \\
\hline 785.01999 & 3.78176147 & 14.7547321 & 3.961244 & 15.05925 & 211.25 & 47.578213 & 69.9 & 69.3 & 68.6 & 69 & 0.000263 \\
\hline 790.06 & 3.78363728 & 14.7532329 & 3.961244 & 15.05925 & 211.175 & 47.553382 & 69.9 & 69.3 & 68.6 & 69 & 0.000262 \\
\hline 795.01999 & 3.77738458 & 14,7562313 & 3.95936768 & 15.06 & 221.125 & 47.205751 & 69.9 & 69.3 & 68.7 & 69 & 0.000263 \\
\hline 800.01999 & 3.76738026 & 14.7539825 & 3.95936768 & 15.05925 & 212.8 & 48.248644 & 69.9 & 69.3 & 68.7 & 69.1 & 0.000263 \\
\hline 805.04999 & 3.77988566 & 14.7547321 & 3.95999312 & 15.06 & 221.525 & 48.000336 & 69.8 & 69.3 & 68.7 & 69.1 & 0.000264 \\
\hline 810.01999 & 3.79239106 & 14.7532329 & 3.95936768 & 15.0585 & 221.475 & 47.528551 & 69.9 & 69.3 & 68.6 & 69.1 & 0.000263 \\
\hline 815.01999 & 3.7748835 & 14.7539825 & 3.95999312 & 15.06 & 221.4 & 47.429228 & 70 & 69.3 & 68.8 & 69 & 0.000264 \\
\hline
\end{tabular}

HNF-SD-WM-TRP-275 Rev, 0 APPENDIX C, Page
Engineering Testing Laboratory 3/13/97 


\begin{tabular}{|c|c|c|c|c|c|c|c|c|c|c|c|}
\hline \begin{tabular}{|l|l|} 
SECONDS & 1 \\
\end{tabular} & \begin{tabular}{|l|} 
MAIN DUCT \\
DIFF (IN H2O)
\end{tabular} & \begin{tabular}{|l|} 
MAIN DUCT \\
ABS (PSIA)
\end{tabular} & \begin{tabular}{|l|} 
GAS INJECT \\
DIFF (IN H2O) \\
\end{tabular} & \begin{tabular}{|l} 
GAS INJECT \\
ABS (PSIA)
\end{tabular} & \begin{tabular}{|l|} 
NEWPORT \\
(PPM) \\
\end{tabular} & \begin{tabular}{|l} 
SMC \\
(PPM)
\end{tabular} & \begin{tabular}{|l} 
AMBBIENT \\
TEMP.(F)
\end{tabular} & \begin{tabular}{|l|} 
GAS INJECT \\
TEMP.(F)
\end{tabular} & \begin{tabular}{|l|} 
MAIN DUCT \\
TEMP.(F) \\
\end{tabular} & \begin{tabular}{|l|} 
WEC CELL \\
TEMP.(F)
\end{tabular} & $\begin{array}{l}\text { WEC CELL } \\
\text { NOLTS) }\end{array}$ \\
\hline & & & & & & & & & & & \\
\hline 820.04999 & 3.78801417 & 14.7547321 & 3.95936768 & 15.05925 & 221.525 & 47.975506 & 70 & 69.3 & 68.8 & 69.1 & 0.000264 \\
\hline 825.01999 & 3.79176579 & 14.75473321 & 3.96061856 & 15.05925 & 221.375 & 47.901013 & 70 & 69.3 & 68.7 & 69.1 & 0.000264 \\
\hline 830.01999 & 3.78801417 & 14.7539825 & 3.95936768 & 15.0585 & 221.525 & 47.627874 & 69.9 & 69.3 & 68.8 & 69.1 & 0.000263 \\
\hline 835.06 & 3.77675931 & 14.7554847 & 3.95936768 & 15.05925 & 211.475 & 47.851352 & 70 & 69.3 & 68.6 & 69 & 0.000264 \\
\hline 840.04999 & 3.77238242 & 14.7539825 & 3.95936768 & 15.05925 & 210.9 & 47.578213 & 70 & 69.3 & 68.7 & 69 & 0.000264 \\
\hline 845.01999 & 3.78176147 & 14.7539825 & 3.961244 & 15.05925 & 221.425 & 47.752028 & 70 & 69.3 & 68.6 & 69 & 0.000263 \\
\hline 850.04001 & 3.79676795 & 14.7539825 & 3.96061856 & 15.05925 & 211.325 & 47.627874 & 69.9 & 69.3 & 68.6 & 69 & 0.000263 \\
\hline 855.01999 & 3.77988566 & 14.7547321 & 3.95999312 & 15.05925 & 221.6 & 47.106428 & 70 & 69.3 & 68.7 & 69.1 & 0.000263 \\
\hline 860.01999 & 3.77926039 & 14.7539825 & 3.95999312 & 15.05925 & 221.1 & 47.851352 & 70 & 69.3 & 68.6 & 69.1 & 0.000262 \\
\hline 865.07001 & 3.80927335 & 14.7554817 & 3.95936768 & 15.05775 & 221.475 & 47.454059 & 69.9 & 69.3 & 68.7 & 69.1 & 0.000263 \\
\hline 870.01999 & 3.78551309 & 14.7547321 & 3.95749136 & 15.05925 & 218.275 & 47.354736 & 70 & 69.3 & 68.6 & 69 & 0.000263 \\
\hline 875.01999 & 3.7686308 & $\$ 4.7547321$ & 3.95749136 & 15.06 & 221.225 & 47.627874 & 70.1 & 69.3 & 68.6 & 69 & 0.000264 \\
\hline 880.04001 & 3.7811362 & 14.7554817 & 3.95749136 & 15.05925 & 221.25 & 47.578213 & 70 & 69.3 & 68.6 & 69 & 0.000263 \\
\hline 885.01999 & 3.7748835 & 14.7547321 & 3.95749136 & 15.05925 & 220.8 & 47.652705 & 70 & 69.3 & 68.7 & 69.1 & 0.000262 \\
\hline 890,01999 & 3.78926471 & 14.7547321 & 3.95749136 & 15.05925 & 221.5 & 47.429228 & 69.9 & 69.3 & 68.6 & 69 & 0.000263 \\
\hline 895.04999 & 3.79551741 & 14.7539825 & 3.9581168 & 15.0585 & 221.125 & 47.329905 & 70 & 69.3 & 68.5 & 69 & 0.000264 \\
\hline 900.01999 & 3.77175715 & 14.7554817 & 3.95749136 & 15.05925 & 210.6 & 48.049998 & 70 & 69.3 & 68.6 & 69 & 0.000263 \\
\hline 905.01999 & 3.78613836 & 14.7554817 & 3.95686592 & 15.0585 & 221.525 & 47.031935 & 70 & 69.3 & 68.6 & 69.1 & 0.000263 \\
\hline 910.04999 & 3.81802713 & 14.7532329 & 3.95749136 & 15.06 & $2 \div 1.475$ & 46.982274 & 70 & 69.3 & 68.6 & 69 & 0.000263 \\
\hline 915.01999 & 3.76988134 & 14.7554817 & 3.95749136 & 15.05925 & 211.15 & 47.131258 & 69.9 & 69.3 & 68.5 & 69 & 0.000264 \\
\hline 920.01999 & 3.7873889 & 14.7539825 & 0.080013536 & 14.60025 & 210.9 & 47.329905 & 69.9 & 69.3 & 68.6 & 69 & 0.000264 \\
\hline
\end{tabular}




\begin{tabular}{|c|c|c|c|c|c|c|c|c|c|c|c|}
\hline TEST:FGI2 & $2222122 \mathrm{Feb} 1$ & 1997 WEC-SP & $9 \mathrm{ppm}$ & & & & & & & & \\
\hline SECOND & \begin{tabular}{|l|} 
MAIN DUCT \\
DIFF IIN H2O
\end{tabular} & $\mid$\begin{tabular}{|l|} 
MAIN DUCT \\
ABS (PSIA)
\end{tabular} & \begin{tabular}{|l|} 
GAS INJECT \\
DIFF INHZO
\end{tabular} & GAS INJECT & NEWPORT & SMC & AMBIENT & GAS INJECT & MAIN DUCT & & WEC CELL \\
\hline & & ABS (PSIA) & DIFF (IN H2O) & ABS (PSIA) & (PPM) & (PPM) & TEMP.(F) & TEMP.(F) & TEMP.(F) & TEMP.(F) & (VOLTS) \\
\hline $\begin{array}{l}0.009979 \\
\end{array}$ & 3.81802713 & 14.7554817 & -0.000167872 & 14.58225! & 24.325 & 3.3249272 & 69.9. & 68.9 & 68.8 & 69.3 & 0.000234 \\
\hline 5.029999 & 3.83240834 & 14.7539825 & 0.9403688 & 14.62575 & 24.15 & -2.48068 & 69.9 & 69 & 68.6 & 69.3 & 0.000234 \\
\hline 10.01999 & 3.81990294 & 14.7547321 & 0.94161968 & 14.62575 & 49.875 & 692.036796 & 70 & 69 & 68.7 & 69.3 & 0.000248 \\
\hline 15.01999 & 3.81990294 & 14.7547321 & 0.94224512 & 14.625 & 251.5 & 1317.02803 & 69.9 & 69 & 68.7 & 69.3 & 0.000284 \\
\hline 20.01999 & 3.82615564 & 14.7547321 & 0.94224512 & 14.62575 & 521.75 & 1610.7764 & 69.8 & 69 & 68.7 & 69.3 & 0.000325 \\
\hline 25.01999 & 3.81990294 & 14.7547321 & 0.94161968 & 14.625 & 782.25 & 1728.971 & 69.7 & 69 & 68.8 & 69.4 & 0.000361 \\
\hline 30.01999 & 3.84241266 & 14.7539825 & 0.94224512 & 14.6265 & 1012.25 & 1776.14952 & 69.7 & 69 & 68.8 & 69.4 & 0.000395 \\
\hline 35.01999 & 3.83240334 & 14.7532329 & 0.94099424 & 14.62575 & 1202.5 & \begin{tabular}{|l|l|}
1799.24217 \\
\end{tabular} & 69.7 & 69 & 68.8 & 69.4 & 0.00042 \\
\hline 40.01999 & 3.83240834 & 14.7532329 & 0.94161968 & 14.625 & 1372.5 & 1811.16095 & 69.8 & 69 & 68.7 & 69.3 & 0.000442 \\
\hline 45.01999 & 3.81052389 & 14.7539825 & 0.94161968 & 14.625 & 1512.25 & 1825.56282 & 69.7 & 69 & 68.9 & 69.4 & 0.000459 \\
\hline 50.01999 & $\mathbf{3 . 8 0 3 0 2 0 6 5}$ & 14.7539825 & 0.94161968 & 14.625 & 1592.25 & 1834.2536 & 69.8 & 69 & 68.9 & 69.3 & 0.000473 \\
\hline 55.01999 & 3.83866104 & 14.7532329 & 0.94099424 & 14.625 & 1672.5 & 1842.94438 & 69.8 & 69 & 68.9 & 69.4 & 0.000485 \\
\hline 60.01999 & $3.84\{78739$ & 14.7547321 & 0.94224512 & 14.625 & 1732.5 & \begin{tabular}{|l|l|}
1853.125 \\
\end{tabular} & 69.8 & 69 & 68.8 & 69.4 & 0.000493 \\
\hline 65.01999 & 3.81802713 & 14.7539825 & 0.93849248 & 14.625 & 1793 & 1857.34624 & 69.9 & 69 & 68.9 & 69.4 & .000501 \\
\hline 70.01999 & 3.84303793 & $\$ 4.7547321$ & 0.94099424 & 14.62575 & 1833 & 1857.09793 & 70.1 & 69 & 69 & 69.4 & 0.000506 \\
\hline 75.01999 & 3.83866104 & 14.7547321 & 0.94099424 & 14.62575 & 1863 & 1861.81578 & 70.1 & 69 & 69 & 69.3 & 0.000512 \\
\hline 80.01999 & 3.81552605 & 14.7547321 & 0.94099424 & 14.625 & 1903 & 1863.80225 & 70.1 & 69 & 69 & 69.4 & 0.000515 \\
\hline 85.01999 & 3.813 & 14.7539825 & 0.93974336 & 14.625 & 1923 & 1874.47949 & 70.1 & 69 & 68.9 & 69.3 & 0.000519 \\
\hline 90.01999 & 3.81615132 & 14.7539825 & 0.9403688 & 14.62575 & 1943 & 1876.21765 & 70 & 69 & 68.7 & 69.3 & 0.000522 \\
\hline 95.01999 & $818027+3$ & \begin{tabular}{|l|l}
3 & 14.7532329 \\
\end{tabular} & 0.93974336 & 4.62575 & 1962.75 & 1875.96934 & 70 & 69 & 68.8 & 69.4 & 0.000525 \\
\hline 100.03 & 3.83428415 & 14.7524832 & 0.93974336 & 14.62575 & 1973 & 1877.45919 & 70.1 & 69 & 68.9 & 69.4 & 0.000526 \\
\hline 105.02 & 3.80677227 & 14.7532329 & 0.93974336 & 14.62425 & 1983 & 1880.43888 & 70.1 & 69 & 68.9 & 69.4 & 0.000529 \\
\hline 110.02 & 3.80677227 & 14.7547321 & 0.93974336 & 14.62575 & 1993 & \begin{tabular}{|l|l|}
1883.41858 \\
\end{tabular} & 70 & 69 & 68.8 & 69.4 & 0.00053 \\
\hline 115.17 & 3.81615132 & 39825 & 0.93974336 & 14.6265 & 2003 & 1895.83398 & 70.1 & 69 & 68.7 & 69.3 & 0.000531 \\
\hline 120.02 & 3.83490942 & 14.7554817 & 0.93974336 & 14.62575 & 2012.75 & 1893.3509 & 70.1 & 69 & 68.9 & 69.4 & 0.000532 \\
\hline 125.02 & 3.83866104 & 14.7539825 & 0.93911792 & 14.625 & 2023 & 1896.3306 & 70.1 & 69 & 68.8 & 69.4 & 0.000533 \\
\hline 130.03 & 3.80864808 & 14.7532329 & 0.93786704 & 14.62425 & 2033.25 & 1898.31706 & 70.1 & 69 & 68.9 & 69.4 & 0.000534 \\
\hline 135.02 & 3.83053253 & 14.7532329 & 0.93849248 & 14.625 & 2043 & 1894.84075 & 70.1 & 69 & 68.8 & 69.3 & 0.000535 \\
\hline 140.02 & 3.79864376 & 14.7532329 & 0.9372416 & 14.625 & 2043.25 & 1893.3509 & 70.1 & 69 & 68.9 & 69.4 & 0.000536 \\
\hline 145.16 & 3.81615132 & 14.7532329 & 0.93786704 & 14.625 & 2053 & 1899.06198 & 70.1 & 69 & 69 & 69.4 & 0.000538 \\
\hline 150.02 & 3.81927767 & 14.7524832 & 0.9372416 & 14.62575 & 2053.25 & \begin{tabular}{|l|}
1899.31029 \\
\end{tabular} & 70.2 & 69 & 69 & 69.4 & 0.000538 \\
\hline 155.02 & 3.8186524 & 14.7547321 & & 14.625 & 2063 & 1897.32383 & 70.2 & 69 & 68.9 & 69.4 & 0.000537 \\
\hline 160.02 & 3.80739754 & 14.7547321 & 0.93661616 & 14.625 & 2062.75 & 1894.84075 & 70.2 & 69 & 68.9 & 69.4 & 0.000538 \\
\hline 165.02 & 3.81927767 & 14.7547321 & 0.93661616 & 14.62575 & 2063 & $\mid 1893.10259$ & 70.2 & 69 & 68.9 & 69.4 & 0.000538 \\
\hline 170.02 & 3.80239538 & 14.7547321 & 0.9372416 & 14.62575 & 2073 & 1897.07552 & 70.2 & 69 & 68.9 & 69.4 & 0.000538 \\
\hline 175.02 & 3.81114916 & 14.7547321 & 0.93661616 & 14.62575 & 2073.25 & \begin{tabular}{|l|}
1897.07552 \\
\end{tabular} & 70.2 & 69 & 68.8 & 69.4 & 0.000539 \\
\hline 180.02 & 3.83303361 & 14.7547321 & 0.9372416 & 14.625 & 2073.25 & 1899.5586 & 70.2 & 69 & 68.9 & 69.4 & 0.00054 \\
\hline 185.02 & 3.82678091 & 14.7539825 & 0.9372416 & 14.625 & 2073.26 & 1895.58567 & 70.1 & 69 & 68.9 & 69.4 & 0.00054 \\
\hline
\end{tabular}




\begin{tabular}{|c|c|c|c|c|c|c|c|c|c|c|c|}
\hline SECOND & \begin{tabular}{|l|} 
MAIN DUCT \\
DIFF (IN H2O
\end{tabular} & \begin{tabular}{|l|} 
MAIN DUCT \\
ABS (PSIA) \\
\end{tabular} & \begin{tabular}{|l|} 
GAS INJECT \\
DIFF (IN H2O)
\end{tabular} & \begin{tabular}{|l|} 
GAS INJECT \\
ABS (PS|A)
\end{tabular} & \begin{tabular}{|l|} 
NEWPORT \\
(PPM)
\end{tabular} & $\begin{array}{l}\text { SMC } \\
\text { (PPM) }\end{array}$ & \begin{tabular}{|l|} 
AMBIENT \\
TEMP.(F) \\
\end{tabular} & \begin{tabular}{|l|} 
GAS INJECT \\
TEMP.(F)
\end{tabular} & \begin{tabular}{|l|} 
MAIN DUCT \\
TEMP.(F)
\end{tabular} & \begin{tabular}{|l|} 
WEC CELL \\
TEMP.(F)
\end{tabular} & \begin{tabular}{|l|} 
WEC CELL \\
(VOLTS)
\end{tabular} \\
\hline & & & & & & & & & & & \\
\hline 190.02 & 3.81114916 & 14.7554817 & 0.9372416 & 14.62425 & 2082.75 & 1896.3306 & 70.1 & 69 & 68,8 & 69.4 & 0.000541 \\
\hline 195.02 & 3.82240402 & 14.7547321 & 0.93599072 & 14.625 & 2083 & 1898.06875 & 70.2 & 69 & 68.9 & 69.3 & 0.000541 \\
\hline 200.28 & 3.82365456 & 14.7547321 & 0.93473984 & 14.62425 & 2082.75 & 1901.79337 & 70.2 & 69 & 68.8 & 69.4 & 0.000541 \\
\hline 205.02 & 3.81802713 & 14.7547321 & 0.93473984 & 14.625 & 2083.25 & 1896.08229 & 70.2 & 69 & 69 & 69.4 & 0.000541 \\
\hline 210.02 & 3.81552605 & 14.7554817 & 0.93536528 & 14.62575 & 2083.25 & 1899.06198 & 70.1 & 69 & 68.8 & 69.4 & 0.000541 \\
\hline 215.02 & 3.8249051 & 14.7547321 & 0.93473984 & 14.62575 & 2083 & 1900.30352 & 70.2 & 69 & 68.8 & 69.3 & 0.000541 \\
\hline 220.02 & 3.82365456 & 14.7547321 & 0.93473984 & 14.62575 & 2083 & 1898.56537 & 70.2 & 69 & 68.8 & 69.3 & 0.000542 \\
\hline 225.02 & 3.83378307 & 14.7547321 & 0.93473984 & 14.625 & 2082.75 & 1900.80014 & 70.3 & 69 & 68.9 & 69.3 & 0.000541 \\
\hline 230.02 & 3.80989862 & 14.7554817 & 0.93473984 & 14.6265 & 2093.5 & 1905.7663 & 70.3 & 69.1 & 68.8 & 69.3 & 0.00054 \\
\hline 235.02 & 3.81490078 & 14.7554817 & 0.93536528 & 14.62575 & 2093.5 & 1907.00784 & 70.2 & 69 & 68.8 & 69.3 & 0.000541 \\
\hline 240.02 & 3.81427551 & 14.7554817 & 0.93536528 & 14.62575 & 2093.5 & 1905.02138 & 70.2 & 69 & 68.8 & 69.3 & 0.000541 \\
\hline 245.02 & 8249051 & 14.7547321 & 0.93536528 & 14.62575 & 2092.75 & 1899.06198 & 70.2 & 69 & 68.9 & 69.4 & 0.000541 \\
\hline 250.02 & 3.82678091 & 14.7547321 & 0.934773984 & 14.625 & 2093 & 1895.08906 & 70.2 & 69 & 68.8 & 69.4 & 0.000541 \\
\hline 255.02 & 3.83053253 & 14.7562313 & 536528 & 14.6265 & 2093 & 1903.03491 & 70.2 & 69 & 68.9 & 69.4 & 0.000543 \\
\hline 260.02 & 3.81114916 & 14.7547321 & 0.93599072 & 14.625 & 2093 & 1905.7663 & 70.2 & 69 & 68.8 & 69.4 & 0.000542 \\
\hline 265.18 & 3.811774443 & 14.7547321 & 0.93473984 & 14.62575 & 2093.25 & 1904.52476 & 70.1 & 69.1 & 68.7 & 69.3 & 0.000542 \\
\hline 270.02 & 3.82177875 & 14.7547321 & 0.93536528 & 14.62575 & 2093 & 1904.52476 & 70.1 & 69 & 68.8 & 69.4 & 0.000541 \\
\hline 275.02 & 3.81552605 & 14.7532329 & 0.93661616 & 14.62575 & 2093 & 1901.54506 & 70.1 & 69.1 & 68.9 & 69.3 & 0.000542 \\
\hline 280.02 & 3.82615564 & 14.7547321 & 0.93536528 & 14.62575 & 2093 & 1904.27645 & 70.1 & 69 & 68.9 & 69.4 & 0.000543 \\
\hline 285.03 & 3.83866104 & 14.7554817 & 0.93473984 & 14.62575 & 2093.5 & 1899.31029 & 70.1 & 69 & 68.9 & 69.4 & 0.000543 \\
\hline 290.02 & 3.82553037 & 14.7539825 & 0.93348896 & 14.62575 & 2093.5 & 1904.52476 & 70.2 & 69 & 68.9 & 69.4 & 0.000543 \\
\hline 295.02 & 3.83303361 & 14.7539825 & 0.93286352 & 14,62425 & 2093 & 1904.52476 & 70.2 & 69.1 & 68.8 & 69.3 & 0.000543 \\
\hline 300.02 & 3.80864808 & 14.7554817 & 0.93348896 & 14.62575 & 2102.75 & 1906.51122 & 70.1 & 69 & 68.7 & 69.4 & 0.000542 \\
\hline 305.02 & 3.80552173 & 14.7532329 & 0.93348896 & 14.62575 & 2093 & 1906.26292 & 70.1 & 69.1 & 68.8 & 69.3 & 0.000543 \\
\hline 310.02 & 3.81427551 & 14.7539825 & 0.93286352 & 14.62575 & 2093 & 1906.75953 & 70.2 & 69.1 & 68.8 & 69.4 & 0.000543 \\
\hline 315.02 & 3.82678091 & 14.7547321 & 0.93286352 & 14.625 & 2093 & 1902.28999 & 70.2 & 69.1 & 68.8 & 69.4 & 0.000542 \\
\hline 320.02 & 3.81615132 & 14.7539825 & 0.93348896 & 14.62575 & 2003.5 & 1900.80014 & 70.2 & 69.1 & 68.8 & 69.4 & 0.000542 \\
\hline 325.02 & 3.83240834 & 14.7532329 & 0.93286352 & 14.625 & 2093.25 & 1903.03491 & 70.2 & 69 & 68.7 & 69.4 & 000543 \\
\hline 330.02 & 3.81990294 & 14.7539825 & 0.93286352 & 14.625 & 2103.25 & 1901.04845 & 70.2 & 69.1 & 68.7 & 69.3 & 0.000542 \\
\hline 335.02 & 3.83303361 & 14.7539825 & 0.93286352 & 14.6265 & 2103.25 & 1895.33736 & 70.2 & 69 & 68.8 & 69.4 & 0,000544 \\
\hline 340.02 & 3.84428847 & 14.7532329 & 0.93223808 & 14.6265 & 2103 & \begin{tabular}{|r|}
1896,3306 \\
\end{tabular} & 70.2 & 69.1 & 68.8 & 69.4 & 0,000543 \\
\hline 345.02 & 3.82052821 & 14.7539825 & 0.93286352 & 14.62575 & 2103.25 & 1897.32383 & 70.2 & 69 & 68.8 & 69.4 & 0.000543 \\
\hline 350.02 & 3.82427983 & 14.7539825 & 0.93161264 & 14.62575 & 2103.25 & 1891.11613 & 70.3 & 69.1 & 68.9 & 69.4 & 0.000544 \\
\hline 355.02 & 3.81052389 & 14.7539825 & 0.93036176 & 14.62575 & 2102.75 & 1891.36444 & 70.2 & 69 & 68.9 & 69.4 & 0.000543 \\
\hline 360.02 & 3.82302929 & 14.7524832 & 0.93036176 & 14.62575 & 2093 & 1890.61951 & 70.2 & 69.1 & 68.9 & 69,4 & 0,000543 \\
\hline 365.02 & 3.81740186 & 14.7517336 & 0.92973632 & 14.62575 & 2103 & 1893.10259 & 70.2 & 69.1 & 68.9 & 69.4 & 0.000543 \\
\hline 370.02 & 3.81927767 & 14.7517336 & 0.9309872 & 14.625 & 2103 & 1897.82044 & 70.1 & 69.1 & 68.8 & 69,4 & 0.000543 \\
\hline 375.02 & 3.82678091 & 14.7539825 & 0.9309872 & 14.62575 & 2103.25 & 1900.80014 & 70 & 69.1 & 68.8 & 69.3 & 0.000543 \\
\hline 380.02 & 3.83053253 & 14.7532329 & 0.93036176 & 14.6265 & 2103.25 & 1904.52476 & 70.1 & 69.1 & 68.8 & 69.4 & 0.000543 \\
\hline 385.02 & 3.83240834 & 14.7517336 & 0.9309872 & 14.6265 & 2103.25 & 1893.59921 & 70.1 & 69.1 & 68.8 & 69.4 & 0.000543 \\
\hline 390.06 & 3.81490078 & 14.7539825 & 0.9309872 & 14.62575 & 2103 & 1894.59244 & 70.1 & 69.1 & 68.8 & 69.4 & 0.000543 \\
\hline 395.02 & 3.83928631 & 14.7547321 & 0.93036176 & 14.62575 & 2103.25 & 1893.84752 & 70.1 & 69.1 & 68.8 & 69.4 & 0.000544 \\
\hline
\end{tabular}




\begin{tabular}{|c|c|c|c|c|c|c|c|c|c|c|c|}
\hline SECOND & MAIN DUCT & MAIN DUCT & GAS INJECT & GAS INJECT & NEWPORT & SMC & AMBIENT & GAS INJECT & MAIN DUCT & WEC CELL & WEC CELL \\
\hline & DIFF (IN H2O & ABS (PSIA) & DIFF (IN H2O) & $A B S$ (PSIA) & (PPM) & (PPM) & TEMP.(F) & TEMP.(F) & TEMP.(F) & TEMP.(F) & (VOLTS) \\
\hline 400.03 & 3.80927335 & 14.7547321 & 0.9309872 & 14.62575 & 2102.75 & $\mid 1897.57214$ & 70.2 & 69.1 & 69 & 69.4 & 0.000544 \\
\hline 405.02 & 3.83428415 & 14.75477321 & 0.93036176 & 14.62575 & 2103.25 & 1892.85428 & 70.2 & 69.1 & 68.8 & 69.3 & 0.000543 \\
\hline 410.02 & 3.80239538 & 14.7539825 & 0.92911088 & 14.625 & 2103 & 1895.33736 & 70.2 & 69.1 & 68.9 & 69.4 & 0.000542 \\
\hline 415.02 & 3.81552605 & 14.7532329 & 0.92848544 & 14.625 & 2102.75 & \begin{tabular}{|l|}
1896.82721 \\
\end{tabular} & 70.2 & 69.1 & 68.9 & 69.4 & 0.000544 \\
\hline 420.07 & 3.80927335 & 14.7524832 & 0.93036176 & 14.62575 & 2102.75 & 1898.06875 & 70.1 & 69.1 & 68.8 & 69.4 & 0.000543 \\
\hline 425.02 & 3.81177443 & 14.7524832 & 0.92911088 & 14.62575 & 2103 & 1896.3306 & 70.1 & 69.1 & 68.9 & 69.4 & 0.000543 \\
\hline 430.02 & 3.80864808 & 14.7539825 & 0.92848544 & 14.62425 & 2103.5 & 1894.34413 & 70.1 & 69.1 & 69 & 69.4 & 0.000543 \\
\hline 435.02 & 3.83240834 & 14.7532329 & 0.92848544 & 14.62575 & 2103.5 & 1895.83398 & 70.1 & 69.1 & 68.9 & 69.4 & 0.000543 \\
\hline 440.07 & 3.81677659 & 14.7547321 & 0.92848544 & 14.62575 & 2103 & 1888.38474 & 70.1 & 69.1 & 69 & 69.4 & 0.000543 \\
\hline 445.02 & 3.81552605 & 14.7539825 & 0.92911088 & 14.6265 & 2103.25 & 1890.1229 & 70.1 & 69.1 & 69 & 69.4 & 0.000543 \\
\hline 450.09 & 3.81602713 & 14.7539825 & 0.92786 & 14.62575 & 2103.25 & 1889.62628 & 70.1 & 69.1 & 68.9 & 69.4 & 0.000543 \\
\hline 455.02 & 3.83866104 & 14.7532329 & 0.92848544 & 14.625 & 2103 & \begin{tabular}{|l|}
1891.36444 \\
\end{tabular} & 70.2 & 69.1 & 68.9 & 69.4 & 0.000544 \\
\hline 460,02 & 3.81740186 & 14.7532329 & 0.92848544 & 14.62575 & 2103.25 & 1894.59244 & 70.1 & 69.1 & 68.9 & 69.4 & 0.000544 \\
\hline 465.02 & 3.85366752 & 14.7539825 & 0.92848544 & 14.625 & 2103 & 1894.59244 & 70.1 & 69.1 & 68.8 & 69.4 & 0.000544 \\
\hline 470.02 & 3.80739754 & 14.7547321 & 0.92848544 & 14.62575 & 2103.25 & 1890.1229 & 70.1 & 69.1 & 68.8 & 69.3 & 0.000543 \\
\hline 475.02 & 3.82427983 & 14.7532329 & 0.92911088 & 14.62575 & 2103 & 1889.87459 & 70.1 & 69.1 & 68.8 & 69.4 & 0.000543 \\
\hline 480.02 & 3.82615564 & 14.7547321 & 0.92848544 & 14.6265 & 2103 & 1894.09582 & 70.1 & 69.1 & 68.9 & 69.4 & 0.000544 \\
\hline 485.02 & 3.81990294 & 14.7532329 & 0.92911088 & 14.625 & 2103.25 & 1892.35767 & 70.1 & 69.1 & 68.9 & 69.4 & 0.000543 \\
\hline 490.02 & 3,81740186 & 14.7524832 & 0.92848544 & 14.62575 & 2103.25 & 1886.64658 & 70.2 & 69.1 & 68.9 & 69.4 & 0.000543 \\
\hline 495.02 & 3.81927767 & 14.7532329 & 0.92848544 & 14.62575 & 2103.25 & 1888.88136 & 70.2 & 69.1 & 68.9 & 69.4 & 0.000543 \\
\hline 500.02 & 3.81990294 & 14.7532329 & 0.92848544 & 14.62575 & 2103 & 1890.86782 & 70.2 & 69.1 & 69 & 69.4 & 0.000543 \\
\hline 505.02 & 3.82553037 & 14.7562313 & 0.92848544 & 14.62575 & 2103 & 1889.87459 & 70.2 & 69.1 & 69 & 69.4 & 0.000543 \\
\hline 510.06 & 3.80677227 & 14.7539825 & 0.92911088 & 14.62575 & 2103 & 1889.12966 & 70.3 & 69.1 & 68.9 & 69.4 & 0.000542 \\
\hline 515.06 & 3.81615132 & 14.7539825 & 0.92973632 & 14.6265 & 2103 & 1886.64658 & 70.2 & 69.1 & 69 & 69,4 & 0.000544 \\
\hline 520.02 & 3.82990726 & 14.7539825 & 0.92848544 & 14.625 & 2103.25 & 1889.62628 & 70.1 & 69.1 & 69 & 69,4 & 0.000543 \\
\hline 525.05 & 3.83365888 & 14.7547321 & 0.92786 & 14.625 & 2102.5 & 1887.88812 & 70.2 & 69.2 & 68,9 & 69.3 & 0.000542 \\
\hline 530.02 & 3.82240402 & 14.7539825 & 0.92911088 & 14.62575 & 2103 & 1884,90843 & 70.1 & 69.1 & 69 & 69.4 & 0.000543 \\
\hline 535.02 & 3.82615564 & 14.7547321 & 0.92911088 & 14.62575 & 2103 & 1887.1432 & 70.1 & 69.1 & 68.9 & 69.4 & 0.000543 \\
\hline 540.02 & 3.81990294 & 14.7532329 & 0.92911088 & 14.62575 & 2103.25 & 1886.89489 & 70.1 & 69.1 & 68.9 & 69.4 & 0.000543 \\
\hline 545.08 & 3.80552173 & 14.7554817 & 0.92848544 & 14.62575 & 2103.25 & 1889.37797 & 70.1 & 69.1 & 68.9 & 69.4 & 0.000543 \\
\hline 550.02 & 3.81615132 & 14.7539825 & 0.92911088 & 14.62575 & 2103.5 & 1884.66012 & 70 & 69.2 & 68.8 & 69.4 & 0.000543 \\
\hline 555.05 & 3.82427983 & 14.7539825 & 0.92598368 & 14.62575 & 2103 & 1880.43888 & 70 & 69.1 & 69 & 69.4 & 0.000544 \\
\hline 560.02 & 3.80927335 & 14.7539825 & 0.92911088 & 14.62575 & 2103 & 1880.43888 & 69.9 & 69.1 & 68.8 & 69.4 & 0.000543 \\
\hline 565.02 & 3.81740186 & 14.7532329 & 0.92848544 & 14.6265 & 2103 & 1881.18381 & 70 & 69.2 & 68.9 & 69.4 & 0.000544 \\
\hline 570.02 & 3.82052821 & 14.7539825 & 0.92660912 & 14.625 & 2103.25 & 1883.9152 & 70.1 & 69.2 & 68.9 & 69.4 & 0.000543 \\
\hline 575.02 & 3.81365024 & 14.7547321 & 0.92660912 & 14.62575 & 2103.25 & 1883.9152 & 70.2 & 69.2 & 69 & 69.4 & 0.000542 \\
\hline 580.02 & 3.81490078 & 14.7539825 & 0.92786 & 14.6265 & 2103.25 & 1882.92196 & 70.2 & 69.2 & 68.9 & 69.4 & 0.000542 \\
\hline 585.05 & 3.83303361 & 14.7539825 & 0.92848544 & 14.62575 & 2103.25 & 1884.90843 & 70.2 & 69.2 & 68.9 & 69.3 & 0.000542 \\
\hline 590.1 & 3.83053253 & 14.7547321 & 0.92786 & 14.6265 & 2103.25 & 1886.64658 & 70.2 & 69.2 & 68.9 & 69.4 & 0.000544 \\
\hline 595.02 & 3.82615564 & 14.7547321 & 0.92911088 & 14.6265 & 2093 & 1886.39828 & 70.2 & 69.2 & 68.9 & 69.4 & 0.000543 \\
\hline 600.06 & 3.81927767 & 14.7530825 & 0.92660912 & 14.625 & 2103 & 1881,43212 & 70.2 & 69.2 & 69 & 69.3 & 0.000542 \\
\hline 605.02 & 3.83178307 & 14.7547321 & 0.92660912 & 14.62575 & 2103.25 & 1884.41181 & 70.2 & 69.2 & 68.9 & 69.4 & 0.000544 \\
\hline
\end{tabular}




\begin{tabular}{|c|c|c|c|c|c|c|c|c|c|c|c|}
\hline SECOND & \begin{tabular}{|l|} 
MAIN DUCT \\
DIFF (IN H2O \\
\end{tabular} & $\begin{array}{l}\text { MAIN DUCT } \\
\text { ABS (PSIA) } \\
\end{array}$ & \begin{tabular}{|l|} 
GAS INJECT \\
DIFF (IN H2O) \\
\end{tabular} & \begin{tabular}{|l|} 
GAS INJECT \\
ABS (PSIA) \\
\end{tabular} & $\begin{array}{l}\text { NEWPORT } \\
\text { (PPM) } \\
\end{array}$ & $\frac{S M C}{(P P M)}$ & \begin{tabular}{|l|} 
AMBIENT \\
TEMP.(F) \\
\end{tabular} & \begin{tabular}{|l|} 
GAS INJECT \\
TEMP.(F)
\end{tabular} & \begin{tabular}{|l|} 
MAIN DUCT \\
TEMP.(F) \\
\end{tabular} & \begin{tabular}{|l|} 
WEC CELL \\
TEMP.(F) \\
\end{tabular} & $\begin{array}{l}\text { WEC CELL } \\
\text { (VOLTS) } \\
\end{array}$ \\
\hline 610.02 & 3.81552605 & 14.7547321 & 0.92723456 & 14.62575 & 2103.25 & 1886.64658 & 70.2 & 69.2 & 68.9 & 69.4 & 0.000543 \\
\hline 615.02 & 3.82302929 & 14.7524832 & 0.92660912 & 14.625 & 2103 & 1887.88812 & 70.2 & 69.2 & 68.9 & 69.4 & 0.000543 \\
\hline 620.02 & 3.81365024 & 14.7539825 & 0.92660912 & 14.625 & 2103 & 1886.14997 & 70.2 & 69.2 & 68.9 & 69.4 & 0.000543 \\
\hline 625.02 & 3.81927767 & 14.7539825 & 0.92598368 & 14.625 & 2103.25 & 1879.94227 & 70.2 & 69.2 & 68.9 & 69.4 & 0.000543 \\
\hline 630.05 & 3.80864808 & 14.7539825 & 0.92723456 & 14.625 & 2103 & 1883.9152 & 70.2 & 69.2 & 69 & 69.4 & 0.000543 \\
\hline 635.02 & 3.82427983 & 14.7547321 & 0.92723456 & 14.62575 & 2103.25 & 1881.18381 & 70.2 & 69.2 & 68.9 & 69.4 & 0.000543 \\
\hline 640.02 & 3.81552605 & 14.7532329 & 0.92786 & 14.6265 & 2103 & 1880.43888 & 70.2 & 69.2 & 68.9 & 69.3 & 0.000542 \\
\hline 645.03 & 3.80864808 & 14.7539825 & 0.92723456 & 14.62575 & 2102.5 & 1882.17704 & 70.2 & 69.2 & 68.9 & 69.4 & 0.000542 \\
\hline 650.02 & 3.81490078 & 14.7517336 & 0.92660912 & 14.62575 & 2103 & 1884.66012 & 70.2 & 69.2 & 68.9 & 69.4 & 0.000543 \\
\hline 655.02 & 3.85491806 & 14.7532329 & 0.92723456 & 14.625 & 2103 & 1879.94227 & 70.2 & 69.2 & 68.9 & 69.3 & 0.000543 \\
\hline 660.05 & 3.81740186 & 14.7539825 & 0.92660912 & 14.62575 & 2103 & 1881.43212 & 70.2 & 69.2 & 69.1 & 69.4 & 0.000544 \\
\hline 665.02 & 3.83615996 & 14.7532329 & 0.92660912 & 14.62575 & 2093.5 & 1877.21088 & 70.2 & 69.2 & 68.9 & 69.4 & 0.000543 \\
\hline 670.02 & 3.82177875 & 14.7532329 & 0.9247328 & 14.62575 & 2093 & 1870.50656 & 70.2 & 69.2 & 68.9 & 69.3 & 0.000543 \\
\hline 675.02 & 3.8249051 & 14.7547321 & 0.92410736 & 14.62575 & 2092.75 & 1868.76841 & 70.3 & 69.2 & 68.9 & 69.4 & 0.000543 \\
\hline 680.02 & 3.83178307 & 14.7532329 & 0.92285648 & 14.62575 & 2093 & 1872.74134 & 70.3 & 69.2 & 68.9 & 69.4 & 0.000543 \\
\hline 685.02 & 3.806147 & 14.7539825 & 0.9247328 & 14.62575 & 2102.75 & 1873.48626 & 70.2 & 69.2 & 68.9 & 69.4 & 0.000543 \\
\hline 690.02 & 3.82240402 & 14.7547321 & 0.9247328 & 14.62575 & 2093.25 & 1875.22442 & 70.2 & 69.2 & 69 & 69.4 & 0.000543 \\
\hline 695.02 & 3.81740186 & 14.7554817 & 0.92223104 & 14.62575 & 2093.25 & 1876.96257 & 70.2 & 69.2 & 69 & 69.4 & 0.000542 \\
\hline 700.02 & 3.84116212 & 14.7532329 & 92285648 & 14.625 & 2092.75 & 1877.9558 & 70.3 & 69.2 & 69.1 & 69.4 & 0.000542 \\
\hline 705.07 & 3.82240402 & 14.7547321 & 92223104 & 14.62575 & 2093 & 1878.20411 & 70.2 & 69.2 & 69 & 69.4 & 0.000543 \\
\hline 710.11 & 3.83178307 & 14.7547321 & 0.92348192 & 14.62575 & 2093 & 1878.70073 & 70.3 & 69.2 & 68.9 & 69.4 & 0.000542 \\
\hline 715.02 & 3.82052821 & 14.7547321 & 0.92285648 & 14.62575 & 2093.25 & 1877.9558 & 70.3 & 69.2 & 69 & 69.4 & 0.000542 \\
\hline 720.02 & 3.79551741 & 14.7517336 & 0.92285648 & 14.62575 & 2093.5 & 1874.97611 & 70.3 & 69.2 & 69 & 69.4 & 0.000542 \\
\hline 725.05 & 3.811774443 & 14.7532329 & 0.92285648 & 14.62575 & 2093.25 & 1879.44565 & 70.3 & 69.2 & 69 & 69.5 & 0.000542 \\
\hline 730.02 & 3.81490078 & 14.7539825 & 0.92223104 & 14.62575 & 2093.25 & 1882.92196 & 70.4 & 69.2 & 68.9 & 69.5 & 0.000541 \\
\hline 735.02 & 3.81802713 & 14.7532329 & 0.92285648 & 14.625 & 2093 & 1880.68719 & 70.3 & 69.2 & 68.9 & 69.4 & 0.000542 \\
\hline 740.02 & 3.83053253 & 14.7539825 & 0.92223104 & 14.62575 & 2093.5 & 1880.19058 & 70.3 & 69.2 & 69 & 69.4 & 0.000543 \\
\hline 745.02 & 3.82615564 & 14.7539825 & 0.92223104 & 14.625 & 2093.5 & 1878.94904 & 70.3 & 69.2 & 69 & 69.4 & 0.000542 \\
\hline 750.05 & 3.82365456 & 14.7554817 & 0.92223104 & 14.625 & 2093.25 & 1874.47949 & 70.4 & 69.3 & 68.9 & 69.4 & 0.000542 \\
\hline 755.08 & 3.82427983 & 14.7547321 & 0.92348192 & 14.62425 & 2093.25 & \begin{tabular}{l|l}
1877.7075 \\
\end{tabular} & 70.3 & 69.2 & 69 & 69.5 & 0.000543 \\
\hline 760.02 & 3.81615132 & 14.7539825 & 0.92285648 & 14.625 & 2093 & \begin{tabular}{|l|l|}
3876.46596 \\
\end{tabular} & 70.2 & 69.2 & 69 & 69.4 & 0.000542 \\
\hline 765.05 & 3.82740618 & 14.7532329 & 0.92285648 & 14.625 & 2093 & 1874.23118 & 70.2 & 69.2 & 68.9 & 69.4 & 0.000542 \\
\hline 770.08 & 3.8249051 & 14.7539825 & 0.92348192 & 14.62425 & 2093.5 & $\begin{array}{ll}882.17704 \\
\end{array}$ & 70.2 & 69.2 & 69 & 69.4 & 0.000542 \\
\hline 775.02 & 3.82553037 & 14.7554817 & 0.92223104 & 14.625 & 2093.5 & 1881.68042 & 70.2 & 69.2 & 68.9 & 69.4 & 0.000542 \\
\hline 780.06 & 3.82803146 & 14.7539825 & 0.9216056 & 14.6265 & 2093.5 & $\begin{array}{ll}1875.96934 \\
\end{array}$ & 70.2 & 69.2 & 69 & 69.5 & 0.000542 \\
\hline 785.02 & 3.82865672 & 14.7524832 & 0,92223104 & 14.6265 & 2093.25 & $\begin{array}{l}1879.69396 \\
\end{array}$ & 70.3 & 69.2 & 68.9 & 69.5 & 0.000540 \\
\hline 790.02 & 3.84491374 & 14.7517336 & 0.92285648 & 14.62575 & 2093 & 1883.66689 & 70.2 & 69.2 & 68.9 & 69.5 & 0.000543 \\
\hline 795.05 & 3.80927335 & 14.7524832 & 0.92285648 & 14.6265 & 2093.25 & 1884.41181 & 70.3 & 69.3 & 69.1 & 69.5 & 0.00054 \\
\hline 800.07 & 3.82865672 & 14.7539825 & 0.92285648 & 14.62575 & 2093 & 1881.92873 & 70.2 & 69.3 & 69 & 69.4 & 0.00054 \\
\hline 805.02 & 3.82115348 & 14.7539825 & 0.92223104 & 14.62575 & 2093.5 & 1879.69396 & 70 & 69.2 & 68.9 & 69.4 & 0.00054 \\
\hline 810.02 & 3.82302929 & 14.7524832 & 0.922233104 & 14.62575 & 2093 & 1880.19058 & 70 & 69.3 & 68.9 & 69.5 & 0.00054 \\
\hline 815.02 & 3.8186524 & 14.7524832 & 0.92223104 & 14.625 & 2093.25 & \begin{tabular}{|l|l|}
3 & 1882.92196 \\
\end{tabular} & 70 & 69.3 & 68.9 & 69.5 & 0.00054 \\
\hline
\end{tabular}




\begin{tabular}{|c|c|c|c|c|c|c|c|c|c|c|c|}
\hline SECOND & \begin{tabular}{|l|} 
MAIN DUCT \\
DIFF (IN HZO \\
\end{tabular} & $\begin{array}{l}\text { MAIN DUCT } \\
\text { ABS (PSIA) }\end{array}$ & \begin{tabular}{|l|} 
GAS INJECT \\
DIFF (IN H2O)
\end{tabular} & $\begin{array}{l}\text { GAS INJECT } \\
\text { ABS (PSIA) }\end{array}$ & \begin{tabular}{|l|} 
NEWPORT \\
(PPM) \\
\end{tabular} & $\begin{array}{l}\text { SMC } \\
\text { (PPM) }\end{array}$ & \begin{tabular}{|l|} 
AMBIENT \\
TEMP.(F) \\
\end{tabular} & \begin{tabular}{|l|} 
GAS INJECT \\
TEMP.(F) \\
\end{tabular} & \begin{tabular}{|l|} 
MAIN DUCT \\
TEMP.(F) \\
\end{tabular} & \begin{tabular}{|l|} 
WEC CELL \\
TEMP.(F)
\end{tabular} & \begin{tabular}{|l} 
WEC CELL \\
(VOLTS) \\
\end{tabular} \\
\hline & & & & & & & & & & & \\
\hline 820,02 & 3.82928199 & 14.7539825 & 0.92223104 & 14.625 & 2093 & 1883.66689 & 70 & 69.2 & 68.9 & 69.5 & 0.000542 \\
\hline 825.02 & 3.821777875 & 14.7524832 & 0.92223104 & 14.62575 & 2093.25 & 1884.41181 & 70.1 & 69.2 & 69 & 69.5 & 0,000543 \\
\hline 830.11 & 3.83178307 & 14.7532329 & 0.92285648 & 14.62575 & 2093 & 1879.69396 & 70.1 & 69.2 & 68.9 & 69.4 & 0.000543 \\
\hline 835.02 & 3.82240402 & 14.7539825 & 0.92223104 & 14.62575 & 2093.25 & 1885.90166 & 70.2 & 69.2 & 69 & 69.4 & 0.000542 \\
\hline 840.07 & 3.81177443 & 14.7532329 & 0.92286648 & 14.625 & 2093 & 1881.68042 & 70.2 & 69.2 & 69 & 69.5 & 0.000542 \\
\hline 845.02 & 3,8186524 & 14.7524832 & 0.92348192 & 14.625 & 2093 & 1880.19058 & 70.2 & 69.2 & 69.1 & 69.4 & 0.000543 \\
\hline 850.02 & 3.81990294 & 14.7532329 & 0.92035472 & 14.62425 & 2093 & 1881.18381 & 70.2 & 69.3 & 69.1 & 69.5 & 0.000543 \\
\hline 855.02 & 3.80239538 & 14.7539825 & 0.9216056 & 14.62425 & 2093 & 1881.18381 & 70.3 & 69.2 & 69 & 69.5 & 0.000542 \\
\hline 860.02 & 3.83178307 & 14.7532329 & 0.92035472 & 14.625 & 2093.25 & 1880.19058 & 70.3 & 69.3 & 69 & 69.4 & 0.000542 \\
\hline 865.02 & 3.81552605 & 14.7524832 & 0.92035472 & 14.625 & 2093 & 1879.94227 & 70.4 & 69.3 & 69 & 69.4 & 0.000542 \\
\hline 870.02 & 3.83178307 & 14.7547321 & 0.92098016 & 14.625 & 2093.25 & 1878.70073 & 70.4 & 69.2 & 69 & 69.4 & 0.000541 \\
\hline 875.04 & 3.82115348 & 14.7554817 & 0.9216056 & 14.62575 & 2003.25 & 1872.98964 & 70.4 & 69.2 & 68.9 & 69.4 & 0.000542 \\
\hline 880.02 & 3.85366752 & 14.7539825 & 0.92035472 & 14.625 & 2093 & 1876.21765 & 70.3 & 69.3 & 68.9 & 69.4 & 0.000542 \\
\hline 885.02 & 3.82177875 & 14.7524832 & 0.92035472 & 14.625 & 2093.5 & 1876.21765 & 70.3 & 69.3 & 68.9 & 69.4 & 0.000542 \\
\hline 890.02 & 3.84241266 & 14.7539825 & 0.92035472 & 14.62425 & 2093.25 & 1878.20411 & 70.3 & 69.3 & 68.9 & 69.4 & 0.000542 \\
\hline 895.02 & 3.81927767 & 14.7539825 & 0.92035472 & 14.62425 & 2093.5 & 1882.92196 & 70.2 & 69.3 & 69 & 69.4 & 0.000542 \\
\hline 900.02 & 3.83428415 & 14.7547321 & 0.92035472 & 14.62575 & 2093 & 1884.66012 & 70.3 & 69.3 & 68.9 & 69.4 & 0.000542 \\
\hline 905.03 & 3.82678091 & 14.7524832 & 0,92035472 & 14.625 & 2093 & 1884.1635 & 70.3 & 69.3 & 68.8 & 69.4 & 0.000541 \\
\hline 910.02 & 3.83240834 & 14.7524832 & 0.92035472 & 14.625 & 2093 & 1880.19058 & 70.2 & 69.3 & 68.9 & 69.4 & 0.000542 \\
\hline 915.02 & 3.83928631 & 14.7539825 & 0.92035472 & 14.625 & 2093 & 1878.94904 & 70.2 & 69.3 & 68.9 & 69.4 & 0.000541 \\
\hline 920.02 & 3.85679387 & 14.7547321 & 0.92098016 & 14.625 & 2093.25 & 1875.72103 & 70.2 & 69.3 & 68.9 & 69.4 & 0,000541 \\
\hline
\end{tabular}




\begin{tabular}{|c|c|c|c|c|c|c|c|c|c|c|c|}
\hline \multicolumn{12}{|c|}{$\begin{array}{l}\text { TEST: FGI22222 } 22 \text { Feb } 1997 \text { WEC-SPOOL } \\
\text { Measured Hydrogen Concentration }=2170 \mathrm{ppm}\end{array}$} \\
\hline 0.0100098 & 3.75550013 & 14.7532329 & 0.232120544 & 14.59125 & 31 & 3.349758 & 70 & 69.1 & 68.8 & 69.4 & 0,000236 \\
\hline 5.0299988 & 3.75174851 & 14.7562313 & 0.9872768 & 14.6295 & 31.65 & -2.8283112 & 70 & 69.2 & 68.9 & 69.4 & 0.000236 \\
\hline 10.02002 & 3.77363296 & 14.7539825 & 0.9872768 & 14.63325 & 102.925 & 734.49746 & 69.9 & 69.1 & 68.8 & 69.4 & 0.000252 \\
\hline 15.02002 & 3.7623781 & 14.7547321 & 0.98477504 & 14.63325 & 281.5 & 1383.3263 & 70 & 69.1 & 68.9 & 69.4 & 0.00029 \\
\hline 20.02002 & 3.76425391 & 14.7547321 & 0.98790224 & 14.63325 & 571.5 & 1688.4968 & 69.9 & 69.1 & 68.9 & 69.5 & 0.000334 \\
\hline 25.02002 & 3.74111892 & 14.7532329 & 0.98602592 & 14.6325 & 882.5 & 1814.1406 & 70 & 69.1 & 68.9 & 69.5 & 0.000376 \\
\hline 30.02002 & 3.7498727 & 14.7524832 & 0.98790224 & 14.63325 & 1149.25 & 1864.5472 & 70.1 & 69.1 & 68.9 & 69.5 & 0.000409 \\
\hline 35.02002 & 3.73924311 & 14.7532329 & 0.98790224 & 14.6325 & 1312.5 & 1895.5857 & 70 & 69.1 & 68.9 & 69.5 & 0.000438 \\
\hline 40,02002 & 3.75112324 & 14.7524832 & 0.98790224 & 14.6325 & 1472.25 & 1919.4232 & 70 & 69.1 & 69 & 69.5 & 0.00046 \\
\hline 45.02002 & 3.75550013 & 14.7554817 & 0.9872768 & 14.63325 & 1622.5 & 1924.6377 & 70.1 & 69.1 & 68.9 & 69.5 & 0.000477 \\
\hline 50.02002 & 3.74737162 & 14.7532329 & 0.98540048 & 14.63325 & 1722.5 & 1939.2879 & 70.1 & 69.1 & 68.9 & 69.5 & 0.000493 \\
\hline 55.02002 & 3.75675067 & 14.7562313 & 0.98477504 & 14.63325 & 1792.5 & 1947.482 & 70.1 & 69.1 & 68.9 & 69.4 & 0.000504 \\
\hline 60.02002 & 3.77113188 & 14.7554817 & 0.98665136 & 14.634 & 1872.5 & 1950.2134 & 70.1 & 69.1 & 68.9 & 6.4 & 0.000513 \\
\hline 65.02002 & 3.75237378 & 14.7532329 & 0.98790224 & 14.6325 & 1921 & 1955.1796 & 70.1 & 69.1 & 68.9 & 69,4 & 0.000522 \\
\hline 70.02002 & 3.74049365 & 14.7562313 & 0.9872768 & 14.63325 & 1973.25 & 1960.6424 & 70.1 & 69.1 & 69 & 69.4 & 0.000527 \\
\hline 75.02002 & 3.74924743 & 14.7532329 & 0.98665136 & 14.6325 & 2002.75 & 1959.4008 & 70.1 & 69.1 & 68.9 & 69.4 & 0.000532 \\
\hline 80.02002 & 3.75112324 & 14.7547321 & 0.9872768 & 14.6325 & 2050.75 & 1974.2993 & 70.2 & 69.1 & 68.9 & 69.4 & 0.000537 \\
\hline 85,02002 & 3.76487918 & 14.7524832 & 0.98540048 & 14.63175 & 2062.5 & 1974.5476 & 70.2 & 69.1 & 68.9 & 69.4 & 0.00054 \\
\hline 90.02002 & 3.75487486 & 14.750984 & 0.98665136 & 14.63175 & 2092.75 & 1973.5544 & 70.3 & 69.1 & 68.9 & 69.5 & 0.000544 \\
\hline 95.02002 & 3.74737162 & 14.7524832 & 0.98602592 & 14.63025 & 2113.5 & 1986.963 & 70.2 & 69.1 & 68.9 & 69.5 & 0.000547 \\
\hline 100.02002 & 3.75362432 & 14.7524832 & 0.98540048 & 14.63325 & 2123 & 1990.9359 & 70.3 & 69.1 & 68.9 & 69.4 & 0.00055 \\
\hline 105.02002 & 3.73861784 & 14.7539825 & 0.98790224 & 14.63325 & 2143.25 & 1994.4123 & 70.2 & 69.1 & 68.9 & 69.5 & 0.000551 \\
\hline 110.02002 & 3.75550013 & 14.7554817 & 0.98665136 & 14.6325 & 2153.25 & 1998.6335 & 70.3 & 69.1 & 69 & 69.5 & 0.000551 \\
\hline 115,02002 & 3.76800553 & 14.7524832 & 0.98665136 & 14.6325 & 2162.5 & 2002.1098 & 70.3 & 69.1 & 69 & 69.5 & 0.000554 \\
\hline 120.02002 & 3.74299473 & 14.7539825 & 0.98602592 & 14.6325 & 2173.25 & 2006.5793 & 70.3 & 69.1 & 68.9 & 69.5 & 0.000555 \\
\hline 125.02002 & 3.79176579 & 14.7539825 & 0.98540048 & 14.63325 & 2172.75 & 2004.5929 & 70.3 & 69.1 & 68.9 & 69.4 & 0.000556 \\
\hline 130.02002 & 3.73986838 & 14.7539825 & 0.98602592 & 14.63325 & 2182.75 & 2005.8344 & 70.3 & 69.1 & 69 & 69.4 & 0.000557 \\
\hline 135.02002 & 3.74737162 & 14.7532329 & 0.98477504 & 14.6325 & 2193.25 & 2009.0624 & 70.3 & 69.1 & 6 & 69.5 & 0.000558 \\
\hline 140.09 & 3.7498727 & 14.7539825 & 0.98540048 & 14.6325 & 2203 & 2008.0692 & 70.4 & 69.1 & 69 & 69.5 & 0.000559 \\
\hline 145.02002 & 3.78801417 & 14.7524832 & 0.98540048 & 14.6325 & 2203.25 & 2005.3378 & 70.4 & 69.2 & 69 & 69.5 & 0.00056 \\
\hline 150.02002 & 3.74549581 & 14.7524832 & 0.98540048 & 14.63325 & 2213.25 & 2012.2904 & 70.4 & 69.1 & 68.9 & 69.4 & 0.000561 \\
\hline 155.02002 & 3.75112324 & 14.7562313 & 0.98602592 & 14.6325 & 2213.25 & 2011.5455 & 70.4 & 69.1 & 68.9 & 69.4 & 0.000561 \\
\hline 160,02002 & 3.7498727 & 14.7532329 & 0.98477504 & 14.63325 & 2223.25 & 2015.5184 & 70.4 & 69.1 & 68.9 & 69,4 & 0.000562 \\
\hline 165.02002 & 3.74612108 & 14.7539825 & 0.98540048 & 14.6325 & 2223.25 & 2014.2769 & 70.4 & 69.1 & 68.8 & 69.4 & 0.000562 \\
\hline 170.02002 & 3.75862648 & 14.7577306 & 0.98540048 & 14.634 & 2223.25 & 2018.2498 & 70.3 & 69.1 & 68.8 & 69.4 & 0.000563 \\
\hline 175.02002 & 3.7498727 & 14.7532329 & 0.98540048 & 14.63325 & 2223.25 & 2021.2295 & 70.4 & 69.1 & 68.9 & 69.4 & 0.000563 \\
\hline 180.02002 & 3.73924311 & 14.7517336 & 0.98540048 & 14.6325 & 2233.25 & 2029.1754 & 70.4 & 69.1 & 68.9 & 69.4 & 0.000563 \\
\hline 185.02002 & 3.74299473 & 14.7524832 & 0.98540048 & 14.63325 & 2233.25 & 2023.216 & 70.4 & 69.1 & 69 & 69.5 & 0.000563 \\
\hline
\end{tabular}




\begin{tabular}{|c|c|c|c|c|c|c|c|c|c|c|c|}
\hline SECONDS & \begin{tabular}{|l|} 
MAIN DUCT \\
DIFF (IN H2 \\
\end{tabular} & \begin{tabular}{|l} 
MAIN DUCT \\
ABS (PSIA)
\end{tabular} & \begin{tabular}{|l|} 
GAS INJECT \\
DIFF (IN H2O \\
\end{tabular} & $\begin{array}{l}\text { GAS INJECT } \\
\text { ABS (PSIA) }\end{array}$ & \begin{tabular}{|l|} 
NEWPORT \\
(PPM) \\
\end{tabular} & \begin{tabular}{|l|} 
SMC \\
(PPM) \\
\end{tabular} & \begin{tabular}{|l|} 
AMBIENT \\
TEMP.(F) \\
\end{tabular} & \begin{tabular}{|l|} 
GAS INJECT \\
TEMP.(F) \\
\end{tabular} & \begin{tabular}{|l} 
MAIN DUCT \\
TEMP.(F) \\
\end{tabular} & \begin{tabular}{|l|} 
WEC CELL \\
TEMP.(F)
\end{tabular} & \begin{tabular}{|l|} 
WEC CELL \\
(VOLTS)
\end{tabular} \\
\hline 190.02002 & 3.73611676 & 14.7547321 & 0.98602592 & 14.634 & 2233.25 & 2026.6923 & 70.4 & 69.1 & 68.8 & 69.4 & 0.000564 \\
\hline 195.02002 & 3.76425391 & 14.7532329 & 0.98540048 & 14.63475 & 2243.25 & 2018.4981 & 70.4 & 69.1 & 68.8 & 69.5 & 0.000564 \\
\hline 200.02002 & 3.75174851 & 14.7532329 & 0.984775504 & 14.63325 & 2243.25 & 2022.2228 & 70.4 & 69.1 & 68.8 & 69.5 & 0.000564 \\
\hline 205.02002 & 3.74924743 & 14.7547321 & 0.98602592 & 14.634 & 2243.25 & 2024.7058 & 70.4 & 69.1 & 68.9 & 69.5 & 0.000564 \\
\hline 210.02002 & 3.74487054 & 14.7532329 & 0.98540048 & 14.63325 & 2243 & 2026.6923 & 70.3 & 69.1 & 69 & 69.5 & 0.000565 \\
\hline 215.02002 & 3.74424527 & 14.7524832 & 0.98540048 & 14.63325 & 2243.25 & 2026.1957 & 70.3 & 69.2 & 68.8 & 69.4 & 0.000566 \\
\hline 220.02002 & 3.74862216 & 14.7532329 & 0.98540048 & 14.63325 & 2253.5 & 2025.6991 & 70.2 & $\begin{array}{l}\infty .2 \\
69.2\end{array}$ & $\begin{array}{l}\infty .0 \\
68.7\end{array}$ & 09.4 & 0.000565 \\
\hline 225.02002 & 3.74049365 & 14.7524832 & 0.98602592 & 14.634 & 2253 & 2024.7058 & 70.2 & 69.2 & 68.7 & 69.4 & 0.000565 \\
\hline 230.02002 & 3.7686308 & 14.7532329 & 0.98540048 & 14.63325 & 2253.5 & 2028.4305 & 70.2 & 69.2 & 68.8 & 69.5 & 0.000565 \\
\hline 235.02002 & 3.75362432 & 14.7524832 & 0.98540048 & 14.63325 & 2253.25 & 2028.4305 & 70.1 & 69.2 & 68.8 & 69.5 & 0.000566 \\
\hline 240.02002 & 3.75424959 & 14.7554817 & 0.98352416 & 14.63325 & 2253.25 & 2028.6788 & 70.1 & 69.1 & 68.9 & 69.5 & 0.000565 \\
\hline 245.02002 & 3.75174851 & 14.7524832 & 0.9841496 & 14.63325 & 2253.25 & 2029.4237 & 70.1 & 69.2 & 68.8 & 69.5 & 0.000566 \\
\hline 250.02002 & 3.77988566 & 14.7539825 & 0.9841496 & 14.634 & 2253.25 & 2027.9338 & 70.1 & 69.2 & 68.9 & 69.5 & 0.000566 \\
\hline 255.03 & 3.7623781 & 14.7554817 & 0.9841496 & 14.634 & 2253.75 & 2029.4237 & 70 & 69.2 & 68.8 & 69.5 & 0.000566 \\
\hline 260.02002 & 3.75487486 & 14.7547321 & 0.98602592 & 14.63325 & 2253.5 & 2026.444 & 70 & 69.2 & 68.9 & 69.5 & 0.000566 \\
\hline 265.02002 & 3.76550445 & 14.7562313 & 0.98540048 & 14.634 & 2258 & 2021.4778 & 70 & 69.2 & 68.9 & 69.4 & 0.000566 \\
\hline 270.02002 & 3.73611676 & 14.7554817 & 0.98352416 & 14.63325 & 2263.25 & 2023.216 & 70.1 & 69.2 & 68.8 & 69.4 & 0,000567 \\
\hline 275.06 & 3.75299905 & 14.7547321 & 0.98352416 & 14.634 & 2252.75 & 2023.4643 & 70.1 & 69.2 & 68.9 & 69.5 & 0.000567 \\
\hline 280.02002 & 3.74236946 & 14.7554817 & 0.98289872 & 14.634 & 2263.25 & 2023.7126 & 70.1 & 69.2 & 68.8 & 69.4 & 0.000567 \\
\hline 285.02002 & 3.73861784 & 14.7547321 & 0.98352416 & 14.63325 & 2263.25 & 2022.9677 & 70.1 & 69.2 & 68.9 & 69.4 & 0.006567 \\
\hline 290.02002 & 3.7561254 & 14.7554817 & 0.9841496 & 14.63325 & 2263.75 & 2025.6991 & 70.1 & 69.2 & 68.9 & 69.5 & 0.000566 \\
\hline 295.02002 & 3.75424959 & 14.7554817 & 0.98352416 & 14.6325 & 2263.25 & 2028.1821 & 70.1 & 69.2 & 69 & 69.5 & 0.000567 \\
\hline 300.02002 & 3.78676363 & 14.7554817 & 0.9841496 & 14.63325 & 2263.25 & 2026.9406 & 70.1 & 69.2 & 69 & 69.5 & 0.000567 \\
\hline 305.02002 & 3.74924743 & 14.7547321 & 0.98477504 & 14.63325 & 2263.75 & 2032.6517 & 70.1 & 69.2 & 69 & 69.5 & 0.000567 \\
\hline 310.03 & 3.76175283 & 14.7562313 & 0.98227328 & 14.634 & 2263 & 2028.9271 & 70.1 & 69.2 & 69 & 69.5 & 0.000567 \\
\hline 315.02002 & 3.76050229 & 14.7547321 & 0.98352416 & 14.6325 & 2264 & 2032.9 & 70.1 & 69.2 & 69 & 69.5 & 0.000567 \\
\hline 320.03 & 3.7561254 & 14.7577306 & 0,98352416 & 14.6325 & 2263.25 & 2034.3898 & 70.1 & 69.2 & 68.8 & 69.4 & 0.000568 \\
\hline 325.02002 & 3.78801417 & 14.7554817 & 0.98352416 & 14.63325 & 2263.5 & 2031.9068 & 70.1 & 69.2 & 68.8 & $\frac{0.4}{69.4}$ & 0.000567 \\
\hline 330.02002 & 3.75550013 & 14.7562313 & 0.98289872 & 14.634 & 2263.75 & 2030.1686 & 70.1 & 69.2 & 68.9 & 69.4 & 0.000567 \\
\hline 335.02002 & 3.74549581 & 14.7554817 & 0.98352416 & 14.63325 & 2263.5 & 2026.9406 & 70 & 69.2 & 68.9 & 69.4 & 0.000568 \\
\hline 340.02002 & 3.73424095 & 14.7532329 & 0.98352416 & 14.634 & 2263.75 & 2031.4101 & 69.9 & 69.2 & 68.9 & 69.5 & 0.000568 \\
\hline 345.02002 & 3.77988566 & 14.7562313 & 0.9841496 & 14.63325 & 2263.25 & 2032.9 & 69.9 & 69.2 & 69 & 69.5 & 0.000568 \\
\hline 350.02002 & 3.75800121 & 14.7554817 & 0.98352416 & 14.6325 & 2263.5 & 2038.8594 & 69.9 & 69.2 & 68.9 & 69.5 & 0.000567 \\
\hline 355.02002 & 3.74737162 & 14.7577306 & 0.98477504 & 14.6325 & 2263.5 & 2039.6043 & 69.9 & 69.2 & 69 & 69.4 & 0.000568 \\
\hline 360.02002 & 3.75362432 & 14.756981 & 0.98352416 & 14.6325 & 2263.5 & 2038.1145 & 70 & 69.2 & 69 & 69.5 & 0.000568 \\
\hline 365.02002 & 3.74674635 & 14.7562313 & 0.98352416 & 14.63325 & 2263.5 & 2037.3695 & $-\frac{10}{70}$ & 69.2 & -6 & 69.4 & 0.000568 \\
\hline 370.02002 & 3.75362432 & 14.756981 & 0.98352416 & 14.634 & 2263.25 & 2035.3831 & 70.1 & 69.2 & 68.9 & 69.4 & 0.000569 \\
\hline 375.02002 & 3.75550013 & 14.756981 & 0.98352416 & 14.63325 & 2263.75 & 2038.6111 & 70.1 & 69.3 & 69 & 69.4 & 0.000568 \\
\hline 380.02002 & 3.74424527 & 14.7547321 & 0.98289872 & 14.6325 & 2263.25 & 2035.6314 & 70.1 & 69.3 & 68.9 & 69.4 & 0.000568 \\
\hline 385.02002 & 3.74487054 & 14.756981 & 0.98289872 & 14.6325 & 2263.5 & 2030.1686 & 70.1 & 69.2 & 69 & 69.4 & 0.000568 \\
\hline 390.04001 & 3.74487054 & 14.7562313 & 0.98352416 & 14.63325 & 2263.75 & 2030.4169 & 70.2 & 69.2 & 69 & 69.5 & 0.000568 \\
\hline 395.02002 & 3.74924743 & 14.7562313 & 0.98352416 & 14.6325 & 2263.5 & 2031.6585 & 70.2 & 69.3 & 68.9 & 69.4 & 0.000567 \\
\hline
\end{tabular}




\begin{tabular}{|c|c|c|c|c|c|c|c|c|c|c|c|}
\hline SECONDS & $\frac{\text { MAIN DUCT }}{\text { DIFF (IN H2 }}$ & \begin{tabular}{|l|} 
MAIN DUCT \\
ABS (PSIA)
\end{tabular} & \begin{tabular}{|l|} 
GAS INJECT \\
DIFF (IN H2O
\end{tabular} & \begin{tabular}{|l|} 
GAS INJECT \\
ABS (PS|A) \\
\end{tabular} & \begin{tabular}{|l|} 
NEWPORT \\
(PPM) \\
\end{tabular} & \begin{tabular}{|l|} 
SMC \\
(PPM)
\end{tabular} & \begin{tabular}{|l} 
AMBIENT \\
TEMP.(F)
\end{tabular} & \begin{tabular}{|l|} 
GAS INJECT \\
TEMP.(F) \\
\end{tabular} & $\begin{array}{l}\text { MAIN DUCT } \\
\text { TEMP.(F) }\end{array}$ & \begin{tabular}{|l|} 
WEC CELL \\
TEMP.(F) \\
\end{tabular} & $\begin{array}{l}\text { WEC CELL } \\
\text { (VOLTS) }\end{array}$ \\
\hline & & & & & & & & & & & \\
\hline 400.02002 & 3.75925175 & 14.7547321 & 0.98352416 & 14.6325 & 2263.75 & 2036.3763 & 70.3 & 69.3 & 68.9 & 69.4 & 0.000568 \\
\hline 405.02002 & 3.74487054 & 14.7562313 & 0.98164784 & 14.634 & 2273.25 & 2038.8594 & 70.3 & 69.3 & 68.9 & 69.4 & 0.000569 \\
\hline 410.02002 & 3.75800121 & 14.756981 & 0.98289872 & 14.63325 & 2273.5 & 2032.6517 & 70.3 & 69.2 & 69 & 69.5 & 0.000569 \\
\hline 415.02002 & 3.74549581 & 14.7547321 & 0.98289872 & 14.63325 & 2273.5 & 2033,8932 & 70.3 & 69.3 & 69 & 69.5 & 0.000568 \\
\hline 420.08002 & 3.7498727 & 14.7547321 & 0.98164784 & 14.6325 & 2273.75 & 2037,8662 & 70.3 & 69.2 & 68.9 & 69.4 & 0.000568 \\
\hline 425.02002 & 3.7561254 & 14.7562313 & 0.98164784 & 14.63325 & 2273.75 & 2039.6043 & 70.4 & 69.3 & 68.9 & 69.4 & 0.000568 \\
\hline 430.09 & 3.74674635 & 14.7554817 & 0.9810224 & 14.6325 & 2263.5 & 2034.8865 & 70.4 & 69.3 & 69 & 69.5 & 0.000568 \\
\hline 435.02002 & 3.7373673 & 14.756981 & 0.98164784 & 14.63325 & 2273 & 2035.8797 & 70.3 & 69.3 & 69 & 69.4 & 0.000569 \\
\hline 440.05002 & 3.77050661 & 14.7552313 & 0.9810224 & 14.6325 & 2273.25 & 2036.6246 & 70.3 & 69.3 & 68.8 & 69.5 & 0.000569 \\
\hline 445.02002 & 3.74299473 & 14.7562313 & 0.98289872 & 14.63325 & 2273.25 & 2040.3492 & 70.3 & 69.2 & 69 & 69.4 & 0.000569 \\
\hline 450.07001 & 3.75112324 & 14.7554817 & 0.98164784 & 14.63325 & 2273.5 & 2041.0942 & 70.3 & 69.3 & 69 & 69.5 & 0.000568 \\
\hline 455.02002 & 3.74737162 & 14.7547321 & 0.98164784 & 14.63325 & 2273.25 & 2037.8662 & 70.3 & 69.3 & 68.9 & 69.5 & 0.000568 \\
\hline 460.11002 & 3.77425823 & 14.7554617 & 0.98164784 & 14.63325 & 2274 & 2041.0942 & 70.3 & 69.2 & 69 & 69.4 & 0.000568 \\
\hline 465,02002 & 3.76612972 & 14.7562313 & 0.98164784 & 14.6325 & 2273.25 & 2041,3425 & 70.3 & 69.3 & 68.9 & 69.4 & 0.000569 \\
\hline 470.02002 & 3.75174851 & 14.756981 & 0.98164784 & 14.6325 & 2273.5 & 2037.3695 & 70.3 & 69.3 & 68.8 & 69.5 & 0.000569 \\
\hline 475.02002 & 3.73424095 & 14.7554817 & 0.98227328 & 14.63325 & 2273.25 & 2040.5975 & 70.3 & 69.3 & 68.9 & 69.5 & 0.000569 \\
\hline 480,02002 & 3.72861352 & 14.7554817 & 0.98227328 & 14.6325 & 2273.5 & 2037.6178 & 70.2 & 69.3 & 69.1 & 69.4 & 0.00057 \\
\hline 485,02002 & 3.74549581 & 14.7562313 & 0.98164784 & 14.63325 & 2273.75 & 2041.0942 & 70.2 & 69.3 & 69.1 & 69.4 & 0.000569 \\
\hline 490.02002 & 3.73486622 & 14.7554817 & 0.98227328 & 14.6325 & 2273.25 & 2042.584 & 70.3 & 69.3 & 69.1 & 69.4 & 0.000568 \\
\hline 495.02002 & 3.75049797 & 14.7562313 & 0.98164784 & 14.6325 & 2273.75 & 2009.1077 & 70.3 & 69.3 & 69.1 & 69.5 & 0.00057 \\
\hline 500.02002 & 3.75800121 & 14.7562313 & 0.98164784 & 14.63325 & 2273.75 & 2041.3425 & 70.3 & 69.3 & 69.4 & 69.5 & 0.000569 \\
\hline 505,02002 & 3.75237378 & 14.7562313 & 0.98227328 & 14.6325 & 2273 & 2040.5975 & 70.3 & 69.3 & 69.1 & 69.5 & 0.000569 \\
\hline 510.07001 & 3.75550013 & 14.7562313 & 0.98164784 & 14.6325 & 2273.5 & 2036.8729 & 70.3 & 69.3 & 69.1 & 69.5 & 0.000569 \\
\hline 515.05002 & 3.7373673 & 14.7562313 & 0.98164784 & 14.6325 & 2273.75 & 2037.1212 & 70.4 & 69.3 & 69 & 69.5 & 0.00057 \\
\hline 520.02002 & 3.76425391 & 14.7554817 & 0.98164784 & 14.6325 & 2274 & 2037.6178 & 70.4 & 69.3 & 69.1 & 69.4 & 0.000569 \\
\hline 525.02002 & 3.74612108 & 14.7554817 & 0.98164784 & 14.6325 & 2273.5 & 2037.8662 & 70.3 & 69.3 & 69 & 69.5 & 0.00057 \\
\hline 530.02002 & 3.74111892 & 14.756981 & 0.98164784 & 14.6325 & 2273.25 & 2042.584 & 70.2 & 69.3 & 69 & 69.5 & 0.00057 \\
\hline 535.02002 & 3.75987702 & 14.7554817 & 0.98227328 & 14.63175 & 2273.75 & 2030.4169 & 70.2 & 69.3 & 69 & 69.5 & 0.00057 \\
\hline 540.07001 & 3.74549581 & 14.7562313 & 0.98164784 & 14.6325 & 2273.5 & 2036.128 & 70.2 & 69.3 & 69 & 69.5 & 0.000569 \\
\hline 545.03 & 3.74612108 & 14.7562313 & 0.98164784 & 14.6325 & 2273.5 & 2031.4101 & 70.2 & 69.3 & 68.9 & 69.5 & 0.000569 \\
\hline 550.02002 & 3.75862648 & 14.756981 & 0.9810224 & 14.63325 & 2273.25 & 2032.4034 & 70.2 & 69.3 & 68.9 & 69.5 & 0.00057 \\
\hline 555.04004 & 3.75424959 & 14.7554817 & 0.9810224 & 14.63325 & 2273.5 & 2040.3492 & 70.1 & 69.3 & 69.1 & 69.5 & 0.00057 \\
\hline 560.02002 & 3.7498727 & 14.7562313 & 0.9810224 & 14.6325 & 2273.5 & 2042.0874 & 70.1 & 69.3 & 69 & 69.5 & 0.000569 \\
\hline 565.02002 & 3.76738026 & 14.7554817 & 0.98164784 & 14.6325 & 2273.25 & 2038.6111 & 70.2 & 69.3 & 68.9 & 69.5 & 0.000569 \\
\hline 570.02002 & 3.75550013 & 14.7554817 & 0.98164784 & 14.6325 & 2273.5 & 2043,8255 & 70.2 & 69.3 & 68.9 & 69.5 & 0.000569 \\
\hline 575.02002 & 3.75987702 & 14.7554817 & 0.98039696 & 14.63325 & 2273.5 & 2040.8459 & 70.2 & 69.3 & 68.9 & 69.5 & 0.00057 \\
\hline 580.02002 & 3.75362432 & 14.7562313 & 0.98164784 & 14.63325 & 2273.75 & 2036.6246 & 70.2 & 69.3 & 69.1 & 69.5 & 0.000569 \\
\hline 585.06 & 3.78988998 & 14.7562313 & 0.98227328 & 14.63325 & 2283.5 & 2037.8662 & 70.2 & 69.3 & 69 & 69.5 & 0.000569 \\
\hline 590.02002 & 3.73236514 & 14.7562313 & 0.98164784 & 14.634 & 2283.5 & 2041.0942 & 70.2 & 69.3 & 69.1 & 69.5 & 0.00057 \\
\hline 595.02002 & 3.7498727 & 14.7547321 & 0.98227328 & 14.6325 & 2283.5 & 2040.3492 & 70.1 & 69.3 & 69.1 & 69.5 & 0.000571 \\
\hline 600.05002 & 3.74612108 & 14.7554817 & 0.98164784 & 14.6325 & 2273.5 & 2033.6449 & 70.2 & 69.3 & 69 & 69.5 & 0.00057 \\
\hline 605.02002 & 3.77988566 & 14.7547321 & 0.98164784 & 14.63325 & 2274 & 2040.8459 & 70.1 & 69.3 & 69 & 69.5 & 0.000569 \\
\hline
\end{tabular}




\begin{tabular}{|c|c|c|c|c|c|c|c|c|c|c|c|}
\hline SECONDS & \begin{tabular}{|l|} 
MAIN DUCT \\
DIFF (IN H2 \\
\end{tabular} & \begin{tabular}{|l|} 
MAIN DUCT \\
ABS (PSIA) \\
\end{tabular} & \begin{tabular}{|l|} 
GAS INJECT \\
DIFF (IN H2O \\
\end{tabular} & \begin{tabular}{|l} 
GAS INJECT \\
ABS (PSIA) \\
\end{tabular} & \begin{tabular}{|l|} 
NEWPORT \\
(PPM) \\
\end{tabular} & \begin{tabular}{|l} 
SMC \\
(PPM)
\end{tabular} & \begin{tabular}{|l|} 
AMBIENT \\
TEMP.(F) \\
\end{tabular} & \begin{tabular}{|l|} 
GAS INJECT \\
TEMP.(F) \\
\end{tabular} & $\begin{array}{l}\text { MAIN DUCT } \\
\text { TEMP.(F) } \\
\end{array}$ & \begin{tabular}{|l|} 
WEC CELL \\
TEMP.(F) \\
\end{tabular} & \begin{tabular}{|l|} 
WEC CELL \\
(VOLTS) \\
\end{tabular} \\
\hline 610.02002 & 3.75049797 & 14,7554817 & 0.9810224 & 14.63325 & 2273.25 & 2036.128 & 70.1 & 69.3 & 69 & 695 & 0,000560 \\
\hline 615.02002 & 3.76425391 & 14.7554817 & 0.9810224 & 14.63325 & 2273 & 2038.1145 & 70.1 & 69.3 & 68.8 & 69.5 & 0.00057 \\
\hline 620.02002 & 3.74487054 & 14.7547321 & 0.9810224 & 14.63325 & 2273.75 & 2040.3492 & 70.1 & 69.3 & 68.8 & 69.5 & 0.000569 \\
\hline 625.02002 & 3.73549149 & 14.7554817 & 0.98227328 & 14.6325 & 2273 & 2040.3492 & 70.1 & 69.3 & 68.9 & 69.5 & 0.00057 \\
\hline 630.04001 & 3.74799689 & 14.7547321 & 0.98164784 & 14.63325 & 2273.5 & 2043.8255 & 70.1 & 69.4 & 68.8 & 69.5 & 0.000569 \\
\hline 635.02002 & 3.76925607 & 14.7562313 & 0.98164784 & 14.6325 & 2273.25 & 2041.5908 & 70 & 69.3 & 69 & 69.5 & 0.000569 \\
\hline 640.02002 & 3.73924311 & 14.756981 & 0.98164784 & 14.63325 & 2273.25 & 2041.3425 & 70 & 69.3 & 69 & 69.5 & 0.00057 \\
\hline 645.06 & 3.76925607 & 14.7554817 & 0.98164784 & 14.6325 & 2273.5 & 2040.5975 & 70.1 & 69.3 & 69 & 69.5 & 0.000569 \\
\hline 650.02002 & 3.73173987 & 14.7554817 & 0.9810224 & 14.63325 & 2273.25 & 2042.8323 & 70.1 & 69.3 & 68.9 & 69.5 & 0.00057 \\
\hline 655.02002 & 3.73299041 & 14.7562313 & 0.98164784 & 14.63325 & 2273.5 & 2036.8729 & 70.1 & 69.3 & 68.8 & 69.5 & 0.00057 \\
\hline 660.06 & 3.73924311 & 14.7577306 & 0.9810224 & 14.6325 & 2273.25 & 2034.8865 & 70.1 & 69.4 & 68.9 & 69.5 & 0.000569 \\
\hline 665.02002 & 3.74487054 & 14.7562313 & 0.98164784 & 14.63325 & 2273.5 & 2040.8459 & 70.1 & 69.3 & 69 & 69.5 & 0.000569 \\
\hline 670.02002 & 3.75550013 & 14.7562313 & 0.98164784 & 14.6325 & 2273.25 & 2037.8662 & 70.1 & 69.3 & 69.1 & 69.5 & 0.000569 \\
\hline 675.03 & 3.76050229 & 14.756981 & 0.98039696 & 14.63325 & 2273.25 & 2037.6178 & 70.1 & 69.4 & 69 & 69.5 & 0.00057 \\
\hline 680.02002 & 3.74549581 & 14.7562313 & 0.97914608 & 14.6325 & 2274 & 2035.1348 & 70.1 & 69.3 & 69 & 69.5 & 0.00057 \\
\hline 685.02002 & 3.75424959 & 14.7577306 & 0.9810224 & 14.63325 & 2273.25 & 2041.8391 & 70.1 & 69.4 & 69 & 69.5 & 0.000569 \\
\hline 60.03 & 3.73236514 & 14.7562313 & 0.97914608 & 14.63325 & 2273.5 & 2034.1415 & 70.1 & 69.4 & 68.9 & 69.5 & 0.000569 \\
\hline 695.02002 & 3.75675067 & 14.7562313 & 0.98227328 & 14.63325 & 2273 & 2041.0942 & 70.2 & 69.4 & 69.1 & 69.5 & 0.00057 \\
\hline 700.02002 & 3.74299473 & 14.7562313 & 0.9810224 & 14.63325 & 2273.5 & 2040.3492 & 70.2 & 69.4 & 69.1 & 69.5 & 0.000569 \\
\hline 705.06 & 3.74737162 & 14.7554817 & 0.9810224 & 14.63325 & 2273.25 & 2040.1009 & 70.2 & 69.3 & 69.1 & 69.5 & 0.000569 \\
\hline 710.06 & 3.75737594 & 14.7547321 & 0.97914608 & 14.63325 & 2273.75 & 2038.6111 & 70.2 & 69.3 & 69.1 & 69.5 & 0.000569 \\
\hline 715.02002 & 3.78176147 & 14.7562313 & 0.9810224 & 14.634 & 2274 & 2031.6585 & 70.2 & 69.4 & 68.9 & 69.5 & 0.00057 \\
\hline 720.03 & 3.74612108 & 14.7554817 & 0.97914608 & 14.6325 & 2273.25 & 2034.6382 & 70.2 & 69.3 & 69 & 69.5 & 0.00057 \\
\hline 725.02002 & 3.74674635 & 14.7524832 & 0.98227328 & 14.63175 & 2273.25 & 2036.6246 & 70.2 & 69.3 & 69 & 69.5 & 0.00057 \\
\hline 730.02002 & 3.77926039 & 14.7547321 & 0.97914608 & 14.63325 & 2273.5 & 2036.3763 & 70.2 & 69.4 & 68.9 & 69.5 & 0.00057 \\
\hline 735.06 & 3.74474419 & 14.7554817 & 0.97977152 & 14,6325 & 2283.25 & 2041.8391 & 70.2 & 69.3 & 68.9 & 69.5 & 0.000569 \\
\hline 740.02002 & 3.75112324 & 14.7562313 & 0.97977152 & 14.6325 & 2283.25 & 2028.6788 & 70.1 & 69.3 & 69.1 & 69.5 & 0.00057 \\
\hline 745.02002 & 3.7686308 & 14.7562313 & 0.97914608 & 14.63325 & 2281 & 2027.1889 & 70.2 & 69.4 & 69.1 & 69.5 & 0.00057 \\
\hline 750.04001 & 3.74236946 & 14.7547321 & 0.97977152 & 14.63175 & 2273.5 & 2027.9338 & 70.2 & 69.4 & 68.9 & 69.5 & 0.00057 \\
\hline 755.03 & 3.7498727 & 14.7554817 & 0.97914608 & 14.63175 & 2273.25 & 2032.1551 & 70 & 69.3 & 68.9 & 69.5 & 0.000569 \\
\hline 760.02002 & 3.75049797 & 14.7539825 & 0.97852064 & 14.63325 & 2273.5 & 2035.6314 & 70.1 & 69.4 & 68.9 & 69.5 & 0.000569 \\
\hline 765.03 & 3.74362 & 14.7554817 & 0.97852064 & 14.63325 & 2273.25 & 2034.3898 & 70.1 & 69.3 & 68.9 & 69.5 & 0.000569 \\
\hline 770.02002 & 3.76050229 & 14.7547321 & 0,97914608 & 14.6325 & 2273.25 & 2035,8797 & 70 & 69.4 & 68.9 & 69.5 & 0.000568 \\
\hline 775.02002 & 3.76175283 & 14.7547321 & 0.97664432 & 14.6325 & 2273.5 & 2033.1483 & 70 & 69.4 & 69 & 69.5 & 0.00057 \\
\hline 780.05002 & 3.76800553 & 14.7539825 & 0.97914608 & 14.63175 & 2273.75 & 2028.9271 & 70 & 69.4 & 69.1 & 69.5 & 0.000569 \\
\hline 785.02002 & 3.74998727 & 14.7547321 & 0.97914608 & 14.63325 & 2273.5 & 2028.1821 & 70 & 69.4 & 69 & 69.5 & 0.000569 \\
\hline 790.02002 & 3.73299041 & 14.7562313 & 0.97914608 & 14.63325 & 2273 & 2031.1618 & 70 & 69.4 & 69 & 6.5 & 0.000568 \\
\hline 795.04001 & 3.74612108 & 14.7547321 & 0.97852064 & 14.6325 & 2273.25 & 2023.9609 & 69.9 & 69.4 & 68.9 & 69.5 & 0.00057 \\
\hline 800.02002 & 3.74674635 & 14.7532329 & 0.97914608 & 14.6325 & 2274 & 2029.9203 & 69.9 & 69.4 & 69 & 69.5 & 0.000569 \\
\hline 805.02002 & 3.7498727 & 14.7562313 & 0.97664432 & 14.63175 & 2273 & 2033.3966 & 69.9 & 69.4 & 69 & 69.5 & 0.000569 \\
\hline 810.05002 & 3.75550013 & 14.7562313 & 0.97914608 & 14.6325 & 2273.25 & 2034.1415 & 70 & 69.4 & 69 & 69.5 & 0.000569 \\
\hline 815.03 & 3.74549581 & 14.756981 & 0.97664432 & 14.63175 & 2273.25 & 2034.1415 & 70 & 69.4 & 68.9 & 69.5 & 0.000568 \\
\hline
\end{tabular}




\begin{tabular}{|c|c|c|c|c|c|c|c|c|c|c|c|}
\hline SECONDS & $\begin{array}{l}\text { MAIN DUCT' } \\
\text { DIFF (IN H2 }\end{array}$ & \begin{tabular}{|l|} 
MAIN DUCT \\
ABS (PSIA)
\end{tabular} & \begin{tabular}{|l|} 
GAS INJECT \\
DIFF (IN H2O \\
\end{tabular} & $\begin{array}{l}\text { GAS INJECT } \\
\text { ABS (PSIA) }\end{array}$ & \begin{tabular}{|l|} 
NEWPORT \\
(PPM) \\
\end{tabular} & \begin{tabular}{|l} 
SMC \\
(PPM) \\
\end{tabular} & $\begin{array}{l}\text { AMBIENT } \\
\text { TEMP.(F) } \\
\end{array}$ & \begin{tabular}{|l|} 
GAS INJECT \\
TEMP.(F) \\
\end{tabular} & \begin{tabular}{|l|} 
MAIN DUCT \\
TEMP.(F) \\
\end{tabular} & \begin{tabular}{|l|} 
WEC CELL \\
TEMP.(F) \\
\end{tabular} & \begin{tabular}{|l|} 
WEC CELL \\
(VOLTS)
\end{tabular} \\
\hline 820.02002 & 3.76612972 & 14.7554817 & 0.97914608 & 14.63325 & 2273.25 & 2031.9068 & 70 & 69.4 & 68.9 & 69.5 & 0.000569 \\
\hline 825.03 & 3.76925607 & 14.7562313 & 0.97664432 & 14.63325 & 2274 & 2035.1348 & 70 & 69.3 & 69.1 & 69.5 & 0.000569 \\
\hline 830.02002 & 3.74549581 & 14.756981 & 0.97664432 & 14.6325 & 2273.5 & 2035.3831 & 70 & 69.4 & 69.1 & 69.5 & 0.000568 \\
\hline 835.02002 & 3.77050661 & 14.7554817 & 0.97726976 & 14.6325 & 2273.75 & 2037.6178 & 70.2 & 69.4 & 69.1 & 69.5 & 0.000569 \\
\hline 840.05002 & 3.74549581 & 14.756981 & 0.97977152 & 14.63325 & 2273.5 & 2037.3695 & 70.2 & 69.4 & 69 & 69.5 & 0.00057 \\
\hline 845.10001 & 3.77363296 & 14.7547321 & 0.97726976 & 14.63175 & 2273.75 & 2034.6382 & 70.2 & 69.4 & 68.9 & 69.5 & 0.000569 \\
\hline 850.02002 & 3.7498727 & 14.7562313 & 0.97601888 & 14,63175 & 2273.5 & 2022.4711 & 70.2 & 69.4 & 68.9 & 69.5 & 0.000569 \\
\hline 855.05002 & 3.75174851 & 14.7577306 & 0.97852064 & 14.6325 & 2273 & 2027.6855 & 70.3 & 69.4 & 68.9 & 69.5 & 0.000669 \\
\hline 860.04001 & 3.77238242 & 14.756981 & 0.97664432 & 14.63325 & 2273.5 & 2028.6788 & 70.3 & 69.4 & 69 & 69.5 & 0.000568 \\
\hline 865.02002 & 3.75925175 & 14.7562313 & 0.97664432 & 14.63325 & 2273.5 & 2023.216 & 70.3 & 69.3 & 69.1 & 69.5 & 0.000569 \\
\hline 870.03 & 3.74236946 & 14.7554817 & 0.97726976 & 14.63325 & 2273.75 & 2023.216 & 70.3 & 69.4 & 69 & 69.5 & 0.000569 \\
\hline 875.02002 & 3.76300337 & 14.7562313 & 0.97914608 & 14.63325 & 2273 & 2026.444 & 70.3 & 69.4 & 68.9 & 69.5 & 0.000569 \\
\hline 880.02002 & 3.73861784 & 14.7554817 & 0.97664432 & 14.6325 & 2273.25 & 2028.4305 & 70.3 & 69.4 & 69 & 69.5 & 0.000569 \\
\hline 885.04001 & 3.74362 & 14.7547321 & 0.97720976 & 14.6325 & 2273.75 & 2032.9 & 70.3 & 69.4 & 69.1 & 69.5 & 0.000569 \\
\hline 890.03 & 3.75862648 & 14.7562313 & 0.97726976 & 14,6325 & 2273 & 2030.9135 & 70.3 & 69.4 & 69 & 69.5 & 0.000569 \\
\hline 895.06 & 3.74862216 & 14.7562313 & 0.97601888 & 14.6325 & 2273.5 & 2028.6788 & 70.3 & 69.4 & 68.9 & 69.5 & 0.00057 \\
\hline 900.03 & 3.75299905 & 14.756981 & 0.97726976 & 14.63175 & 2273.75 & 2030.1686 & 70.3 & 69.4 & 69.1 & 69.5 & 0.000569 \\
\hline 905.09 & 3.75174851 & 14.7554817 & 0.9778952 & 14.6325 & 2273.5 & 2028.9271 & 70.3 & 69.4 & 69.1 & 69.5 & 0.000569 \\
\hline 910.02002 & 3.7561254 & 14.756981 & 0.97726976 & 14.6325 & 2274 & 2022.7194 & 70.3 & 69.4 & 69.1 & 69.6 & 0.000569 \\
\hline 915.02002 & 3.74924743 & 14.7562313 & 0.9778952 & 14.6325 & 2273.25 & 2019.988 & 70.3 & 69.4 & 69.2 & 69.6 & 0.00057 \\
\hline 920,02002 & 3.76112756 & 14.7562313 & 0.9778952 & 14.6325 & 2274 & 2024.7058 & 70.3 & 69.4 & 69.2 & 69.5 & 0.00057 \\
\hline 925.03 & 3.74924743 & 14.756981 & 0.97726976 & 14.6325 & 2273.25 & 2027.4372 & 70.4 & 69.4 & 69 & 69.5 & 0.000569 \\
\hline 930.02002 & 3.74549581 & 14.7562313 & 0.97726976 & 14.6325 & 2273 & 2025.2024 & 70.3 & 69.4 & 68.9 & 69.5 & 0.00057 \\
\hline 935.03 & 3.76362864 & 14.7562313 & 0.708956 & 14.62725 & 2273.5 & 2027.4372 & 70.3 & 69.4 & 69 & 69.6 & 0.000569 \\
\hline
\end{tabular}




\begin{tabular}{|c|c|c|c|c|c|c|c|c|c|c|c|}
\hline \multicolumn{12}{|c|}{$\begin{array}{l}\text { TEST: FG122223 } 22 \text { Feb } 1997 \text { WEC-SPOOL } \\
\text { Measured Hydrogen Concentration = 2159ppm }\end{array}$} \\
\hline SECONOS & $\frac{\text { MAIN DUCT }}{\text { DIFF IN H2O }}$ & \begin{tabular}{|l|} 
MAIN DUCT \\
ABS (PSIA)
\end{tabular} & \begin{tabular}{|l|} 
GAS INJECT \\
DIFF (IN H2O
\end{tabular} & $\begin{array}{l}\text { GAS INJECT } \\
\text { ABS (PSIA) }\end{array}$ & \begin{tabular}{|l|} 
NEWPORT \\
(PPM)
\end{tabular} & \begin{tabular}{|l|} 
SMC \\
(PPM)
\end{tabular} & $\mid \frac{\text { AMBIENT }}{\text { TEMP }\left({ }^{\circ} \mathrm{F}\right.}$ & $\frac{\text { GAS INJECT }}{\text { TEMP }\left({ }^{\circ} \mathrm{F}\right)}$ & $\mid$\begin{tabular}{|l|} 
MAIN DUCT \\
TEMP $\left({ }^{\circ} \mathrm{F}\right)$
\end{tabular} & $\begin{array}{l}\text { WEC CELL } \\
\left.\mid \text { TEMP ( }{ }^{\circ} \mathrm{F}\right)\end{array}$ & \begin{tabular}{|l} 
WEC CELL \\
(VOLTS)
\end{tabular} \\
\hline & & & & & & & & & & & \\
\hline 0.0100098 & 3.76800553 & 14.7562313 & 0.382288688 & 14.6325 & 31.475 & -3.349758 & 70.3 & 69.3 & 69 & 69.6 & 0.000235 \\
\hline 5.0200195 & 3.75299905 & 14.7547321 & 0.98039696 & 14.63175 & 30.95 & 3.2504348 & 70.3 & 69.1 & 69 & 69.5 & 0.000235 \\
\hline 10.02002 & 3.76925607 & 14.7562313 & 0.97664432 & 14.63325 & 41.55 & 615.06132 & 70.4 & 69.3 & 69.1 & 69.6 & 0.000249 \\
\hline 15.02002 & 3.7373673 & 14.7554847 & 0.97726976 & 14.63325 & 271.5 & 1293.1905 & 70.3 & 69.4 & 69 & 69.5 & 0.000285 \\
\hline 20.02002 & 3.7561254 & 14.7562313 & 0.97726976 & 14.6325 & 541.75 & 1628.9029 & 70.4 & 69.4 & 69 & 69.6 & 0.00033 \\
\hline 25.059998 & 3.74549581 & 14.7554817 & 0.97664432 & 14.6325 & 632 & 1774.908 & 70.3 & 69.4 & 69 & 69.5 & 0.00037 \\
\hline 30.02002 & 3.74549581 & 14.7562313 & 0.97664432 & 14.6325 & 1072.25 & 1838.9714 & 70.3 & 69.4 & 69.1 & 69.5 & 0.000404 \\
\hline 35.02002 & 3.77425823 & 14.7547321 & 0.97664432 & 14.63175 & 1263.75 & 1875.2244 & 70.3 & 69.4 & 69 & 69.5 & 0.000432 \\
\hline 40.02002 & 3.74487054 & 14.7547321 & 0.97726976 & 14.6325 & 1432.25 & 1891.3644 & 70.3 & 69.4 & 69 & 69.5 & 0.000455 \\
\hline 45.02002 & 3.73924311 & 14.7562313 & 0.97664432 & 14.63175 & 1572.25 & 1901.5451 & 70.4 & 69.4 & 69.1 & 69.5 & 0.000473 \\
\hline 50.02002 & 3.74549581 & 14.7554817 & 0.97726976 & 14.631 & 1682.25 & 1914.2088 & 70.3 & 69.3 & 69.1 & 69.5 & 0.000488 \\
\hline 55.02002 & 3.74674635 & 14.7562313 & 0.97726976 & 14.63325 & 1762.75 & 1927.6174 & 70.3 & 69.4 & 69 & 69.5 & 0.0005 \\
\hline 60.02002 & 3.76925607 & 14.7554817 & 0.97664432 & 14.63175 & 1832.75 & 1927.6174 & 70.3 & 69.4 & 69 & 69.5 & 0.000509 \\
\hline 65.02002 & 3.74362 & 14.7554817 & 0.97726976 & 14.6325 & 1892.5 & 1932.0869 & 70.3 & 69.4 & 69 & 69.5 & 0.000517 \\
\hline 70.02002 & 3.75800121 & 14.7562313 & 0.97852064 & 14.6325 & 1943 & 1937.798 & 70.3 & 69.4 & 69 & 69.5 & 0.000523 \\
\hline 75.02002 & 3.75550013 & 14.7554817 & 0.97664432 & 14.63325 & 1983 & 1944.7507 & 70.3 & 69.4 & 69.1 & 69.5 & 0.000528 \\
\hline 80.02002 & 3.74924743 & 14.756981 & 0.97601888 & 14.63175 & 2012.5 & 1947.9787 & 70.3 & 69.4 & 69 & 69.5 & 0.000532 \\
\hline 85.02002 & 3.74737162 & 14.7577306 & 0.97664432 & 14.6325 & 2033 & 1953.9381 & 70.3 & 69.4 & 69.1 & 69.6 & 0.000536 \\
\hline 90.02002 & 3.74549581 & 14.7554817 & 0.97664432 & 14.6325 & 2063 & 1957.6627 & 70.3 & 69.4 & 69.1 & 69.5 & 0.000541 \\
\hline 95.02002 & 3.75362432 & 14.7547321 & 0.97664432 & 14.6325 & 2082.75 & 1958.1593 & 70.3 & 69.4 & 69.1 & 69.5 & 0.000542 \\
\hline 100.02002 & 3.74674635 & 14.7562313 & 0.97664432 & 14.63175 & 2093 & 1965.1119 & 70.3 & 69.4 & 69.1 & 69.5 & 0.000545 \\
\hline 105.02002 & 3.75174851 & 14.7554817 & 0.97726976 & 14.6325 & 2112.75 & 1971.5679 & 70.3 & 69.4 & 69.1 & 69.5 & 0.000546 \\
\hline 110.02002 & 3.74487054 & 14.7562313 & 0.97664432 & 14.6325 & 2132 & 1972.5612 & 70.2 & 69.4 & 69 & 69.6 & 0.000549 \\
\hline 115.02002 & 3.75112324 & 14.756981 & 0.97664432 & 14.63325 & 2133.5 & 1972.3128 & 70.2 & 69.4 & 69 & 69.5 & 0.00065 \\
\hline 120.03 & 3.77613404 & 14.756981 & 0.97664432 & 14.63325 & 2142.5 & 1973.5544 & 70.2 & 69.4 & 69 & 69.5 & 0.000551 \\
\hline 125.02002 & 3.77238242 & 14.756981 & 0.97726976 & 14.6325 & 2153.5 & 1967.595 & 70.2 & 69.4 & 69 & 69.5 & 0.000552 \\
\hline 130.02002 & 3.76362864 & 14.7554817 & 0.97726976 & 14.63325 & 2163.25 & 1977.5273 & 70.2 & 69.3 & 69.1 & 69.5 & 0.000553 \\
\hline 135.02002 & 3.75737594 & 14.7562313 & 0.97726976 & 14.63175 & 2172.75 & 1977.279 & 70.2 & 69.4 & 69 & 69.5 & 0.000555 \\
\hline 140.02002 & 3.739868838 & 14.756981 & 0.97664432 & 14.63325 & 2173.25 & 1983.9833 & 70.2 & 69.4 & 68.9 & 69.5 & 0.000556 \\
\hline 145.02002 & 3.74674635 & 14.7547321 & 0.97726976 & 14.6325 & 2183.25 & 1985.9698 & 70.2 & 69.4 & 68.9 & 69.5 & 0.000556 \\
\hline 150.05002 & 3.75299905 & 14.756981 & 0.97726976 & 14.63175 & 2183.5 & 1993.1707 & 70.2 & 69.4 & 68.9 & 69.5 & 0.000557 \\
\hline 155.02002 & 3.76550445 & 14.7577306 & 0.97664432 & 14.6325 & 2193.5 & 1994.4123 & 70.2 & 69.4 & 69.1 & 69.5 & 0.000558 \\
\hline 160.02002 & 3.72548717 & 14.7554817 & 0.97726976 & 14.6325 & 2203 & 1983.9833 & 70.2 & 69.4 & 69.1 & 69.6 & 0.000558 \\
\hline 165.02002 & 3.74924743 & 14.7562313 & 0.97726976 & 14.6325 & 2203 & 1988.9495 & 70.3 & 69.4 & 68.9 & 69.5 & 0.000558 \\
\hline 170.02002 & 3.75800121 & 14.7562313 & 0.97726976 & 14.6325 & 2203.25 & 1987.2113 & 70.3 & 69.4 & 68.9 & 69.6 & 0.000559 \\
\hline 175.02002 & 3.7561254 & 14.7562313 & 0.97664432 & 14.6325 & 2203 & 1992.4258 & 70.3 & 69.4 & 68.9 & 69.5 & 0.000558 \\
\hline 180.02002 & 3.74799689 & 14.756981 & 0.97664432 & 14.6325 & 2213.25 & 1994.4123 & 70.3 & 69.4 & 69 & 69.6 & 0.00056 \\
\hline 185.02002 & 3.77425823 & 14.756981 & 0.97664432 & 14.6325 & 2213.25 & 2002.6064 & 70.3 & 69.4 & 69 & 69.5 & 0.00056 \\
\hline
\end{tabular}




\begin{tabular}{|c|c|c|c|c|c|c|c|c|c|c|c|}
\hline SECONDS & \begin{tabular}{|l} 
MAIN DUCT \\
DIFF (IN H2O \\
\end{tabular} & \begin{tabular}{|l|} 
MAIN DUCT \\
ABS (PSIA) \\
\end{tabular} & $\begin{array}{l}\text { GAS INJECT } \\
\text { DIFF (IN H2O }\end{array}$ & $\begin{array}{l}\text { GAS INJECT } \\
\text { ABS (PSIA) } \\
\end{array}$ & $\begin{array}{l}\text { NEWPORT } \\
\text { (PPM) } \\
\end{array}$ & \begin{tabular}{|l} 
SMC \\
(PPM)
\end{tabular} & $\begin{array}{l}\text { AMBIENT } \\
\text { TEMP ( }{ }^{\circ} \mathrm{F} \\
\end{array}$ & $\begin{array}{l}\text { GAS INJECT } \\
\text { TEMP ( ("F) }\end{array}$ & $\begin{array}{l}\text { MAIN DUCT } \\
\text { TEMP (*F) } \\
\end{array}$ & $\begin{array}{l}\text { WEC CELL } \\
\left.\text { TEMP ( }{ }^{\circ} \mathrm{F}\right) \\
\end{array}$ & $\begin{array}{l}\text { WEC CELL } \\
\text { (VOLTS) }\end{array}$ \\
\hline 190.02002 & 3.75174851 & 14.7562313 & 0.97664432 & 14,6325 & 2213 & 2006.331 & 70.3 & 69.4 & 69.1 & 69.5 & 0.00056 \\
\hline 195.02002 & 3.7498727 & 14.7562313 & 0.97601888 & 14.63325 & 2213.25 & 2006.5793 & 70.3 & 69.4 & 69.1 & 69.5 & 0.000561 \\
\hline 200.02002 & 3.75174851 & 14.756981 & $0.9766 \overline{4432}$ & 14.6325 & 2223 & 2003.5997 & 70.4 & 69.4 & 69.1 & 69.6 & 0.000562 \\
\hline 205.02002 & 3.77550877 & 14.7577306 & 0.97664432 & 14.6325 & 2213.75 & 2002.3581 & 70.4 & 69.4 & 69.2 & 69.5 & 0.000561 \\
\hline 210.02002 & 3.7498727 & 14.7577306 & 0.97726976 & 14.63325 & 2223.25 & 1999.6267 & 70.4 & 69.4 & 69.1 & 69.5 & 0.000561 \\
\hline 215.02002 & 3.74549581 & 14.756981 & 0.97664432 & 14.63325 & 2223 & 2004.5929 & 70.4 & 69.4 & 69.2 & 69.6 & 0.000562 \\
\hline 220.02002 & 3.74362 & 14.7577306 & 0.97664432 & 14.6325 & 2223.25 & 2009.3107 & 70.3 & 69.4 & 69.1 & 69.5 & 0.000562 \\
\hline 225.02002 & 3.7498727 & 14.7577306 & 0.97726976 & 14.6325 & 2223.5 & 2010.5523 & 70.3 & 69.4 & 69 & 69.6 & 0.000562 \\
\hline 230.02002 & $3.755500 \div 3$ & 14.756981 & 0.97601888 & 14.63175 & 2223 & 2011.0489 & 70.4 & 69.4 & 69 & 69.6 & 0.000563 \\
\hline 235.02002 & 3.73924314 & 14.7577306 & 0.97664432 & 14.63325 & 2223.25 & 2018.4981 & 70.3 & 69.4 & 69.1 & 69.5 & 0.000562 \\
\hline 240.02002 & 3.76612972 & 14.7562313 & 0.97601888 & 14.63175 & 2233.25 & 2016.2634 & 70.3 & 69.4 & 69.1 & 69.5 & 0.000562 \\
\hline 245.02002 & 3.74487054 & 14.7562313 & 0.97726976 & 14.6325 & 2233 & 2017.7532 & 70.4 & 69.4 & 69.1 & 69.6 & 0.000563 \\
\hline 250.02002 & 3.73861784 & 14.756981 & 0.97726976 & 14.63175 & 2233.25 & 2015.0218 & 70.3 & 69.4 & 69 & 69.6 & 0.000563 \\
\hline 255.02002 & 3.76362864 & 14.7554817 & 0.97726976 & 14.6325 & 2233.25 & 2018.9947 & 70.3 & 69.4 & 69.2 & 69.5 & 0.000564 \\
\hline 260.02002 & 3.73048933 & 14.7562313 & 0.97726976 & 14.6325 & 2233 & 2014.2769 & 70.3 & 69.4 & 69.2 & 69.6 & 0.000563 \\
\hline 265.02002 & 3.74612108 & 14.7539825 & 0.97726976 & 14.63175 & 2233.25 & 2018.4981 & 70.3 & 69.4 & 69.2 & 69.5 & 0.000564 \\
\hline 270.02002 & 3.76612972 & 14.7547321 & 0.9778952 & 14.63175 & 2233 & 2018.0015 & 70.4 & 69.5 & 69.2 & 69.5 & 0.000564 \\
\hline 275.02002 & 3.7498727 & 14.7562313 & 0.97726976 & 14.63175 & 2233 & 2014.0286 & 70.4 & 69.5 & 69.2 & 69.5 & 0.000564 \\
\hline 280.02002 & 3.74236946 & 14.7562313 & 0.97726976 & 14.6325 & 2243.25 & 2013.2837 & 70.4 & 69.4 & 69.1 & 69.6 & 0.000564 \\
\hline 285.02002 & 3.75112324 & 14.7562313 & 0.97726976 & 14.6325 & 2243 & 2019.7397 & 70.4 & 69.4 & 69.1 & 69.5 & 0.000564 \\
\hline 290.02002 & 3.74924743 & 14.756981 & 0.97726976 & 14.6325 & 2243.25 & 2019.7397 & 70.3 & 69.4 & 68.9 & 69.6 & 0.000564 \\
\hline 295.02002 & 3.7561254 & 14.756981 & 0.97726976 & 14.6325 & 2243.25 & 2006.331 & 70.4 & 69.4 & 68.9 & 69.6 & 0.000565 \\
\hline 300.02002 & 3.76362864 & 14.7562313 & 0.97726976 & 14.63175 & 2243 & 2007.076 & 70.3 & 69.4 & 68.9 & 69.6 & 0.000565 \\
\hline 305.03 & 3.7686308 & 14.7554817 & 0.97726976 & 14.63175 & 2243.25 & 2012.0421 & 70.3 & 69.4 & 69.1 & 69.6 & 0.000565 \\
\hline 310.02002 & 3.74862216 & 14.7562313 & 0.97664432 & 14.631 & 2243.25 & 2010.0557 & 70.3 & 69.5 & 69.1 & 69.6 & 0.000564 \\
\hline 315.02002 & 3.73799257 & 14.7562313 & 0.97664432 & 14.6325 & 2243.25 & 2011.2972 & 70.3 & 69.4 & 69.1 & 69.6 & 0.000565 \\
\hline 320.02002 & 3.74549581 & 14.7562313 & 0.97664432 & 14.6325 & 2243.25 & 2019.7397 & 70.4 & 69.5 & 69.1 & 69.6 & 0.000565 \\
\hline 325.02002 & 3.74487054 & 14.7562313 & 0.97726976 & 14.63175 & 2243.25 & 2012.2904 & 70.4 & 69.5 & 69.1 & 6.6 & 0.000565 \\
\hline 330.02002 & 3.75174851 & 14.7554817 & 0.97664432 & 14.63175 & 2243.5 & 2009.0624 & 70.4 & 69.5 & 69 & 69.6 & 0.000564 \\
\hline 335.02002 & 3.78613836 & 14.7554817 & 0.97726976 & 14.63175 & 2243.5 & 2013.7803 & 70.3 & 69.5 & 69.1 & 69.6 & 0.000565 \\
\hline 340.02002 & 3.7561254 & 14.7562313 & 0.97726976 & 14.63175 & 2243.5 & 2015.0218 & 70.3 & 69.4 & 69 & 69.5 & 0.000565 \\
\hline 345.02002 & 3.73861784 & 14.7562313 & 0.9778952 & 14.63175 & 2243.5 & 2021.9744 & 70.3 & 69.5 & 69 & 69.6 & 0.000565 \\
\hline 350.02002 & 3.76675499 & 14.7554817 & 0.97726976 & 14.63175 & 2243.25 & 2021.2295 & 70.3 & 69.5 & 69 & 69.6 & 0.000565 \\
\hline 355.02002 & 3.74612108 & 14.7554817 & 0.97601888 & 14.63175 & 2253.75 & 2019.2431 & 70.3 & 69.4 & 69 & 69.6 & 0.000565 \\
\hline 360.02002 & 3.75362432 & 14.756981 & 0.97726976 & 14.631 & 2243.5 & 2021.9744 & 70.3 & 69.4 & 69.1 & 69.6 & 0.000565 \\
\hline 365.02002 & 3.73674203 & 14.756981 & 0.97664432 & 14.63175 & 2253.5 & 2022.7194 & 70.3 & 69.5 & 69 & 69.5 & 0.000565 \\
\hline 370.03 & 3.74799689 & 14.7554817 & 0.97664432 & 14.6325 & 2253.25 & 2022.7194 & 70.4 & 69.4 & 68.9 & 69.5 & 0.000565 \\
\hline 375.02002 & $\mathbf{3 . 7 4 5 4 9 5 8 1}$ & 14.7554817 & 0.97664432 & 14.63175 & 2253.5 & 2025,9474 & 70,3 & 69.5 & 68.9 & $69 . \overline{6}$ & 0.000565 \\
\hline 380.02002 & 3.74924743 & 14.756981 & 0.97726976 & 14.63175 & 2253.75 & 2020.9812 & 70.2 & 69.5 & 69 & 69.6 & 0.000565 \\
\hline 385.02002 & 3.74924743 & 14.7562313 & 0.974768 & 14.6325 & 2243.75 & 2019.988 & 70.2 & 69.5 & 68.9 & 69.6 & 0.000565 \\
\hline 390.02002 & 3.74862216 & 94.7562313 & 0.97664432 & 14.63175 & 2243.25 & 2024.4575 & 70.2 & 69.5 & 69.1 & 69.6 & 0.000565 \\
\hline 395.02002 & 3.74612108 & 14.756981 & 0.97414256 & 14.6325 & 2243 & 2017.7532 & 70.2 & 69.5 & 69.1 & 69.6 & 0.000565 \\
\hline
\end{tabular}




\begin{tabular}{|c|c|c|c|c|c|c|c|c|c|c|c|}
\hline SECONDS & $\begin{array}{l}\text { MAIN DUCT } \\
\text { DIFF (IN H2O }\end{array}$ & \begin{tabular}{|l|} 
MAIN DUCT \\
ABS (PSIA) \\
\end{tabular} & $\begin{array}{l}\text { GAS INJECT } \\
\text { DIFF (IN H2O }\end{array}$ & \begin{tabular}{|l} 
GAS INJECT \\
ABS (PSIA) \\
\end{tabular} & \begin{tabular}{|l|} 
NEWPORT \\
(PPM) \\
\end{tabular} & \begin{tabular}{|l|} 
SMC \\
(PPM) \\
\end{tabular} & \begin{tabular}{|l|} 
AMBIENT \\
TEMP $\left({ }^{\circ} \mathrm{F}\right.$ \\
\end{tabular} & \begin{tabular}{|l|} 
GAS INJECT \\
TEMP (" $F)$
\end{tabular} & \begin{tabular}{|l|} 
MAIN DUCT \\
TEMP $\left({ }^{\circ} \mathrm{F}\right)$ \\
\end{tabular} & $\begin{array}{l}\text { WEC CELL } \\
\text { TEMP ('F) } \\
\end{array}$ & $\begin{array}{l}\text { WEC CELL } \\
\text { (VOLTS) }\end{array}$ \\
\hline 400.03 & 3.75112324 & 14.7554817 & 0.97726976 & 14.63175 & 2253 & 2017.7532 & 70.1 & 69.5 & 69.2 & 69.7 & 0.000567 \\
\hline 405.02002 & 3.74049365 & 14.7562313 & 0.974768 & 14.6325 & 2254 & 2021.2295 & 70.2 & 69.5 & 69.1 & 69.6 & 0.000566 \\
\hline 410.05002 & 3.74799689 & 14.7562313 & 0.97664432 & 14.6325 & 2253.5 & 2018.7464 & 70.2 & 69.5 & 69.1 & 69.6 & 0.000566 \\
\hline 415.02002 & 3.74924743 & 14.7554817 & 0.974768 & 14.6325 & 2253.75 & 2020.2363 & 70.2 & 69.5 & 69.1 & 69.6 & 0.000566 \\
\hline$\$ 20.06$ & 3.76675499 & 14.7554817 & 0.97726976 & 14.63325 & 2253.25 & 2024.9541 & 70.2 & 69.5 & 69.1 & 69.6 & 0.000566 \\
\hline 425.02002 & 3.74799689 & 14.756981 & 0.97414256 & 14.634 & 2253.25 & 2023.4643 & 70.2 & 69.5 & 69.1 & 69.6 & 0.000567 \\
\hline 430.02002 & 3.75424959 & 14.756981 & $0.97 \varnothing 01888$ & 14.63325 & 2253.75 & 2020.4646 & 70.2 & 69.5 & 69.1 & 69.6 & 0.000565 \\
\hline 435.02002 & 3.74049365 & 14.7562313 & 0.97414256 & 14.63325 & 2253.25 & 2016.2634 & 70.2 & 69.5 & 69.1 & 69.6 & 0.000565 \\
\hline 440.07001 & 3.76800553 & 14.756981 & 0.97664432 & 14.63325 & 2253.5 & 2014.2769 & 70.2 & 69.5 & 69.1 & 69.6 & 0.000566 \\
\hline 445.02002 & 3.76925607 & 14.7562313 & 0.97726976 & 14.63325 & 2253.5 & 2016.5117 & 70.2 & 69.5 & 69 & 69.6 & 0.000566 \\
\hline 450.10001 & 3.75550013 & 14.7562313 & 0.97539344 & 14.6325 & 2254 & 2005.8344 & 70.2 & 69.5 & 69 & 69.6 & 000567 \\
\hline 455.02002 & 3.75112324 & 14.7554817 & 0.97726976 & 14.63325 & 2253.5 & 2006.5793 & 70.3 & 69.5 & 69 & 69.6 & 000566 \\
\hline 460.02002 & 3.73799257 & 14.756981 & 0.97726976 & 14.6325 & 2253.25 & 2008.0092 & 70.3 & 69.5 & 69 & 69.6 & 000566 \\
\hline 465.02002 & 3.74424527 & 14.7562313 & 0.97726976 & 14.63325 & 2253.5 & 2009.559 & 70.3 & 69.5 & 69 & 69.6 & 0.000566 \\
\hline 470.02002 & 3.74236946 & 14.7562313 & 0.97726976 & 14.6325 & 2253.5 & 2015.2701 & 70.3 & 69.5 & 6 & 69.6 & 0.000566 \\
\hline 475.02002 & 3.75925175 & 14.756981 & 0.97726976 & 14.6325 & 2253.5 & 2015.2701 & 70.2 & 69.5 & 69.1 & 69.6 & 0.000565 \\
\hline 480.02002 & 3.73861784 & 14.756981 & 0.9778952 & 14,63325 & 2253.25 & 2016.2634 & 70.3 & 69.5 & 69.1 & 69.6 & 0.000565 \\
\hline 485.02002 & 3.74737162 & 14.7562313 & 0.97539344 & 14.6325 & 2253.5 & 2016.0151 & 70.2 & 69.5 & 69.1 & 69.6 & 0.000566 \\
\hline 490.02002 & 3.74924743 & 14.7562313 & 0.974768 & 14.6325 & 2253.75 & 2014.7735 & 70.1 & 69.5 & 69.1 & 69.6 & 0.000566 \\
\hline 495.02002 & 3.73861784 & 14.7577306 & 0.974768 & 14.63325 & 2253 & 2016.76 & 70.2 & 69.5 & 69.1 & 69.6 & 0.000566 \\
\hline 500.02002 & 3.7623781 & 14.7562313 & 0.97726976 & 14.63325 & 2253.5 & 2012.2904 & 70.3 & 69.5 & 69.1 & 69.6 & 0.000566 \\
\hline 505.02002 & 3.77926039 & 14.756981 & 0.97539344 & 14.6325 & 2253.25 & 2016.2634 & 70.3 & 69.5 & 6 & 69.6 & 0.000565 \\
\hline 510.02002 & 3.75800121 & 14.756981 & 0.97664432 & 14.6325 & 2253 & 2017.2566 & 70.3 & 69.5 & 68.9 & 69.6 & 0.000565 \\
\hline $\mathbf{5 1 5 . 0 7 0 0 1}$ & 3.75112324 & 14.7562313 & 0.97539344 & 14.6325 & 2253.5 & 2014.0286 & 70.3 & 69.5 & 69 & 69.6 & 000567 \\
\hline 520.02002 & 3.75174851 & 14.7562313 & 0.97726976 & 14.63325 & 2253 & 2014.5252 & 70.3 & 69.5 & 68.9 & 69.6 & 0.000566 \\
\hline 525.06 & 3.76988134 & 14.756981 & 0.974768 & 14.6325 & 2253.5 & 2003.848 & 70.3 & 69.5 & 69 & 69.6 & 0.000567 \\
\hline 530.02002 & 3.76487918 & 14.7562313 & 0.974768 & 14.63325 & 2253.25 & 1997.392 & 70.2 & 69.5 & 69.1 & 69.6 & 0.000567 \\
\hline 535.02002 & 3.74674635 & 14.7562313 & 0.97539344 & 14.63325 & 2263.25 & 2005.3378 & 70.2 & 69.5 & 69.1 & 69.6 & 0,000566 \\
\hline 540.02002 & 3.73674203 & 14.7562313 & 0.97664432 & 14.63175 & 2263.75 & 2013.2837 & 70.1 & 69.5 & 69 & 69.5 & 0.000566 \\
\hline 545,06 & 3.74487054 & 14.7562313 & $0.976 \overline{66432}$ & 14.6325 & 2263.25 & 2012.787 & 70.1 & 69.5 & 69.1 & 69.6 & 0.000567 \\
\hline 550.02002 & 3.7498727 & 14.7562313 & 0,97539344 & 14.6325 & 2263.5 & 2012.5387 & 70.1 & 69.5 & 69.1 & 69.6 & 0.000567 \\
\hline 555.05002 & 3.74487054 & 14.7577306 & 0.97539344 & 14.6325 & 2264 & 2009.0624 & 70.1 & 69.5 & 69 & 69.6 & 0.000567 \\
\hline 560.02002 & 3.75550013 & 14.7562313 & 0.974768 & 14.63175 & 2263.5 & 2010.0557 & 70.1 & 69.5 & 69.1 & 69.6 & 0.000567 \\
\hline 565.02002 & 3.75352432 & 14.756981 & 0.97601888 & 14.6325 & 2263.25 & 2009.3107 & 70.2 & 69.5 & 69 & 69.6 & 0.000566 \\
\hline 570.02002 & 3.74737162 & 14.7562313 & 0.974768 & 14.63175 & 2263.5 & 2008.0692 & 70.3 & 69.5 & 69 & 69.6 & 0.000567 \\
\hline 575.05002 & 3.74924743 & 14.7562313 & 0.974768 & 14.6325 & 2255.5 & 2014.2769 & 70.3 & 69.5 & 69.1 & 69.6 & 0.000567 \\
\hline 580.02002 & 3.75174851 & 14.756981 & 0.97539344 & 14.63325 & 2263.5 & 2013.0354 & 70.3 & 69.5 & 69 & 69.6 & 0.000566 \\
\hline 585.06 & 3.75987702 & 14.7562313 & 0.97664432 & 14.6325 & 2263.5 & 2010.5523 & 70.5 & 69.5 & 69.2 & 69.6 & 0.000566 \\
\hline 590.07001 & 3.74924743 & 14.7552313 & 0.974768 & 14.63175 & 2263 & 2010.0557 & 70.5 & 69.5 & 69 & 69.6 & 0.000568 \\
\hline 595.02002 & 3.74424527 & 14.7547321 & 0.97539344 & 14.6325 & 2263.5 & 2013.0354 & 70.5 & 69.5 & 69 & 69.6 & 0.000566 \\
\hline 600.03 & 3.75112324 & 14.7554817 & 0.974768 & 14.6325 & 2264 & 2010.0557 & $\overline{70.5}$ & 69.5 & 69.1 & 69.6 & 0.000566 \\
\hline 605.02002 & 3.76300337 & 14.75544817 & 0.97539344 & 14.6325 & 2263 & 2011.5455 & 70.4 & 69.5 & 69 & 69.6 & 0.000566 \\
\hline
\end{tabular}




\begin{tabular}{|c|c|c|c|c|c|c|c|c|c|c|c|}
\hline SECONDS & \begin{tabular}{|l|} 
MAIN DUCT \\
DIFF (IN H2O \\
\end{tabular} & \begin{tabular}{|l|} 
MAIN DUCT \\
ABS (PSIA) \\
\end{tabular} & $\begin{array}{l}\text { GAS INJECT } \\
\text { DIFF (IN H2O } \\
\end{array}$ & \begin{tabular}{|l} 
GAS INJECT \\
ABS (PSIA) \\
\end{tabular} & $\begin{array}{l}\text { NEWPORT } \\
\text { (PPM) } \\
\end{array}$ & $\begin{array}{l}\text { SMC } \\
\text { (PPM) }\end{array}$ & $\begin{array}{l}\text { AMBIENT } \\
\text { TEMP }\left({ }^{\circ} \mathrm{F}\right. \\
\end{array}$ & \begin{tabular}{|l|} 
GAS INJECT \\
TEMP ("F) \\
\end{tabular} & \begin{tabular}{|l|} 
MAIN DUCT \\
TEMP ('F) \\
\end{tabular} & \begin{tabular}{|l|} 
WEC CELLL \\
TEMP $\left({ }^{\circ} \mathrm{F}\right)$ \\
\end{tabular} & $\begin{array}{l}\text { WEC CELL } \\
\text { (VOLTS) }\end{array}$ \\
\hline 610.02002 & 3.75550013 & 14.7562313 & 0.974768 & 14.6325 & 2264 & 2010.0557 & 70.4 & 69.5 & 69 & 69.6 & 0.000566 \\
\hline 615.02002 & 3.76425391 & 14.7599794 & 0.97664432 & 14.634 & 2263.5 & 2007.5726 & 70.4 & 69.5 & 69 & 69.6 & 0.000567 \\
\hline 620.03 & 3.7561254 & 14.756981 & 0.97539344 & 14.6325 & 2253.25 & 2004.0963 & 70.4 & 69.5 & 69.1 & 69.6 & 0.000565 \\
\hline 625.02002 & 3.7498727 & 14.756981 & 0.97601888 & 14.63325 & 2253.5 & 2009.559 & 70.5 & 69.5 & 69.2 & 69.6 & 0.000565 \\
\hline 630.04001 & 3.76487918 & 14.756981 & 0.97539344 & 14.6325 & 2263.25 & 2010.8006 & 70.5 & 69.5 & 69.1 & 69.6 & 0.000567 \\
\hline 635.02002 & 3.74799689 & 14.7562313 & 0.97414256 & 14.63175 & 2263.75 & 2008.0692 & 70.4 & 69.5 & 69.1 & 69.6 & 0.000567 \\
\hline 640.02002 & 3.74487054 & 14.7554817 & 0.974768 & 14.634 & 261 & 2010.0557 & 70.4 & 69.5 & 69.1 & 69.5 & 0.000567 \\
\hline 645.05002 & 3.75550013 & 14.756981 & 0.974768 & 14.53325 & 2263.5 & 2010.0557 & 70.4 & 69.5 & 69 & 69.6 & 0.000567 \\
\hline 650.04001 & 3.74674635 & 14.756981 & 0.97539344 & 14.6325 & 2263.5 & 2007.3243 & 70.3 & 69.5 & 69.1 & 69.6 & 0.000566 \\
\hline 655.02002 & 3.75174851 & 14.7584802 & 0.974768 & 14.63325 & 2263.5 & 2009.0624 & 70.3 & 69.5 & 69.2 & 69.6 & 0.000567 \\
\hline 660.06 & 3.75987702 & 14.7562313 & 0.97601888 & 14.6325 & 2263.75 & 2013.532 & 70.3 & 69.5 & 69.1 & 69.6 & 0.000568 \\
\hline 665.02002 & 3.76050229 & 14.756981 & 0.974768 & 14.63175 & 2263.25 & 2013.2837 & 70.2 & 69.5 & 69.1 & 69.6 & 0.000567 \\
\hline 670.02002 & 3.74737162 & 14.7577306 & 0.97601888 & 14.63325 & 2264 & 2008.8141 & 70.3 & 69.5 & 69.1 & 69.6 & 0.000567 \\
\hline 675.06 & 3.75800121 & 14.7562313 & 0.97539344 & 14.6325 & 2263.75 & 2000.62 & 70.4 & 69.5 & 69.1 & 69.6 & 0.000567 \\
\hline 680.02002 & 3.74299473 & 14.7562313 & 0.974768 & 14.63325 & 2263.5 & 1999.6267 & 70.3 & 69.6 & 69.1 & 69.5 & 0.000567 \\
\hline 685.02002 & 3.76175283 & 14.7592298 & 0.974768 & 14.63325 & 2263.75 & 1999.875 & 70.3 & 69.5 & 69 & 69.5 & 0.000566 \\
\hline 690.02002 & 3.74549581 & 14.7554817 & 0.97539344 & 14.63325 & 2263.5 & 2008.5658 & 70.3 & 69.5 & 69 & 69.6 & 0.000567 \\
\hline 695.02002 & 3.77238242 & 14.7554817 & 0.97539344 & 14.63325 & 2263.75 & 2011.7938 & 70.3 & 69.5 & 69 & 69.6 & 0.000568 \\
\hline 700.02002 & 3.76175283 & 14.7562313 & 0.97289168 & 14.63325 & 2263.5 & 2002.1098 & 70.3 & 69.5 & 69.1 & 69.6 & 0.000567 \\
\hline 705.03 & 3.75987702 & 14.7577306 & 0.97414256 & 14.634 & 2263.75 & 2001.3649 & 70.3 & 69.5 & 69.1 & 69.6 & 0.000567 \\
\hline 710.05002 & 3.75112324 & 14.7577306 & 0.974768 & 14.6325 & 2264 & 2003.3513 & 70.2 & 69.5 & 69.1 & 69.6 & 0.000567 \\
\hline 715.02002 & 3.7498727 & 14.7562313 & 0.97539344 & 14.63175 & 2263.25 & 2008.0692 & 70.2 & 69.5 & 69.1 & 69.6 & 0.000567 \\
\hline 720.04001 & 3.73986838 & 14.756981 & 0.97414256 & 14.6325 & 2263.25 & 2007.076 & 70,3 & 69.5 & 69.1 & 69.6 & 0.000567 \\
\hline 725.02002 & 3.72611244 & 14.7577306 & 0.97539344 & 14.6325 & 2263.5 & 2002.8547 & 70.3 & 69.5 & 69.1 & 69.6 & 0,000567 \\
\hline 730.02002 & 3.75800121 & 14.756981 & 0.97351712 & 14.63325 & 2263.75 & 2005.8344 & 70.3 & 69.5 & 69.1 & 69.6 & 0.000567 \\
\hline 735.07001 & 3.74487054 & 14.756981 & 0.974768 & 14.6325 & 2263.75 & 2003.848 & 70.3 & 69.5 & 69.1 & 69.5 & 0.000567 \\
\hline 740.02002 & 3.74549581 & 14.7562313 & 0.974768 & 14.63325 & 2263.5 & 2001.3649 & 70.2 & 69.6 & 69 & 69.6 & 0.000568 \\
\hline 745.02002 & 3.74549581 & 14.7562313 & 0.974768 & 14.6325 & 2263.5 & 2003.5997 & 70.2 & 69.5 & 69.1 & 69.6 & 0.000567 \\
\hline 750.04001 & 3.76425391 & 14.7554817 & 0.97414256 & 14.63325 & 2263.5 & 1998.8818 & 70.2 & 69.6 & 69.2 & 69.6 & 0.000567 \\
\hline 755.06 & 3.75049797 & 14.756981 & 0.974768 & 14.634 & 2263.25 & 2003.5997 & 70.3 & 69.5 & 69.1 & 69.6 & 0.000568 \\
\hline 760.02002 & 3.75487486 & 14.756981 & 0.974768 & 14.63325 & 2263.5 & 2000.62 & 70.4 & 69.5 & 69.1 & 69.6 & 0.000568 \\
\hline 765.03 & 3.73799257 & 14.756981 & 0.97539344 & 14.63325 & 2263.5 & 1998.8818 & 70.4 & 69.6 & 69.2 & 69.6 & 0.000569 \\
\hline 770.02002 & 3.74549581 & 14.7554817 & 0.974768 & 14.6325 & 2263.5 & 2001.6132 & 70.4 & 69.6 & 69.2 & 69.6 & 0.000567 \\
\hline 775.03 & 3.76612972 & 14.7562313 & 0.97289168 & 14.6325 & 2263 & 2003.5997 & 70.4 & 69.6 & 6 & 69.6 & 0.000567 \\
\hline 780.02002 & 3.75925175 & 14.7554817 & 0.974768 & 14.63325 & 2263.75 & 2003.3513 & 70.4 & 69.6 & 69.1 & 69.6 & 0.000567 \\
\hline 785.08002 & 3.74737162 & 14.7554817 & 0.974768 & 14.63325 & 2264 & 2004,0963 & 70.4 & 69.6 & 69.1 & 69.6 & 0.000566 \\
\hline 790.02002 & 3.73924311 & 14.7562313 & 0.97414256 & 14.63175 & 2263.75 & 2003.5997 & 70.3 & 69.6 & 69.1 & 69.5 & 0.000567 \\
\hline 795.02002 & 3.74862216 & 14.756981 & 0.97226624 & 14.6325 & 2263.5 & 1993.6673 & 70.4 & 69.6 & 69.1 & 69.6 & 0.000566 \\
\hline 800.06 & 3.75049797 & 14.7562313 & 0.97226624 & 14.63325 & 2263.5 & 1999.875 & 70.5 & 69.6 & 69 & 69.6 & 0.000568 \\
\hline 805.02002 & 3.77175745 & 14.7562313 & 0.97539344 & 14.63325 & 2263.5 & 2002.6064 & 70.4 & 69.6 & 69.1 & 69.6 & 0.000567 \\
\hline 810.02002 & 3.76550445 & 14.7554817 & 0.97351712 & 14.63325 & 2263.5 & 2000.8683 & 70.5 & 69.6 & 69 & 69.5 & 0.000567 \\
\hline 815.07001 & 3.76362864 & 14.7547321 & 0.97289168 & 14.63325 & 2263.25 & 1998.6335 & 70.5 & 69.6 & 69.2 & 69.6 & 0.000567 \\
\hline
\end{tabular}




\begin{tabular}{|c|c|c|c|c|c|c|c|c|c|c|c|}
\hline SECONDS & MAIN DUCT & MAIN DUCT & GAS INJECT & GAS INJECT & NEWPORT & SMC & AMBIENT & GAS INJECT & MAIN DUCT & WEC CELL & WEC CELL \\
\hline & & ABS (PSIA) & DIFF (IN H2O & ABS (PS|A) & (PPM) & $($ PPM) & TEMP ( ${ }^{\circ} \mathrm{F}$ & TEMP ( $\left.{ }^{\circ} \mathrm{F}\right)$ & TEMP $\left({ }^{\circ} \mathrm{F}\right)$ & TEMP ( $\left.{ }^{\circ} \mathrm{F}\right)$ & (VOLTS) \\
\hline 820.02002 & 3.74487054 & 14.7554817 & 0.97226524 & 14,6325 & 2263.5 & 1993.9156 & 70.5 & 69.6 & 69 & 69.6 & 0,000567 \\
\hline 825.02002 & 3.7686308 & 14.7562313 & 0.97351712 & 14.6325 & 2263.25 & 1998,3852 & 70.4 & 69.6 & 69.1 & 69.6 & 0,000568 \\
\hline 830.02002 & 3.74674635 & 14.7554817 & 0.97226624 & 14.63325 & 2263.75 & 2003.3513 & 70.4 & 69.6 & 69 & 69.6 & 0.000568 \\
\hline 835.05002 & 3.74737162 & 14.7547321 & 0.97289168 & 14.63325 & 2263.25 & 1999.3784 & 70.3 & 69.6 & 69.2 & 69.6 & 0.000567 \\
\hline 840.02002 & 3.76425391 & 14.7562313 & 0.97351712 & 14,63325 & 2263.75 & $1988.20 \overline{46}$ & 70.4 & 69.6 & 68.9 & 69,6 & 0.000567 \\
\hline 845.02002 & 3.75112324 & 14.7554817 & 0.97289168 & 14.6325 & 2263.5 & 1986.4664 & 70.3 & 69.6 & 69.2 & 69.6 & 0.000567 \\
\hline 850.02002 & 3.75299905 & 14.7562313 & 0.97351712 & 14.63175 & 2263.75 & 1991.9292 & 70.3 & 69.6 & 69.1 & 69.6 & 0.000566 \\
\hline 855.02002 & 3.7498727 & 14.7554817 & 0.97226624 & 14.63175 & 2263.25 & 1993.9156 & 70.4 & 69.6 & 69.1 & 69.6 & 0.000567 \\
\hline 860.04001 & 3.74549581 & 14.756981 & 0.97351712 & 14.63325 & 2263.5 & 1996.1504 & 70.3 & 69.6 & 69.2 & 69.6 & 0.000566 \\
\hline 865.04001 & 3.75174851 & 14,7562313 & 0.97289168 & 14.63325 & 2263.5 & 2000.1233 & 70.2 & 69.6 & 69 & 69.6 & 0.000566 \\
\hline 870.02002 & 3.73861784 & 14.7554817 & 0.97351712 & 14.63325 & 2263.25 & 1988.7012 & 70.2 & 69.6 & 69.2 & 69.6 & 0.000567 \\
\hline 875.04001 & 3.76112756 & 14.7532329 & 0.97289168 & 14.6325 & 2263.75 & 1988.9495 & 70.1 & 69.6 & 69 & 69.6 & 0.000567 \\
\hline 880.06 & 3.73861784 & 14.7547321 & 0.97289168 & 14.6325 & 2264 & 1996.8953 & 70.1 & 69.6 & 69.1 & 69.6 & 0.000567 \\
\hline 885.02002 & 3.75112324 & 14.7584802 & 0.97289168 & 14.6325 & 2263.5 & 2002.6064 & 70.1 & 69.6 & 69.1 & 69.6 & 0.000567 \\
\hline 890.05002 & 3.74424527 & 14.7554817 & 0.97226624 & 14.634 & 2263.75 & 1998.8818 & 70.2 & 69.6 & 69 & 69.6 & 0.000567 \\
\hline 895.02002 & 3.74487054 & 14.7562313 & 0.97226624 & 14.6325 & 2263.25 & 1997.392 & 70.2 & 69.6 & 69 & 69.6 & 0.000566 \\
\hline 900.02002 & 3.74924743 & 14.7562313 & 0.97226624 & 14.63325 & 2263.75 & 1998.6335 & 70.1 & 69.6 & 68.9 & 69.6 & 0.000567 \\
\hline 905.04001 & 3.75550013 & 14.7547321 & 0.97289168 & 14.6325 & 2263.75 & 2002.1098 & 70.2 & 69.6 & 69.1 & 69.6 & 0.000567 \\
\hline 910.02002 & 3.77300769 & 14.7592298 & 0.97289168 & 14.634 & 2263.5 & 2002.6064 & 70.2 & 69.6 & 68.9 & 69.6 & 0.000567 \\
\hline 915.02002 & 3.76175283 & 14.756981 & 0.97289168 & 14.63325 & 2263.5 & 2003.103 & 70.3 & 69.6 & 68.9 & 69.6 & 0.000566 \\
\hline 920.04001 & 3.7623781 & 14.7554817 & 0.97226624 & 14.6325 & 2263.5 & 2000.1233 & 70.3 & 69.6 & 69 & 69.6 & 0.000566 \\
\hline
\end{tabular}




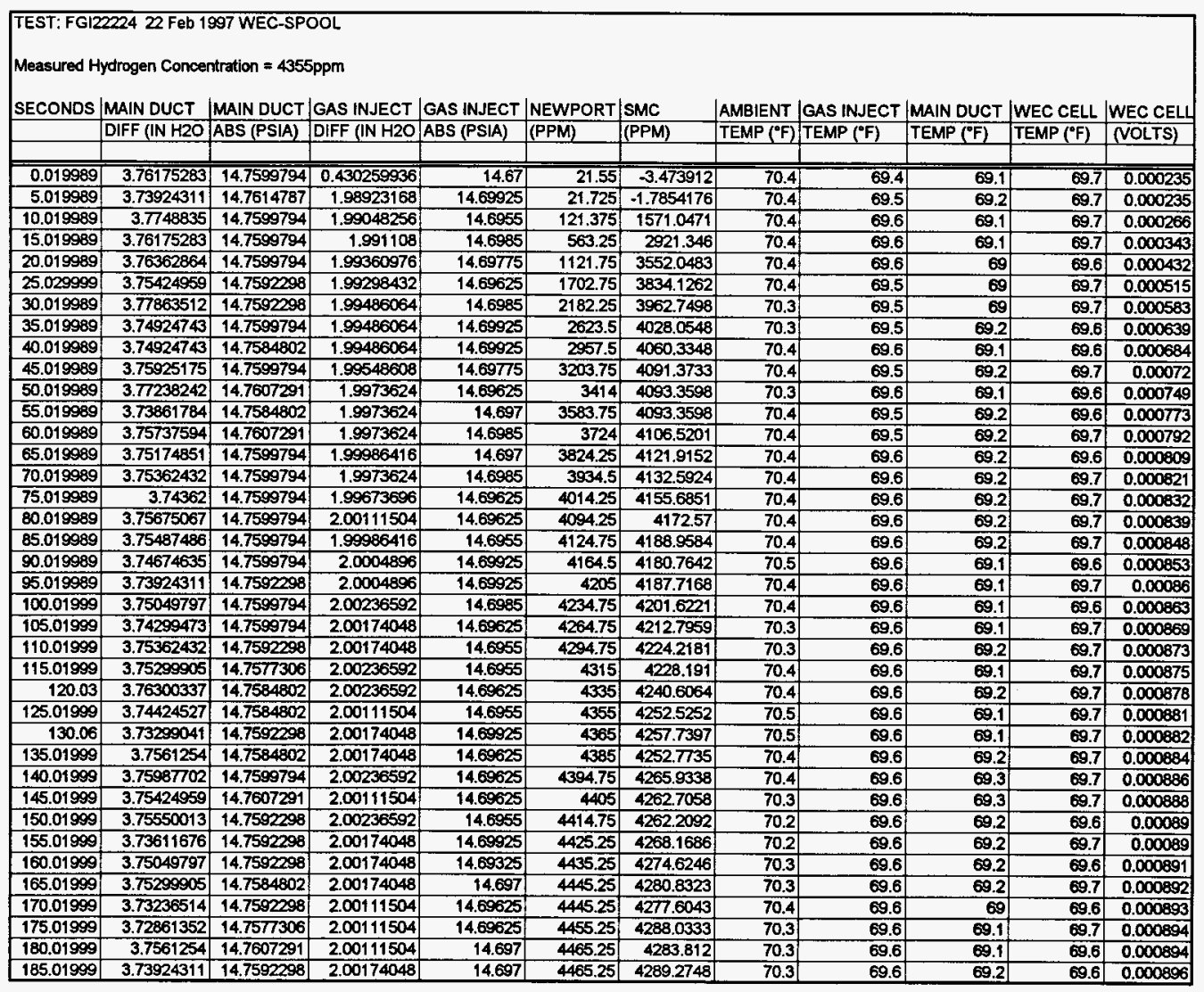




\begin{tabular}{|c|c|c|c|c|c|c|c|c|c|c|c|}
\hline SECONDS & $\begin{array}{l}\text { MAIN DUCT } \\
\text { DIFF (IN H2O }\end{array}$ & \begin{tabular}{|l|} 
MAIN DUCT \\
ABS (PSIA) \\
\end{tabular} & $\begin{array}{l}\text { GAS INJECT } \\
\text { DIFF (IN H2O }\end{array}$ & $\begin{array}{l}\text { |GAS INJECT } \\
\text { ABS (PSIA) }\end{array}$ & \begin{tabular}{|l|} 
NEWPORT \\
(PPM)
\end{tabular} & $\frac{\text { SMC }}{\text { (PPM) }}$ & \begin{tabular}{|l|} 
AMBIENT \\
TEMP ( $\left.{ }^{\circ} \mathrm{F}\right)$
\end{tabular} & $\begin{array}{l}\text { GAS INJECT } \\
\text { TEMP }\left({ }^{\circ} \mathrm{F}\right)\end{array}$ & $\begin{array}{l}\text { MAIN DUCT } \\
\left.\text { TEMP ( }{ }^{\circ} \mathrm{F}\right)\end{array}$ & $\frac{\text { WEC CELL }}{\text { TEMP ("F) }}$ & \begin{tabular}{|l} 
WEC CELL \\
(VOLTS)
\end{tabular} \\
\hline & & & & & & & & & & & \\
\hline 190.01999 & 3.77300769 & 14.7599794 & 200174048 & 14.69625 & 4475.25 & 4283.812 & 70.3 & 69.7 & 69.1 & 69.6 & 0.000897 \\
\hline 195.01999 & 3.74236946 & 14.7584802 & 2.00111504 & 14.6955 & 4475.25 & 4277.356 & 70.4 & 69.7 & 69.1 & 69.7 & 0.000897 \\
\hline 200.01999 & 3.73674203 & 14.7592298 & 2.00474048 & 14.69625 & 4485.25 & 4282.0739 & 70.4 & 69.7 & 69.1 & 69.6 & 0.000898 \\
\hline 205.01999 & 3.74487054 & 14.7599794 & 200236592 & 14,6955 & 4485.25 & 4280.3357 & 70.3 & 69.7 & 69.2 & 69.7 & 0.000898 \\
\hline 210.01999 & 3.79426687 & 14.7592298 & 2.00111504 & 14.697 & 4485 & 4290.5163 & 70.4 & 69.7 & 69.2 & 69.7 & 0.000898 \\
\hline 215.01999 & 3.73861784 & 14.7592298 & 2.00174048 & 14.6955 & 4485.25 & 4290.268 & 70.4 & 69.7 & 69.3 & 69.7 & 0.000899 \\
\hline 220.01999 & 3.74236946 & 14,7599794 & 2.00236592 & 14.69625 & 4495.25 & 4300.4487 & 70.5 & 69.7 & 69.2 & 69.6 & 0.000899 \\
\hline 225.01999 & 3.74924743 & 14.7599794 & 2.00174048 & 14.697 & 4495.25 & 4305.4148 & 70.5 & 69.7 & 69.3 & 69.7 & 0.000901 \\
\hline 230,01999 & 3.74424527 & 14.7592298 & 2.00236592 & 14.69625 & 4502.25 & 4309.3877 & 70.5 & 69.7 & 69.2 & 69.7 & 0.000901 \\
\hline 235.01999 & 3.74924743 & 14.7592298 & 2.00174048 & 14.69625 & 4504.75 & 4306.9047 & 70.6 & 69.7 & 69.2 & 69.7 & 0.000002 \\
\hline 240.01999 & 3.77300769 & 14.7607291 & 2.00174048 & 14.69625 & 4505.25 & 4310.8776 & 70.6 & 69.7 & 69.2 & 69.6 & 0.000002 \\
\hline 245.03998 & 3.74924743 & 14.7584802 & 2.00174048 & 14.697 & 4515.75 & 4321.3065 & 70.5 & 69.7 & 69.3 & 69.7 & 0.000903 \\
\hline 250.01999 & 3.76175283 & 14.7584802 & 2.00111504 & 14.6985 & 4515 & 4326.2727 & 70.5 & 69.7 & 69.1 & 69.6 & 0.000903 \\
\hline 255.01999 & 3.74424527 & 14.7584802 & 1.99986416 & 14.6955 & 4525,75 & 4327.2659 & 70.4 & 69.8 & 69.1 & 69.6 & 0.000904 \\
\hline 260.01999 & 3.74549581 & 14.7592298 & 2.00174048 & 14.69625 & 4525.5 & 4331.7355 & 70.3 & 69.8 & 69.2 & 69.7 & 0.000904 \\
\hline 265.01999 & 3.74924743 & 14.7584802 & 2.00174048 & 14.69625 & 4526 & 4321.0582 & 70.4 & 69.8 & 69.1 & 69.7 & 0.000905 \\
\hline 270.03 & 3.74487054 & 14.7584802 & 200236592 & 14.697 & 4535.5 & 4329.5007 & 70.3 & 69.8 & 69.2 & 69.7 & 0.000004 \\
\hline 275.03998 & 3.72548717 & 14.7592298 & 2.00236592 & 14.697 & 4535.25 & 4333.2253 & 70.3 & 69.8 & 69.2 & 69.7 & 0.000905 \\
\hline 280.01999 & 3.75362432 & 14.7584802 & 2.0004896 & 14.694 & 4535.75 & 4338.4398 & 70.4 & 69.8 & 69.2 & 69.7 & 0.000905 \\
\hline 285.01999 & 3.75049797 & 14.7584802 & 2.00111504 & 14.69625 & 4535.25 & 4338.9364 & 70.4 & 69.8 & 69.2 & 69.7 & 0,000905 \\
\hline 290.01999 & 3.74299473 & 14.7599794 & 2.0004896 & 14.697 & 4536 & 4330.4939 & 70.4 & 69.8 & 69.3 & 69.7 & 0.000005 \\
\hline 295.01999 & 3.74799689 & 14.7607291 & 200174048 & 14.69625 & 4535.5 & 4324.0379 & 70.4 & 69.8 & 69.2 & 69.6 & 0.000904 \\
\hline 300.01999 & 3.75925175 & 14.7584802 & 200111504 & 14.6955 & 4535.5 & 4331.2388 & 70.4 & 69.8 & 69.2 & 69.7 & 0.000905 \\
\hline 305.01999 & 3.74487054 & 14.7607291 & 1.99986416 & 14.60625 & 4535.5 & 4332.7287 & 70.4 & 69.8 & 69.2 & 69.7 & 0.000905 \\
\hline 310.01999 & 3.75675067 & 14.7607291 & 2.00174048 & 14.69625 & 4535.5 & 4341.1712 & 70.5 & 69.9 & 69.2 & 69.7 & 0.000906 \\
\hline 315.01999 & 3.74924743 & 14.7599794 & 2.00174048 & 14.697 & 4536 & 4341.4195 & 70.6 & 69.8 & 69.2 & 69.7 & 0.000905 \\
\hline 320.01999 & 3.75737594 & 14.7584802 & 2.0004896 & 14.69625 & 4545.25 & 4327.0176 & 70.6 & 69.8 & 69.2 & 69.7 & 0.000905 \\
\hline 325.01999 & 3.74737162 & 14.7599794 & 2.0004896 & 14.697 & 4545.5 & 4335.4601 & 70.5 & 69.9 & 69.3 & 69.7 & 0.000906 \\
\hline 330.01999 & 3.75675067 & 14.7584802 & 200174048 & 14.69625 & 4545.25 & 4324.7828 & 70.6 & 69.8 & 69.3 & 69.7 & 0.000907 \\
\hline 335.01999 & 3.75987702 & 14.7592298 & 1.99798784 & 14.697 & 4545.5 & 4338.9364 & 70.7 & 69.9 & 69.3 & 69.7 & 0.000906 \\
\hline 340.01999 & 3.74424527 & 14.7607291 & 1.99923872 & 14.69625 & 4545.25 & 4334.2185 & 70.7 & 69.9 & 69.3 & 69.8 & 0.000907 \\
\hline 345.01999 & 3.75112324 & 14.7607291 & 2.00111504 & 14.69625 & 4555.5 & 4338.6881 & 70.6 & 69.9 & 69.3 & 69.7 & 0.000907 \\
\hline 350.01999 & 3.76550445 & 14.7607291 & 2.00111504 & 14.697 & 4555.5 & 4350.1103 & 70.6 & 69.9 & 69.3 & 69.7 & 0.000907 \\
\hline 355.01999 & 3.73799257 & 14.7592298 & 1.9973624 & 14.697 & 4554.75 & 4341.4195 & 70.6 & 69.9 & 69.3 & 69.7 & 0.000908 \\
\hline 360.01999 & 3.74487054 & 14.7592298 & 1.9973624 & 14.69625 & 4555.5 & 4330.4939 & 70.6 & 69.9 & 69.2 & 69.7 & 0.000908 \\
\hline 365.01999 & 3.73986838 & 14.7599794 & 1.99923872 & 14.6955 & 4565.75 & 4321.0582 & 70.6 & 69.9 & 69.2 & 69.7 & 0.000009 \\
\hline 370.01999 & 3.73674203 & 14.7607291 & 1.99861328 & 14.69625 & 4565 & 4334.9635 & 70.6 & 69.9 & 69.2 & 69.7 & 0.000909 \\
\hline 375.01999 & 3.74549581 & 14.7607291 & 1.99986416 & 14.6955 & 4566 & 4342.9093 & 70.6 & 69.9 & 69.2 & 69.7 & 0.000909 \\
\hline 380.01999 & 3.74862216 & 14.7607291 & 1.99923872 & 14.69325 & 4565.25 & 4343.1576 & 70.6 & 70 & 69.3 & 69.7 & 0.000909 \\
\hline 385.01999 & 3.73861784 & 14.7599794 & 1.9973624 & 14.694 & 4565 & 4341.6678 & 70.6 & 70 & 69.3 & 69.7 & 0.00091 \\
\hline 390.06 & 3.74362 & 14.7599794 & 1.99861328 & 14.697 & 4565.75 & 4328.5075 & 70.6 & 69.9 & 69.2 & 69.7 & 0.000009 \\
\hline 395.01999 & 3.74799689 & 14.7599794 & 1.99923872 & 14.697 & 4565.5 & 4324.7828 & 70.6 & 69.9 & 69.2 & 69.7 & 0.000909 \\
\hline
\end{tabular}




\begin{tabular}{|c|c|c|c|c|c|c|c|c|c|c|c|}
\hline SECONDS & MAIN DUCT & MAIN DUCT & GAS INJECT & GAS INJECT & NEWPORT & SMC & AMBIENT & GAS INJECT & MAIN DUCT & WEC CELL & WEC CELU \\
\hline & DIFF (IN H2O & ABS (PS|A) & DIFF (IN H2O & ABS (PS|A) & (PPM) & (PPM) & TEMP ( $\left.{ }^{\circ} \mathrm{F}\right)$ & TEMP ( $\left.{ }^{\circ} \mathrm{F}\right)$ & TEMP $\left({ }^{\circ} \mathrm{F}\right)$ & TEMP ('F) & (NOLTS) \\
\hline 400,01999 & 3.74362 & 14.7607291 & 1.99923872 & 14.69625 & 4565.5 & 4337.4465 & 70.5 & 70 & 69.3 & 69.7 & 0.000909 \\
\hline 405.01999 & 3.74737162 & 14.7592298 & 1.99673696 & 14.697 & 4565.5 & 4337.4465 & 70.5 & 70 & 69.4 & 69.7 & 0.00091 \\
\hline 410.01999 & 3.75737594 & 14.7614787 & 1.9973624 & 14.69625 & 4565.75 & 4342.1644 & 70.5 & 70 & 69.3 & 69.7 & 0.000909 \\
\hline 415,01999 & 3.74612108 & 14.7607291 & 1.99986416 & 14.6955 & 4565.5 & 4346.1373 & 70.5 & 70 & 69.3 & 69.7 & 0.000908 \\
\hline 420.01999 & 3.77425823 & 14.7592298 & 1.99673696 & 14.69625 & 4565.5 & 4338.4398 & 70.5 & 70 & 69.3 & 69.7 & 0.000909 \\
\hline 425.09 & 3.74612108 & 14.7607291 & 1.9973624 & 14.6955 & 4565.5 & 4342.4127 & 70.4 & 70 & 69.2 & 69.7 & 0.000909 \\
\hline 430.01999 & 3.75487486 & 14.7607291 & 1.99923872 & 14.69325 & 4565.5 & 4348.8687 & 70.5 & 70 & 69.2 & 69.7 & 0.000909 \\
\hline 435.06998 & 3.74487054 & 14.7599794 & 1.9973624 & 14.69775 & 4565.25 & 4350.6069 & 70.5 & 70 & 69.2 & 69.7 & 0.000909 \\
\hline 440.01999 & 3.75362432 & 14.7599794 & 1.99673696 & 14.69625 & 4565.25 & 4335.2118 & 70.5 & 70 & 69.4 & 69.7 & 0.000909 \\
\hline 445.01999 & 3.74549581 & 14.7599794 & 1.99798784 & 14.694 & 4565.25 & 4336.7016 & 70.5 & 70 & 69.3 & 69.7 & 0.000909 \\
\hline 450.01999 & 3.74737162 & 14.756981 & 1.9973624 & 14.694 & 4565.5 & 4345.3924 & 70.6 & 70 & 69.3 & 69.7 & 0.00091 \\
\hline 455.01999 & 3.80177011 & 14.7584802 & 1.99673696 & 14.69625 & 4565.5 & 4349.6136 & 70.7 & 70 & 69.3 & 69.7 & 0.00091 \\
\hline 460.01999 & 3.749224743 & 14.7584802 & 1.99986416 & 14.694 & 4565.5 & 4352.8416 & 70.7 & 70 & 69.3 & 69.7 & 0.00091 \\
\hline 465.01999 & 3.751112324 & 14.7592298 & 1.9973624 & 14.69325 & 4575.75 & 4356.318 & 70.7 & 70.1 & 69.2 & 69.7 & 0.000911 \\
\hline 470.01999 & 3.74549581 & 14.7599794 & 1.99673696 & 14.697 & 4575.5 & 4350.6069 & 70.7 & 70.1 & 69.2 & 69.7 & 0.00091 \\
\hline 475.01999 & 3.73299041 & 14.7599794 & 1.99673696 & 14.69475 & 4575.75 & 4351.3518 & 70.7 & 70.1 & 69.2 & 69.7 & 0.00091 \\
\hline 480.01999 & 3.74549581 & 14.7599794 & 1.99673696 & 14.697 & 4576 & 4334.2185 & 70.7 & 70 & 69.2 & 69.7 & 0.000911 \\
\hline 485.01999 & 3.75487486 & 14.7599794 & 1.9973624 & 14.697 & 4575.5 & 4331.2388 & 70.6 & 70.1 & 69.3 & 69.7 & 0.00091 \\
\hline 490.01999 & 3.74924743 & 14.7614787 & 1.99611152 & 14.69775 & 4575.25 & 4328.2591 & 70.7 & 70.1 & 69.3 & 69.7 & 0.000911 \\
\hline 495.01999 & 3.74487054 & 14.7599794 & 1.99798784 & 14.697 & 4575.5 & 4346.1373 & 70.7 & 70.1 & 69.4 & 69.7 & 0.000911 \\
\hline 500.01999 & 3.73861784 & 14.7592298 & 1.9973624 & 14,69625 & 4575.25 & 4340.9229 & 70.7 & 70.1 & 69.4 & 69.7 & 0.000912 \\
\hline 505.01999 & 3.75550013 & 14.7614787 & 1.99673696 & 14.69475 & 4575.5 & 4335.2118 & 70.7 & 70.1 & 69.3 & 69.7 & 0.000911 \\
\hline 510.01999 & 3.75174851 & 14.7607291 & 1.99798784 & 14.697 & 4576 & 4336.4533 & 70.6 & 70.1 & 69.3 & 69.7 & 0.000911 \\
\hline 515.01999 & 3.76050229 & 14.7592298 & 1.9973624 & 14.69625 & 4575.75 & 4326.0244 & 70.6 & 70.1 & 69.3 & 69.7 & 0.000912 \\
\hline 520.01999 & 3.74924743 & 14.7607291 & 1.99673696 & 14.69475 & 4576 & 4338.9364 & 70.6 & 70.1 & 69.3 & 69.7 & 0.000912 \\
\hline 525.01999 & 3.7623781 & 14.7599794 & 1.99673696 & 14.69775 & 4575.75 & 4340.4262 & 70.5 & 70.1 & 69.1 & 69.7 & 0.000912 \\
\hline 530.01999 & 3.72673771 & 14.7599794 & 1.99673696 & 14.69775 & 4575.75 & 4340.4262 & 70.5 & 70.1 & 6.1 & 69.7 & 0.000912 \\
\hline 535.01999 & 3.74862216 & 14.7614787 & 1.99673696 & 14.69475 & 4577.25 & 4334.7152 & 70.5 & 70.1 & 69.1 & 69.7 & 0.000912 \\
\hline 540.01999 & 3.73236514 & 14.7614787 & 1.99673696 & 14.697 & 4585.75 & 4346.3856 & 70.5 & 70.1 & 69.2 & 69.7 & 0.000912 \\
\hline 545.01999 & 3.75550013 & 14.7599794 & 1.9973624 & 14.697 & 4585.75 & 4337.4465 & 70.5 & 70.1 & 69.2 & 69.7 & 0.000912 \\
\hline 550.06998 & 3.78051093 & 14.7607291 & 1.9973624 & 14.69475 & 4585.75 & 4339.6813 & 70.5 & 70.1 & 69.2 & 69.7 & 0.000913 \\
\hline 555.01999 & 3.7686308 & 14.7607291 & 1.99673696 & 14.69325 & 4585.75 & 4345.6407 & 70.5 & 70.1 & 69.2 & 69.7 & 0.000913 \\
\hline 560.03 & 3.74487054 & 14.7607291 & 1.9973624 & 14.694 & 4586 & 4336.205 & 70.6 & 70.1 & 69.4 & 69.7 & 0.000913 \\
\hline 565.01999 & 3.74549581 & 14.7607291 & 1.9973624 & 14.69625 & 4586 & 4345.889 & 70.5 & 70.1 & 69.3 & 69.8 & 0.000913 \\
\hline 570.01999 & 3.74799689 & 14.7577306 & 1.9973624 & 14.694 & 4586 & 4339.433 & 70.5 & 70.1 & 69.3 & 69.7 & 0.000912 \\
\hline 575.04999 & 3.78551309 & 14.7592298 & 1.9973624 & 14.697 & 4586 & 4340.9229 & 70.5 & 70.1 & 69.2 & 69.8 & 0.000913 \\
\hline 580.01999 & 3.74487054 & 14.7599794 & 1.9973624 & 14.694 & 4585.75 & 4350.3586 & 70.5 & 70.1 & 69.1 & 69.7 & 0.000913 \\
\hline 585.01999 & 3.74049365 & 14.75922298 & 1,99673696 & 14.69625 & 4585.5 & 4343.4059 & 70.5 & 70.1 & 69.1 & 69.7 & 0.000912 \\
\hline 590.01999 & 3.74862216 & 14.7584802 & 1.99798784 & 14.694 & 4586 & 4348.3721 & 70.5 & 70.1 & 69.3 & 69.7 & 0.000911 \\
\hline 595.03998 & 3.77300769 & 14.7592298 & 1.99673696 & 14.697 & 4585.25 & 4334.9635 & 70.5 & 70.1 & 69.2 & 69.7 & 0.000911 \\
\hline 600.01999 & 3.73799257 & 14.7599794 & 1.99673696 & 14.69625 & 4586 & 4330.7422 & 70.5 & 70.2 & 69.4 & 69.8 & 0.000912 \\
\hline 605.01999 & 3.75174851 & 14.7592298 & 1.9973624 & 14.697 & 4585.75 & 4329.9973 & 70.5 & 70.2 & 69.2 & 69.7 & 0.000912 \\
\hline
\end{tabular}




\begin{tabular}{|c|c|c|c|c|c|c|c|c|c|c|c|}
\hline SECONDS & \begin{tabular}{|l|} 
MAIN DUCT \\
DIFF (IN H2O \\
\end{tabular} & \begin{tabular}{|l|} 
MAIN DUCT \\
ABS (PSIA)
\end{tabular} & \begin{tabular}{|l|} 
GAS INJECT \\
DIFF (IN H2O
\end{tabular} & \begin{tabular}{|l|} 
GAS INJECT \\
ABS (PSIA)
\end{tabular} & \begin{tabular}{|l|} 
NEWPORT \\
(PPM)
\end{tabular} & $\begin{array}{l}\text { SMC } \\
\text { (PPM) }\end{array}$ & \begin{tabular}{|l|} 
AMBIENT \\
TEMP $\left({ }^{\circ} \mathrm{F}\right)$ \\
\end{tabular} & \begin{tabular}{|l|} 
GAS INJECT \\
TEMP $\left({ }^{\circ} \mathrm{F}\right)$ \\
\end{tabular} & \begin{tabular}{|l|} 
MAIN DUCT \\
TEMP $\left({ }^{\circ} \mathrm{F}\right)$
\end{tabular} & \begin{tabular}{|l|} 
WEC CELL \\
TEMP $\left({ }^{\circ} \mathrm{F}\right)$ \\
\end{tabular} & \begin{tabular}{|l|} 
WEC CELL \\
(VOLTS
\end{tabular} \\
\hline & & & & & & & & & & & \\
\hline 610.06 & 3.73299041 & 14.7592298 & 1.99673696 & 14.697 & 4585.75 & 4336.205 & 70.4 & 70.2 & 69.3 & 69.7 & 0.000912 \\
\hline 615.01999 & 3.74111892 & 14.7577306 & 1.99673696 & 14.697 & 4585.75 & 4331.4872 & 70.2 & 70.2 & 69.1 & 69.7 & 0.000942 \\
\hline 620.01999 & 3.76612972 & 14.7592298 & 1.99673696 & 14.697 & 4586 & 4324.7828 & 70.2 & 70.2 & 69.1 & 69.8 & 0.000911 \\
\hline 625.04999 & 3.74487054 & 14.7584802 & 1.99673696 & 14.6955 & 4585.5 & 4313.3607 & 70.3 & 70.2 & 69.2 & 69.8 & 0.000912 \\
\hline 630.01999 & 3.741111892 & 14.7584802 & 1.9973624 & 14.69625 & 4586 & 4321.5548 & 70.4 & 70.2 & 69.2 & 69.8 & 0.000913 \\
\hline 635.01999 & 3.7498727 & 14.7577306 & 1.9973624 & 14.69325 & 4585.75 & 4335.9567 & 70.4 & 70.2 & 69.2 & 69.7 & 0.000912 \\
\hline 640,03998 & 3.74049365 & 14.7584802 & 1.99673696 & 14.69625 & 4595.75 & 4330.2456 & 70.4 & 70.2 & 69.1 & 69.7 & 0.000913 \\
\hline 645.06 & 3.74549581 & 14.7592298 & 1.9973624 & 14.69325 & 4595.25 & 4337.6949 & 70.4 & 70.2 & 69.1 & 69.7 & 0.000913 \\
\hline 650.01999 & 3.74424527 & 14.7599794 & 1.9973624 & 14.69475 & 4595.25 & 4333.2253 & 70.5 & 70.2 & 69.3 & 69.8 & 0.000913 \\
\hline 655.04999 & 3.74924743 & 14,7592298 & 1.9973624 & 14.6955 & 4595.5 & 4337.9432 & 70.5 & 70.2 & 69.2 & 69.8 & 0.000913 \\
\hline 660.01999 & 3.75737594 & 14.7592298 & 1.99673696 & 14.6925 & 4596 & 4348.1238 & 70.5 & 70.2 & 69.1 & 69.7 & 0.000913 \\
\hline 665.01999 & 3.73924311 & 14.7599794 & 1.9973624 & 14.694 & 4585.75 & 4338.6881 & 70.5 & 70.2 & 69.1 & 69.7 & 0.000912 \\
\hline 670.01999 & 3.76550445 & 14.7584802 & 1.99673696 & 14.69625 & 4585.5 & 4341.9161 & 70.5 & 70.2 & 69.1 & 69.8 & 0.000913 \\
\hline 675.01999 & 3.74299473 & 14.7584802 & 1.99673696 & 14.69325 & 4585.75 & 4340.4262 & 70.5 & 70.2 & 69.1 & 69.8 & 0.000913 \\
\hline 680.01999 & 3.75800121 & 14.7599794 & 1.9973624 & 14.694 & 4585.25 & 4338.9364 & 70.6 & 70.2 & 69.1 & 69.8 & 0.000913 \\
\hline 685.06998 & 3.77863512 & 14.75922298 & 1.99673696 & 14.694 & 4585.75 & 4345.1441 & 70.5 & 70.2 & 69.2 & 69.7 & 0.000913 \\
\hline 690.01999 & 3.74862216 & 14.7599794 & 4.9973624 & 14.697 & 4586 & 4334.2185 & 70.6 & 70.2 & 69.2 & 69.7 & 0.000913 \\
\hline 695.01999 & 3.73611676 & 14.7599794 & 1.9973624 & 14.69625 & 4586 & 4329.9973 & 70.5 & 70.2 & 69.2 & 69.7 & 0.000913 \\
\hline 700.01999 & 3.77238242 & 14.7599794 & 1.99798784 & 14.69325 & 4585.75 & 4335.9567 & 70.5 & 70.2 & 69.1 & 69.7 & 0.000913 \\
\hline 705.01999 & 3.74924743 & 14.7592298 & 1.99673696 & 14.694 & 4586 & 4331.9838 & 70.5 & 70.2 & 69.1 & 69.7 & 0.000914 \\
\hline 710.01999 & 3.74424527 & 14.7599794 & 1.9973624 & 14.69475 & 4595.25 & 4330.2456 & 70.5 & 70.2 & 69.1 & 69.7 & 0.000914 \\
\hline 715.04999 & 3.76175283 & 14.7592298 & 1.9973624 & 14.697 & 4595.5 & 4339.9296 & 70.6 & 70.2 & 69.3 & 69.7 & 0.000914 \\
\hline 720.01999 & 3.7623781 & 14.7592298 & 1.9973624 & 14.694 & 4596 & 4332.2321 & 70.5 & 70.2 & 69.2 & 69.8 & 0.000914 \\
\hline 725.01999 & 3.75112324 & 14.7599794 & 1.9973624 & 14.69775 & 4595.75 & 4329.0041 & 70.5 & 70.2 & 69.2 & 69.8 & 0.000914 \\
\hline 730.03 & 3.73861784 & 94.7592298 & 1.99673696 & 14.69625 & 4595.5 & 4328.5075 & 70.5 & 70.2 & 69.3 & 69.8 & 0.000914 \\
\hline 735.01999 & 3.74612108 & 14.75999794 & 1.99673696 & 14.69325 & 4595.75 & 4325.2795 & 70.5 & 70.2 & 69.3 & 69.8 & 0.000915 \\
\hline 740.01999 & 3.74924743 & 14.7607291 & 1.9973624 & 14.6955 & 4595.25 & 4328.2591 & 70.4 & 70.2 & 69.3 & 69.8 & 0.000915 \\
\hline 745.06 & 3.76925607 & 14.7607291 & 1.9973624 & 14.69325 & 4596 & 4324.0379 & 70.5 & 70.2 & 69.2 & 69.8 & 0.000915 \\
\hline 750.01999 & 3.75550013 & 14.7599794 & 1.9973624 & 14.694 & 4596 & 4317.3336 & 70.5 & 70.2 & 69.2 & 69.8 & 0.000915 \\
\hline 755.01999 & 3.74924743 & 14.7599794 & 1.9973624 & 14.694 & 4604.25 & 4323.0447 & 70.5 & 70.2 & 69.3 & 69.7 & 0.000915 \\
\hline 760.06998 & 3.74924743 & 14.7592298 & 1.99673696 & 14.69325 & 4606 & 4322.2998 & 70.5 & 70.3 & 69.4 & 69.7 & 0.000914 \\
\hline 765.01999 & 3.75424959 & 14.7592298 & 1.99548608 & 14.694 & 4605.75 & 4328.2591 & 70.5 & 70.2 & 69.3 & 69.8 & 0.000915 \\
\hline 770.01999 & 3.75362432 & 14.7592298 & 1.99548608 & 14.69325 & 4605.5 & 4321.8031 & 70.6 & 70.3 & 69.2 & 69.7 & 0.000915 \\
\hline 775.03998 & 3.74174419 & 14.7607291 & 1.99673696 & 14.694 & 4605.5 & 4334.7152 & 70.6 & 70.2 & 69.4 & 69.7 & 0.000916 \\
\hline 780.01999 & 3.74862216 & 14.7592298 & 1.99611152 & 14.69325 & 4605.75 & 4336.4533 & 70.6 & 70.2 & 69.3 & 69.7 & 0.000915 \\
\hline 785.01999 & 3.75049797 & 14.7577306 & 1.99548608 & 14.694 & 4606 & 4336.9499 & 70.5 & 70.3 & 69.3 & 69.8 & 0.000916 \\
\hline 790.01999 & 3.75737594 & 14.7599794 & 1.99548608 & 14.69325 & 4605.75 & 4344.6475 & 70.5 & 70.3 & 69.4 & 69.8 & 0.000916 \\
\hline 796.04999 & 3.76175283 & 14.7607291 & 1.99548608 & 14.6955 & 4605.75 & 4331.7355 & 70.5 & 70.3 & 69.2 & 69.8 & 0.000916 \\
\hline 800.01999 & 3.76175283 & 14.7592298 & 1.99486064 & 14.6925 & 4605.5 & 4319,3201 & 70.5 & 70.3 & 69.3 & 69.7 & 0.000915 \\
\hline 805.01999 & 3.73924311 & 14.7599794 & 1.99611152 & 14.69325 & 4606 & 4332.7287 & 70.5 & 70.3 & 69.2 & 69.7 & 0.000915 \\
\hline 810.01999 & 3.74924743 & 14.7592298 & 1.99486064 & 14.69325 & 4606.25 & 4327.2659 & 70.6 & 70.3 & 69.2 & 69.8 & 0.000914 \\
\hline 815.01999 & 3.7498727 & 14.7592298 & 1.99548608 & 14.69325 & 4606 & 4316.0921 & 70.6 & 70.3 & 69.2 & 69.7 & 0.000914 \\
\hline
\end{tabular}




\begin{tabular}{|c|c|c|c|c|c|c|c|c|c|c|c|}
\hline SECONDS & \begin{tabular}{|l|} 
MAIN DUCT \\
DIFF
\end{tabular} & \begin{tabular}{|l|} 
MAIN DUCT \\
ABS (PSIA)
\end{tabular} & \begin{tabular}{|l|} 
GAS INJECT \\
DIFF (IN H2O
\end{tabular} & \begin{tabular}{|l|} 
GAS INJECT \\
ABS (PSIA)
\end{tabular} & \begin{tabular}{|l|} 
NEWPORT \\
(PPM)
\end{tabular} & \begin{tabular}{|l|} 
SMC \\
(PPM)
\end{tabular} & AMBIENT & \begin{tabular}{|l|} 
GAS INJECT \\
TEMP ("F) \\
\end{tabular} & \begin{tabular}{|l|} 
MAIN DUCT \\
TEMP ( $\left.^{\circ} \mathrm{F}\right)$
\end{tabular} & \begin{tabular}{|l|} 
WEC CELL \\
TEMP $\left({ }^{\circ} \mathrm{F}\right)$
\end{tabular} & \begin{tabular}{|l|} 
WEC CELI \\
NOLTS)
\end{tabular} \\
\hline & & & & & & & & & & & \\
\hline 820.03 & 3.79051525 & 14.7599794 & 1.99548608 & 14.69325 & 4605.75 & 4304.1733 & 70.6 & 70.3 & 69.3 & 69.8 & 0.000915 \\
\hline 825.01999 & 3.75675067 & 14.7592298 & 1.99548608 & 14.69325 & 4605.75 & 4309.3877 & 70.6 & 70.3 & 69.3 & 69.8 & 0.000915 \\
\hline 830.01999 & 3.73861784 & 14.7607291 & 1.99548608 & 14.69325 & 4605.5 & 4320.8099 & 70.4 & 70.3 & 69.3 & 69.8 & 0.000915 \\
\hline 835.06998 & 3.72986406 & 14.7592298 & 1.99548608 & 14.6955 & 4605.75 & 4317.3336 & 70.4 & 70.3 & 69.2 & 69.8 & 0.000916 \\
\hline 840.04999 & 3.7561254 & 14.7599794 & 1.99548608 & 14.6955 & 4605.75 & 4333.2253 & 70.3 & 70.3 & 69.2 & 69.8 & 0.000915 \\
\hline 845.01999 & 3.74487054 & 14.7584802 & 1.99486064 & 14.6955 & 4606 & 4333.2253 & 70.4 & 70.3 & 69 & 69.8 & 0.000916 \\
\hline 850.03 & 3.75424959 & 14.7592298 & 1.99548608 & 14.69325 & 4605.75 & 4338.4398 & 70.3 & 70.3 & 69.2 & 69.7 & 0.000916 \\
\hline 855.01999 & 3.73674203 & 14.7607291 & 1.9942352 & 14.6925 & 4605.75 & 4319.5684 & 70.3 & 70.4 & 69.2 & 69.8 & 0.000915 \\
\hline 860.01999 & 3.74549581 & 14.7599794 & 1.9942352 & 14.6955 & 4605.75 & 4320.5616 & 70.3 & 70.3 & 69.2 & 69.7 & 0.000915 \\
\hline 865.03 & 3.74111892 & 14.7607291 & 1.9942352 & 14.69625 & 4605.75 & 4334.4668 & 70.3 & 70.4 & 69.1 & 69.8 & 0.000915 \\
\hline 870.01999 & 3.76425391 & 14.7599794 & 1.99486064 & 14.69325 & 4605.75 & 4324.5345 & 70.2 & 70.3 & 69 & 69.7 & 0.000915 \\
\hline 875.01999 & 3.74487054 & 14.7599794 & 1.99486064 & 14.6955 & 4605.75 & 4311.1259 & 70.2 & 70.4 & 69.1 & 69.7 & 0.000915 \\
\hline 880.01999 & 3.732990041 & 14.7607291 & 1.99548608 & 14.69325 & 4605.75 & 4304.6699 & 70.3 & 70.4 & 69.2 & 69.8 & 0.000915 \\
\hline 885.01999 & 3.75862648 & 14.7599794 & 1.9942352 & 14.69325 & 4605.75 & 4318.0785 & 70.3 & 70.3 & 69.2 & 69.7 & 0.000915 \\
\hline 890.01999 & 3.7748835 & 14.7592298 & 1,99548608 & 14.69625 & 4605.75 & 4313.609 & 70.3 & 70.3 & 69.2 & 69.8 & 0.000915 \\
\hline 895.03 & 3.73924311 & 14.7607291 & 1.9942352 & 14.69625 & 4605.75 & 4322.0514 & 70.3 & 70.4 & 69.2 & 69.7 & 0.000915 \\
\hline 900.01999 & 3.75800121 & 14.7599794 & 1.99486064 & 14.694 & 4605.5 & 4335.2118 & 70.3 & 70.4 & 69.1 & 69.7 & 0.000915 \\
\hline 905.01999 & 3.74487054 & 14.7584802 & 1.99486064 & 14.69625 & 4605.75 & 4327.5142 & 70.3 & 70.4 & 69.2 & 69.7 & 0.000915 \\
\hline 910.06 & 3.75550013 & 14.7592298 & 1.99486064 & 14.69325 & 4605.25 & 4321.5548 & 70.4 & 70.4 & 69.3 & 69.7 & 0.000915 \\
\hline 915.01999 & 3.74487054 & 14.7592298 & 1.99548608 & 14,69625 & 4605.75 & 4322.0514 & 70.4 & 70.4 & 69.2 & 69.7 & 0.000915 \\
\hline 920.01999 & 3.74862216 & 14.7599794 & 1.99548608 & 14.69625 & 4606 & 4325.5278 & 70.4 & 70.3 & 69.2 & 69.8 & 0.000916 \\
\hline 925.03998 & 3.7373673 & 14.7599794 & 1.99548608 & 14.6955 & 4605.75 & 4318.8234 & 70.3 & 70.4 & 69.1 & 69.8 & 0.000916 \\
\hline
\end{tabular}




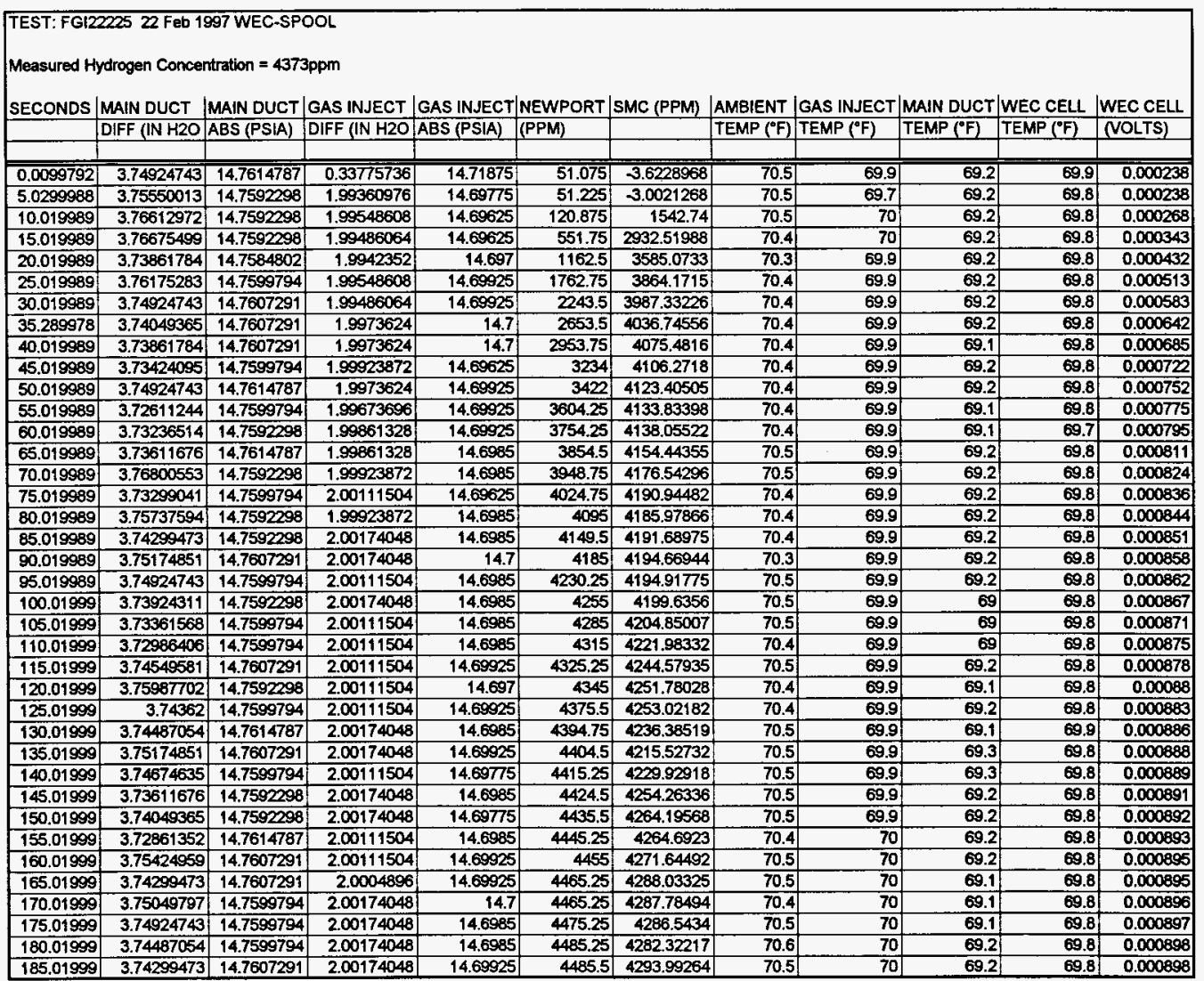




\begin{tabular}{|c|c|c|c|c|c|c|c|c|c|c|c|}
\hline SECONDS & MAIN DUCT & MAIN DUCT & GAS INJECT & GAS INJECT & NEWPORT & SMC (PPM) & AMBIENT & GAS INJECT & MAIN DUCT & WEC CELL & WEC CELL \\
\hline & DIFF (IN H2O & ABS (PSIA) & DIFF (IN H2O & ABS (PSIA) & (FPM) & & TEMP $\left({ }^{\circ} \mathrm{F}\right)$ & TEMP $\left({ }^{\circ} \mathrm{F}\right)$ & TEMP $\left({ }^{\circ} \mathrm{F}\right)$ & TEMP $\left({ }^{\circ} \mathrm{F}\right)$ & (VOLTS) \\
\hline 190.01999 & 3.73924311 & 14.7599794 & 2.00111504 & 14.69625 & 4485.25 & 4277.60432 & 70.5 & 70 & 69.3 & 69.8 & 0.000897 \\
\hline 195.01999 & 3.75174851 & 14.7599794 & 2.00174048 & 14,69925 & 4495.25 & 4279.83909 & 70.6 & 70 & 69.3 & 69.8 & 0.000899 \\
\hline 200.01999 & 3.73986838 & 14.7599794 & 2.00174048 & 14.69925 & 4495.25 & 4289.77141 & 70.6 & 70 & 69.3 & 69.8 & 0.0009 \\
\hline 205.01999 & 3.74612108 & 14.7629779 & 2.00111504 & 14,6985 & 4505.25 & 4295.48249 & 70.6 & 70 & 69.3 & 69.8 & 0.0009 \\
\hline 210.01999 & 3.73236514 & 14.7614787 & 2.00111504 & 14.6985 & 4505.5 & 4294.73757 & 70.7 & 70 & 69.3 & 69.8 & 0.000901 \\
\hline 215.01999 & 3.78613836 & 14.7599794 & 2.00174048 & 14.69625 & 4515.25 & 4290.26802 & 70.5 & 70 & 69.3 & 69.8 & 0.000903 \\
\hline 220.01999 & 3.73986838 & 14.7599794 & 2.00174048 & 14.69925 & 4515.25 & 4289.77141 & 70.5 & 70.1 & 69.3 & 69.8 & 0.000903 \\
\hline 225.01999 & 3.72798825 & 14.7592298 & 2.00111504 & 14.6985 & 4526 & 4273.8797 & 70.5 & 70 & 69.2 & 69.7 & 0.000903 \\
\hline 230.01999 & 3.76050229 & 14.7599794 & 2.00236592 & 14.69925 & 4525.25 & 4285.05356 & 70.5 & 70 & 69.2 & 69.7 & 0.000904 \\
\hline 235.01999 & 3.74487054 & 14.7584802 & 2.00111504 & 14.69925 & 4525.5 & 4292.7511 & 70.5 & 70.1 & 69.2 & 69.7 & 0.000904 \\
\hline 240.01999 & 3.72986406 & 14.7599794 & 2.00174048 & 14.6985 & 4535.5 & 4289.5231 & 70.5 & 70.1 & 69.2 & 69.8 & 0.000905 \\
\hline 245.01990 & 3.76800553 & 14.7607291 & 2.00111504 & 14.69625 & 4535.5 & 4289.02648 & 70.5 & 70.1 & 69.2 & 69.8 & 0.000905 \\
\hline 250.01999 & 3.75112324 & 14.7584802 & 2.00174048 & 14.69625 & 4535.25 & 4299.45542 & 70.5 & 70 & 69.3 & 69.8 & 0.000906 \\
\hline 255.01999 & 3.7623781 & 14.7584802 & 2.00174048 & 14.697 & 4545.5 & 4299.95204 & 70.5 & 70 & 69.3 & 69.8 & 0.000906 \\
\hline 260.01999 & 3.77926039 & 14.7577306 & 2.00174048 & 14.69625 & 4545.75 & 4283.06709 & 70.5 & 70.1 & 69.4 & 69.8 & 0.000907 \\
\hline 265.01999 & 3.75174851 & 14.7584802 & 2.00174048 & 14.5985 & 4554.75 & 4288.28156 & 70.5 & 70.1 & 69.4 & 69.8 & 0.000909 \\
\hline 270.01999 & 3.75174851 & 14.7599794 & 2.0004896 & 14.69925 & 4555.5 & 4288.77818 & 70.4 & 70.1 & 69.4 & 69.8 & 0.000908 \\
\hline 275.01999 & 3.73924311 & 14.75922298 & 200236592 & 14.6985 & 4555.75 & 4279.59078 & 70.5 & 70.1 & 69.3 & 69.8 & 0.000908 \\
\hline 280,03 & 3.75174851 & 14.7599794 & 2.00174048 & 14.69625 & 4555 & 4276.61108 & 70.6 & 70.1 & 69.2 & 69.8 & 0.000908 \\
\hline 285.03 & 3.72611244 & 14.7584802 & 1.99986416 & 14.69925 & 4555.25 & 4292.5028 & 70.5 & 70.1 & 69.2 & 69.8 & 0.000909 \\
\hline 290.01999 & 3.75424959 & 14.7607291 & 2.00174048 & 14.69925 & 4555.25 & 4295.7308 & 70.5 & 70.1 & 69.2 & 69.7 & 0.000908 \\
\hline 295.01999 & 3.76175283 & 14.7607291 & 1.99986416 & 14.7 & 4555 & 4300.20034 & 70.4 & 70.1 & 69.2 & 69.8 & 0.000908 \\
\hline 300.01999 & 3.76550445 & 14.7607291 & 2.00174048 & 14.7 & 4563.25 & 4310.38097 & 70.4 & 70.1 & 69.3 & 69.8 & 0.000909 \\
\hline 305.01999 & 3.74924743 & 14.7607291 & 2.00236592 & 14.69925 & 4565.5 & 4299.95204 & 70.4 & 70.1 & 69.2 & 69.8 & 0.000909 \\
\hline 310.01999 & 3.76050229 & 14.7592298 & 1.99923872 & 14.69925 & 4565.5 & 4297.46896 & 70.4 & 70.1 & 69.3 & 69.8 & 0.000908 \\
\hline 315.03 & 3.74737162 & 14.7599794 & 2.00174048 & 14.69925 & 4565.5 & 4288.77818 & 70.3 & 70.1 & 69.3 & 69.8 & 0.00091 \\
\hline 320.01999 & 3.73861784 & 14.7592298 & 2.00111504 & 14.7 & 4565.75 & 4287.53664 & 70.3 & 70.1 & 69.3 & 69.8 & 0.000909 \\
\hline 325.01999 & 3.73299041 & 14.7614787 & 2.0004896 & 14.69625 & 4565 & 4289.5231 & 70.3 & 70.2 & 69.2 & 69.8 & 0.000909 \\
\hline 330.01999 & 3.74111892 & 14.7607291 & 2.00174048 & 14.6985 & 4565.5 & 4296.22742 & 70.4 & 70.1 & 69.3 & 69.8 & 0.000909 \\
\hline 335.01999 & 3.74612108 & 14.7607291 & 1.99923872 & 14.6985 & 4565.25 & 4291.75787 & 70.4 & 70.1 & 69.3 & 69.8 & 0.00091 \\
\hline 340.01999 & 3.73299041 & 14.7592298 & 2.00111504 & 14.69925 & 4565.5 & 4306.40804 & 70.3 & 70.2 & 69.3 & 69.8 & 0.00091 \\
\hline 345.03 & 3.7561254 & 14.7599794 & 2.00111504 & 14.69925 & 4566 & 4307.15297 & 70.4 & 70.2 & 69.1 & 69.7 & 0.00091 \\
\hline 350.01999 & 3,75424959 & 14.7592298 & 2.00174048 & 14.697 & 4575.5 & 4304.9182 & 70.4 & 70.1 & 69.1 & 69.8 & 0.000911 \\
\hline 355.01999 & 3.74299473 & 14.7607291 & 1.99923872 & 14.69625 & 4575.5 & 4303.67666 & 70.5 & 70.1 & 69.2 & 69.8 & 0.000911 \\
\hline 360.01999 & 3.73236514 & 14.7599794 & 1.99861328 & 14.69925 & 4576 & 4296,97234 & 70.5 & 70.1 & 69.2 & 69.8 & 0.000911 \\
\hline 365.01999 & 3.74737162 & 14.7592298 & 1.99673696 & 14.7 & 4575.75 & 4292.00618 & 70.6 & 70.2 & 69.3 & 69.8 & 0.000912 \\
\hline 370.01999 & 3.73799257 & 14.7592298 & 1.99923872 & 14.6985 & 4576 & 4293.74434 & 70.5 & 70.1 & 69.2 & 69.8 & 0.000912 \\
\hline 375.01999 & 3.739866838 & 14.7577306 & 1.99861328 & 14.69925 & 4575.75 & 4302.93173 & 70.6 & 70.2 & 69.3 & 69.8 & 0.000911 \\
\hline 380.01999 & 3.73861784 & 14.7592298 & 1.9973624 & 14.69925 & 4585.25 & 4295.23418 & 70.6 & 70.2 & 69.3 & 69.8 & 0.000912 \\
\hline 385.01999 & 3.7561254 & 14.7592298 & 1.99923872 & 14.6985 & 4585.75 & 4296.97234 & 70.6 & 70.2 & 69,3 & 69.8 & 0,000913 \\
\hline 390.01999 & 3.74424527 & 14.7592298 & 1.99986416 & 14.69925 & 4586 & 4302.93173 & 70.6 & 70.2 & 69.3 & 69.8 & 0.000912 \\
\hline 395.01999 & 3.75987702 & 14.7584802 & 1.99986416 & 14.7 & 4585.75 & 4304.66989 & 70.5 & 70.2 & 69.3 & 69.8 & 0.000913 \\
\hline
\end{tabular}




\begin{tabular}{|c|c|c|c|c|c|c|c|c|c|c|c|}
\hline SECONDS & MAIN DUCT & MAIN DUCT & GAS INJECT & GAS INJECT & NEWPORT & SMC (PPM) & AMBIENT & GAS INJECT & MAIN DUCT & WEC CELL & WEC CELL \\
\hline & DIFF (iN H2O & ABS (PSIA) & DIFF (IN H2O & ABS (PSIA) & (PPM) & & TEMP $\left({ }^{\circ} \mathrm{F}\right)$ & TEMP ( $\left.{ }^{\circ} \mathrm{F}\right)$ & TEMP $\left({ }^{\circ} \mathrm{F}\right)$ & TEMP $\left({ }^{\circ} \mathrm{F}\right)$ & (VOLTS) \\
\hline 40001999 & 373236514 & 14.7592298 & 1.99986416 & 1469925 & 4585.5 & 4301,44188 & 70.6 & 702 & 693 & 698 & 0.000912 \\
\hline 405.01999 & \begin{tabular}{|l|l|}
.75112324 \\
.7512
\end{tabular} & 14.7584802 & 1.9973624 & 14.69625 & 4585.5 & 4298.9588 & 70.5 & 70.2 & 69,3 & 69.8 & 0.000913 \\
\hline 410.01999 & 3.74924743 & 14.7584802 & 1.99673696 & 14.697 & 4585.25 & 4302.68342 & 70.6 & 70.2 & 69.3 & 69.8 & 0.000914 \\
\hline 415.01999 & 3.77550877 & 14.7599794 & 2.0004896 & 14.69925 & 4585.5 & 4297.96557 & 70.5 & 70.2 & 69.3 & 69.8 & 0.000913 \\
\hline 420.01999 & 3.7811362 & 14.7599794 & 1.99861328 & 14.69925 & 4586 & 4293.49603 & 70.5 & 70.2 & 69.3 & 69.8 & 0.000913 \\
\hline 425.01999 & 3.74362 & 14.7592298 & 1.99986416 & 14.6955 & 4585.75 & 4298.46219 & 70.6 & 70.2 & 69.2 & 69.8 & 0.000912 \\
\hline 430.01999 & 3.74737162 & 14.7607291 & 1.99923872 & 14.6985 & 4586 & 4295.7308 & 70.5 & 70.2 & 69.3 & 69.8 & $\overline{0.000912}$ \\
\hline 435.10999 & 3.74549581 & 14.7584802 & 1.99986416 & 14.6985 & 4585.75 & 4311.87082 & 70.6 & 70.2 & 69.3 & 69.8 & 0.000913 \\
\hline 440.01999 & 3.73299041 & 14.7592298 & 1.99986416 & 14.6985 & 4585.25 & 4307.40128 & 70.6 & 70.3 & 69.2 & 69.8 & 0.000913 \\
\hline 445.01999 & 3.76612972 & 14.7607291 & 1.99673696 & 14.69625 & 4585.5 & 4302.68342 & 70.6 & 70.2 & 69.3 & 69.8 & 0.000911 \\
\hline 450.01999 & 3.74924743 & 14.7599794 & 1.9973624 & 14.69925 & 4586 & 4294.98588 & 70.5 & 70.2 & 69.3 & 69.8 & 0.000913 \\
\hline 455.01999 & 3.74549581 & 14.7599794 & 1.99798784 & 14.69925 & 4585.75 & 4287.78494 & 70.5 & 70.2 & 69.3 & 69.8 & 0.000913 \\
\hline 460.01999 & 3.73924311 & 14.7599794 & 1.99798784 & 14.6985 & 4586 & 4295.97911 & 70.5 & 70.2 & 69.2 & 69.8 & 0.000913 \\
\hline 465.01999 & 3.741111892 & 14.7599794 & 1.9973624 & 14.69775 & 4595.5 & 4303.92496 & 70.5 & 70.3 & 69.2 & 69.8 & 0.000913 \\
\hline 470.01999 & 3.73924311 & 14.7599794 & 1.9973624 & 14.69925 & 4585.75 & 4297.96557 & 70.5 & 70.3 & 69.3 & 69.8 & 0.000913 \\
\hline 475.01999 & 3.75800121 & 14.7507291 & 1.9973624 & 14.6985 & 4595.75 & 4306.40804 & 70.5 & 70.3 & 69.3 & 69.8 & 0.000914 \\
\hline 480.01999 & 3.75362432 & 14.7599794 & 1.9973624 & 14.6985 & 4595.75 & 4318.07852 & 70.5 & 70.3 & 69.3 & 69.8 & 0.000913 \\
\hline 485.01999 & 3.74049365 & 14.7584802 & 1.99986416 & 14.69925 & 4596 & 4319.07175 & 70.3 & 70.3 & 69.3 & 69.8 & 0.000914 \\
\hline 490.01999 & 3.74487054 & 14.7592298 & 1.99673696 & 14.6955 & 4595.5 & 4319.07175 & 70.4 & 70.2 & 69.4 & 69.8 & 0.000914 \\
\hline 495.01999 & 3.75737594 & 14.7592298 & 1.99986416 & 14.6985 & 4595.75 & 4322.05145 & 70.4 & 70.3 & 69.3 & 69.8 & 0.000915 \\
\hline 500.01999 & 3.74236946 & 14.7599794 & 1.99986416 & 14.7 & 4595.25 & 4315.84375 & 70.5 & 70.3 & 69.4 & 69.8 & 0.000915 \\
\hline 505.01999 & 3.74862216 & 14.7599794 & 1.99861328 & 14.69625 & 4595.75 & 4323.7896 & 70.5 & 70.3 & 69.3 & 69.7 & 0.000914 \\
\hline 510.01999 & 3.74862216 & 14.7614787 & 1.9973624 & 14.69625 & 4596 & 4319.56837 & 70.5 & 70.3 & 69.3 & 69.8 & 0.000914 \\
\hline 515.01999 & 3.73236514 & 14.7607291 & $1.9986 \div 328$ & 14.6985 & 4605.75 & 4316.58867 & 70.5 & 70.3 & 69.3 & 69.8 & 0.000916 \\
\hline 520.01999 & 3.73361568 & 14.7607291 & 1.9973624 & 14.7 & 4605.5 & 4315.34713 & 70.5 & 70.3 & 69.2 & 69.8 & 0.000915 \\
\hline 525.01999 & 3.75487486 & 14.7599794 & 1.99923872 & 14.6985 & 4595.75 & 4321.30652 & 70.6 & 70.3 & 69.2 & 69.8 & 0.000914 \\
\hline 530.04999 & 3.75112324 & 14.7599794 & 1.99923872 & 14.697 & 4595.75 & 4320.80991 & 70.6 & 70.3 & 69.4 & 69.8 & 0.000915 \\
\hline 535.01999 & 3.7373673 & 14.7607291 & 1.99611152 & 14.6985 & 4595.75 & 4303.42835 & 70.6 & 70.3 & 69.2 & 69.8 & 0.000915 \\
\hline 540.01989 & 3.77175715 & 14.7607291 & 1.99673696 & 14.697 & 4596.25 & 4307.89789 & 70.6 & 70.3 & 69.2 & 69.8 & 0.000915 \\
\hline 545.01999 & 3.78551309 & 14.7592298 & 1.99923872 & 14.69925 & 4605.75 & 4310.38097 & 70.6 & 70.3 & 69.2 & 69.8 & 0.000915 \\
\hline 550.01999 & 3.7498727 & 14.7607291 & 1.99673696 & 14.69925 & 4606 & 4316.83698 & 70.6 & 70.3 & 69.2 & 69.8 & 0.000915 \\
\hline 555.01999 & 3.74487054 & 14.7599794 & 1.9973624 & 14.6985 & 4606 & 4329.9973 & 70.6 & 70.3 & 69.1 & 69.8 & 0.000915 \\
\hline 560.01999 & 3.74862216 & 14.7592298 & 1.99673696 & 14.6985 & 4605.5 & 4322.54806 & 70.6 & 70.3 & 69.3 & 69.8 & 0.000916 \\
\hline 565.01999 & 3.74299473 & 14.7592298 & 1.99673696 & 14.697 & 4605.75 & 4319.56837 & 70.6 & 70.3 & 69.3 & 69.8 & 0.000915 \\
\hline 570.01999 & 3.76800553 & 14.7599794 & 1.99673696 & 14.69925 & 4605.75 & 4321.05822 & 70.6 & 70.3 & 69.3 & 69.8 & 0.000915 \\
\hline 575.01999 & 3.73299041 & 14.7607291 & 1.99798784 & 14.69625 & 4605.75 & 4321.55483 & 70.6 & 70.3 & 69.2 & 69.8 & 0.000915 \\
\hline 580.01999 & 3.75174851 & 14.7599794 & 1.9973624 & 14.697 & 4607.75 & 431211913 & 70.6 & 70.3 & 69.2 & 69.8 & 0.000916 \\
\hline 585.01999 & 3.74487054 & 14.7607291 & 1.9973624 & 14.6985 & 4616 & 4316.83698 & 70.6 & 70.3 & 69.2 & 69.8 & 0.000916 \\
\hline 590.09 & 3.806147 & 14.7599794 & 1.99798784 & 14.697 & 4615.5 & 4319.32006 & 70.6 & 70.4 & 69.1 & 69.8 & 0.000915 \\
\hline 595.09 & 3.739868338 & 14.7592298 & 1.9973624 & 14.6985 & 4616 & 4326.27268 & 70.5 & 70.3 & 69.2 & 69.8 & 0.000916 \\
\hline 600.01999 & 3.74549581 & 14.7599794 & 1.99673696 & 14.6985 & 4606 & 4326.02438 & 70.5 & 70.4 & 69.2 & 69.8 & 0.000915 \\
\hline 605.03 & 3.74487054 & 14.7592298 & 1.99798784 & 14.69625 & 4605.5 & 4337.94316 & 70.5 & 70.3 & 69.3 & 69.8 & 0.000916 \\
\hline
\end{tabular}




\begin{tabular}{|c|c|c|c|c|c|c|c|c|c|c|c|}
\hline SECONDS & \begin{tabular}{|l|} 
MAIN DUCT \\
DIFF (IN H2O \\
\end{tabular} & \begin{tabular}{|l|} 
MAIN DUCT \\
ABS (PS|A)
\end{tabular} & \begin{tabular}{|l|} 
GAS INJECT \\
DIFF (IN H2O \\
\end{tabular} & \begin{tabular}{|l|} 
GAS INJECT \\
ABS (PSIA) \\
\end{tabular} & \begin{tabular}{|l|} 
NEWPORT \\
(PPM)
\end{tabular} & SMC (PPM) & \begin{tabular}{|l|} 
AMBIENT \\
TEMP ( $\left.{ }^{\circ} \mathrm{F}\right)$ \\
\end{tabular} & $\begin{array}{l}\text { GAS INJECT } \\
\left.\text { TEMP ( }{ }^{\circ} \mathrm{F}\right)\end{array}$ & $\begin{array}{l}\text { MAIN DUCT } \\
\text { TEMP }\left({ }^{\circ} \mathrm{F}\right) \\
\end{array}$ & $\begin{array}{l}\text { WEC CELL } \\
\left.\text { TEMP ( }{ }^{\circ} \mathrm{F}\right)\end{array}$ & \begin{tabular}{|l|} 
WEC CELL \\
(VOLTS)
\end{tabular} \\
\hline 610.01999 & 3.74424527 & 14.7607291 & 1.9973624 & 14.6985 & 4606 & 4341.17116 & 70.5 & 70.4 & 69.3 & 69.8 & 0.000915 \\
\hline 615.01999 & 3.74924743 & 14.7599794 & 1.99673696 & 14.697 & 4606 & 4334.71516 & 70.5 & 70.4 & 69.4 & 69.8 & 0.000915 \\
\hline 620.06 & 3.7498727 & 14.75999794 & 1.9973624 & 14.6955 & 4605.75 & 4333.97023 & 70.5 & 70.4 & 69.4 & 69.8 & 0.000916 \\
\hline 625.01999 & 3.7686308 & 14.7607291 & 1,99673696 & 14.69925 & 4605.5 & 4336.205 & 70.5 & 70.3 & 69.2 & 69.8 & 0.000917 \\
\hline 630.04999 & 3.75362432 & 14.7584802 & 1.99673696 & 14.69625 & 4605.75 & 4319.32006 & 70.5 & 70.4 & 69.2 & 69.8 & 0.000916 \\
\hline 635.03 & 3.75487486 & 14.7584802 & 1.9973624 & 14.69625 & 4615.5 & 4319.07175 & 70.5 & 70.4 & 69.3 & 69.8 & 0.000916 \\
\hline 640.01999 & 3.7561254 & 14.7592298 & 1.99673696 & 14.6955 & 4616 & 4333.72192 & 70.5 & 70.4 & 69.3 & 69.8 & 0.000916 \\
\hline 645.01999 & 3.74674635 & 14.7599794 & 1.9973624 & 14.6985 & 4606 & 4336.70162 & 70.5 & 70.4 & 69.3 & 69.8 & 0.000916 \\
\hline 650.06 & 3.75237378 & 14.75922298 & 1.9973624 & 14.69625 & 4615.75 & 4341.66778 & 70.4 & 70.4 & 69.3 & 69.8 & 0.000917 \\
\hline 655.01999 & 3.74924743 & 14.7584802 & 1.9973624 & 14.6985 & 4615.75 & 4347.13056 & 70.5 & 70.4 & 69.2 & 69.8 & 0.000917 \\
\hline 660.01999 & 3.755500013 & 14.7584802 & 1.99798784 & 14.6985 & 4615.5 & 4341.66778 & 70.4 & 70.4 & 69.3 & 69.8 & 0.000917 \\
\hline 665.03998 & 3.74737162 & 14.7599794 & 1.9973624 & 14.6985 & 4615.5 & 4326.52099 & 70.5 & 70.4 & 69.4 & 69.8 & 0.000917 \\
\hline 670.01999 & 3.7811362 & 14.7592298 & 1.99486064 & 14.69925 & 4626 & 4321.55483 & 70.5 & 70.4 & 69.2 & 69.8 & 0.000918 \\
\hline 675.01999 & 3.75550013 & 14.7599794 & 1.99486064 & 14.69325 & 4625.75 & 4325.77607 & 70.5 & 70.4 & 69.2 & 69.8 & 0.000918 \\
\hline 680.01999 & 3.7561254 & 14.75999794 & 1.99486064 & 14.69625 & 4625.25 & 4331.48715 & 70.5 & 70.4 & 69.3 & 69.8 & 0.000918 \\
\hline 685.01999 & 3.74862216 & 14.7599794 & 1.9973624 & 14.69525 & 4625.5 & 4323.04468 & 70.5 & 70.4 & 69.4 & 69.8 & 0.000918 \\
\hline 690.01999 & 3.74111892 & 14.7599794 & 1.9973624 & 14.6985 & 4625.5 & 4330.74223 & 70.5 & 70.4 & 69.4 & 69.8 & 0.000918 \\
\hline 695.03 & 3.74487054 & 14.7607291 & 1.9973624 & 14.69625 & 4625.5 & 4342.4127 & 70.5 & 70.4 & 69.3 & 69.8 & 0.000917 \\
\hline 700.01999 & 3.7561254 & 14.7592298 & 1.99673696 & 14.69625 & 4626 & 4350.35856 & 70.5 & 70.4 & 69.3 & 69.8 & 0,000918 \\
\hline 705.01999 & 3.73924311 & 14.7599794 & 1.9973624 & 14.697 & 4625.75 & 4339.68132 & 70.4 & 70.4 & 69.3 & 69.8 & 0.000918 \\
\hline 710.03998 & 3.73924311 & 14.7592298 & 1.99798784 & 14.694 & 4626 & 4334.46685 & 70.3 & 70.4 & 69.3 & 69.8 & 0.000918 \\
\hline 715.01999 & 3.73924311 & 14.7599794 & 1.99548608 & 14.69625 & 4615.75 & 4346.63394 & 70.3 & 70.4 & 69.3 & 69.8 & 0.000917 \\
\hline 720.01999 & 3.74236946 & 14.7607291 & 1.99673696 & 14.6955 & 4615.75 & 4337.19824 & 70.3 & 70.5 & 69.2 & 69.8 & 0.000917 \\
\hline 725.06 & 3.7561254 & 14.7592298 & 1.99673696 & 14.6985 & 4615.5 & 4335.21177 & 70.3 & 70.4 & 69.3 & 69.8 & 0.000918 \\
\hline 730.01999 & 3.72548717 & 14.7607291 & 1.99486064 & 14.6955 & 4616 & 4339.43301 & 70.3 & 70.4 & 69.2 & 69.8 & 0.000917 \\
\hline 735.01999 & 3.74549581 & 14.7599794 & 1.99486064 & 14.6955 & 4616 & 4352.09672 & 70.3 & 70.4 & 69.3 & 69.8 & 0.000918 \\
\hline 740.03998 & 3.74049365 & 14.7599794 & 1.9973624 & 14.694 & 4615.25 & 4351.10348 & 70.3 & 70.4 & 69.4 & 69.8 & 0.000918 \\
\hline 745.03 & 3.75800121 & 14.7592298 & 1.9973624 & 14.6955 & 4615.75 & 4347.13056 & 70.4 & 70.4 & 69.4 & 69.8 & 0.000917 \\
\hline 750.01999 & 3.75800121 & 14.7592298 & 1.99673696 & 14.69625 & 4615.5 & 4351.35179 & 70.3 & 70.4 & 69.3 & 69.8 & 0.000917 \\
\hline 755.01999 & 3.74487054 & 14.7599794 & 1.99673696 & 14.697 & 4616 & 4340.17793 & 70.4 & 70.4 & 69.2 & 69.8 & 0.000917 \\
\hline 760.01999 & 3.74737162 & 14.7599794 & 1.99798784 & 14.60625 & 4625.75 & 4343.40594 & 70.4 & 70.5 & 69.3 & 69.8 & 0.000918 \\
\hline 765.03 & 3.76988134 & 14.7599794 & 1.99486064 & 14.694 & 4625.5 & 4345.88902 & 70.4 & 70.4 & 69.1 & 69.8 & 0.000918 \\
\hline 770.01999 & 3.74924743 & 14.7599794 & 1.99486064 & 14.697 & 4625.75 & 4340.17793 & 70.4 & 70.4 & 69.3 & 69,8 & 0.000918 \\
\hline 775.01999 & 3.74111892 & 14.7592298 & 1.9942352 & 14.697 & 4625.75 & 4344.15086 & 70.4 & 70.5 & 69.3 & 69.8 & 0.000917 \\
\hline 780.03998 & 3.77238242 & 14.7614787 & 4.99486064 & 1469475 & 4625.75 & 4339.92962 & 70.4 & 70.5 & 69.1 & 69.8 & 0.000918 \\
\hline 785.01999 & 3.73236514 & 14.7599794 & 1.9942352 & 14.697 & 4625.75 & 4333.97023 & 70.4 & 70.4 & 69.1 & 69.8 & 0.000919 \\
\hline 790.01999 & 3.73486622 & 14.7614787 & 1.99486064 & 14.69775 & 4626 & 4336.205 & 70.4 & 70.5 & 69.2 & 69.8 & 0.000918 \\
\hline 755.06 & 3.74111892 & 14.7599794 & 1.99673696 & 14.697 & 4625.75 & 4352.09672 & 70.5 & 70.4 & 69.2 & 69.8 & 0.000918 \\
\hline 800.01999 & 3.74174419 & 14.7592298 & 1.99486064 & 14.69325 & 4625.75 & 4341.91609 & 70.5 & 70.5 & 69.1 & 69.8 & 0.000918 \\
\hline 805.01999 & 3.75925175 & 14.7599794 & 1.9942352 & 14.697 & 4626 & 4345.14409 & 70.5 & 70.5 & 69.1 & 69.8 & 0.000019 \\
\hline 810.04999 & 3.74924743 & 14.7577306 & 1,99548608 & 14.69625 & 4626 & 4337.94316 & 70.4 & 70.4 & 69.1 & 69.8 & 0.000918 \\
\hline 815.01999 & 3.74924743 & 14.7584802 & 1.99673696 & 14.697 & 4625.75 & 4329.25238 & 70.2 & 70.5 & 69.2 & 69.8 & 0.000918 \\
\hline
\end{tabular}




\begin{tabular}{|c|c|c|c|c|c|c|c|c|c|c|c|}
\hline SECONDS & \begin{tabular}{|l|} 
MAIN DUCT \\
DIFF (IN H2O
\end{tabular} & \begin{tabular}{|l|} 
MAIN DUCT \\
ABS (PSIA)
\end{tabular} & \begin{tabular}{|l|} 
GAS INJECT \\
DIFF (IN H2O
\end{tabular} & \begin{tabular}{|l|} 
GAS INJECT \\
ABS (PSIA)
\end{tabular} & \begin{tabular}{|l|} 
NEWPORT \\
(PPM)
\end{tabular} & \begin{tabular}{|l|} 
SMC (PPM) \\
\end{tabular} & \begin{tabular}{|l|} 
AMBIENT \\
TEMP $\left({ }^{\circ} \mathrm{F}\right)$
\end{tabular} & \begin{tabular}{|l|} 
GAS INJECT \\
TEMP ( $\left.{ }^{\circ} F\right)$
\end{tabular} & $\frac{\mid \text { MAIN DUCT }}{\left.\mid \text { TEMP }^{\circ} \mathrm{F}\right)}$ & \begin{tabular}{|l} 
WEC CELL \\
TEMP ( $^{\circ}$ )
\end{tabular} & \begin{tabular}{|l} 
WEC CELL \\
NOLTS)
\end{tabular} \\
\hline & & & & & & & & & & & \\
\hline 820.01999 & 3.73861784 & 14.7599794 & 1.9973624 & 14.697 & 4625.75 & 4346.63394 & 70.1 & 70.5 & 69.3 & 69.8 & 0.000918 \\
\hline 825.03998 & 3.73173987 & 14.7607291 & 1.99673696 & 14.6955 & 4625.75 & 4343.90255 & 70.2 & 70.5 & 69.2 & 69.8 & 0.000918 \\
\hline 830.01999 & 3.73424095 & 14.7592298 & 1.9973624 & 14.69925 & 4625.75 & 4328,50746 & 70.2 & 70.4 & 69.1 & 69.8 & 0.000919 \\
\hline 835.01999 & 3.73361568 & 14.7577306 & 1.9973624 & 14.697 & 4625.75 & 4325.27945 & 70.2 & 70.5 & 69.1 & 69.8 & 0.000919 \\
\hline 840.03998 & 3.72861352 & 14.7577306 & 1.99673696 & 14.69625 & 4626 & 4328.01084 & 70.3 & 70.4 & 69.1 & 69.8 & 0.000918 \\
\hline 845.03 & 3.73299041 & 14.7599794 & 1.99673696 & 14.697 & 4626 & 4342.1644 & 70.4 & 70.5 & 69.1 & 69.8 & 0.000919 \\
\hline 850.01999 & 3.75550013 & 14.7607291 & 1.99673696 & 14.69625 & 4632.25 & 4346.88225 & 70.3 & 70.5 & 69.2 & 69.8 & 0.000919 \\
\hline 855.03998 & 3.75299905 & 14.7614787 & 1.99673696 & 14.697 & 4635.75 & 4333.97023 & 70.3 & 70.5 & 69.3 & 69.8 & 0.00092 \\
\hline 860.01999 & 3.74737162 & 14.7599794 & 1.9973624 & 14.69475 & 4635.25 & 4326.7693 & 70.4 & 70.5 & 69.3 & 69.8 & 0.000919 \\
\hline 865.01999 & 3.74737162 & 14.7599794 & 1.99673696 & 14.697 & 4635.75 & 4330.24561 & 70.4 & 70.5 & 69.3 & 69.9 & 0.00092 \\
\hline 870.03 & 3.74799689 & 14.7599794 & 1.9973624 & 14.69475 & 4636 & 4324.03791 & 70.4 & 70.5 & 69.2 & 69.8 & 0.00092 \\
\hline 875.01999 & 3.76425391 & 14.7599794 & 1.99798784 & 14.694 & 4635.75 & 4338.68808 & 70.5 & 70.5 & 69.2 & 69.8 & 0.00092 \\
\hline 880.01999 & 3.75800121 & 14.7607291 & 1.99548608 & 14.69475 & 4635.75 & 4343.15763 & 70.5 & 70.5 & 69.2 & 69.8 & 0.00092 \\
\hline 885.01999 & 3.74924743 & 14.7599794 & 1.90611152 & 14.69475 & 4635.5 & 4333.47362 & 70.5 & 70.5 & 69.3 & 69.8 & 0.00092 \\
\hline 890.01999 & 3.74549581 & 14.7599794 & 1.99486064 & 14.697 & 4635.5 & 4337.44654 & 70.4 & 70.5 & 69.2 & 69.8 & 0.000919 \\
\hline 895.01999 & 3.74487054 & 14.7599794 & 1.9973624 & 14.69625 & 4635.5 & 4336.70162 & 70.5 & 70.5 & 69.2 & 69.8 & 0.000919 \\
\hline 900.07001 & 3.75424959 & 14.7584802 & 1.99798784 & 14.697 & 4635.75 & 4348.3721 & 70.5 & 70.5 & 69.3 & 69.9 & 0.00092 \\
\hline 905.01999 & 3.74737162 & 14.7592298 & 1.99486064 & 14.697 & 4635.5 & 4338.19147 & 70.6 & 70.5 & 69.2 & 69.8 & 0.000919 \\
\hline 910.01999 & 3.76175283 & 14.7584802 & 1.99673696 & 14.697 & 4636 & 4325.77607 & 70.5 & 70.5 & 69.2 & 69.9 & 0.000921 \\
\hline 915.01999 & 3.75925175 & 14.75999794 & 1.99548608 & 14.697 & 4635.75 & 4334.21854 & 70.5 & 70.5 & 69.2 & 69.8 & 0.000919 \\
\hline 920.01999 & 3.73361568 & 14.7599794 & 1.9942352 & 14.69625 & 4636 & 4329.9973 & 70.5 & 70.5 & 69.2 & 69.8 & 0.00092 \\
\hline 925.01999 & 3.74236946 & 14.7592298 & 1.9942352 & 14.69775 & 4635.75 & 4323.7896 & 70.5 & 70.5 & 69.3 & 69.9 & 0.000921 \\
\hline 930.06 & 3.75550013 & 14.7599794 & 1.99548608 & 14.697 & 4635.5 & 4327.76253 & 70.5 & 70.5 & 69.2 & 69.8 & 0.00092 \\
\hline 935.07001 & 3.75862648 & 14.7599794 & 1.9973624 & 14.69625 & 4635.75 & 4331.98377 & 70.6 & 70.5 & 69.2 & 69.8 & 0.00092 \\
\hline 940.10001 & 3.7686308 & 14.7584802 & 8.6864432 & 16.593 & 4635.75 & 4348.6204 & 70.5 & 70.6 & 69.2 & 69.8 & 0.00092 \\
\hline
\end{tabular}




\begin{tabular}{|c|c|c|c|c|c|c|c|c|c|c|c|}
\hline \multicolumn{12}{|c|}{$\begin{array}{l}\text { TEST: FGI22226 } 22 \text { Feb } 1997 \text { WEC-SPOOL } \\
\text { Measured Hydrogen Concentration }=4377 \mathrm{ppm}\end{array}$} \\
\hline & DIFF (IN H2O) & ABS (PSIA) & DIFF (IN H2O) & ABS (PS|A) & (PPM) & (PPM) & TEMP.(F) & TEMP. (F) & TEMP. (F) & TEMP. (F) & (VOLTS) \\
\hline 0.01001 & 3.768631 & 14.75998 & 0.654543 & 14.7225 & 41.2 & -3.72202 & 70.5 & 70.1 & 69.2 & 69.8 & 0,000238 \\
\hline 5.019989 & 3.748622 & 14.75548 & 1.977974 & 14.69325 & 41.1 & -1.98406 & 70.6 & 70.1 & 69.3 & 69.9 & 0.000239 \\
\hline 10.01999 & 3.773008 & 14.75698 & 1.978599 & 14.69325 & 121.375 & 1655.72 & 70.5 & 70.3 & 69.3 & 69.8 & 0.00027 \\
\hline 15.01999 & 3.733616 & 14.75698 & 1.977974 & 14.69325 & 577.25 & 2999.315 & 70.4 & 70.3 & 69.3 & 69.9 & 0.000347 \\
\hline 20.01999 & 3.752374 & 14.75623 & 1.978599 & 14.694 & 1182 & 3625.299 & 70.4 & 70.3 & 69.3 & 69.8 & 0.000436 \\
\hline 25.01999 & 3.745496 & 14.75698 & 1,977974 & 14.694 & 1762.75 & 3877.58 & 70.5 & 70.2 & 69.2 & 69.8 & 0.000518 \\
\hline 30.01999 & 3.744871 & 14.75848 & 1.977974 & 14.6955 & 2243 & 3973.675 & 70.5 & 70.2 & 69.2 & 69.8 & 0.000586 \\
\hline 35.01999 & 3.762378 & 14.75773 & 1.977974 & 14.6925 & 2623 & 4036.994 & 70.5 & 70.2 & 69.3 & 69.8 & 0.000643 \\
\hline 40.01999 & 3.760502 & 14.75623 & 1.979225 & 14.69325 & 2953.75 & 4074.24 & 70.5 & 70.2 & 69.3 & 69.8 & 0.000689 \\
\hline 45.01999 & 3.762378 & 14.75998 & 1.980476 & 14.69325 & 3269.75 & 4084.917 & 70.5 & 70.2 & 69.3 & 69.8 & 0.000726 \\
\hline 50.01999 & 3.759877 & 14.75623 & 1.981101 & 14.69325 & 3463.5 & 4108.755 & 70.5 & 70.2 & 69.2 & 69.8 & 0.000754 \\
\hline 55.01999 & 3.744871 & 14.75773 & 1.981101 & 14.69325 & 3619.25 & 4131.599 & 70.5 & 70.2 & 69.2 & 69.8 & 0.000779 \\
\hline 60.01999 & 3.749247 & 14.75548 & 1.979225 & 14.69325 & 3754.5 & 4154.444 & 70.6 & 70.2 & 69.3 & 69.8 & 0.000798 \\
\hline 65.01999 & 3.759252 & 14.75698 & 1.97985 & 14.69325 & 3864 & 4198.394 & 70.6 & 70.2 & 69.3 & 69.8 & 0.000813 \\
\hline 70.01999 & 3.752999 & 14.75698 & 1.97985 & 14.694 & 3964.5 & 4194.918 & 70.6 & 70.2 & 69.3 & 69.8 & 0.000826 \\
\hline 75.13998 & 3.74362 & 14.75623 & 1.980476 & 14.69325 & 4044.25 & 4182.006 & 70.6 & 70.2 & 69.3 & 69.8 & 0.000836 \\
\hline 80.01999 & 3.768631 & 14.75998 & 1.97985 & 14.69325 & 4104 & 4193.428 & 70.6 & 70.2 & 69.3 & 69.8 & 0.000845 \\
\hline 85.01999 & 3.73299 & 14.75698 & 1.980476 & 14.69325 & 4154.75 & 4204.602 & 70.5 & 70.2 & 69.3 & 69.8 & 0.000852 \\
\hline 90.01999 & 3.732365 & 14.75623 & 1.982352 & 14.69325 & 4194.75 & 4226.949 & 70.6 & 70.2 & 69.3 & 69.9 & 0.00086 \\
\hline 95.01999 & 3.726112 & 14.75698 & 1.982352 & 14.69625 & 4234.5 & 4229.433 & 70.6 & 70.2 & 69.3 & 69.8 & 0.000864 \\
\hline 100.02 & 3.745496 & 14.75623 & 1.981726 & 14.69325 & 4274.75 & 4234.647 & 70.7 & 70.2 & 69.3 & 69.8 & 0.000869 \\
\hline 105.02 & 3.744871 & 14.75623 & 1.981726 & 14.694 & 4305 & 4240.11 & 70.7 & 70.2 & 69.4 & 69.9 & 0.000873 \\
\hline 110.02 & 3.759252 & 14.75548 & 1.982352 & 14.69325 & 4324.75 & 4251.78 & 70.7 & 70.2 & 69.4 & 69.8 & 0.000876 \\
\hline 115.02 & 3.72111 & 14.75623 & 1.981726 & 14.694 & 4345 & 4257.988 & 70.7 & 70.2 & 69.4 & 69.9 & 0.00088 \\
\hline 120.02 & 3.756125 & 14.75998 & 1.981726 & 14.697 & 4365.25 & 4267.92 & 70.8 & 70.1 & 69.3 & 69.8 & 0.000862 \\
\hline 125.05 & 3.73174 & 14.75773 & 1.982352 & 14.69325 & 4385.25 & 4274.376 & 70.7 & 70.1 & 69.3 & 69.8 & 0.000885 \\
\hline 130.02 & 3.730489 & 14.75773 & 1.981726 & 14.697 & 4395 & 4283.564 & 70.7 & 70.1 & 69.3 & 69.8 & 0.000866 \\
\hline 135.02 & 3.751749 & 14.75623 & 1.981726 & 14.694 & 4415.25 & 4274.376 & 70.7 & 70.1 & 69.3 & 69.7 & 0.000888 \\
\hline 140.02 & 3.742995 & 14.75548 & 1.983603 & 14.69325 & 4424.5 & 4279.591 & 70.7 & 70.1 & 69.3 & 69.8 & 0.00089 \\
\hline 145.02 & 3.762378 & 14.75773 & 1.984228 & 14.69325 & 4435.25 & 4289.523 & 70.7 & 70.1 & 69.3 & 69.7 & 0.000892 \\
\hline
\end{tabular}




\begin{tabular}{|c|c|c|c|c|c|c|c|c|c|c|c|}
\hline SECONDS & MAIN DUCT & MAIN DUCT & GAS INJECT & GAS INJECT & NEWPORT & SMC & AMBIENT & GAS INJECT & MAIN DUCT & WEC CELL & WEC CELL \\
\hline & DIFF (IN H2O) & ABS (PS|A) & DIFF (IN H2O) & ABS (PS|A) & (PPM) & (PPM) & TEMP.(F) & TEMP. (F) & TEMP. (F) & TEMP. (F) & (NOLTS) \\
\hline 0.01001 & 3.768631 & 14.75998 & 0.654543 & 14.7225 & 41.2 & -3.72222 & 70.5 & 70.1 & 69.2 & 69.8 & 0.000238 \\
\hline 150.02 & 3.751749 & 14.75548 & 1.982977 & 14.69325 & 4445 & 4290.516 & 70.7 & 70.1 & 69.3 & 69.8 & 0.000893 \\
\hline 155.02 & 3.739243 & 14.75623 & 1.983603 & 14.694 & 4454.75 & 4287.785 & 70.7 & 70.1 & 69.2 & 69.8 & 0.000694 \\
\hline 160.02 & 3.736742 & 14.75923 & 1.984228 & 14.694 & 4465.25 & 4289.523 & 70.8 & 70.1 & 69.2 & 69.8 & 0.000895 \\
\hline 165.02 & 3.749247 & 14.75623 & 1.984228 & 14.694 & 4475 & 4282.074 & 70.7 & 70.1 & 69.2 & 69.9 & 0.000897 \\
\hline 170.02 & 3.734866 & 14.75623 & 1.984854 & 14.69325 & 4475.25 & 4278.101 & 70.8 & 70,1 & 69.3 & 69.8 & 0.000897 \\
\hline 175.02 & 3.732365 & 14.75623 & 1.984228 & 14.69475 & 4485.25 & 4294.986 & 70.8 & 70.1 & 69.4 & 69.9 & 0.000898 \\
\hline 180.02 & 3.753624 & 14.75623 & 1.984854 & 14.69325 & 4494.5 & 4284.805 & 70.8 & 70.1 & 69.5 & 69.8 & 0.0009 \\
\hline 185.02 & 3.761753 & 14.75473 & 1.984228 & 14.694 & 4495.25 & 4294.241 & 70.8 & 70.1 & 69.4 & 69.8 & 0.0009 \\
\hline 190.02 & 3.750498 & 14.75698 & 1.983603 & 14.694 & 4505.25 & 4303.677 & 70.8 & 70.1 & 69.3 & 69.8 & 0.000901 \\
\hline 195.02 & 3.747997 & 14.75698 & 1.983603 & 14.69325 & 4505.75 & 4306.905 & 70.8 & 70.1 & 69.4 & 69.8 & 0.000901 \\
\hline 200.02 & 3.764879 & 14.75623 & 1.984228 & 14.69325 & 4515.25 & 4313.361 & 70.8 & 70.1 & 69.4 & 69.8 & 0.000903 \\
\hline 205.02 & 3.734241 & 14.75698 & 1.984228 & 14.694 & 4515.75 & 4309.884 & 70.8 & 70.1 & 69.3 & 69.8 & 0.000904 \\
\hline 210.02 & 3.739243 & 14.75623 & 1.983603 & 14.69475 & 4525.5 & 4307.65 & 70.8 & 70.1 & 69.2 & 69.8 & 0.000903 \\
\hline 215.02 & 3.744871 & 14.75698 & 1.984854 & 14.694 & 4525.25 & 4308.146 & 70.8 & 70.1 & 69.3 & 69.8 & 0.000904 \\
\hline 220.02 & 3.761753 & 14.75698 & 1.984228 & 14.694 & 4535.5 & 4305.911 & 70.8 & 70.1 & 69.3 & 69.8 & 0.000905 \\
\hline 225.02 & 3.742369 & 14.75623 & 1.984228 & 14.69325 & 4535.25 & 4310.878 & 70.8 & 70.1 & 69.4 & 69.8 & 0.000907 \\
\hline 230.02 & 3.740494 & 14.75698 & 1.983603 & 14.69325 & 4545.5 & 4315.099 & 70.8 & 70.1 & 69.4 & 69.8 & 0.000905 \\
\hline 235.02 & 3.736742 & 14.75773 & 1.984854 & 14.69325 & 4545.25 & 4318,823 & 70.7 & 70.1 & 69.4 & 69.8 & 0.000907 \\
\hline 240.02 & 3.749247 & 14.75848 & 1.983603 & 14.69325 & 4545 & 4306.408 & 70.8 & 70.1 & 69.3 & 69.8 & 0.000008 \\
\hline 245.02 & 3.756751 & 14.75773 & 1.984228 & 14.694 & 4545.75 & 4309.884 & 70.8 & 70.1 & 69.4 & 69.8 & 0.000007 \\
\hline 250.02 & 3.741119 & 14.75623 & 1.984854 & 14.694 & 4545.5 & 4317.83 & 70.8 & 70.1 & 69.4 & 69.8 & 0.000008 \\
\hline 255.02 & 3.768631 & 14.75698 & 1.983603 & 14.69475 & 4555.5 & 4322.548 & 70.8 & 70.1 & 69.4 & 69.8 & 0.000908 \\
\hline 260.02 & 3.746121 & 14.75773 & 1.984854 & 14.69475 & 4555.5 & 4327.266 & 70.8 & 70.1 & 69.4 & 69.8 & 0.000000 \\
\hline 265.02 & 3.745496 & 14.75773 & 1.984228 & 14.69325 & 4565.5 & 4331.487 & 70.8 & 70.1 & 69.4 & 69.8 & 0.00091 \\
\hline 270.02 & 3.752999 & 14.75773 & 1.984854 & 14.694 & 4564.75 & 4331.239 & 70.8 & 70.1 & 69.4 & 69.8 & 0.00091 \\
\hline 275.03 & 3.76613 & 14.75923 & 1.984854 & 14.69475 & 4565 & 4327.018 & 70.8 & 70.1 & 69.3 & 69.8 & 0.000009 \\
\hline 280.02 & 3.744871 & 14.75773 & 1.984854 & 14.694 & 4575.5 & 4325.776 & 70.8 & 70.1 & 69.3 & 69.8 & 0.00091 \\
\hline 285.02 & 3.741119 & 14.75698 & 1.985479 & 14.694 & 4575.5 & 4320.81 & 70.8 & 70.1 & 69.4 & 69.8 & 0.00091 \\
\hline 290.02 & 3.739243 & 14.75848 & 1.984854 & 14.69475 & 4575.75 & 4320.313 & 70.7 & 70.1 & 69.3 & 69.8 & 0.00091 \\
\hline 295.03 & 3.7555 & 14.75548 & 1.984228 & 14.694 & 4575.75 & 4318.823 & 70.7 & 70.1 & 69.2 & 69.8 & 0.00091 \\
\hline 300.02 & 3.737993 & 14.75698 & 1.984228 & 14.694 & 4575.25 & 4323.045 & 70.6 & 70.1 & 69.3 & 69.8 & 0.000911 \\
\hline 305.02 & 3.769256 & 14.75548 & 1.984228 & 14.694 & 4575.5 & 4334.963 & 70.7 & 70.1 & 69.3 & 69.8 & 0.000912 \\
\hline 310.03 & 3.747372 & 14.75548 & 1.984854 & 14.694 & 4575.75 & 4330.742 & 70.6 & 70.1 & 69.3 & 69.9 & 0.000911 \\
\hline
\end{tabular}




\begin{tabular}{|c|c|c|c|c|c|c|c|c|c|c|c|}
\hline SECONDS & MAIN DUCT & MAIN DUCT & GAS INJECT & GAS INJECT & NEWPORT & SMC & AMBIENT & GAS INJECT & MAIN DUCT & WEC CELL & WEC CELL \\
\hline & DIFF (IN H2O) & ABS (PSIA) & DIFF (IN H2O) & ABS (PS|A) & (PPM) & (PPM) & TEMP.(F) & TEMP. (F) & TEMP. (F) & TEMP. (F) & (VOLTS) \\
\hline 0.01001 & 3.768631 & 14.75998 & 0.654543 & 14.7225 & 41.2 & 3.72222 & 70.5 & 70.1 & 69.2 & 69.8 & 0.000238 \\
\hline 315.02 & 3.7555 & 14.75548 & 1.984228 & 14.69475 & 4585.25 & 4323.293 & 70.6 & 70.1 & 69.3 & 69.8 & 0.000911 \\
\hline 320.02 & 3.758004 & 14.75548 & 1.986104 & 14.694 & 4586 & 4322.548 & 70.6 & 70.1 & 69.2 & 69.8 & 0.000912 \\
\hline 325.02 & 3.744871 & 14.75623 & 1.984228 & 14.69475 & 4586 & 4328.011 & 70.5 & 70.1 & 69.2 & 69.9 & 0.000912 \\
\hline 330.02 & 3.759877 & 14.75773 & 1.986104 & 14.694 & 4585.5 & 4320.313 & 70.5 & 70.1 & 69.2 & 69.8 & 0.000912 \\
\hline 335.04 & 3.7555 & 14.75698 & 1.98673 & 14.694 & 4585.75 & 4317.582 & 70.4 & 70.1 & 69.3 & 69.8 & 0.000912 \\
\hline 340.04 & 3.744871 & 14.75698 & 1.985479 & 14.694 & 4586 & 4327.266 & 70.3 & 70.1 & 69.3 & 69.9 & 0.000912 \\
\hline 345.02 & 3.753624 & 14.75623 & 1.98673 & 14.694 & 4585 & 4311.623 & 70.3 & 70.1 & 69.2 & 69.8 & 0.000913 \\
\hline 350.09 & 3.742369 & 14.75623 & 1.987355 & 14.69325 & 4585.5 & 4312.616 & 70.3 & 70.1 & 69.3 & 69.9 & 0.000913 \\
\hline 355.02 & 3.756125 & 14.75698 & 1.98673 & 14.69325 & 4585.75 & 4314.851 & 70.3 & 70.1 & 69.3 & 69.8 & 0.000913 \\
\hline 360.02 & 3.756125 & 14.75698 & 1.98673 & 14.69325 & 4595.25 & 4320.81 & 70.5 & 70.1 & 69.3 & 69.8 & 0.000914 \\
\hline 365.02 & 3.744871 & 14.75623 & 1.986104 & 14.694 & 4595.75 & 4322.796 & 70.5 & 70.1 & 69.3 & 69.8 & 0.000913 \\
\hline 370.02 & 3.749247 & 14.75698 & 1.987355 & 14.694 & 4595.5 & 4322.548 & 70.6 & 70.1 & 69.3 & 69.8 & 0.000914 \\
\hline 375.02 & 3.740494 & 14.75698 & 1.98673 & 14.69325 & 4595.25 & 4324.038 & 70.6 & 70.1 & 69.4 & 69.8 & 0.000914 \\
\hline 380.02 & 3.778635 & 14.75773 & 1.98673 & 14.69325 & 4595.75 & 4323.541 & 70.5 & 70.1 & 69.4 & 69.8 & 0.000914 \\
\hline 385.02 & 3.737367 & 14.75623 & 1.988606 & 14.694 & 4605.75 & 4308.146 & 70.6 & 70.1 & 69.4 & 69.8 & 0.000914 \\
\hline 390.04 & 3.745496 & 14.75698 & 1.988606 & 14.694 & 4605.75 & 4310.133 & 70.7 & 70.1 & 69.4 & 69.8 & 0.000914 \\
\hline 395.02 & 3.749247 & 14.75773 & 1.989232 & 14.69475 & 4605.5 & 4326.273 & 70.6 & 70.1 & 69.4 & 69.8 & 0.000915 \\
\hline 400.02 & 3.751123 & 14.75548 & 1.986104 & 14.60325 & 4605.5 & 4332.48 & 70.7 & 70.2 & 69.3 & 69.8 & 0.000915 \\
\hline 405.02 & 3.748622 & 14.75698 & 1.98673 & 14.694 & 4605.5 & 4330.742 & 70.6 & 70.1 & 69.4 & 69.9 & 0.000914 \\
\hline 410.03 & 3.754875 & 14.75623 & 1.988606 & 14.69325 & 4606 & 4321.307 & 70.5 & 70.1 & 69.4 & 69.8 & 0.000914 \\
\hline 415.02 & 3.753624 & 14.75698 & 1.988606 & 14.694 & 4606 & 4313.112 & 70.5 & 70.1 & 69.4 & 69.9 & 0.000915 \\
\hline 420.03 & 3.751749 & 14.75548 & 1.987355 & 14.69325 & 4605.25 & 4321.058 & 70.5 & 70.1 & 69.5 & 69.9 & 0.000915 \\
\hline 425.02 & 3.774884 & 14.75548 & 1.988606 & 14.694 & 4605.5 & 4328.756 & 70.6 & 70.4 & 69.5 & 69.8 & 0.000915 \\
\hline 430.1 & 3.791766 & 14.75698 & 1.988606 & 14.694 & 4605.75 & 4319.072 & 70.6 & 70.1 & 69.3 & 69,8 & 0.000915 \\
\hline 435.02 & 3.758001 & 14.75623 & 1.989232 & 14.694 & 4605.5 & 4308.891 & 70.6 & 70.1 & 69.5 & 69.8 & 0.000916 \\
\hline 440.05 & 3.76613 & 14.75548 & 1.988606 & 14.694 & 4605.25 & 4312.119 & 70.6 & 70.1 & 69.4 & 69.9 & 0.000915 \\
\hline 445.02 & 3.753624 & 14.75623 & 1.989232 & 14.694 & 4606 & 4317.085 & 70.7 & 70.1 & 69.4 & 6.8 & 0.000916 \\
\hline 450.09 & 3.749873 & 14.75623 & 1.987355 & 14.69025 & 4605 & 4316.837 & 70.8 & 70.1 & 69.4 & 69.9 & 0.000917 \\
\hline 455.02 & 3.739243 & 14.75623 & 1.988606 & 14.69325 & 4605.75 & 4315.347 & 70.8 & 70.1 & 69.4 & 69.9 & 0.000916 \\
\hline 460.02 & 3.746121 & 14.75698 & 1.989232 & 14.694 & 4605.75 & 4317.085 & 70.8 & 70.1 & 69.4 & 69.8 & 0.000916 \\
\hline 465.02 & 3.7555 & 14.75773 & 1.987981 & 14.691 & 4615.5 & 4315.099 & 70.9 & 70.2 & 69.4 & 6.8 & 0.000916 \\
\hline 470.02 & 3.756125 & 14.75623 & 1.987981 & 14.69325 & 4616 & 4305.167 & 70.9 & 70.1 & 69.5 & 69.9 & 0.000918 \\
\hline 475.02 & 3.74362 & 14.75623 & 1.987981 & 14.69175 & 4616 & 4304.173 & 70.9 & 70.1 & 69.4 & 69.9 & 0.000918 \\
\hline
\end{tabular}




\begin{tabular}{|c|c|c|c|c|c|c|c|c|c|c|c|}
\hline SECONDS & MAIN DUCT & MAIN DUCT & GAS INJECT & GAS INJECT & NEWPORT & SMC & AMBIENT & GAS INJECT & MAIN DUCT & WEC CELL & WEC CELL \\
\hline & DIFF (IN H2O) & ABS (PSIA) & DIFF (IN H2O) & ABS (PSIA) & (PPM) & (PPM) & TEMP.(F) & TEMP. (F) & TEMP. (F) & TEMP. (F) & (VOLTS) \\
\hline 0.01001 & 3.768631 & 14.75998 & 0.654543 & 14.7225 & 41.2 & -3.72222 & 70.5 & 70.1 & 69.2 & 69.8 & 0.000238 \\
\hline 480.02 & 3.747997 & 14.75773 & 1.987981 & 14.694 & 4616 & 4305.663 & 70.9 & 70.1 & 69.4 & 69.9 & 0.000918 \\
\hline 485.02 & 3.744871 & 14.75773 & 1.987981 & 14.69325 & 4616 & 4311.126 & 70.8 & 70.1 & 69.4 & 69.9 & 0.000917 \\
\hline 490.02 & 3.738618 & 14.75398 & 1.987981 & 14.6925 & 4615.75 & 4310.629 & 70.8 & 70.1 & 69.5 & 69,8 & 0.000918 \\
\hline 495.02 & 3.764254 & 14.75698 & 1.988606 & 14.69175 & 4615.5 & 4320.065 & 70.8 & 70.1 & 69.5 & 69.9 & 0.000917 \\
\hline 500.02 & 3.766755 & 14.75698 & 1.987981 & 14.6925 & 4615.75 & 4334.467 & 70.8 & 70.1 & 69.5 & 69.9 & 0.000916 \\
\hline 505.02 & 3.746746 & 14.75848 & 1.988606 & 14.69475 & 4615.25 & 4324.783 & 70.8 & 70.1 & 69.5 & 69.9 & 0.000917 \\
\hline 510.06 & 3.74362 & 14.75623 & 1.989232 & 14.6925 & 4616 & 4314.602 & 70.7 & 70.1 & 69.5 & 69.9 & 0.000916 \\
\hline 515.02 & 3.739243 & 14.75623 & 1.987981 & 14.69175 & 4615.75 & 4320.065 & 70.6 & 70.1 & 69.4 & 69.9 & 0.000917 \\
\hline 520.02 & 3.732365 & 14.75698 & 1.989857 & 14.69475 & 4615.25 & 4323.79 & 70.6 & 70.1 & 69.4 & 69.9 & 0.000917 \\
\hline 525.03 & 3.744871 & 14.75848 & 1.989232 & 14.69475 & 4615.5 & 4315.844 & 70.6 & 70.1 & 69.2 & 69.8 & 0.000916 \\
\hline 530.02 & 3.765504 & 14.75698 & 1.988606 & 14.69175 & 4615.75 & 4317.83 & 70.7 & 70.1 & 69.3 & 69.9 & 0.000917 \\
\hline 535.02 & 3.747372 & 14.75773 & 1.987981 & 14.691 & 4615.25 & 4318.079 & 70.7 & 70.1 & 69.4 & 69.9 & 0.000916 \\
\hline 540.09 & 3.749873 & 14.75698 & 1.988606 & 14.691 & 4615.5 & 4320.562 & 70.7 & 70.1 & 69.5 & 69.9 & 0.000916 \\
\hline 545.05 & 3.746121 & 14.75698 & 1.987981 & 14.69325 & 4615.75 & 4310.381 & 70.7 & 70.1 & 69.4 & 69.9 & 0.000917 \\
\hline 550.02 & 3.73299 & 14.75773 & 1.988606 & 14.691 & 4615.25 & 4324.286 & 70.6 & 70.1 & 69.5 & 69.9 & 0.000917 \\
\hline 555.05 & 3.766755 & 14.75698 & 1.988606 & 14.691 & 4615.75 & 4322.051 & 70.6 & 70.1 & 69.4 & 69.9 & 0.000917 \\
\hline 560.02 & 3.744871 & 14.75848 & 1,989232 & 14.69175 & 4616 & 4314,602 & 70.6 & 70.1 & 69.4 & 69.9 & 0,000918 \\
\hline 565.02 & 3.741119 & 14.75623 & 1.988606 & 14.694 & 4615.75 & 4316.837 & 70.6 & 70.2 & 69.4 & 69.9 & 0.000919 \\
\hline 570.02 & 3.741119 & 14.75623 & 1.991108 & 14.691 & 4626 & 4317.334 & 70.6 & 70.1 & 69.4 & 69.8 & 0.000918 \\
\hline 575.02 & 3.744871 & 14.75623 & 1.990483 & 14.69325 & 4625.5 & 4312.119 & 70.7 & 70.1 & 69.3 & 69.8 & 0.000917 \\
\hline 580.02 & 3.744871 & 14.75623 & 1.990483 & 14.69175 & 4625.5 & 4313.361 & 70.7 & 70.1 & 69.2 & 69.8 & 0.000919 \\
\hline 585.04 & 3.736742 & 14.75548 & 1.991108 & 14.69475 & 4625.5 & 4311.871 & 70.7 & 70.1 & 69.4 & 69.9 & 0.000919 \\
\hline 590.06 & 3.745496 & 14.75548 & 1.989232 & 14.691 & 4625.5 & 4312.119 & 70.8 & 70.1 & 69.4 & 69.8 & 0.000919 \\
\hline 595.02 & 3.751123 & 14.75698 & 1.989232 & 14.694 & 4626 & 4302.932 & 70.7 & 70.2 & 69.5 & 69.9 & 0.000919 \\
\hline 600,02 & 3.739243 & 14.75548 & 1.988606 & 14.69475 & 4636 & 4289.026 & 70.7 & 70.2 & 69.5 & 69.9 & 0.000919 \\
\hline 605.02 & 3.744871 & 14.75698 & 1.988606 & 14.691 & 4635.75 & 4294.986 & 70.7 & 70.1 & 69.5 & 69.9 & 0.000919 \\
\hline 610.02 & 3.749873 & 14.75773 & 1.988606 & 14.694 & 4635.5 & 4292.503 & 70.7 & 70.1 & 69.4 & 69.8 & 0.000918 \\
\hline 615.02 & 3.784888 & 14.75623 & 1.988606 & 14.691 & 4635.5 & 4300.2 & 70.6 & 70.1 & 69.4 & 69.8 & 0.000919 \\
\hline 620.02 & 3.738618 & 14.75623 & 1.988606 & 14.69025 & 4635.5 & 4309.139 & 70.6 & 70.2 & 69.4 & 69.9 & 0.00092 \\
\hline 625.02 & 3.749247 & 14.75548 & 1.987981 & 14.69325 & 4635.25 & 4308.146 & 70.6 & 70.2 & 69.4 & 69.9 & 0.00002 \\
\hline 630.02 & $\mathbf{3 . 7 3 2 9 9}$ & 14.75548 & 1.990483 & 14.691 & 4635.75 & 4316.589 & 70.6 & 70.2 & 69.3 & 69.8 & 0.000921 \\
\hline 635.02 & 3.759252 & 14.75623 & 1.988606 & 14.691 & 4635.75 & 4304.67 & 70.6 & 70.1 & 69.3 & 69.9 & 0.00092 \\
\hline 640.02 & 3.739243 & 14.75623 & 1.989232 & 14.691 & 4635.25 & 4305.167 & 70.5 & 70.2 & 69.2 & 69.9 & 0.00092 \\
\hline
\end{tabular}




\begin{tabular}{|c|c|c|c|c|c|c|c|c|c|c|c|}
\hline SECONDS & MAIN DUCT & MAIN DUCT & GAS INJECT & GAS INJECT & NEWPORT & SMC & AMBIENT & GAS INJECT & MAIN DUCT & WEC CELL & WEC CELL \\
\hline & DIFF (IN H2O) & ABS (PSIA) & DIFF (IN H2O) & ABS (PSIA) & (PPM) & (PPM) & TEMP.(F) & TEMP. (F) & TEMP. (F) & TEMP, (F) & (VOLTS) \\
\hline 0.01001 & 3.768631 & 14.75998 & 0.654543 & 14.7225 & 41.2 & -3.72222 & 70.5 & 70.1 & 69.2 & 69.8 & 0.000238 \\
\hline 645.02 & 3.758001 & 14.75698 & 1.991108 & 14.69175 & 4635.5 & 4320.81 & 70.4 & 70.1 & 69.3 & 69.9 & 0.000921 \\
\hline 650.02 & 3.733616 & 14.75698 & 1.990483 & 14.69325 & 4635.75 & 4327.763 & 70.6 & 70.1 & 69.3 & 69.9 & 0.00092 \\
\hline 655.02 & 3.747372 & 14.75773 & 1.990483 & 14.691 & 4635.25 & 4327.018 & 70.5 & 70.1 & 69.4 & 69.9 & 0.00092 \\
\hline 660.05 & 3.740873 & 14.75698 & 1.990483 & 14.691 & 4635.75 & 4324.783 & 70.6 & 70.2 & 69.3 & 69.9 & 0.00092 \\
\hline 665.02 & 3.723611 & 14.75698 & 1.991733 & 14.69325 & 4635.5 & 4311.623 & 70.6 & 70.1 & 69.4 & 69.9 & 0.00092 \\
\hline 670.02 & 3.747372 & 14.75548 & 1.991733 & 14.69175 & 4635.25 & 4314.354 & 70.6 & 70.2 & 69.4 & 69.9 & 0.000921 \\
\hline 675.03 & 3.751749 & 14.75623 & 1.991108 & 14.691 & 4635.5 & 4306.408 & 70.7 & 70.2 & 69.4 & 69.9 & 0.000921 \\
\hline 680.02 & 3.736117 & 14.75323 & 1.991733 & 14.69175 & 4635.5 & 4299.952 & 70.7 & 70.1 & 69.4 & 69.9 & 0.000919 \\
\hline 685.02 & 3.729864 & 14.75623 & 1.991733 & 14.694 & 4635.5 & 4301.69 & 70.7 & 70.2 & 69.4 & 69.9 & 0.00092 \\
\hline 690.02 & 3.744245 & 14.75623 & 1.991108 & 14.69025 & 4636 & 4287.288 & 70.7 & 70.1 & 69.4 & 69.9 & 0.000919 \\
\hline 695.08 & 3.760502 & 14.75548 & 1.990483 & 14.69025 & 4635.5 & 4302.683 & 70.7 & 70.2 & 69.4 & 69.9 & 0.00092 \\
\hline 700.02 & 3.744871 & 14.75623 & 1.991108 & 14.691 & 4635.75 & 4311.623 & 70.7 & 70.2 & 69.4 & 69.9 & 0.000919 \\
\hline 705.06 & 3.744871 & 14.75698 & 1.991108 & 14.694 & 4635.5 & 4305.663 & 70.7 & 70.2 & 69.3 & 69.9 & 0.00092 \\
\hline 710.02 & 3.737993 & 14.75548 & 1.991108 & 14.69325 & 4635.75 & 4310.629 & 70.7 & 70.2 & 69.2 & 69.8 & 0.000921 \\
\hline 715.02 & 3.749247 & 14.75548 & 1.991108 & 14.691 & 4635.5 & 4309.388 & 70.7 & 70.2 & 69.2 & 69.8 & 0.000921 \\
\hline 720.03 & 3.753624 & 14.75473 & 1.991108 & 14.691 & 4646.25 & 4300.945 & 70.7 & 70.2 & 69.4 & 69.8 & 0.000921 \\
\hline 725.02 & 3.741119 & 14.75548 & 1.992984 & 14.691 & 4646 & 4293.744 & 70.8 & 70.2 & 69.4 & 69.9 & 0.000921 \\
\hline 730.02 & 3.747907 & 14.75623 & 1.991733 & 14.691 & 4646 & 4294.489 & 70.7 & 70.2 & 69.4 & 69.8 & 0.000921 \\
\hline 735.05 & 3.764879 & 14.75698 & 1.992984 & 14.69025 & 4646 & 4296.227 & 70.7 & 70.1 & 69.4 & 69.9 & 0.000921 \\
\hline 740.09 & 3.742369 & 14.75623 & 1.991108 & 14.69175 & 4646 & 4311.126 & 70.7 & 70.1 & 69.3 & 69,9 & 0.00092 \\
\hline 745.02 & 3.749873 & 14.75623 & 1.99361 & 14.691 & 4646 & 4313.857 & 70.7 & 70.2 & 69.3 & 69.9 & 0.000921 \\
\hline 750.02 & 3.747372 & 14.75698 & 1.991108 & 14.69175 & 4646 & 4303.677 & 70.7 & 70.1 & 69.3 & 69.8 & 0.000921 \\
\hline 755.02 & 3.744871 & 14.75623 & 1.991733 & 14.691 & 4646 & 4294.241 & 70.7 & 70.1 & 69.3 & 69.8 & 0.000921 \\
\hline 760.02 & 3.744245 & 14.75698 & 1.990483 & 14.691 & 4645.75 & 4297.221 & 70.7 & 70.1 & 69.3 & 69.9 & 0.000921 \\
\hline 765.02 & 3.747997 & 14.75698 & 1,991733 & 14.691 & 4646 & 4301.194 & 70.7 & 70.2 & 69.3 & 69.8 & 0.000921 \\
\hline 770.03 & 3.749873 & 14.75698 & 1.991733 & 14.691 & 4646 & 4310.878 & 70.7 & 70.2 & 69.4 & 69.9 & 0.000921 \\
\hline 775.02 & 3.7555 & 14.75773 & 1.992359 & 14.691 & 4645.25 & 4306.656 & 70.6 & 70.1 & 69.5 & 69.9 & 0.00092 \\
\hline 780.02 & 3.770507 & 14.75698 & 1.991108 & 14.691 & 4646 & 4315.099 & 70.6 & 70.2 & 69.4 & 69.9 & 0.000921 \\
\hline 785.07 & 3.730489 & 14.75548 & 1.991733 & 14.691 & 4645.75 & 4321.555 & 70.4 & 70.2 & 69.4 & 69.8 & 0.000921 \\
\hline 790.09 & 3.742995 & 14.75773 & 1.991108 & 14.691 & 4646.25 & 4312.616 & 70.5 & 70.1 & 69.3 & 69.9 & 0.000923 \\
\hline 795.02 & 3.739868 & 14.75773 & 1.992984 & 14.691 & 4646 & 4303.428 & 70.6 & 70.2 & 69.3 & 69.9 & 0.000921 \\
\hline 800.03 & 3.746121 & 14.75698 & 1.992984 & 14.69175 & 4645.75 & 4306.656 & 70.5 & 70.2 & 69.2 & 69.8 & 0.00092 \\
\hline 805.02 & 3.759877 & 14.75773 & 1.992359 & 14.69175 & 4646 & 4297.221 & 70.5 & 70.2 & 69.3 & 69.9 & 0.000921 \\
\hline
\end{tabular}




\begin{tabular}{|c|c|c|c|c|c|c|c|c|c|c|c|}
\hline SECONDS & MAIN DUCT & MAIN DUCT & GAS INJECT & GAS INJECT & NEWPORT & SMC & AMBIENT & GAS INJECT & MAIN DUCT & WEC CELL & WEC CELL \\
\hline & DIFF (IN H2O) & ABS (PSIA) & DIFF (IN H2O) & ABS (PSIA) & (PPM) & $(\mathrm{PPM})$ & TEMP.(F) & TEMP. (F) & TEMP. (F) & TEMP. (F) & (VOLTS) \\
\hline 0.01001 & 3.768631 & 14.75998 & 0.654543 & 14.7225 & 41.2 & -3.72222 & 70.5 & 70.1 & 69.2 & 69.8 & 0.000238 \\
\hline 810.02 & 3.741744 & 14.75773 & 1.992984 & 14.69175 & 4645.75 & 4298.214 & 70.4 & 70.2 & 69.2 & 69.9 & 0.00092 \\
\hline 815.02 & 3.73174 & 14.75848 & 1.99361 & 14.691 & 4646 & 4301.194 & 70.5 & 70.2 & 69.3 & 69.9 & 0.000921 \\
\hline 820.02 & 3.747997 & 14.75398 & 1.99361 & 14.6925 & 4645.75 & 4306,905 & 70.5 & 70.2 & 69.4 & 69.8 & 0.000921 \\
\hline 825.02 & 3.751749 & 14.75548 & 1.992984 & 14.69175 & 4646 & 4303.677 & 70.5 & 70.2 & 69.4 & 69.8 & 0.000921 \\
\hline 830.03 & 3.738618 & 14.75623 & 1.991733 & 14.69175 & 4646 & 4305.167 & 70.6 & 70.2 & 69.4 & 69.8 & 0.000922 \\
\hline 835.02 & 3.740494 & 14.75473 & 1.991108 & 14.69175 & 4645.5 & 4303.428 & 70.6 & 70.2 & 69.4 & 69.8 & 0.000922 \\
\hline 840.02 & 3.749873 & 14.75548 & 1.991733 & 14.69175 & 4646 & 4294.241 & 70.6 & 70.2 & 69.4 & 69.8 & 0.000922 \\
\hline 845.08 & 3.759877 & 14.75698 & 1.992984 & 14.691 & 4646 & 4292.999 & 70.6 & 70.2 & 69.4 & 69.8 & 0.000922 \\
\hline 850.02 & 3.749247 & 14.75698 & 1.99361 & 14.691 & 4646.25 & 4286.792 & 70.5 & 70.1 & 69.4 & 69.9 & 0.000921 \\
\hline 855.02 & 3.744871 & 14.75623 & 1.99361 & 14.691 & 4645.75 & 4292.999 & 70.5 & 70.2 & 69.3 & 69.9 & 0.000922 \\
\hline 860.02 & 3.746746 & 14.75698 & 1.992984 & 14.694 & 4645.75 & 4301.69 & 70.6 & 70.2 & 69.5 & 69.8 & 0.000922 \\
\hline 865.02 & 3.744871 & 14.75773 & 1.994235 & 14.69175 & 4656.25 & 4301.69 & 70.6 & 70.2 & 69.4 & 69.8 & 0.000922 \\
\hline 870.02 & 3.761753 & 14.75773 & 1.992984 & 14.69175 & 4655.75 & 4278.101 & 70.5 & 70.2 & 69.4 & 69.8 & 0.000922 \\
\hline 875.04 & 3.756125 & 14.75698 & 1.992984 & 14.69175 & 4655.5 & 4287.785 & 70.5 & 70.2 & 69.3 & 69.8 & 0.000923 \\
\hline 880.02 & 3.751123 & 14.75698 & 1.992984 & 14.69175 & 4655.75 & 4289.771 & 70.5 & 70.2 & 69.4 & 69.8 & 0.000922 \\
\hline 885.02 & 3.747997 & 14.75398 & 1.992984 & 14.69175 & 4656 & 4294.738 & 70.5 & 70.2 & 69.5 & 69.9 & 0.000922 \\
\hline 890.05 & 3.737993 & 14.75698 & 1.992984 & 14.69175 & 4656.25 & 4290.765 & 70.5 & 70.2 & 69.4 & 69.9 & 0.000922 \\
\hline 895.09 & 3.740494 & 14.75548 & 1.992984 & 14.691 & 4656.25 & 4296.227 & 70.5 & 70.2 & 69.4 & 69.9 & 0.000923 \\
\hline 900.02 & 3.742369 & 14.75773 & 1.992984 & 14.69175 & 4656 & 4303.925 & 70.5 & 70.2 & 69.3 & 69.8 & 0.000921 \\
\hline 905.06 & 3.742995 & 14.75848 & 1.992984 & 14.69175 & 4656.25 & 4306.905 & 70.6 & 70.2 & 69.4 & 69.8 & 0.000923 \\
\hline 910.07 & 3.738618 & 14.75773 & 1.99361 & 14.691 & 4656 & 4311.623 & 70.5 & 70.2 & 69.2 & 69.9 & 0.000923 \\
\hline 915.02 & 3.749247 & 14.75698 & 1.99361 & 14.69175 & 4656 & 4300.945 & 70.6 & 70.2 & 69.3 & 69.9 & 0.000923 \\
\hline 920.02 & 3.758001 & 14.75698 & 1.992984 & 14.69175 & 4655.5 & 4308.146 & 70.5 & 70.2 & 69.3 & 69.9 & 0.000922 \\
\hline 925.02 & 3.736742 & 14.75923 & 1.99361 & 14.69175 & 4655.75 & 4312.367 & 70.6 & 70.2 & 69.4 & 69.9 & 0.000922 \\
\hline 930.17 & 3.737993 & 14.75473 & 1.992359 & 14.69175 & 4656.25 & 4319.072 & 70.6 & 70.2 & 69.2 & 69.8 & 0.000922 \\
\hline 935.02 & 3.749247 & 14.75698 & 1.992984 & 14.69175 & 4655.75 & 4311.374 & 70.6 & 70.2 & 69.4 & 69.8 & 0.000922 \\
\hline
\end{tabular}




\begin{tabular}{|c|c|c|c|c|c|c|c|c|c|c|c|}
\hline \multicolumn{12}{|c|}{$\begin{array}{l}\text { TEST: FGI22227 22 Feb } 1997 \text { WEC-SPOOL } \\
\text { Measured Hydrogen Concentration = 6432ppm }\end{array}$} \\
\hline & DIFF (IN H2O) & ABS (PSIA) & DIFF (IN H2O) & ABS (PSIA) & (PPM) & (PPM) & TEMP. (F) & TEMP. (F) & TEMP. (F) & TEMP. (F) & (VOLTS) \\
\hline 0.009979 & 3.746746 & 14.75398 & 0.217298 & 14.655 & 51.225 & -3.32493 & 70.4 & 69.9 & 69.5 & 69.8 & 0.00024 \\
\hline 5.019989 & 3.737993 & 14.75548 & 2.961165 & 14.7585 & 51,35 & 10.18303 & 70.5 & 69.9 & 69.4 & 69.8 & 0.000241 \\
\hline 10.01999 & 3.7555 & 14.75473 & 2.958664 & 14.75925 & 200.575 & 2399.899 & 70.4 & 69.9 & 69.5 & 69.8 & 0.000286 \\
\hline 15.01999 & 3.764254 & 14.75473 & 2.959289 & 14.7585 & 882.5 & 4415.415 & 70.5 & 69.9 & 69.5 & 69.8 & 0.000399 \\
\hline 20.01999 & 3.725487 & 14.75473 & 2.961165 & 14.7585 & 1773.25 & 5366.683 & 70.5 & 69.9 & 69.5 & 69.9 & 0.000533 \\
\hline 25.01999 & 3.739868 & 14.75473 & 2.963042 & 14.76 & 2573.25 & 5748.084 & 70.6 & 69.9 & 69.4 & 69.8 & 0.000654 \\
\hline 30,01999 & 3.759877 & 14.75473 & 2.962416 & 14.7585 & 3344.75 & 5919.417 & 70.6 & 69.9 & 69.4 & 69.8 & 0.000757 \\
\hline 35.01999 & 3.73299 & 14.75548 & 2.966794 & 14.75925 & 3942.5 & 5988.695 & 70.6 & 69.9 & 69.4 & 69.8 & 0.000841 \\
\hline 40.01999 & 3.724237 & 14.75398 & 2.966169 & 14.7585 & 4374.25 & 6074.609 & 70.7 & 69.9 & 69.4 & 69.8 & 0.000908 \\
\hline 45.01999 & 3.786764 & 14.75398 & 2.966169 & 14.75925 & 4803.75 & 6112.6 & 70.6 & 69.9 & 69.4 & 69.8 & 0.000962 \\
\hline 50.01999 & 3.742369 & 14.75323 & 2.96742 & 14.75925 & 5135 & 6133.458 & 70.6 & 69.9 & 69.3 & 69.8 & 0.001006 \\
\hline 55.01999 & 3.747372 & 14.75473 & 2.968045 & 14.7585 & 5365.5 & 6169.463 & 70.6 & 69.9 & 69.4 & 69.8 & 0.001042 \\
\hline 60.01999 & 3.744871 & 14.75398 & 2.968671 & 14.75925 & 5575.5 & 6186.845 & 70.7 & 69.9 & 69.3 & 69.8 & 0.001069 \\
\hline 65.01999 & 3.732365 & 14.75248 & 2.968671 & 14.7585 & 5745.75 & 6188.334 & 70.7 & 69.9 & 69.4 & 69.8 & 0.001092 \\
\hline 70.01999 & 3.749247 & 14.75323 & 2.968671 & 14.75925 & 5865.75 & 6222.104 & 70.7 & 69.9 & 69.3 & 69.8 & 0.001111 \\
\hline 75.01999 & 3.742995 & 14.75398 & 2.968671 & 14.7585 & 5995.75 & 6248.177 & 70.7 & 70 & 69.4 & 69.8 & 0.001127 \\
\hline 80.01999 & 3.745496 & 14.75398 & 2.969922 & 14.75925 & 6066 & 6253.143 & 70.7 & 70 & 69.4 & 69.8 & 0.001139 \\
\hline 85.01999 & 3.751123 & 14.75323 & 2.970547 & 14.75775 & 6146.25 & 6286.913 & 70.7 & 70 & 69.5 & 69.8 & 0.00115 \\
\hline 90.01999 & 3.721736 & 14.75398 & 2.971172 & 14.7585 & 6206.25 & 6309.509 & 70.7 & 70 & 69.5 & 69.8 & 0.001159 \\
\hline 95.01999 & 3.7555 & 14.75473 & 2.970547 & 14.75925 & 6266.25 & 6319.193 & 70.6 & 70 & 69.5 & 69.8 & 0.001167 \\
\hline 100.02 & 3.747372 & 14.75398 & 2.970547 & 14.7585 & 6316.5 & 6325.649 & 70.6 & 70 & 69.3 & 69.8 & 0.001174 \\
\hline 105.02 & 3.736742 & 14.75548 & 2.971172 & 14.7585 & 6366.25 & 6318.448 & 70.7 & 70 & 69.4 & 69.8 & 0.001179 \\
\hline 110.02 & 3.747359 & 14.75398 & 2.971172 & 14.7585 & 6396.5 & 6340.051 & 70.7 & 70 & 69.4 & 69.8 & 0.001185 \\
\hline 115.02 & 3.73299 & 14.75398 & 2.971172 & 14.7585 & 6436.75 & 6362.15 & 70.7 & 70 & 69.4 & 69.8 & 0.00119 \\
\hline 120.02 & 3.757376 & 14.75173 & 2.971172 & 14.75925 & 6466.5 & 6373.324 & 70.7 & 70 & 69.3 & 69.8 & 0.001194 \\
\hline 125.02 & 3.732365 & 14.75473 & 2.969296 & 14.75925 & 6497 & 6375.807 & 70.7 & 70 & 69.3 & 69.8 & 0.001198 \\
\hline 130.02 & 3.749247 & 14.75323 & 2.970547 & 14.76 & 6506.5 & 6395.672 & 70.7 & 70 & 69.4 & 69.8 & 0.001201 \\
\hline 135.02 & 3.744871 & 14.75323 & $2.96867 t$ & 14.7585 & 6526.5 & 6383.504 & 70.6 & 70.1 & 69.6 & 69.8 & 0.001203 \\
\hline 140.02 & 3.737993 & 14.75323 & 2.970547 & 14.76 & 6555.5 & 6378.042 & 70.7 & 70.1 & 69.4 & 69.8 & 0.001205 \\
\hline 145.02 & 3.745496 & 14.75398 & 2.971172 & 14.76 & 6576.75 & 6347.996 & 70.7 & 70.1 & 69.5 & 69.8 & 0.001209 \\
\hline
\end{tabular}




\begin{tabular}{|c|c|c|c|c|c|c|c|c|c|c|c|}
\hline SECONDS & MAIN DUCT & MAIN DUCT & GAS INJECT & GAS INJECT & NEWPORT & SMC & AMBIENT & GAS INJECT & MAIN DUCT & WEC CELL & WEC CELL \\
\hline & DIFF ( $\mathrm{N} \mathrm{H} 2 \mathrm{O})$ & ABS (PSIA) & DIFF (IN H2O) & ABS (PSIA) & (PPM) & (PPM) & TEMP. (F) & TEMP. (F) & TEMP. (F) & TEMP. (F) & (VOLTS) \\
\hline 0.009979 & 3.746746 & 14.75398 & 0.217298 & 14.655 & 51.225 & 3.32493 & 70.4 & 69.9 & 69.5 & 69.8 & 0.00024 \\
\hline 150.02 & 3.751749 & 14.75398 & 2.971798 & 14.7585 & 6586.25 & 6379.532 & 70.7 & 70.1 & 69.4 & 69.8 & 0.001211 \\
\hline 155.02 & 3.749247 & 14.75623 & 2.971172 & 14.76 & 6596.75 & 6404.859 & 70.7 & 70.1 & 69.6 & 69.8 & 0.001213 \\
\hline 160.02 & 3.734866 & 14.75548 & 2.968045 & 14.7585 & 6607 & 6401.631 & 70.7 & 70.1 & 69.6 & 69.9 & 0.001214 \\
\hline 165.02 & 3.732365 & 14.75473 & 2.970547 & 14.75925 & 6617 & 6394.678 & 70.6 & 70.1 & 69.6 & 69.8 & 0.001216 \\
\hline 170.02 & 3.749247 & 14.75623 & 2.968671 & 14.75925 & 6635.75 & 6410.073 & 70.7 & 70.1 & 69.6 & 69.8 & 0.001219 \\
\hline 175.02 & 3.736742 & 14.75398 & 2.969296 & 14.75925 & 6647.25 & 6397.41 & 70.6 & 70.1 & 69.4 & 69.8 & 0.00122 \\
\hline 180.02 & 3.729864 & 14.75398 & 2.969922 & 14.75925 & 6656.75 & 6405.107 & 70.7 & 70.2 & 69.5 & 69.8 & 0.001221 \\
\hline 185.02 & 3.74362 & 14.75473 & 2.969296 & 14.75925 & 6667 & 6424.724 & 70.7 & 70.2 & 69.4 & 69.8 & 0.001223 \\
\hline 190.02 & 3.726112 & 14.75398 & 2.959296 & 14.75925 & 6667.25 & 6442.85 & 70.7 & 70.2 & 69.3 & 69.8 & 0.001224 \\
\hline 195.02 & 3.73174 & 14.75473 & 2.969296 & 14.7585 & 6677 & 6447.568 & 70.7 & 70.2 & 69.4 & 69.8 & 0.001224 \\
\hline 200.02 & 3.747997 & 14.75323 & 2.968671 & 14.75925 & 6687 & 6450.299 & 70.7 & 70.2 & 69.3 & 69.8 & 0.001226 \\
\hline 205.02 & 3.728614 & 14.75473 & 2.96742 & 14.75925 & 6687.25 & 6457.997 & 70.8 & 70.2 & 69.4 & 69.9 & 0.001226 \\
\hline 210.02 & 3.734866 & 14.75548 & 2.968045 & 14.75925 & 6687.25 & 6417.771 & 70.8 & 70.2 & 69.3 & 69.8 & 0.001226 \\
\hline 215.02 & 3.738618 & 14.75623 & 2.968671 & 14.7585 & 6697.25 & 6441.112 & 70.8 & 70.2 & 69.3 & 69.8 & 0.001227 \\
\hline 220.02 & 3.747997 & 14.75398 & 2.960296 & 14.75925 & 6697.25 & 6443.595 & 70.7 & 70.2 & 69.4 & 69.9 & 0.001227 \\
\hline 225.02 & 3.744245 & 14.75398 & 2.966169 & 14.75925 & 6707 & 6432.421 & 70.7 & 70.2 & 69.4 & 69.9 & 0.001228 \\
\hline 230.02 & 3.732365 & 14.75398 & 2.966794 & 14.75925 & 6707.25 & 6440.615 & 70.8 & 70.3 & 69.5 & 69.8 & 0.001229 \\
\hline 235.02 & 3.73299 & 14.75398 & 2.96742 & 14.75925 & 6707.5 & 6456.755 & 70.8 & 70.3 & 69.5 & 69.9 & 0.001229 \\
\hline 240.02 & 3.726738 & 14.75398 & 2.96742 & 14.75925 & 6717.25 & 6461.225 & 70.8 & 70.3 & 69.5 & 69.9 & 0.001231 \\
\hline 245.02 & 3.732365 & 14.75473 & 2.966169 & 14.76 & 6727.5 & 6462.466 & 70.9 & 70.3 & 69.3 & 69.8 & 0.001232 \\
\hline 250.02 & 3.741119 & 14.75398 & 2.96742 & 14.7585 & 6727 & 6474.882 & 70.8 & 70.3 & 69.4 & 69.8 & 0.001232 \\
\hline 255.02 & 3.741744 & 14.75473 & 2.96742 & 14.75925 & 6737.25 & 6497.478 & 70.8 & 70.3 & 69.4 & 69.8 & 0.001233 \\
\hline 260.02 & 3.754875 & 14.75398 & 2.966169 & 14.76 & 6737.25 & 6486.304 & 70.8 & 70.3 & 69.4 & 69.8 & 0.001234 \\
\hline 265.02 & 3.734241 & 14.75473 & 2.966794 & 14.75925 & 6747 & 6477.117 & 70.9 & 70.3 & 69.3 & 69.8 & 0.001234 \\
\hline 270.02 & 3.741119 & 14.75548 & 2.966794 & 14.7585 & 6747.5 & 6466.688 & 70.9 & 70.3 & 69.4 & 69.8 & 0.001233 \\
\hline 275.02 & 3.749247 & 14.75398 & 2.966169 & 14.75925 & 6747.25 & 6481.338 & 70.8 & 70.3 & 69.3 & 69.8 & 0.001234 \\
\hline 280.02 & 3.738618 & 14.75473 & 2.966169 & 14.75925 & 6747 & 6473.392 & 70.8 & 70.3 & 69.4 & 69.8 & 0.001235 \\
\hline 285.04 & 3.726112 & 14.75548 & 2.966169 & 14.75925 & 6747 & 6486.056 & 70.8 & 70.4 & 69.4 & 69.9 & 0.001235 \\
\hline 290.04 & 3.747372 & 14.75398 & 2.966794 & 14.75925 & 6757.5 & 6474.634 & 70.8 & 70.4 & 69.3 & 69.8 & 0.001234 \\
\hline 295.02 & 3.727988 & 14.75473 & 2.966794 & 14.75925 & 6757.75 & 6487.546 & 70.8 & 70.4 & 69.4 & 69.8 & 0.001236 \\
\hline 300.02 & 3.736117 & 14.75548 & 2.96742 & 14.75925 & 6766.25 & 6508.403 & 70.8 & 70.4 & 69.4 & 69.8 & 0.001237 \\
\hline 305.03 & 3.741119 & 14.75398 & 2.96742 & 14.75925 & 6767.5 & 6498.719 & 70.8 & 70.4 & 69.3 & 69.8 & 0.001237 \\
\hline 310.02 & 3.753624 & 14.75398 & 2.966794 & 14.75925 & 6767 & 6489.035 & 70.8 & 70.4 & 69.4 & 69.9 & 0.001238 \\
\hline
\end{tabular}




\begin{tabular}{|c|c|c|c|c|c|c|c|c|c|c|c|}
\hline SECONDS & MAIN DUCT & MAIN DUCT & GAS INJECT & GAS INJECT & NEWPORT & SMC & AMBIENT & GAS INJECT & MAIN DUCT & WEC CELL & WEC CELL \\
\hline & DIFF (IN H2O) & ABS (PS|A) & DIFF (IN H2O) & ABS (PSIA) & (PPM) & (PPM) & TEMP. (F) & TEMP. (F) & TEMP. (F) & TEMP. (F) & (VOLTS) \\
\hline 0.009979 & 3.746746 & 14.75398 & 0.217298 & 14.655 & 51.225 & -3.32493 & 70.4 & 69.9 & 69.5 & 69.8 & 0.00024 \\
\hline 315.02 & 3.734866 & 14.75473 & 2.966794 & 14.7585 & 6777.25 & 6476.123 & 70.8 & 70.4 & 69.4 & 69.8 & 0.001239 \\
\hline 320.02 & 3.738618 & 14.75473 & 2.966794 & 14.7585 & 6778 & 6477.117 & 70.8 & 70.4 & 69.3 & 69.9 & 0.001238 \\
\hline 325.02 & 3.741744 & 14.75398 & 2.96742 & 14.76 & 6777 & 6473.392 & 70.8 & 70.4 & 69.4 & 69.9 & 0.001239 \\
\hline 330.02 & 3.763629 & 14.75473 & 2.966794 & 14.7585 & 6778 & 6473.64 & 70.8 & 70.4 & 69.3 & 69.9 & 0.001239 \\
\hline 335.04 & 3.749873 & 14.75248 & 2.968045 & 14.75925 & 6787.75 & 6483.076 & 70.8 & 70.4 & 69.4 & 69.9 & 0.00124 \\
\hline 340.02 & 3.734241 & 14.75473 & 2.966169 & 14.75925 & 6787.5 & 6503.437 & 70.8 & 70.4 & 69.5 & 69.8 & 0.001241 \\
\hline 345.02 & 3.744871 & 14.75548 & 2.966794 & 14.75925 & 6787.5 & 6501.202 & 70.9 & 70.4 & 69.5 & 69.9 & 0.001241 \\
\hline 350.02 & 3.746121 & 14.75548 & 2.966169 & 14.75925 & 6797.5 & 6499.713 & 70.9 & 70.4 & 69.4 & 69.8 & 0.001241 \\
\hline 355.02 & 3.737993 & 14.75398 & 2.966794 & 14.75925 & 6798 & 6506.665 & 70.9 & 70.4 & 69.5 & 69.8 & 0.001241 \\
\hline 360.02 & 3.75425 & 14.75548 & 2.966794 & 14.75925 & 6797.25 & 6480.841 & 70.9 & 70.4 & 69.4 & 69.9 & 0.001242 \\
\hline 365.02 & 3.749247 & 14.75548 & 2.96742 & 14.75925 & 6808 & 6501.699 & 70.8 & 70.5 & 69.4 & 69.8 & 0.001242 \\
\hline 370.02 & 3.732365 & 14.75548 & 2.96742 & 14.7585 & 6807.5 & 6459.238 & 70.6 & 70.4 & 69.2 & 69.8 & 0.001243 \\
\hline 375.02 & 3.741119 & 14.75473 & 2.966169 & 14.7585 & 6807.25 & 6465.694 & 70.6 & 70.4 & 69.2 & 69.8 & 0.001242 \\
\hline 380.02 & 3.747372 & 14.75473 & 2.96742 & 14.76 & 6807.5 & 6468.922 & 70.6 & 70.4 & 69.2 & 69,9 & 0.001243 \\
\hline 385.02 & 3.739868 & 14.75398 & 2.966794 & 14.75925 & 6807.5 & 6475.875 & 70.6 & 70.5 & 69.2 & 69.9 & 0.001243 \\
\hline 390.02 & 3.733616 & 14.75473 & 2.966169 & 14.75925 & 6817.75 & 6502.196 & 70.6 & 70.5 & 69.3 & 69.9 & 0.001244 \\
\hline 395.02 & 3.747372 & 14.75473 & 2.966794 & 14.75925 & 6817.75 & 6514.859 & 70.6 & 70.5 & 69.4 & 69.9 & 0.001245 \\
\hline 400.02 & 3.736742 & 14.75398 & 2.966794 & 14.7585 & 6817.75 & 6516.846 & 70.5 & 70.5 & 69.4 & 69.9 & 0.001245 \\
\hline 405.05 & 3.756751 & 14.75548 & 2.966169 & 14.75925 & 6822 & 6516.598 & 70.6 & 70.5 & 69.3 & 69.9 & 0.001245 \\
\hline 410.02 & 3.734241 & 14.75248 & 2.966169 & 14.7585 & 6828 & 6514.859 & 70.7 & 70.5 & 69.4 & 69.8 & 0.001245 \\
\hline 415.02 & 3.746746 & 14.75398 & 2.965544 & 14.75925 & 6817.75 & 6502.444 & 70.8 & 70.5 & 69.5 & 69.9 & 0.001244 \\
\hline 420.02 & 3.752374 & 14.75398 & 2.966794 & 14.75775 & 6827.25 & 6511.135 & 70.8 & 70.5 & 69.3 & 69.8 & 0.001245 \\
\hline 425.02 & 3.738618 & 14.75473 & 2.966169 & 14.75925 & 6827.75 & 6512.625 & 70.8 & 70.5 & 69.4 & 69.9 & 0.001245 \\
\hline 430.02 & 3.741119 & 14,75473 & 2.964293 & 14.75925 & 6828 & 6505.175 & 70.8 & 70.5 & 69.3 & 69.8 & 0.001245 \\
\hline 435.02 & 3.749873 & 14.75548 & 2.965544 & 14.75925 & 6827.75 & 6496.733 & 70.8 & 70.5 & 69.3 & 69.8 & 0.001245 \\
\hline 440.02 & 3.7555 & 14.75473 & 2966169 & 14.7585 & 6827.25 & 6504.182 & 70.7 & 70.5 & 69.4 & 69.9 & 0.001245 \\
\hline 445.04 & 3.751123 & 14.75623 & 2.966169 & 14.75925 & 6827.75 & 6519.577 & 70.8 & 70.5 & 69.4 & 69.8 & 0.001245 \\
\hline 450.02 & 3.741119 & 14.75473 & 2.966794 & 14.7585 & 6827.75 & 6498.968 & 70.8 & 70.6 & 69.4 & 69.9 & 0.001245 \\
\hline 455.06 & 3.738618 & 14.75623 & 2.966169 & 14.7585 & 6828 & 6477.117 & 70.8 & 70.6 & 69.4 & 69.9 & 0.001245 \\
\hline 460.02 & 3.744871 & 14.75548 & 2.965544 & 14.75925 & 6828.25 & 6491.022 & 70.7 & 70.5 & 69.5 & 69.9 & 0.001247 \\
\hline 465.02 & 3.751749 & 14.75473 & 2.963667 & 14.75925 & 6834.75 & 6489.035 & 70.7 & 70.6 & 69.4 & 69.9 & 0.001246 \\
\hline 470.02 & 3.751123 & 14.75548 & 2.962416 & 14.75925 & 6838 & 6493.753 & 70.6 & 70.6 & 69.4 & 69.9 & 0.001246 \\
\hline 475.04 & 3.742369 & 14.75323 & 2.966794 & 14.75925 & 6837.5 & 6485.807 & 70.7 & 70.6 & 69.3 & 69.9 & 0.001247 \\
\hline
\end{tabular}




\begin{tabular}{|c|c|c|c|c|c|c|c|c|c|c|c|}
\hline SECONDS & MAIN DUCT & MAIN DUCT & GAS INJECT & GAS INJECT & NEWPORT & SMC & AMBIENT & GAS INJECT & MAIN DUCT & WEC CELL & WEC CELL \\
\hline & DIFF (IN H2O) & ABS (PSIA) & DIFF (IN H2O) & ABS (PSIA) & (PPM) & (PPM) & TEMP. (F) & TEMP. (F) & TEMP. (F) & TEMP. (F) & (NOLTS) \\
\hline 0.009979 & 3.746746 & 14.75398 & 0.217298 & 14.655 & 51.225 & -3.32493 & 70.4 & 69.9 & 69.5 & 69.8 & 0.00024 \\
\hline 480.02 & 3.748622 & 14.75173 & 2.966169 & 14.7585 & 6837.75 & 6486.056 & 70.6 & 70.6 & 69.4 & 69.8 & 0.001247 \\
\hline 485.02 & 3.741744 & 14.75173 & 2.965544 & 14.7585 & 6837.25 & 6496.485 & 70.7 & 70.6 & 69.4 & 69.9 & 0.001247 \\
\hline 490.02 & 3.739868 & 14.75323 & 2.961791 & 14.75925 & 6837.75 & 6495.988 & 70.7 & 70.6 & 69.4 & 69.8 & 0,001246 \\
\hline 495.08 & 3.754875 & 14.75473 & 2.966169 & 14.7585 & 6837.75 & 6517.342 & 70.7 & 70.6 & 69.4 & 69.9 & 0.001246 \\
\hline 500.02 & 3.729239 & 14.75398 & 2.963042 & 14.7585 & 6838 & 6479.103 & 70.7 & 70.6 & 69.3 & 69.8 & 0.001247 \\
\hline 505.1 & 3.735491 & 14.75323 & 2.966169 & 14.7585 & 6837.75 & 6476.123 & 70.7 & 70.6 & 69.4 & 69.9 & 0.001247 \\
\hline 510.02 & 3.725487 & 14.75473 & 2.966169 & 14.75925 & 6838 & 6482.828 & 70.8 & 70.6 & 69.3 & 69.9 & 0.001247 \\
\hline 515.02 & 3.727988 & 14.75548 & 2.966794 & 14.75925 & 6847.5 & 6493.753 & 70.8 & 70.6 & 69.4 & 69.9 & 0.001247 \\
\hline 520.02 & 3.736117 & 14.75473 & 2.966169 & 14.75925 & 6847.75 & 6494.995 & 70.8 & 70.6 & 69.3 & 69.9 & 0.001247 \\
\hline 525.02 & 3.748622 & 14.75398 & 2.965544 & 14.7585 & 6848 & 6515.356 & 70.8 & 70.6 & 69.3 & 69.9 & 0.001249 \\
\hline 530.05 & 3.736742 & 14.75173 & 2.966794 & 14.75925 & 6847.75 & 6491.518 & 70.7 & 70.6 & 69.2 & 69.8 & 0.001249 \\
\hline 535.02 & 3.746746 & 14.75248 & 2.966794 & 14.7585 & 6858 & 6483.821 & 70.8 & 70.6 & 6.4 & 69.9 & 0.00125 \\
\hline 540.02 & 3.7555 & 14.75473 & 2.966169 & 14.76 & 6858 & 6451.044 & 70.8 & 70.6 & 69.4 & 69.9 & 0.00125 \\
\hline 545.05 & 3.719234 & 14.75098 & 2.96742 & 14.75925 & 6858.25 & 6448.313 & 70.9 & 70.6 & 6.5 & 69.9 & 0.00125 \\
\hline 550.02 & 3.733616 & 14.75548 & 2.966794 & 14.75925 & 6857.75 & 6464.453 & 70.8 & 70.7 & 69.4 & 69.9 & 0.00125 \\
\hline 555.02 & 3.725487 & 14.75473 & 2.966169 & 14.75925 & 6858 & 6476.372 & 70.9 & 70.6 & 69.3 & 69.9 & 0.001251 \\
\hline 560.03 & 3.759252 & 14.75098 & 2.966794 & 14.7585 & 6858.25 & 6489.284 & 70.9 & 70.6 & 69.5 & 69.8 & 0.001251 \\
\hline 565.03 & 3.748622 & 14.75398 & 2.966169 & 14.7585 & 6867.75 & 6483.076 & 70.8 & 70.6 & 69.4 & 69.9 & 0.001251 \\
\hline 570.02 & 3.752999 & 14.75473 & 2966794 & 14.76 & 6868 & 6496.236 & 70.8 & 70.7 & 69.4 & 69.9 & 0.001252 \\
\hline 575.05 & 3.739868 & 14.75248 & 2.968045 & 14.75925 & 6868.25 & 6511.631 & 70.8 & 70.7 & 69.4 & 69.9 & 0.001251 \\
\hline 580.02 & 3.734241 & 14.75549 & 2.96742 & 14.7585 & 6867.5 & 6517.342 & 70.8 & 70.7 & 69.3 & 69.9 & 0.001251 \\
\hline 585.02 & 3.738618 & 14.75398 & 2.966794 & 14.75925 & 6868 & 6499.713 & 70.8 & 70.7 & 69.4 & 69.9 & 0.001251 \\
\hline 590.02 & 3.740494 & 14.75398 & 2.968045 & 14.75925 & 6868 & 6511.135 & 70.7 & 70.7 & 69.3 & 69.9 & 0.001251 \\
\hline 595.02 & 3.752999 & 14.75398 & 2.966794 & 14.75925 & 6867.75 & 6509.397 & 70.7 & 70.7 & 69.3 & 69.9 & 0.001252 \\
\hline 600.02 & 3.753624 & 14.75473 & 2.966794 & 14.75925 & 6868.25 & 6524.543 & 70.7 & 70.7 & 69.4 & 69.9 & 0.001252 \\
\hline 605.03 & 3.738618 & 14.75173 & 2.965544 & 14.75775 & 6867.75 & 6500.706 & 70.7 & 70.7 & 69.4 & 69.9 & 0.001253 \\
\hline 610.07 & 3.753624 & 14.75323 & 2.966169 & 14.75925 & 6877.75 & 6500.706 & 70.7 & 70.7 & 69.3 & 69.9 & 0.001254 \\
\hline 615.02 & 3.761128 & 14.75323 & 2.965544 & 14.75925 & 6877.75 & 6497.974 & 70.7 & 70.7 & 69.4 & 69.9 & 0.001254 \\
\hline 620.02 & 3.751749 & 14.75323 & 2.966169 & 14.75925 & 6878.25 & 6488.042 & 70.7 & 70.7 & 69.4 & 69.8 & 0.001254 \\
\hline 625.02 & 3.736742 & 14.75248 & 2.965544 & 14.75925 & 6884.75 & 6486.056 & 70.7 & 70.7 & 69.4 & 69.8 & 0.001254 \\
\hline 630.02 & 3.746746 & 14.75323 & 2.966169 & 14.75925 & 6888 & 6513.121 & 70.8 & 70.7 & 69.4 & 69.9 & 0.001254 \\
\hline 635.05 & 3.742369 & 14.75323 & 2.966794 & 14.75925 & 6887.75 & 6500.706 & 70.7 & 70.7 & 69.5 & 69.9 & 0,001254 \\
\hline 640.07 & 3.754875 & 14.75398 & 2.966169 & 14.75925 & 6887.5 & 6477.613 & 70.8 & 70.7 & 69.4 & 69.9 & 0.001254 \\
\hline
\end{tabular}




\begin{tabular}{|c|c|c|c|c|c|c|c|c|c|c|c|}
\hline SECONDS & MAIN DUCT & MAIN DUCT & GAS INJECT & GAS INJECT & NEWPORT & SMC & AMBIENT & GAS INJECT & MAIN DUCT & WWEC CELL & WEC CELL \\
\hline & DIFF (IN H2O) & ABS (PSIA) & DIFF (IN H2O) & ABS (PS|A) & (PPM) & (PPM) & TEMP. (F) & TEMP. (F) & TEMP. $(F)$ & TEMP. (F) & (NOLTS) \\
\hline 0.009979 & 3.746746 & 14.75398 & 0.217298 & 14.655 & 51.225 & -3.32493 & 70.4 & 69.9 & 69.5 & 69.8 & 0.00024 \\
\hline 645.02 & 3.746746 & 14.75398 & 2.966169 & 14.75925 & 6887.75 & 6487.546 & 70.8 & 70.7 & 69.4 & 69.9 & 0.001253 \\
\hline 650.02 & 3.745496 & 14.75473 & 2.965544 & 14.75925 & 6878.25 & 6494.498 & 70.8 & 70.7 & 69.5 & 69.9 & 0.001252 \\
\hline 655.02 & 3.751123 & 14.75473 & 2,966169 & 14.75925 & 6878.25 & 6475.627 & 70.8 & 70.7 & 69.5 & 69.9 & 0.001253 \\
\hline 660.02 & 3.761753 & 14.75398 & 2.966169 & 14.75925 & 6877.75 & 6486.801 & 70.8 & 70.7 & 69.5 & 69.9 & 0.001253 \\
\hline 665.02 & 3.730489 & 14.75098 & 2.966169 & 14.75925 & 6878.25 & 6489.532 & 70.8 & 70.8 & 69.5 & 69.9 & 0.001253 \\
\hline 670.02 & 3.77801 & 14.75323 & 2.966169 & 14.7585 & 6877.75 & 6499.464 & 70.8 & 70.7 & 69.4 & 69.9 & 0.001253 \\
\hline 675.02 & 3.744871 & 14.75398 & 2.966794 & 14.75925 & 6887.75 & 6490.277 & 70.8 & 70.7 & 69.5 & 69.9 & 0.001254 \\
\hline 680.09 & 3.768006 & 14.75323 & 2.966794 & 14.76 & 6888.25 & 6485.559 & 70.8 & 70.7 & 69.5 & 69.8 & 0.001252 \\
\hline 685.02 & 3.734866 & 14.75098 & 2.966794 & 14.7585 & 6883.5 & 6483.076 & 70.8 & 70.7 & 69.5 & 69.9 & 0.001253 \\
\hline 690.02 & $3.75800 t$ & 14.75323 & 2.966794 & 14.75925 & 6888 & 6477.365 & 70.7 & 70.8 & 69.4 & 69.9 & 0.001254 \\
\hline 695.1 & 3.739243 & 14.75398 & 2.96742 & 14.7585 & 6887.75 & 6486.304 & 70.7 & 70.8 & 69.4 & 69.9 & 0.001255 \\
\hline 700.04 & 3.737993 & 14.75473 & 2.966169 & 14.75925 & 6887.75 & 6485.311 & 70.8 & 70.8 & 69.4 & 69.9 & 0.001254 \\
\hline 705.02 & 3.732365 & 14.75248 & 2.966794 & 14.75925 & 6887.5 & 6470.909 & 70.8 & 70.8 & 69.4 & 69.9 & 0.001256 \\
\hline 710.02 & 3.742995 & 14.75323 & 2.966794 & 14.75925 & 6887.5 & 6481.586 & 70.9 & 70.8 & 69,4 & 69.9 & 0.001255 \\
\hline 715.06 & 3.751123 & 14.75398 & 2.966169 & 14.75775 & 6887.75 & 6494.002 & 70.9 & 70.8 & 69.4 & 69.9 & 0.001255 \\
\hline 720.02 & 3.744245 & 14.75173 & 2.96742 & 14.7585 & 6898 & 6487.546 & 70.8 & 70.8 & 69.4 & 69.9 & 0.001255 \\
\hline 725.02 & 3.764254 & 14.75398 & 2.968045 & 14.7585 & 6897.75 & 6457.997 & 70.8 & 70.8 & 69.5 & 69.9 & 0.001256 \\
\hline 730.02 & 3.729864 & 14.75098 & 2.964918 & 14.75925 & 6898 & 6466.439 & 70.7 & 70.8 & 69.5 & 69.9 & 0.001256 \\
\hline 735.02 & 3.750498 & 14.75323 & 2.9667994 & 14.75925 & 6898.25 & 6487.297 & 70.7 & 70.8 & 69.4 & 69.9 & 0.001257 \\
\hline 740.02 & 3.738618 & 14.75308 & 2.968671 & 14.75925 & 6897.75 & 6483.821 & 70.7 & 70.8 & 69.4 & 69.8 & 0.001256 \\
\hline 745.06 & 3.739868 & 14.75023 & 2.968671 & 14.75925 & 6898 & 6482.083 & 70.7 & 70.8 & 69.4 & 69.9 & 0.001257 \\
\hline 750.02 & 3.750498 & 14.75323 & 2.968671 & 14.75925 & 6898 & 6470.412 & 70.7 & 70.8 & 69.4 & 69.8 & 0.001256 \\
\hline 755.02 & 3.742369 & 14.75398 & 2.968671 & 14.75925 & 6898.25 & 6477.862 & 70.7 & 70.8 & 69.4 & 69.9 & 0.001256 \\
\hline 760.06 & 3.726112 & 14.75323 & 2.969296 & 14.7585 & 6908.25 & 6489.78 & 70.5 & 70.8 & 69.4 & 69.9 & 0.001258 \\
\hline 765.08 & 3.748622 & 14.75173 & 2.968045 & 14.76 & 6908.25 & 6480.593 & 70.4 & 70.8 & 69.3 & 69.9 & 0.001258 \\
\hline 770.02 & 3.741119 & 14.75473 & 2.969296 & 14.75925 & 6908 & 6505.92 & 70.4 & 70.8 & 69.4 & 69.9 & 0.001257 \\
\hline 775.03 & 3.744871 & 14.75023 & 2.969296 & 14.75925 & 6908 & 6489.532 & 70.4 & 70.8 & 69.4 & 69.8 & 0.001257 \\
\hline 780.02 & 3.740494 & 14.75248 & 2.968671 & 14.7585 & 6908.25 & 6482.579 & 70.4 & 70.8 & 69.4 & 69.9 & 0.001257 \\
\hline 785.02 & 3.73174 & 14.75173 & 2.968671 & 14.7585 & 6907.75 & 6467.433 & 70.4 & 70.8 & 69.4 & 69.9 & 0.001257 \\
\hline 790.03 & 3.744871 & 14.75173 & 2.968045 & 14.75925 & 6907.75 & 6471.405 & 70.4 & 70.8 & 69.4 & 69.8 & 0.001256 \\
\hline 795.02 & 3.741744 & 14.75173 & 2.970547 & 14.75925 & 6908.25 & 6453.279 & 70.5 & 70.8 & 69.3 & 69.8 & 0.001256 \\
\hline 800.02 & 3.736117 & 14.75323 & 2.968671 & 14.76075 & 6907.75 & 6485.062 & 70.5 & 70.9 & 69.4 & 69.9 & 0.001256 \\
\hline 805.03 & 3.749247 & 14.75173 & 2.970547 & 14.75925 & 6898 & 6489.78 & 70.6 & 70.8 & 69.4 & 69.9 & 0.001255 \\
\hline
\end{tabular}




\begin{tabular}{|c|c|c|c|c|c|c|c|c|c|c|c|}
\hline SECONDS & MAIN DUCT & MAIN DUCT & GAS INJECT & GAS INJECT & NEWPORT & SMC & AMBIENT & GAS INJECT & MAIN DUCT & WEC CELL & WEC CELL \\
\hline & DIFF (IN H2O) & ABS (PSIA) & DIFF (IN H2O) & ABS (PS|A) & (PPM) & (PPM) & TEMP. (F) & TEMP. (F) & TEMP. (F) & TEMP. (F) & (VOLTS) \\
\hline 0.009979 & 3.746746 & 14.75398 & 0.217298 & 14.655 & 51.225 & -3.32493 & 70.4 & 69.9 & 69.5 & 69.8 & 0.00024 \\
\hline 810.02 & 3.748622 & 14.75548 & 2.969922 & 14.75775 & 6898 & 6505.672 & 70.6 & 70.8 & 69.5 & 69.9 & 0.001257 \\
\hline 815.02 & 3.736117 & 14.75248 & 2.968671 & 14.75925 & 6898 & 6504.43 & 70.6 & 70.8 & 69.4 & 70 & 0.001256 \\
\hline 820.03 & 3.7555 & 14.75548 & 2.971172 & 14.75925 & 6897.75 & 6508.403 & 70.7 & 70.8 & 69.4 & 69.9 & 0.001256 \\
\hline 825.02 & 3.768006 & 14.75473 & 2.970547 & 14.7585 & 6908.5 & 6493.505 & 70.8 & 70.8 & 69.4 & 69.9 & 0.001257 \\
\hline 830.02 & 3.741119 & 14.75173 & 2.971172 & 14.75925 & 6908.25 & 6470.909 & 70.9 & 70.8 & 69.5 & 69.8 & 0.001257 \\
\hline 835.04 & 3.757376 & 14.75548 & 2.971172 & 14.75925 & 6908.25 & 6472.647 & 70.9 & 70.8 & 69.5 & 69.9 & 0.001257 \\
\hline 840.1 & 3.747997 & 14.75248 & 2.969922 & 14.7585 & 6908.25 & 6498.719 & 70.8 & 70.9 & 69.5 & 69.9 & 0.001257 \\
\hline 845.02 & 3.736742 & 14.75248 & 2.971172 & 14.7585 & 6908 & 6498.223 & 70.9 & 70.9 & 69.4 & 69.9 & 0.001258 \\
\hline 850.03 & 3.738618 & 14.75623 & 2.970547 & 14.7585 & 6908.25 & 6494.498 & 70.9 & 70.9 & 69.5 & 69.9 & 0.001257 \\
\hline 855.02 & 3.725487 & 14.75098 & 2.971798 & 14.76 & 6908.25 & 6475.13 & 70.9 & 70.9 & 69.4 & 69.9 & 0.001259 \\
\hline 860.02 & 3.732365 & 14.75248 & 2.973049 & 14.75925 & 6918.25 & 6476.868 & 70.8 & 70.9 & 69.4 & 69.9 & 0.001258 \\
\hline 865.02 & 3.737993 & 14.75173 & 2.972423 & 14.7585 & 6918 & 6470.164 & 70.9 & 70.8 & 69.4 & 69.9 & 0.001259 \\
\hline 870.04 & 3.751749 & 14.75173 & 2.971798 & 14.7585 & 6917.75 & 6476.62 & 70.9 & 70.9 & 69.5 & 69.9 & 0.00126 \\
\hline 875.02 & 3.748622 & 14.75398 & 2.973049 & 14.75925 & 6918.25 & 6494.002 & 70.9 & 70.9 & 69.4 & 69.9 & 0.00126 \\
\hline 880.02 & 3.73174 & 14.75248 & 2.971798 & 14.7585 & 6928.25 & 6497.23 & 70.9 & 70.9 & 69.4 & 69.9 & 0.001259 \\
\hline 885.02 & 3.775509 & 14.75473 & 2.972423 & 14.75925 & 6928 & 6471.405 & 70.8 & 70.9 & 69.4 & 69.9 & 0.00126 \\
\hline 890.02 & 3.734866 & 14.75248 & 2.972423 & 14.7585 & 6928 & 6477.862 & 70.7 & 70.9 & 69.4 & 69.9 & 0.00126 \\
\hline 895.04 & 3.744871 & 14.75623 & 2.973674 & 14.7585 & 6927.75 & 6493.008 & 70.8 & 70.9 & 69.5 & 69.9 & 0.00126 \\
\hline 900.03 & 3.73174 & 14.75248 & 2.972423 & 14.7585 & 6927.75 & 6484.566 & 70.8 & 70.9 & 69.5 & 69.9 & 0.001261 \\
\hline 905.02 & 3.745496 & 14.75173 & 2.973049 & 14.75925 & 6928 & 6484.566 & 70.8 & 70.9 & 69.5 & 69.9 & 0.00126 \\
\hline 910.03 & 3.737993 & 14.75398 & 2.972423 & 14.7585 & 6928 & 6481.09 & 70.8 & 70.9 & 69.4 & 69.9 & 0.001261 \\
\hline 915.05 & 3.756125 & 14.75473 & 2.972423 & 14.75925 & 6928.25 & 6472.647 & 70.8 & 70.9 & 69.4 & 69.9 & 0.001261 \\
\hline 920.02 & 3.723611 & 14.75323 & 2.972423 & 14.75925 & 6928.5 & 6481.09 & 70.8 & 70.9 & 69.4 & 69.9 & 0.001262 \\
\hline 925.04 & 3.732365 & 14.75173 & 2.973049 & 14.75925 & 6928.5 & 6487.794 & 70.8 & 70.9 & 69.3 & 69.9 & 0.00126 \\
\hline
\end{tabular}




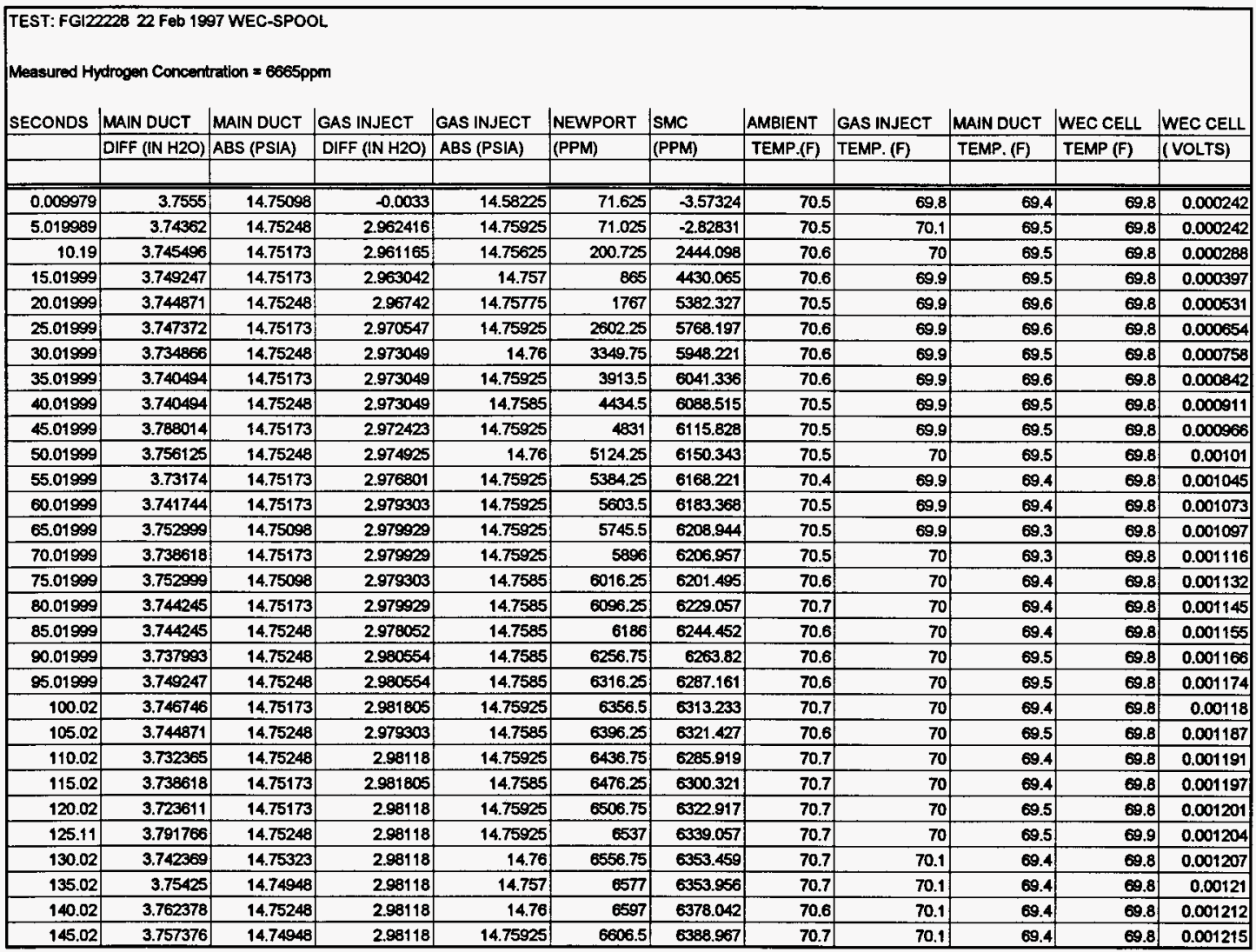




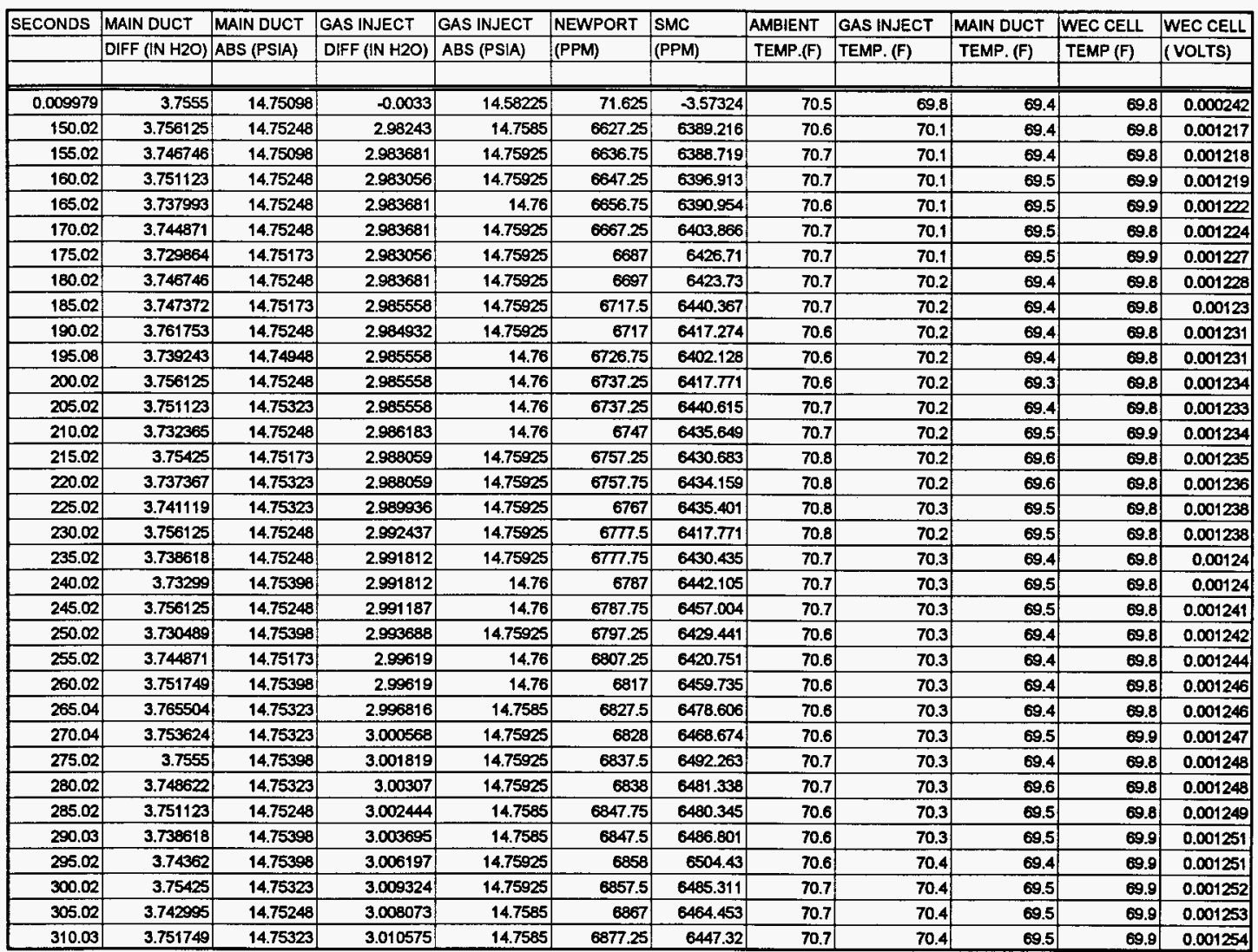




\begin{tabular}{|c|c|c|c|c|c|c|c|c|c|c|c|}
\hline SECONDS & MAIN DUCT & MAIN DUCT & GAS INJECT & GAS INJECT & NEWPORT & SMC & AMBIENT & GAS INJECT & MAIN DUCT & WEC CELL & WEC CELL \\
\hline & DIFF (IN H2O) & ABS (PSIA) & DIFF (IN H2O) & ABS (PS|A) & (PPM) & (PPM) & TEMP.(F) & TEMP. (F) & TEMP. (F) & TEMP (F) & (VOLTS) \\
\hline 0.009979 & 3.7555 & 14.75098 & -0.0003 & 14.58225 & 71.625 & -3.57324 & 70.5 & 69.8 & 69.4 & 69.8 & 0.000242 \\
\hline 315.02 & 3.7555 & 14.75398 & 3.013077 & 14.7585 & 6877.75 & 6461.97 & 70.7 & 70.4 & 69.5 & 69.9 & 0.001254 \\
\hline 320.03 & 3.76613 & 14.75248 & 3.014953 & 14.75925 & 6887.5 & 6497.23 & 70.7 & 70.4 & 69.5 & 69.8 & 0.001256 \\
\hline 325.02 & 3.736742 & 14.75248 & 3.015579 & 14.76 & 6898 & 6527.026 & 70.7 & 70.4 & 69.5 & 69.8 & 0.001255 \\
\hline 330.03 & 3.751123 & 14.75398 & 3.017455 & 14.7585 & 6898 & 6534.972 & 70.8 & 70.4 & 69.4 & 69.9 & 0.001257 \\
\hline 340.02 & 3.742995 & 14.75323 & 3.021833 & 14.76 & 6908 & 6501.202 & 70.7 & 70.5 & 69.4 & 69.9 & 0.001259 \\
\hline 345.03 & 3.768631 & 14.75398 & 3.021208 & 14.7585 & 6917.75 & 6506.665 & 70.7 & 70.5 & 69.5 & 69.9 & 0.001259 \\
\hline 350.02 & 3.744245 & 14.75098 & 3.023709 & 14.7585 & 6927.75 & 6517.839 & 70.7 & 70.4 & 69.5 & 69.8 & 0.001261 \\
\hline 355.02 & 3.742995 & 14.75398 & 3.02496 & 14.7615 & 6927.75 & 6534.724 & 70.7 & 70.5 & 69.5 & 69.9 & 0.001262 \\
\hline 360.02 & 3.749247 & 14.75023 & 3.025586 & 14.75925 & 6937.5 & 6543.167 & 70.7 & 70.5 & 69.3 & 69.8 & 0,001262 \\
\hline 365.04 & 3.732365 & 14.74948 & 3.027462 & 14.7615 & 6938 & 6559.307 & 70.7 & 70.5 & 69.4 & 69.9 & 0.001263 \\
\hline 370.02 & 3.769881 & 14.75098 & 3.029964 & 14.75775 & 6947.25 & 6555.582 & 70.7 & 70.5 & 69.4 & 69.8 & 0.001262 \\
\hline 380.02 & 3.751749 & 14.75173 & 3.03184 & 14.7615 & 6947.75 & 6587.614 & 70.8 & 70.5 & 69.4 & 69.9 & 0.001264 \\
\hline 385.02 & 3.757376 & 14.75098 & 3.034967 & 14.75925 & 6957.75 & 6593.325 & 70.9 & 70.5 & 69.4 & 69.8 & 0,001264 \\
\hline 390.02 & 3.740494 & 14.75248 & 3.036218 & 14.76075 & 6957.75 & 6573.46 & 70.9 & 70.5 & 69.4 & 69.9 & 0.001266 \\
\hline 396.02 & 3.7555 & 14.74948 & 3.037469 & 14.7615 & 6968 & 6553.099 & 70.8 & 70.5 & 69.5 & 69.9 & 0.001267 \\
\hline 400.02 & 3.747372 & 14.74948 & 3.038095 & 14.763 & 6967.75 & 6575.943 & 70.8 & 70.5 & 69.4 & 69.9 & 0.001267 \\
\hline 405.02 & 3.732365 & 14.75023 & 3.039971 & 14.7615 & 6978 & 6587.862 & 70.8 & 70.5 & 69.5 & 69.9 & 0.001269 \\
\hline 410.02 & 3.737993 & 14.75173 & 3.044349 & 14.76375 & 6988 & 6593.076 & 70.8 & 70.6 & 69.4 & 69.9 & 0.00127 \\
\hline 415.02 & 3.737993 & 14.74874 & 3.044349 & 14.76375 & 6987.75 & 6615.424 & 70.8 & 70.6 & 69.6 & 69.9 & 0.001273 \\
\hline 420.02 & 3.747372 & 14.75248 & 3.046851 & 14.7645 & 6998 & 6613.438 & 70.8 & 70.6 & 69.5 & 69.9 & 0.001273 \\
\hline 425.02 & 3.774258 & 14.75248 & 3.048727 & 14.7645 & 7008 & 6601.271 & 70.8 & 70.6 & 69.6 & 69.9 & 0.001274 \\
\hline 430.02 & 3.744871 & 14.75398 & 3.048727 & 14.76375 & 7017.5 & 6617.411 & 70.8 & 70.6 & 69.6 & 69.9 & 0.001275 \\
\hline 435.09 & 3.738618 & 14.74948 & 3.051222 & 14.7645 & 7018 & 6629.578 & 70.8 & 70.6 & 69.4 & 69.8 & 0.001275 \\
\hline 440.02 & 3.749873 & 14.75023 & 3.050603 & 14.76375 & 7028 & 6623.618 & 70.7 & 70.6 & 69.4 & 69.9 & 0.001276 \\
\hline 445.05 & 3.761753 & 14.75248 & 3.065607 & 14.76375 & 7028 & 6652.67 & 70.7 & 70.6 & 69.5 & 69.9 & 0.001276 \\
\hline 450.02 & 3.760502 & 14.75023 & 3.055607 & 14.76375 & 7038 & 6621.632 & 70.7 & 70.6 & 69.5 & 69.9 & 0.001276 \\
\hline 465.02 & 3.732365 & 14.75398 & 3.061236 & 14.7645 & 7048 & 6649.691 & 70.8 & 70.7 & 69.5 & 69.9 & 0.001279 \\
\hline 470.02 & 3.746746 & 14.75248 & 3.061861 & 14.76525 & 7058.25 & 6635.537 & 70.8 & 70.6 & 69.5 & 69.9 & 0.00128 \\
\hline 475.02 & 3.745496 & 14.74874 & 3.063112 & 14.7645 & 7057.75 & 6643.731 & 70.8 & 70.7 & 69.4 & 69.8 & 0.001281 \\
\hline
\end{tabular}




\begin{tabular}{|c|c|c|c|c|c|c|c|c|c|c|c|}
\hline SECONDS & MAIN DUCT & MAIN DUCT & GAS INJECT & GAS INJECT & NEWPORT & SMC & AMBIENT & GAS INJECT & MAIN DUCT & WEC CELL & WEC CELL \\
\hline & DIFF (IN H2O) & ABS (PS|A) & DIFF (IN H2O) & ABS (PSIA) & (PPM) & (PPM) & TEMP.(F) & TEMP. (F) & TEMP. $(F)$ & TEMP (F) & (VOLTS) \\
\hline 0.009979 & 3.7555 & 14.75098 & -0.0033 & 14.58225 & 71.625 & 3.57324 & 70.5 & 69.8 & 69.4 & 69.8 & 0.000242 \\
\hline 480.02 & 3.753624 & 14.75173 & 3.065614 & 14.7645 & 7067.5 & 6662.106 & 70.7 & 70.6 & 69.4 & 69.8 & 0.001281 \\
\hline 485.02 & 3.7555 & 14.75023 & 3.06749 & 14.7645 & 7068.25 & 6661.113 & 70.7 & 70.7 & 69.6 & 69.8 & 0.001281 \\
\hline 490.02 & 3.808648 & 14.75248 & 3.068741 & 14.76675 & 7077.75 & 6656.643 & 70.7 & 70.7 & 69.6 & 69.9 & 0.001282 \\
\hline 495.02 & 3.761753 & 14.75323 & 3.071868 & 14.76675 & 7077.75 & 6677.005 & 70.7 & 70.7 & 69.5 & 69.9 & 0.001283 \\
\hline 500.02 & 3.739243 & 14.75023 & 3.071868 & 14.766 & 7078 & 6681.971 & 70.7 & 70.6 & 69.6 & 69.9 & 0.001283 \\
\hline 505.02 & 3.751123 & 14.75323 & 3.07437 & 14.76675 & 7078 & 6687.682 & 70.8 & 70.7 & 69.6 & 69.9 & 0.001284 \\
\hline 510.02 & 3.739868 & 14.75023 & 3.076246 & 14.7675 & 7088.25 & 6678.494 & 70.7 & 70.7 & 69.6 & 69.8 & 0.001285 \\
\hline 515.07 & 3.7555 & 14.75098 & 3.078123 & 14.766 & 7097.75 & 6685.199 & 70.7 & 70.7 & 69.5 & 69.9 & 0.001286 \\
\hline 520.02 & 3.745496 & 14.75098 & 3.079999 & 14.76675 & 7098.25 & 6717.727 & 70.7 & 70.7 & 69.6 & 69.9 & 0.001286 \\
\hline 525.02 & 3.742995 & 14.75023 & 3.079999 & 14.766 & 7108.25 & 6692.896 & 70.7 & 70.7 & 69.6 & 69.9 & 0.001287 \\
\hline 530.04 & 3.768006 & 14.75023 & 3.081875 & 14.76675 & 7108.25 & 6688.427 & 70.7 & 70.7 & 69.4 & 6.9 & 0.001288 \\
\hline 535.02 & 3.759877 & 14.75023 & 3.084377 & 14.766 & 7118.25 & 6705.063 & 70.7 & 70.7 & 69.6 & 69.8 & 0.00129 \\
\hline 545.04 & 3.760502 & 14.75098 & 3.086253 & 14.766 & 7127.75 & 6729.398 & 70.8 & 70.8 & 69.5 & 69.9 & 0.001291 \\
\hline 550.02 & 3.76738 & 14.75398 & 3.08813 & 14.766 & 7138.25 & 6724.928 & 70.8 & 70.7 & 69.5 & 69.9 & 0.001292 \\
\hline 555.02 & 3.746746 & 14.75098 & 3.090632 & 14.766 & 7148 & 6719.465 & 70.8 & 70.7 & 69.5 & 69.9 & 0.001294 \\
\hline 560.02 & 3.745496 & 14.75173 & 3.090632 & 14.766 & 7148 & 6734.612 & 70.8 & 70.8 & 69.5 & 69.9 & 0.001295 \\
\hline 565.04 & 3.751749 & 14.75398 & 3.093759 & 14.76675 & 7157.75 & 6732.874 & 70.8 & 70.8 & 69.6 & 69.9 & 0.001295 \\
\hline 570.02 & 3.737993 & 14.75023 & 3.09501 & 14.766 & 7158.5 & 6747.524 & 70.7 & 70.8 & 69.4 & 69.9 & 0.001296 \\
\hline 575.03 & 3.740494 & 14.75098 & 3.097511 & 14.766 & 7158 & 6747.524 & 70.6 & 70.8 & 69.6 & 69.8 & 0.001295 \\
\hline 580.05 & 3.759877 & 14.75173 & 3.096886 & 14.766 & 7168 & 6771.362 & 70.6 & 70.8 & 69.5 & 69.9 & 0.001296 \\
\hline 585.02 & 3.752374 & 14.75098 & 3.099388 & 14.76525 & 7168 & 6756.215 & 70.6 & 70.8 & 69.5 & 69.9 & 0.001297 \\
\hline 590.04 & 3.766755 & 14.75323 & 3.101889 & 14.766 & 7178.5 & 6768.878 & 70.6 & 70.8 & 69.5 & 69.9 & 0.001297 \\
\hline 595.02 & 3.749873 & 14.75248 & 3.10314 & 14.766 & 7178 & 6772.355 & 70.6 & 70.8 & 69.4 & 69.9 & 0.001299 \\
\hline 600.02 & 3.751123 & 14.75023 & 3.10314 & 14.766 & 7188.5 & 6787.75 & 70.6 & 70.8 & 69.4 & 69.9 & 0.0013 \\
\hline 605.05 & 3.724237 & 14.75173 & 3.105017 & 14.7675 & 7188.5 & 6770.368 & 70.7 & 70.8 & 69.4 & 69.9 & 0.0013 \\
\hline 610.09 & 3.742369 & 14.75098 & 3.109395 & 14.76675 & 7198 & 6776.079 & 70.7 & 70.8 & 69.4 & 69.9 & 0.001301 \\
\hline 615.02 & 3.742995 & 14.74948 & 3.108769 & 14.766 & 7198.25 & 6764.657 & 70.8 & 70.8 & 69.6 & 69.9 & 0.001302 \\
\hline 630.02 & 3.741119 & 14.75023 & 3.113773 & 14.766 & 7218 & 6794.703 & 70.8 & 70.8 & 69.5 & 69.9 & 0.001305 \\
\hline 635.02 & 3.742995 & 14.75098 & 3.116275 & 14.766 & 7218.5 & 6804.883 & 70.8 & 70.8 & 69.5 & 69.9 & 0.001304 \\
\hline 640.02 & 3.742369 & 14.74948 & 3.116275 & 14.766 & 7228 & 6812.084 & 70.8 & 70.8 & 69.4 & 69.8 & 0.001306 \\
\hline
\end{tabular}




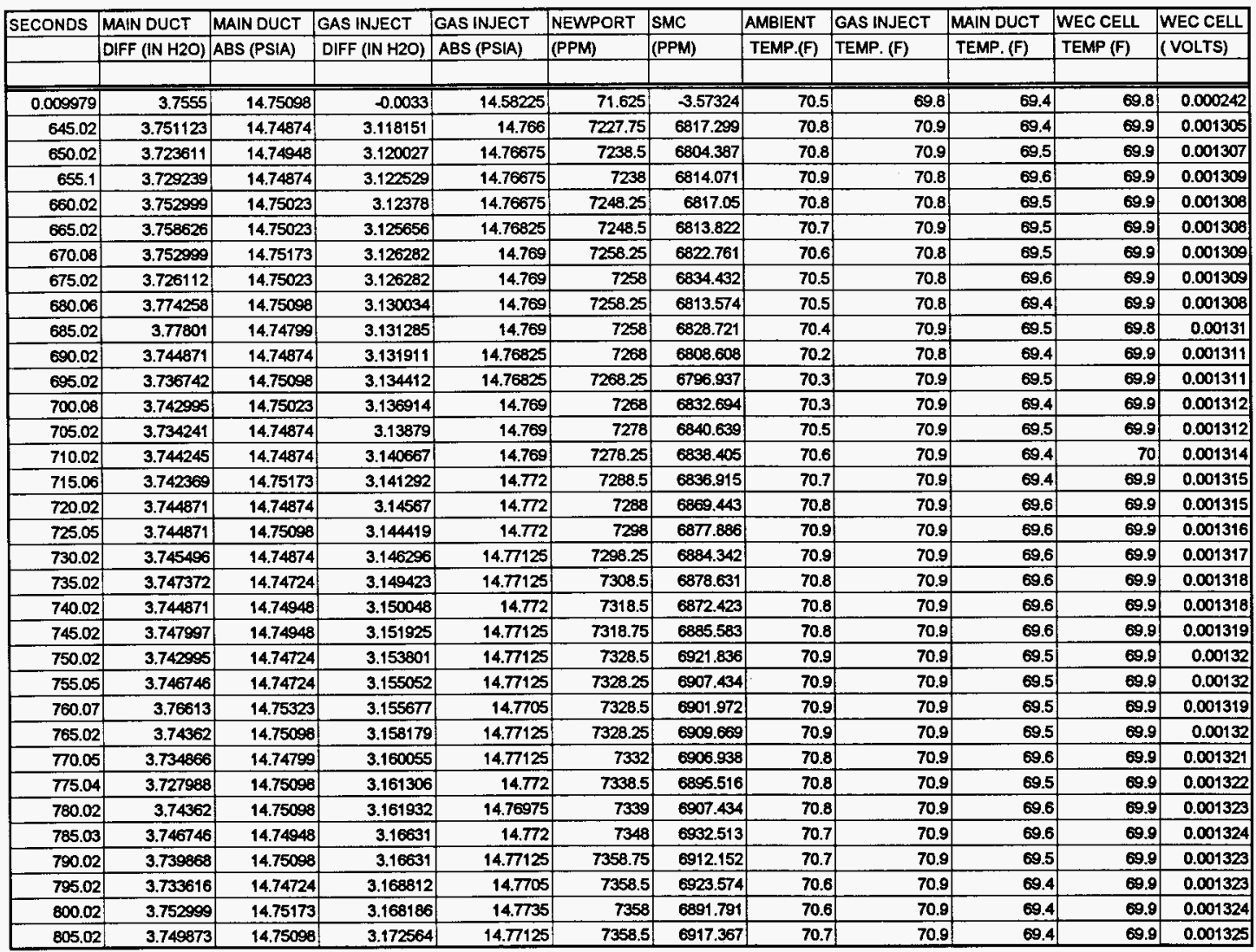




\begin{tabular}{|c|c|c|c|c|c|c|c|c|c|c|c|}
\hline SECONDS & MAIN DUCT & MAIN DUCT & GAS INJECT & GAS INJECT & NEWPORT & SMC & AMBIENT & GAS INJECT & MAIN DUCT & WEC CELL & WEC CELL \\
\hline & DIFF (IN H2O) & ABS (PS|A) & DIFF (IN H2O) & ABS (PSIA) & (PPM) & (PPM) & TEMP.(F) & TEMP. (F) & TEMP. ( $F$ ) & TEMP (F) & (VOLTS) \\
\hline & & & & & & & & & & & \\
\hline 0.009979 & 3.7555 & 14.75098 & -0.0033 & 14.58225 & 71.625 & -3.57324 & 70.5 & 69.8 & 69.4 & 69.8 & 0.000242 \\
\hline 810.02 & 3.742995 & 14.74874 & 3.17319 & 14.77125 & 7368.25 & 6929.037 & 70.8 & 70.9 & 69.5 & 69.9 & 0.001326 \\
\hline 815.06 & 3.744871 & 14.74948 & 3.17444 & 14.77425 & 7368.25 & 6936.486 & 70,8 & 70.9 & 69.5 & 69.9 & 0.001328 \\
\hline 820.07 & 3.746746 & 14.74799 & 3.176317 & 14.77125 & 7378.75 & 6942.942 & 70.8 & 70.9 & 69.5 & 70 & 0.001328 \\
\hline 825.02 & 3.772382 & 14.75023 & 3.175066 & 14.7735 & 7388.5 & 6944.184 & 70.7 & 70.9 & 69.6 & 69.9 & 0.001329 \\
\hline 830.07 & 3.749247 & 14.74724 & 3.178193 & 14.77425 & 7388.5 & 6935.245 & 70.7 & 70.9 & 69.6 & 69.9 & 0.00133 \\
\hline 835.02 & 3.76738 & 14.74948 & 3.179444 & 14.7735 & 7398.25 & 6950.888 & 70.6 & 70.9 & 69.5 & 69.9 & 0.001332 \\
\hline 840.02 & 3.748622 & 14.74649 & 3.183822 & 14.7735 & 7398.75 & 6932.513 & 70.6 & 71 & 69.5 & 70 & 0.001332 \\
\hline 845.04 & 3.751749 & 14.74649 & 3.185698 & 14.7735 & 7408.5 & 6946.915 & 70.6 & 71 & 69.5 & 69.9 & 0.001331 \\
\hline 850.07 & 3.749247 & 14.74874 & 3.185073 & 14.77275 & 7408.5 & 6962.31 & 70.6 & 71 & 69.5 & 69.9 & 0.001332 \\
\hline 855.02 & 3.745496 & 14.74874 & 0.00446 & 14.5815 & 7419 & 6960.821 & 70.6 & 70.9 & 69.4 & 69.9 & 0.001333 \\
\hline 860.03 & 3.7555 & 14.74649 & 3.094384 & 14.7585 & 7389 & 5040.655 & 70.6 & 70.9 & 69.4 & 69.9 & 0.001309 \\
\hline 865.07 & 3.734866 & 14.74799 & 3.201334 & 14.7765 & 6818.75 & 3504.622 & 70.6 & 71 & 69.5 & 69.9 & 0.001213 \\
\hline
\end{tabular}




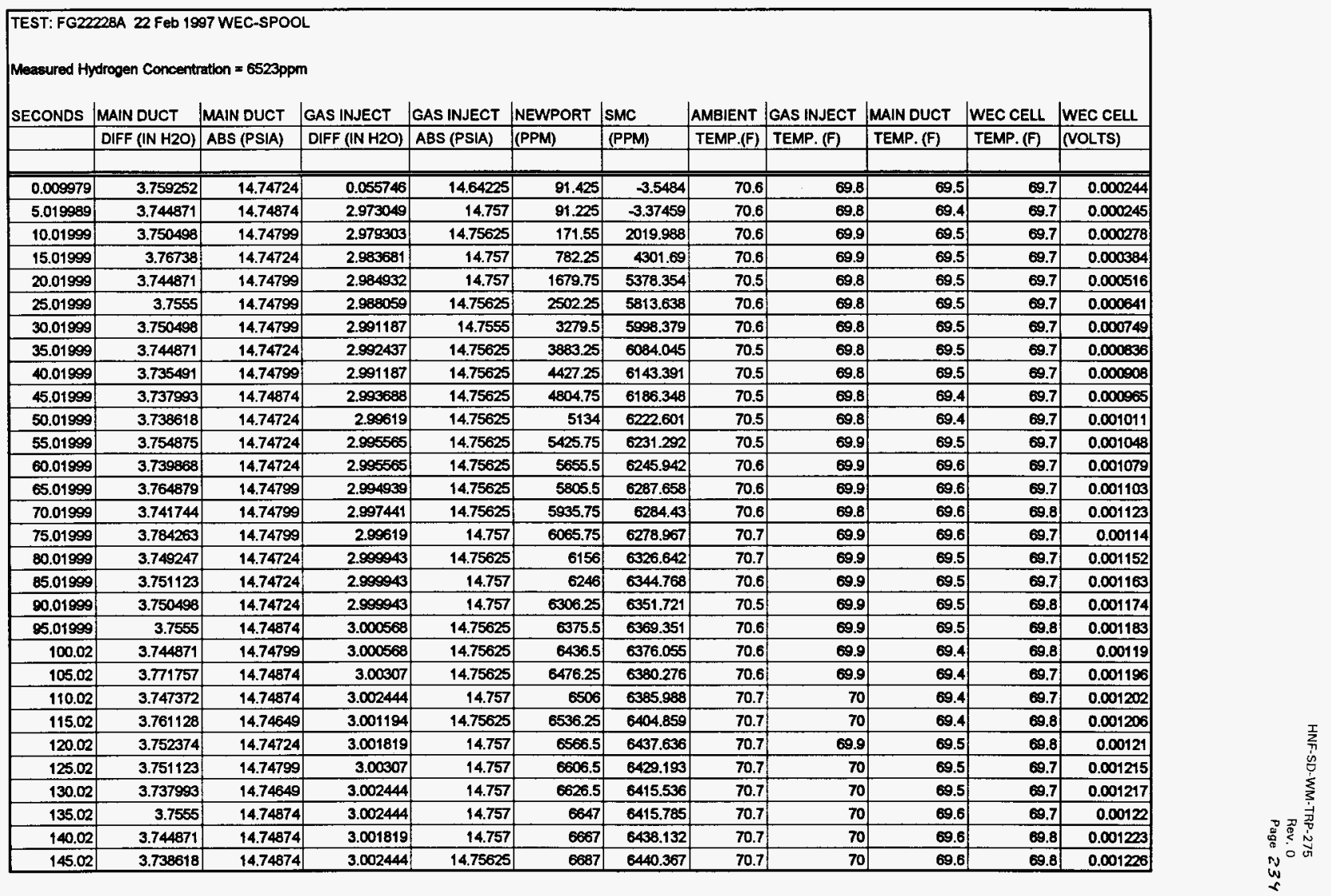




\begin{tabular}{|c|c|c|c|c|c|c|c|c|c|c|c|}
\hline SECONDS & MAIN DUCT & MAIN DUCT & GAS INJECT & GAS INJECT & NEWPORT & SMC & AMBIENT & GAS INJECT & MAIN DUCT & WEC CELL & WEC CELL \\
\hline & DIFF (IN H2O) & ABS (PSIA) & DIFF (IN H2O) & ABS (PSIA) & (PPM) & (PPM) & TEMP.(F) & TEMP. (F) & TEMP. (F) & TEMP. (F) & (NOLTS) \\
\hline & & & & & & & & & & & \\
\hline 0.009979 & 3.759252 & 14.74724 & 0.055746 & 14.64225 & 91.425 & -3.5484 & 70.6 & 69.8 & 69.5 & 69.7 & 0.000244 \\
\hline 150.02 & 3.776134 & 14.74724 & 3.002444 & 14.757 & 6697 & 6453.527 & 70.7 & 70 & 69.6 & 69.7 & 0.001228 \\
\hline 155.02 & 3.784888 & 14.74874 & 3.001819 & 14.7555 & 6717 & 6456.259 & 70.7 & 70 & 69.5 & 69.8 & 0.00123 \\
\hline 160.02 & 3.756125 & 14.74948 & 3.002444 & 14.7555 & 6726.75 & 6465.198 & 70.7 & 70 & 69.5 & 69.8 . & 0.001233 \\
\hline 165.02 & 3.735491 & 14.74948 & 3.001819 & 14.75625 & 6747 & 6461.473 & 70.7 & 70.1 & 69.5 & 69.8 & 0.001235 \\
\hline 170.02 & 3.744871 & 14.74874 & 3.002444 & 14.757 & 6757 & 6454.521 & 70.5 & 70 & 69.5 & 69.8 & 0.001238 \\
\hline 175.02 & 3.7555 & 14.74724 & 3.004321 & 14.757 & 6767.25 & 6455.514 & 70.6 & 70 & 69.4 & 69.7 & 0.001239 \\
\hline 180.02 & 3.759252 & 14.74649 & 3.003695 & 14.7555 & 6776.75 & 6493.008 & 70.6 & 70.1 & 69.5 & 69.8 & 0.00124 \\
\hline 185.02 & 3.744245 & 14.74649 & 3.004321 & 14.75625 & 6787.5 & 6497.478 & 70.6 & 70.1 & 69.5 & 69.7 & 0.001241 \\
\hline 190.02 & 3.740494 & 14.74724 & 3.004946 & 14.75775 & 6796.75 & 6482.331 & 70.6 & 70.1 & 69.6 & 69.8 & 0.001243 \\
\hline 195,02 & 3.757376 & 14.74874 & 3.004946 & 14.75625 & 6807.25 & 6487.049 & 70.7 & 70.1 & 69.6 & 69.7 & 0.001244 \\
\hline 200,02 & 3.749247 & 14.74724 & 3.004321 & 14.75625 & 6817 & 6498.968 & 70.7 & 70.1 & 69.6 & 69.7 & 0.001244 \\
\hline 205.08 & 3.7555 & 14.74799 & 3.006823 & 14.757 & 6827.25 & 6506.417 & 70.7 & 70.1 & 69.4 & 69.8 & 0.001246 \\
\hline 215.02 & 3.7555 & 14.74724 & 3.006197 & 14.75775 & 6837.25 & 6499.216 & 70.8 & 70.2 & 69.6 & 69.8 & 0.001248 \\
\hline 220.02 & 3.732365 & 14.74724 & 3.006197 & 14.757 & 6837 & 6507.658 & 70.8 & 70.2 & 69.6 & 69.8 & 0.001249 \\
\hline 225.02 & 3.742369 & 14.74799 & 3.006823 & 14.757 & 6847.5 & 6514.114 & 70.8 & 70.2 & 69.5 & 69.8 & 0.00125 \\
\hline 230.02 & 3.749247 & 14.74799 & 3.007448 & 14.757 & 6847.5 & 6534.972 & 70.8 & 70.2 & 69.5 & 69.8 & 0.001251 \\
\hline 235.02 & 3.75425 & 14.74724 & 3.006823 & 14.757 & 6857.25 & 6523.798 & 70.8 & 70.2 & 69.4 & 69.8 & $0.00125 t$ \\
\hline 240.02 & 3.750498 & 14.74724 & 3.008073 & 14.757 & 6857.5 & 6514.114 & 70.8 & 70.2 & 69.4 & 69.7 & 0.001251 \\
\hline 245.02 & 3.745496 & 14.74799 & 3.009324 & 14.757 & 6867.5 & 6511.631 & 70.8 & 70.3 & 69.5 & 69.8 & 0.001253 \\
\hline 250.02 & 3.744871 & 14.74799 & 3.008699 & 14.75625 & 6867.75 & 6507.907 & 70.8 & 70.2 & 69.5 & 69.7 & 0.001254 \\
\hline 255.28 & 3.740494 & 14.74724 & 3.008699 & 14.757 & 6877.5 & 6511.88 & 70.8 & 70.2 & 69.5 & 69.8 & 0.001255 \\
\hline 260.02 & 3.73174 & 14.74724 & 3.008699 & 14.75775 & 6887.75 & 6536.214 & 70.8 & 70.2 & 69.5 & 69.8 & 0.001256 \\
\hline 265.02 & 3.753624 & 14.74724 & 3.008699 & 14.75625 & 6887.25 & 6551.857 & 70.7 & 70.3 & 69.6 & 69.8 & 0.001256 \\
\hline 270.02 & 3.740494 & 14.74799 & 3.008699 & 14.757 & 6897.25 & 6552.602 & 70.7 & 70.3 & 69.5 & 69.8 & 0.001256 \\
\hline 275.02 & 3.751123 & 14.74649 & 3.008073 & 14.75625 & 6897.25 & 6520.322 & 70.8 & 70.3 & 69.5 & 69.8 & 0.001257 \\
\hline 280.02 & 3.735491 & 14.74649 & 3.006699 & 14.75625 & 6908 & 6516.101 & 70.7 & 70.3 & 69.4 & 69.8 & 0.001259 \\
\hline 285.02 & 3.741119 & 14.74874 & 3.008699 & 14.75625 & 6907.25 & 6528.516 & 70.7 & 70.3 & 69.4 & 69.8 & 0.001259 \\
\hline 300.02 & 3.749247 & 14.74649 & 3.00995 & 14.75925 & 6927.25 & 6542.67 & 70.7 & 70.4 & 69.4 & 69.8 & 0.00126 \\
\hline 305.02 & 3.751123 & 14.74724 & 3.010575 & 14.75625 & 6927.75 & 6551.112 & 70.7 & 70.3 & 69.4 & 69.8 & 0.001261 \\
\hline 310.02 & 3.7555 & 14.74874 & 3.00995 & 14.75625 & 6927,25 & 6547.388 & 70.7 & 70.3 & 69.4 & 69.8 & 0.001261 \\
\hline
\end{tabular}




\begin{tabular}{|c|c|c|c|c|c|c|c|c|c|c|c|}
\hline SECONDS & MAIN DUCT & MAIN DUCT & GAS INJECT & GAS INJECT & NEWPORT & SMC & AMBIENT & GAS INJECT & MAIN DUCT & WEC CELL & WEC CELL \\
\hline & DIFF (IN H2O) & ABS (PSIA) & DIFF (IN H2O) & ABS (PSIA) & (PPM) & (PPM) & TEMP.(F) & TEMP. (F) & TEMP. (F) & TEMP. (F) & (VOLTS) \\
\hline 0.009979 & 3.759252 & 14.74724 & 0.055746 & 14.64225 & 91.425 & -3.5484 & 70.6 & 69.8 & 69.5 & 69.7 & 0.000244 \\
\hline 315.02 & 3.763629 & 14.74574 & 3.010575 & 14.75625 & 6937.75 & 6530.503 & 70.7 & 70.4 & 69.6 & 69.8 & 0.001261 \\
\hline 320.02 & 3.748622 & 14.74724 & 3.012452 & 14.75625 & 6937.5 & 6517.094 & 70.7 & 70.4 & 69.5 & 69.8 & 0.001262 \\
\hline 325.02 & 3.758001 & 14.74799 & 3.013077 & 14.7555 & 6947.5 & 6525.785 & 70.7 & 70.4 & 69.5 & 69.8 & 0.001263 \\
\hline 330.02 & 3.744871 & 14.74724 & 3.013077 & 14.757 & 6947.25 & 6555.83 & 70.7 & 70.4 & 69.5 & 69.8 & 0.001264 \\
\hline 335.02 & 3.745496 & 14.74724 & 3.013077 & 14.757 & 6957.25 & 6539.69 & 70.6 & 70.4 & 69.5 & 69.8 & 0.001265 \\
\hline 340.02 & 3.744871 & 14.74649 & 3.012452 & 14.75625 & 6967.75 & 6535.469 & 70.6 & 70.4 & 69.5 & 69.8 & 0.001266 \\
\hline 345.02 & 3.741119 & 14.74574 & 3.011826 & 14.75625 & 6967.75 & 6526.778 & 70.7 & 70.4 & 69.5 & 69.8 & 0.001266 \\
\hline 350.02 & 3.751123 & 14.74724 & 3.012452 & 14.75625 & 6967.75 & 6515.853 & 70.7 & 70.5 & 69.5 & 6.8 & 0.001266 \\
\hline 355.02 & 3.753624 & 14.74799 & 3.012452 & 14.75625 & 6967.75 & 6546.891 & 70.7 & 70.4 & 69.4 & 69.8 & 0.001265 \\
\hline 360.02 & 3.758001 & 14.74724 & 3.015579 & 14.75625 & 6967.75 & 6543.663 & 70.7 & 70,4 & 69.4 & 69.8 & 0.001265 \\
\hline 365.02 & 3.764254 & 14.74724 & 3.013077 & 14.757 & 6967.5 & 6560.548 & 70.6 & 70.4 & 69.4 & 69.8 & 0.001266 \\
\hline 370.02 & 3.736742 & 14.74799 & 3.012452 & 14.757 & 6968 & 6550.616 & 70.6 & 70.4 & 69.4 & 69.9 & 0.001267 \\
\hline 380.02 & 3.749247 & 14.74574 & 3.014328 & 14.757 & 6967.75 & 6565.266 & 70.5 & 70.5 & 69.4 & 69.8 & 0.001267 \\
\hline 385.02 & 3.745496 & 14.74574 & 3.013077 & 14.75625 & 6978 & 6575.943 & 70.5 & 70.5 & 69.4 & 69.8 & 0.001268 \\
\hline 390.02 & 3.750498 & 14.74724 & 3.00995 & 14.75775 & 6978 & 6588.855 & 70.4 & 70.5 & 69.4 & 69.8 & 0.001268 \\
\hline 395.02 & 3.746746 & 14.74724 & 3.012452 & 14.75625 & 6977.75 & 6569.735 & 70.4 & 70.5 & 69.4 & 69.8 & 0.001268 \\
\hline 400.02 & 3.745496 & 14.74724 & 3.013077 & 14.757 & 6988 & 6574.702 & 70.4 & 70.5 & 69.4 & 69.8 & 0.001269 \\
\hline 405.07 & 3.745496 & 14.74874 & 3.013702 & 14.75625 & 6987.75 & 6566.259 & 70.4 & 70.5 & 69.4 & 69.8 & 0.001268 \\
\hline 410.02 & 3.744245 & 14.74649 & 3.013077 & 14.75625 & 6988 & 6558.065 & 70.4 & 70.5 & 69.5 & 69.8 & 0.001268 \\
\hline 415.02 & 3.746121 & 14.74649 & 3.013702 & 14.75625 & 6987.75 & 6534.476 & 70.5 & 70.5 & 69.5 & 69.8 & 0.001268 \\
\hline 420.02 & 3.751749 & 14.74649 & 3.014953 & 14.757 & 6988 & 6541.925 & 70.5 & 70.5 & 69.5 & 69.8 & 0.001269 \\
\hline 425.06 & 3.773008 & 14.74724 & 3.014328 & 14.75625 & 6987.5 & 6554.34 & 70.5 & 70.5 & 69.5 & 69.8 & 0.001268 \\
\hline 430.02 & 3.761128 & 14.74799 & 3.014953 & 14.75625 & 6977.75 & 6561.293 & 70.5 & 70.5 & 69.5 & 69.8 & 0.001268 \\
\hline 435.1 & 3.736742 & 14.74799 & 3.014953 & 14.75625 & 6978 & 6586.372 & 70.5 & 70.5 & 69.4 & 69.8 & 0.001268 \\
\hline 440.02 & 3.75425 & 14.74724 & 3.014953 & 14.7555 & 6978 & 6582.647 & 70.5 & 70.5 & 69.5 & 6.8 & 0.001268 \\
\hline 445.02 & 3.73174 & 14.74799 & 3.014953 & 14.75625 & 6977.75 & 6604.747 & 70.6 & 70.6 & 69.4 & 69.8 & 0.001269 \\
\hline 450.02 & 3.747372 & 14.74799 & 3.014953 & 14.75625 & 6978 & 6588.11 & 70.6 & 70.5 & 69.5 & 69.8 & 0.001269 \\
\hline 465.02 & 3.7555 & 14.74874 & 3.014953 & 14.75625 & 6998 & 6564.273 & 70.6 & 70.6 & 69.5 & 69.9 & 0.001272 \\
\hline 470.02 & 3.732365 & 14.74799 & 3.014953 & 14.757 & 6998 & 6548.629 & 70.6 & 70.6 & 69.5 & 69.8 & 0.001272 \\
\hline 475.02 & 3.727363 & 14.74874 & 3.014328 & 14.75625 & 7007.5 & 6529.758 & 70.6 & 70.5 & 69.5 & 69.8 & 0.001273 \\
\hline
\end{tabular}




\begin{tabular}{|c|c|c|c|c|c|c|c|c|c|c|c|}
\hline SECONDS & MAIN DUCT & MAIN DUCT & GAS INJECT & GAS INJECT & NEWPORT & SMC & AMBIENT & GAS INJECT & MAIN DUCT & WEC CELL & WEC CELL \\
\hline & DIFF (IN H2O) & ABS (PSIA) & DIFF (IN H2O) & ABS (PSIA) & (PPM) & (PPM) & TEMP,(F) & TEMP. (F) & TEMP. (F) & TEMP. (F) & (VOLTS) \\
\hline & & & & & & & & & & & \\
\hline 0.009979 & 3.759252 & 14.74724 & 0.055746 & 14.64225 & 91.425 & -3.5484 & 70.6 & 69.8 & 69.5 & 69.7 & 0.000244 \\
\hline 480.02 & 3.744871 & 14.74649 & 3.014953 & 14.75625 & 7007.5 & 6528.02 & 70.6 & 70.6 & 69.5 & 69.9 & 0.001274 \\
\hline 485.02 & 3.746121 & 14.74799 & 3.014953 & 14.757 & 7008 & 6544.656 & 70.6 & 70.6 & 69.6 & 69.8 & 0.001273 \\
\hline 490.02 & 3.759877 & 14.74799 & 3.015579 & 14.757 & 7017.75 & 6546.891 & 70.7 & 70.6 & 69.6 & 69.9 & 0.001274 \\
\hline 495.02 & 3.744871 & 14.74874 & 3.014953 & 14.75625 & 7018 & 6542.918 & 70.7 & 70.6 & 69.5 & 69.9 & 0.001275 \\
\hline 505.02 & 3.764254 & 14.74874 & 3.014953 & 14.75775 & 7028 & 6556.823 & 70.7 & 70.6 & 69.5 & 69.9 & 0.001275 \\
\hline 510.02 & 3.734241 & 14.74724 & 3.014953 & 14.75625 & 7028 & 6560.548 & 70.7 & 70.6 & 69.5 & 69.8 & 0.001275 \\
\hline 515.02 & 3.738618 & 14.74799 & 3.014953 & 14.757 & 7028 & 6564.521 & 70.8 & 70.6 & 69.5 & 69.8 & 0.001276 \\
\hline 520.02 & 3.735491 & 14.74874 & 3.014328 & 14.75625 & 7028 & 6560.796 & 70.7 & 70.6 & 69.4 & 69.8 & 0.001274 \\
\hline 525.02 & 3.77926 & 14.74948 & 3.014953 & 14.75625 & 7027.75 & 6564.769 & 70.7 & 70.6 & 69.5 & 69.9 & 0.001276 \\
\hline 530.04 & 3.751749 & 14.74724 & 3.014953 & 14.757 & 7028 & 6554.092 & 70.7 & 70.6 & 69.5 & 69.9 & 0.001277 \\
\hline 535.02 & 3.762378 & 14.74649 & 3.014328 & 14.75625 & 7037.75 & 6542.918 & 70.8 & 70.6 & 69.5 & 69.9 & 0.001276 \\
\hline 545.02 & 3.751749 & 14.74799 & 3.014328 & 14.75625 & 7028.25 & 6538.697 & 70.7 & 70.7 & 69.5 & 69.9 & 0.001276 \\
\hline 550.02 & 3.757376 & 14.74799 & 3.014953 & 14.75775 & 7028 & 6554.837 & 70.7 & 70.6 & 69.4 & 69.9 & 0.001276 \\
\hline 555.02 & 3.73174 & 14.74724 & 3.014953 & 14.757 & 7028.25 & 6558.065 & 70.7 & 70.6 & 69.5 & 69.8 & 0.001275 \\
\hline 560.06 & 3.760502 & 14.74574 & 3.014953 & 14.757 & 7028 & 6559.307 & 70.7 & 70.7 & 69.5 & 69.8 & 0.001276 \\
\hline 565.02 & 3.749247 & 14.74799 & 3.013077 & 14.75625 & 7028 & 6551.609 & 70.6 & 70.7 & 69.5 & 69.9 & 0.001276 \\
\hline 570.02 & 3.738618 & 14.74724 & 3.012452 & 14.757 & 7027.5 & 6574.205 & 70.6 & 70.7 & 69.5 & 69.9 & 0.001276 \\
\hline 575.06 & 3.748622 & 14.74649 & 3.012452 & 14.757 & 7038 & 6535.966 & 70.7 & 70.7 & 69.5 & 69.8 & 0.001276 \\
\hline 580.02 & 3.751123 & 14.74724 & 3.011201 & 14.757 & 7038.5 & 6537.952 & 70.7 & 70.7 & 69.4 & 69.8 & 0.001277 \\
\hline 585.02 & 3.745496 & 14.74724 & 3.012452 & 14.757 & 7037.75 & 6552.354 & 70.6 & 70.7 & 69.4 & 69.8 & 0.001277 \\
\hline 590.07 & 3.734241 & 14.74724 & 3.010575 & 14.757 & 7038 & 6545.65 & 70.6 & 70.7 & 69.3 & 69.8 & 0.001277 \\
\hline 595.08 & 3.746746 & 14.74724 & 3.010575 & 14.757 & 7038.25 & 6557.072 & 70.6 & 70.7 & 69.4 & 69.8 & 0.001276 \\
\hline 600.02 & 3.742369 & 14.74649 & 3.010575 & 14.75775 & 7038.25 & 6550.119 & 70.7 & 70.7 & 69.5 & 69.8 & 0.001276 \\
\hline 605.03 & 3.745496 & 14.74724 & 3.010575 & 14.75625 & 7037.25 & 6540.435 & 70.7 & 70.7 & 69.5 & 69.9 & 0.001276 \\
\hline 610.04 & 3.762378 & 14.74649 & 3.00995 & 14.75625 & 7037.75 & 6535.469 & 70.7 & 70.7 & 69.4 & 69.8 & 0.001276 \\
\hline 615.02 & 3.747372 & 14.74574 & 3.011201 & 14.75625 & 7038.25 & 6536.462 & 70.6 & 70.7 & 69.4 & 69.9 & 0.001277 \\
\hline 630.02 & 3.732365 & 14.74724 & 3.011201 & 14.757 & 7048 & 6561.541 & 70.6 & 70.7 & 69.4 & 69.9 & 0.001277 \\
\hline 635.05 & 3.763003 & 14.74799 & 3.010575 & 14.757 & 7048 & 6551.609 & 70.6 & 70.7 & 69.6 & 69.9 & 0.001276 \\
\hline 640.04 & 3.752999 & 14.74574 & 3.010575 & 14.75625 & 7048 & 6542.173 & 70.6 & 70.7 & 69.4 & 69.8 & 0.001277 \\
\hline
\end{tabular}

HNF-SD-WM-TRP-275 Rev. 0 


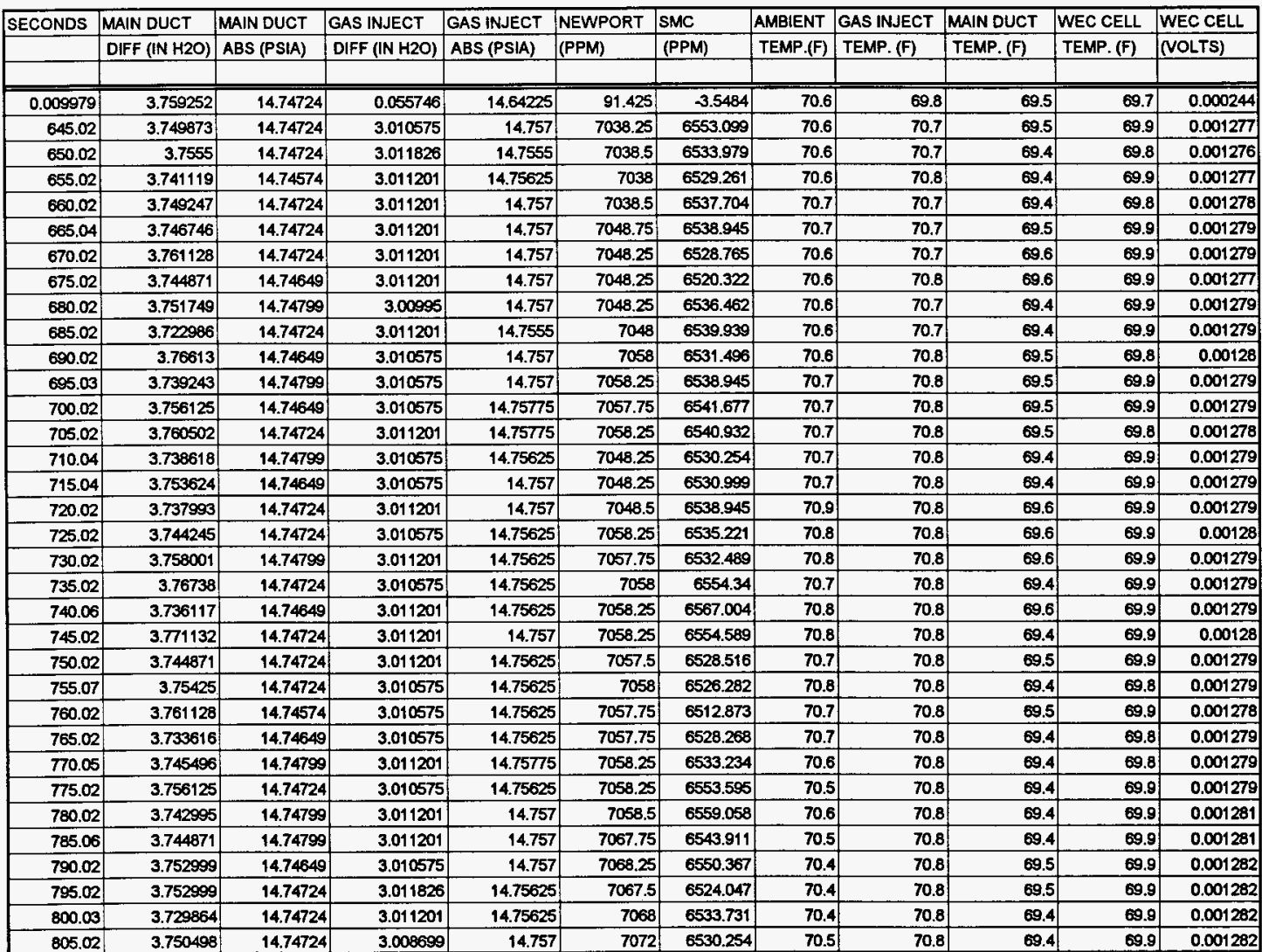




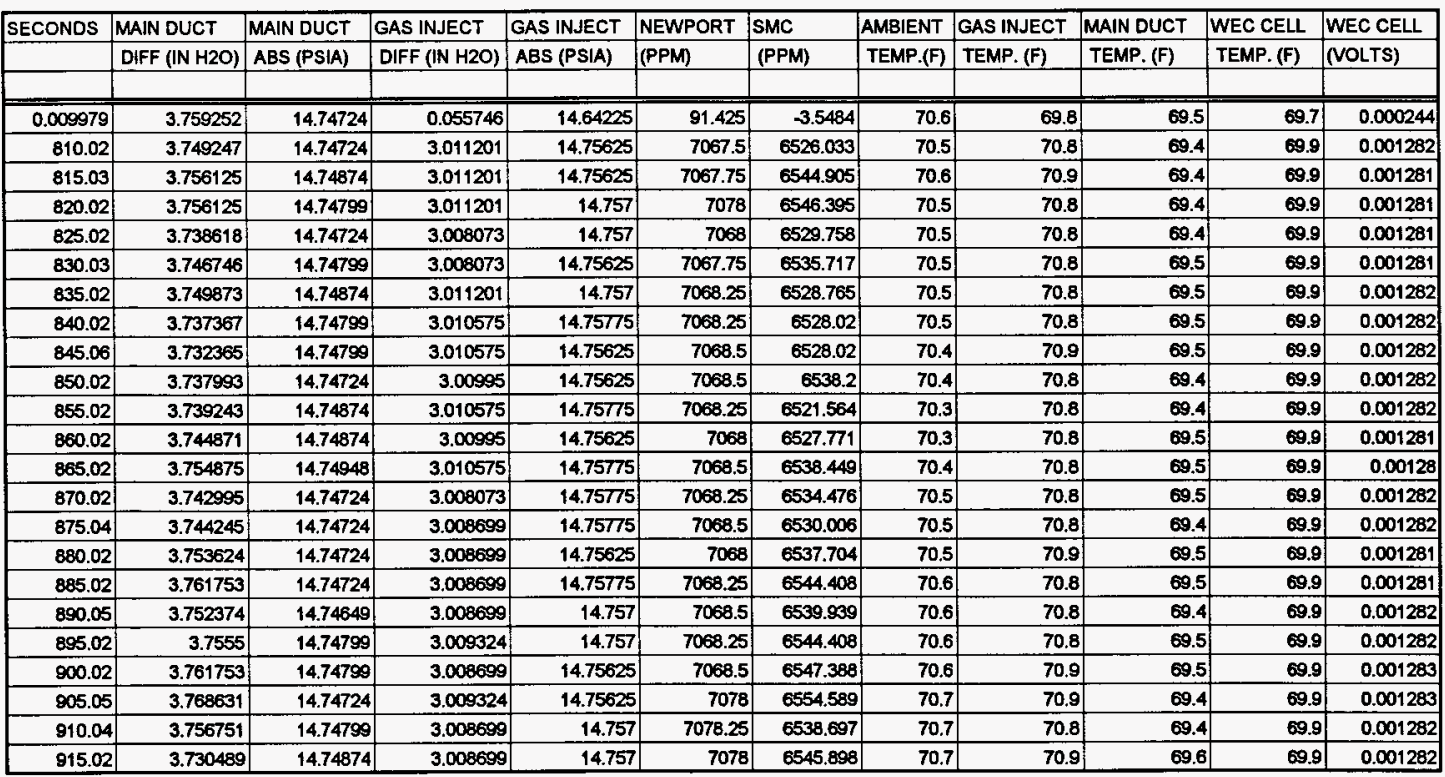




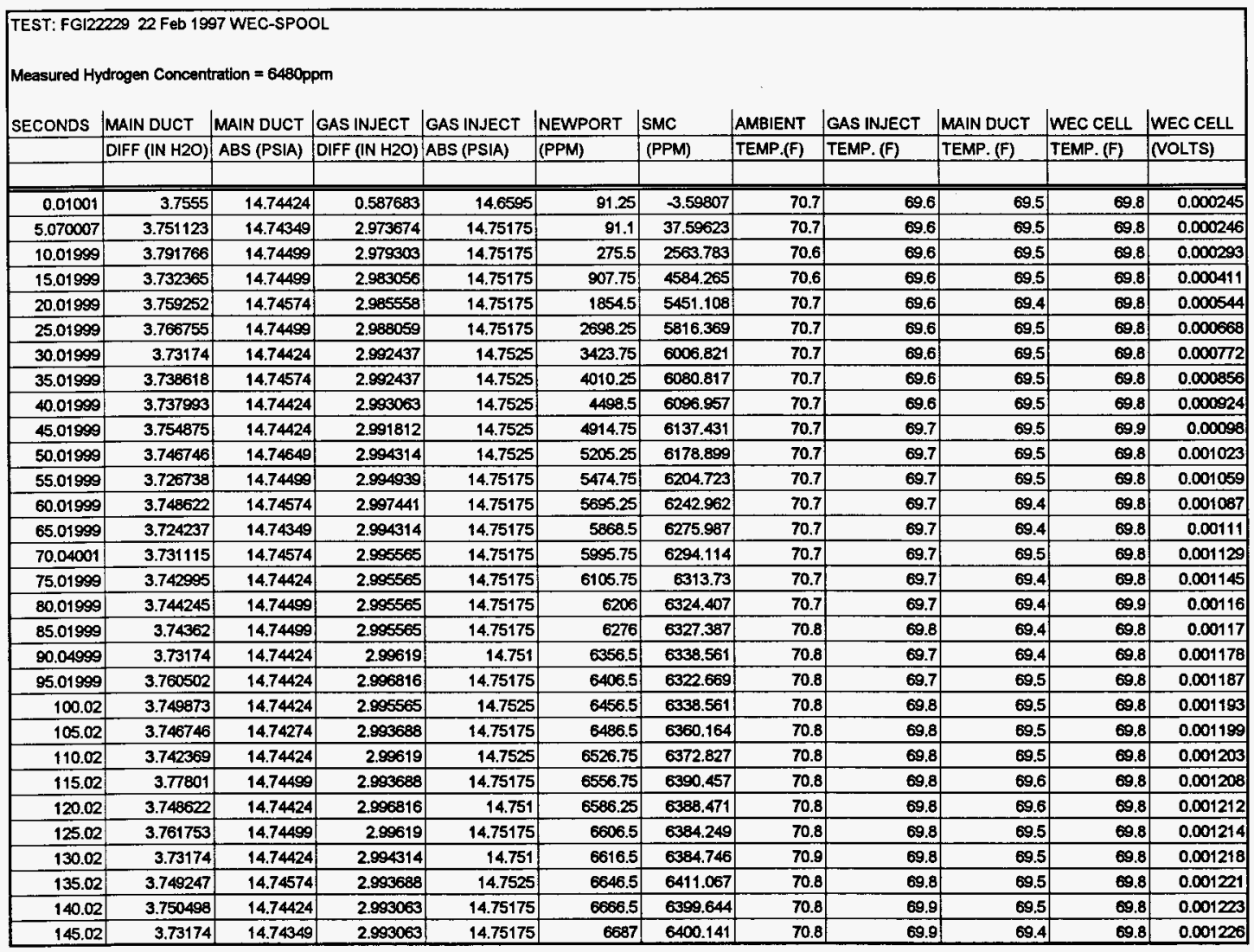




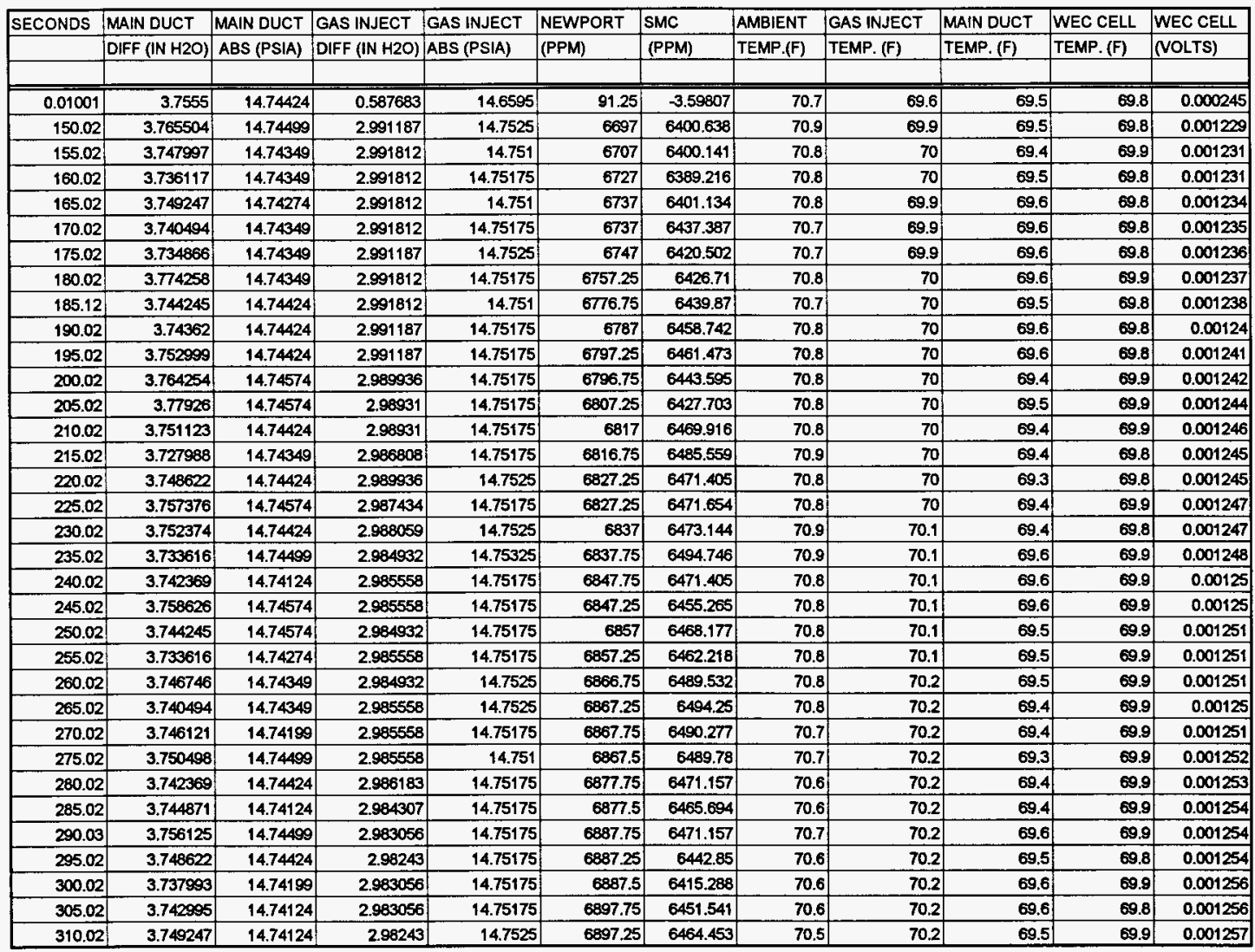




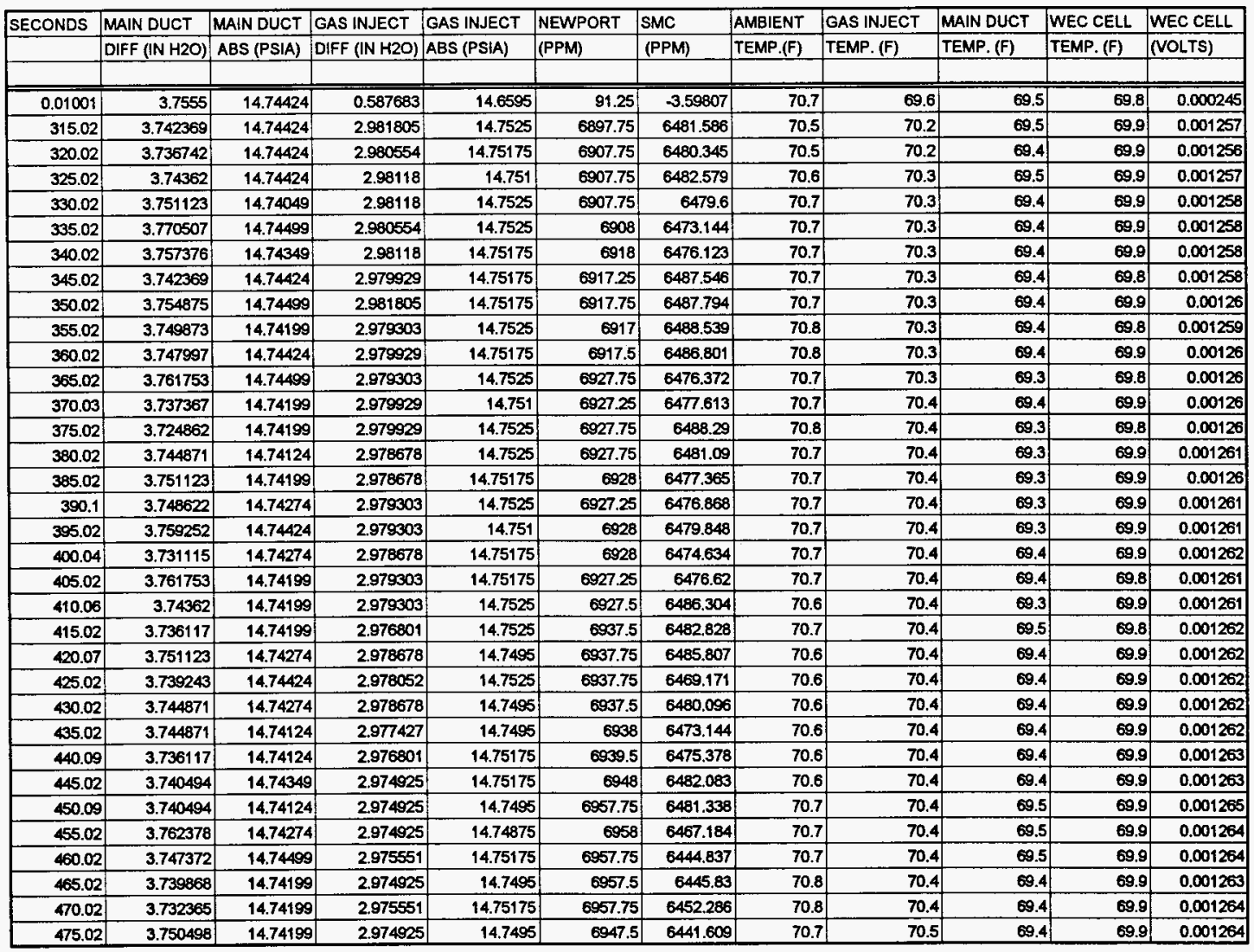




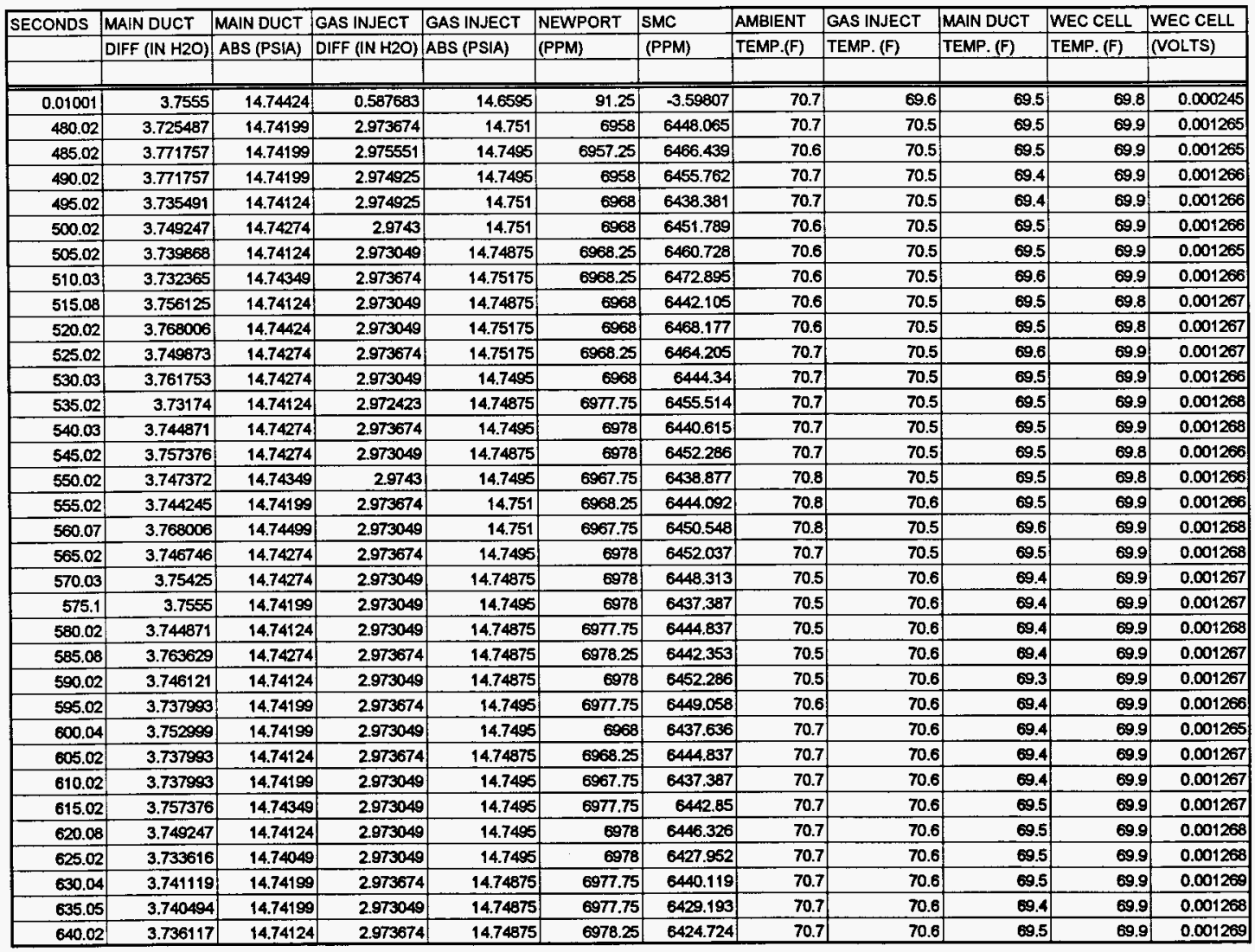




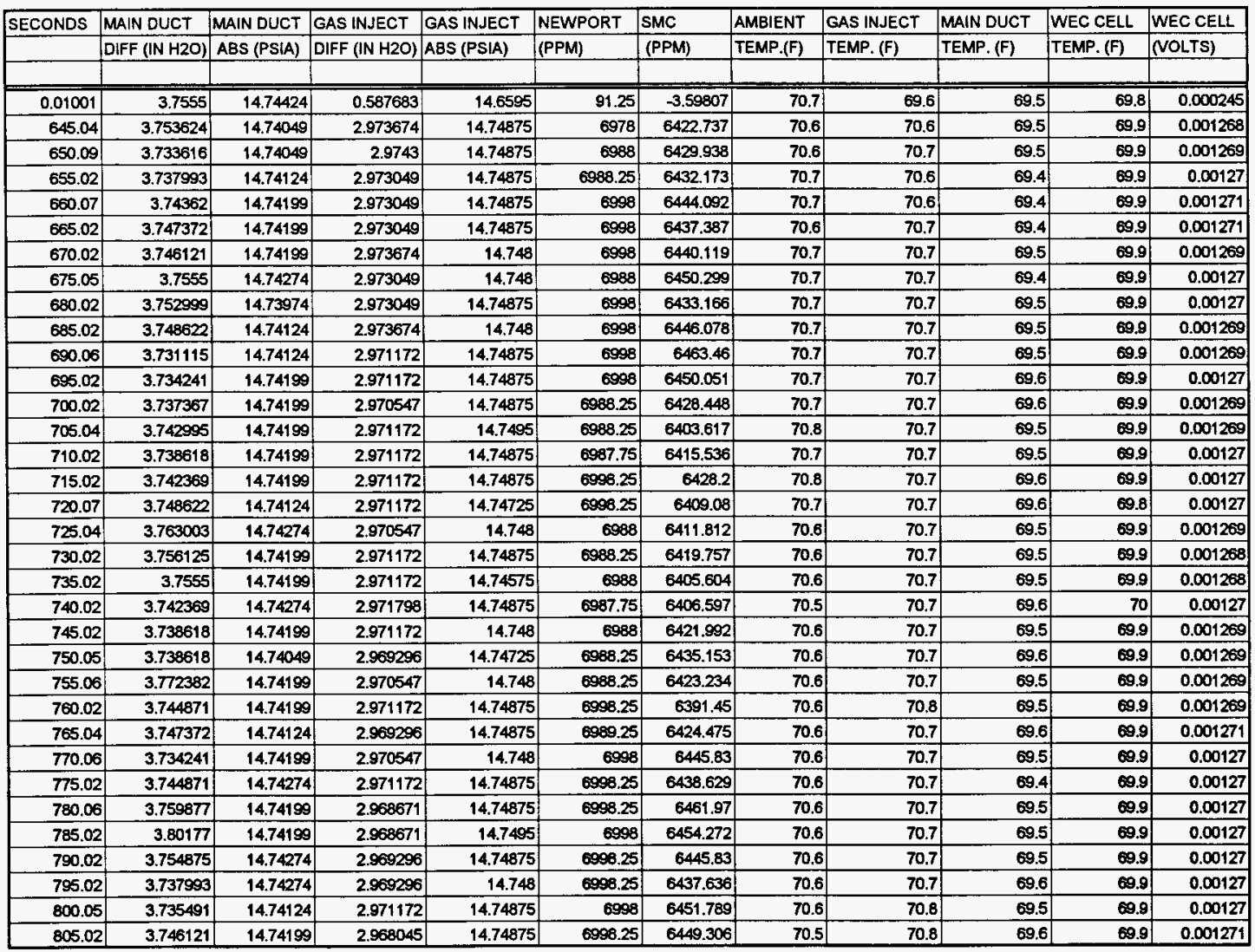




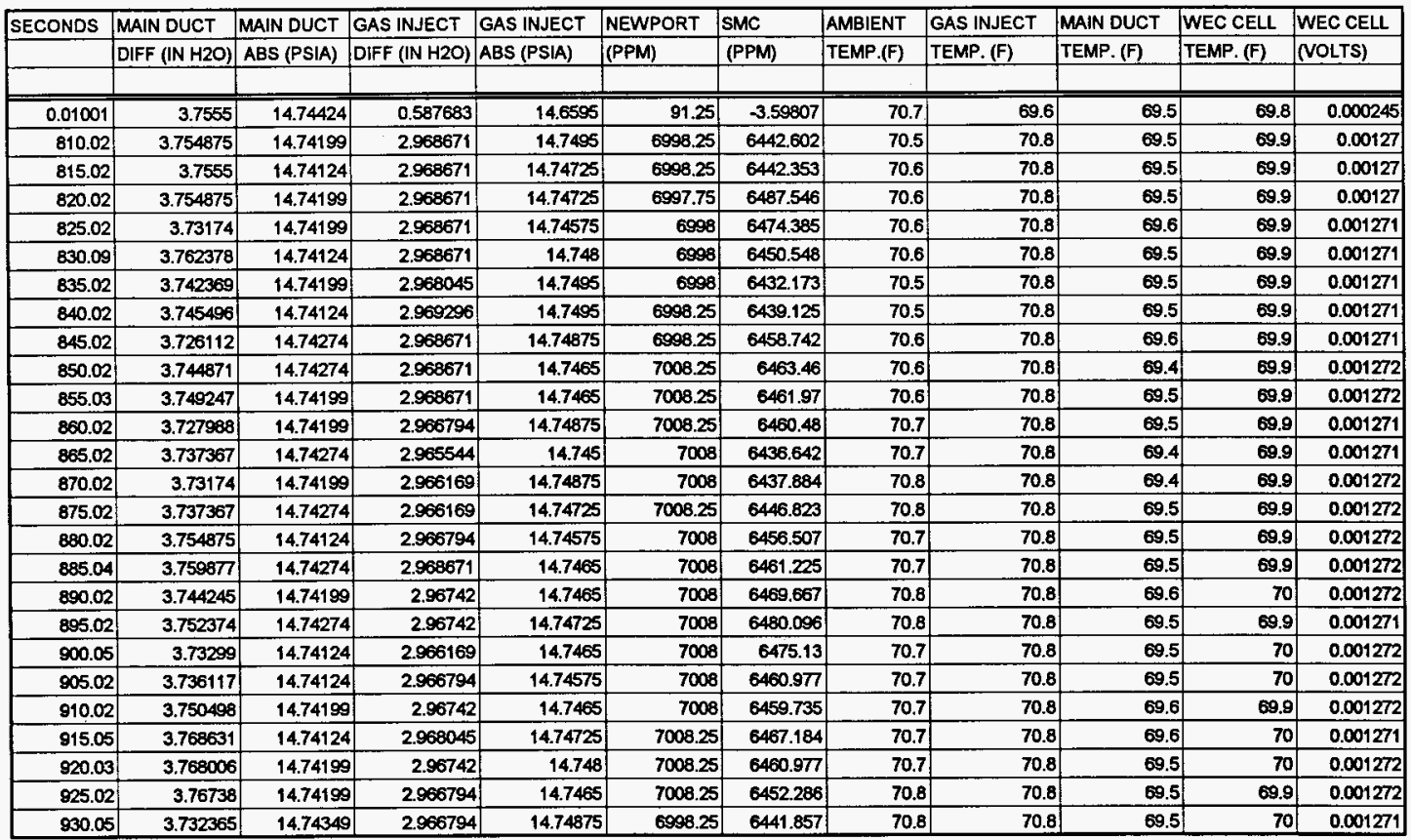


HNF-SD-WM-TRP-275

Rev. 0

Page ZYE

APPENDIX D - EXCEPTION RECORDS 


\section{TEST EXCEPTION SHEET}

\begin{tabular}{|c|c|c|c|c|c|}
\hline Test & Exce & & \multicolumn{3}{|c|}{$\begin{array}{l}\text { Title of Test: HNF-SD-WM-TC- } \\
\text { RMCS FGI FLOW RESPONSE TEST }\end{array}$} \\
\hline \multicolumn{3}{|r|}{ EXCEPTIONS } & \multicolumn{3}{|c|}{ CORRECTION APPROVAL } \\
\hline \multirow{2}{*}{$\begin{array}{l}\text { Procedure } \\
\text { Step } \\
\text { Number }\end{array}$} & \multirow[t]{2}{*}{ Date } & \multirow[t]{2}{*}{ Description } & \multicolumn{3}{|c|}{ Initials/Date } \\
\hline & & & $\begin{array}{c}\text { Test } \\
\text { Engineer }\end{array}$ & 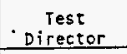 & Witnesses \\
\hline 10.2 .3 .5 & $|2-13-9\rangle \mid$ & 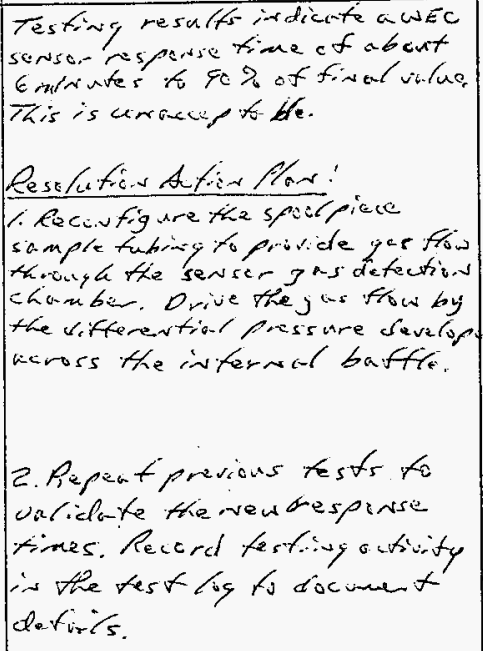 & d & $\begin{array}{l}\text { ged. } \\
2=-17-97\end{array}$ & ' \\
\hline & & 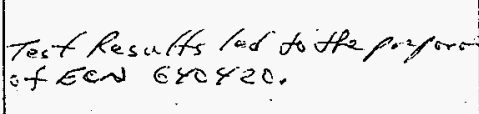 & 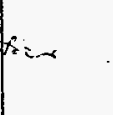 & & \\
\hline
\end{tabular}

IEST APPROVED WITH EXCEPTIONS

EXCEPTION RESOLVED

tephiend 12-17-97 Grie Viald

Test Director / Date

FGI Cognizant Engineer / Date

Miedul 1. Mequry $13-10-97$

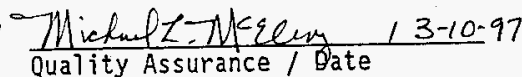




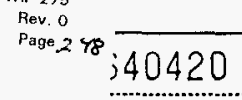

ENGINEERING CHANGE NOTICE

Poos 1 of 7 Proj.

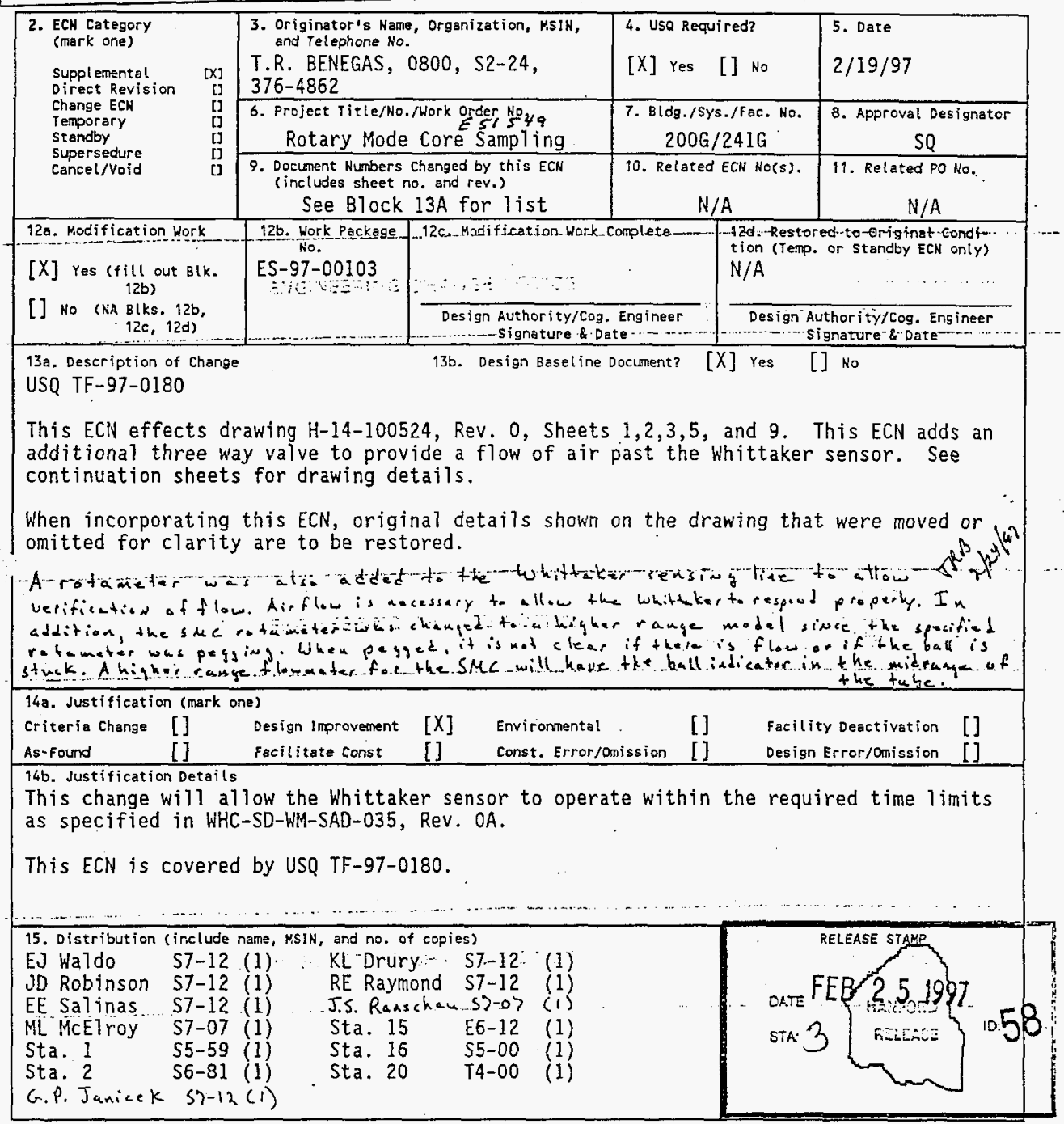

A-7900-013-2(05/96) GEF095 


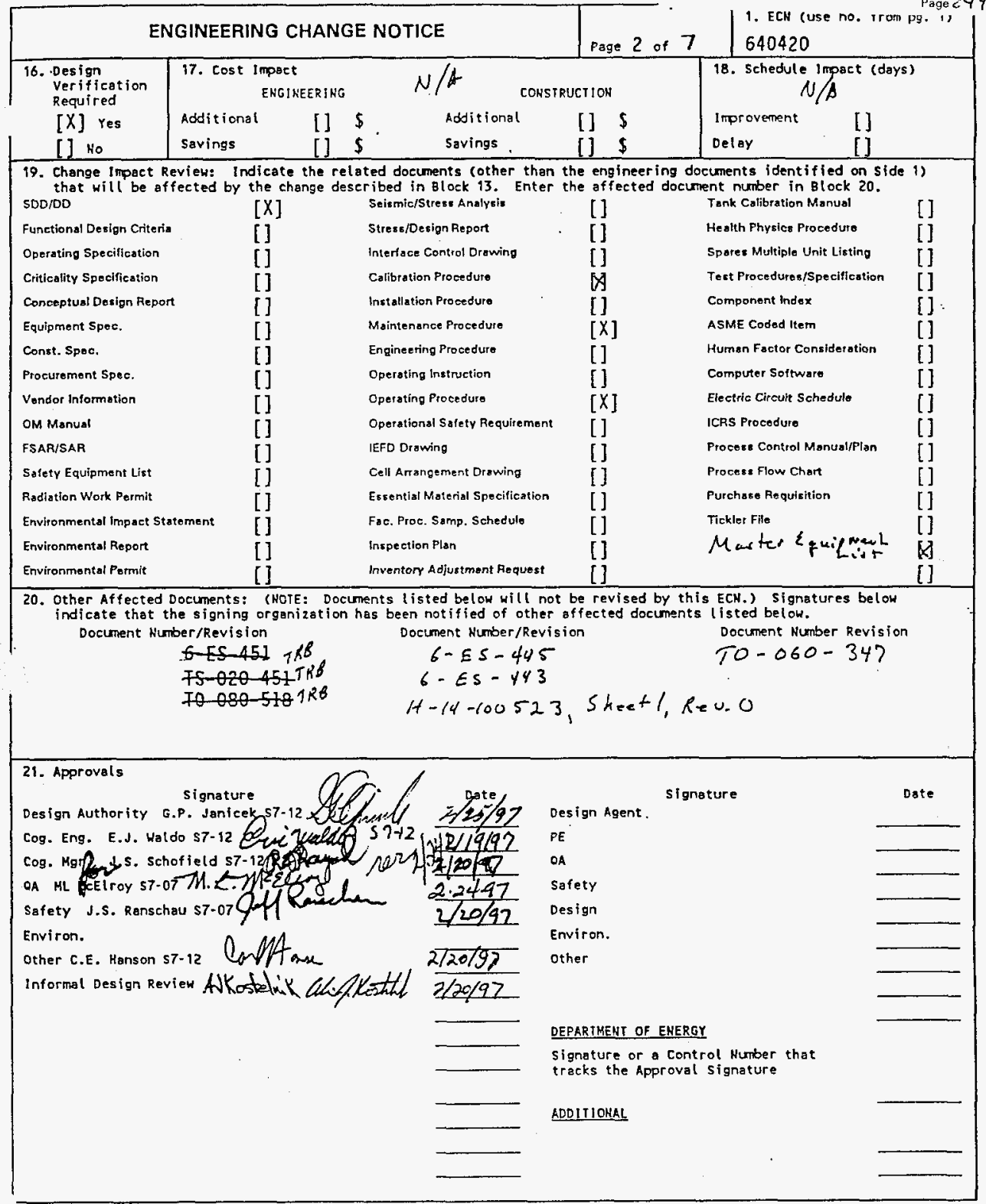


ENGINEERING CHANGE NOTICE CONTINUATION SHEE I

1. Change the quantity on the "PARTS/MATERIAL LIST", H-14-100524, Sheet 1, Rev. 0:

a). Item 12 from " 3 " to "2", (reduces number of bulkhead fittings by one).

b). Item 21 from "1" to "2", (increases number of 3-way valves by one).

c). Item 38 from "14" to "18", (updates number of labels generated to date).

d). Item 74 from "2" to "3" (increases number of unions by one, Reference ECN 639109 ).

e). Item 72 from "2" to "4" (increases number of male connectors from 2 to 4 for the use of an additional rotameter).

2. Change the "PARTS/MATERIAL LIST", H-14-100524, Sheet 1, Rev. 0, part number 58 as follows:

Was:

\begin{tabular}{|l|l|l|l|}
\hline GASKET, I/8 THK & NEOPRENE, DURO $40 \pm 5$ & 3 & 58 \\
\hline
\end{tabular}

is:

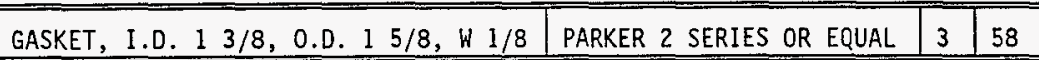

3. Change Item 69 from the "PARTS/MATERIAL LIST", H-14-100524, Sheet 1, Rev. 0, from "RMA-12" to "RMA-13". Refer to ECN 636576.

4. Change the quantity on the "PARTS/MATERIAL LIST", H-14-100524, Sheet 1, Rev. 0, for Item 69 from " 1 " to "2". Refer to ECN 636576.

5. Delete detail 58 on H-14-100524, Sheet 3, Rev. 0.

6. Add the following Item to the SCHEDULE, Item 38, Drawing H-14-100524, Rev. 0, Sheet 5.

\begin{tabular}{|c|c|c|c|}
\hline ALPHA & TEXT & $\begin{array}{c}\text { MINIMUM PLATE SIZE } \\
(H \times W)\end{array}$ & MINIMUM TEXT HEIGHT \\
\hline$T$ & $\begin{array}{c}\text { VTP-V-2207T } \\
\text { WHITTAKER H2 CELL } \\
\text { RETURN ISOLATION }\end{array}$ & $1 \times 2$ & $7 / 32$ \\
\hline
\end{tabular}

7. Add flag note 28 to $\mathrm{H}-14-100524$, Sheet 1 .

"28. Place flowmeter approximately midway between Whittaker sensor and Whittaker Valve and as close as practical to spool piece, i.e., provide maximum protection for flowmeter as practical."

8. See additional pages for detailed changes. 
H-14-100524, SHEET 2, ZONE A-F, 2-6

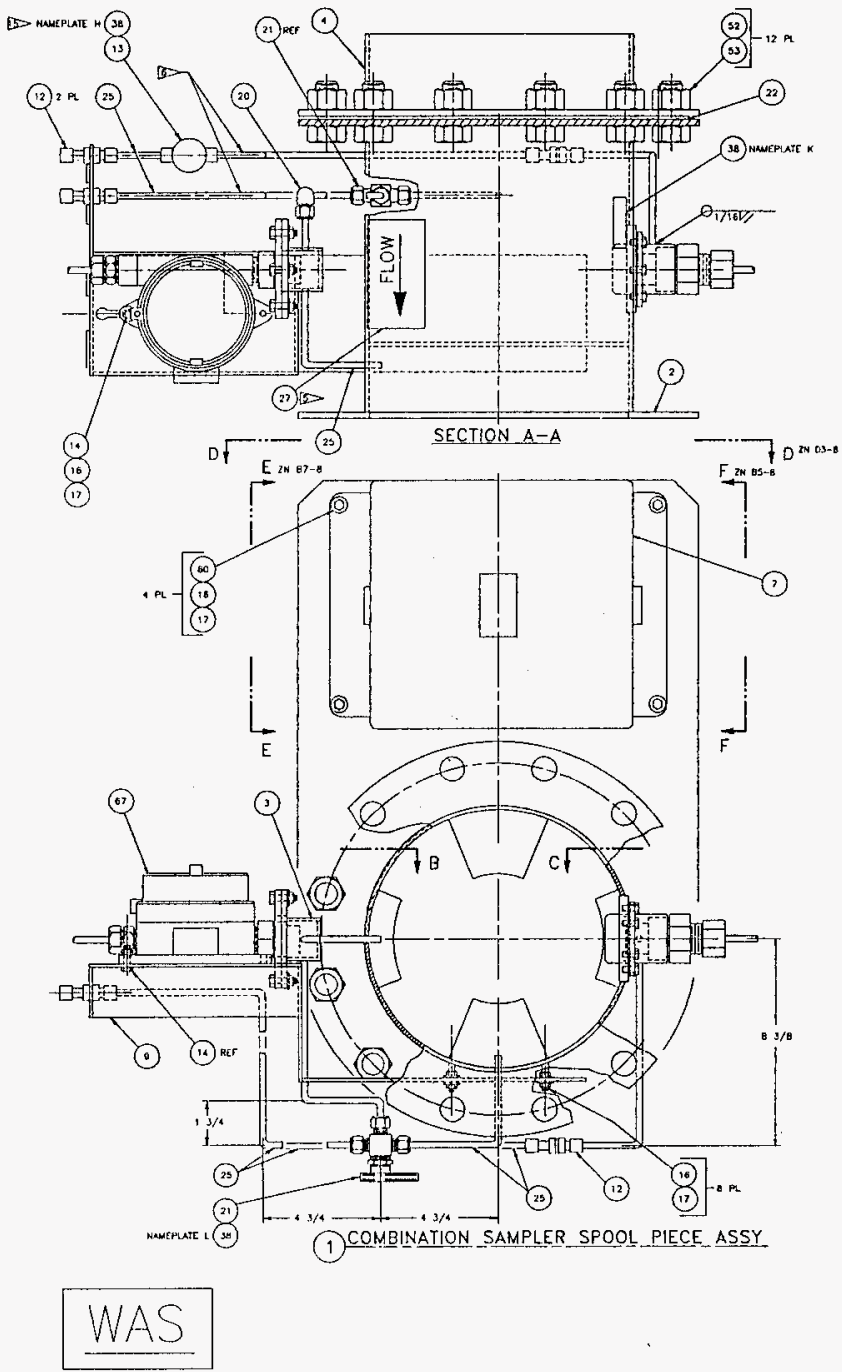




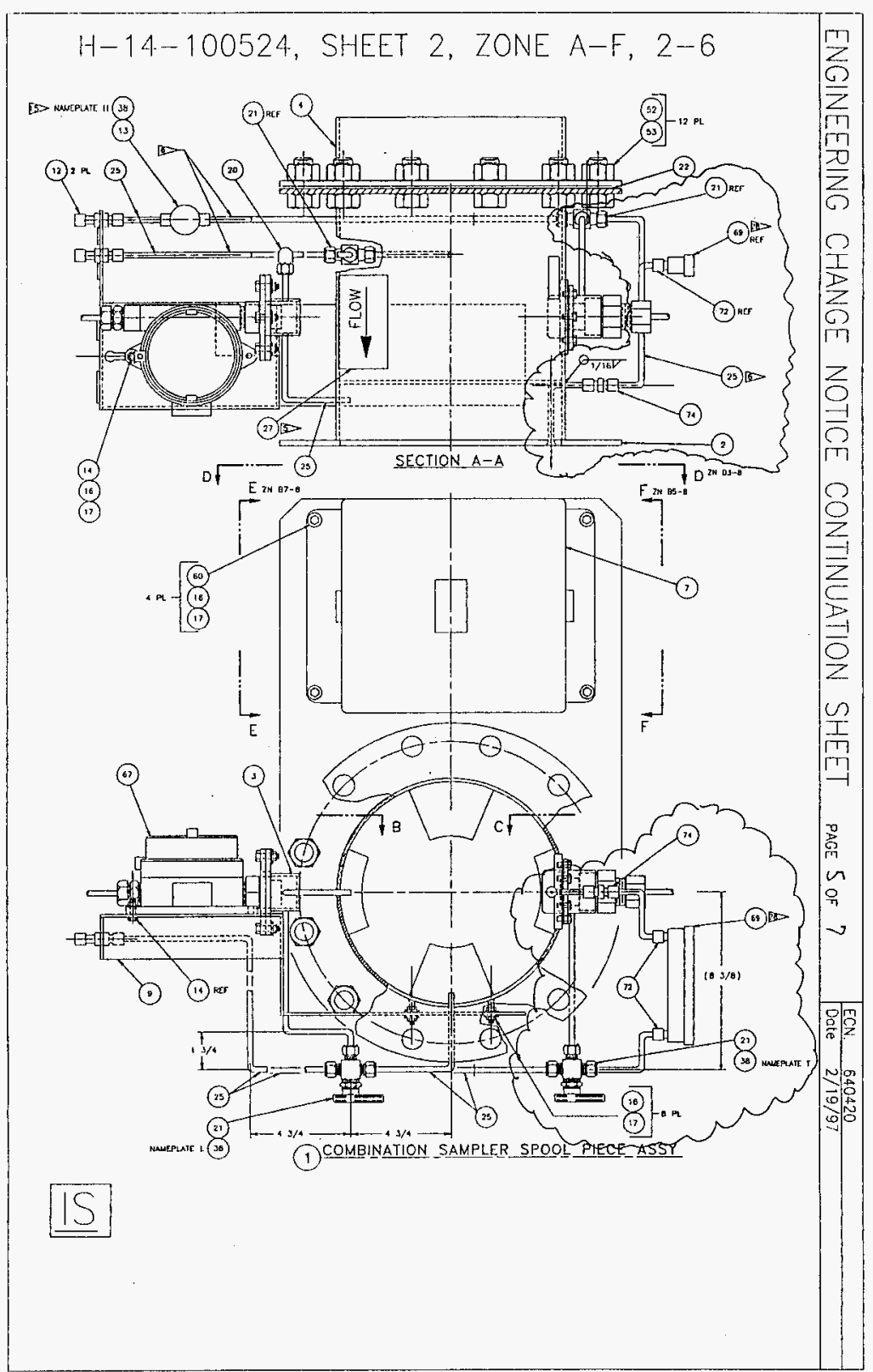

HNF-SD-WM-TRP- 275

Rev. 0

Page 252 


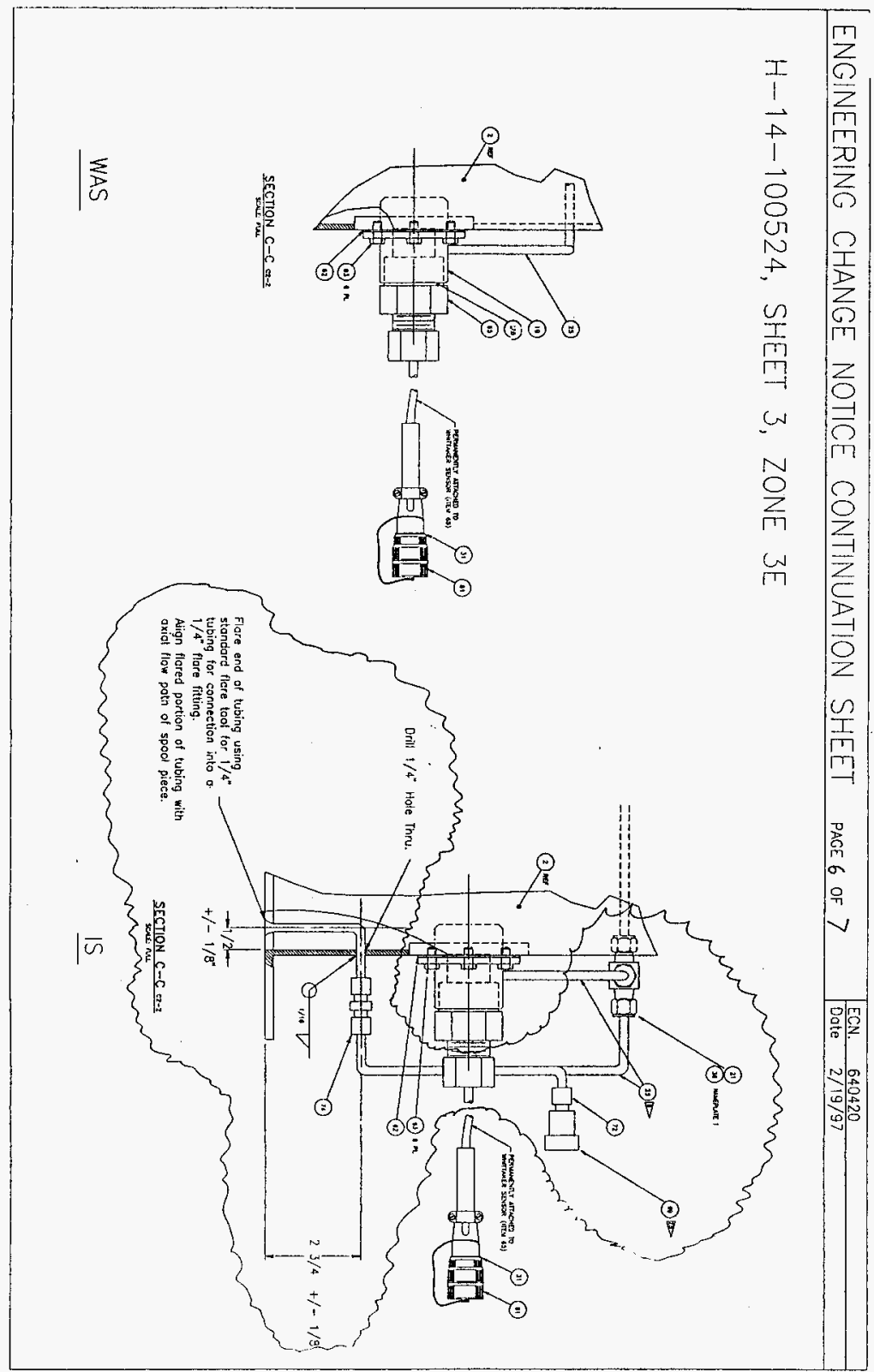




\section{WAS}

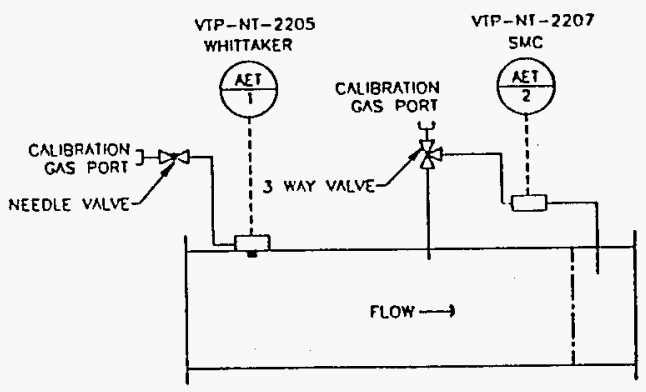

SAMPLER SPOOL $P$ \& ID

\section{IS}

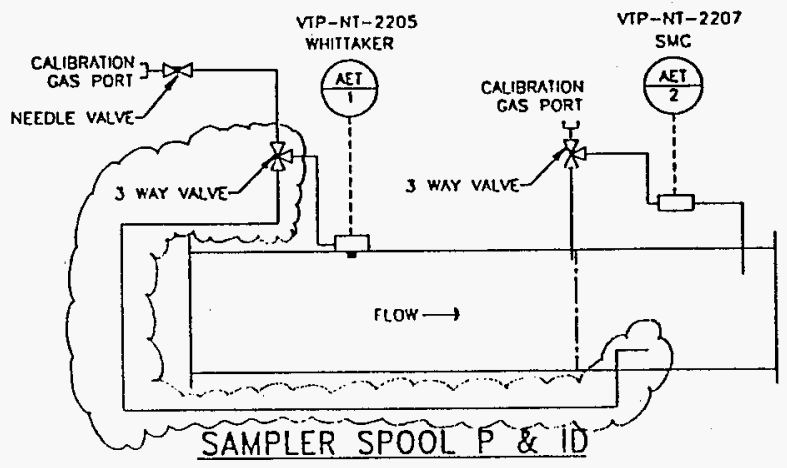


TEST EXCEPTION SHEET

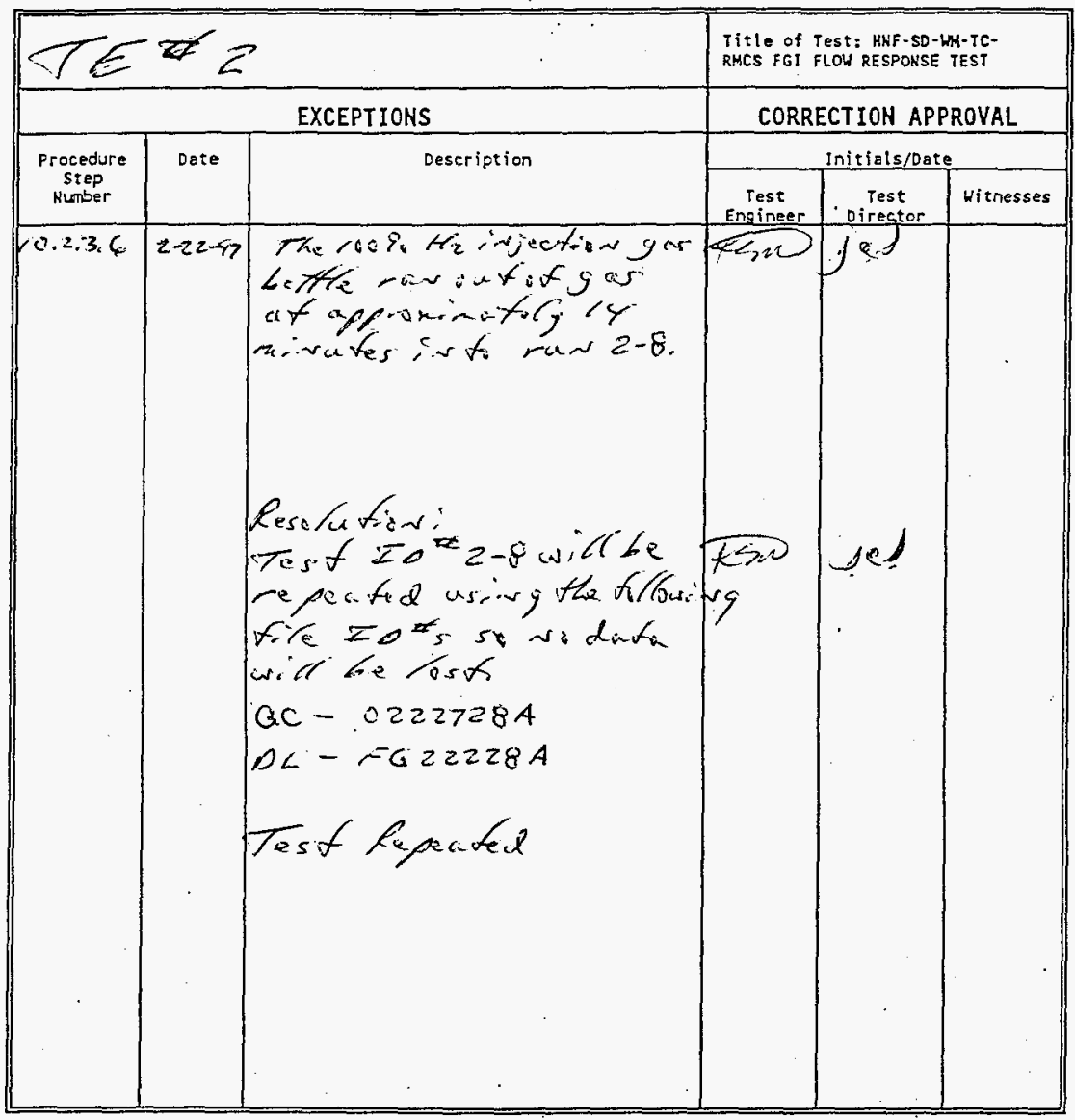

\section{TEST APPROVED WITH EXCEPTIONS}

EXCEPTION RESOLVED

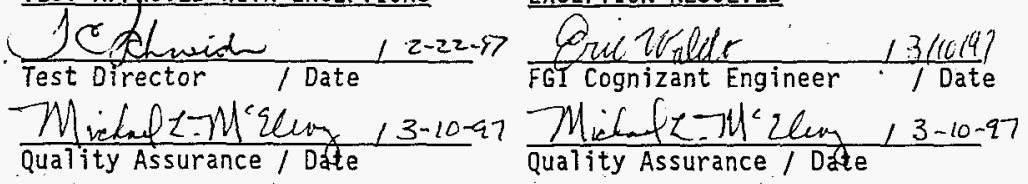


HNF-SD-WM-TRP- 275

Rev. 0
Page $25 \%$

TEST EXCEPTION SHEET

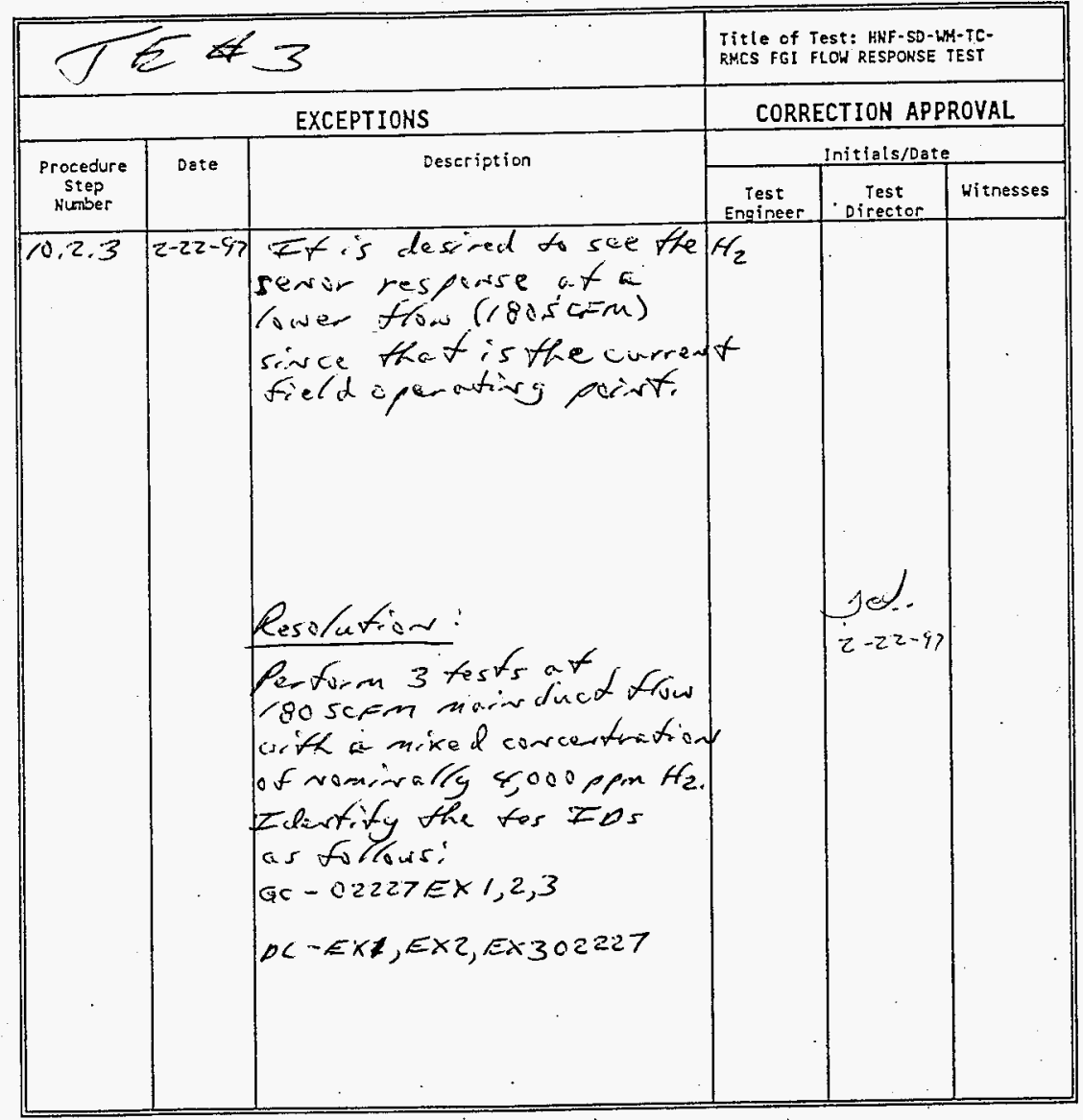

TEST APPROVED WITH EXCEPTIONS EXCEPTION RESOLVED

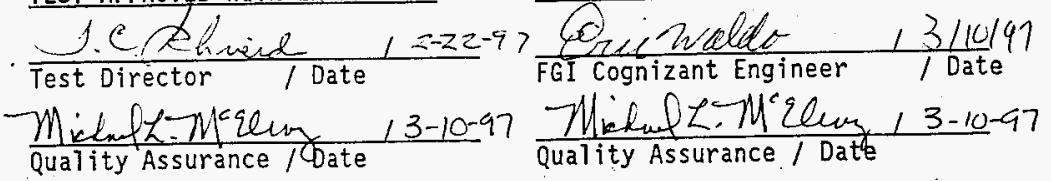


TEST EXCEPTION SHEET

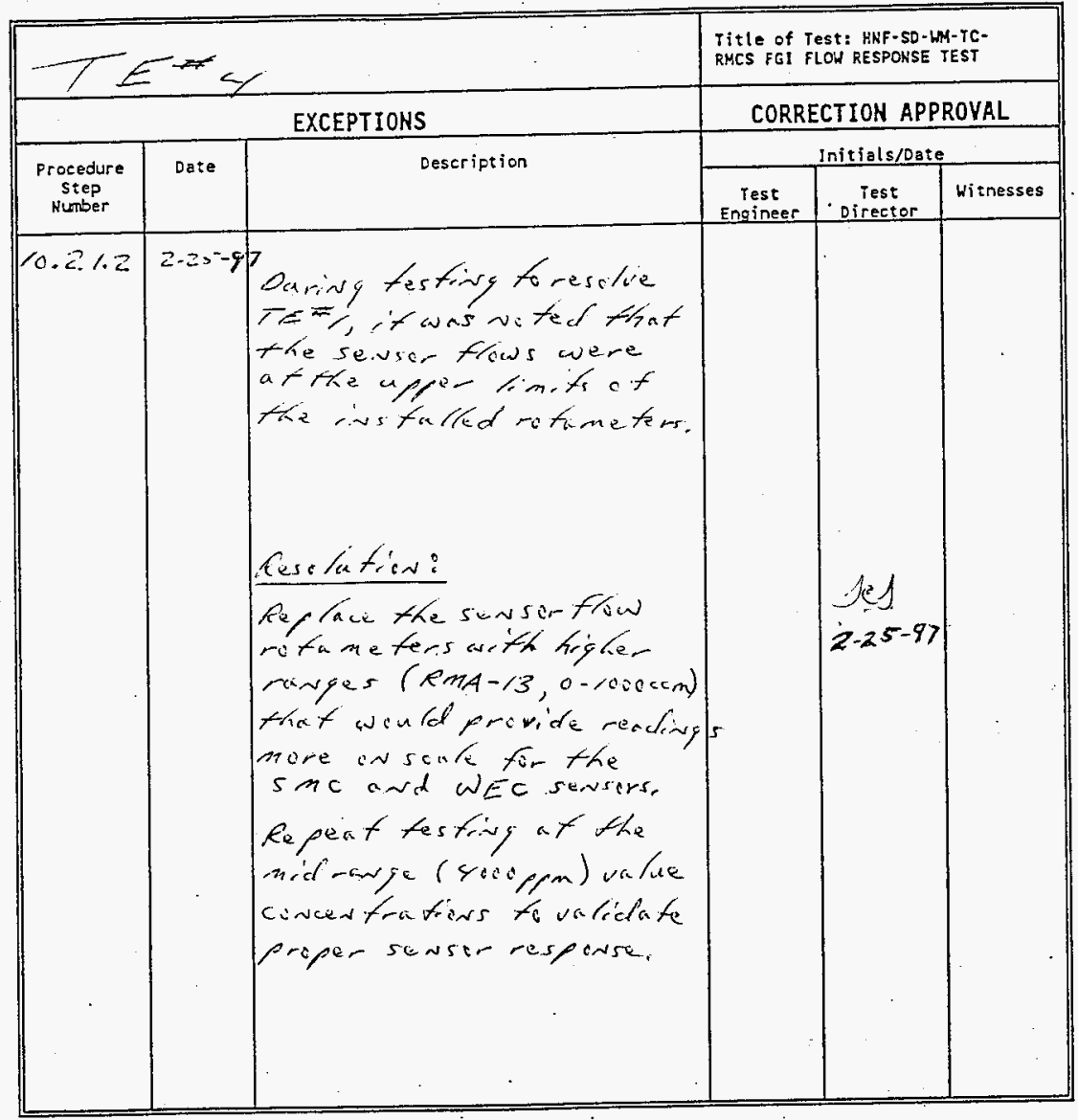

\section{TEST APPROVED WITH EXCEPTIONS}

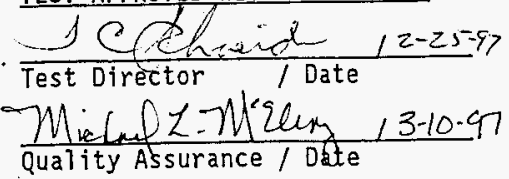

EXCEPTION RESOLVED

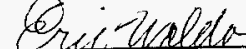

FGI Cognizant Engineer / Date

Milud $2-7$ McEew $3-10-97$ 
HNF-SD-WM-TRP- 275

Rev. 0

Page 258

\section{APPENDIX E - TEST LOG RECORDS}




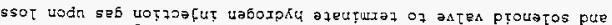

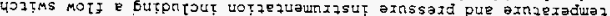

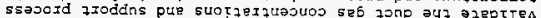

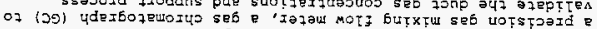

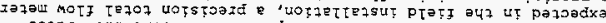

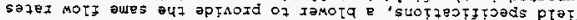

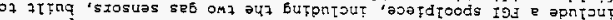

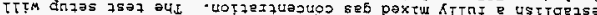

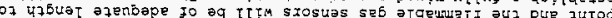

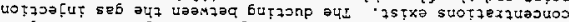

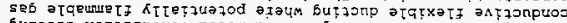

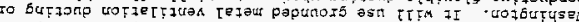

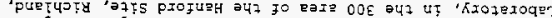

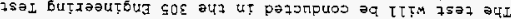

NOIIdIYวง 0.2

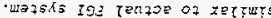

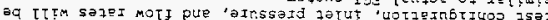

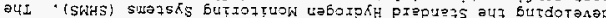

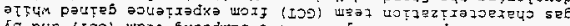

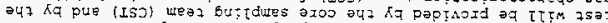

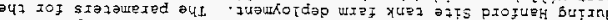

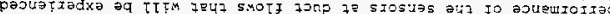

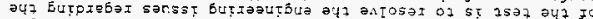

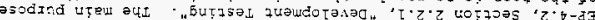

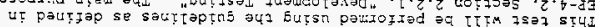

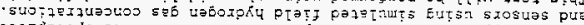

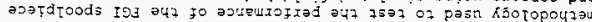

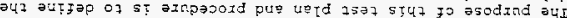

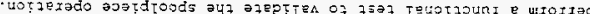
of KI

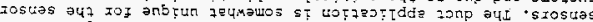

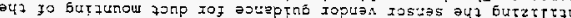

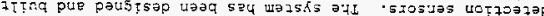

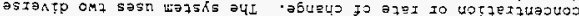

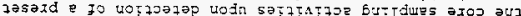

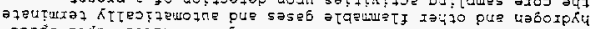

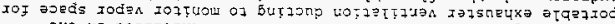

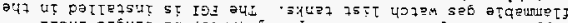

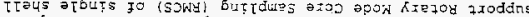

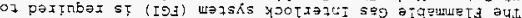

NOIDOCOLEN 0.1

IYก0

JSLOdS3U MOTJ aszidTOOdS

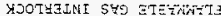

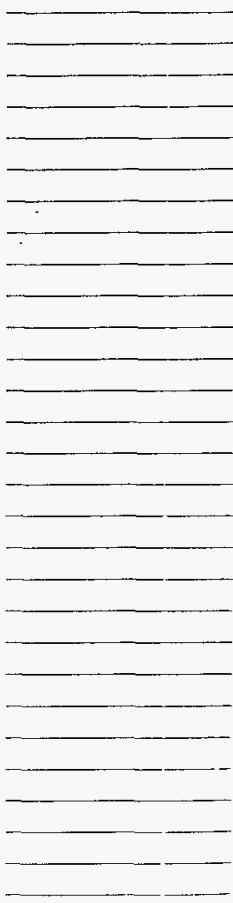

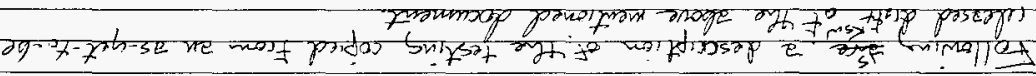

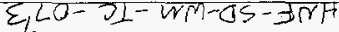

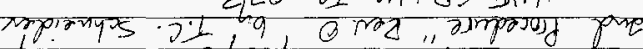

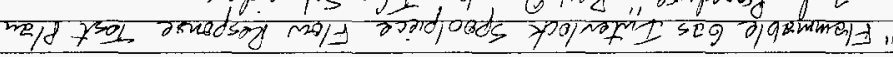

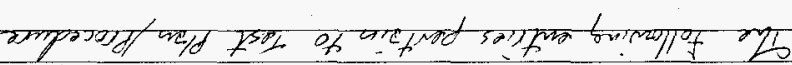

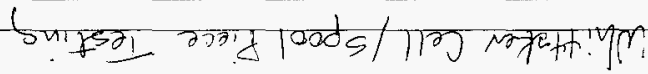


sอţ25txอ200x840

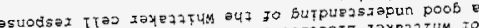

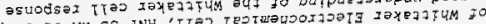

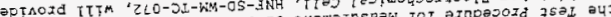

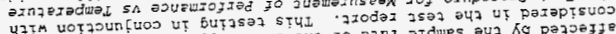

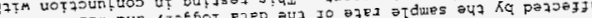

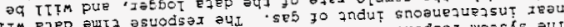

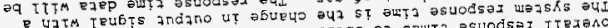

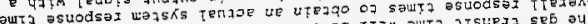
әч

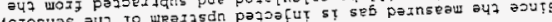

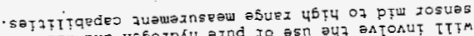

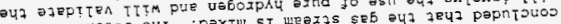

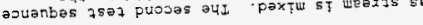

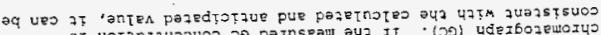

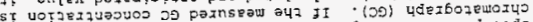
506

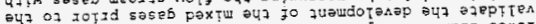
o7 pesก

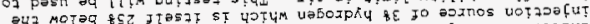

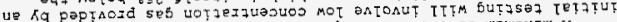

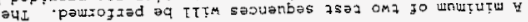

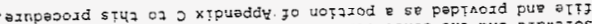

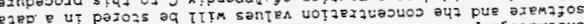
The ses

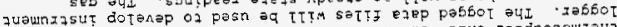

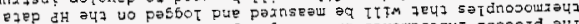

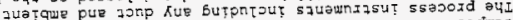

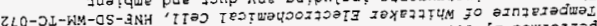

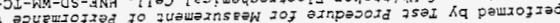

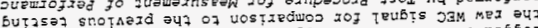
aznsegur os

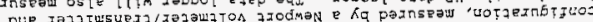

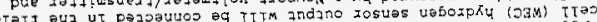
I

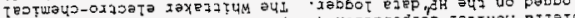

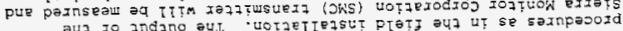

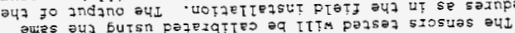

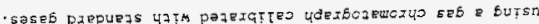
a sos

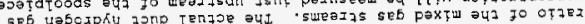

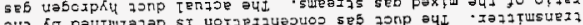

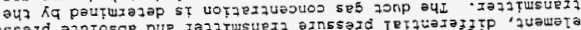

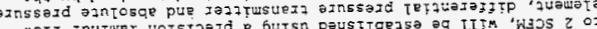

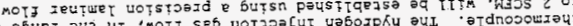

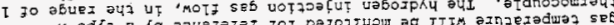

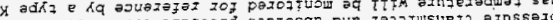

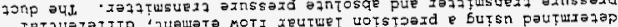

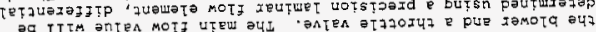

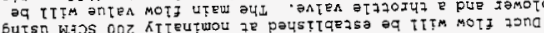

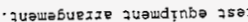

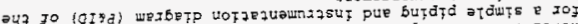

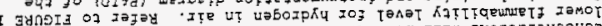

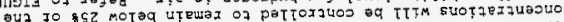

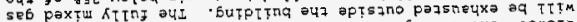

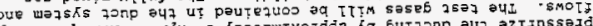

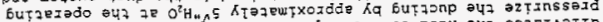

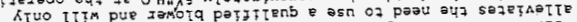

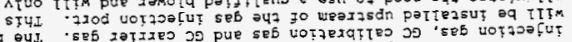
ardues doj pañotdua aq oste titim seseb pessezduos motz jomp jo

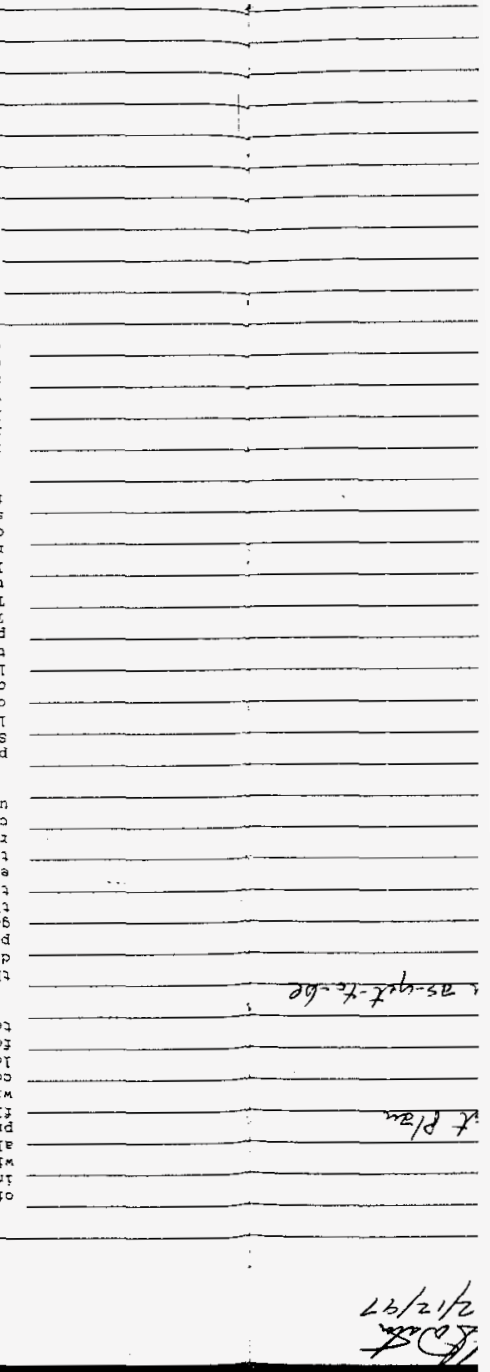

$+2$

단.

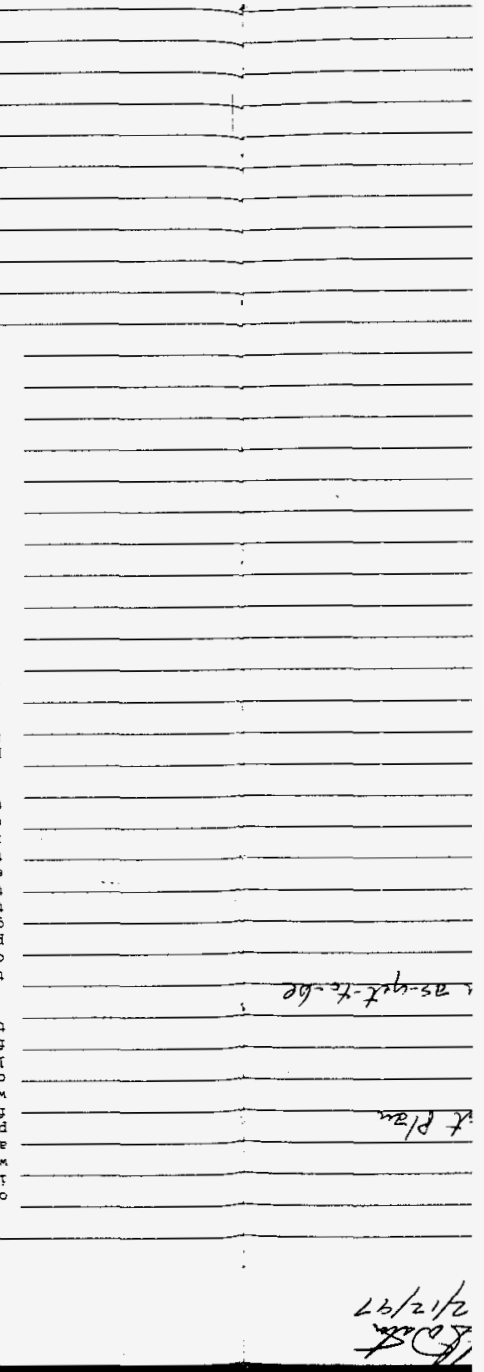

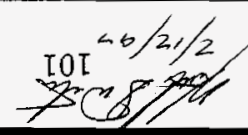

(1) 


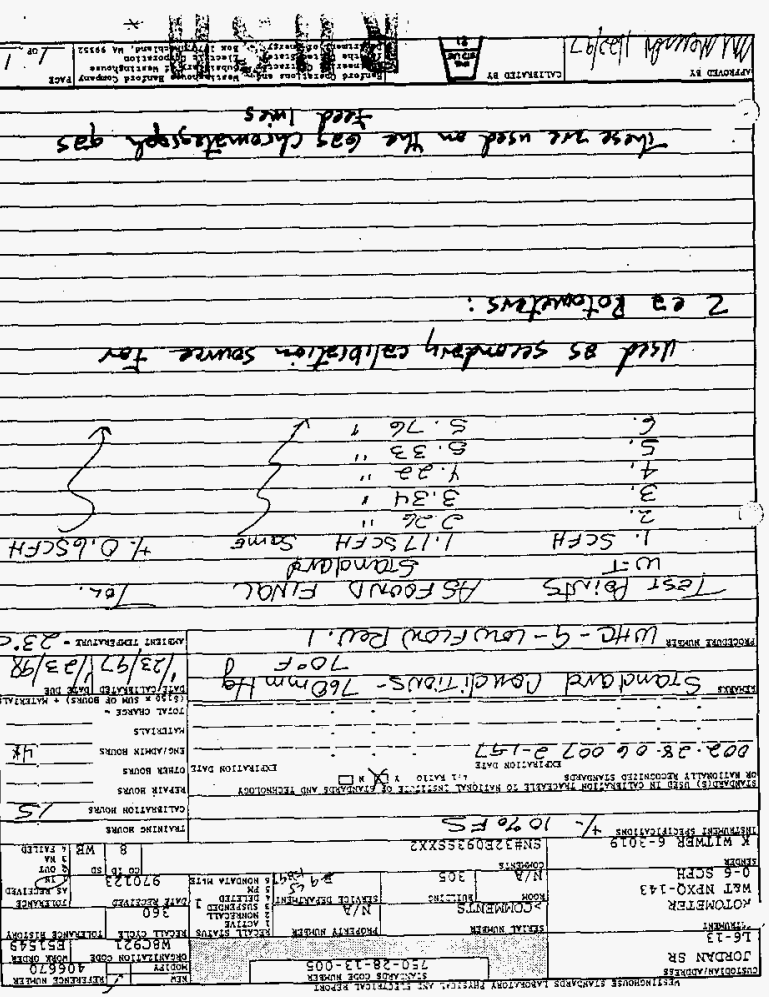

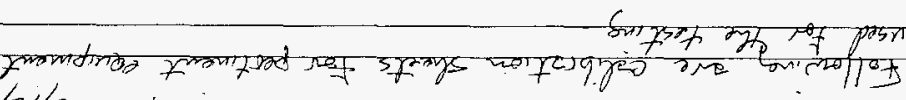
C6/21/2 
多少盾

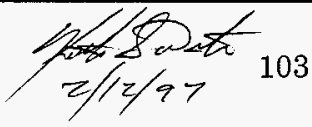

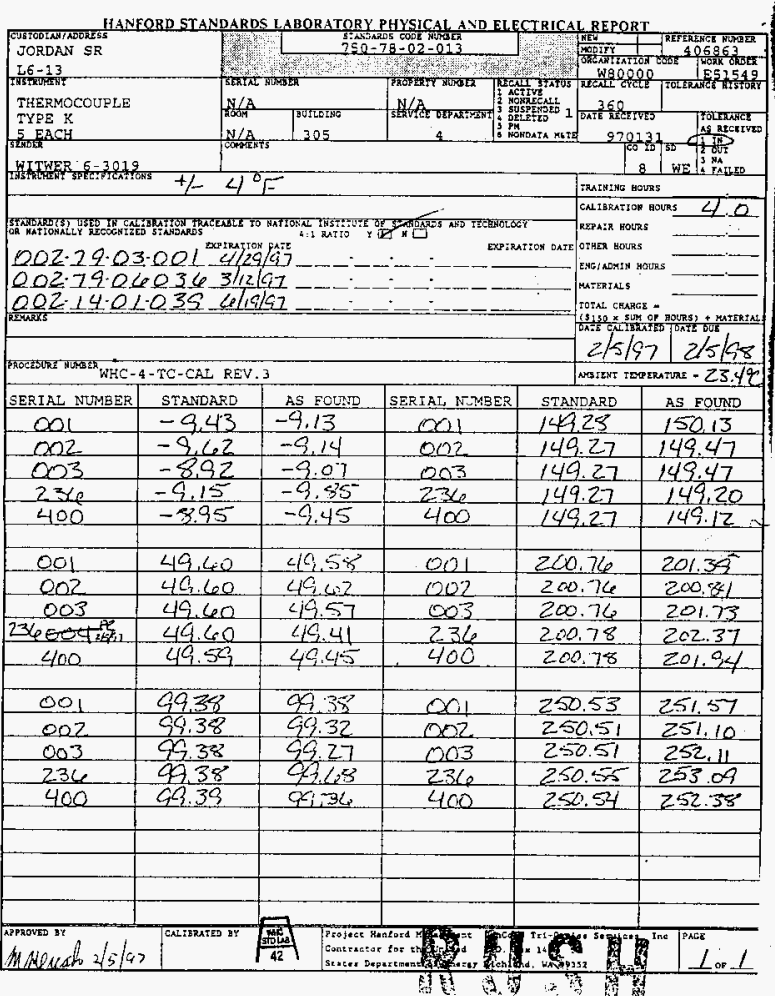



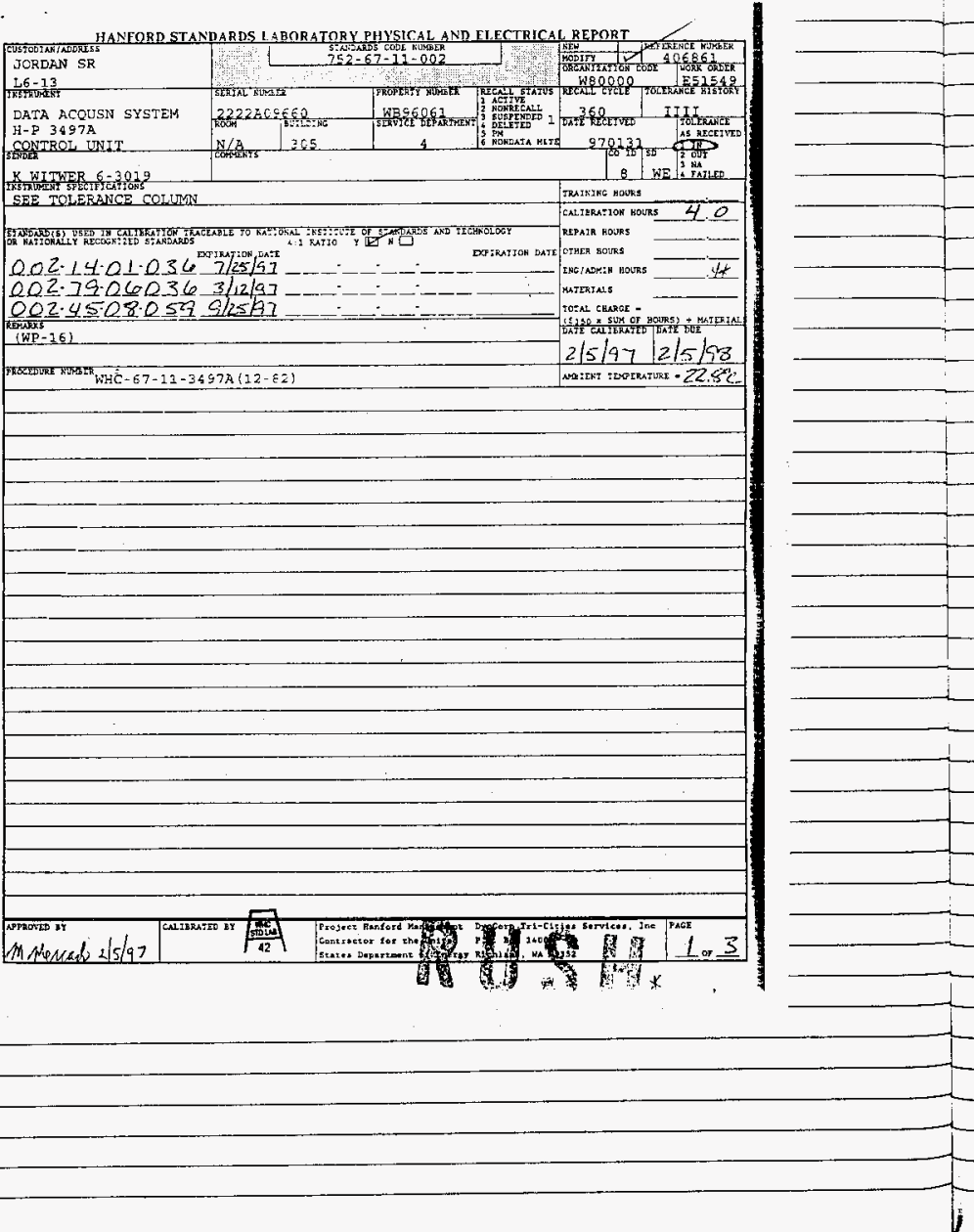

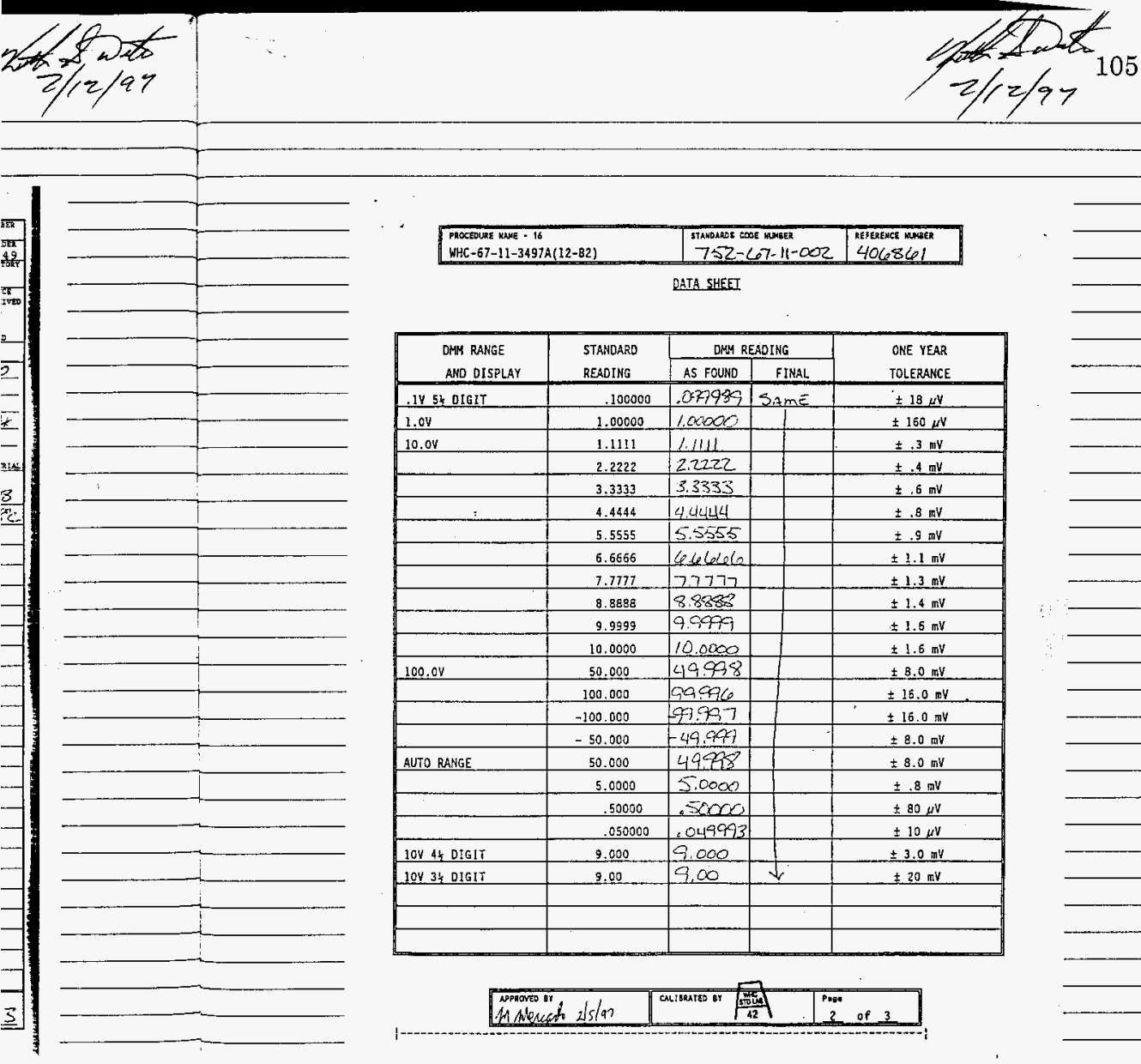

\begin{tabular}{|c|c|c|}
\hline $\begin{array}{l}\text { PROCEDURE WRAE - } 16 \\
\text { WHC-67-11-3497A(12-82) }\end{array}$ & $\begin{array}{l}\text { STAAOALSE COOE HAGER } \\
752-67-11-002\end{array}$ & $\begin{array}{l}\text { REFEREAE WMBQER } \\
406861\end{array}$ \\
\hline
\end{tabular}

\begin{tabular}{|c|c|c|c|c|}
\hline \multirow{2}{*}{$\begin{array}{l}\text { DMH RANGE } \\
\text { AND DISPLAY }\end{array}$} & \multirow{2}{*}{$\begin{array}{l}\text { STANDARD } \\
\text { READING }\end{array}$} & \multicolumn{2}{|c|}{ DMM READING } & \multirow{2}{*}{$\begin{array}{l}\text { ONE YEAR } \\
\text { TOLERANCE }\end{array}$} \\
\hline & & AS FOUND & FINAL & \\
\hline $.145 \&$ DIGIT & .100000 & .077935 & S.zmE & $\pm 18 \mu V$ \\
\hline $1.0 \mathrm{~V}$ & 1.00000 & 1.00000 & & $\pm 160 \mu V$ \\
\hline \multirow[t]{3}{*}{$10.0 \mathrm{~V}$} & 1.1111 & 1.1111 & & $=.3 \mathrm{mV}$ \\
\hline & 2.2222 & 2.2222 & & $\pm .4 \mathrm{mV}$ \\
\hline & 3.3333 & 3.3333 & & $\pm .6 \mathrm{mV}$ \\
\hline \multirow[t]{7}{*}{$\therefore$} & 4.4444 & 4.4444 & & $\pm .8 \mathrm{mV}$ \\
\hline & 5.5555 & 5.5555 & & $\pm .9 \mathrm{mV}$ \\
\hline & 6.6665 & 6066106 & & $\pm 1.1 \mathrm{mV}$ \\
\hline & 7.7777 & ר77.77 & & $\pm 1.3 \mathrm{mV}$ \\
\hline & 8.8888 & 8.8838 & & $\pm 1.4 \mathrm{mV}$ \\
\hline & 9.9999 & 9.9999 & & $\pm 1.6 \mathrm{mV}$ \\
\hline & 10.0000 & 10.0000 & & $\pm 1.6 \mathrm{mV}$ \\
\hline \multirow[t]{4}{*}{$100.0 \mathrm{~V}$} & 50.000 & 49.938 & & $\pm 8.0 \mathrm{mV}$ \\
\hline & 100,000 & 99,996 & & $\pm 16.0 \mathrm{mV}$ \\
\hline & -100.000 & .9 .97 & & $\pm 16.0 \mathrm{\pi V}$ \\
\hline & -50.000 & -49.997 & & $\pm 8.0 \mathrm{mV}$ \\
\hline \multirow[t]{4}{*}{ AUTO RANGE } & 50.000 & 49923 & & $\pm 8.0 \mathrm{mV}$ \\
\hline & 5.0000 & 5.0000 & & $\pm .8 \mathrm{mV}$ \\
\hline & .50000 & $\triangle 50000$ & & $\pm 80 \mu \mathrm{V}$ \\
\hline & .050000 & .049993 & & $\pm 10 \mu \nu$ \\
\hline $1044+\frac{1}{2}$ DIGIT & 9.000. & 9,000 & & $\pm 3.0 \mathrm{mv}$ \\
\hline LOY 3! DIGIT & 3.00 & 9.00 & $v$ & $\pm 20 \mathrm{mV}$ \\
\hline & & & & \\
\hline & & & & \\
\hline & & & & \\
\hline
\end{tabular}



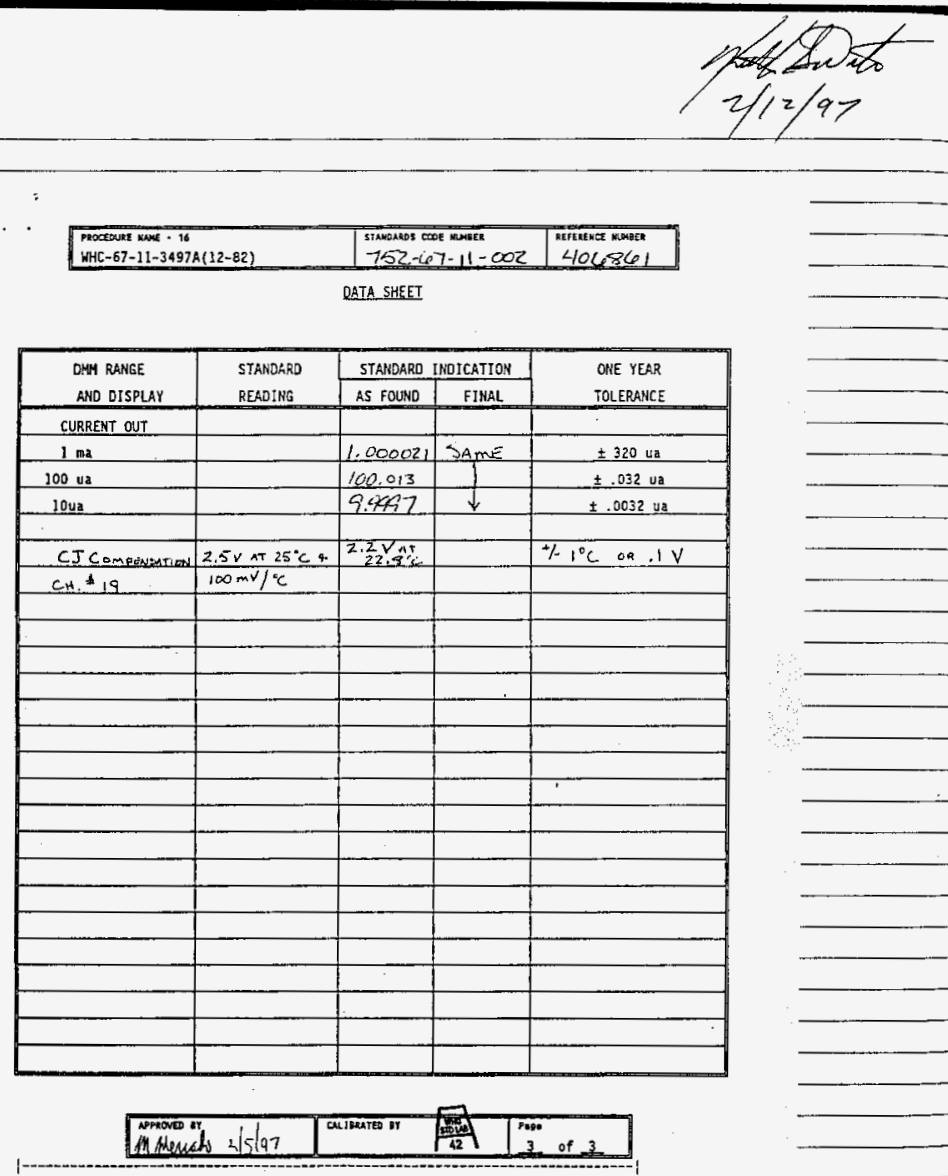

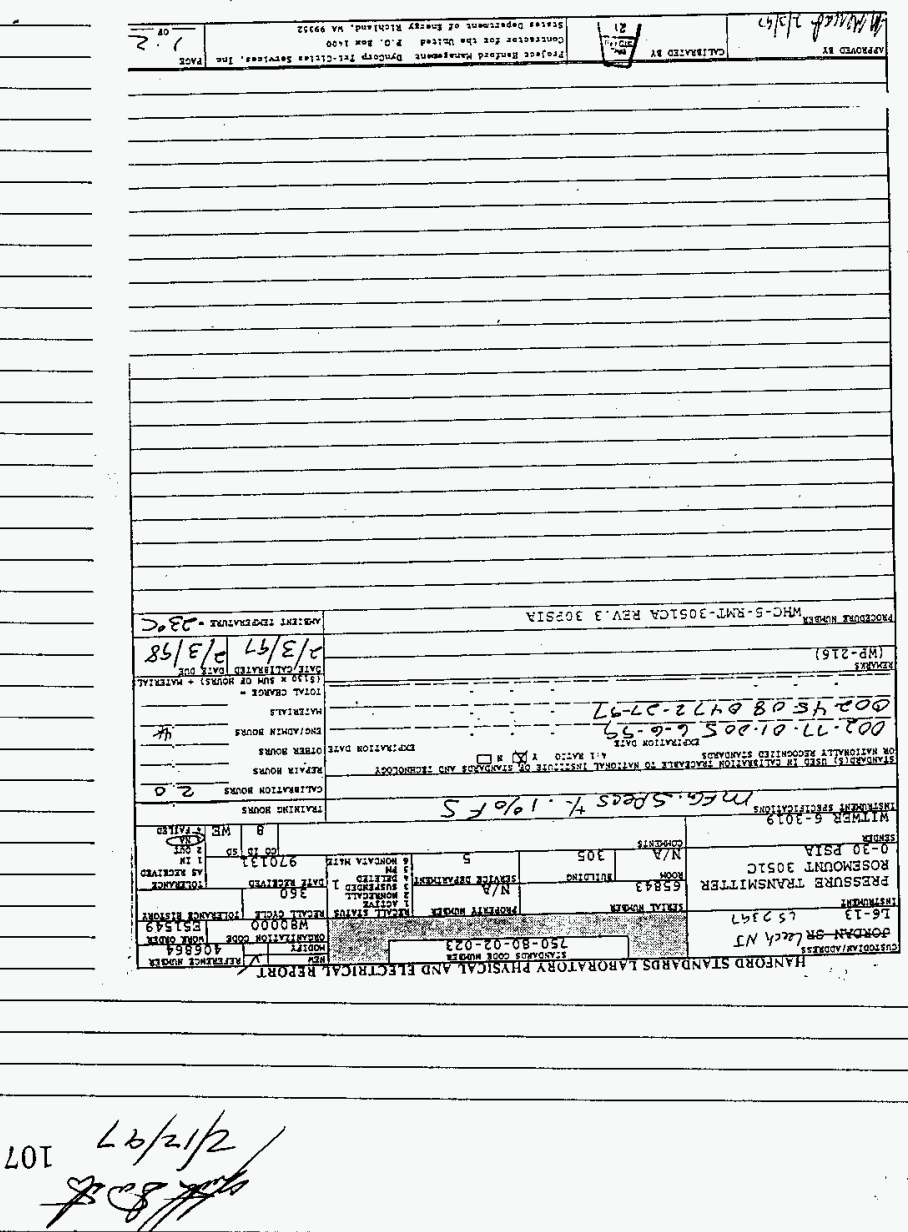

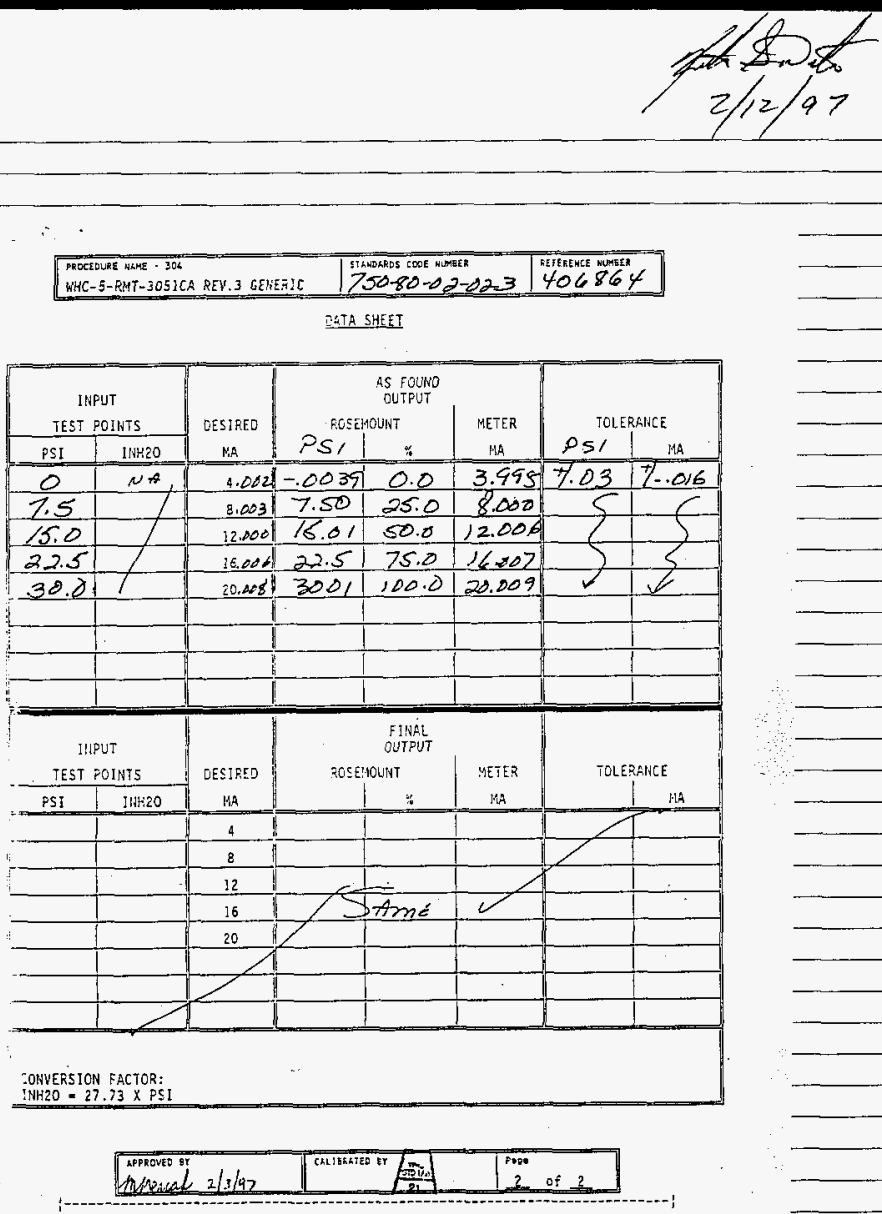


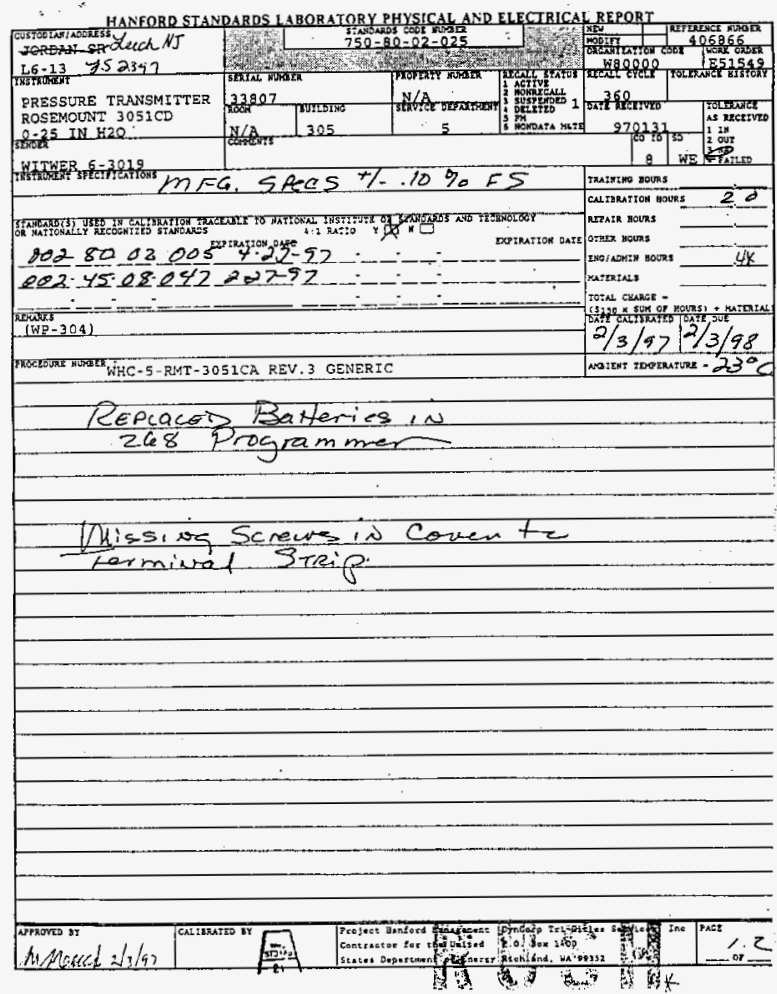



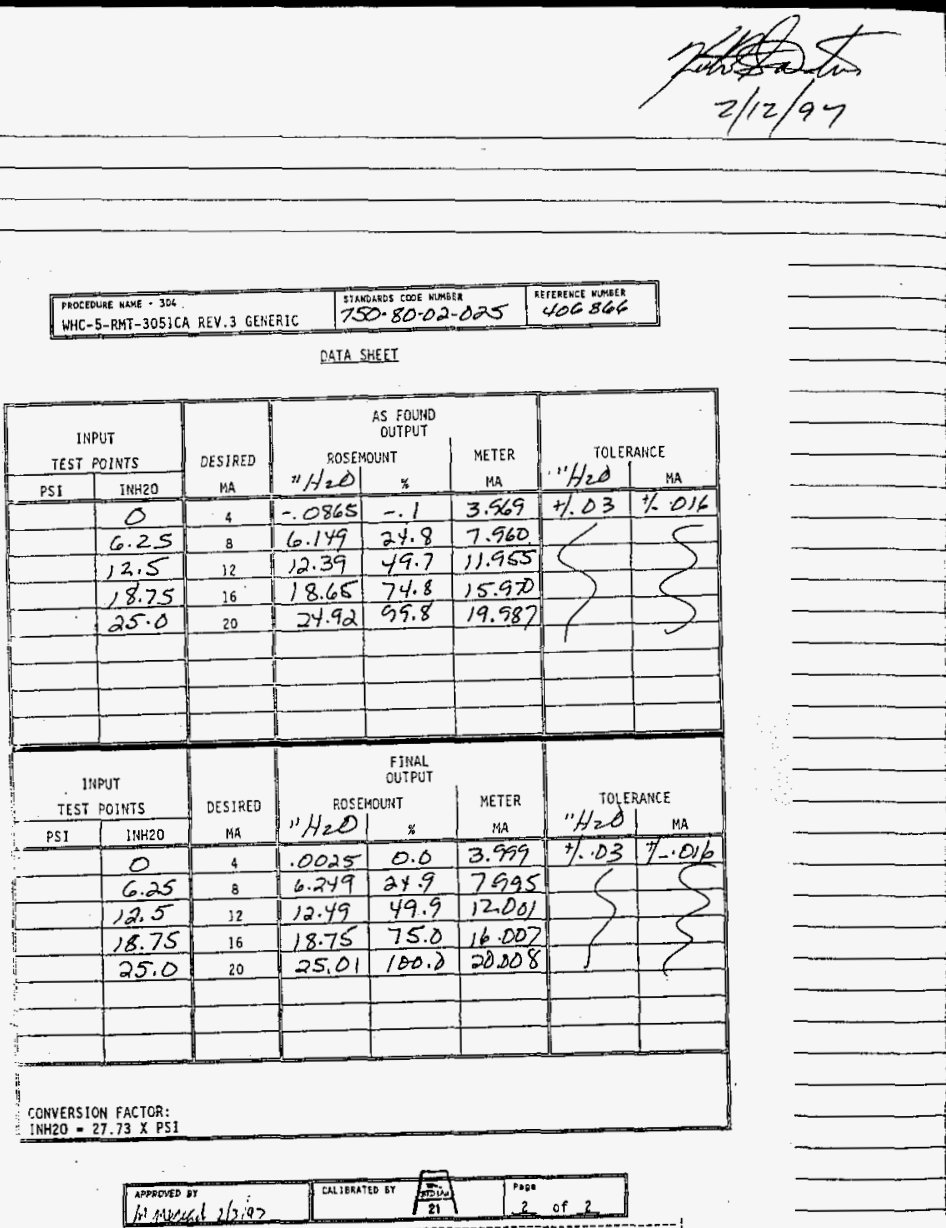

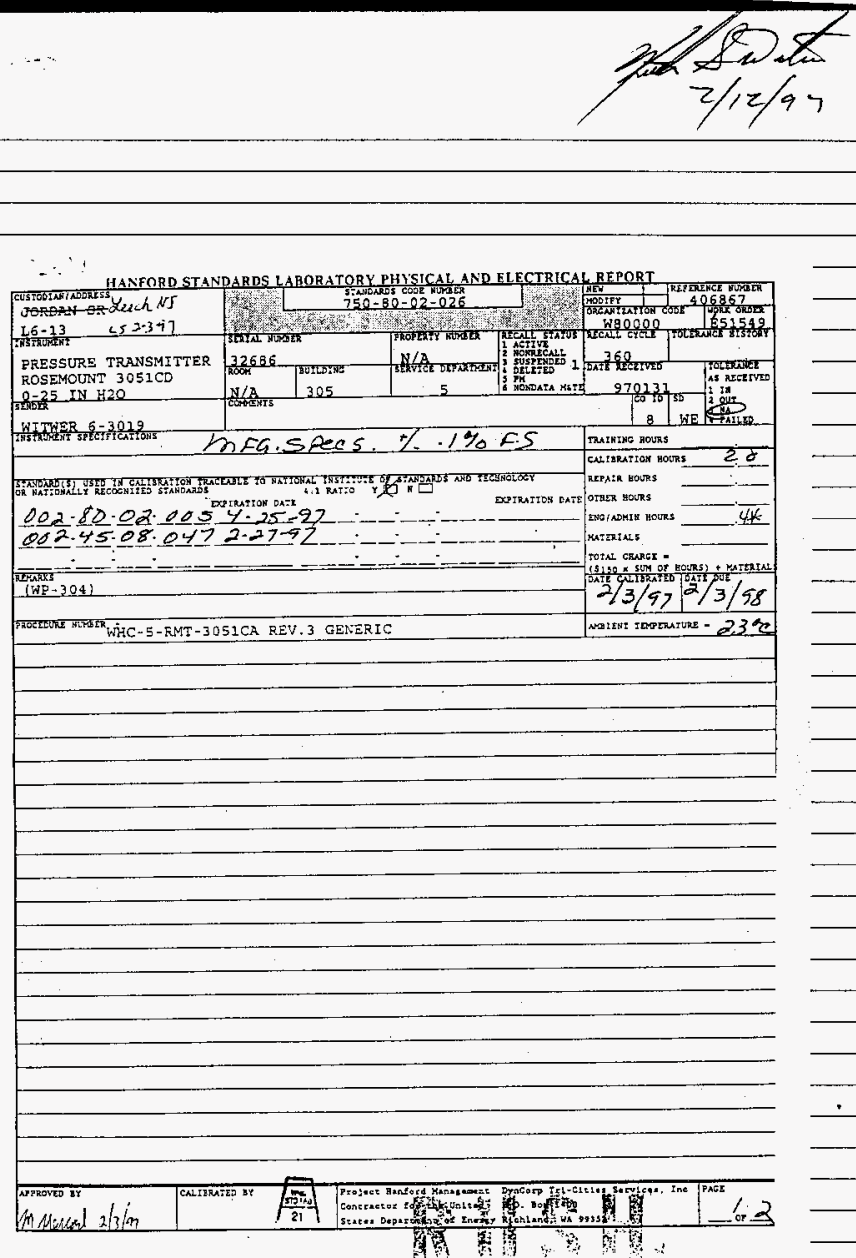


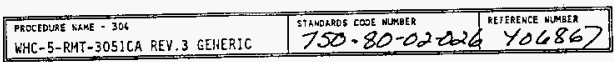

QATA SHEET

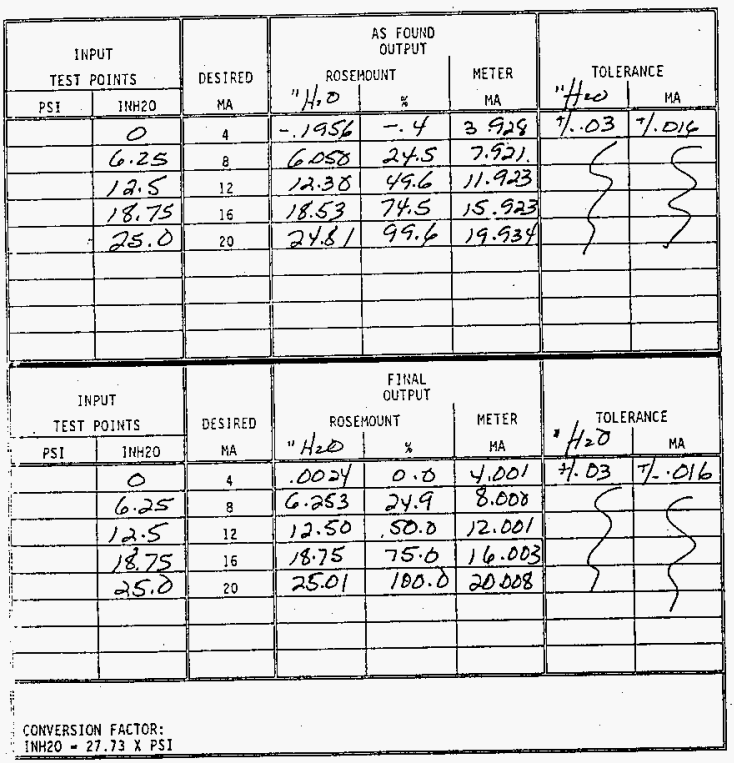

calibrator

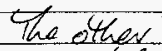
by both lag usings 
Temparature inputs Fo $\mathrm{Te}$-1 through 4 verctied is

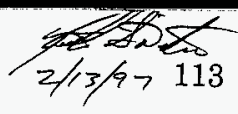

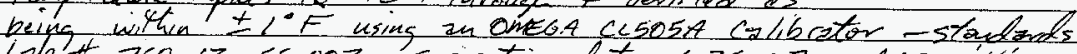

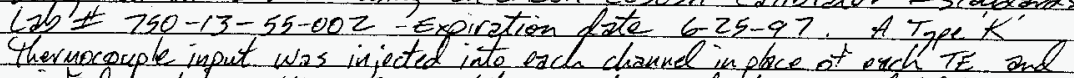

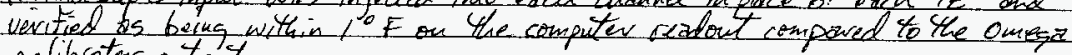
calibrator ontpist.

The other sigual iuputs are straigla voltage inpats and were veritied by bith wisul comparison with Resemonim trowtpanel desplay or by using the calibiation data tam each unit.

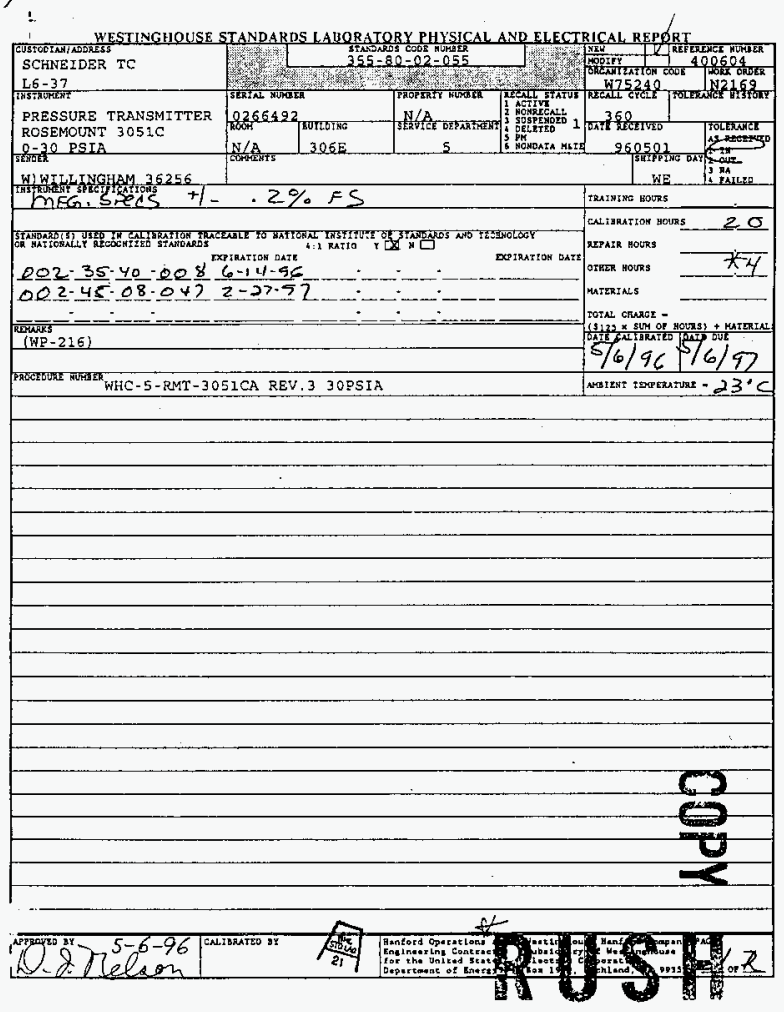




\section{4}
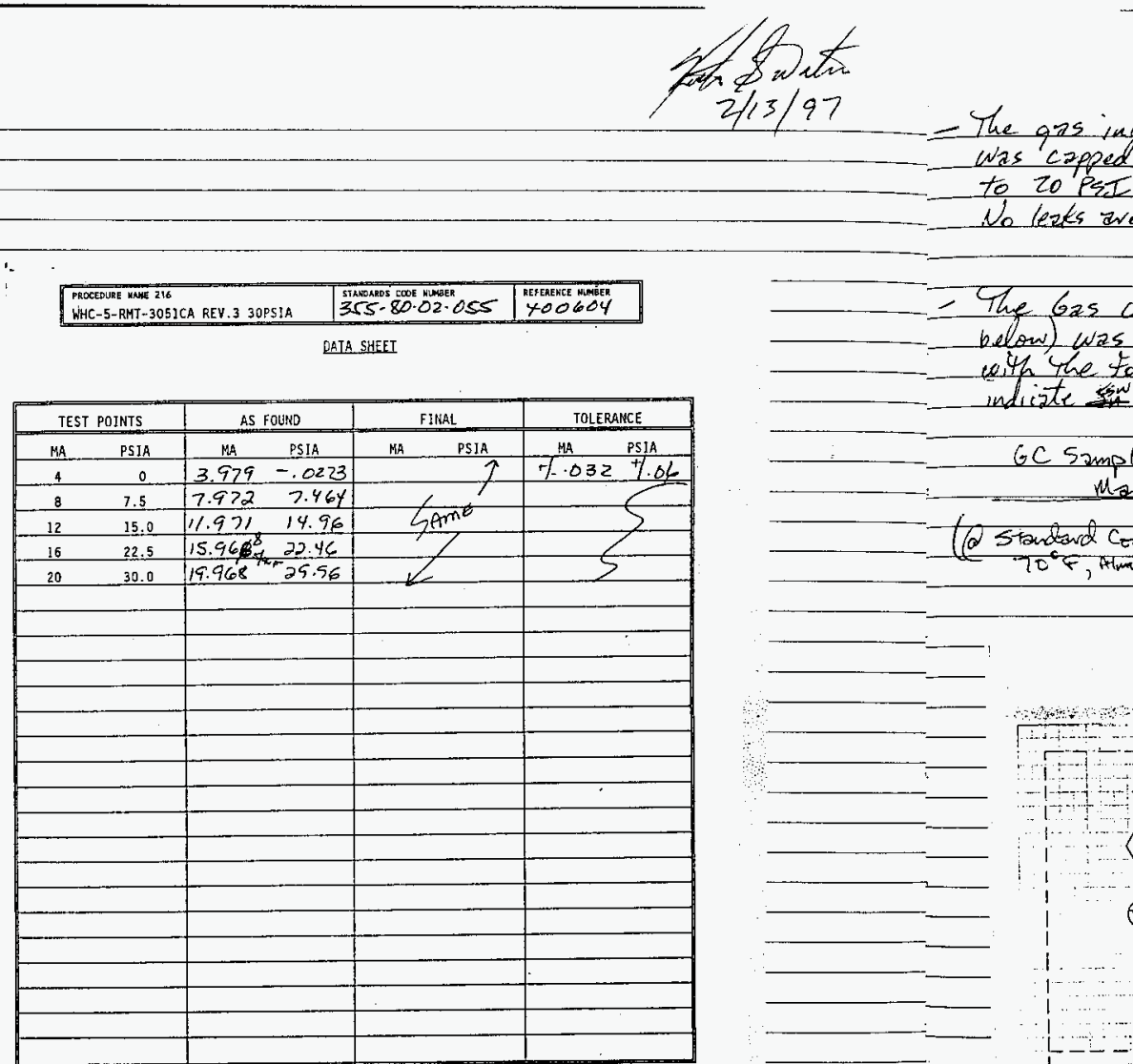

\begin{tabular}{|c|c|c|c|c|}
\hline \multicolumn{2}{|c|}{ TEST POINTS } & AS FOUND & FINAL & TOLERANCE \\
\hline MA & PSIA & MA PSIA & PSIA & MA PSIA \\
\hline 4 & 0 & $3.979-.0223$ & $\tau$ & $7.032+1.06$ \\
\hline 8 & 7.5 & $7.972 \quad 7.464$ & 10 & \\
\hline 12 & 15.0 & $11.971,14.96$ & Seme & 2 \\
\hline 16 & 22.5 & $15.960_{4}^{8} 22.46$ & & $\angle$ \\
\hline 20 & 30.0 & $19.968^{74}-25.56$ & $2<$ & 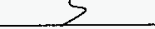 \\
\hline & & & & \\
\hline & & & & \\
\hline & & & & \\
\hline & & & & \\
\hline & & & & \\
\hline & & & & \\
\hline & & & & \\
\hline & & & & \\
\hline & & & & \\
\hline & & & & \\
\hline & & & & \\
\hline & & & & \\
\hline & & & & \\
\hline & & & & \\
\hline & & & & \\
\hline & & & & \\
\hline & & & & \\
\hline & & & & \\
\hline & & & & \\
\hline
\end{tabular}

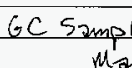
$M_{2}$ $\left(10\right.$ Standeard $C_{\tau}$

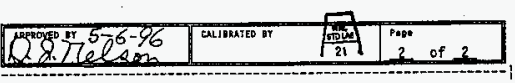




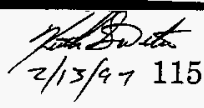

- The gas inlet line from the Flaunable gas bottle (to or some toation) was capped at the fitting next to the main duct The line was presseerized to zo Psi with $N_{2}$ and lezk cherted using sozpig water it each fittings.

- The Gas chromatagraph Sample klow Indiciator (Et-4 on fardo Diasram below) was charoctirized using an HP 0101-0113-504P Ficm FlowneTE ic with the tollowing results. Note that this flowmeter is primaric used to indiate 2 flow/No-trow condition-104 tor an exed flow dalue.

GC Sumple Flow Indertor Marks (oo of Full sale)

\begin{tabular}{|c|c|c|}
\hline (2) Standzand condtions) & 25 & 7.73 \\
\hline (a Standird lenditions & 50 & $24.60 \mathrm{cc} / \mathrm{min}$ \\
\hline & 75 & 63.29 \\
\hline & 100 & 111.10 \\
\hline
\end{tabular}

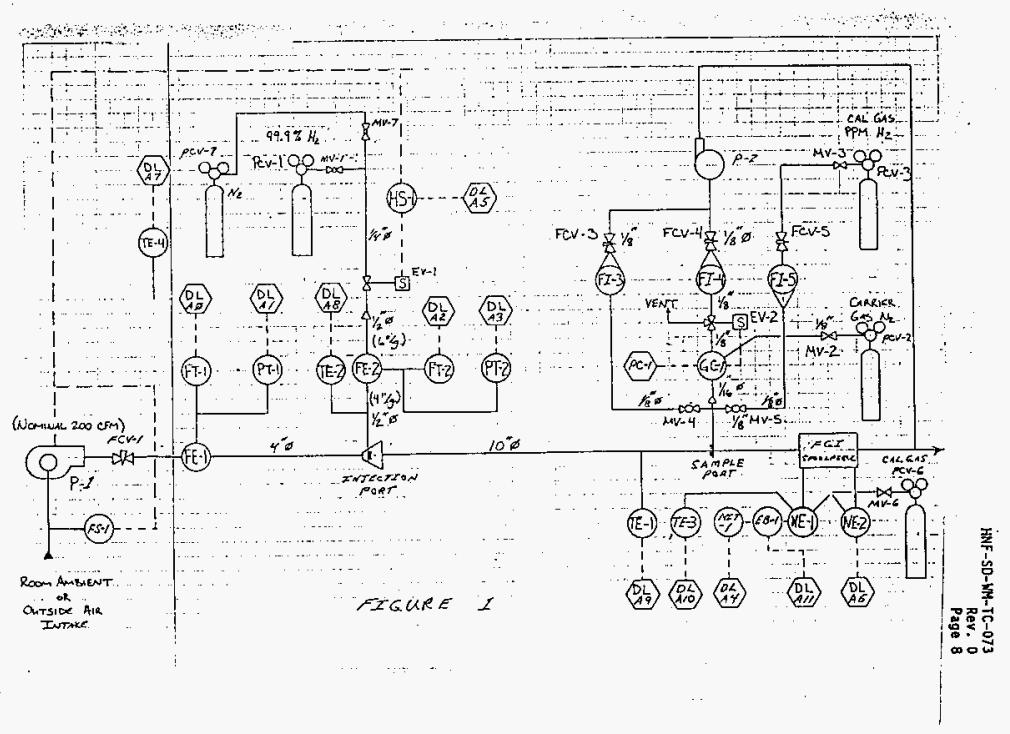

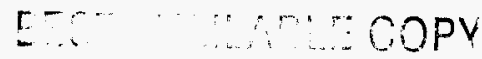


116

FI-3 \& FI-5, Petometers used with the bas Giromatasrach unit were calibrated asing a third rotometer

which was calibrated at the siandards lall - Cal 250-28-13-005 Range $=0-6$ SCFH - AIR. Following are, the results fiom the calibration fcharaterization (Cal Expires (-27-98)

Primary Flowmeter $C x+150-28-13-005$ Indicstion (SCFH)

0.5
1.1
1.6
2.1
2.5

Flowmeter FI-3 Indication (SCFH)

$$
\begin{array}{c|c}
0.5 & 0.5 \\
1.1 & 1.0 \\
1.6 & 1.5 \\
2.1 & 2.0 \\
2.5 & 2.5 \\
\hline & \\
\hline \text { Primzern Flowmeter } & \text { Flowme } \\
\text { Imdication } & \text { Ind } \\
\text { (SCEH) } & \text { (SCH) }
\end{array}
$$

\begin{tabular}{l|l}
0.4 & 0.5 \\
1.1 & 1.0 \\
1.6 & 1.5 \\
2.0 & 2.0 \\
2.4 & 2.5
\end{tabular}

These values were obtained bu Marty Casito, SESC 


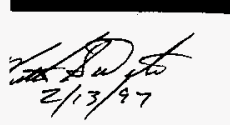

Following is a summary listing of the test exiupment used

$50-28-13-005$ iets trom
MEASUREMENT APPENDIX A

MEASUREMENT AND EQUIPYENT CATA SHEET

FE-1 Main Duct flow Elemert Laninar flow Element, Meriam Inst. Model 50MC24, Seriat No. $757200-\mathrm{K}$

Range: $0-424.5$ SCFM air at std. temp./press.

Accuracy: $1.0 \%$ of reading.

Cal. Std.: Vendor Cal. Record Curve

FT-1 Main Duct Flow Xmitter Diff. Press., Rosemount, Model 305:CD, Serial

No. 32686

Range: $0-25$ " $\mathrm{H}_{2} \mathrm{O}$

Range: $0-25 \mathrm{H}_{2} \mathrm{O}$

CaI. Std.: Std. Lab. No. 750-80-02-026

Cal. Due Date: $2-3-98$

PT-1 Main Duct Press Xnitter Abs. Press., Rosemount, Model 3051CD, Serial No. 65843

Range: 0-30 psia

Ascuracy: $0.1 \%$ FS

Cal. Std.: Std. Lab. No. 750-80-02-023

Cal. Due Date: 2-3-9B

FE-2 Injection Flow Elenent Laminar Flow Element Merian inst. Model somJ1010, Seriai No. 753510 - 33 Range: $0-1.5087$ SCFM air at sto. temp./press. Accuracy: $1.0 \%$ of reading

Ca1. Std, : Vendor Cal. Record curve

FT-2 Injection Flow Xmitter Olff. Press,, Rosemount, Model 3051Co, Serial No. 33807

Range: $0-25 * \mathrm{H}_{2} \mathrm{O}$

Accuracy: 0.1\% FS

Cal. Std.: Std, Lab. No, 750-80-02-025

Ca7. Due Date: 2-3-98

PT-2 Injection Press Xmitter Abs. Press., Rosenount, Model 3051CD, Serial No. 0266492

Ràngq: $0-30$ psia

Accuracy: $0.1 \%$ FS

Ca1. Std.: Std. Lab. Ho. 355-80-02-055

Ca1. Due Date: 5-6-97

FI/FCV-3 GC Bypass Flow Rotameter with outlet control valve, Wallace \&

Tiernan, Model 32E083sixx

Range: $0-2.5$ CFH air

Accuracy: iox Fs

Cal.: Test Log Comparison (Indication Only)

FI-4 GC Sample Flow

Rotameter, Matheson, Model FM-1050 E]-4Y10L-E910 Range: 0-100 cern air

Accuracy: $5 \%$ is

Cal.: Test Log Comparison (Indication only)

\section{That

. 
118

cant:
FI/FCY-5 oC Cal. Flow

TE-1

TE-2

TE-3

TE-4

GC-1 $\mathrm{H}_{2}$ Concentration Std.

PC-1 GC Control Computer

DL-1 System Data Logger

NE-1 Whittaker Sensor

NE-2 SMC SEnsor

Handheld Digital Multineter

Rotameter with iniet control valve, Wallace a Tiernan, Model 32E083s1XX2

Range: $0-2.5 \mathrm{CFH}$ air

Accuracy: 10x Fs

Cal.: Test Log Comparison (Indication onfy)

Type $K$ stainless steel jacketed thermocouple Cal.: Std. Lab. No. 750-78-02-013 S/N 002

Cal. Due Date: $2-5-98$

Type $X$ stainiess steel jacketed thermocouple Cal.: Std. Lab. 110. 750-78-02-013 S/N 236

Cal. Due Date: 2-5-98

Type K stainless steel jacketed thermocouple Cal.: Std. Lab. No. 750-78-02-013 S/N O01 Cal. Due Date: $2-5-98$

Type $K$ stainiess steel jacketed thernocouple Ca1.: Std. Lab. No. 750-78-02-013 $5 / \mathrm{N} 400$

Cal. Due Date: $2-5-08$

Dual Column GC, Micro-sensor Tech. Inc., Model H200D, Serial No. 150313

Range: $100-30.000 \mathrm{ppr}$

Accuracy: 10\% Recóing

Cal.: Standard Eas per Appendix $F-3$

Lap Top PC, Toshiba Satelifte Pro Nodel 405CS, Serial No. 02528992-1

Range: N/A

Aecuracy: $N / A$

H-P Data logger, Mode] 3497A, Serial No. 2222409650

Range and Accuracy: $0-0.1 \mathrm{Vdc}( \pm 18 \mathrm{uVdc}), 0-100$

Vdc $( \pm 0.3 n V o c), T / C\left( \pm 1^{\circ} \mathrm{C}\right)$

Ga1.: Std. Lab. No. 752-67-1]-002

Cal. Due Date: 2-5-98

Electro-Chemical Cell, Whittaker, Made] $1090020-$

2. Serial No. HS-1226

2, Serial No. HS-1226

Range: 0-10x $\mathrm{H}_{2}$ by volum

Cal.: Standard Gas per Appendix F-2

Combustible Gas Module, Sierra Monitor Corp., Model 410]-02, Serial ino. 96-8-4455

Range: $0-100 \%$ LFL $\mathrm{H}_{2}$

Aceuracy: $3 x$ Fs

Cal.: Standard Gas per Appendix $F-1$

Fluke Model 702, Serial No. 6240608

[al.: 5td. Lab. No. 750-13-71-001

Cal. Due Date: 32-06-97

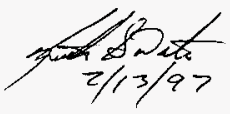

$2 / 13 / 97$ , 
Correction Factors used b3, uP Bto Ace sustem to change inpet volteges to the proper engueeriong units are as tollows

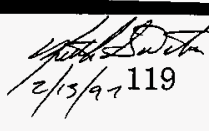

\begin{tabular}{|c|c|c|}
\hline ch $L$ & Iuput Measured & slope \\
\hline 0 & Main Duct DifF Pess $\left(\frac{14}{47}\right)$ & 6.2527 \\
\hline 1 & M/ain Duct Abs Press (PSiA) & 7.49622 \\
\hline 2 & 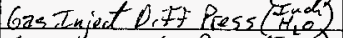 & 6.2544 \\
\hline 3 & Gas Inied Alos Press ( $\left.B^{\prime}{ }^{\prime} A\right)$ & 2.5 \\
\hline 4 & Neweart I Itimitontant (PPM) & 250 \\
\hline 6 & $S M C\left(P P_{M}\right)$ & 2483.08 \\
\hline 7 & Ambient tamp (oF) & (N/A) \\
\hline 8 & Fltrmuable Gas Temp $(C F)$ & $N / A$ \\
\hline 9 & Main Duct Tamp ( ${ }^{\circ}$ ) & $(N / A)$ \\
\hline 10 & Whittaker Cell Teup ( $\left.{ }^{\circ} \mathrm{F}\right)$ & $(N / A)$ \\
\hline 11 & Whittater Cell Oulput (Volss) & 1 \\
\hline
\end{tabular}

\begin{tabular}{|c|c|}
\hline offset & \\
\hline-6.2607 & \\
-7.4918 & \\
-6.2647 & \\
-7.5 & \\
-250 & \\
-2557.57 & \\
& \\
& \\
& \\
\hline
\end{tabular}

- Correction Value used to calibrate Newport Iutinaty miter trom WEC cell imput was bosed on the tollowing Initial voltage $=0.206 \mathrm{~V} \rightarrow 0$ PPM Final $V_{0} t_{\text {tag }}=1.406 \mathrm{~W} \geqslant 8070 \mathrm{PPM}$

- This would give a correction Factor of PPM $=6725\left(V_{\text {in }}\right)-1385.35$ The aps flow rate was 2 scfH to the lec cell tor 15 minutes.

- Corraction Value used to calibrate SMC input was based on the following values

$$
\begin{aligned}
& \text { Iritial Veltage }=1.03 \mathrm{~V} \rightarrow 0 \mathrm{PPM} \\
& \text { Fingl Voltage }=4.28 \mathrm{~V} \rightarrow 8070 \mathrm{PPM}
\end{aligned}
$$

- This quves the cerrection factor of. $P P M=2483.08\left(v_{i m}\right)-2557.57$

The Gas flow for This $8070 \mathrm{fm} \mathrm{H}_{2} \mathrm{Cal}$ qas was $550 \mathrm{cc} / \mathrm{min}$ to the $\mathrm{ks}$.

$$
\text { SMC }
$$

- CaC Cofibrotion porometur:

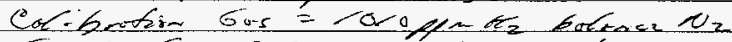

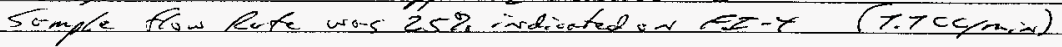


Ge-Crlitedosor Cortivaed?

Colibrotion fite $N_{0} 02 / 37 \mathrm{cat}$

Ther seguentiat Cot urlues are:

$10045^{3} 6$ ftr

1008.98 ApM

100956 pen

1009.18 epm

Te 8070 cheger was terted by the $Q S$

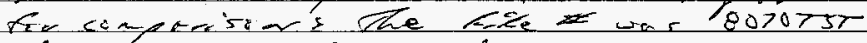

the refuetior volues rend were:

\section{4 \\ $8040.09 \mathrm{pa}$ \\ $8032,3 \mathrm{fer}$}

Complets the secow corcentuos The ges

Ciscosit Evitien

$0.850 \mathrm{Fm}$ taturif Mace a presen the rec ET-2 reap

Since were using $3 \% \mathrm{H}_{2}$ we take $3 \%$ of the difference ot these two Ualues $\quad 03(181.7-87.6)=2.823 \rightarrow$ Subtract this amenest from the Air factor $7(131.87-2.823)=778.88^{150} /$ The air volue is what the thow senser curve is bosed on

Thus a correction foctor of $179.047 / 481.82=-284$

We know correction tator for pure $H_{2}=87.6$

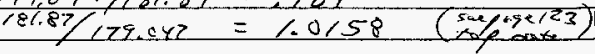

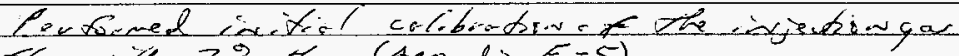
Fir writh $39 \pi_{2}$. (Appendix $<-5$ )

PT-2 $=6.937 \mathrm{r} / \mathrm{kz} \quad \quad P T-z=15.89$ p5s

Tonpe TE-2 $=71{ }^{\circ}$ PCV-Z $=6$ psig

SCFA equivalest $=1.269 \mathrm{SCFM}$

G'C meascival Otlue $=237.8 \mathrm{ppm}$.

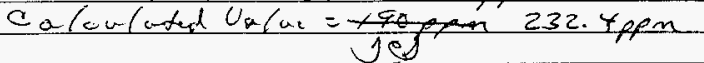


is $\Omega_{1}$ 757

Completand the tow curcertuation testiug ard bagen the seward fhase testing wrth the s,000-5000 ffm

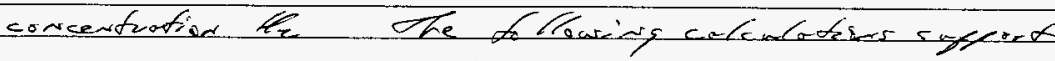
The gas mixing.

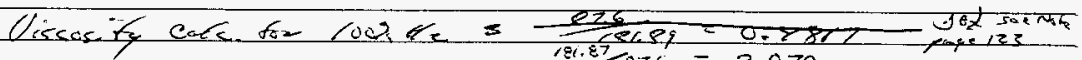

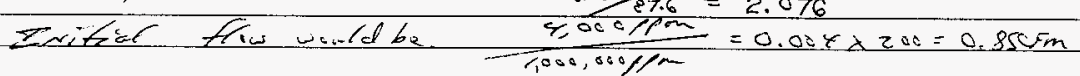
$18 i .8787 .6=2.076$

$0.85 \mathrm{Fm}=$ Sesiond ffow $\quad \frac{0.8 \mathrm{CF}}{1.6 .087} \times 8^{*}=3.978^{*} / \mathrm{F}_{2} 0$

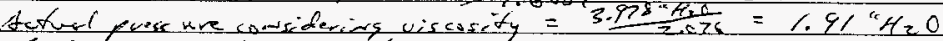

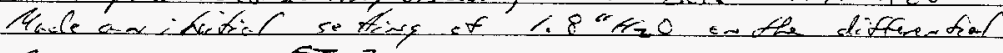
persena gouge FE-Z?

The radi-ys ind

$F T-2=1.78 \quad C F=2=18.73$

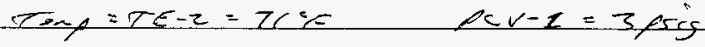

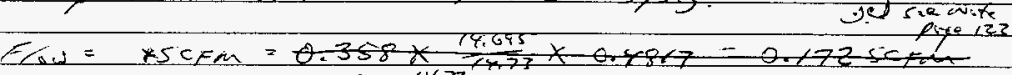

ef these two ame air volue

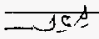

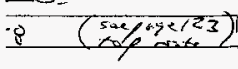
zingar

$=0.358 \times 24.73 \times 2.076=0.745565$

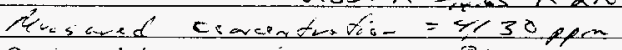

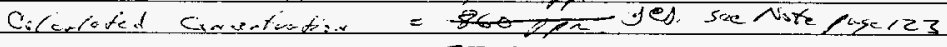
$3725 \mathrm{ppm}$

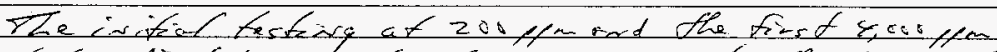

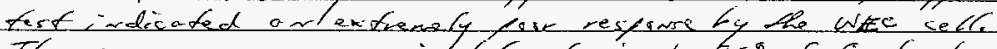

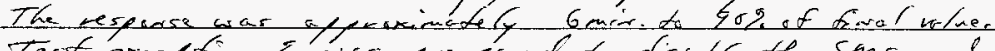
Test exicettion 5 was prepared to dist bt the $5 \mathrm{mc}$ ard

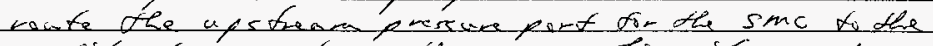

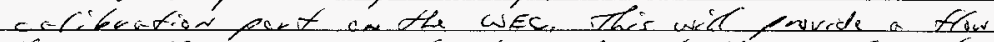

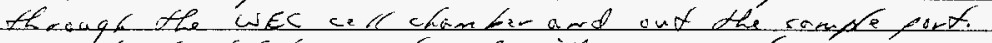

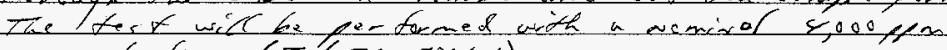
concartrotions. (Tat ID. $E X /-1$ )

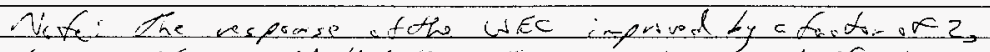

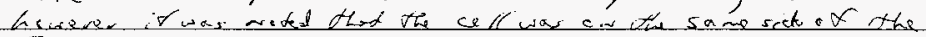

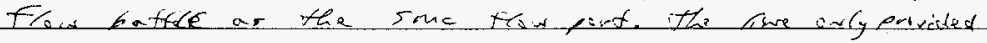


arrother condinction foth. A dowers trean (efthe kefole)

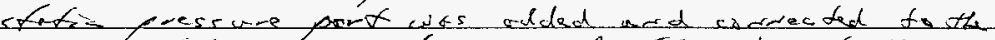
wee culitertien port to provede frow thaingt the

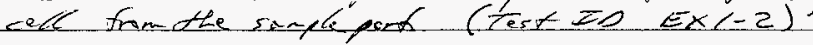

The cell cesperar was sjoridicurtly

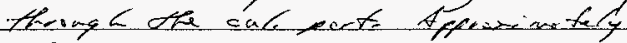
veluos. rinferved with a fin

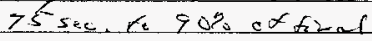$$
\frac{2(14) 7}{x+2}
$$

$22 \theta-57160$

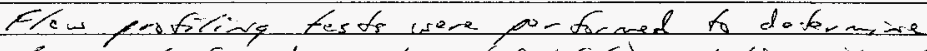

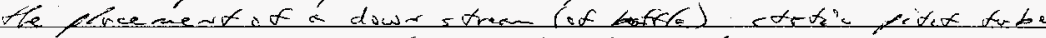

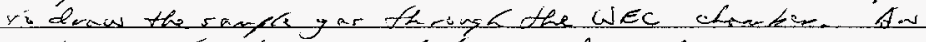

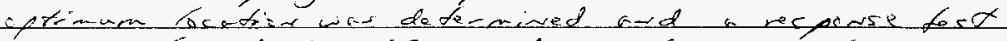

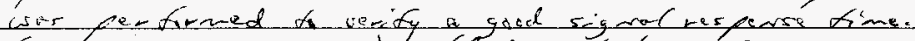

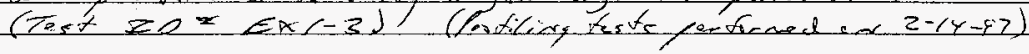

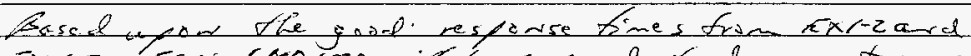

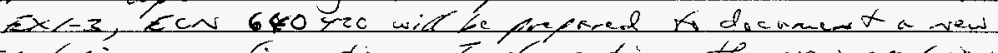

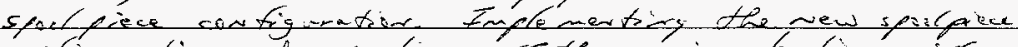

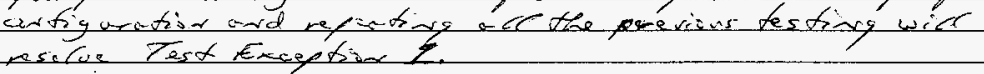

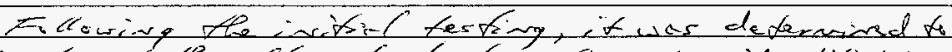

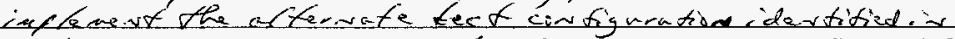

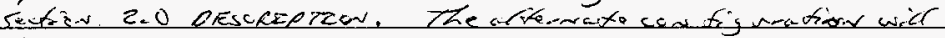

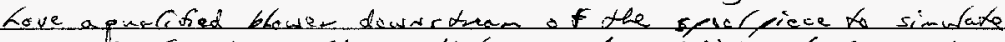

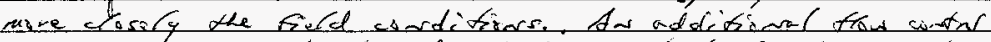
carce FCV-2 with be lacted at the intet of the blawer to

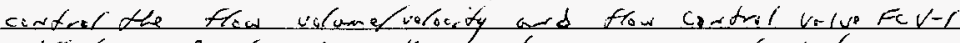
will be ured $\alpha$ cedvee the sys tem fress and to ketween

- zand-4"reo, the - 3t4 "Azo is the anticipated field regotive system porsuns (par telecenwith Jim Ritiverer, $S \in S \in, 2$.

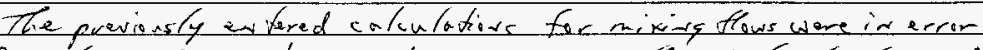

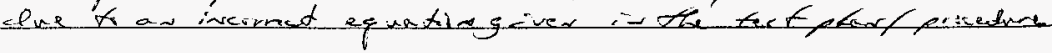




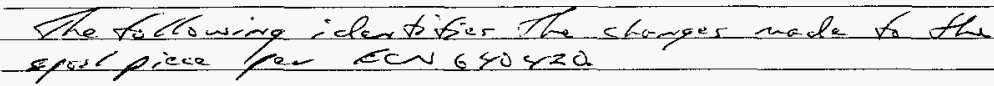
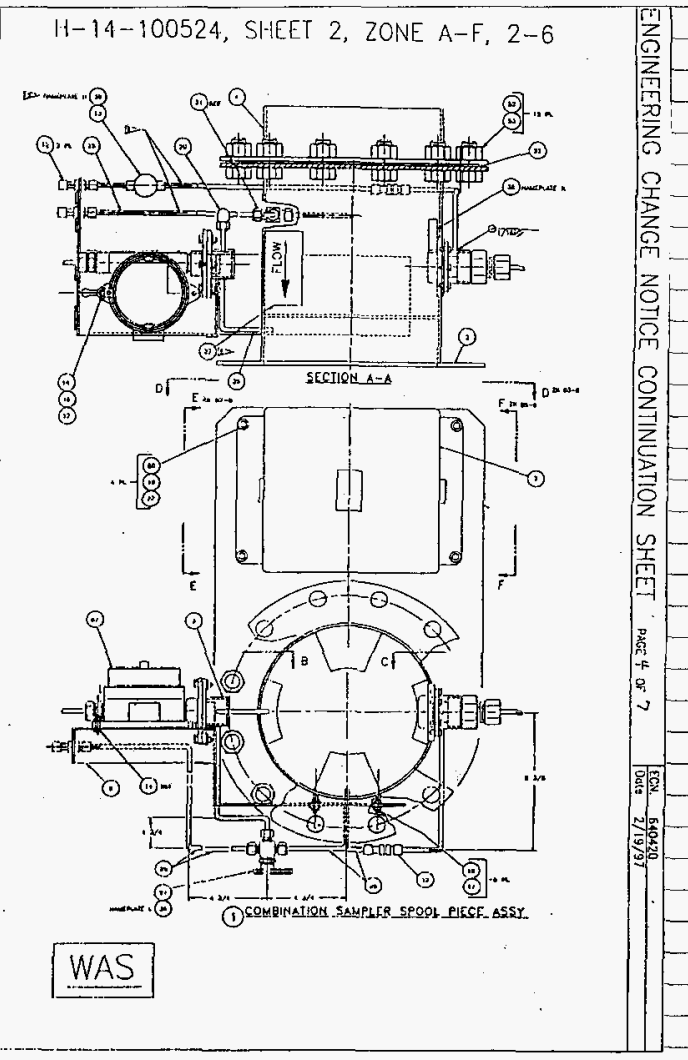

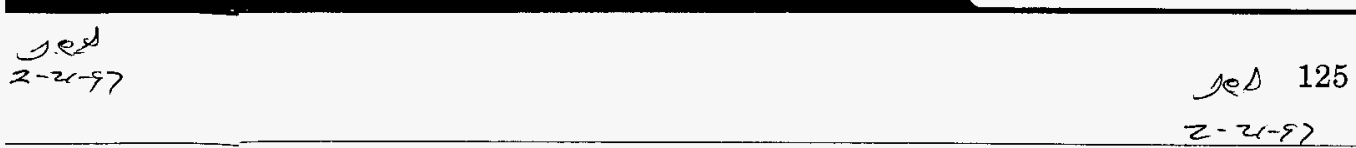

the

11-14-100524, SHEET 2, ZONE A-F, 2-6

$$
\text { (1) }
$$<smiles>C1CO1</smiles>
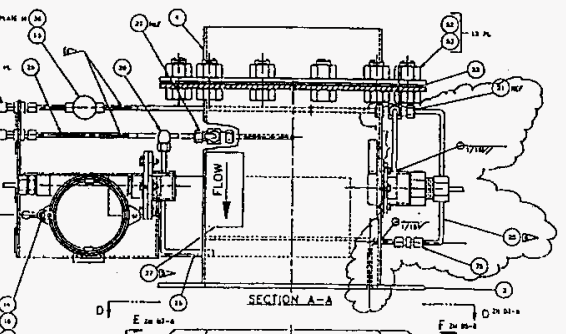

of Exa.-
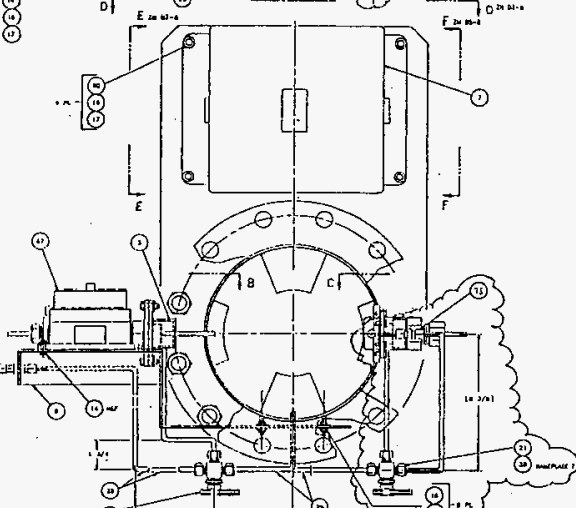

(i) E.

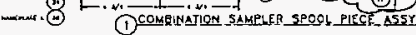

IS

Instructions: Field route tubing as shown \& as required by general notes. Required dimensions shown on page 6 . 


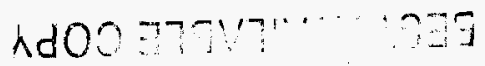

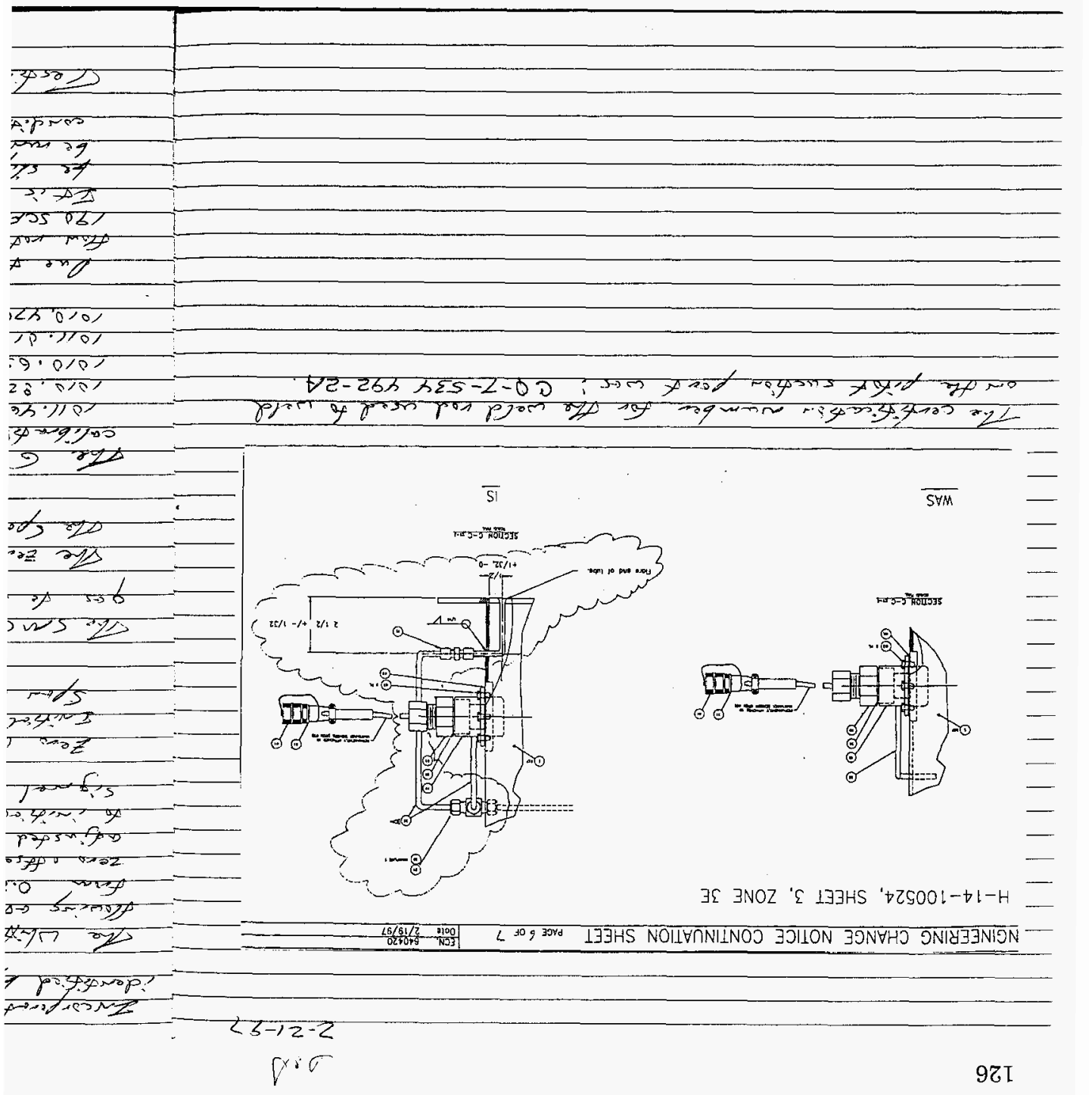




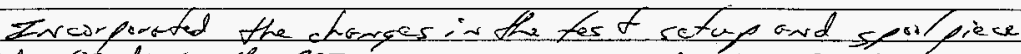
idartified by the Afro on page 123 aral ECN 680 y20.

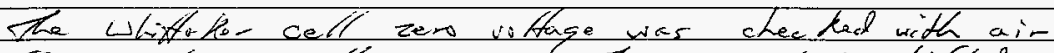

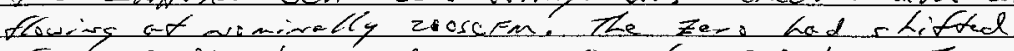

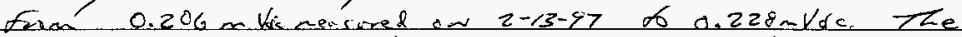
zees offsat was idjusded a d the fieal value or sporowas

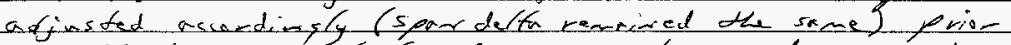

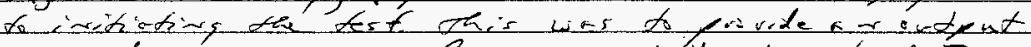

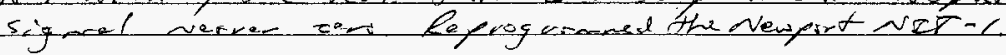

Zero voltoge $=0.228$

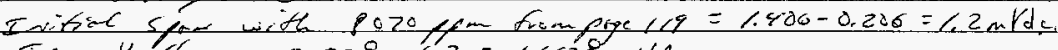

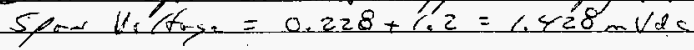

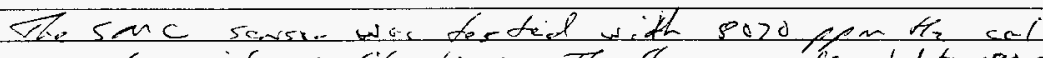

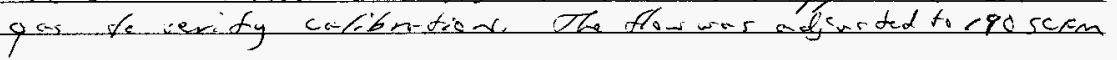
The Zers wis adjisded to 0.8 fen ote Goor was adjust o 8065 pem

The GCius set ufand ciolibroted with the reropen

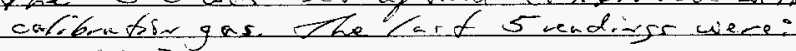

to 1 ineld coll. $407 \mathrm{fem}$

ros. izs'ppo

10,0. estepen

rocr.dro fer

$10,0,876$ ffem

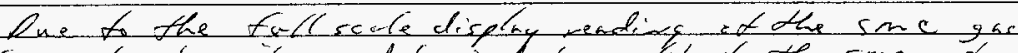

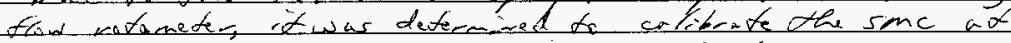

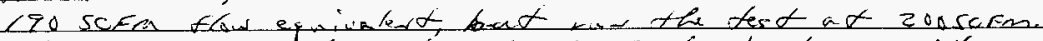
It is articipated that or la the tioni owtpat sped will te sightily a ffested kut witf the rire time. Tes of writ alsi

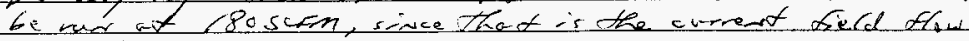
conclitions.

Cesting was termivaded elve to evel ofshift. 
128

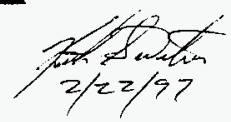

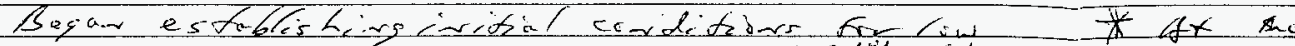

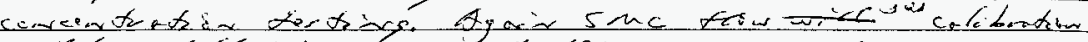

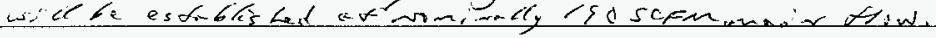

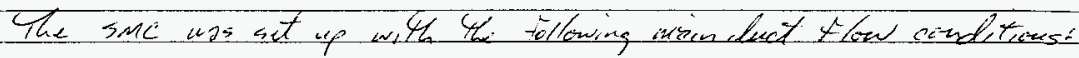

Ditf Press $=3.55$

A6s Press $=14.73$ Dant tamp $-65^{\circ} \mathrm{F}$

ilsing 9070 fpm Cat gas the suc wos callerated to read

c. $=$ So45 PPu with ral gas (8070 Ppa) Flowing and

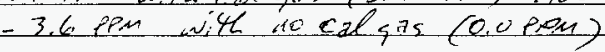

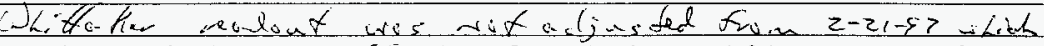

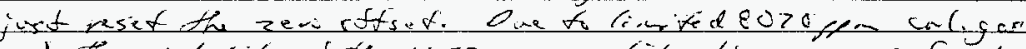

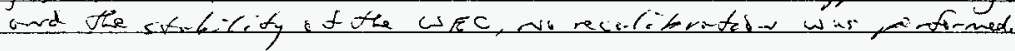

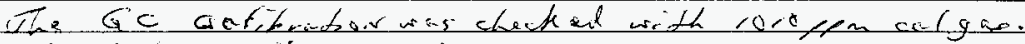
the $c_{1}+5$ pertings serex:

1009.363 fen, $2089.599,1010.336,1009.890$ and 1000.70 iffm

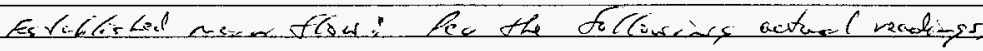

Zsegar ard the The dun The fi-t

$\dot{Q}_{s, n p l e f t}$ fro $r e$ EET-1 = $=3.75^{\circ}+120$

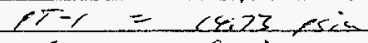
$T E-1=68 \%$ स्वृतs

$0.8 Y=$

Esteflished an injectis ffeur of wianiorelly d. 8 serem of

0.904

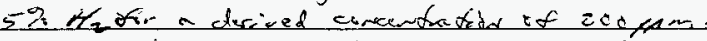

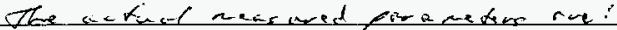

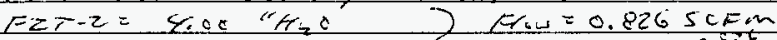

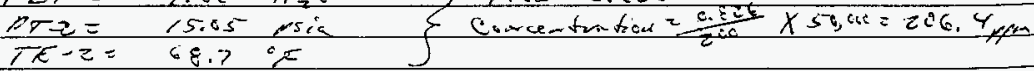

Sc reads $=2 / 8,0 \times m$ 


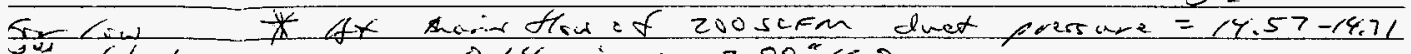
cols broten or $-0.144^{\circ} c^{\circ} a--3.88$ " $t_{2} 0$

in.

$x e^{2} z d$
189.6

Complated $200 \mathrm{Hm}$ ferting owd homed $H_{2}$ cil. gas

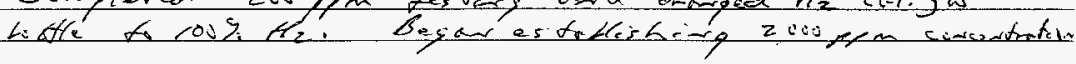
Ci-lovlated FAT-1 rending fix 2,000 pea =

react

a

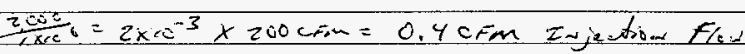

$$
0.4=x\left(\frac{18 \pi .57}{57.6}\right)=c \quad x=0.193 \mathrm{scFm}
$$$$
\frac{0.793}{1.6087} \times 8.0^{\circ} \mathrm{H} / 2 \mathrm{O}=0.960 \mathrm{~K} / 2 \mathrm{O}
$$

FIT-2 $=0.954 \longrightarrow F C u=0.39755 \mathrm{cFm}$

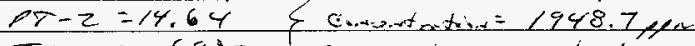

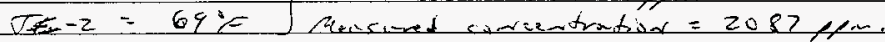

$-21-57-6 i k 6$ $\operatorname{coc} \cos$ ainimad

$\sec \operatorname{cgas}$.

10.0.70 ifem.

Creculis $\rightarrow 5$.

$50,00: 20.4,4,2$
Zeyar zos fen. The duct flow

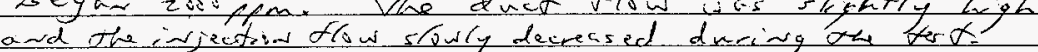

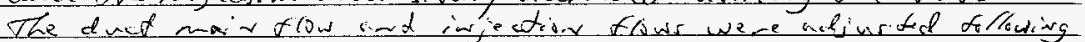

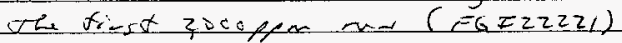

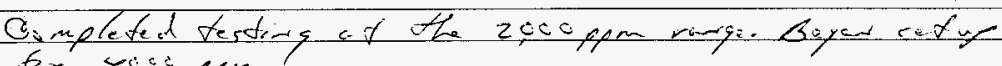
fex resisen.

$\frac{2308}{1 \times 0 c^{2}}=4.2 \times 10^{-3} \times 200 \mathrm{~cm}=0.845 \mathrm{cFm}$ irjection f/4

$$
0.8 y=x\left(\frac{787.87}{8.6}\right) \quad x=0.40465 \mathrm{cmin}
$$

Seren ef

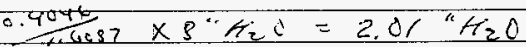

$$
\text { FIT }=2.00 \quad \text { Flw }=0.8315 \mathrm{cFm}
$$$$
P T=14.63\} \text { conientriatice }=4156
$$

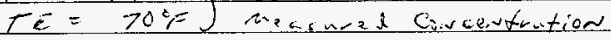

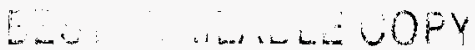


130

$$
\text { sid } 2-2 z-79
$$

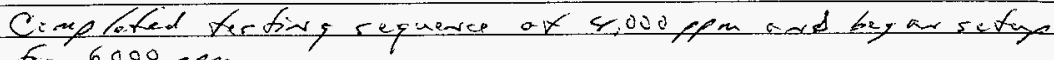
fer $6,000 \mathrm{ppm}$.

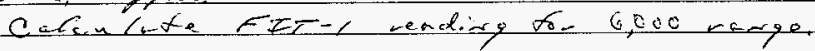
$\frac{6.2 \times 0^{3}}{1 \times 60^{3}}=6.2 \times 10^{-3} \times 2 i=c F M=1.245 \mathrm{cFm}$

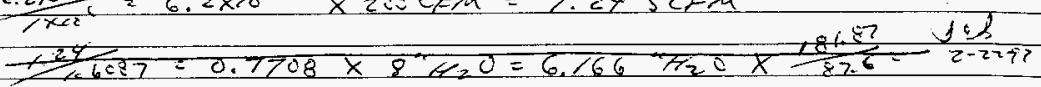

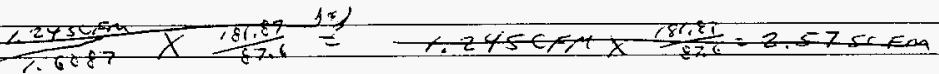

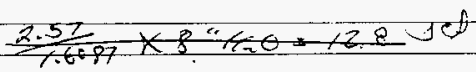

$1+24501=x\left(\frac{134.57}{876}\right)$

$$
K=0.59756 \pi
$$

$\overrightarrow{\mathrm{En}},+2$

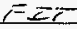
tpTE. Maine d $-0.63$

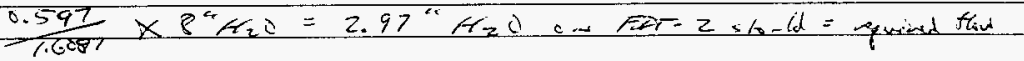
Setict UrGues. $=27-2=2.97 \mathrm{H} / 2 \mathrm{O}$

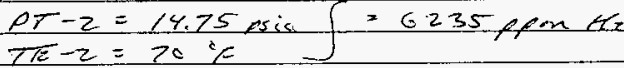
$6+1996500 \mathrm{~m} M$ Min

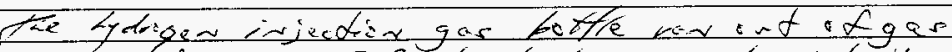

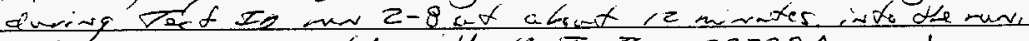
The ran was repated with the Zp $0222728 \mathrm{~A}$ and

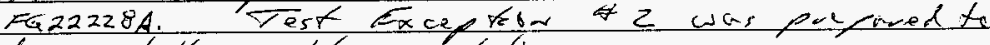
docament the publem resulution.

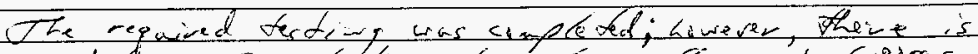

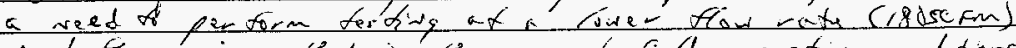

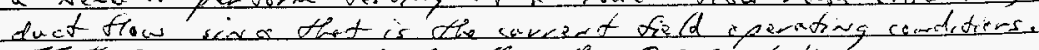

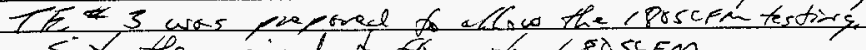

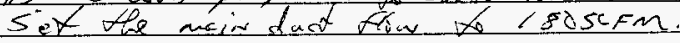

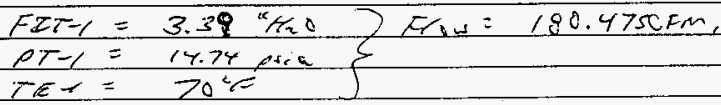




\section{$5 \sqrt{8}$
26
$2-2797$}

7 -
Establisted Sirjection Gas ffiw. for wimivally $4,000 \mathrm{Apm}$ on $x$ C C Fan.

Enjefitid Pormeders

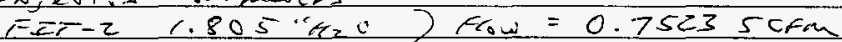

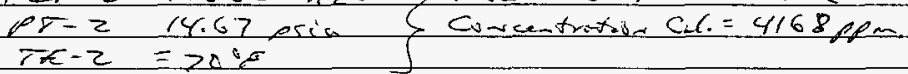

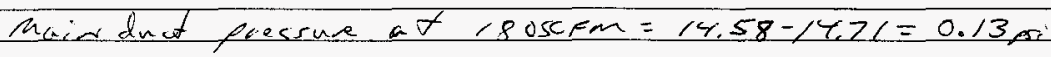
$-0.13,5 i=-3.6 " \% i c 0$

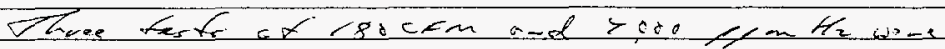

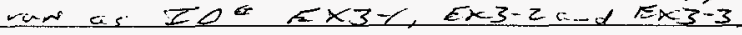

reviend fland

$19265 \mathrm{cem}$ Muiw
$1 \alpha \cos$

$-\alpha_{0}$ dL rune

ond

traped tc

私ent

(180ses)

is cemtidiers. 


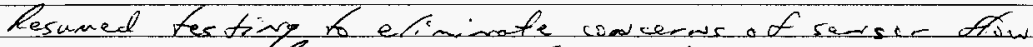
indicaters. norgs

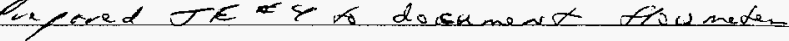

The $5 \mu \mathrm{f}$ with becatikested useivy the tisws rend by a vew mita netew $R M A-r 3(0-1000 \mathrm{spm})$ instarlel in place et tee RmA-C $(0-250 \mathrm{~cm})$.

The WEC and wewpint -eadsat with be zero

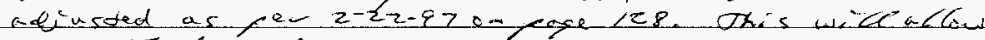

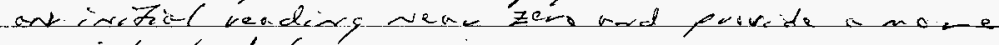
coursistort data comparsin

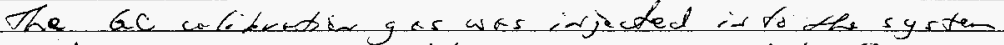

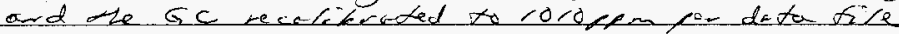
UTZCAL. DEE
S tetac a. $2-25-8$ ses expa Tley uac weed to

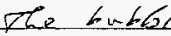
"spikes" the Ue kive Cis:tra buble a crigsers terof (sur (Note.' stionted ectont supte-ts

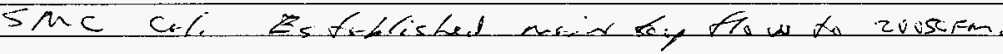
$F Z T=3.76 \% \%$

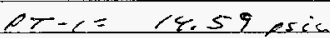 $T E-1=$ $70^{\circ} \mathrm{C}$

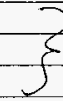

\section{Flow $=\angle 585 \mathrm{Fm}$}

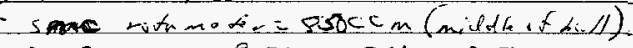 $0=2 \mathrm{ffm}$

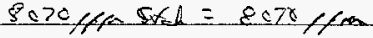

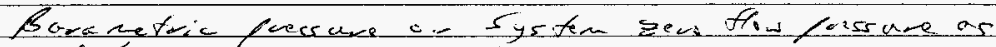

nacl ty $P T=2=14.55 / 5 i a$.

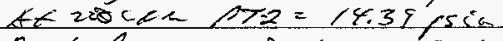

Orut Aressmee $=0.16$ psic. $z-4.432$ "4zo

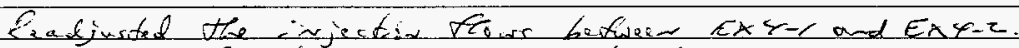

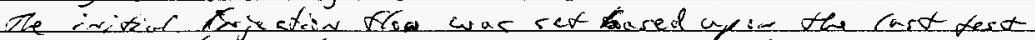

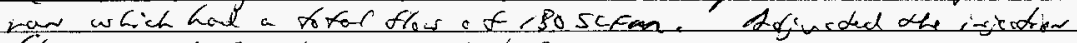

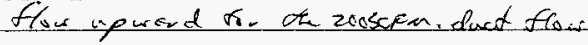

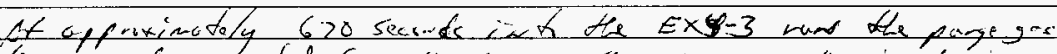

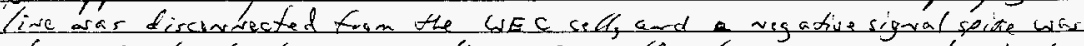

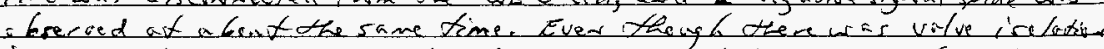

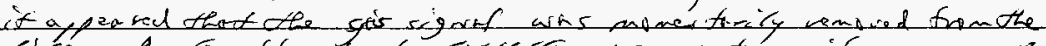

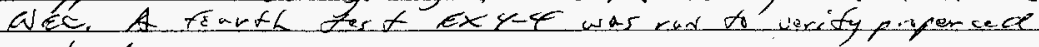
ontons. 


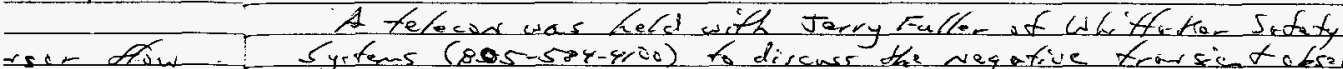
Asumedar

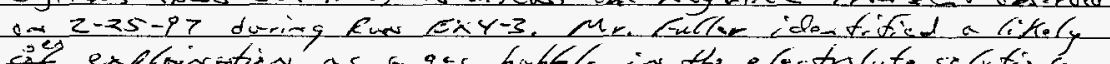

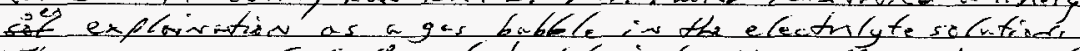

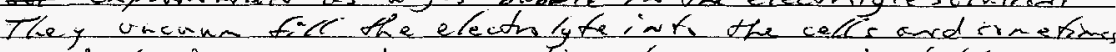

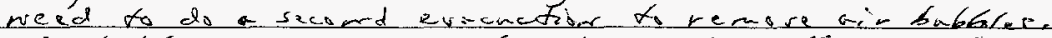
The bubler cruse are irstability in the cell in the firm if "spities" i- the entput signal M- Furte otso r foted Whet the suggested it ariet trtior tre the cell in wiemal

Zisus read $\therefore=5 d x h e d$

$z e \cdot r$

Crablaw

a $m$ are

$5 y \leq x e$

sice

$200 \mathrm{seF}$

$m($ maitdle:f(i./1)

$i=807 \mathrm{ftm}$

-are as

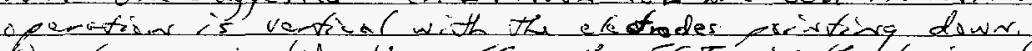

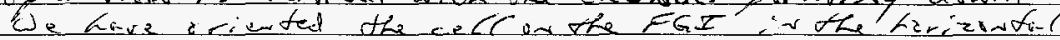

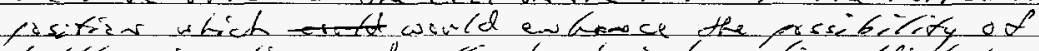

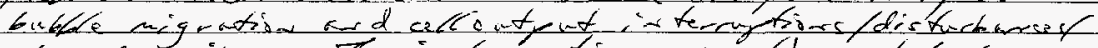
rigred spiths. The intercyptejers wauld uet/ast

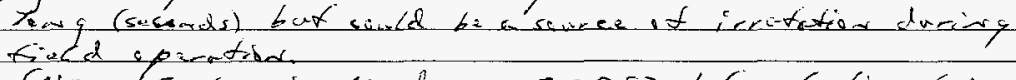

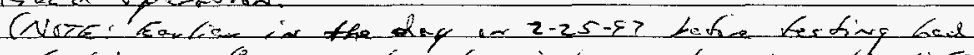

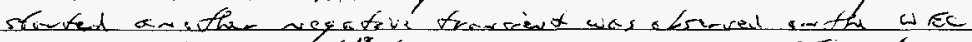

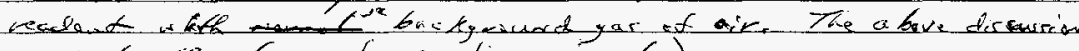
suppets tio chrerved vegative regaral.)

dEAYr.

$\cos \alpha \operatorname{tes} \alpha$ dhe isisction

Z2.

the fingegos -

ignal seite wis s vilue irelation.

in from the

figfon $c . c l$

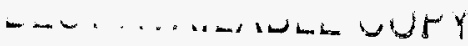


HNF-SD-WM-TRP- 275

Rev, 0

Page 293

APPENDIX F - SUPPORTING DOCUMENTATION 


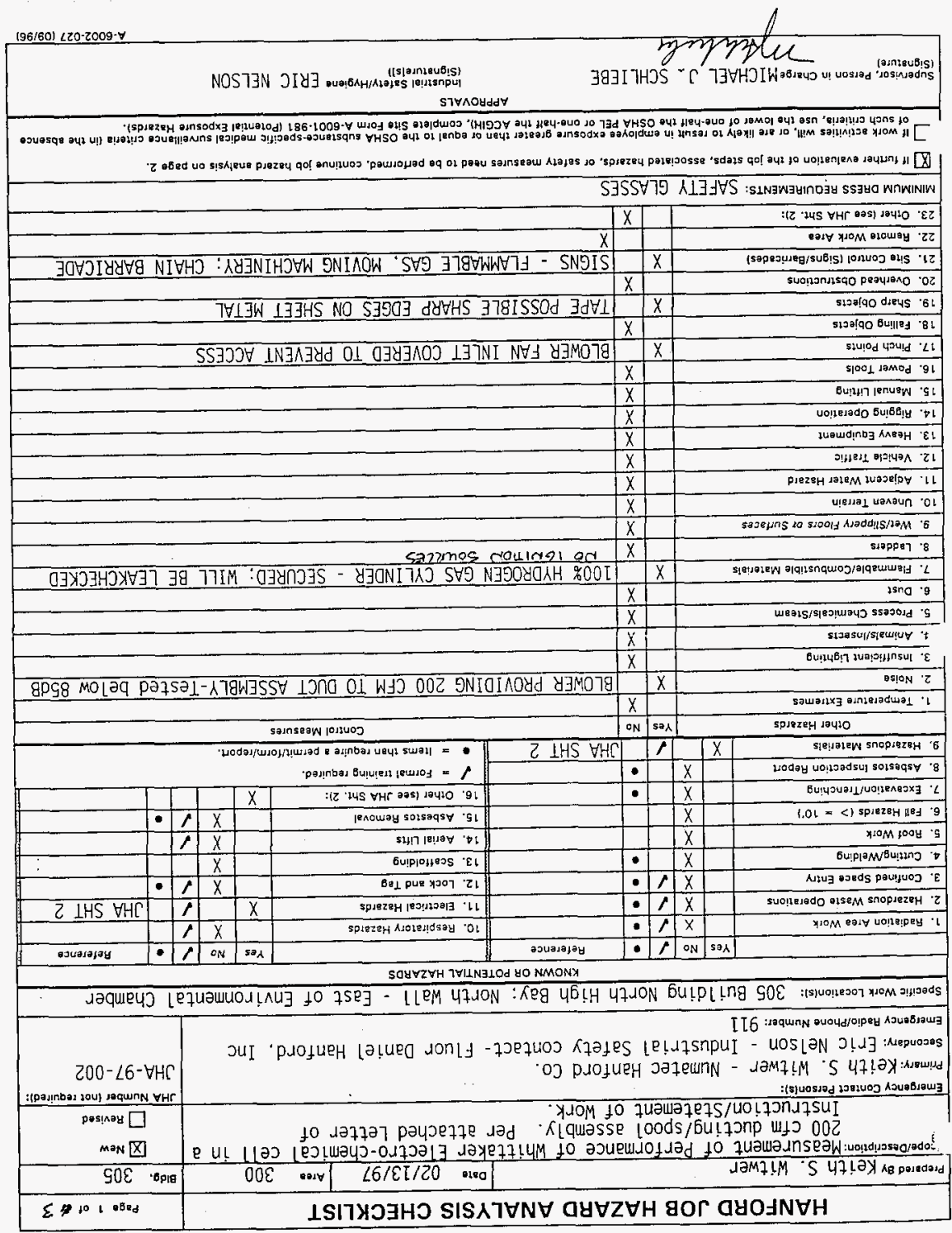

$h b z \cdot{ }^{20} \mathrm{ed}$

0 'say

SLZ-dVI-WM-IS- - NNH 


\begin{tabular}{|c|c|c|}
\hline \multicolumn{3}{|c|}{ HANFORD JOB HAZARD ANALYSIS (Continuation Sheet) } \\
\hline $\begin{array}{c}\text { Sequence of Basic Job Steps } \\
\text { or Work Activity }\end{array}$ & Hazards Present & How to Eliminate Hazards \\
\hline $\begin{array}{l}\text { 1.0 Pre-startup: } \\
\text { Configuration and Assembly of } \\
\text { Test Apparatus }\end{array}$ & $\begin{array}{l}\text { A) Control Voltages (24vdc) } \\
\text { B)Equipment Line Voltages. } \\
\text { (110vAC) } \\
\text { C) Pressurized Flammable Gas } \\
\text { Cylinder-gas feed lines - } \\
\text { up to initial isolation } \\
\text { valve. } \\
\text { D) Air Ducting w/ } 200 \mathrm{cfm} \\
\text { air flow }\end{array}$ & $\begin{array}{l}\text { A) Insure power off when making connections to equipment. } \\
\text { B)Insure power cords are in good condition and insure no } \\
\text { standing water near cord. } \\
\text { C) Use proper gas cylinder procedure when moving/storing } \\
\text { cylinders. Use proper cylinder holders when stored. } \\
\text { Insure no flame source is nearby and insure lines are } \\
\text { leakproof (using soap test) before starting test. } \\
\text { D) Use caution when working around blower and insure } \\
\text { others working nearby are aware of blower inlet point. }\end{array}$ \\
\hline 2.0 Startup sequence & Pressurized gas (flammable) & $\begin{array}{l}\text { Insure outlet lines are tight and pressure regulator is of } \\
\text { proper type and functioning properly. }\end{array}$ \\
\hline 3.0 Operations sequence & $\begin{array}{l}\text { A) Blower Fan } \\
\text { B)Potentially flammable gas } \\
\text { source (when exposed to } \\
\text { atmosphere) } \\
\text { C)Flammable Gas }\end{array}$ & $\begin{array}{l}\text { A)Chain barricade will be up around fan. } \\
\text { B)Sign stating "Caution - Flammable Gas - No Sparks or } \\
\text { Open Flames" will be posted. } \\
\text { C)Use Gas chromatograph unit to verify concentration is } \\
\text { below LFL - shut off gas flow if not. } \\
\text { Purge gas line immediately downstream of gas solenoid } \\
\text { control valve with nitrogen after test. } \\
\text { Gas insertion point positioned in a } 200 \text { SCFM flow stream } \\
\text { will provide rapid dilution to below LFL ( } 4 \% \\
\text { hydrogen)after mixing. } \\
\text { Air flow ducting near and downstream of flammable gas } \\
\text { insertion point will be electrically tied to building } \\
\text { ground. Flammable gas solenoid valve will shut off if ma' } \\
\text { air flow is lost - via air flow switch. }\end{array}$ \\
\hline
\end{tabular}

G: IKEITH'SILETTERSIWEC_JHA2. FRM 


\begin{tabular}{|c|c|c|c|}
\hline \multicolumn{3}{|c|}{ HANFORD JOB HAZARD ANALYSIS (Continuation Sheet) } & Page 3 of 3 \\
\hline $\begin{array}{c}\text { Sequence of Basic Job Steps } \\
\text { or Work Activity }\end{array}$ & Hazards Present & \multicolumn{2}{|c|}{ How to Eliminate Hazards } \\
\hline 4.0 Shutdown sequence & $\begin{array}{l}\text { A)Pressurized Gas } \\
\text { (flammable) } \\
\text { B)Residual H2 in Ducting }\end{array}$ & \multicolumn{2}{|c|}{$\begin{array}{l}\text { A) Insure gas cylinder are properly closed before } \\
\text { disconnecting gas lines } \\
\text { B)Purge gas line immediately downstream of gas solenoid } \\
\text { control valve with nitrogen after test. }\end{array}$} \\
\hline 5.0 Emergency procedures & $\begin{array}{l}\text { A)Gas line leak } \\
\text { B)Gas cylinder rupture }\end{array}$ & \multicolumn{2}{|c|}{$\begin{array}{l}\text { A)Frequently monitor gas flow - if excessive flow. is } \\
\text { observed - shut off gas supply } \\
\text { B) Mount cylinder in position where catastrophic failure } \\
\text { cannot harm personnel - call } 911 \text { if accident occurs. }\end{array}$} \\
\hline 6.0 Cleanup and dismantling & $\begin{array}{l}\text { A) Damage to Pressurized Gas } \\
\text { cylinder when moving to } \\
\text { cylinder dock. } \\
\text { B)Electrical hazards }\end{array}$ & \multicolumn{2}{|c|}{$\begin{array}{l}\text { A) Use proper gàs cylinder handling procedure } \\
\text { B) Power down equipment before dismantling. }\end{array}$} \\
\hline
\end{tabular}

G: KKEITH'SILETTERSIWEC_JHA2.FRM 
Meeting Description Pre Job Meeting for Whittaker Cell / Spool Piece Testing

Results Desired insure attendees are aware of hazards. control points. site safety info, etc

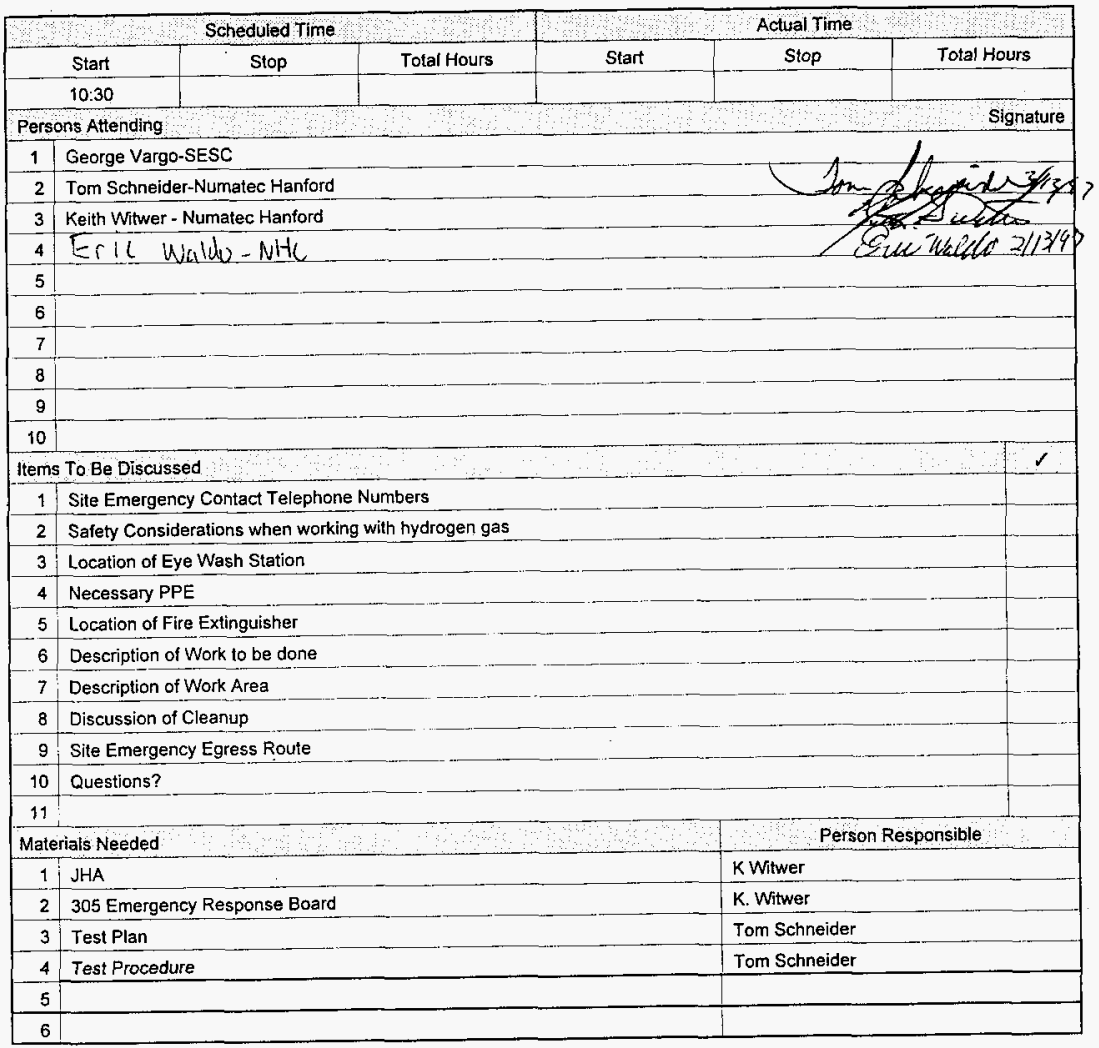




\subsection{SCHEDULE}

In order to support the earliest deployment of the RMCS Truck 4, the assembly of the test equipment will begin during the preparation of this test plan/procedure. The testing will begin as soon as the plan/procedure is approved and any modifications to the test assembly arrangement has been made. The test report will be prepared and released as soon as the test data has been compiled and evaluated.

\subsection{QUALITY ASSURANCE}

Quality Assurance will review the documentation associated with this testing as part of the review process. There are no QC hold points or verifications required for this testing.

\subsection{SAFETY}

Safety will review the test $p l a n$ and procedure as part of the review process. A Job hazards Analys is will be prepared as a part of this testing program and will be included in the test report. All work will be performed under the guidel ines of WHC-CM-1-10, Safety Manual, WHC-CM-1-11 and WHC-CM-440 , Industrial Hygiene Manuals.

\subsection{TEST PROCEDURE}

\subsection{Test Prerequisites}

Prior to the initiation of testing the following conditions shall be verified:

10.1.1 The job hazards analysis (JHA), for the performance of this testing in $305 \mathrm{ETL}$ /has been prepared and approved.

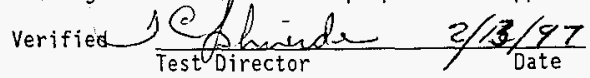

10.1.2 The information for all instruments and test equipment has been recorded in Appendix $A$.

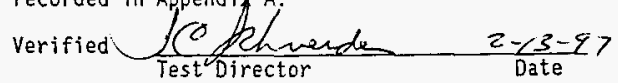

10.1.3 All bottled gases initially required for the testing have been staged. The information for all gas bottles has been recorded in Appendix B.

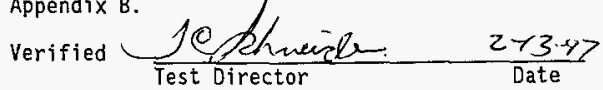


10.1.4 The test apparatus has been assembled, configured, connected and initially verified operational, according to FIGURE $I$ and Appendix A. Verify that the exhaust ducting has been routed outside of the building.

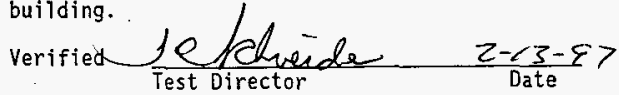

10.1.5 The hydrogen injection piping has been pressure tested, to 20 psig, for leaks using a soap bubble solution (Snoop) and documented in the tegt $\log$.

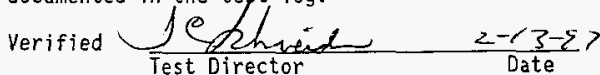

10.1.6 A pretest safety briefing has been held with the testing personnel and recorded in the test log. The briefing will be conducted by the 305 ETL cognizant engineer.

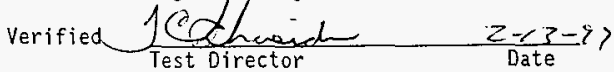

\subsection{Test Procedure}

If any steps in this section cannot be performed as required or results in an abnormal condition, then prepare a sequentially numbered Test Exception Record in Appendix $D$ and a log entry identifying the problem. The resolution of the Exception will determine what testing, if any will need to be repeated.

The testing may be suspended at any time at the request of the 305 ETL facility manager, cognizant engineer or test director when an unsafe condition exists that could harm the facility, equipment or personnel.

10.2.1 Test Equipment Calibration

Calibrate the test equipment in preparation for testing.

10.2.1.1 Verify that the Whittaker hydrogen monitoring system is calibrated per the directions in Appendix F-2.

10.2.1.2 Verify that the SMC combustible gas monitor is calibrated per the directions in Appendix F-1.

10.2.1.3 Verify that the $G C$ system is calibrated per the directions in Appendix $\mathrm{F}-3$.

10.2.2 Low Hydrogen Copcentration Testing

The low hydrogen concentration testing will use hydrogen injection standard gas that is below the lower flamability limit of hydrogen. This testing is to determine if the sensors will detect a low concentration of hydrogen present in the flow duct. 
Fuor Daniel Northwest, Ing.

P.O. Bax toto

Rilehtend, tah os35-1050

$\begin{array}{ll}\text { To: } & \text { Ruskin } \\ & \text { Fax (816)765-8955 }\end{array}$

APPLICATION FOR PERMISSION TO YSE YOUR COPYRIGHTED MATERIAL

Dato: March 24, 1997

Permisaion is requested to reproduce the following copyrighted matalal from:

Catalog cut sheats for CDRr92 Heavy Duty Round Isolation Damper. Spec CDRI92-894. Seloctions from text (speolfy by dote of lesue, pege, peregraph, or Ilustratton; ff decired, attach a copy of the materid in queation):

Title of work or project in which thle moteidel wis bo included:

Letter Report, "340 Vault XI Exhaust System HEPA F17tor Evaluation", prepared by Fluor Dante] Northwest, Inc. for Rust Foderal Services of Hanford, Inc.

Estimated publloation data: March 1997 : Authar. , TIa E. Arndt

Publishor (if applicabie): $n / a$

If the copyighted material is not to bo ased in a publighed work, ploese provide a butel dacoripton of how it is to be usad:

Copyrighted material wtll be included in the engtneering letter report listed above. The engineering study will be copled for the customer and records managament archives.

PLEABE REPLY A8 8OON A8 POBBIBLE VIA FAX AT 509-373-0313.

Name; Lori B. Weidnur

signature: Lovi B. Lleidnen

MatN: 63-17 Date: March 24, 1997

Fupr Danied Northwest, Ine.

Credit line (if required):

NA

Application epproved by:

Name:

T.w. Arnoce

Company Nama:

Signature:

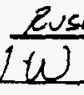

skin $x \frac{f}{5}$

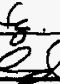

Date: $\quad 3-24-97$

Signatory' Podition: Project Manager (on bahalf of) 
Fluor Denied Nortinwest, lne. P.c. $\operatorname{cox} 1050$ Richiand, im sess2-10s0

\section{APPLICATION FOR PERMISSION TO USE YOUR COPYRIGHTED MATERIAL}

Ta: Flanders Filters, Ine.
Date: March 21, 1997

Permiasion is requeated to reproduce the foltowing copyrighted material from:

Catalog cut sheets: Dimensions and Test Section functions for E-Series or NBC-Series Housings". "Selection Table, Test Sections for E-Sertes or NBC-Series Housings", "Qualified Products List", "Notes on Filter Salection", and "Standard Sizes and Capacities".

Solactions from sext lapecify by date of lasue, page, paragraph, or illustration; if destred, attaoh a copy of the materiel in (uestion);

Title of woik or project in which this maserial will be includad:

Lattar Report, "340 Vault KI Exhaust System HEPA Fqlter Evaluation", prepared by Fluor Daniel Northwest, Ine. for Rust Federal Services of Hanford, Inc.

Estimatod publication date: March 1997

Author: Tim E. Arndt

Publisher (if applicable): $n / a$

If the copyrighted material is not to be usod in a publiahed work, plase provide o briof dascription of how it is to be used:

Copyrighted materiat will be included in the engineering letter report listed above. The engineering study will be copled for the customer and records managenent archives only.

\section{PLEASE REPLY AS SOON AS P088IBLE VIA FAX AT 609-373-0313.}

$\begin{array}{ll}\text { Name: Tim E. Arndt } & \text { MSIN: G3-17 Date: March 21, I997 } \\ \text { Signawre: } & \text { Wattinghoure Henford Company }\end{array}$

Credit line (if requirad):

Application approved by:

Name: GLeN W. Moore

Company Name: FlandERS/CSC

Signabure:

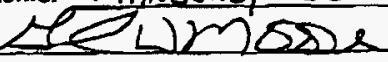

Dato: MARCh $24 \quad 1997$ signatory's Posicion: Mratroting
(on bohal of) 


\section{DISTRIBUTION SHEET}

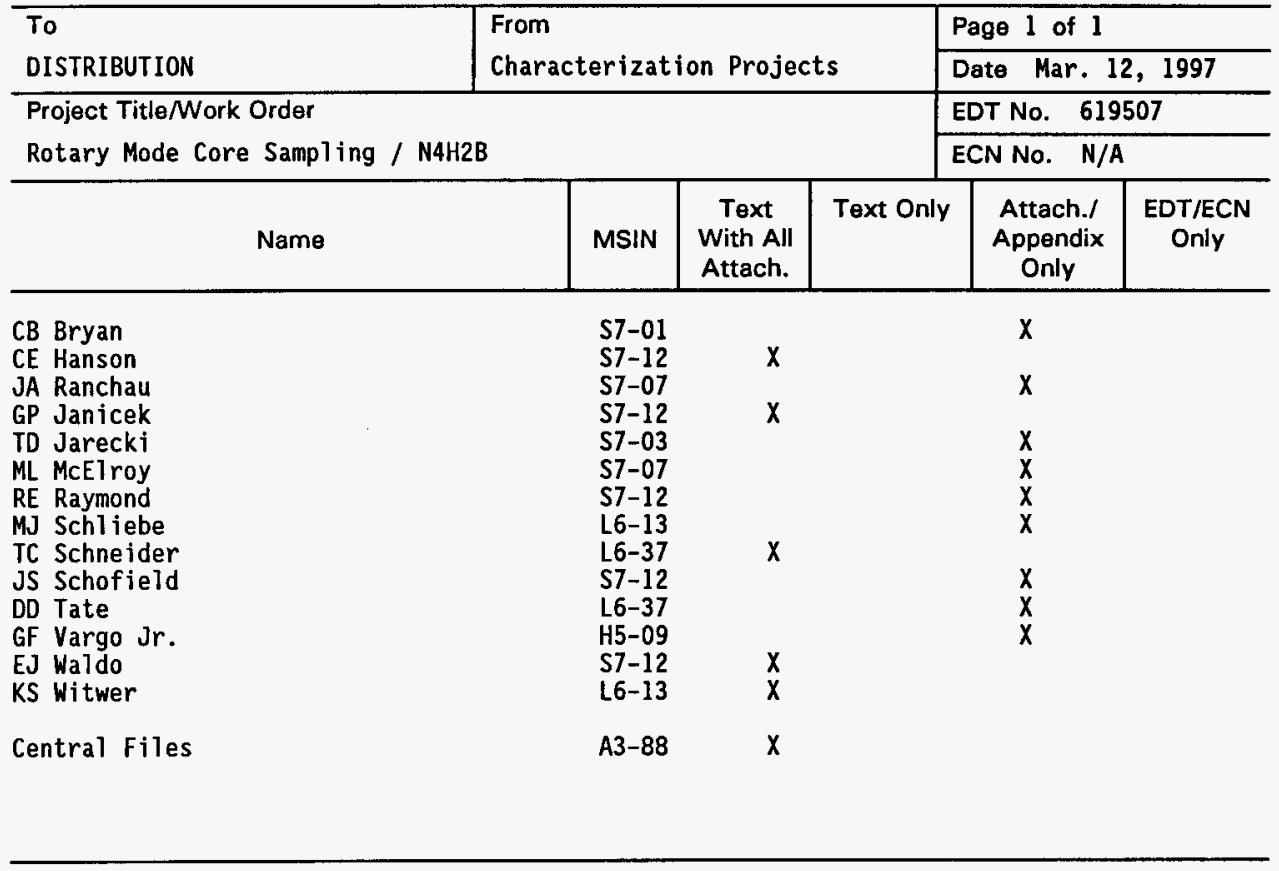

